\title{
Verbindungen von Molybdän und Wolfram in den Oxidationsstufen IV - VI als Modelle für Molybdän-und Wolfram-Cofaktoren
}

\author{
Dissertation
}

Alexander Döring

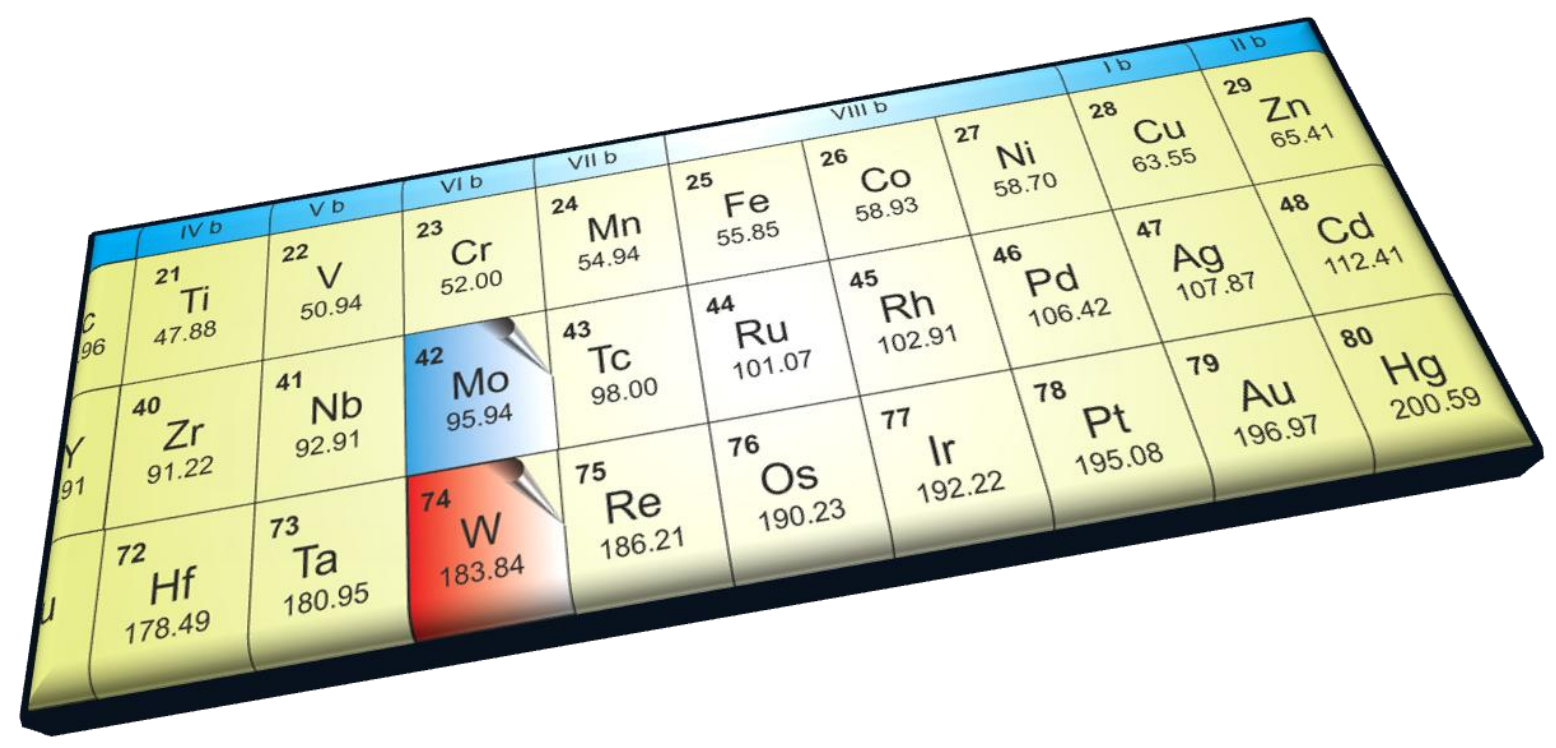

Georg-August-Universität Göttingen 


\title{
Verbindungen von Molybdän und Wolfram in den
}

\section{Oxidationsstufen IV - VI als Modelle für}

\section{Molybdän-und Wolfram-Cofaktoren}

\author{
Dissertation \\ zur Erlangung des Doktorgrades \\ der Mathematisch-Naturwissenschaftlichen Fakultäten \\ der Georg-August-Universität zu Göttingen
}

vorgelegt von

Diplom-Chemiker

Alexander Döring

aus

Kassel

Göttingen 2010 

D7

Referent: Prof. Dr. Carola Schulzke

Korreferent: Prof. Dr. Oliver Wenger

Tag der mündlichen Prüfung: 11.10.10 
Die vorliegende Arbeit entstand im Zeitraum von Juni 2007 bis Juni 2010 unter Anleitung von Frau Prof. Dr. Carola Schulzke am Anorganisch-Chemischen Institut der Georg-AugustUniversität Göttingen. 
für Kerstin 


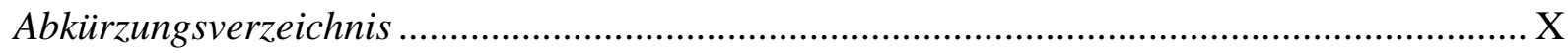

Verwendete Dithiolenliganden und Koordinationsweise zu einem Metall ..............................II

Verwendete Nicht-Dithiolenverbindungen ...........................................................................

Kapitel 1 - Biologischer Hintergrund .................................................... 1

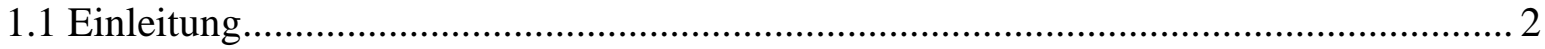

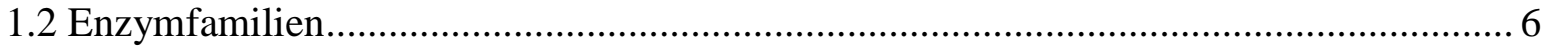

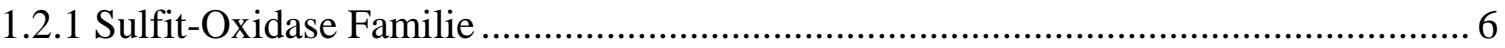

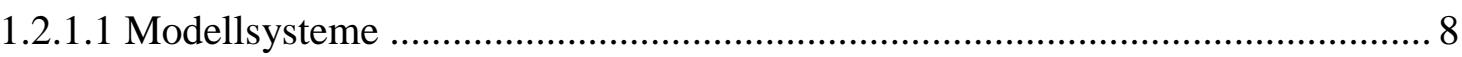

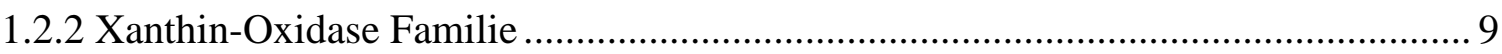

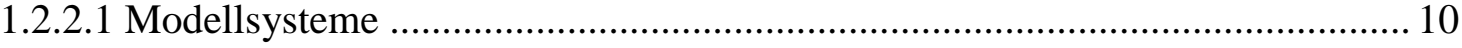

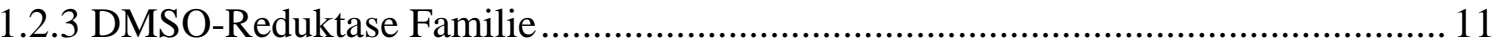

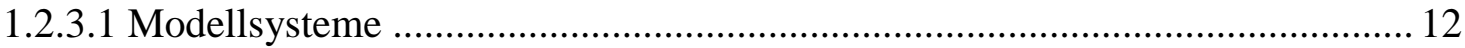

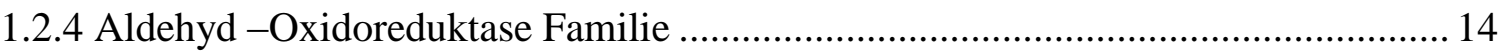

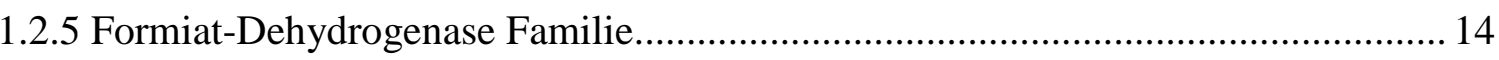

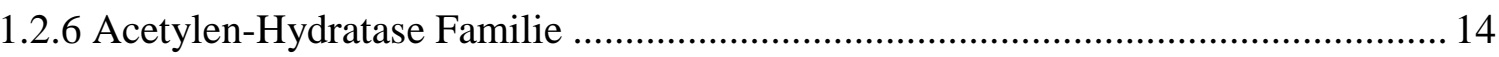

1.2.7 Modellsysteme für Wolframenzyme.................................................................. 15

Kapitel 2 - Synthesestrategien und Methoden ................................................ 16

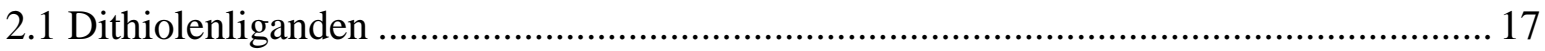

2.2 Verwendete Methoden zur Herstellung von Dithiolensystemen .................................. 18

2.2.1 Dithiolensysteme aus ketonhaltigen Verbindungen........................................... 18

2.2.2 Dithiolensysteme aus Dibromierten Verbindungen ........................................... 22

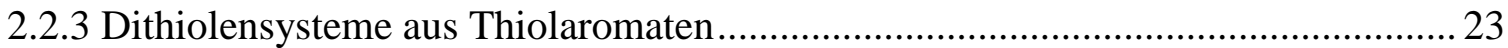

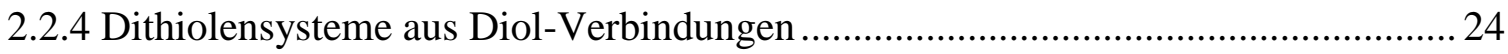

Kapitel 3 - Modelle für Mo- und W-Cofaktoren ............................................. 25

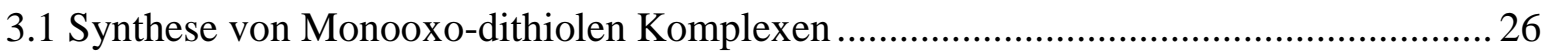

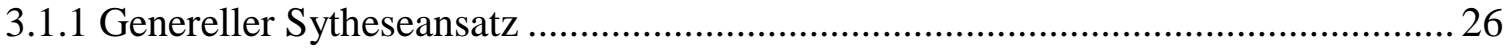

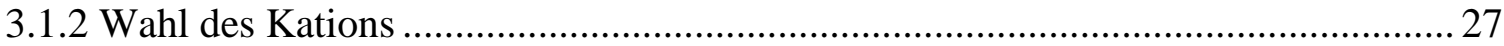

3.2 Synthesen von Modellkomplexen für die DMSO-Reduktase Familie .......................... 28

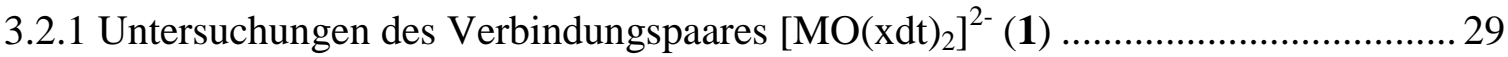

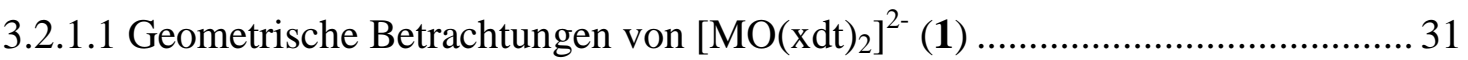

3.2.1.2 Herstellung von Verbindungen in Oxidationsstufe $\mathrm{V}-\left[\mathrm{MO}(\mathrm{xdt})_{2}\right]^{-}(\mathbf{1 c})$.......... 33

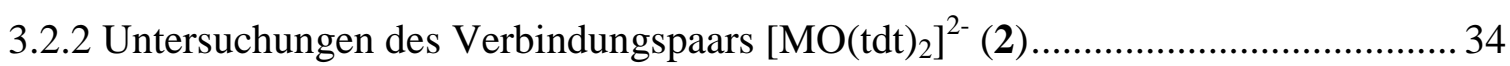

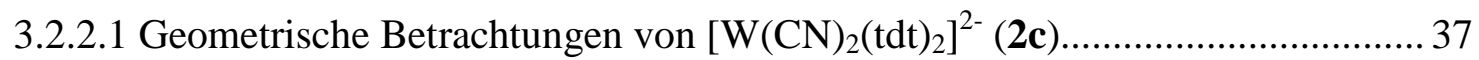

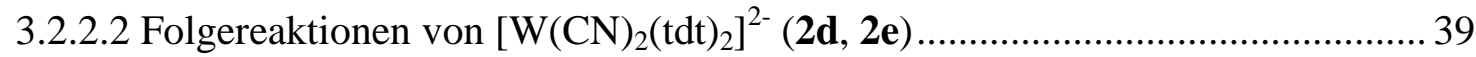




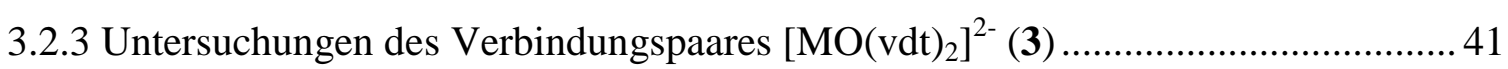

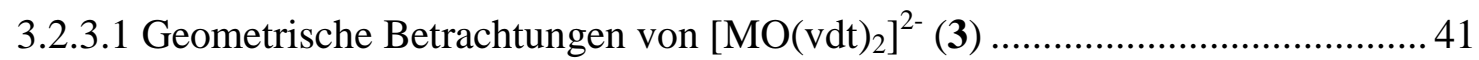

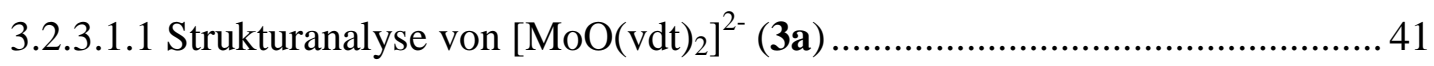

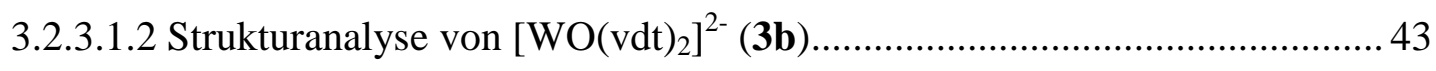

3.2.3.1.3 Herstellung von Verbindungen in Oxidationsstufe V - [WO(vdt $\left.)_{2}\right]^{-}(3 \mathrm{c})$.. 45

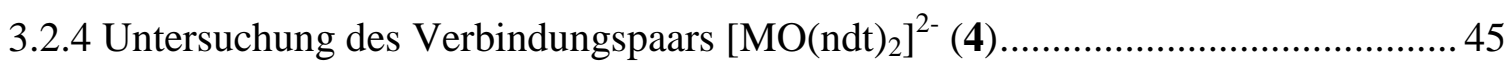

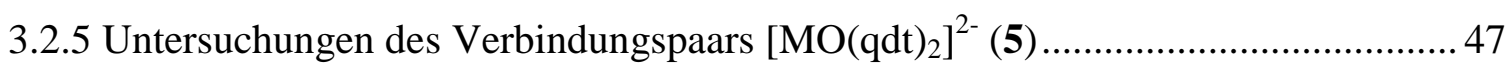

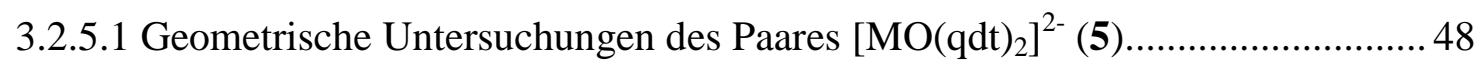

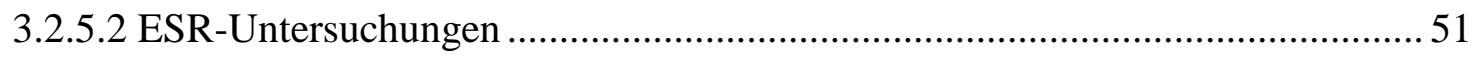

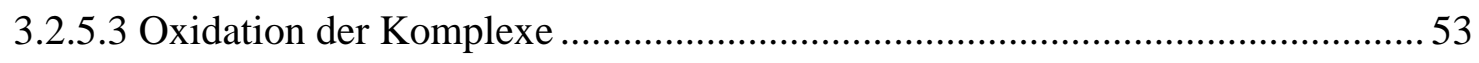

3.2.6 Vergleich der Verbindungen mit aromatischen Dithiolenligandensystemen .......... 55

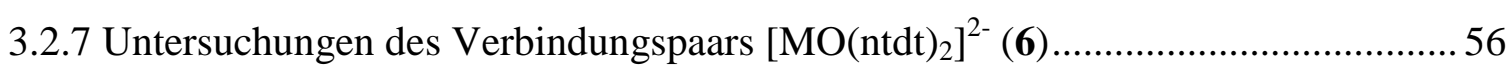

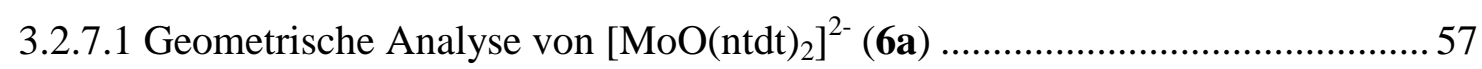

3.2.8 Verbindungspaare mit verschiedenen Ringatomen in Nachbarschaft zur

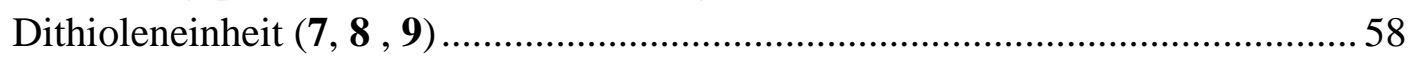

3.2.9 Synthese von Verbindungen mit nicht aromatischen Dithiolensystemen (10) ........59

3.3.1 Modellkomplexe für die reduzierte Form der DMSO-Reduktase Familie.................. 62

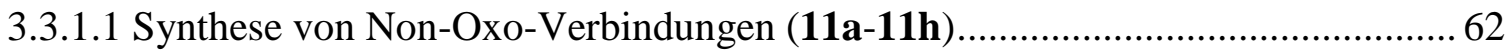

3.4 Synthese von Modellsystemen für die reduzierte Form der Xanthin-Oxidase Familie . 66

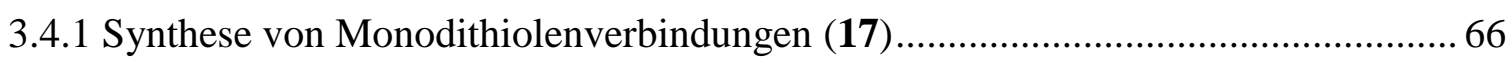

3.4.1.1 Herstellung von unsymmetrischen Dithiolenkomplexen (18/19) ..................... 68

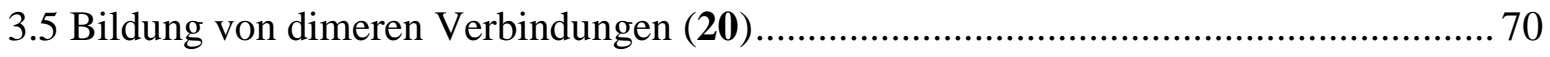

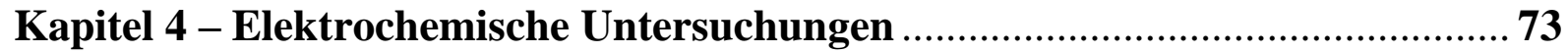

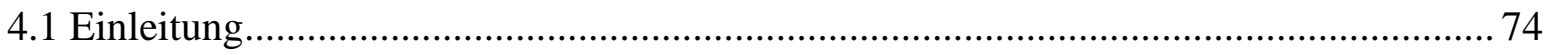

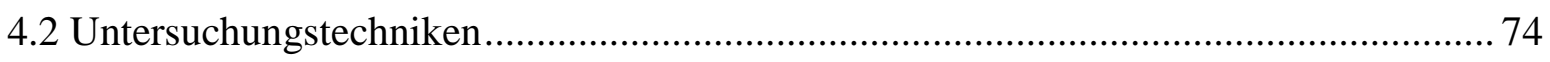

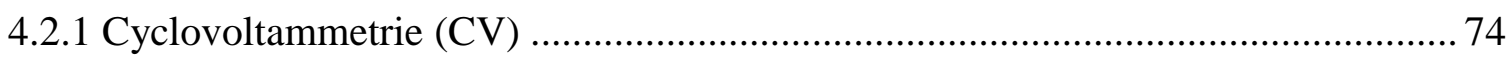

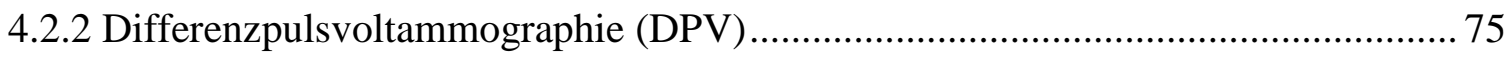

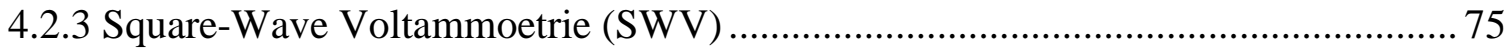

4.3 Temperaturabhängige elektrochemische Messungen ............................................. 77

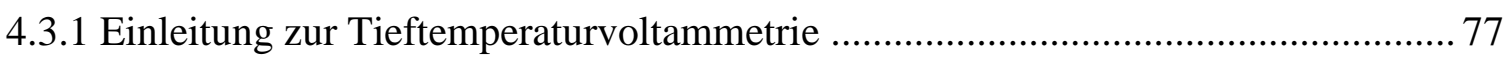

4.3.2 Beeinflussende Faktoren der elektrochemischen Messungen................................. 77

4.3.2.1 Zeitraum in dem der Messzyklus aufgenommen wird .................................. 77

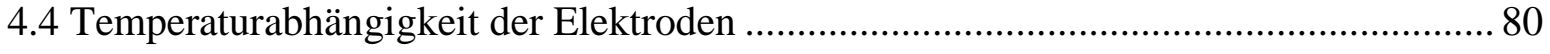

4.4.1 Temperaturabhängigkeit der Silber/Silberchlorid-Elektrode................................ 81 


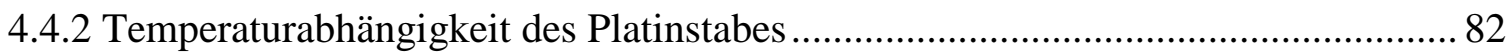

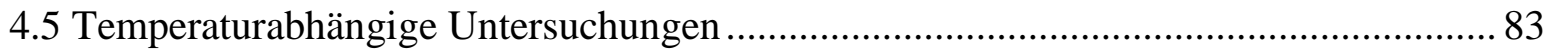

4.6 Unterschiede zwischen Molybdän und Wolframverbindungen ................................... 88

4.7 Geometische Änderungen während des Redoxprozesses........................................... 89

4.8 Weitere Beeinflussungen der Redoxpotentiale..................................................... 90

4.8.1 Beeinflussung der Potentiale durch das Lösungsmittel ......................................... 90

4.8.2 Beeinflussung durch den Umgebungsdruck .................................................... 90

4.8.3 Beeinflussung durch Messgeschwindigkeit und Konzentrationen ......................... 91

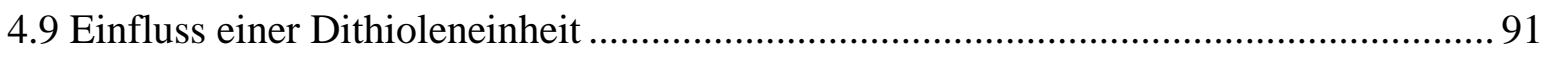

4.10 Wirkung von unsymmetrisch substituerten Dithiolenkomplexen auf die

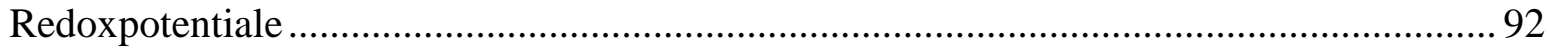

4.12 Wirkung von wechselnden Ringatomen im Ligandensystem ................................... 94

Kapitel 5 - Theoretische Untersuchungen................................................96

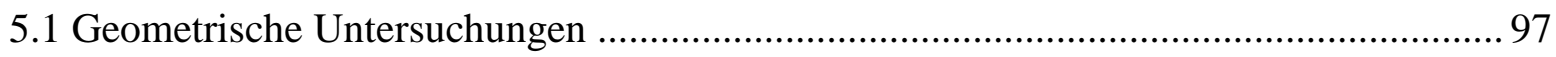

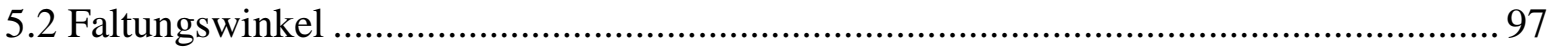

5.3 Elektronische Verhältnisse in den Komplexen ........................................................... 99

5.3.1 Vergleich der Verbindungen mit aromatischen Dithiolenverbindungen ............... 100

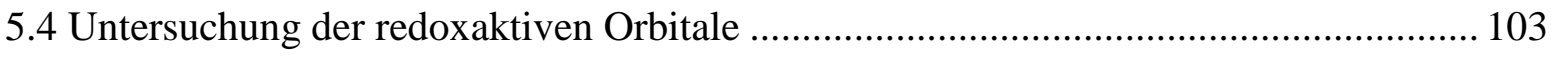

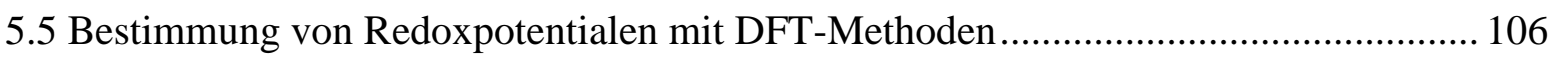

5.5.1 Möglichkeiten zur Bestimmungen von Redoxpotentialen .................................. 106

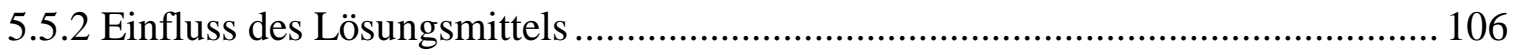

5.5.3 Referenzierung der berechneten Parameter........................................................ 107

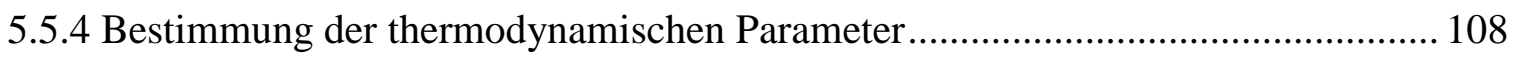

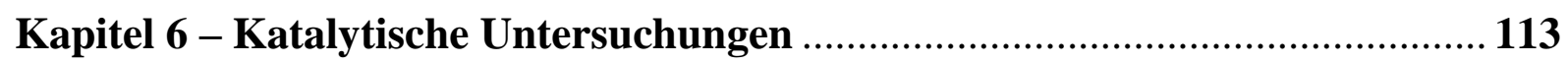

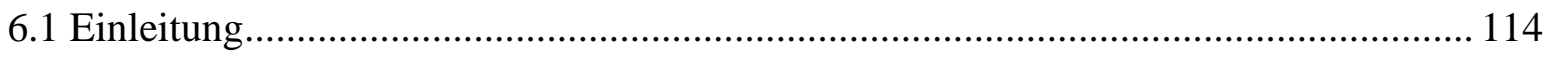

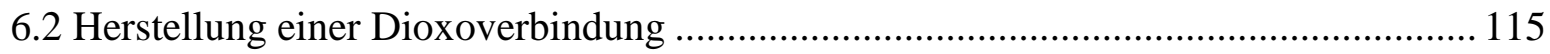

6.2.1 Vergleich der verschiedenen Sauerstoffübertragungen ..................................... 118

6.3 Geometrische Aspekte von Dioxo-Verbindungen...................................................... 119

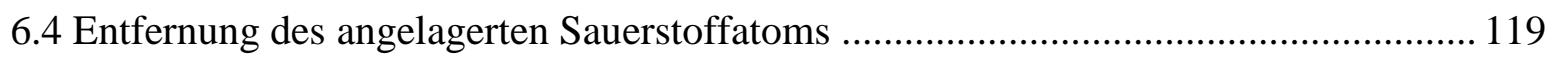

6.5 Temperaturabhängige katalytische Untersuchungen ............................................. 121

6.5.1 Untersuchung der Paare $\left[\mathrm{MO}(\mathrm{xdt})_{2}\right]^{2-} ;\left[\mathrm{MO}(\mathrm{vdt})_{2}\right]^{2-}$ und $\left[\mathrm{MO}(\mathrm{qdt})_{2}\right]^{2-}(\mathbf{1}, \mathbf{3}, \mathbf{5}) \ldots 121$

6.5.2 Bestimmung der kinetischen Parameter........................................................... 122

6.6 Untersuchungen des katalytischen Zyklus mit Hilfe von DFT-Rechnungen ............... 124

6.6.1 Untersuchungen zur Anlagerung des Sauerstoffatoms ..................................... 124 
6.6.2 Untersuchungen der Übertragung des Sauerstoffatoms auf $\mathrm{PR}_{3}$

6.7 Konzentrationsabhänge katalytische Untersuchungen

6.7.1 Untersuchungen der katalytischen Sauerstoffübertragung von $\left[\mathrm{MO}(\mathrm{ntdt})_{2}\right]^{2-}(\mathbf{6}) .129$

6.7.2 Einfluss auf die katalytische Aktivität durch bestimmte Ringatome

6.7.2.1 Untersuchung der katalytischen Sauerstoffübertragung $\left[\mathrm{MO}(\mathrm{tldt})_{2}\right]^{2-}(7) \ldots \ldots 133$

6.7.2.2 Untersuchung der katalytischen Sauerstoffübertragung $\left[\mathrm{MO}(\mathrm{tcdt})_{2}\right]^{2-}(\mathbf{9}) \ldots . . .135$

6.7.2.3 Vergleichende der Katalysen mit $\left[\mathrm{MO}(\mathrm{tldt})_{2}\right]^{2-}$ und $\left[\mathrm{MO}(\mathrm{tcdt})_{2}\right]^{2-}(\mathbf{7}, \mathbf{9}) \ldots \ldots . .137$

6.8 Katalytische Untersuchungen von Non-Oxo-Metall(IV)komplexen (11a, 11b).......... 138

6.9 Katalytische Einfluss durch eine Dithioleneinheit (17) ........................................ 141

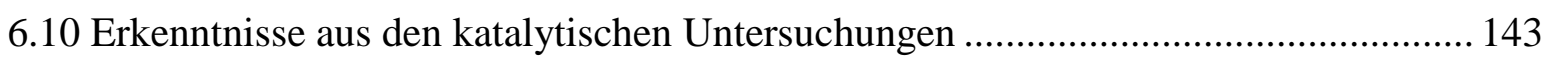

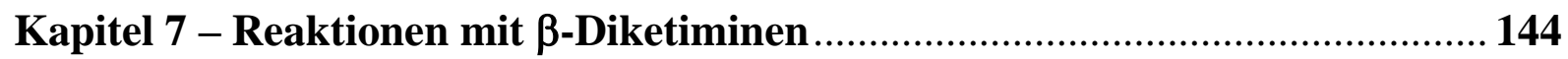

7.1 Einleitung

7.2 Reaktionen mit $\beta$-Diketimin-Komplexen mit Molybdän- und Wolframverbindungen 145

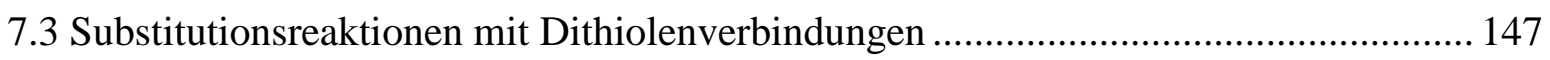

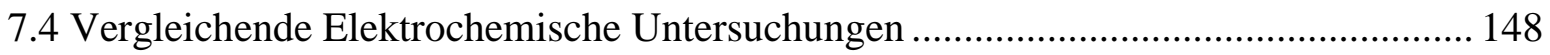

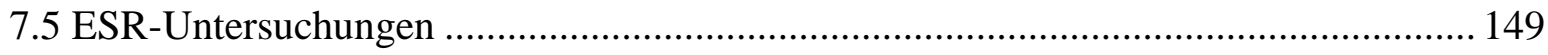

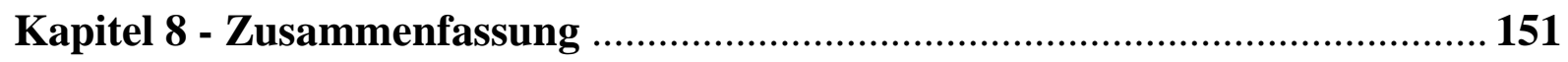

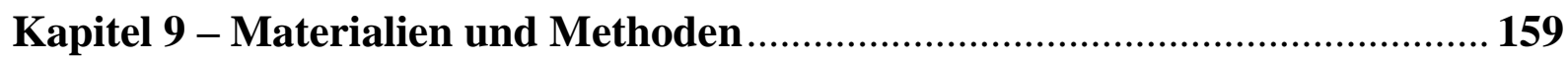

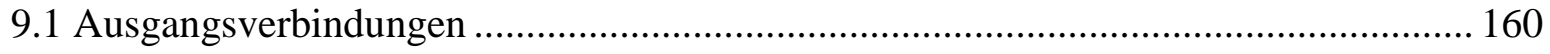

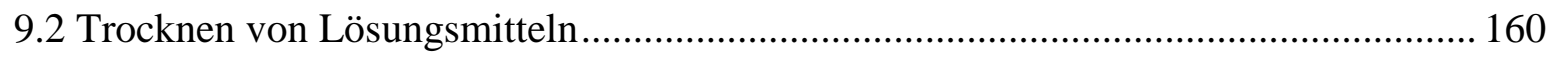

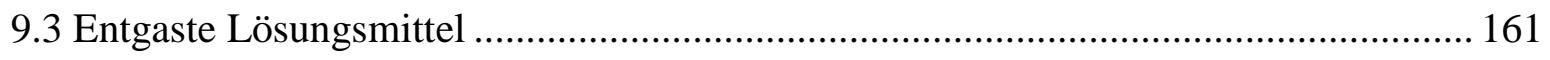

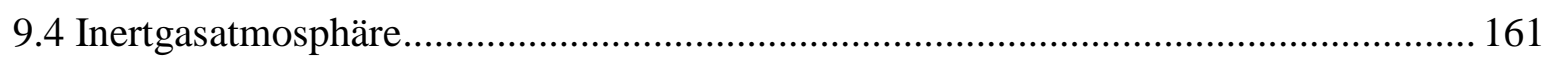

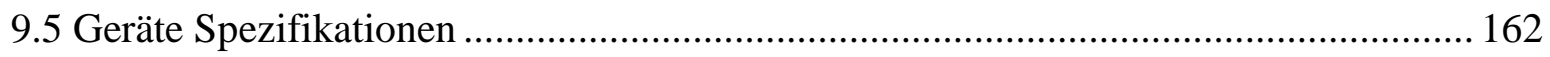

9.5.1 Kernresonanzspektroskopische Untersuchungen .............................................. 162

9.5.2 Massenspektrometrische Untersuchungen ........................................................ 162

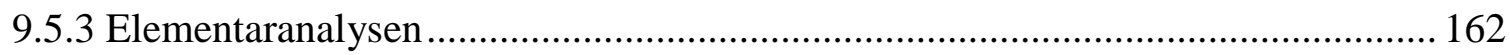

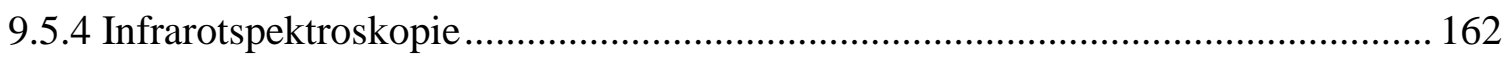

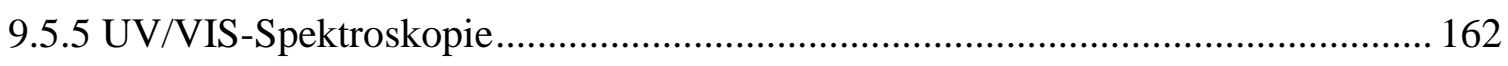

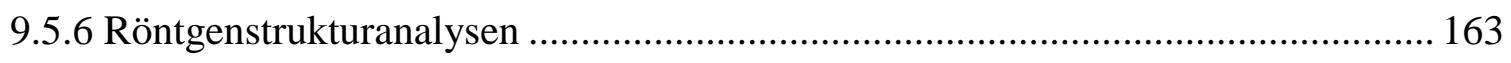

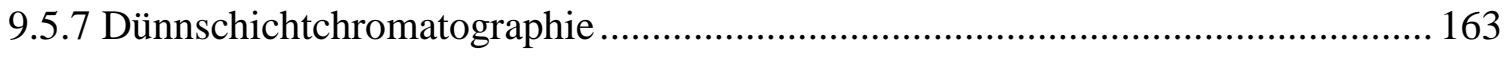

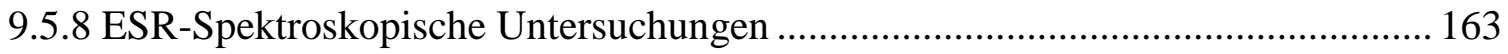

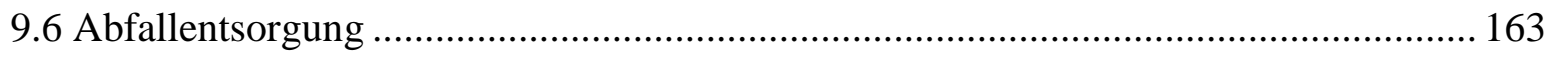

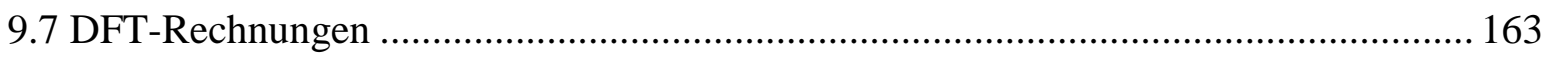

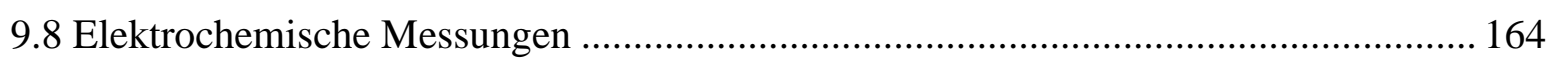




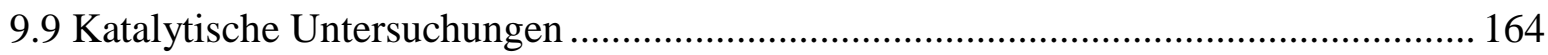

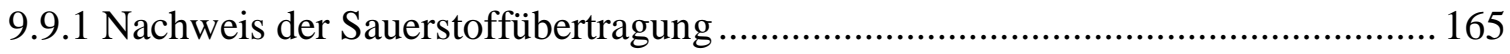

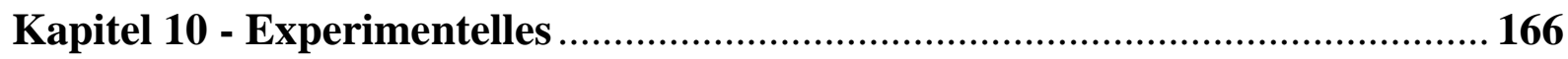

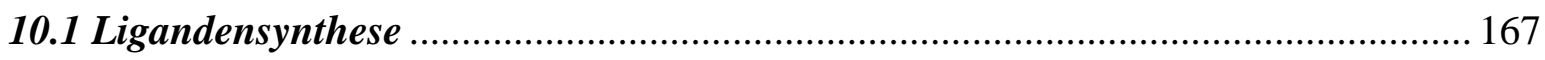

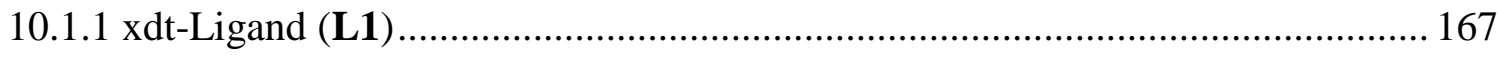

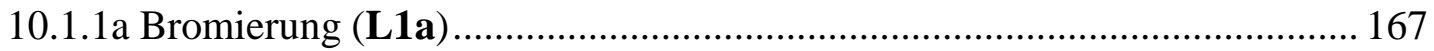

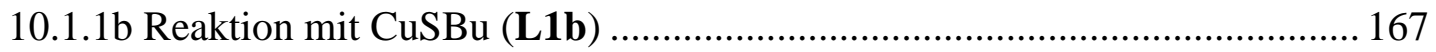

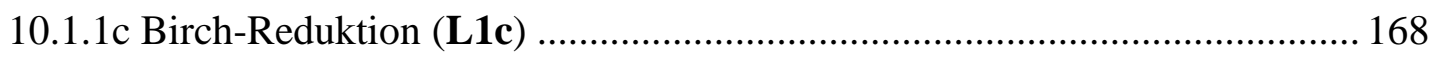

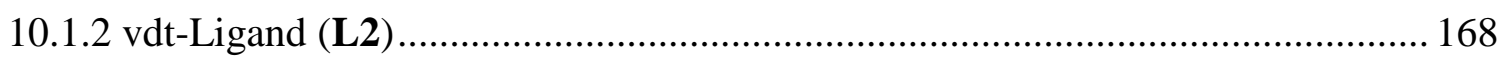

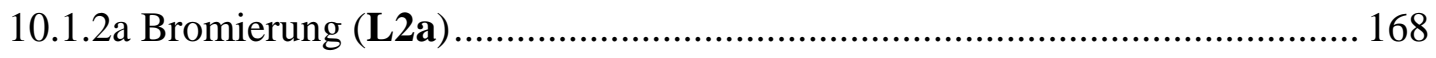

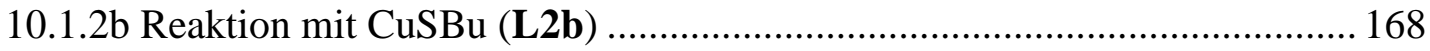

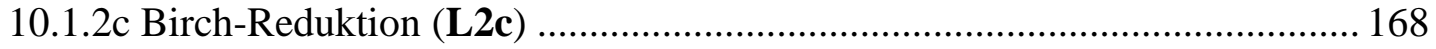

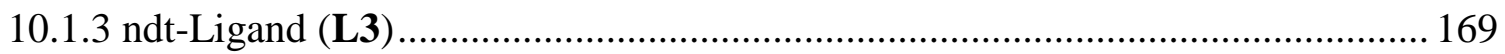

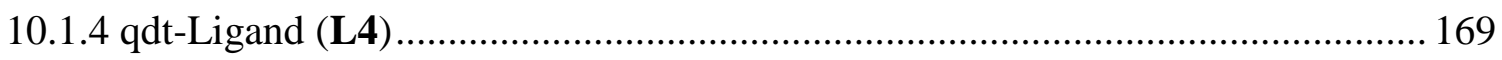

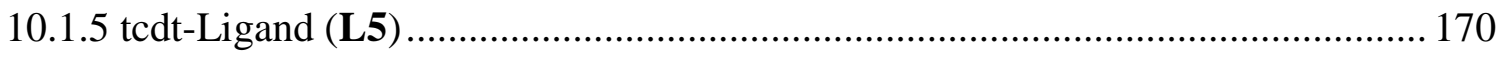

10.1.5a Bromierung (L5a) Methode A........................................................... 170

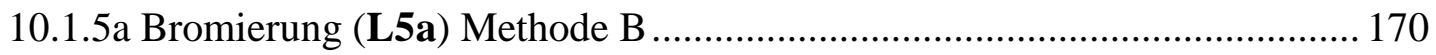

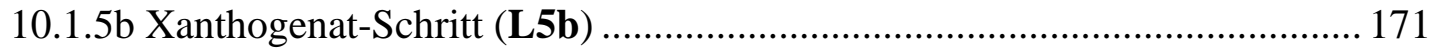

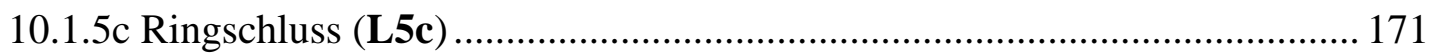

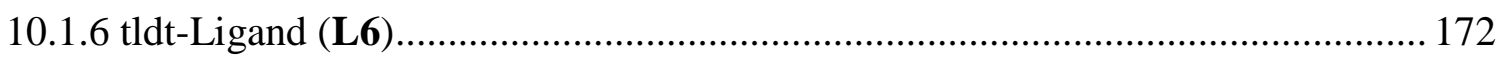

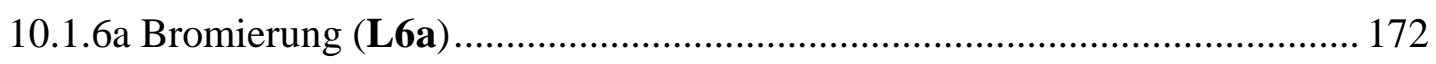

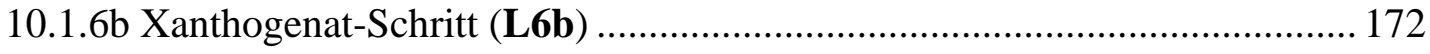

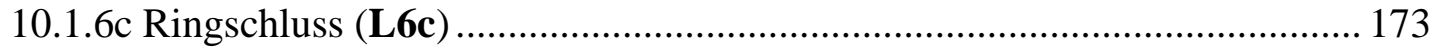

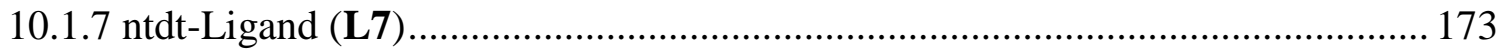

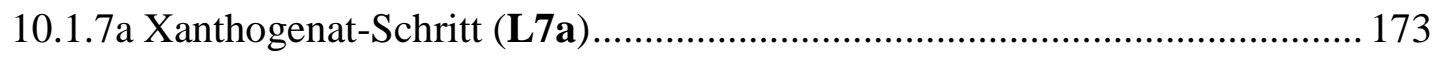

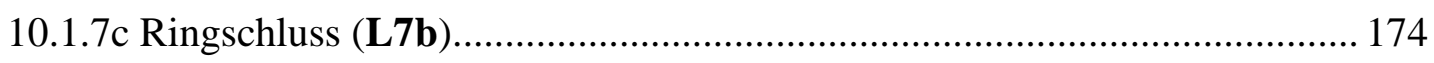

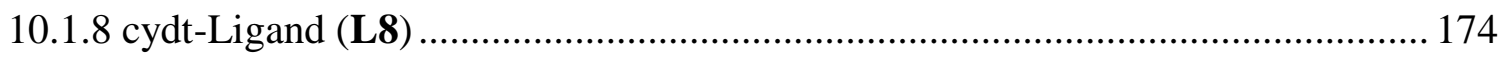

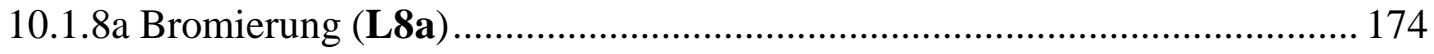

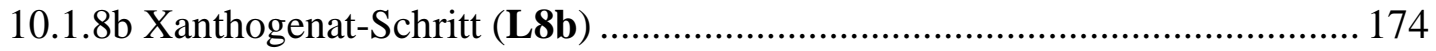

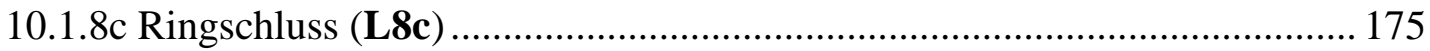

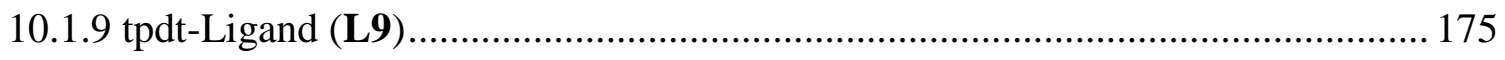

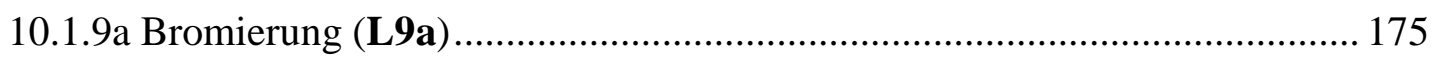

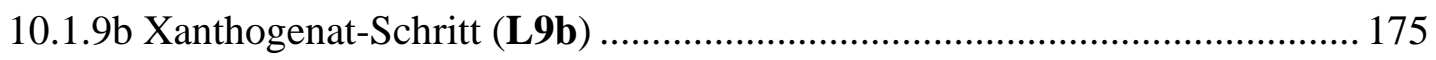

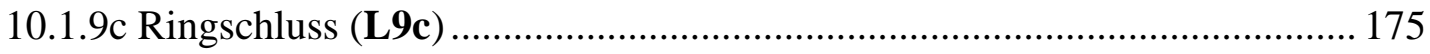




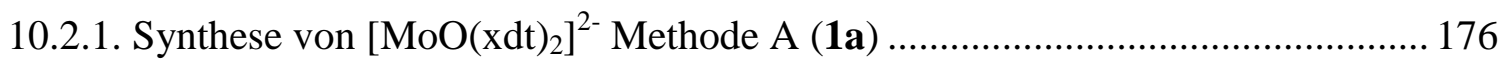

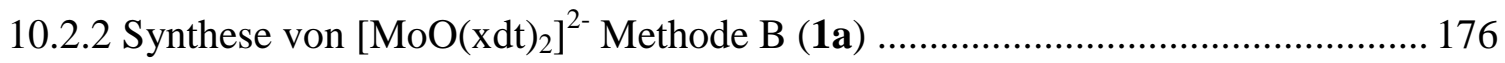

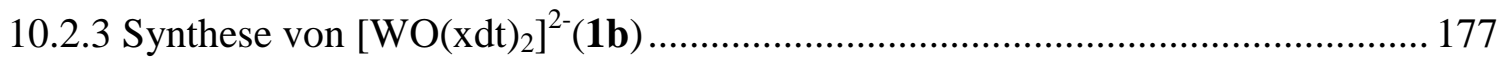

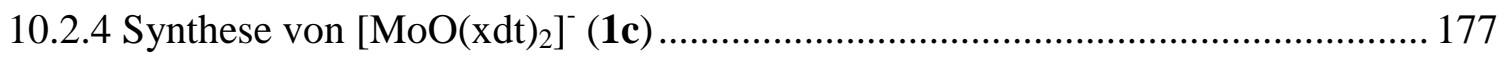

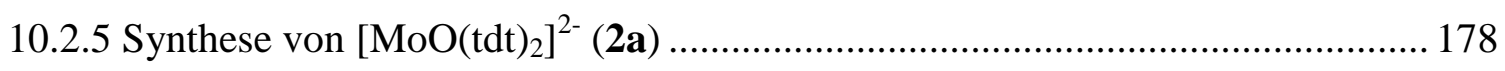

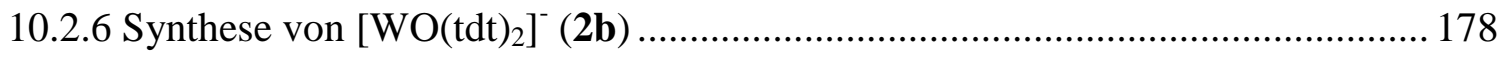

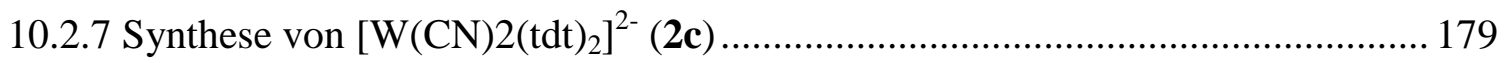

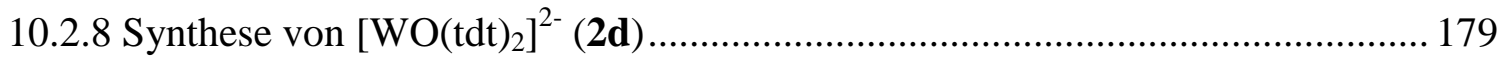

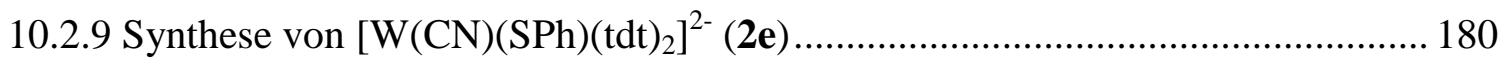

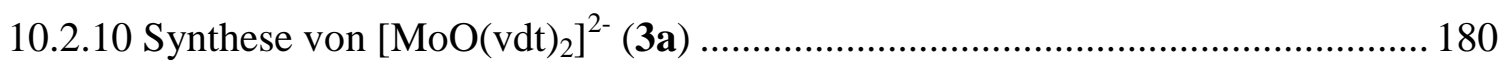

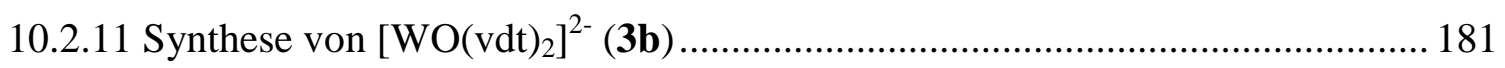

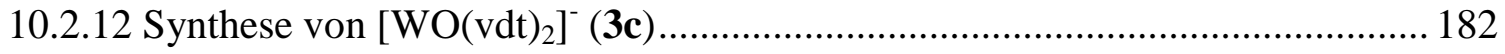

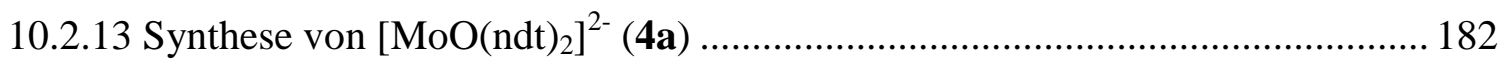

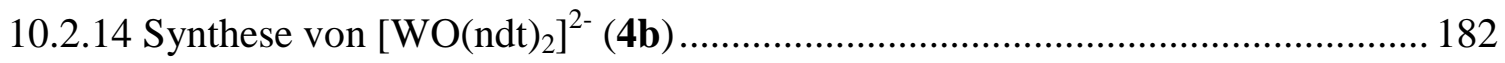

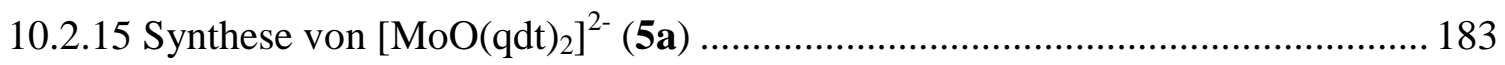

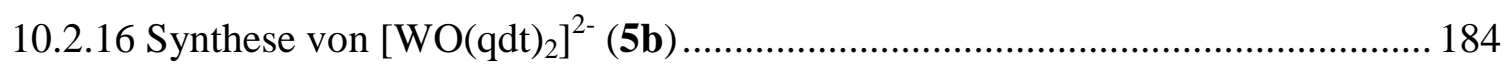

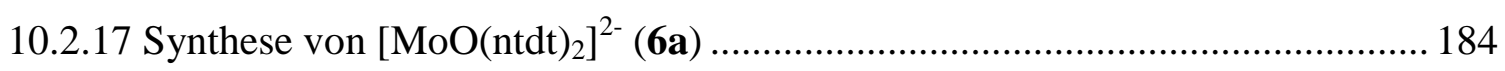

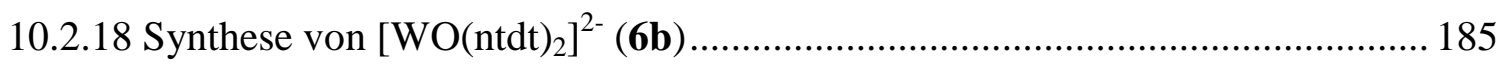

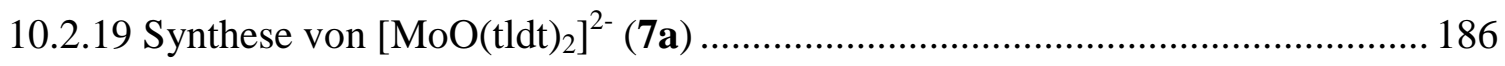

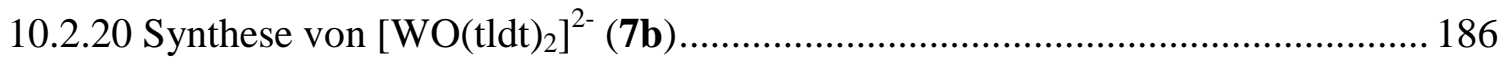

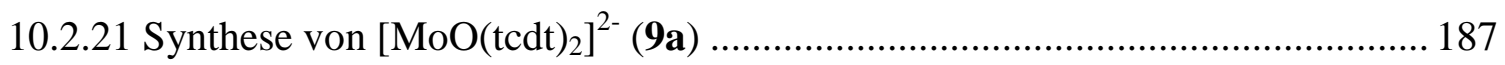

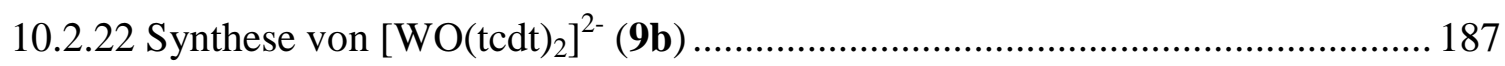

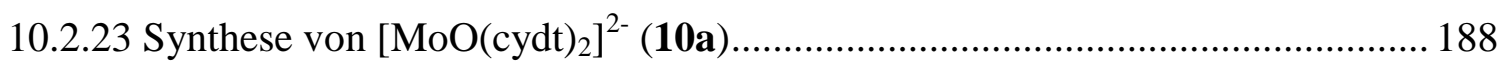

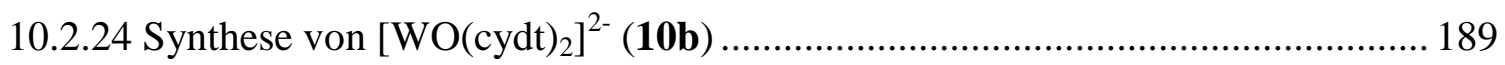

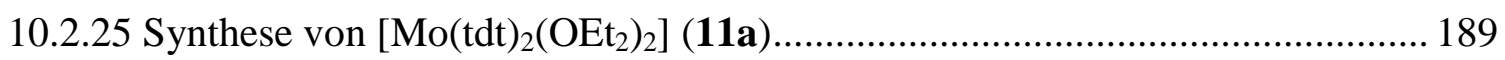

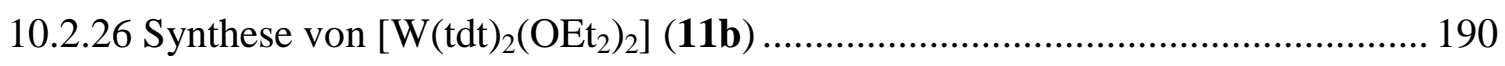

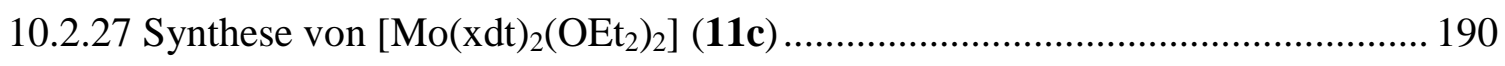

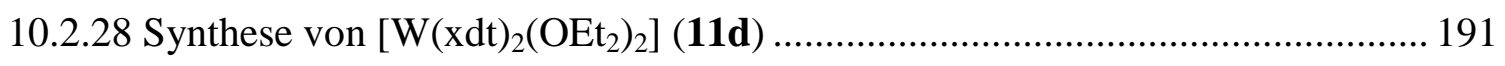

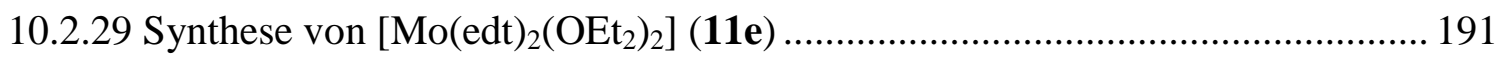

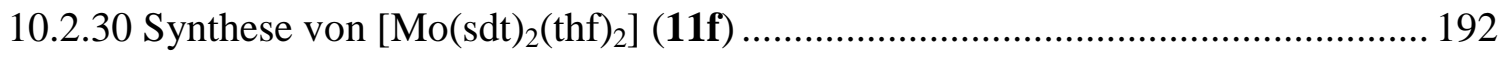

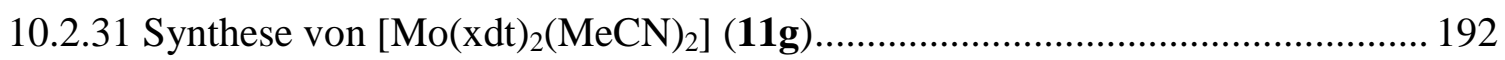

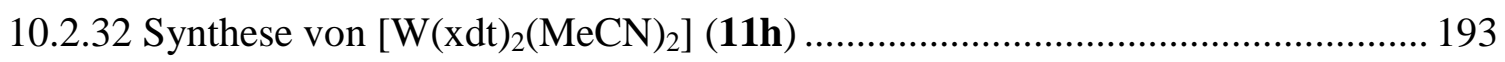

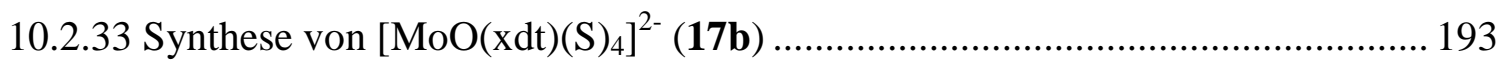




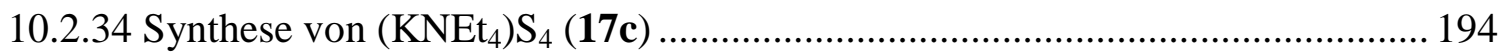

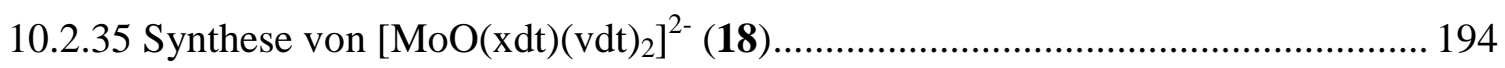

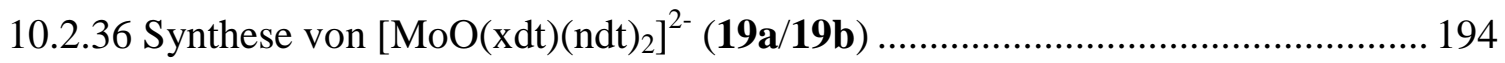

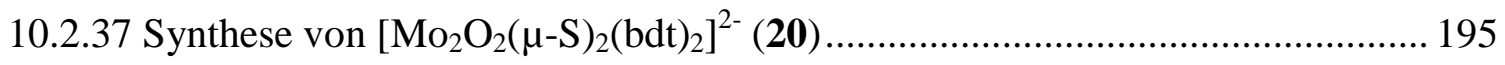

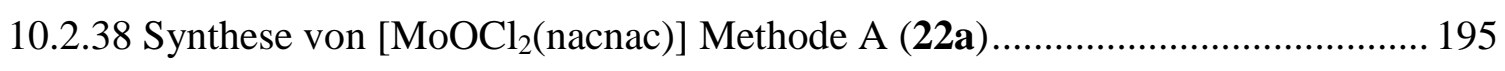

10.2.39 Synthese von $\left[\mathrm{MoOCl}_{2}(\right.$ nacnac) $]$ Methode B (22b) ...................................... 196

10.2.40 Synthese von $\left[\mathrm{WOCl}_{2}(\right.$ nacnac) $]$ Methode A (23a) ........................................ 196

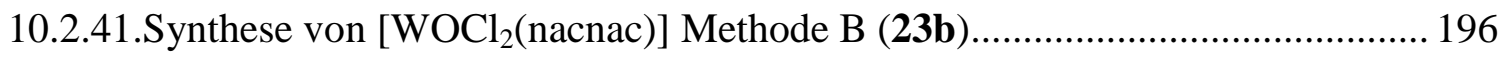

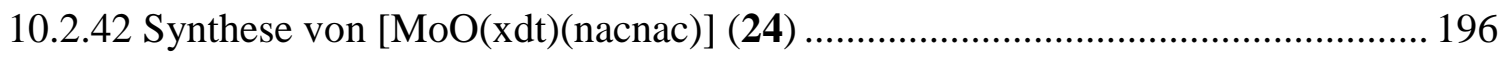

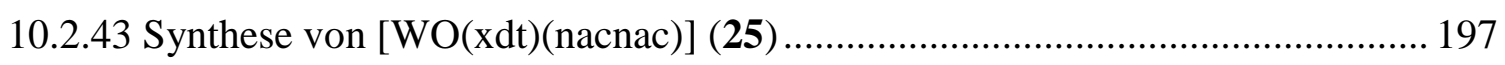

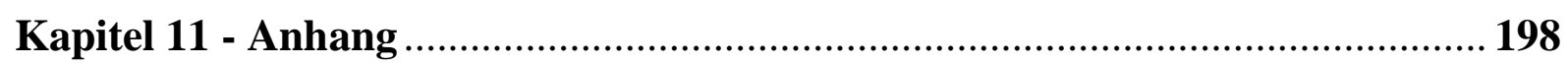

11.1 Auftragungen der elektrochemischen Untersuchungen .......................................... 199

11.2 Auftragungen der temperaturabhängigen katalytischen Reaktionen ......................... 201

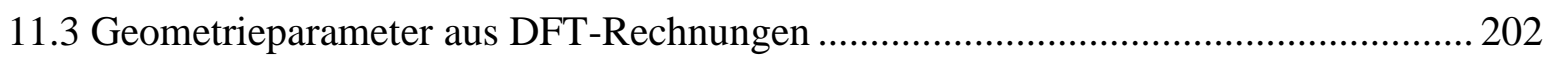

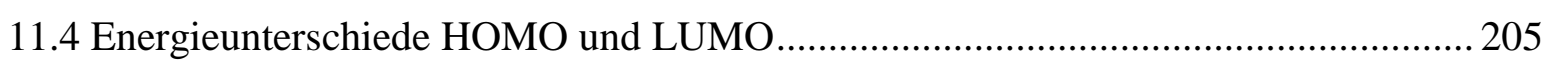

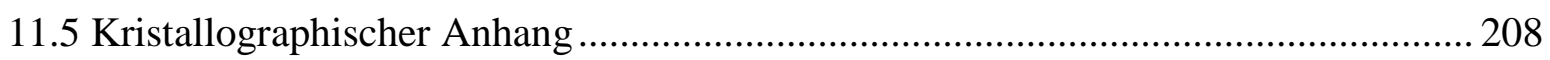

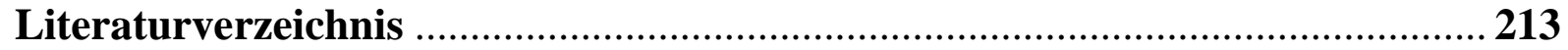

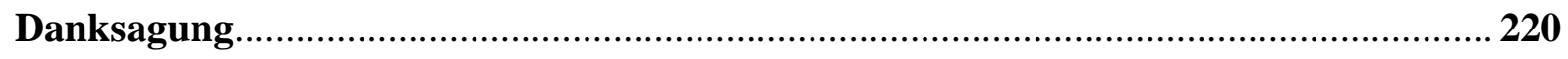

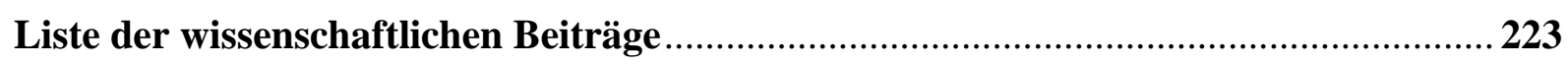

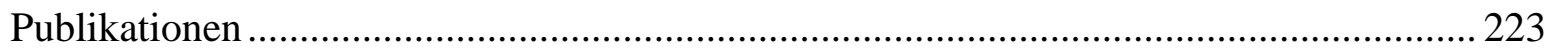

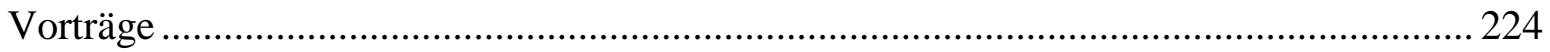

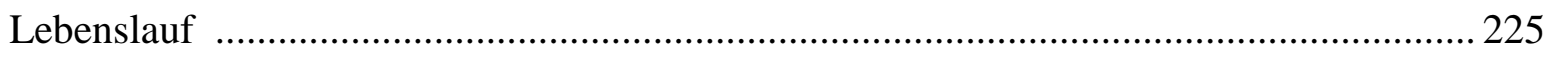




\begin{tabular}{|c|c|c|}
\hline \multicolumn{3}{|c|}{ Abkürzungsverzeichnis } \\
\hline$\AA$ & - & Ångstrøm \\
\hline abs & - & absolut (Sauerstoff sowie Wasserfreiheit) \\
\hline $\mathrm{AH}$ & - & Acetylenhydratase \\
\hline $\mathrm{AO}$ & - & Arsenit-Oxidase \\
\hline AOR & - & Aldehyd-Oxidoreduktase \\
\hline Arom & - & aromatisch \\
\hline$n-\mathrm{Bu}$ & - & $n$-Butyl \\
\hline$n$-BuLi & - & $n$-Butyllithium \\
\hline $\mathrm{CHCl}_{3}$ & - & Chloroform \\
\hline $\mathrm{CH}_{2} \mathrm{Cl}_{2}$ & - & Dichlormethan \\
\hline$c p^{*}$ & - & Pentamethylcyclopentadienyl \\
\hline $\mathrm{CV}$ & - & Cyclovoltammetrie \\
\hline DCM & - & Dichlormethan \\
\hline $\mathrm{DMF}$ & - & $N, N$-Dimethylformamid \\
\hline DMSO & - & Dimethylsulfoxid \\
\hline DPV & - & Differenzpulsvoltammetrie \\
\hline $\mathrm{e}$ & - & molarer Extinktionskoeffizent \\
\hline EA & - & Elementaranalyse \\
\hline edt & - & Ethylendithiolen \\
\hline EI & - & Electron Inpact \\
\hline entg & - & entgast \\
\hline ESI & - & Electron Spray Ionisation \\
\hline $\mathrm{Et}_{2} \mathrm{O}$ & - & Diethylether \\
\hline FAB & - & Fast Atom Bombardment \\
\hline FDH & - & Formiat-Dehydrogenase \\
\hline FW & - & Faltungswinkel \\
\hline g-Wert & - & Landé-Faktor \\
\hline HOMO & - & highest occupied molecular orbial \\
\hline HV & - & Hochvakuum \\
\hline $\mathrm{Hz}$ & - & Hertz \\
\hline i.V. & - & im Vakuum \\
\hline IR & - & Infrarot \\
\hline$J$ & - & Kopplungskonstante \\
\hline
\end{tabular}


K

LUMO

$\mathrm{m}$

$\mathrm{Me}$

$\mathrm{MeCN}$

$\min$

MS

NBA

NMR

NPA

NR

OS

PBE

$\mathrm{Ph}$

RT

TS

SO

THF

$\mathrm{UV} / \mathrm{Vis}$

XOR
Kelvin

lowest unoccupied molecular orbital

Masse

Methyl

Acetonitril

Minute

Massenspektrometrie

Nitrobenzylalkohol

Nuclear Magnetic Resonance

Natural Population Analysis

Nitrat-Reduktase

Oxidationsstufe

Perdew-Burke-Ernzerhof

Phenyl

Raumtemperatur

Transition state

Sulfit-Oxidase

Tetrahydrofuran

Ultraviolett/Visible

Xanthin-Oxidoreduktase 
Verwendete Dithiolenliganden und Koordinationsweise zu einem Metall<smiles>COSc1cc(C)c(C)cc1SO[SH]1OSc2cc(OC)c(OC)cc21</smiles><smiles>c1cc2c(s1)CCCC2</smiles>

cydt<smiles></smiles>

tpdt<smiles>C1CC2=C(CO1)COS2</smiles>

pdt<smiles>c1ccc2c(c1)CCc1soc1-2</smiles>

tldt<smiles>c1ccc2c(c1)SCC1=C2SOC1</smiles>

tedt<smiles>c1ccc2nc3c(nc2c1)OOS3</smiles><smiles>C1=C(c2ccccc2)COS1</smiles>

edt<smiles>C1=C(c2ccc3ccccc3c2)COS1</smiles>

qdt

ntdt

Verwendete Nicht-Dithiolenverbindungen<smiles>CCCc1cccc(P)c1N1OC(c2c(F)cccc2P)=C(C)C=C1C</smiles>

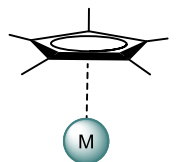

cp*

cp 


\section{Kapitel 1}

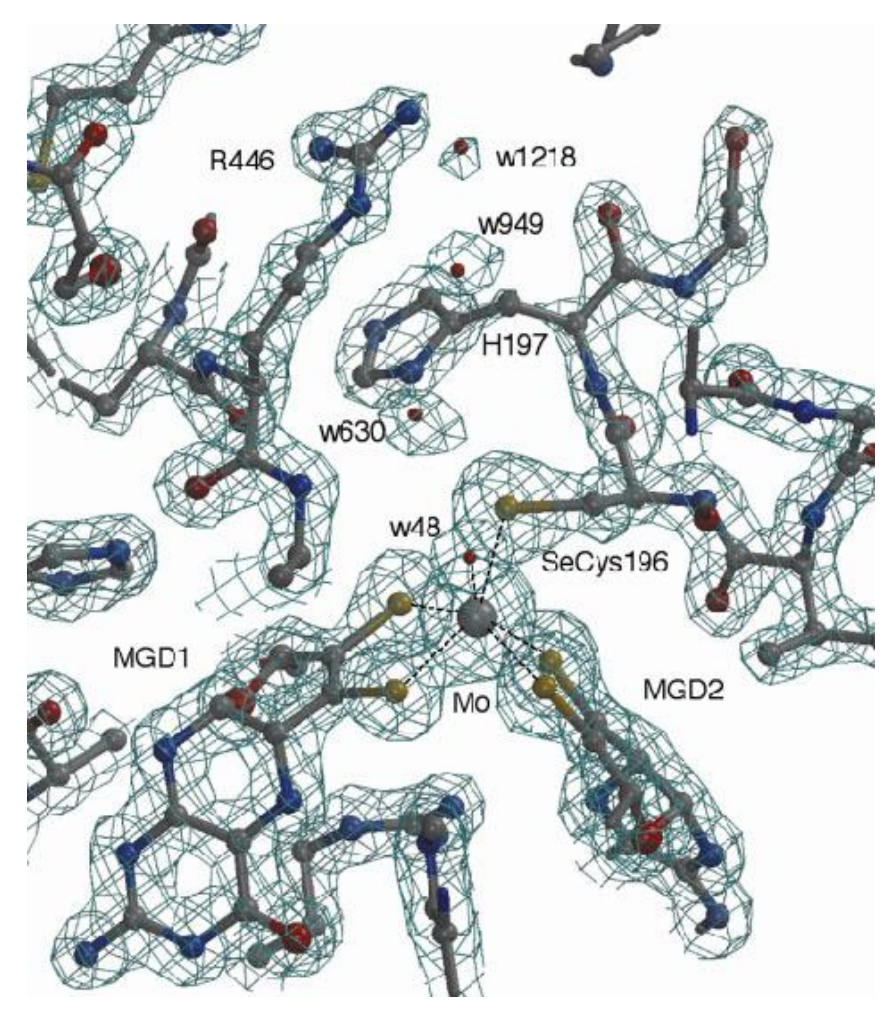

\section{Biologischer Hintergrund}

\section{Zusammenfassung}

In diesem Kapitel werden die aktiven Zentren der verschiedenen Enzymfamilien vorgestellt und es werden die aktuell dazu synthetisierten literaturbekannten Modellverbindungen aufgeführt. 


\subsection{Einleitung}

Molybdän und Wolfram nehmen eine besondere Stellung im Periodensystem ein, da sie die einzigen Elemente der fünften bzw. sechsten Periode sind, die in fast allen Organismen vorkommen, angefangen von den Archaea-Bakterien bis hin zu menschlichen Lebewesen. Sie sind chemisch nahezu identisch und werden deshalb in sehr ähnlichen Enzymen verwendet, mit Ausnahme der Nitrogenase, da von dieser die analoge Form des Wolframs nicht vorkommt bzw. noch unbekannt ist. Die schrittweise Entstehung von Molybdän- und Wolframenzymen war vermutlich sehr stark an die Bioverfügbarkeit beider Metalle, während der Evolution gekoppelt. ${ }^{[1]}$ Es wird angenommen, dass Leben auf der Erde unter sehr heißen und schwefelhaltigen anaerobischen Bedingungen entstanden ist. Unter diesen Bedingungen war die Verfügbarkeit von Molybdän sehr gering, weil das meiste Molybdän als Molybdänsulfid ausgefällt wurde. Da unter den gleichen Bedingungen die analogen Wolframmetalle hauptsächlich in Oxidationsstufe VI vorkamen und dianionische und lösliche $\left[\mathrm{WO}_{\mathrm{X}} \mathrm{S}_{\mathrm{x}-\mathrm{n}}\right]^{2-}(\mathrm{n}=0-4)$ Verbindungen formten, war eine erheblich bessere Bioverfügbarkeit des Wolframs gegeben. Die biologische Verfügbarkeit dieser Metalle für die aktiven Zentren der biologischen Systeme unter den gegebenen Bedingungen war nun entscheidend für den Einbau in die ersten Mikroorganismen. ${ }^{[2]}$

Mit der Abkühlung der Erdkruste und dem Beginn der photosynthetischen Aktivität der Organismen begann auch die vermehrte Sauerstoffproduktion und es kam damit zum Absinken der hohen freien Schwefel-/Sulfidkonzentrationen. Mit der Zeit wurde die Bioverfügbarkeit von Molybdän immer besser und es kam zu einer relativen Verschlechterung der Verfügbarkeit an Wolfram, aufgrund der guten Löslichkeit des $\left[\mathrm{MoO}_{4}\right]^{2-}$-Ions in Wasser. Deshalb stellt Molybdän heute eines der am häufigsten vorkommenden Übergangsmetalle in den Ozeanen dar. Die Entstehung des Molybdats kam durch die oxidative Verwitterung der Erdoberfläche, während der Evolution zustande. Dadurch ergeben sich heute nur noch sehr kleine Konzentrationen (1-2 ppm) an Molybdän in der kontinentalen Kruste. ${ }^{[3]}$ Diese steigende Verbesserung der Verfügbarkeit an löslichem Molybdän ermöglichte den gezielten Einbau in vielerlei aktiven Zentren, in den verschiedensten Enzymen, während der Evolution. Lediglich frühe Organismen (hauptsächlich Archaea), die immer noch unter ähnlichen Bedingungen der frühen Erde leben, verwendeten in ihren aktiven Zentren Wolfram.

Auch ein weiterer Grund für den Wechsel von Wolfram zu Molybdän wird diskutiert: Hierbei werden die sich verändernden Redoxpotentiale bei Temperaturschwankungen als ein 
entscheidendes Kriterium herangezogen. ${ }^{[4]}$ Dabei wurden erste Hinweise auf das unterschiedliche Verhalten beider Metalle bei Temperaturänderungen gefunden. Im Laufe dieser Arbeit wird versucht die Tendenzen weiter zu untersuchen.

Molybdän und Wolframenzyme (mit Ausnahme der Nitrogenase) katalysieren Reaktionen des folgenden Typs.

$$
\mathrm{X}+\mathrm{H}_{2} \mathrm{O} \leftrightarrows \mathrm{XO}+2 \mathrm{H}^{+}+2 \mathrm{e}^{-}
$$

Hierbei findet eine Übertragung von Sauerstoff $\left(\mathrm{O}^{2-}\right)$ auf ein Substrat statt oder die Abspaltung von Sauerstoff unter Bildung von Wasser. ${ }^{[5]}$ Wenn nur eine Sauerstoffatom während dieser katalytischen Reaktion seinen Bindungspartner wechselt, spricht man von Oxo-Transfer-Reaktionen und die Enzyme, die diese Reaktion katalysieren, werden als Oxotransferasen bezeichnet.

Untersuchungen der aktiven Zentren dieser Oxotransferasen haben vor mehr als drei Jahrzehnten begonnen. Die Koordination von Schwefel zu dem Molybdänzentrum ist schon seit der Entdeckung dieser Oxotransferasen bekannt. Es wurde angenommen, dass der Schwefel des Cysteins ausschließlich für die Metallkoordination verantwortlich ist. Doch im Jahre 1982 konnten Rajagopalan und seine Mitarbeiter nachweisen, dass die koordinierten Schwefelatome Teil eines speziellen Ligandensystems, was als Molybdopterin (MPT) bezeichnet wird, sind. Diese Entdeckung entkräftete alle zuvor entstandenen Annahmen. In dieser bemerkenswerten Arbeit isolierten und charakterisierten sie das Di(carboxyamido)Derivat des Molybdopterins durch Fluoreszenz- und Massenspektrometrie. ${ }^{[6]}$ Die erste vorgestellte Struktur inklusive der Pterin-dithiolen Chelateinheit konnte von der isolierten Sulfit-Oxidase aus Hühnerleber charakterisiert werden und ist in Abb.la gezeigt. Hierbei wurde eine nicht indifizierbare Gruppe in Position sechs des Pyranzins gefunden. Die Abbildung zeigt die erste vorgeschlagene Struktur mit einem bizyklischen Ringsystem mit einem Seitenarm der die Dithioleneinheit, sowie eine Phosphatfunktion trägt. Im Jahre 1987 veröffentlichten die gleichen Autoren ein verfeinertes Modell des Molybdäncofaktors in dem sich ein 6-alkylpterin mit einem aus vier Kohlenstoffatomen bestehenden Seitenarm befindet. An diesem Seitenarm ist das Dithiolen in 1'sowie 2'-Position angebunden, außerdem befindet sich ein sekundärer Alkohol in der $3^{\prime}$-Position und ist in Abb.1b gezeigt. ${ }^{[7]}$ 


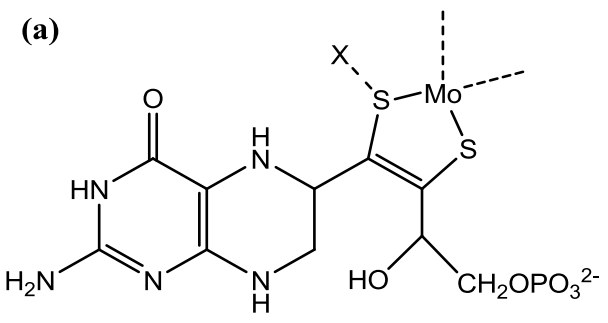<smiles></smiles>

Abb. 1: erste vorgeschlagene Struktur; b) verfeinerte Struktur

1995 gelang es Chan et al. eine Oxotransferase, mit Wolfram im aktiven Zentrum aus der Aldehydferredoxin-Oxidoreduktase (AOR) von Pyrococcus furiosus zu isolieren und durch eine erhaltene Kristallstruktur zu charakterisieren. Die erhaltene Struktur ist in Abb.2a und die gegenwertig akzeptierte Struktur des Molybdopterins in $A b b .2 b$ gezeigt. Diese Struktur basiert auf verschiedensten kristallographischen Studien.

(a)

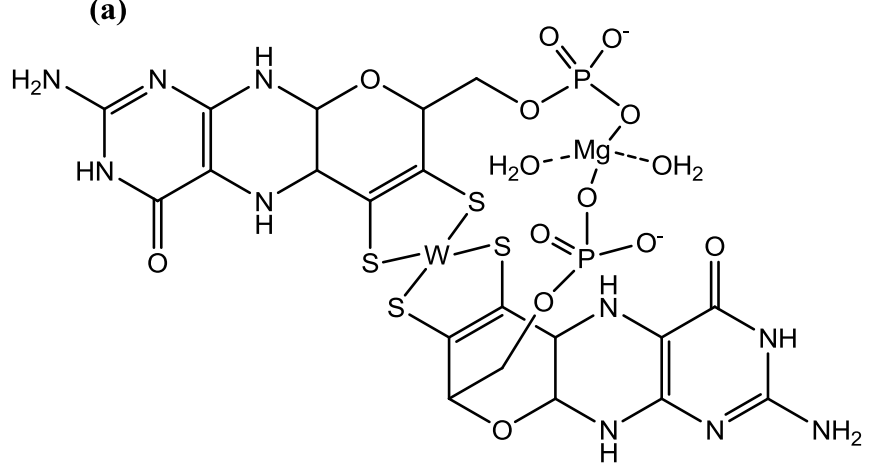

(b)

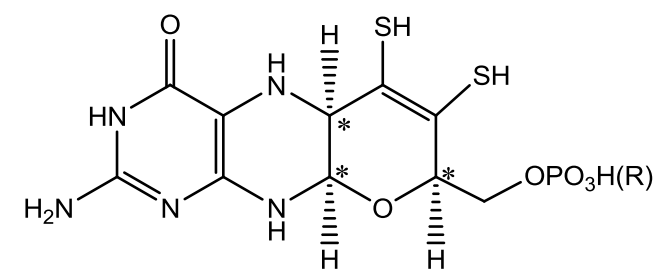

Abb. 2: a) erste Kristallstruktur der AOR; b) gegenwertig akzeptierte Struktur des Molybdopterins,

* kennzeichnet Chirale Zentren, $\mathrm{R}=\mathrm{H}$ oder ein Dinucleotid

Mehr als fünfzig Molybdän- und Wolframenzyme sind bis zum heutigen Tage bekannt. Eine Außnahme unter den molybdänabhängigen Enzymen stellt die Nitrogenase dar. Hierbei ist das Molybdän nicht an Molybdopterinliganden gebunden, sondern an einen EisenSchwefelcluster, Histidin und Homocitrat. ${ }^{[8]}$ In allen anderen Enzymen wird die typische Koordination des Molybdopterinligandens an ein einkerniges Molybdänzentrum gefunden. Bei manchen Enzymzentren wird nur die Anbindung eines Molybdopterinligandens, bei anderen die von zwei beobachtet. Dabei weist der Name Molybdopterin auf das rein organische Gerüst hin und zeigt keinen Zusammenhang mit der Koordination zu Molybdänzentren. In einigen prokaryotischen Organismen sind außerdem an das Ende der Phosphatkette Cytosin, Guanosin, Adenosin oder Tymin gebunden.

Anhand der chemischen Struktur der aktiven Zentren und der Reaktivitäten, wurde durch Hille eine Einteilung der molybdän- sowie der wolframhaltigen Enzyme in Familien 
durchgeführt $^{[2,9]}$ Es wurden die Molybdänenzyme in die Xanthine-Oxidase Familie, die Sulfit-Oxidase Familie und die DMSO-Reduktase Familie eingeteilt.

Die zum gegenwertigen Zeitpunkt bekannten Wolframenzyme wurden in die AldehydFerredoxin-Oxidoreduktase Familie und die Formiat-Dehydrogenase Familie eingeteilt. In 2007 wurde von Einsle et al. noch eine weiteres wolframhaltiges Enzym gefunden, wodurch eine neue Familienklasse, die Acetylen-Hydratase Familie, eingeführt wurde. ${ }^{[10]}$ Ein Überblick der Enzymfamilien ist in $A b b .3$ gegeben.

\section{Molvbdänhaltige Enzyme}

Sulfit-Oxidase Familie
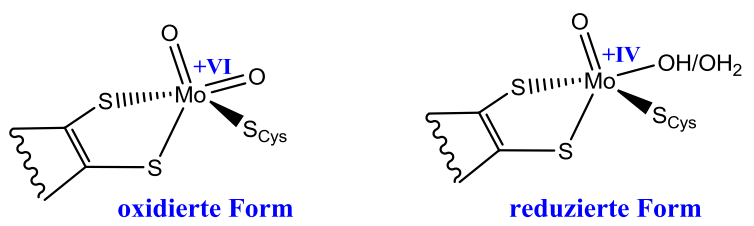

Xanthin-Oxidase Familie
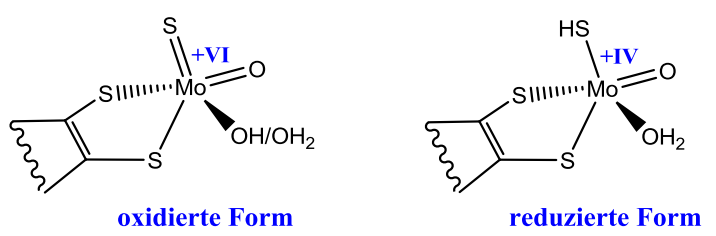

DMSO-Reduktase Familie

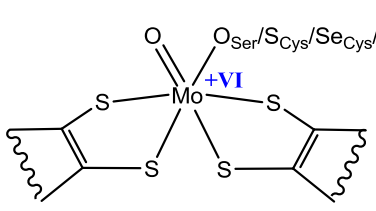

oxidierte Form

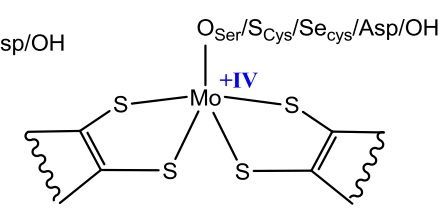

reduzierte Form

(Arsenit-Oxidase Familie)

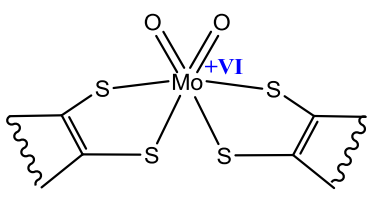

oxidierte Form

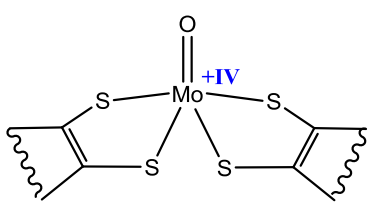

reduzierte Form

\section{Wolframhaltige Enzyme}

Aldehyd-Ferredoxin-Oxidoreduktase Familie

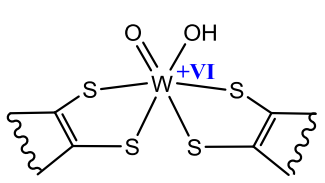

oxidierte Form

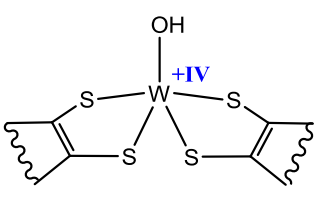

reduzierte Form
Formiat-Dehydrogenase Familie

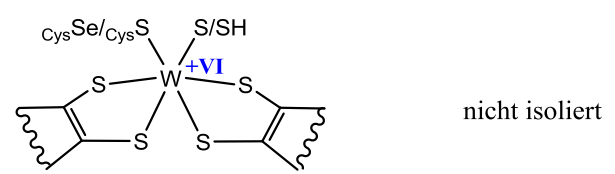

oxidierte Form

reduzierte Form

Acetylen-Hydratase Familie

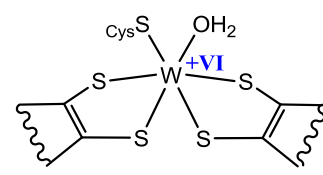

oxidierte Form

Abb. 3: Enzymfamilien

Bei der Namensgebung bestimmte immer der prominenteste Vertreter der Familie den Namen. Enzyme der Xanthin-Oxidase Familie haben ein aktives Zentrum der Zusammensetzung (MPT) $\mathrm{Mo}^{\mathrm{VI}} \mathrm{OS}(\mathrm{OH})$ und es existiert eine gewisse strukturelle Übereinstimmung zur Sulfit-Oxidase Familie des Strukturtyps (MPT)Mo ${ }^{\mathrm{VI}} \mathrm{O}_{2}\left(\mathrm{~S}_{\mathrm{Cys}}\right)$, da in beiden Familien nur ein Molybdopterinligand an das Metallzentrum koordiniert ist. Dies ist 
der größte Unterschied zur DMSO-Reduktase Familie, in der zwei MPT-Liganden an das Metallzentrum koordiniert sind. Weiterhin ist die Anbindung von verschiedenen Aminosäureresten (Serin, Cystein, Selenocystein, Aspartat) vorhanden. Eine weitere Gruppe von Molybdänenzymen mit dem Strukturtyp $(\mathrm{MPT})_{2} \mathrm{MoO}_{2}$ wird als Arsenit-Oxidase Familie bezeichnet. Dieser Familientyp wird zur Oberklasse der DMSO-Reduktase Familie gezählt, doch bei dieser Klasse wurde keine Bindung von Amminosäurerest zum Metallzentrum festgestellt. Eine weitere Untergruppe der Xanthin-Oxidase Familie bildet die CO Dehydrogenasen (nicht gezeigt), die das ungewöhnliche Strukturmotiv $\mathrm{MoO}(\mathrm{OH})(\mathrm{MPT})(\mu-$ $\mathrm{S}) \mathrm{Cu}^{\mathrm{I}} \mathrm{S}_{\mathrm{Cys}}$ aufweist. Diese Enzyme katalysieren die Reaktion von $\mathrm{CO} \mathrm{zu} \mathrm{CO}_{2}$. Die wolframhaltigen Enzyme der Aldehyd-Ferredoxin-Oxidoreduktase Familie haben ein Zentrum des folgenden Typs $(\mathrm{MPT})_{2} \mathrm{~W}^{\mathrm{VI}} \mathrm{O}(\mathrm{OH})$. Die zweite Klasse der Wolframenzyme stellen die Enzyme der Format-Dehydrogenase Familie. Hierbei liegen die Zentren in Oxidationsstufe VI am Metall als $(\mathrm{MPT})_{2} \mathrm{~W}^{\mathrm{VI}}(\mathrm{O})(\mathrm{X})$ vor, wobei das X, für Selenocystein oder Cystein stehen kann. Beide Familien zeigen eine Ähnlichkeit zur molybdänhaltigen DMSOReduktase Familie. Die dritte Familie und relativ junge Familie der Wolframenzyme besteht allein aus der sehr ungewöhnlichen Acetylen Hydrase. Diese katalysiert die Hydration von Acetylen zu Acetaldeyd.

Nach der Entdeckung von Molybdän- und Wolfram-Cofaktoren wurde es für viele bioanorganische Arbeitsgruppen zum Ziel, die Funktion und Struktur dieser Enzyme besser verstehen zu können. Deshalb wurde eine Vielzahl von Modellsystemen für die biologischen Zentren synthetisiert, um detaillierte Aussagen über die Wirkungsweise dieser aktiven Zentren treffen zu können. Im Folgenden werden die einzelnen Enzymfamilien im Detail vorgestellt und den dazu hergestellten Modellsystemen gegenübergestellt.

\subsection{Enzymfamilien}

\subsubsection{Sulfit-0xidase Familie}

Zur Sulfit-Oxidase Familie gehört die eukaryotischen Molybdoproteine Sulfit-Oxidase (SO), welche ubiquitär in Tieren verbreitet ist und meist aus Hühner- oder Rattenleber isoliert wurde. Außerdem wurde dieser Familie die Nitrat-Reduktase (NR) zugeordnet, welche in Algen, Pilzen und höheren Pflanzen vorkommt.

Das aktive Zentrum der Mitglieder dieser Familie besteht aus einem Molybdänzentrum, das von drei Schwefelatomem sowie zwei Oxoliganden in einer annährend quadratisch 
pyramidalen Geometrie umgeben ist. In Abb.4 ist die Kristallstruktur des aktiven Zentrums gezeigt, die von Rajagopalan et. al. aus Hühnerleber erhalten werden konnte. $^{[11]}$

Dabei stammen zwei der Schwefelatome vom Molybdopterin und der einzeln gebundene Schwefel wird durch einen Cysteinrest des Peptids bereitgestellt. Das Vorkommen von Cystein wurde in allen SOs

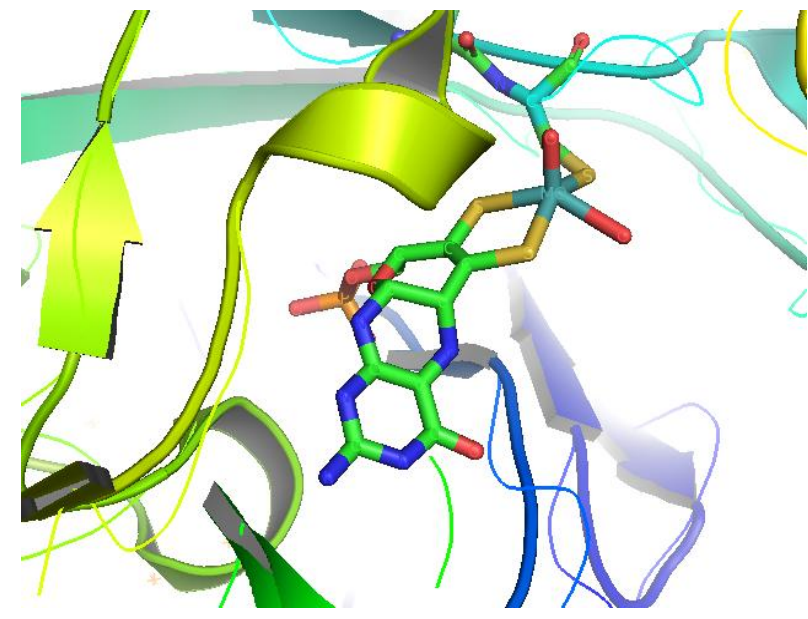

Abb. 4: Aktives Zentrum PDB: 2A99 und NRs gefunden und stellt für die Gruppe der Enzyme ein Charakteristikum dar. Die Bedeutung des Thiolatliganden konnte durch Austausch mit Serinat, welcher zu inaktiven Enzymen führte, nachgewiesen werden. ${ }^{[12]}$

In der Nähe der aktiven Zentren wurden außerdem andere redoxaktive Cofaktoren nachgewiesen. Diese sind bei der SO eine Cytochrom $b_{5}$-Einheit ${ }^{[13]}$ während sich bei der NR ein FAD-Zentrum befindet. ${ }^{[14]}$

Die Sulfit-Oxidase katalysiert die Transformation von Sulfit zu Sulfat, wobei das Molybdänzentrum seinen Oxidationszustand von VI zu IV im katalytischen Zyklus ändert. Die Rückoxidation zur Oxidationsstufe VI findet schrittweise über einen intramolekularen Elekronentransfer zum Cytochrom $b_{5}$ statt. $^{[15]}$ Im Folgenden ist ein Bild des Cytochroms (grün) in Nachbarschaft zum vorhandenen Molybdopterinzentrum (rot) gezeigt.

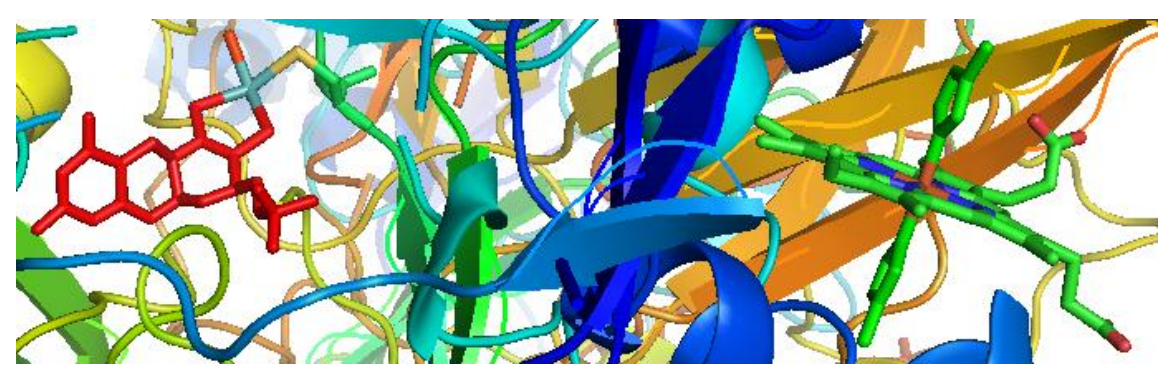

Abb. 5: Einblick in das Enzymsystem; PDB: 1SOX

Bei der Nitratreduktase hingegen wird der Zyklus in entgegengesetzter Richtung zur SO durchlaufen. Für die Reduktion von Nitrat zu Nitrit ist eine Oxdiation des Metallzentrums von IV zu VI nötig. 


\subsubsection{Modellsysteme}

Um das aktive Zentrum dieser Enzymklasse mit Hilfe von Modellverbindungen näher untersuchen zu können, haben sich viele Arbeitsgruppen mit der Herstellung von verschiedensten Systemen beschäftigt. Es wurden erste Systeme von Holm et al. synthetisiert, die zwei Oxogruppen am Metallzentrum trugen und bei denen das Metallzentrum durch sterisch anspruchsvolle Ligandensystemen geschützt wurde. ${ }^{[16]}$ Diese Verbindungen zeigten eine gute Sauerstoffübertragungsfähigkeit und eröffneten das Feld für eine große Anzahl an unterschiedlichen Modellen. Im Jahre 2000 wurde dann von Enemark et al. die erste Modellverbindung mit einem zusätzlichen angelagerten Benzylthiol synthetisiert. ${ }^{[17]}$ Hierbei konnte zwar nicht die Ligandenumgebung des SO vollkommen nachgebildet werden, doch es konnten zwei benachbarte p-Orbitale des Schwefels parallel zu der Mo=O-Bindung sowie ein drittes liegendes p-Orbital in der äquatorialen Ebene erhalten werden, dass in dieser Koordination auch in der biologischen SO gefunden wird. (Abb.6)

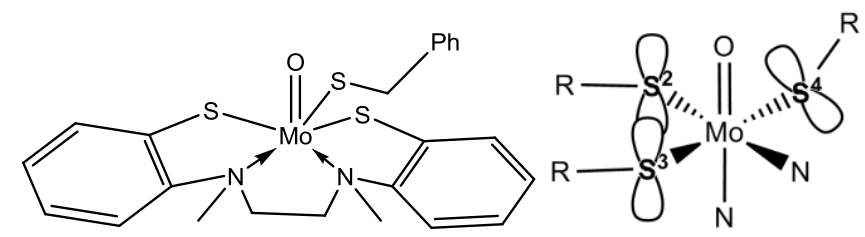

Abb. 6: (links) Modellverbindung von Enemark et al. als Modellsystem für die Sulfitoxidase ${ }^{[17]}$, (rechts) Anordnung der p-Orbitale

Außerdem wurden noch einige Modellkomplexe von Holm et al. synthetisiert, in denen es gelang nur einen Dithiolenliganden an das Metallzentrum zu binden. Dazu gehören Modelle wie $\left[\mathrm{MoO}(\mathrm{SR})_{2}(\mathrm{bdt})\right]^{-}\left(\mathrm{R}=2-\mathrm{Ad}, 2,4,6-i \mathrm{PrC}_{6} \mathrm{H}_{2}\right)$, das auch das beste Modellsystem ist, was bis zum heutigen Zeitpunkt für das aktive Zentrum in der oxidierten Form dargestellt wurde. Für die Synthese wurde $\left[\mathrm{Mo}^{\mathrm{VI}} \mathrm{O}_{2}\left(\mathrm{OSiPh}_{3}\right)_{2}\right]$ mit $\mathrm{Li}_{2}(\mathrm{bdt})$ umgesetzt, und die daraus entstandene $\left[\mathrm{Mo}^{\mathrm{VI}} \mathrm{O}_{2}\left(\mathrm{OSiPh}_{3}\right)(\mathrm{bdt})\right]^{-}-$Verbindung wurde mit sterisch anspruchsvollen Thiolverbindungen zur Zielverbindung umgesetzt ${ }^{[18]}(A b b .7)$
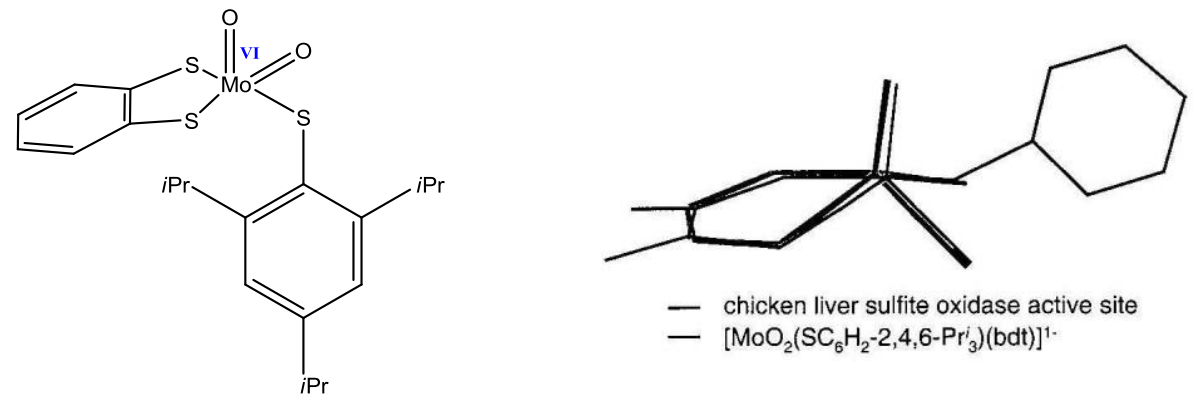

Abb. 7: Die bis zum heutigen Zeitpunkt beste Modellverbindung für die Sulfitoxidase in der oxidierten Form, sowie ein geometrischer Vergleich mit der aus Hühnerleber erhaltenen Kristallstruktur 
Dabei dient das angelagerte Benzoldithiolen als Modell für den Molybdopterinliganden und das Thiolat als Imitator für das Cysteinat. Um einen Vergleich zu der aus Hühnerleber isolierten Verbindung anstellen zu können, wurden beide Strukturen auf ihre geometrische Ähnlichkeit hin untersucht. In Abb.7 links ist zu erkennen, dass die Koordinationsumgebung der synthetisierten Verbindung in sehr guter Übereinstimmung mit dem biologischen Vorbild ist.

\subsubsection{Xanthin-0xidase Familie}

Diese Familie stellt mit mehr als zwanzig charakteristischen Enzymen die größte Gruppe der einkernigen Molybdänenzyme. Hierzu zählen die Xanthin-Oxidoreduktase (XOR), AldehydOxidoreduktase (AOR), Aldehyd-Oxidase (AO) sowie die Formiat-Dehydrogenase (FDH). Das erste durch Röntgenbeugung charakterisierte, aktive Zentrum dieser Familie wurde aus der Aldehydoxidase bei Desulfovibrio gigas isoliert. ${ }^{[19]}$

Das aktive Zentrum dieser Familie ist durch (MPT)Mo ${ }^{\mathrm{VI}} \mathrm{O}(\mathrm{S})(\mathrm{OH})$-Einheiten charakterisiert. Abhängig davon, ob die Enzyme in prokaryotischen oder eukaryotischen Zellen vorkommen, sowie von den einzelnen Reaktionen die katalysiert werden, liegen einkernige Formen des Molybdopterins, sowie auch in der sogenannten „dinukleotider“ Form in Kombination mit Cytosin, Guanin und Adenosin vor. ${ }^{[9]}$

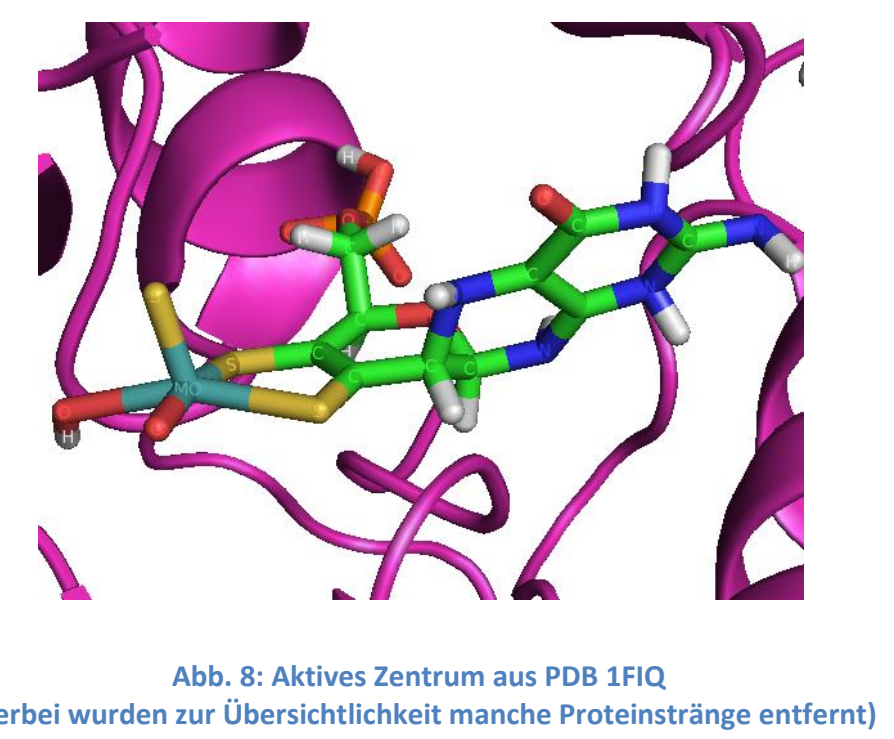

Die Reaktion die durch alle dieser Familie angehörigen Mitglieder katalysiert wird, ist die Spaltung einer R-H Bindung und daraus die Ausbildung einer R-O-Bindung.

$$
\mathrm{RH}+\mathrm{H}_{2} \mathrm{O} \leftrightarrows \mathrm{ROH}+2 \mathrm{H}^{+}+2 \mathrm{e}^{-}
$$


Aufgrund der durch die Enzyme katalysierten Reaktion sollten die Enzyme eigentlich besser mit der Bezeichnung Hydroxylasen versehen werden. Durch diese Enzyme werden oxidative Hydroxylierungen verschiedener Aldehyde, aromatischer Heterozyklen und Kohlenstoffmonoxid erzielt. ${ }^{[9]}$

Allen Enzymen dieser Familie ist gemein, dass sie neben Molybdän auch über weitere redoxaktive Zentren, wie z.B. Fe-S-Cluster, verfügen. Die aus dem Redoxprozess stammenden Elektronen können damit, über einen intermolekularen Weg, zu externen Akzeptoren geleitet werden. Im Folgenden ist ein Ausschnitt aus dem Enzym gezeigt, der die enthaltenen Fe-S-Cluster (orange/gelb), FAD (rot) und Salicylat (grün) in Nachbarschaft zum aktiven Zentrum (blau) zeigt.

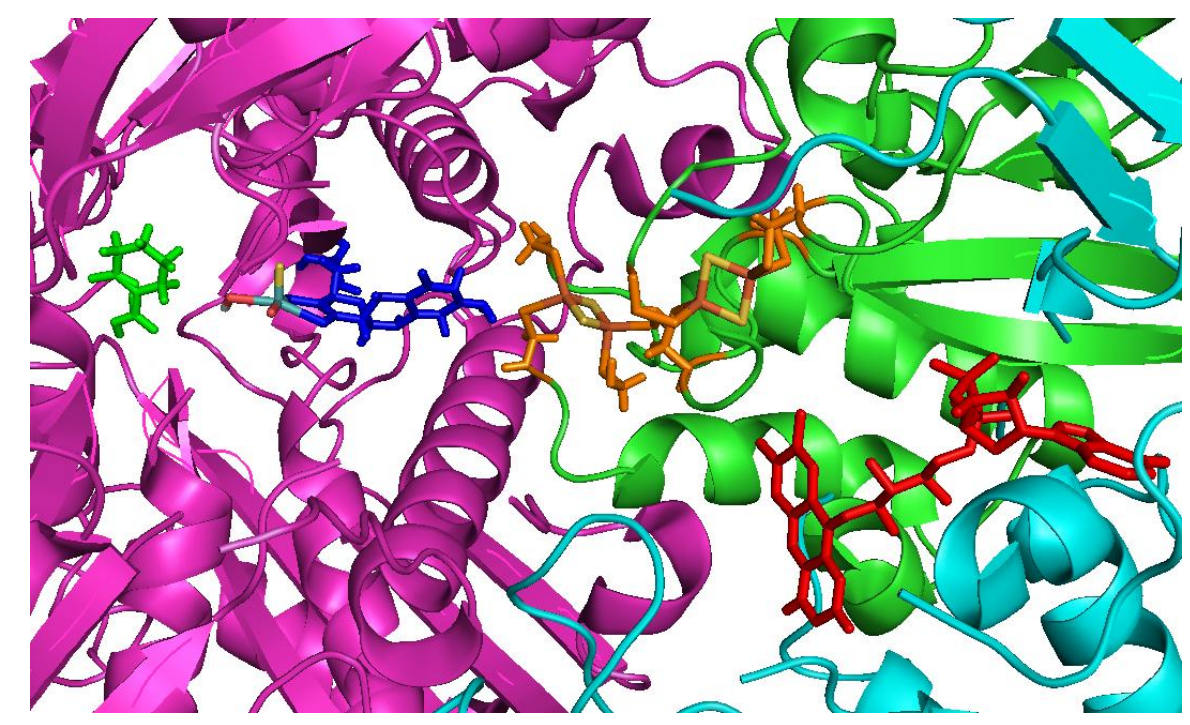

Abb. 9: Einblick in die Enzymsysteme der Xanthine-Oxidase aus PDB 1FIQ

Die Herstellung von Modellsystemen dieses Enzymtyps steckt noch in den Anfängen, da das vorgegebene MoOS-Zentrum synthetisch nicht sehr einfach zugänglich ist.

\subsubsection{Modellsysteme}

Einige Modelle wurden von Young et al. hergestellt, denen es gelang Verbindungen des Typs $\left[\mathrm{Tp}^{i \mathrm{Pr}} \mathrm{MoOS}(\mathrm{OPh})\right]\left(\mathrm{Tp}^{i \mathrm{Pr}}=\right.$ Hydrotris(3-isopropylpyrazol-1-yl)borat) herzustellen. ${ }^{[20]}$ 


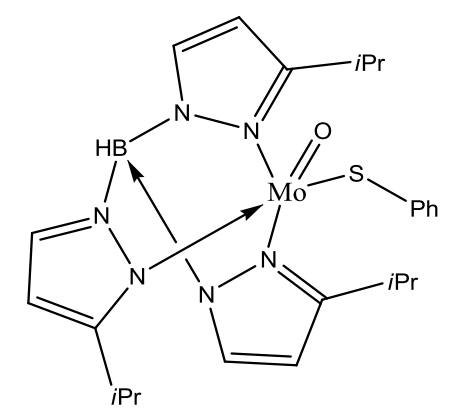

Abb. 10: Modellsystem von Young et al.

In $A b b .10$ ist zum gegenwertigen Zeitpunkt das beste Modell für die Molybdänhydroxylase Familie gezeigt, ausgenommen ganz einfacher Verbindungen wie $\left[\mathrm{MoO}_{4-n} \mathrm{~S}_{\mathrm{n}}\right]^{2-}$ oder $\left[\mathrm{MoOS}\left(\mathrm{ONPh}_{2}\right)_{2}\right]$. Deshalb bietet diese Familienklasse noch viel Spielraum zur Synthese von Modellverbindungen.

\subsubsection{DMSO-Reduktase Familie}

Enzyme der DMSO-Reduktase Familien verfügen über Molybdänzentren und katalysieren eine große Bandbreite an verschiedenen Sauerstoffübertragungs Reaktionen. Im Vergleich zu den anderen Enzymfamilien werden in dieser Familie die meisten Mitglieder gefunden. Typische Vertreter dieser Familie sind Enzyme aus Bakterien und Archaea, wie der DMSOReduktase, Biotin-S-Oxid-Reduktase, Trimethylamin-Oxidoreduktase (TMAOR), ArsenitOxidase (AO) und viele mehr. Es wird ein generelles aktives Zentrum bei den Vertreten gefunden, das im oxidierten Zustand die Form $(\mathrm{MPT})_{2} \mathrm{Mo}^{\mathrm{VI}} \mathrm{O}(\mathrm{R})$ bzw. $(\mathrm{MPT})_{2} \mathrm{Mo}^{\mathrm{IV}}(\mathrm{R})$ im reduzierten Zustand hat $[\mathrm{R}=$ Aminosäurerest $]$. Die erste Kristallstruktur einer DMSOReduktase aus Rhodobacter sphaeroides wurde als eine Mono-Ox-Molybdän(VI) Form mit zwei angelagerten Molybdopteringuanindinucleotiden (MGD) sowie einem Serinrest verfeinert. ${ }^{[21]}$ In der oxidierten Form dieser Enzyme ist das $\mathrm{Mo}^{\mathrm{VI}}$-Zentrum von sechs Liganden umgeben und es wird eine verzerrt trigonalprismatische Geometrie beobachtet. Hierbei werden vier Koordinationsstellen durch die beiden Dithiolenfunktionen der zwei MPT besetzt. Die Bindung von Aminosäureresten wie z.B. Aspartat, Serinat ${ }^{[21]}$, Selenocysteinat ${ }^{[22]}$ oder Cysteinat ${ }^{[23]}$ komplimentieren die Koordinationsphäre. Während des katalytischen Zyklus findet außerdem die Anlagerung eines Oxo-Liganden statt. Nach der Abgabe des Sauerstoffs zur Regenerierung kommt es zur Geometrieänderung in eine quadratisch planare Struktur und zur intrazellulären schrittweisen Reduktion des Molybdänzentrums zur Oxidationsstufe IV, wobei außerdem eine Koordination von Hydroxid- bzw. Wasser auftritt. ${ }^{[24]}$ 
In den Fällen, in denen das Metall an eine Aminosäure gebunden ist, ist die Mo-Oxo-Bindung länger als gewöhnlich und erleichtert damit die Protonierung zu dem Fragment Mo-OH. Die Mo-O Bindung des aminosäurefreien aktiven Zentrums dagegen entspricht hinsichtlich der Bindungslänge eher einer Mo-O-Doppelbindung. Enzyme wie die in Rhodobacter (DMSOReduktase, TMAO-Reduktase, Biotin-S-Oxid-Reduktase) besitzen neben dem Molybdän keine weiteren redoxaktiven Zentren. Die Arsenit-Oxidase hingegen, verfügen über weitere Fe-S-Zentren innerhalb des Proteins. Bei der Arsenit-Oxidase wird sogar eine weitere Untereinheit gefunden, die ein Rieske-Typ 2Fe-2S Zentrum beinhaltet.

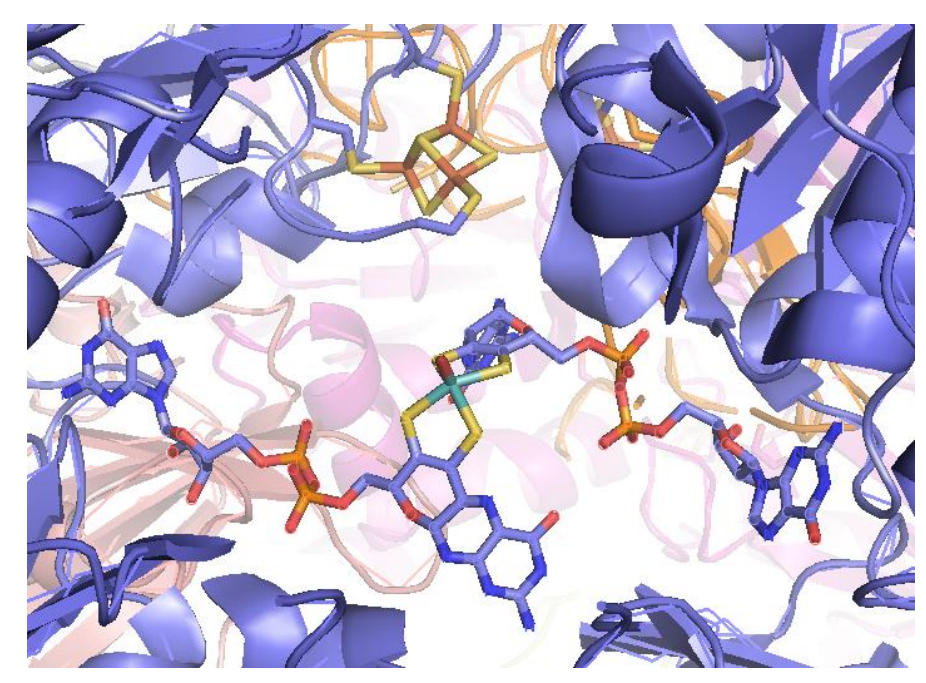

Abb. 11: Aktives Zentrum aus Alcaligenes Faecalis - PGB: 1G8K

Die Kristallstruktur der Arsenit-Oxidase aus Alcaligenes faecalis zeigt eine fünffache Koordination eines $\mathrm{Mo}^{\mathrm{IV}}=\mathrm{O}$ Zentrums wobei die restlichen vier Koordinationsstellen durch die üblichen zwei Molybddopterin-Liganden besetzt sind. ${ }^{[23]}$ In der oxidierten Form wird ein Dioxozentrum gefunden. Der größte Unterschied der Arsenit-Oxidase gegenüber den anderen Mitgliedern der DMSO-Reduktase Familie ist, dass keine Koordination des Metalls durch das Protein existiert. Außerdem stellt das Vorhandensein einer Oxogruppe in der reduzierten Form eine Ausnahme dar. In der Arsenit-Oxidase wird in der Position der sonst koordinierenden Aminosäure ein Alaninrest gefunden, der nicht fähig ist an das Molybdänzentrum zu binden. Nach der Entdeckung dieser Unterart wurde darüber diskutiert, ob die Arsenit-Oxidase als eine eigene Familie betrachtet werden sollte. ${ }^{[25]}$

\subsubsection{Modellsysteme}

Um einen besseren Einblick in die Enzyme der DMSO-Reduktase Familie zu bekommen, wurde eine Vielzahl von Modellverbindungen hergestellt. Es wurde in den Anfängen eine große Anzahl von Systemen mit einem einfach zu erhaltenden $\mathrm{MoO}_{2}$-Zentrum hergestellt, um 
somit die oxidierten Formen der Systeme besser verstehen zu können. ${ }^{[26]}$ Außerdem beschäftigten sich viele Gruppen damit, Modellverbindungen in der Oxidationsstufe V herzustellen, da diese das Bindeglied zwischen dem Oxidationszustand IV und VI im katalytischen Zyklus darstellt. Es wurden anfangs einfache Systeme wie $\left[\mathrm{MoO}(\mathrm{SPh})_{4}\right]^{-}$ hergestellt, die als erste Vertreter dieser Modelle anzusehen sind. ${ }^{[27]}$

Die ersten Oxo-bisdithiolenmetallkomplexe wie $\left[\mathrm{MoO}(\mathrm{bdt})_{2}\right]^{2-}$ und $\left[\mathrm{MoO}(\mathrm{bdt})_{2}\right]^{-}$konnten von der Garner Gruppe hergestellt werden und durch Röntgendiffraktometrie charakterisiert werden. $^{[28]}$ Nach dieser Entdeckung wurde eine große Anzahl an weiteren Dithiolenverbindungen hergestellt. ${ }^{[29]}$ Bisdithiolenmonooxo $\mathrm{Mo}^{\mathrm{IV}}-$ Verbindungen können durch die kontrollierte Oxidation mit z.B. $\mathrm{Me}_{3} \mathrm{NO}$ zur Dioxo-Verbindung mit $\mathrm{Mo}^{\mathrm{VI}}$-Zentrum oxidiert werden. Zur Modulation der Molybdopterinliganden wurden hauptsächlich Benzolderivate mit vorhandenen Dithioleneinheiten verwendet. Im Jahre 2005 wurde von unserer Arbeitsgruppe das erste Komplexsystem mit angelagertem Pyranringsystem hergestellt. Es zeigte sich, dass es wahrscheinlich durch die Bildung von diastereoisomeren Gemischen, während der Reaktion, zu einer schlechten Kristallisierbarkeit der Verbindungen kam. Dadurch konnte keine Kristallstruktur dieser Verbindungen erhalten werden. ${ }^{[4]} \mathrm{Im}$ gleichen Jahr wurden von Sugimoto et al. ähnliche Komplexe mit Pyranringsystemliganden hergestellt. Durch die erhaltenen Kristallstrukturen konnte eine quadratisch pyramidale Umgebung mit schwachen $\mathrm{Mo}=\mathrm{O}$ Bindungen verifiziert werden. ${ }^{[30]}$

Im Folgenden sind die beiden Verbindungen aufgezeigt. (Abb.12)

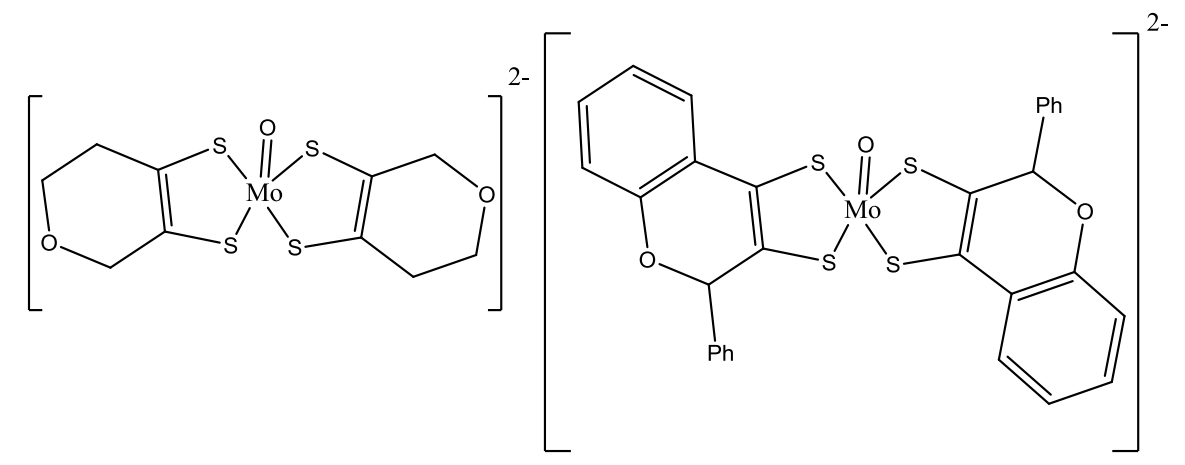

Abb. 12: (links) Modellsystem Sugimoto et al ${ }_{\circ}{ }^{[30]}$ (rechts) Modellsystem von Schulzke et al。 ${ }^{[4]}$

Andere Modellverbindungen wurden dadurch erhalten, dass vorhandene Precursor wie $\left[\mathrm{M}(\mathrm{CO})_{2}(\text { dithiolen })_{2}\right]$ mit den verschiedensten anionischen Liganden wie z.B. $\mathrm{PhO}^{-}, \mathrm{PhS}^{-}$, $\mathrm{PhSe}^{-}, \mathrm{MeO}^{-}$usw. umgesetzt wurden. Hiermit konnten gute Modellsysteme für aktive Zentren der DMSO-Redukatase Familie erhalten werden, da durch die Anlagerung der anionischen Liganden die Koordination durch die Aminosäureresten simuliert wurde. ${ }^{[31]}$ 


\subsubsection{Aldehyd -0xidoreduktase Familie (AOR)}

Die AOR Familie stellt eine Gruppe von Enzymen dar, zu der ausschließlich wolframhaltige aktive Zentren gehören. Derzeit sind 14 Enzyme dieser Gruppe aus anaeroben, meist thermophilen Organismen strukturell charakterisiert worden. Durch die Vertreter der AOR wird die in folgenden gezeigte Reaktion katalysiert, wobei es zur Umwandlung von Aldehyden zu Carbonsäuren kommt (Glg.3). Neben Wolfram als redoxaktivem Zentrum, liegen noch weitere Cofaktoren vor, wie z.B. 4Fe-4S-Cluster.

$$
\mathrm{RCHO}+\mathrm{H}_{2} \mathrm{O} \rightarrow \mathrm{RCOOH}+2 \mathrm{H}^{+}+2 \mathrm{e}^{-}
$$

Die erhaltenen Kristallstrukturen der AOR Familie zeigen, dass das Wolframzentrum von zwei Molybdopterinliganden umgeben ist. Es wird eine verzerrte quadratisch pyramidale Koordinationsumgebung beobachtet, wobei die Molybdopterinliganden in einem Winkel von ca. $97^{\circ}$ von der Ebene gebunden ist. Die beiden Liganden sind nicht nur mit dem Wolframzentrum verbunden, sondern sind außerdem durch ein Magnesiumion über die zwei vorhandenen Phosphatgruppen miteinander fixiert. Anders als bei den Mitgliedern der DMSO-Redukatase Familie wurde keine Koordination von Proteinliganden zum Metallzentrum festgestellt. Somit wird in der oxidierten Form folgende Zusammensetzung gefunden $(\mathrm{MPT})_{2} \mathrm{~W}^{\mathrm{VI}} \mathrm{O}(\mathrm{OH})$ und bei der reduzierten Form $(\mathrm{MPT})_{2} \mathrm{~W}^{\mathrm{IV}}(\mathrm{OH}) .{ }^{[2]}$

\subsubsection{Formiat-Dehydrogenase Familie}

Mitglieder der Familie dieser wolframhaltigen Enzyme sind die Formiatdehydrogenase (katalysiert die Reaktion von Formiat $\mathrm{zu} \mathrm{CO}_{2}$ ) und die $\mathrm{N}$-Formylmethanofurandehydrogenase (katalysiert die Reaktion von $N$-Formylmethanofuran zu Kohlendioxid). Dabei sind beide katalysierten Reaktionen reversibel, wodurch diese Art von Reaktionen von äußerster Wichtigkeit bei der Lösung der Klimaproblemen sein könnten.

\subsubsection{Acetylen-Hydratase Familie}

Eine weitere Gruppe stellt die Acetylenhydratase dar, die keine Redoxreaktionen katalysiert. Das bedeutet, dass das Wolframzentrum während der katalytischen Reaktionen keinen Wechsel der Oxidationstufen zeigt. Durch die Enzyme wird die Umwandlung von Acetylen zu Acetaldehyd katalysiert, was formal die Addition von Wasser an Acetylen darstellt. Seifert et al. publizierten die erste Kristallstruktur dieser Acetylenhydratase aus $P$. acetylenicus. ${ }^{[10]}$ Hierbei wurde eine Koordination von zwei Molybdopterinliganden zum Metallzentrum, sowie eine Anbindungen eines Schwefelatom aus einem Cystein gefunden werden. Außerdem ist 
Wasser an das Wolframzentrum gebunden, wodurch sich eine verzerrt oktaedrische Koordinationsumgebung ergibt.

\subsubsection{Modellsysteme für Wolframenzyme}

Im Vergleich zum Molybdän sind die Wolframenzyme viel weniger intensiv untersucht worden, was sich darin wiederspiegelt, dass auch nicht so viele wolframhaltige Modellverbindungen synthetisiert worden sind. Um die unterschiedliche Wirkung von Wolframenzymen untersuchen zu können wurden zu den Modellverbindungen des Molybdäns vielerlei analoge Wolframverbindungen hergestellt, um vergleichende Untersuchungen durchzuführen. Es konnte gezeigt werden, dass der Komplex $\left[\mathrm{Et}_{4} \mathrm{~N}\right]_{2}\left[\mathrm{~W}^{\mathrm{VI}} \mathrm{O}(\mathrm{mnt})_{2}\right]$ in der Lage ist, $\mathrm{CO}_{2} / \mathrm{HCO}_{3}{ }^{-}$bei $\mathrm{pH}=7.5 \mathrm{zu} \mathrm{HCOO}^{-}$umzuwandeln, was eine Modellreaktion für die Formiatdehydrogenase ist. $^{[32]}$ Außerdem wurden durch unseren Arbeitskreis Wolframverbindungen hergestellt, die zur Simulation des Molybdopterinliganden Pyranring enthaltende Systeme verwendeten. ${ }^{[4]}$ Kristallstrukturen dieser Verbindungen konnten allerdings nicht erhalten werden. Erst später wurden von Sugimoto et al. einfach Pyranligandensysteme (analog zu den Ligandensystemen die er bereits 2005 für Molybdän publizierte ${ }^{[30]}$ ) gebunden an Wolframzentren beschrieben, die auch durch Kristallstrukturen charakterisiert werden konnten. ${ }^{[33]}$ Außerdem konnte Holm et al. Verbindungen des Typs $\left[\mathrm{W}^{\mathrm{IV}}(\mathrm{OMe})\left(\mathrm{S}_{2} \mathrm{C}_{2} \mathrm{Me}_{2}\right)\right]^{-}$herstellen, die im Vergleich mit dem aktiven Zentren der AORFamilie eine sehr gute Übereinstimmung lieferten. ${ }^{[31 \mathrm{~b}]}$

Die FDH Familie konnte ebenfalls von Holm et al. moduliert werden durch Modelle wie $\left(\mathrm{Et}_{4} \mathrm{~N}\right)\left[\mathrm{W}^{\mathrm{VI}} \mathrm{S}(\mathrm{SeAd})\left(\mathrm{C}_{2} \mathrm{~S}_{2} \mathrm{Me}_{2}\right)_{2}\right]$. Es konnten aber weder Sauerstoffübertragungskatalysen untersucht, noch eine Kristallstruktur dieser Verbindungen erhalten werden. ${ }^{[34]}$ 


\section{Kapitel 2}

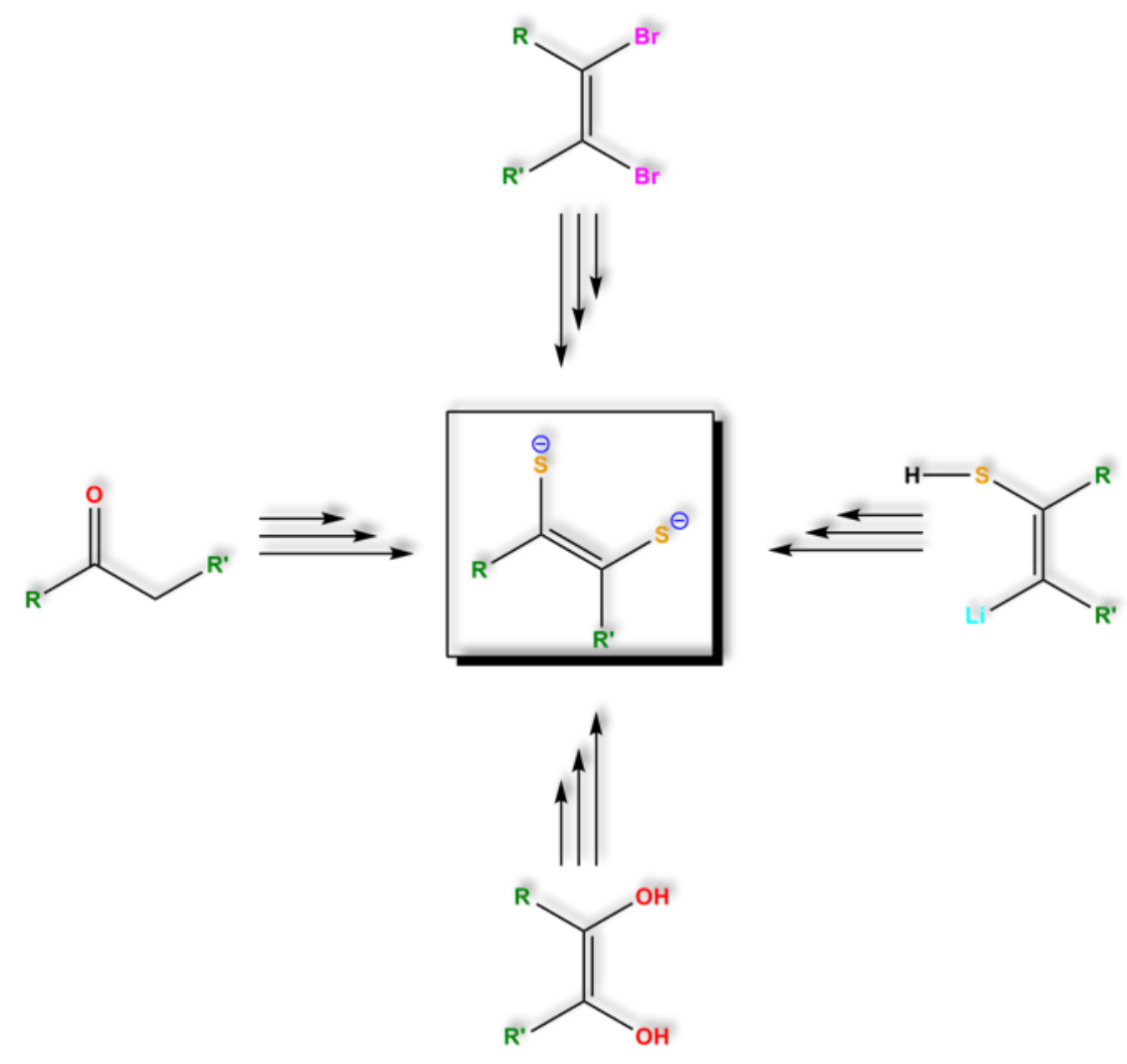

\section{Synthesestrategien und Methoden}

\section{Zusammenfassung}

In diesem Kapitel wird von den Anfängen der Dithiolenchemie berichtet und der Einzug dieser Chemie in die Bereiche der Bioanorganischen Modellchemie. Außerdem werden die von mir verwendeten Synthesestrategien zur Darstellung von Dithiolenverbindungen vorgestellt, sowie die hiermit erhaltenen Ligandensysteme. 


\subsection{Dithiolenliganden}

Die Erforschung von Metalldithiolenkomplexen geht auf die frühen 60er Jahre des 20.Jahrhunderts zurück. Hierbei sind insbesondere die Arbeitsgruppen von Schrauzer (München), Davison und Holm (Harward) und Gray (Columbia) zu nennen. ${ }^{[35]}$ Anfänglich standen meist Redoxaktivitätsbestimmungen und strukturelle Untersuchungen der Bindungen im Vordergrund. Nach dieser Untersuchungsphase zeigten sich weitere nützliche Anwendungen dieser Dithioleneinheiten, z.B. in der Tetrathiafulvalen Chemie. Durch die potentielle Nutzbarkeit als Molekularschalter wurde die Erforschung dieser Systeme stark beschleunigt. ${ }^{[36]}$ Anfang der 90er Jahre konnte aufgrund der Entdeckung der biologischen Molybdän- und Wolframenzyme ein weiteres Feld der Dithiolenchemie eröffnet werden. ${ }^{[37]}$

Die ersten veröffentlichten und vollständig charakterisierten homoleptischen Bisdithiolenverbindungen waren $\left[\mathrm{Ni}(\mathrm{mnt})_{2}\right]$ und $\left[\mathrm{Ni}\left(\mathrm{S}_{2} \mathrm{C}_{2} \mathrm{Ph}_{2}\right)_{2}\right]^{[38]}$. Hierbei konnte eine quadratisch planare Koordinationsumgebung des Nickels beobachtet werden. Außerdem wurden Trisdithiolenverbindungen in Kombination mit anderen Übergangsmetallen gefunden, wie z.B. $\left[\operatorname{Re}\left(\mathrm{S}_{2} \mathrm{C}_{2} \mathrm{Ph}_{2}\right)_{3}\right]$, $\left[\mathrm{Mo}(\mathrm{edt})_{3}\right]$ und $\left[\mathrm{V}\left(\mathrm{S}_{2} \mathrm{C}_{2} \mathrm{Ph}_{2}\right)_{3}\right]^{[39]}$. Diese Verbindungen wiesen hauptsächlich eine ungewöhnliche trigonal prismatische Koordinationsgeometrie auf.

Die aufregende Eigenschaft von Dithiolenmetallkomplexen ist hauptsächlich auf das Zusammenspiel des redoxaktiven Dithiolenligandenes mit dem redoxaktiven Metallzentrum zurückzuführen. Dies wird hauptsächlich auf die starke Non-Innocence der Dithiolenliganden geschoben. Dithiolene können als neutrale Dithioketone, in dianionischer Form sowie als gemischte Radikale an ein Metallzentrum gebunden sein.

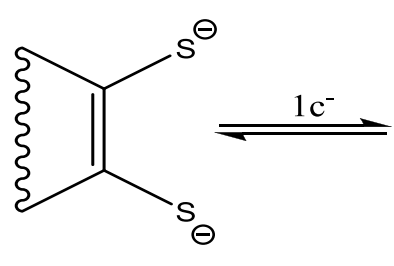

$6 \pi \mathrm{e}^{-}$

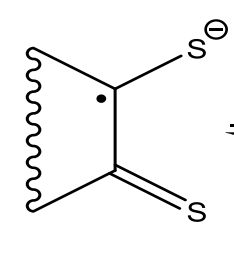

$5 \pi \mathrm{e}^{-}$
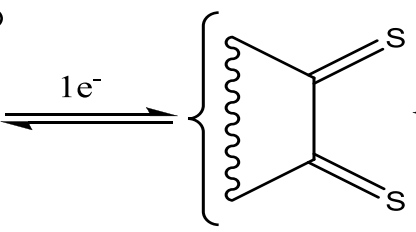

$4 \pi \mathrm{e}^{-}$

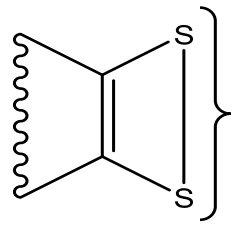

Abb. 13: Non-Innocence Charakter von Dithiolenliganden

Hierbei muss die tatsächliche Valenz aus einer Mischung von Dithion/Dithiete und Dianionischer (Dithiolat) bestehen. Die formal vorhandene Oxidationsstufe unterscheidet sich zu der analogen Sauerstoffstruktur des Quinon/Semiquinon/Catecholat, bei der eine diskrete Oxidationsstufe vorhanden ist. Bei den Dithiolenliganden findet sich meistens eine 
Resonanzstruktur zwischen dem 1,2-Dithion und dem 1,2-Dithiet. Der Unterschied zwischen der dianionischen und der Dithionstruktur ist die Anzahl der vorhandenen $\pi$-Elektronen. Somit findet man beim dianionischen Komplex $6 \pi$-Elektronen, während bei der Thioketonform nur noch $4 \pi$-Elektronen vorhanden sind. Die Anzahl der Sigma-Elektronen von sechs bleibt bei beiden Zustandsformen konstant. Hoffmann und seine Mitarbeiter beschäftigten sich mit der genauen Berechnung der Orbitale in beiden möglichen Elektronenkonfigurationen $^{[40]}$. Die aktuelle Struktur eines Bis-Dithiolenkomplexes stellt eine Mischung aus einem Resonanzhybrid dar. Es wird angenommen, dass die Redoxaktivität entweder nur vom Metall hervorgerufen wird, oder nur vom Ligandensystem.

\subsection{Verwendete Methoden zur Herstellung von Dithiolensystemen}

\subsubsection{Dithiolensysteme aus ketonhaltigen Verbindungen}

Eine Methode die ursprünglich von Garner et al. entwickelt wurde, bietet eine ausgezeichnete Methode zur Herstellung von Dithiolenliganden ${ }^{[29 a}$. Im Folgenden sind die einzelnen Reaktionsschritte aufgeführt.

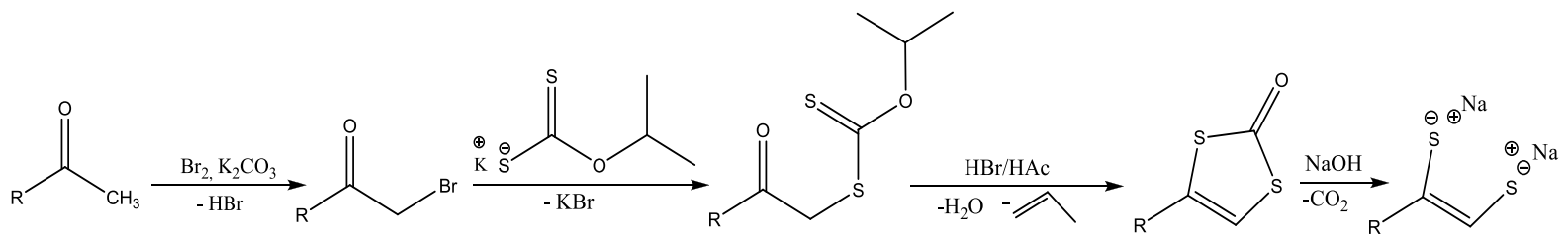

Abb. 14: Reaktionsschema exemplarisch für die Syntheseschritte zu einem Dithiolenliganden

Um diese Reaktionschritte durchführen zu können, muss der herzustellende Dithiolenligand in der Position des gebildeten Dithiolens eine Keto oder Thio Funktion tragen. Außerdem müssen in der $\alpha$-Position zur Carbonylgruppe zwei freie Wasserstoffatome vorhanden sein, da sonst die Bromierung nicht durchgeführt werden kann. Dabei können sowohl Bromierungen mit elementarem Brom sowie Bromierungen mit NBS ( $N$-Bromsuccinimid) durchgeführt werden. Um die Bromierung durchführen zu können, muss es zur säurekatalytischen Enolisierung der Carbonylverbindung kommen, da diese dann als Nucleophil mit Elektrophilen wie $\mathrm{Br}^{+}$reagieren kann. Die Stabilisierung wird durch die mögliche Resonanz der Grenzstrukturen erhalten. 


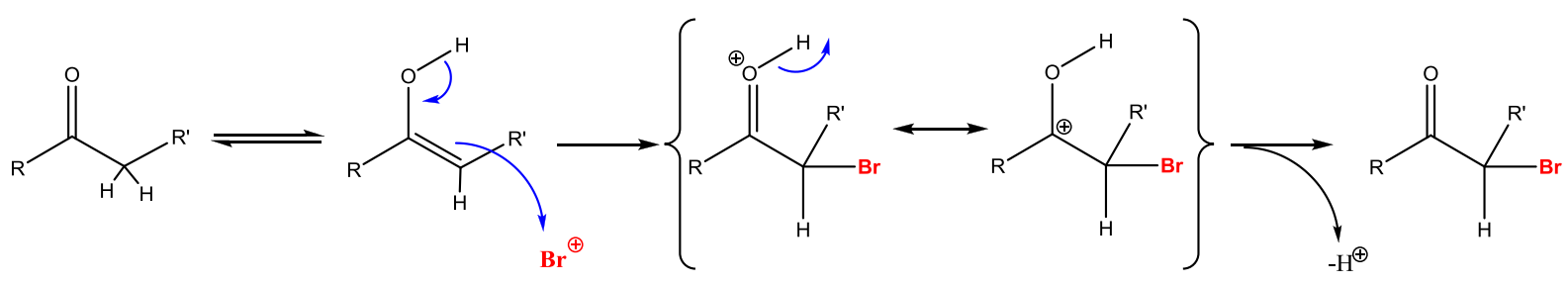

Abb. 15: Bromierung der Carbonylgruppe in $\alpha$-Position

Das abgespaltene Proton reagiert mit dem noch vorhanden $\mathrm{Br}^{-} \mathrm{zu}$ Bromwasserstoff, der dann durch die Verwendung von Kaliumcarbonat als Base wieder abgefangen werden kann. Da die Bildung des entstehenden HBr's nicht zu stark sein darf wird diese Bromierung bei Temperaturen um die $-20^{\circ} \mathrm{C}$ durchgeführt. Bei Verwendung von größeren Mengen Brom (> $5 \mathrm{ml}$ ) müssen die Reaktionszeiten erhöht und die Temperatur drastisch abgesenkt werden; $-80^{\circ} \mathrm{C}$, mind. $24 \mathrm{~h}$ Reaktionszeit. Außerdem besteht das Problem der Mehrfachbromierung. Deshalb muss unter Lichtausschluss gearbeitet werden, da die durch Belichtung entstehenden radikalischen Substanzen sehr stark mit den Edukten reagieren. Wenn die Verbindungen nicht durch elektronenschiebende Substituenten stark aktiviert werden, verläuft die Reaktion bei Verwendung richtiger stöchiometrischer Zusammensetzung sehr sauber. Die Ausbeuten der Reaktion belaufen sich im Mittel auf etwa 75\%, doch die vollständige Charakterisierung ist vielfach nicht möglich, da es sehr schnell zum Zerfall der Verbindungen kommt. Deshalb bietet es sich oft an, die entstandenen Reaktionsprodukte direkt zur nächsten Stufe umzusetzen.

Um die Ausbeuten weiter zu erhöhen, wurden außerdem Bromierungen nach einer Methode von S. Paul durchgeführt. Dabei wurde eine Kieselgelkatalysator in Kombination mit NBS verwendet. ${ }^{[41]}$ Reaktionen mit ausschließlich NBS führten zu geringeren Ausbeuten und einer größeren Anzahl an Nebenprodukten. Mit Hilfe dieser Methode konnten Ausbeuten um die 90\% erzielt werden. Einzige Bedingung ist, dass sich das Edukt in Hexan lösen muss. Bei Verwendung von anderen Lösungsmitteln konnten keine Produkte erhalten werden. Das Kieselgel wird zuvor durch Verwendung von Perchlorsäure aktiviert, wodurch der enthaltene heterogene Katalysator auch nach mehreren Katalyseschritten seine Aktivität nicht verliert.

Den nächsten Schritt der Reaktionsfolge stellt die Reaktion mit einem sogenannten Xanthogenatsalz dar. Dabei kommt es zur Bildung einer $\sigma$-Bindung zwischen Kohlenstoff und Schwefel. Entstandenes Kaliumbromid kann sehr gut durch Extraktion mit Wasser abgetrennt werden. Die Xanthogenatsalze sind sehr leicht zugänglich und können aus den entsprechenden Alkoholen durch Umsetzung mit $\mathrm{KOH}$ und $\mathrm{CS}_{2}$ erhalten werden. ${ }^{[42]}$ Die 
hieraus erhalten Produkte sind stabil und können ohne Probleme an der Luft aufbewahrt werden. Vielfach verläuft diese Reaktion mit Ausbeuten um die 95\% ab, doch bei den von mir hergestellten tldt-Liganden konnten bei diesem Reaktionschritt viele Nebenprodukte beobachtet werden. Eine chromatographische Reinigung an Kieselgel lieferte dann gute Ausbeuten. Außerdem ist das Ansäuern der erhaltenen Lösungen mit ca. 10\% $\mathrm{HCl}$ wichtig, um nicht reagiertes Xanthogenatsalz abzutrennen. Folgende Reaktion findet mit dem Xanthogenatsalz statt.

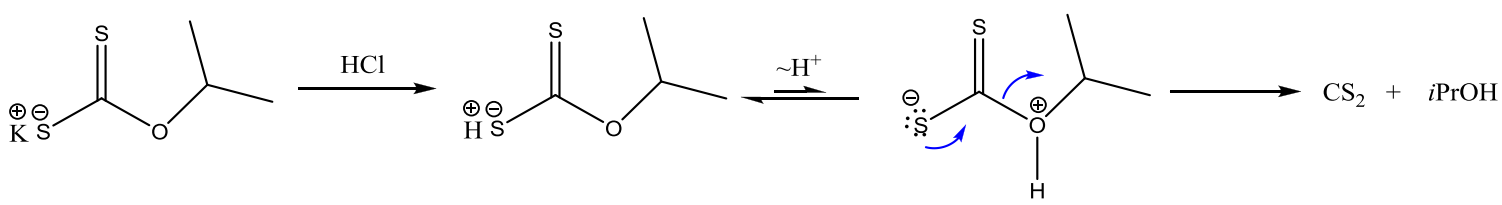

Abb. 16: Abtrennungsreaktion des Xanthogenatsalzes

Die gebildeten flüchtigen Produkte wie Schwefelkohlenstoff und iso-Propanol können nun leicht im Vakuum abgetrennt werden.

Der letzte Schritt, der zu einer Dithiocarbamat-Verbindung führt, verläuft über eine säurekatalysierte Tschugaeff-Ringschluss Reaktion. Dieser Reaktionsschritt stellt den empfindlichsten Schritt der Reaktionsreihe dar, da für diese Ringschlussreaktion starke Säuren wie $\mathrm{HBr}$ oder $\mathrm{H}_{2} \mathrm{SO}_{4}$ nötig sind, wodurch empfindliche Gruppen am Ligandengerüst zerstört werden können. Im Folgenden ist der Mechanismus der Ringschlussreaktion aufgeführt.

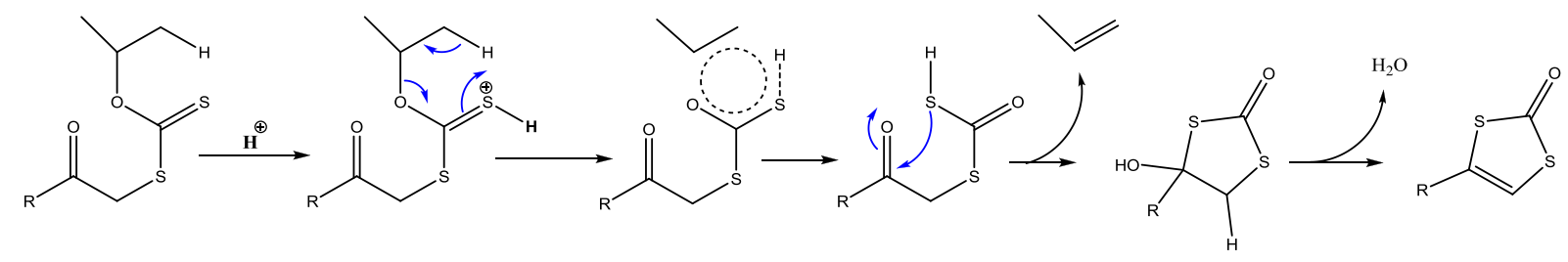

Abb. 17: Ringschlussreaktion nach Tschugaeff

Die entstehende Nebenprodukten Propen und $\mathrm{H}_{2} \mathrm{O}$ können sehr einfach von dem Produkt abgetrennt werden, dass im neutralen $\mathrm{pH}$-Bereich vollkommene Unlöslichkeit in Wasser aufweist. Die angelagerten Gruppen am Xanthogenat (hier iso-Propyl) können variiert werden. Es zeigte sich, dass es bei Gruppen mit größeren sterischen Anspruch zu einer Erleichterung dieser Ringschlussreaktion kommt. Die erhaltenen Liganden sind luftstabil, sollten aber am Besten unter Inertgas-Atmosphäre aufbewahrt werden, da leichte Verfärbungen der Substanzen nach längerem Aufenthalt an Luft auftreten. Diese Art 
Dithiocarbonateinheit stellt eine sehr gute Schutzgruppe für die Dithiolenverbindungen dar. Somit können vielerlei Reaktionen an Gerüstatomen durchgeführt werden, ohne dass es zur Zerstörung der Funktionalität kommt. Einzige Vorsicht ist bei Verwendungen von Basen gegeben, da es zur Ringöffnung unter Kohlendioxidabspaltung kommt. Daher bietet es sich auch an, diese Verbindungstypen in situ bei der Verwendung in Reaktionen zum dianionischen Dithiolat umzusetzen, da diese Dithiolate verschiedene unerwünschte Nebenreaktionen eingehen können.

Mit Hilfe dieser Methode konnte eine Vielzahl von Ligandensystemen erhalten werden.

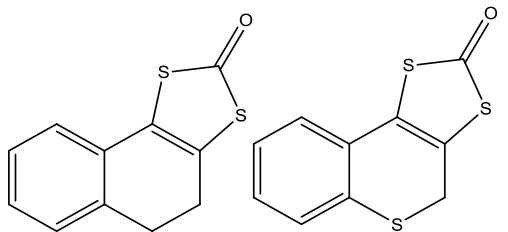

tldt

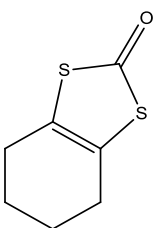

cydt

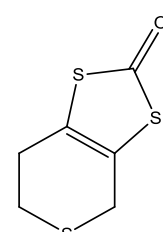

tpdt

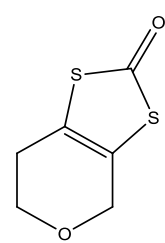

pdt

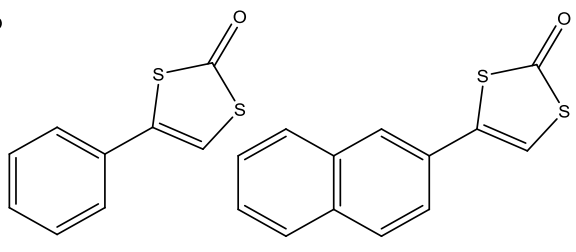

sdt

Abb. 18: Hergestellte Liganden nach der vorgestellten Methode

Der tldt- bzw. tcdt-Ligand wurde ausgehende von $\alpha$-Tetralon sowie Thiochromanon synthetisiert. In beiden Fällen wurde die Isopropylxanthogenatverbindung verwendet, da die Ausbeuten des Ringschlusses hierbei um ca. 5\% besser waren.

Der Cyclohexandithiolenligand wurde ausgehend von Cyclohexanon und anschließender Bromierung in Anwesenheit von $\mathrm{I}_{2}$ als Aktivierungskatalysator nach Literaturangaben bromiert. ${ }^{[43]}$ Der Ringschluss konnte nicht mehr mit der üblich verwendeten $\mathrm{HBr} /$ Eisessigmischung erzielt werden, stattdessen wurde Perchlorsäure verwendet, die das Produkt nach säulenchromatographischer Reinigung an Kieselgel lieferte. Die Thiopyranverbindung wurde aus Methylacrylat hergestellt. Im Folgenden ist die Synthese bis zum Dihydro-2H-thiopyran-4(3H)-on gezeigt

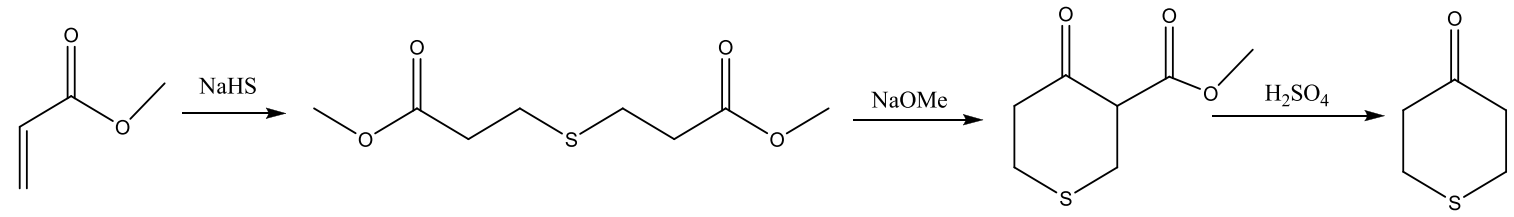

Abb. 19: Synthese bis zum Dihydro-2H-thiopyran-4(4H)-on

Nach den normalen Bromierungsschritten und Anlagerung der Xanthogenats konnte mit Hilfe von Perchlorsäure der Ringschluss durchgeführt werden. Die bromierte Zwischenstufe ist sehr instabil und kann nur direkt und ohne weitere Analysen mit dem Xanthogenatsalz umgesetzt 
werden. Die zuletzt verwendeten sdt und ntdt-Liganden wurden aus den schon kommerziell erhaltenen bromierten Ausgangsverbindungen hergestellt. Alle erhaltenen Liganden konnten sowohl durch NMR-Spektroskopische, massenspektrometrische, infrarotspektroskopische sowie Elementaranalysen charakterisiert werden. Außerdem war es möglich diesen Verbindungstyp über eine Reaktion einer 1,2-Dichlorierten oder bromierter Verbindungen mit Hilfe von Schwefelkohlenstoff $\mathrm{zu}$ einem Trithiocarbonat in einem Reaktionsschritt umzuwandeln. Dabei stellte sich heraus, dass der angelagerte weitere Schwefel zu einer erhöhten Stabilität, gegenüber den Dithiocarbonaten führt und die Ringöffnung mit $\mathrm{KOH}$ bzw. $\mathrm{NaOH}$ nicht mehr möglich war. Deshalb müssen zuvor erstelle Trithiocarbonate mit Hilfe von Quecksilberacetat und Essigsäure zum Dithiocarbonat umgesetzt werden. ${ }^{[4]}$

\subsubsection{Dithiolensysteme aus Dibromierten Verbindungen}

Da zur Verwendung der obigen Reaktionsfolge eine Carbonylgruppe vorhanden sein muss, lassen sich Aromaten, in denen jeder Kohlenstoff bereits drei Bindungen zu benachbarten Atomen aufweist, nicht verwenden. Deshalb hat sich folgende Methode von Klingsberg als sehr hilfreich herausgestellt. ${ }^{[4]}$ Der erste Schritt dieser Reaktionsfolge stellt eine Dibromierung des aromatischen Systems dar. Dabei müssen die eingebrachten Bromatome in Position des späteren Dithiolens angebracht werden.

Es gibt verschiedenste Möglichkeiten aromatische Systeme zu bromieren, doch die Bromierung mit elementarem Brom und Zugabe von einigen Iodkristallen als Katalysator lieferte gute Ergebnisse. ${ }^{[45]}$

Den nächsten Schritt stellt ein Austausch der Bromatome durch Thiole dar. Beim direkten Austausch der Bromatome durch R-SH-Verbindungen, konnte es aufgrund des Bromwasserstoff-Nebenproduktes zu Problemen in der Reaktionsfolge kommen. Deshalb habe ich auf eine Übertragung durch Kupfer-alkylmercaptide zurückgegriffen die nach folgendem Schema erhalten werden können.

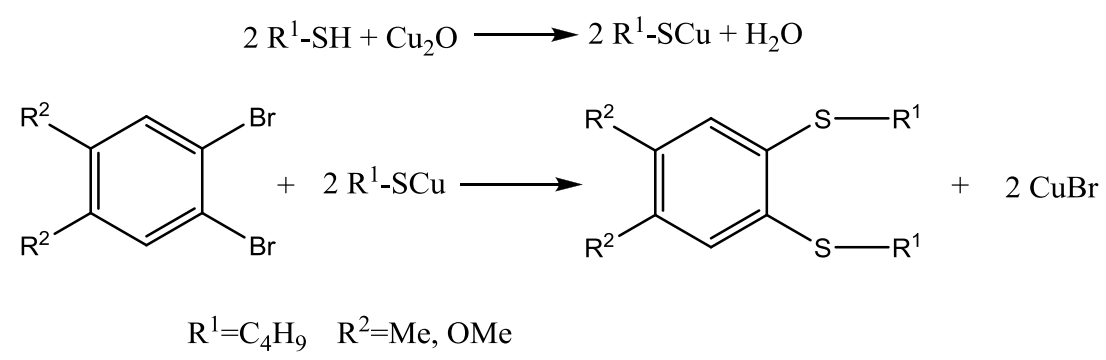

Abb. 20: Übertragung der Thiole

Der Vorteil dieser Methode ist, dass man das entstehende $\mathrm{CuBr}$ und auch andere Kupfernebenprodukte gut durch Extraktion mit Ammoniaklösung abtrennen kann. 
Als letzter Schritt dieser Synthesefolge schließt sich eine Birch-Reduktion an. Hierbei findet eine Reduktion der Thioetherfunktion statt, wobei im letzten Schritt die Dithiolenverbindung erhalten wird.

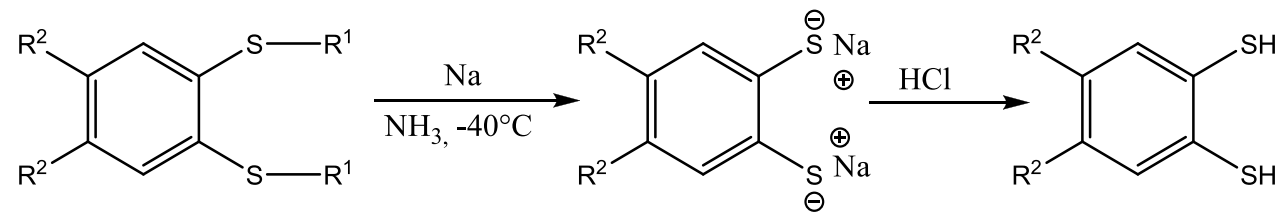

Abb. 21: Birch-Reduktion zum Erhalt des Dithiolenliganden

Mit Hilfe dieser Methode konnten folgende zwei Ligandensysteme (links) erhalten werden.<smiles>COc1cc(S)c(S)cc1OC</smiles>

$\mathrm{vdt}$<smiles>Cc1cc(S)c(S)cc1C</smiles>

$\mathrm{xdt}$

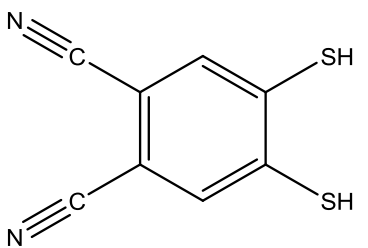

ndt

Abb. 22: (links) hergestellte Ligandensysteme nach der Reduktionsmethode, (rechts) erhalten aus Reaktion mit $\mathrm{AlCl}_{3}$

Befinden sich am aromatischen Gerüst aber empfindlichere Substituenten oder stark desaktivierende Substituenten kann diese Methode zur Herstellung nicht mehr verwendet werden. Bei solchen Gegebenheiten hat sich die Reaktion mit $\mathrm{AlCl}_{3}$ in Toluol angeboten. Mit Hilfe dieses Schritts konnte beim Phtalonitil-Ligand eine Reduktion von der angebundenen Thioetherfunktionen zur Dithiolenverbindungen erhalten werden, siehe Abb.22 rechts.

\subsubsection{Dithiolensysteme aus Thiolaromaten}

Eine neuere Methode in der Herstellung von Dithiolensystemen stellt die Methode von Kirschbaum und Martin da. ${ }^{[46]}$ Hierbei werden Benzolthiole mit $n$-BuLi zu 2- $\mathrm{LiC}_{6} \mathrm{H}_{4}(\mathrm{SLi})$ umgesetzt, welches dann mit elementaren Schwefel zu dem gewünschten Dithiolen reagiert.

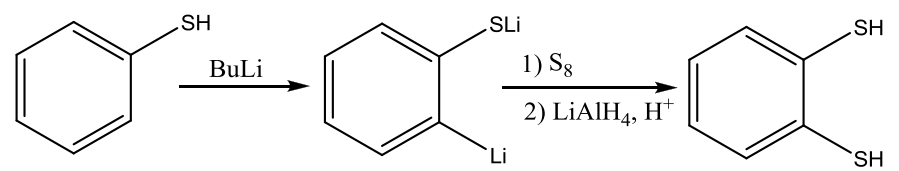

Abb. 23: Herstellung von Dithiolen nach der Methode von Krischbaum und Martin

Bei der Herstellung von Benzoldithiolen bietet diese Synthese einen Vorteil im Vergleich zu den oben beschriebenen Synthesen. Bei Veränderung der Aktivität des aromatischen Systems durch elektronenschiebende sowie elektronenziehende Substituenten kommt es jedoch zur Bildung von einigen Nebenprodukten, die schlecht von den Hauptprodukten abgetrennt werden können. Deshalb wurde in diesen Fällen auf die Methode 2.2.2 zurückgegriffen. 


\subsubsection{Dithiolensysteme aus Diol-Verbindungen}

Eine weitere Methode zur Herstellung von Dithiolenverbindungen ist die Umwandlung von Alkoholgruppen mit Phosphorpentasulfid.

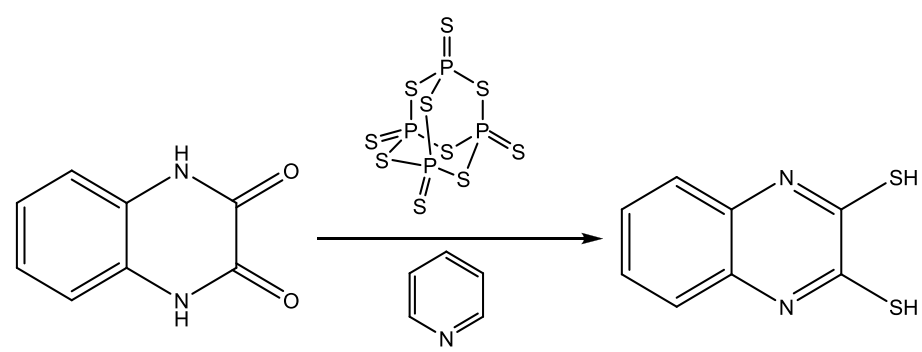

Abb. 24: Syntheseschritte zur Umwandlung von Diol-Gruppen

Dieser Ligand wurde nach Literaturvorschriften ${ }^{[47]}$ synthetisiert. Es zeigte sich, dass zur Anwendung der Umwandlung durch $\mathrm{P}_{4} \mathrm{O}_{10}$ auf andere Ligandensysteme eine Konjugation der $\pi$-Elektronen der Dithioleneinheit auf andere Molekülbereiche möglich sein muss. Diese Reaktionsmethode wurde mit verschieden Diolverbindungen durchgeführt, doch es konnten nicht die Dithiolensysteme in ausreichenden Ausbeuten erhalten werden. Deshalb müssen die vorhandenen Stickstoffatome in Konjugation auch einen Einfluss auf die Reaktion gehabt haben. Da die Reaktionsbedingungen (Refluxieren in trocknem Pyridin) nicht nennenswert verändert werden können, ist es nicht möglich diese Reaktionsmethode für Reaktionen mit empfindlichen Funktionen zu benutzen. Aber sie kann durchaus für Eingangsstufen von Modellverbindungen benutzt werden, die dann in weiteren Syntheseschritten weiter durch Einführen empfindlicher Gruppen verfeinert werden. 


\section{Kapitel 3}

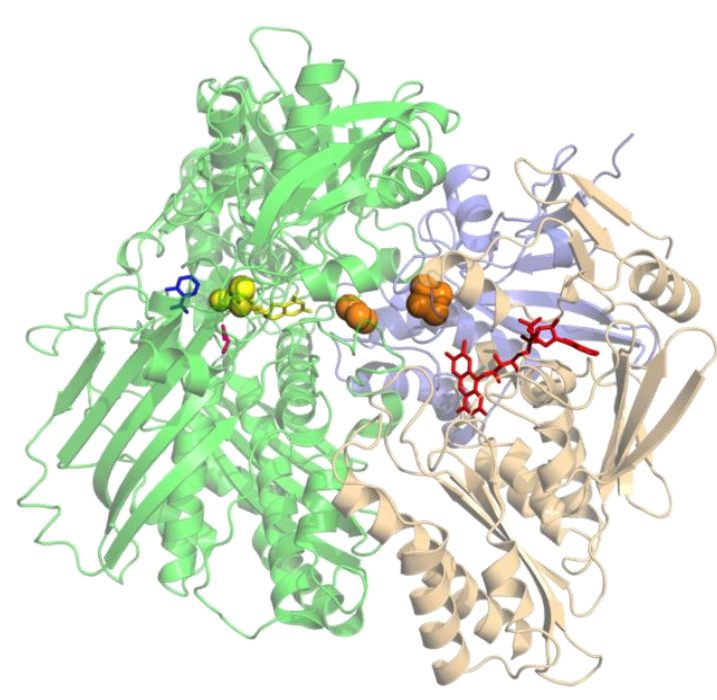

\section{Modelle für Mo- und W-Cofaktoren}

\section{Zusammenfassung}

In diesem Kapitel werden die von mir angewendeten Methoden zur Darstellung der Komplexverbindungen vorgestellt und in die unterschiedlichen Anwendungsbereiche eingeteilt. Außerdem wird beschrieben, wie die vorgestellten Verbindungspaare charakterisiert wurden. 
Hauptanliegen war es eine Reihe von diversen Molybdän- und Wolframkomplexen zu synthetisieren, die als Modelle für Mo- und W- Cofaktoren dienen können. Diese sollten auf verschiedene strukturelle Aspekte, aber vorallem in vergleichenden temperaturabhängigen elektrochemischen Untersuchungen verwendet werden können. Voraussetzung hierfür war eine gewisse Stabilität bei den jeweiligen Untersuchungsbedingungen, moderate Löslichkeit und eine reversibles bzw. quasireversibles elektrochemisches Verhalten. Aufgrund der noninnocence der Dithiolenliganden wurden weiterhin Studien zur elektronischen Struktur der Komplexe wie z.B. UV/Vis und gegeben falls ESR-Spektroskopie durchgeführt. Außerdem wurde DFT-Rechnungen erstellt, um bestimmte Fragen der Struktur zu beantworten. Sofern es möglich war, wurden Kristallstrukturen der Verbindungen bestimmt.

\subsection{Synthese von Monooxo-dithiolen Komplexen}

\subsubsection{Genereller Syntheseansatz}

In der Anwendung hat sich das Ausgangsmaterial $\mathrm{K}_{3} \mathrm{Na}\left[\mathrm{MoO}_{2} \mathrm{CN}_{4}\right] \cdot 6 \mathrm{H}_{2} \mathrm{O}$ als sehr geeignet für eine Reihe von Reaktionen herausgestellt. Ein großer Vorteil ist, dass das Ausgangsmaterial relativ luftstabil ist und auch im Gramm-Maßstab sowohl für Molybdän als auch für Wolfram synthetisiert werden kann. Nachteilig sind die Verwendung von Kaliumcyanid im Herstellungsprozess und die schlechte Löslichkeit der Metallkomplexe. Die beste Löslichkeit wird in entgastem Wasser erzielt. Die Verwendung von anderen Lösungsmitteln bei der Synthese, lieferte nicht die gewünschten Produkte. Ein weiterer Vorteil der Verwendung von Wasser ist, dass bei der Aufarbeitung die ionischen Nebenprodukte gut in der wässrigen Phase verbleiben. Außerdem ist die Verwendung von $\mathrm{NaOH}$ bzw. $\mathrm{KOH}$ möglich, womit die bestmögliche Ringöffnung am effizientesten erzielt werden kann. Die vorhandenen Cyanide werden als gute Abgangsgruppe leicht durch die Alkalisalzdithiolenverbindungen ausgetauscht. Aufgrund der Größe der Liganden und der damit verbundenen geometrischen Änderung der Metallumgebung kommt es während der Reaktion zu einem Verlust der zweiten Oxofunktion. Dennoch wird durch diesen Platzhalter die Bildung von Trisdithiolenkomplexen verhindert.

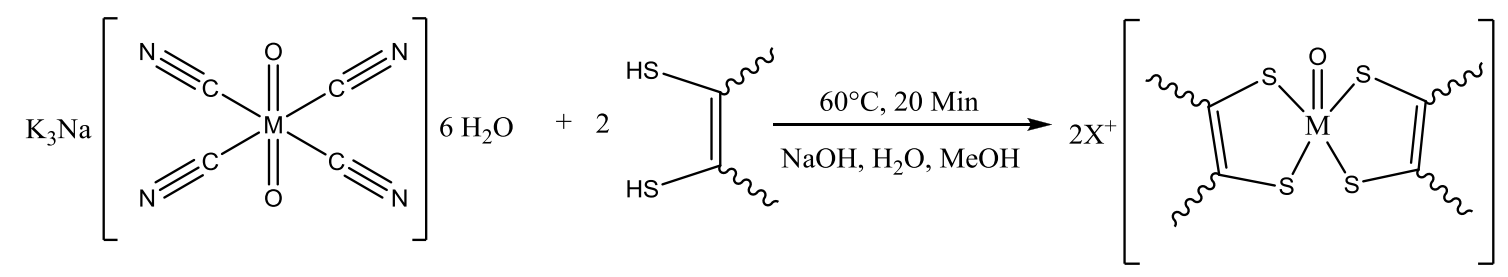


Mit dieser Synthese können sowohl 1,2-Dithiolliganden als auch Dithiocarbonatliganden an Metallzentren koordiniert werden. Die Koordination verschiedenster Sauerstoffliganden schlug nach dieser Methode fehl. Dies ist wohlmöglich auf die unterschiedliche Größe, oder auch auf Unterschiede in der Elektronegativiät der Sauerstoffatome, im Vergleich zu den Schwefelatomen, zurückzuführen.

Wird die Reaktion in sehr gut entgastem Wasser durchgeführt, können auch sauerstoffinstabile Verbindungen synthetisiert werden. Ein Nachteil dieser Reaktion ist aber, dass zur Substitution der $\mathrm{CN}^{-}$-Liganden etwas Reaktionswärme erforderlich ist. Es zeigt sich, dass für die Synthese von Wolframkomplexen eine deutlich größere Wärmezufuhr erforderlich ist. Als Zweitlösungsmittel für die Liganden-Precursorn bietet sich Methanol an, da sich in der Praxis gezeigt hat, dass bei Rückflusstemperaturen von $65^{\circ} \mathrm{C}$ die besten Ausbeuten bei Verwendung von Dithiocarbonaten erreicht werden. Meist kommt es durch die Erwärmung zu einem Farbumschlag von blau(Mo)/violett(W) zu dunkelrot. Von großer Wichtigkeit ist, dass kein überschüssiger Ligand in der Reaktionslösung verbleibt. In Anwesenheit des freien Liganden kann es zu einer Oxidation des Metallausgangsmaterials kommen und somit $\mathrm{K}_{2}\left[\mathrm{M}^{\mathrm{VI}} \mathrm{O}_{2}(\mathrm{CN})_{4}\right]$ entstehen. Da dieses Nebenprodukt ähnliche Lösungseigenschaften wie die Hauptprodukte aufweist, erwies es sich als sehr schwer diese vom Endprodukt abzutrennen. Um dieser Komplikation auszuweichen, wurden die Metallkomplexe in einem leichtem Überschuss (1.1 eq Metall zu 2.0 eq Ligand) verwendet. Nach der Reaktion wurde eine Extraktion mit Chloroform unter Inertgasatmosphäre durchgeführt, um noch vorhandene Liganden bzw. Ligandenzersetzungsprodukte zu entfernen.

\subsubsection{Wahl des Kations}

Da bei dieser Synthese ionische Komplexe hergestellt werden, ist die Wahl des verwendeten Kations ausschlaggebend für die Lösungseigenschaften des entstehenden Produkts. Im Laufe meiner Doktorarbeit verwendete ich $\mathrm{Bu}_{4} \mathrm{~N}^{+}, \mathrm{NEt}_{3} \mathrm{Bz}^{+}, \mathrm{NEt}_{4}{ }^{+}, \mathrm{PPh}_{4}{ }^{+}$als Gegenionen zu den anionischen Metallkomplexen. Der Vorteil der Verwendung des Tetrabutylammonium-, sowie Tetraphenylphoshonium-Kationen ist, die vollkommene Unlöslichkeit der Komplexe in Wasser. Die erhaltenen Verbindungen können erneut mit Wasser gewaschen werden, wodurch nicht reagiertes Metallausgangsmaterial, sowie noch vorhandene Basenreste, sauber abgetrennt werden können. Nachteil bei der Verwendung des $\mathrm{Bu}_{4} \mathrm{~N}^{+}$-Kation ist, dass es vielfach zur Bildung von Ölen kommt, die erst nach mehreren Umkristallisationen als 
Feststoffe oder Kristalle erhalten werden. Um eine Reihe von Nebenprodukten, sowie basische Salzgemische abzutrennen, modifizierte ich die ursprüngliche Synthesemethode von Garner et al. ${ }^{[29 \mathrm{a}]}$ durch den Einsatz von Acetonitril während der Reaktion. Vielfach bot es sich an, das komplette Lösungsmittelgemisch (Wasser/Methanol) von der Reaktionslösung abzutrennen und den erhaltenen basischen Rückstand in Acetontril auszunehmen. Somit konnte in Acetonitril unlösliches $\mathrm{NaOH}$ bzw. $\mathrm{KOH}$ durch Filtration abgetrennt werden. Nach erneutem Einengen und Extraktion unter Schutzgas mit Chloroform wurden sehr reine Produkte erhalten. Bei Verwendung des $\mathrm{PPh}_{4}{ }^{+}$-Kations kann Acetonitril nicht eingesetzt werden, da die entstehenden Komplexe hierin schlecht löslich sind. Bei Verwendung des $\mathrm{PPh}_{4}{ }^{+}$-Kations bietet es sich an, die Reaktionslösung ohne weitere Extraktionen direkt mit einem Überschuss an entgastem Wasser zu versetzen. Die Reaktionslösung wird nun bei $4^{\circ} \mathrm{C}$ im Kühlschrank über Nacht aufbewahrt, wodurch es zum Ausfall des Produkts kam. Nach Filtration und waschen mit entgastem Wasser konnten gute Ausbeuten und reine Produkte erhalten. Bei Verwendung von $\mathrm{NEt}_{4}{ }^{+}$sowie $\mathrm{NEt}_{3} \mathrm{Bz}^{+}$als Kation sind die Komplexe in der Regel in Wasser löslich. Die Salze können durch Aufnahme in Acetonitril und Zugabe von Diethylether direkt als Feststoffe erhalten werden. Nach mehrfachem Umkristallisieren und Verwendung der „Vapor-Diffusions“-Methode mit Methanol bzw. Acetonitril und Dithethylether konnte kristallines oder mikrokristallines Material erhalten werden.

\subsection{Synthesen von Modellkomplexen fürr die DMSO-Reduktase Familie}

Um die Anbindung der Dithioleneinheit des in den biologischen Systemen vorhandenen Molybdopterinligandens chemisch nachzubilden, wurden verschiedene Dithiolenliganden verwendet. Die hierdurch hergestellten Verbindungen stellen besonders gute Modelle für die erst kürzlich entdeckte Unterklasse der DMSO-Redukatase Familie, der Arsenit-Oxidase Familie dar.

Da bis auf wenige Ausnahmen alle Metallkomplexpaare aus dem Ausgangsmaterial $\left[\mathrm{MO}_{2}(\mathrm{CN})_{4}\right]^{4-}$ hergestellt wurden, sind die durchgeführten Reaktionen in folgendem Schema aufgeführt. 


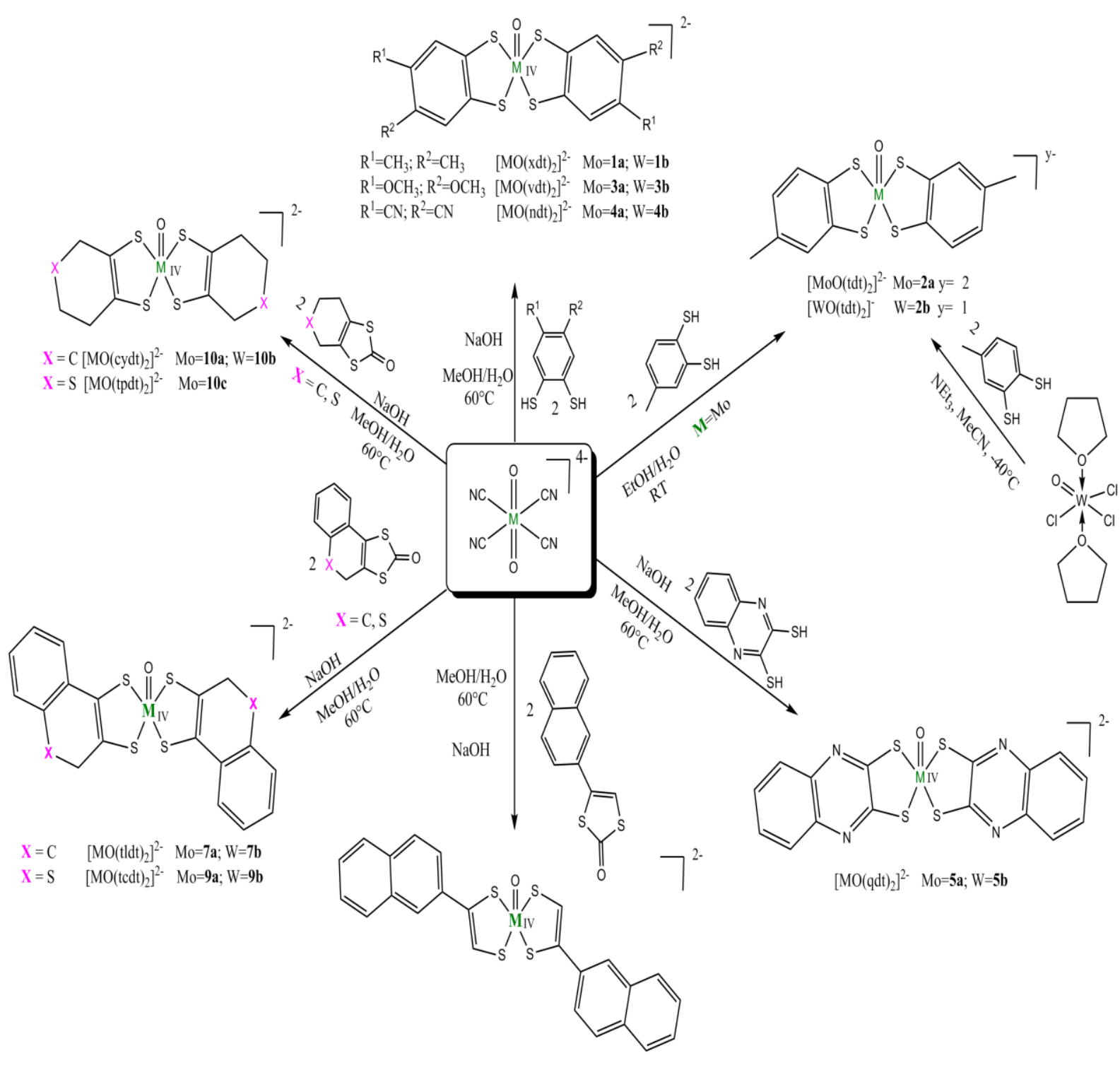

$\left[\mathrm{MO}(\mathrm{ntdt})_{2}\right]^{2-} \mathrm{Mo}=6 \mathrm{a} ; \mathrm{W}=6 \mathbf{b}$

Abb. 26: Syntheserouten zur Darstellungen der Komplexe 1-10

\subsubsection{Untersuchungen des Verbindungspaares $\left[\mathrm{MO}(\mathrm{xdt})_{2}\right]^{2-}$}

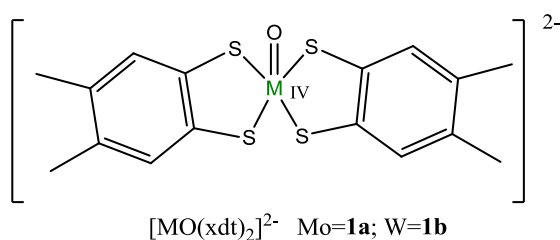

Es konnten sowohl die Molybdän- als auch die Wolframverbindung als hellbraun/orange Kristalle erhalten werden. Die Verbindungen wurden durch eine Reihe von analytischen Methoden identifiziert. Es konnten im $\mathrm{FAB}^{-}$-Massenspektrum Signale bei $450 \mathrm{amu}$ [Mo] bzw. $536 \mathrm{amu}[\mathrm{W}]$ gefunden werden, wodurch der anionischen Molekülteil $\left[\mathrm{MO}(\mathrm{xdt})_{2}\right]^{2-}$ nachgewiesen werden konnte. Elementaranalysen der Atomsorten C, H, N, S lieferten gute Übereinstimmungen mit den theoretisch berechneten Massenprozenten. NMR-Spektren der 
Komplexe zeigten ein Verschwinden der SH-Signale bei $3.67 \mathrm{ppm}$ im ${ }^{1} \mathrm{H}$-Spektrum im Vergleich zu den Ligandenspektren, sowie einen Wegfall des $\mathrm{CN}^{-}$-Signals der Metallausgangsverbindungen im ${ }^{13} \mathrm{C}$-Spektrum. Außerdem ist eine Verschiebung der $\mathrm{M}=\mathrm{O}$ Schwingung im IR-Spektrum im Vergleich zum Ausgangsmaterial von $728 \mathrm{~cm}^{-1} \mathrm{zu} 913 \mathrm{~cm}^{-1}$ bei der Molybdänverbindung und von $742 \mathrm{~cm}^{-1} \mathrm{zu} 911 \mathrm{~cm}^{-1}$ bei der Wolframverbindung festzustellen. UV-Vis-Spektren der Substanz 1a in MeOH zeigen eine Absorption bei $467 \mathrm{~nm}$ mit einem Extinktionskoeffizenten von $2473 \mathrm{l} /(\mathrm{mol} \cdot \mathrm{cm})$, der bei diesen nichtzentrosymmetrischen Verbindungen auf einen d-d-Übergang $\left(d_{x y}\right.$ nach $\left.d_{y z}\right)$ hinweist. Außerdem ist im UV-VIS Spektrum ein Signal bei $777 \mathrm{~nm}[249$ 1/(mol·cm $)]$ festzustellen, das auf die Dioxoverbindung in Oxidationsstufe VI hinweist. Dieser Peak konnte trotz mehrfach Aufnahme der UV-VIS-Spektren, auch bei frisch entgasten Lösungsmitteln, beobachtet werden. Somit muss während der Reaktion oder im Laufe der Messung der Spektren ein geringer Teil der Verbindung mit zwei Oxofunktionen in der Oxidationsstufe VI entstehen. Etweilige Anteile an Reaktionsprodukt müssen sehr gering sein, da die durchgeführten Elementaranalysen gute Ergebnisse lieferten.

Beide Verbindungen konnten mittels DPV für den Übergang $\mathrm{M}^{\mathrm{IV}} \leftrightarrow \mathrm{M}^{\mathrm{V}}$ sauber und reversibel oxidiert werden.

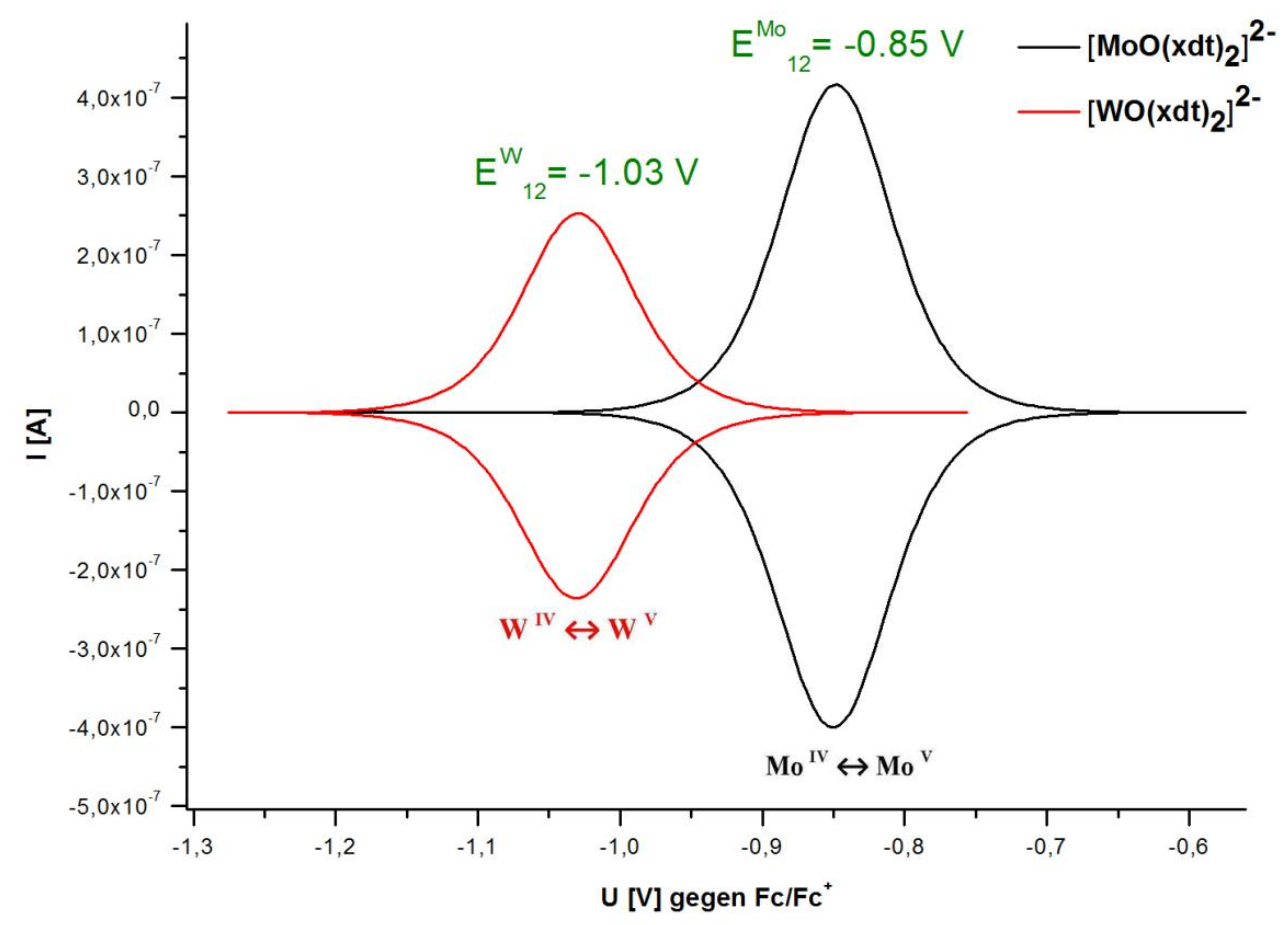

Abb. 27: DPV von $\left[\mathrm{MO}(\mathrm{xdt})_{2}\right]^{2-}$ in $\mathrm{MeCN}$

In einem alternativen Syntheseansatz konnte die Molybdänverbindung aus der Reaktion mit $\left[\mathrm{MoO}_{2} \mathrm{~S}_{2}\right]^{2-}$ mit dem protonierten Liganden ohne Einsatz einer Base gewonnen werden. 


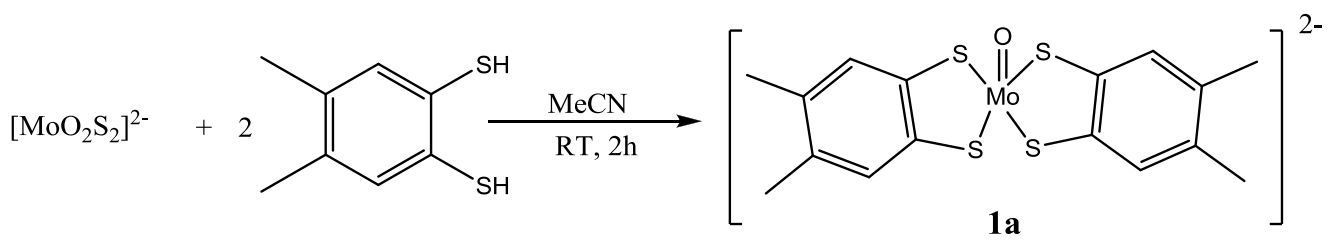

Abb. 28: Reaktion von $\left[\mathrm{MoO}_{2} \mathrm{~S}_{2}\right]^{2-}$ mit dem xdt-Liganden

Es findet eine Reduktion des Metallzentrums, durch das Koordinieren der Dithioleneinheit, begleitend durch Oxidation der beiden Sulfid-Liganden zum Disulfid statt. Einkristalle dieser Verbindung konnten aus Acetonitril/Diethylether bei $-25^{\circ} \mathrm{C}$ erhalten werden. Reaktionen mit Wolframverbindungen des Typs $\left[\mathrm{WO}_{2} \mathrm{~S}_{2}\right]^{2-}$ zeigten keine Reaktion mit dem Liganden.

\subsubsection{Geometrische Betrachtungen des Verbindungspaars}

Von beiden Verbindungen konnten Einkristalle erhalten werden, die durch Röntgenbeugung untersucht wurden. Im Folgenden sind die erhaltenen Kristallstrukturen in der EllipsoidDarstellung mit einer Wahrscheinlichkeit von 50\% gezeigt.

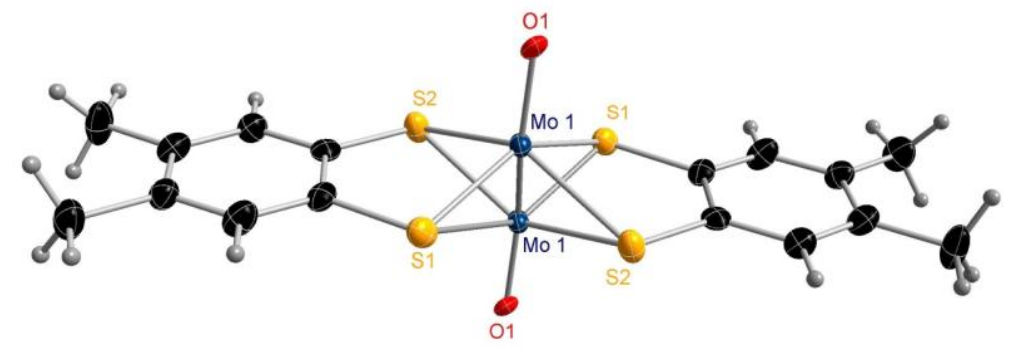

Abb. 29: Kristallstruktur von $\left(\mathrm{NEt}_{4}\right)_{2}\left[\mathrm{MoO}(\mathrm{xdt})_{2}\right]$, Kationen sind nicht dargestellt (1a)

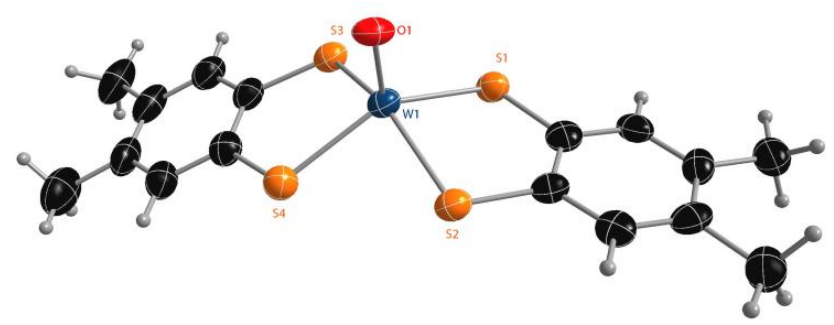

Abb. 30: Kristallstruktur von $\left(\mathrm{BzNEt}_{3}\right)_{2}\left[\mathrm{WO}(\mathrm{xdt})_{2}\right]$, Kationen sind nicht dargestellt (1b)

Die Molybdänverbindung zeichnet sich durch eine für quadratisch pyramidale nicht untypische Fehlordnung aus. Die $\mathrm{Mo}=\mathrm{O}$-Einheit ist über die $\mathrm{S}_{4}$-Ebene gespiegelt, so dass sich jeweils $50 \%$ von $\mathrm{Mo}=\mathrm{O}$ über bzw. unterhalb dieser Ebene befinden. Auf die äquatorialen Liganden hat diese Fehlordnung keinen Einfluss. 
Diese geometrische Fehlordnung wurde bei der Wolframverbindung nicht festgestellt. Um die Unterschiede zwischen den beiden Metallkomplexen aufzuzeigen, werden im Folgenden beide Strukturen in Bezug auf geometrische Parameter detailliert analysiert. Außerdem wurden die Strukturen mit Hilfe von DFT-Rechnungen berechnet und im Folgenden wird ein Vergleich zwischen den berechneten und den erhaltenen Bindungsparametern aus den Kristallstrukturen durchgeführt.

\begin{tabular}{lcccc}
\hline & Mo & Mo-DFT-Rechnung & $\begin{array}{c}\text { W } \\
\text { Kristallstruktur }\end{array}$ & W-DFT-Rechnung \\
\hline $\mathbf{M}=\mathbf{0}$ & Kristallstruktur & & $1.7190(4)$ & 1.72227 \\
$\mathbf{M}-\mathbf{S}_{\mathbf{1}}$ & $2.4548(6)$ & 1.71098 & $2.3574(13)$ & 2.44222 \\
$\mathbf{M}-\mathbf{S}_{\mathbf{2}}$ & $2.4364(5)$ & 2.45690 & $2.3723(6)$ & 2.44222 \\
$\mathbf{M}-\mathbf{S}_{\mathbf{3}}$ & $2.3029(4)$ & 2.45972 & $2.3856(10)$ & 2.44222 \\
$\mathbf{M}-\mathbf{S}_{\mathbf{4}}$ & $2.3967(5)$ & 2.45969 & $2.3769(6)$ & 2.44222 \\
$\mathbf{M}-\mathbf{S}$ & $2.4090(5)$ & 2.45972 & $2.3731(9)$ & 2.44222 \\
$\mathbf{O}-\mathbf{M}-\mathbf{S}_{\mathbf{1}}$ & $108.442(11)$ & 107.8105 & $109.311(24)$ & 107.2695 \\
$\mathbf{O}-\mathbf{M}-\mathbf{S}_{\mathbf{2}}$ & $108.997(9)$ & 107.8014 & $104.695(26)$ & 107.2695 \\
$\mathbf{O}-\mathbf{M}-\mathbf{S}_{\mathbf{3}}$ & $109.124(12)$ & 107.8099 & $109.856(32)$ & 107.2695 \\
$\mathbf{O}-\mathbf{M}-\mathbf{S}_{\mathbf{4}}$ & $108.289(11)$ & 107.8012 & $104.644(40)$ & 107.2695 \\
$\mathbf{O}-\mathbf{M}-\bar{S}$ & $108.713(11)$ & 107.8058 & $107.127(31)$ & 107.2695 \\
$\mathbf{C}=\mathbf{C}$ & $1.4057(3)$ & 1.41480 & $1.4033(6)$ & 1.41156 \\
$\mathbf{C}=\mathbf{C}$ & $1.4057(3)$ & 1.41479 & $1.3822(5)$ & 1.41156 \\
\hline
\end{tabular}

Beim Vergleich der Bindungsparameter aus beiden Kristallstrukturen fallen bei der Molybdänverbindung größere Bindungslängen, sowie größere Bindungswinkel auf. Dies ist wahrscheinlich auf den geringfügig größeren Atomradius des Wolframatoms zurückzuführen. Hierbei ist besonders ein großer Unterschied bei den Metallsauerstoffbindungen festzustellen. Aufgrund der kürzeren Bindungen des Wolframs zum Sauerstoffatom sollte eine größere Elektronendichte beim Wolframatom, im Vergleich zum Molybdän, vorhanden sein. Die gleiche Tendenz ist bei der Doppelbindung der Dithioleneinheit festzustellen, wodurch es aufgrund der kürzeren Doppelbindung zu einer größeren Elektronendichte in der Bindung kommt und somit die geringe non-innocence aufweist. Vergleicht man nun die durch DFT berechneten Bindungslängen und -winkel mit denen aus den erhaltenen Kristallstrukturen, stellt man fest, dass die Metall-Schwefelbindungen durch DFT-Rechnung regelmäßig als zu lang bestimmt werden. Bei beiden Metallen werden durch die Rechnungen ähnliche Bindungsabstände zwischen Schwefel und dem Metall gefunden. Eine Unterschätzung der Bindungsstärke und entsprechende Bestimmung $\mathrm{zu}$ größeren Abständen für die $\mathrm{M}-\mathrm{S}$ Bindungen stellt ein generelles Problem von DFT-Rechnungen dar. ${ }^{[48]}$ Die Bindungswinkel der Sauerstoff-Metall-Schwefelbindung werden bei beiden Metallen durch DFT-Rechnungen 
zu klein bestimmt, wodurch die Abstoßung durch die vorhandenen freien Elektronenpaare der Schwefelatome, als durch die Rechnungen als zu gering eingeschätzt wird. Generell muss festgestellt werden, dass die berechneten Bindungsparameter für Rechnungen in der Gasphase durchgeführt wurden, während es sich bei den Kristallstrukturen um Bindungsabstände bzw. -winkel in Festkörpern handelt, wobei die Beweglichkeit im Vergleich zur Gasphase stark eingeschränkt ist.

\subsubsection{Herstellung von Verbindungen in Oxidationsstufe $\mathrm{V}$}

Um auch Komplexe in der Oxidationsstufe V mit diesem Ligandensystem herzustellen wurde der Ausgangskomplex $\left[\mathrm{Mo}^{\mathrm{V}} \mathrm{OCl}_{3}(\mathrm{thf})_{2}\right]$ unter Zugabe von Triethylamin als Base bei $-40^{\circ} \mathrm{C}$ mit dem Xyloldithiolenliganden in Acetonitril umgesetzt. Der Komplex ([MoO(xdt $\left.)_{2}\right]^{-}(\mathbf{1 c})$ ) wurde als dunkelroter/brauner Feststoff erhalten. Die Verbindung konnte durch Massenspektrometrie sowie durch Elementaranalyse charakterisiert werden. Aufgrund der Oxidationsstufe $\mathrm{V}$ und dem Vorhandensein eines ungepaarten Elektrons ist die Verbindung ESR-aktiv.

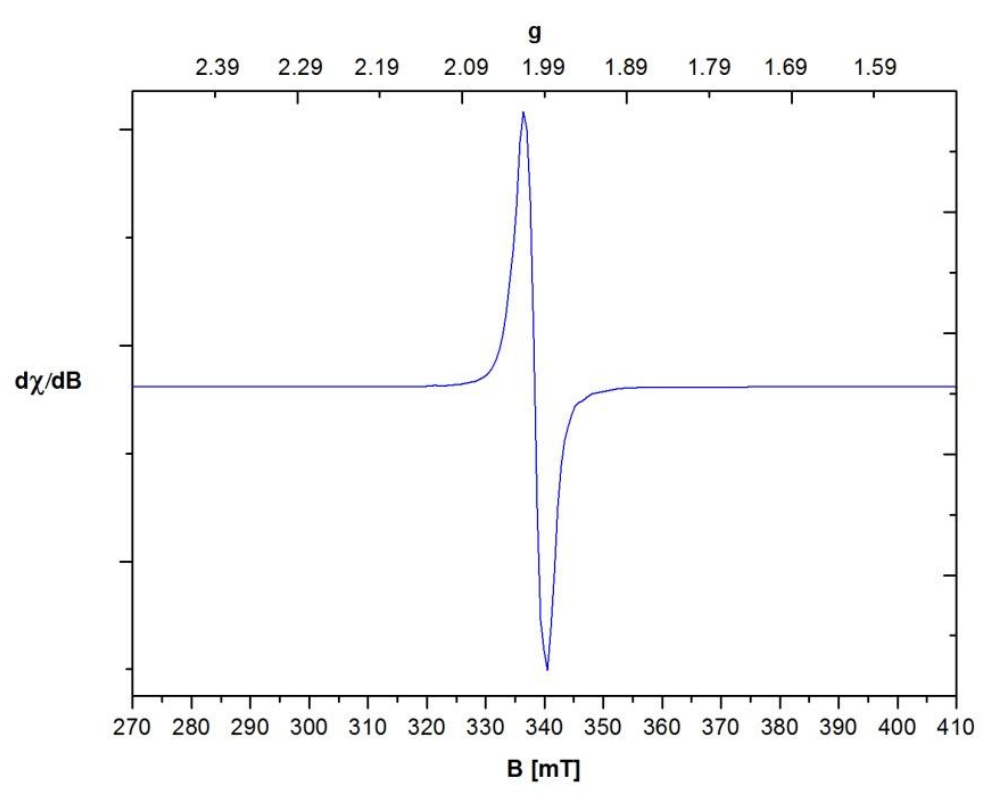

Abb. 31: ESR-Spektrum von $\left[\mathrm{MoO}(\mathrm{xdt})_{2}\right]^{-}$in DMF-Lösung bei $-140^{\circ} \mathrm{C}$

Das vorliegende Signal deutet auf das Vorhandensein eines ungepaarten Elektrons, ausschließlich am Metallzentrum, hin. Es handelt sich um ein isotropes Signal bei dem keine Hyperfeinkopplung vorkommt. Es gilt also $g_{x}=g_{y}=g_{z}$, mit einem Landé-Faktor von 1.99. Es findet kein Eindringen des ungepaarten Elektrons in das aromatische Ringsystem statt, somit kann sicher auf eine Lokalisation des ungepaarten Elektrons am Metallzentrum geschlossen 
werden. Da die Intensität dieses Signals nicht sehr stark ist, können die mit viel geringerer Intensität auftretenden Signale des ${ }^{97}$ Mo-Isotops nur schlecht identifiziert werden.

\subsubsection{Untersuchungen des Verbindungspaars $\left[\mathrm{MO}(\mathrm{tdlt})_{2}\right]^{\mathrm{x}-}$}

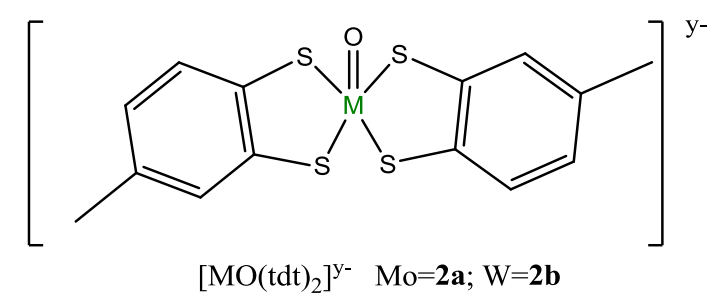

Die Molybdänverbindung (2a) konnte aus dem Ausgangsmaterial $\left[\mathrm{MoO}_{2}(\mathrm{CN})_{4}\right]^{4-}$ aus einer Ethanol/Wasser-Mischung bei RT erhalten werden. Bei der Synthese wurde auf die Verwendung einer weiteren Base verzichtet und die Thiolfunktion wurde direkt mit dem Komplex umgesetzt. Somit sollte als Reaktionsprodukt HCN entstehen, das leicht als gasförmige Komponente aus dem Reaktionsgemisch vertrieben werden kann. Die Verbindung wurde durch die üblichen Analysemethoden NMR, EA, MS sowie IR identifiziert. Außerdem konnte eine Kristallstruktur dieser Verbindung mit dem Tetraethylammoniumkation erhalten werden.

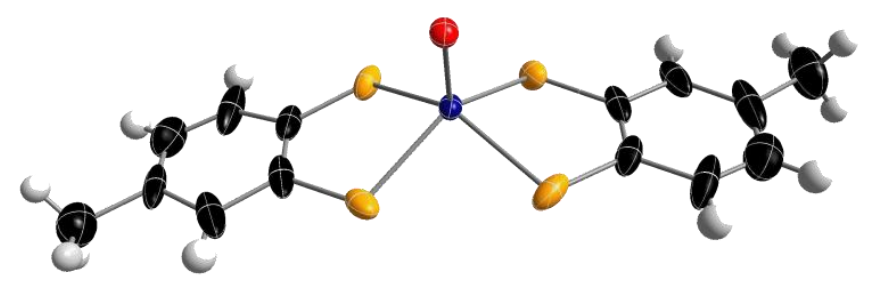

Abb. 32: Kristallstruktur von $\left[\mathrm{MoO}(\mathrm{tdt})_{2}\right]^{2-}$, Kationen sind nicht dargestellt

Die Kristallstruktur wurde schon 1994 von Nakamura et al. publiziert, ${ }^{[29 b]}$ doch im Vergleich zu der von mir erhaltenen Struktur zeigen sich insbesondere Unterschiede bei der MetallSauerstoffbindung. Im Folgenden sind die Bindungsparameter vergleichend dargestellt.

\begin{tabular}{|c|c|c|}
\hline & $2 a$ & $2 \mathbf{a}^{[29 b]}$ \\
\hline $\mathbf{M o}=\mathbf{O}[\stackrel{\AA}{\mathrm{A}}]$ & $1.702(3)$ & $1.666(5)$ \\
\hline $\operatorname{Mo}-\bar{S}[\AA ̊]$ & $2.383(8)$ & $2.384(3)$ \\
\hline $\mathbf{S}-\mathbf{C}[\AA ̊ \AA]$ & $1.743(4)$ & $1.780(2)$ \\
\hline Mo-S-C [ $\left.{ }^{\circ}\right]$ & $106.03(6)$ & $107.15(98)$ \\
\hline O-Mo-S $\left[^{\circ}\right]$ & $107.52(11)$ & $107.75(7)$ \\
\hline
\end{tabular}

Tab. 2: Vergleich der Bindungslängen und -winkel 
Aufgrund der cis und trans Anordnung der Methylgruppen am Dithiolenliganden sind zwei Isomere möglich. Da beide Isomere simultan kristallisieren, treten in den Strukturen Fehlordnungen in den zwei möglichen Positionen am Aromaten auf. Bei den von mir erhaltenen Kristallen wurden Untersuchungstemperaturen von $-140^{\circ} \mathrm{C}$ verwendet, während die Kristalle von Nakamura et al. bei $23^{\circ} \mathrm{C}$ analysiert wurden. Daher wird in meiner Kristallstruktur die Hauptspezies des Isomerengemisches mit einer Wahrscheinlichkeit von $80 \%$ gefunden, während diese nur zu $60 \%$ bei der publizierten Struktur beobachtet werden konnte. Die Methylgruppen am Liganden wirken als Elektronendonorgruppen, wodurch es zu einer Verlängerung aller Bindungen im Molekül, im Vergleich zu Systemen mit nur einem Benzoldithiolenliganden, kommt. Eine weitere Verstärkung des Elektronenschubs durch die Methylgruppen ist bei den Xyloldithiolenliganden in den Komplexen 1a und 1b festzustellen, wobei weitere Bindungsverlängerungen auftreten. Beim Vergleich der erhaltenen Bindungslängen fällt auf, dass bei der von mir erhaltenen Struktur längere MetallSauerstoffbindungen nachgewiesen werden, dies wahrscheinlich auch auf die unterschiedlichen Messtemperaturen der beiden Untersuchungen zurückzuführen. Die MetallSchwefelbindungen werden fast identisch bestimmt. Aufgrund der kürzer bestimmten S-CBindungen resultieren Abweichungen in den Bindungswinkeln.

Bei der Anwendung der gleichen Synthesemethode für Wolfram konnte die analoge Wolframverbindung nicht erhalten werden. Das Produkt dieser Reaktion ist im qualitativen Umsatz eine Verbindung mit zwei verbleibenden $\mathrm{CN}^{-}$-Liganden am Wolframzentrum.

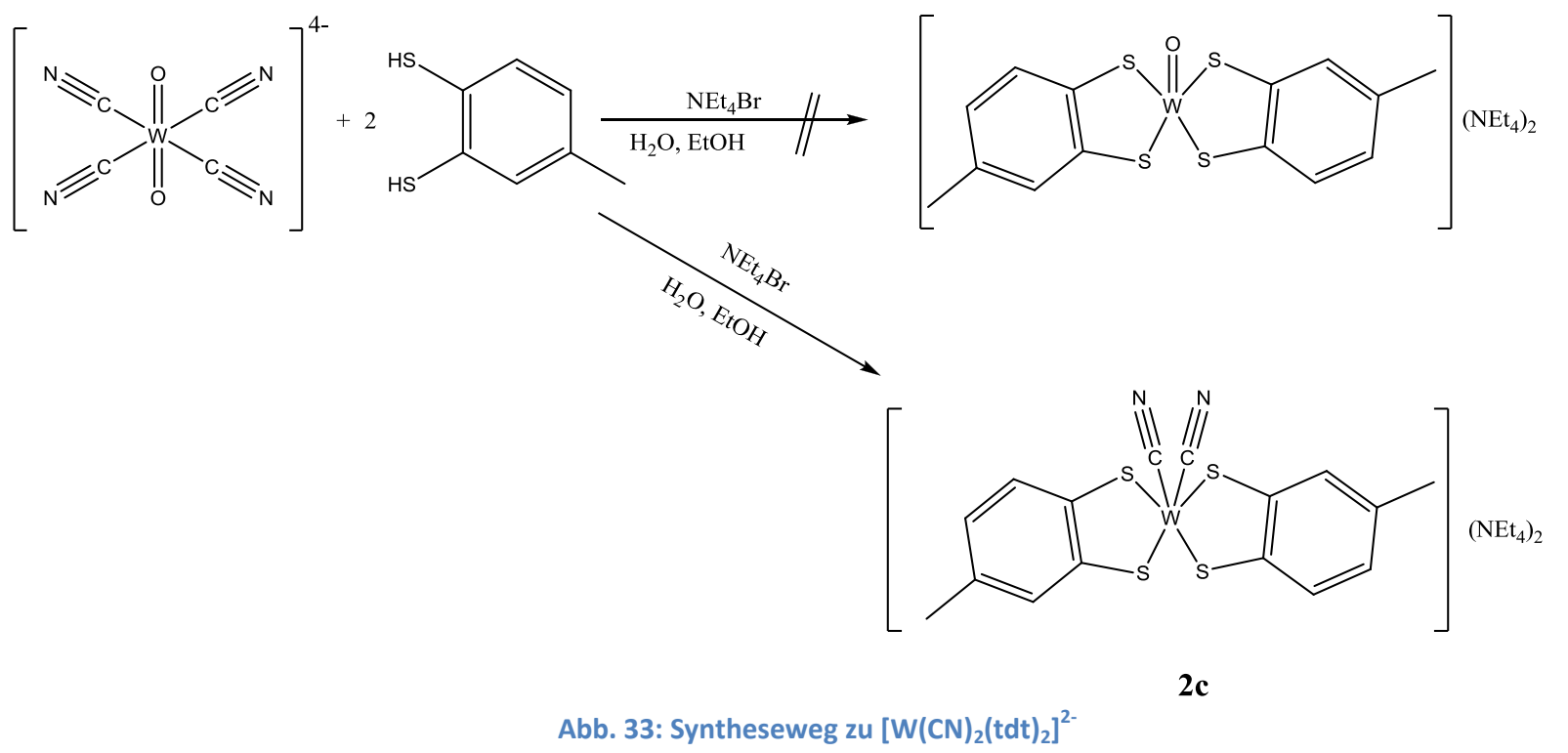


Die erhaltene Verbindung konnte sowohl durch das Vorhandensein des Molmassenpeaks im ESI $I^{-}$Spektrum in Acetonitril bei 543.8, sowie im $\mathrm{FAB}^{-}$in 3-NBA identifiziert werden. Die vorhandenen CN-Schwingungen sind gut im IR-Spektrum bei $1479 \mathrm{~cm}^{-1} \mathrm{zu}$ beobachten. Außerdem sind keine Metallsauerstoffschwingungen $\mathrm{zu}$ erkennen. Im ${ }^{13} \mathrm{C}-\mathrm{NMR}$-Spektrum konnte deutlich ein Signal bei 124.3 ppm beobachtet werden, dass auf die vorhanden CNGruppen im Molekül hinweist. C, H, N,S - Elementaranalysen zeigten gute Übereinstimmungen mit den theoretisch berechneten Massenprozenten.

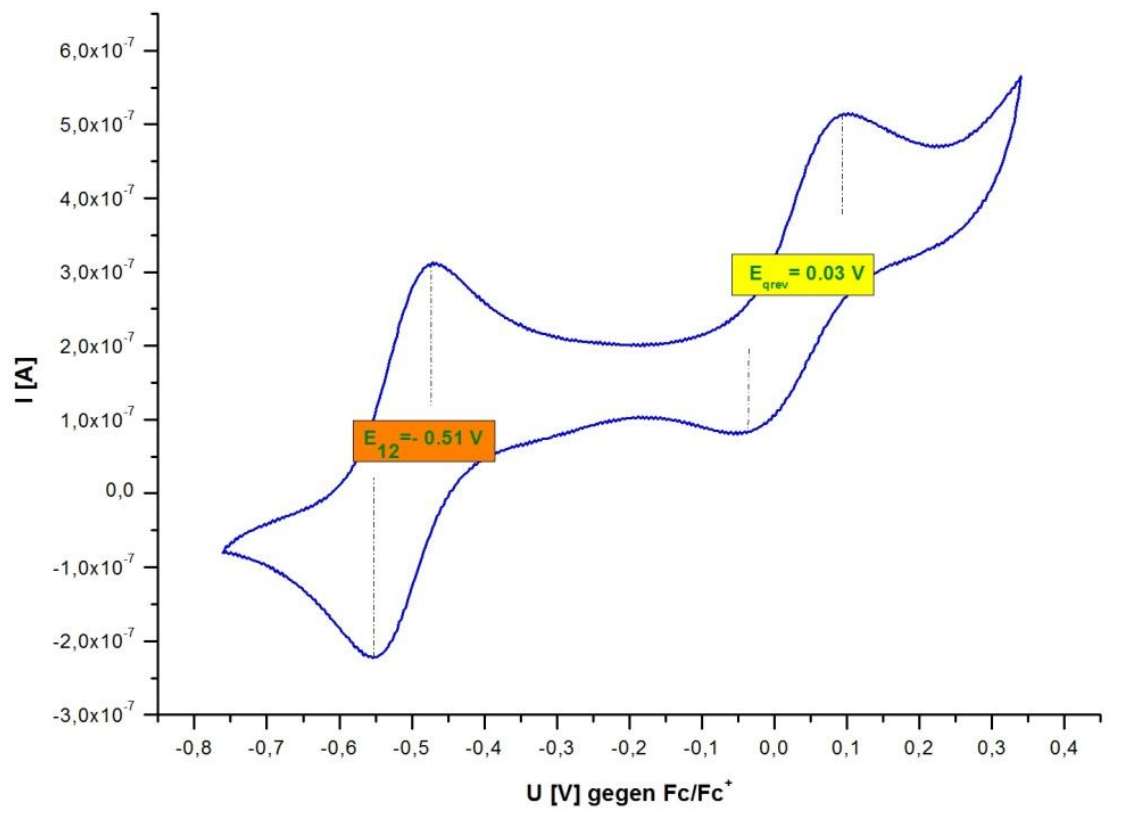

Abb. 34: CV-Spektrum der Vebindung $\left[\mathrm{W}(\mathrm{CN})_{2}(\mathrm{tdt})_{2}\right]^{2-}$

Es wurden Cyclovoltammogramme der Verbindung (2c) in einer Acetonitrillösung aufgenommen. Es ist bei $-0.51 \mathrm{~V}$ ein reversibles Signal zu beobachten, das der Oxidation von Oxidationsstufe IV nach V zugeordnet werden kann. Bei einem Potential von $0.03 \mathrm{~V}$ ist ein quasireversibler Peak zu erkennen, der einer Oxidation von Oxidationsstufe V nach VI entsprechen sollte.

Im UV-VIS Spektrum konnten drei Banden bei 479, 388 und bei $359 \mathrm{~nm}$ beobachtet werden. Vergleicht man dieses Spektrum mit dem der Verbindung (2b), wobei eine Absorption bei $461 \mathrm{~nm}$ gefunden wird, so stellt man eine Verschiebung des Peaks zu kürzeren Wellenlängen fest. Da das Vorhandensein eines Cyanidliganden zu einer Vergrößerung des energetischen Abstands zwischen dem LUMO und dem HOMO-Orbital führt, beobachtet man die Anregung der d-Orbitale des Metallzentrums bei kürzerwelligem Licht $(388 \mathrm{~nm})$. Die anderen Peaks werden wahrscheinlich durch Ligand-Metallchargetransfer-Übergange zustande kommen, wodurch Elektronen vom CN-Liganden auf das Metallzentrum übertragen werden. 
Da die Abspaltung von zwei Oxo-Funktionen ungewöhnlich ist, wurden die Bindungsenergien der $\mathrm{CN}^{-}$und Oxofunktionen mit Hilfe von DFT-Rechnungen bestimmt. Dazu wurden die $\mathrm{CN}^{-}$bzw. die Oxo-Liganden in kleinsten Schritten von $0.1 \AA$ von der zuvor geometrieoptimierten Struktur entfernt. Hierbei wurden alle anderen Bindungslängen und Winkel als „starre Körper“-Molekülteile betrachtet. Nach einer Entfernung der $\mathrm{CN}^{-}$-Liganden von $2.964 \AA$ bzw. $2.243 \AA$ des Sauerstoffatoms konnte ein Bruch der Ligand-Metall Bindung beobachtet werden. Deshalb wurde eine Sattelpunktsuche durchgeführt, bei der als Startpunkt eine Struktur mit gebundenen Gruppen benutzt wurde und als Endstruktur eine Verbindung, bei der keine Bindung mehr zwischen dem Metallatom und der untersuchenden Gruppe bestand. Da es beim Erreichen des Übergangszustands zum Wegfall eines Translationsfreiheitsgrads kommt, kann man diesen im Auftauchen einer imaginären Frequenz beobachten. Die Bindungsenergie wurde durch die Differenzbildung der Struktur im Übergangszustand zu der Ausgangsstruktur ermittelt. In der nachfolgenden Tabelle sind die Bindungsenergien mit den dazu bestimmten Energiewerten aufgeführt.

\begin{tabular}{ccccc}
$\begin{array}{c}\text { Betrachtetes } \\
\text { Atom }\end{array}$ & $\begin{array}{c}\text { Gebundene } \\
\text { Form[Hartreé] }\end{array}$ & $\begin{array}{c}\text { Nicht gebundene } \\
\text { Form [Hartreé] }\end{array}$ & $\begin{array}{c}\Delta \mathbf{E} \\
{[\text { Hartreé] }}\end{array}$ & $\begin{array}{c}\text { Bindungsenergie } \\
{[\mathbf{k J} / \mathbf{m o l}]}\end{array}$ \\
\hline $\mathbf{O}$ & -588.94282589 & -588.69477200 & 0.2480539 & 651.27 \\
CN & -588.94282589 & -589.17710095 & 0.2342751 & 615.08
\end{tabular}

Tab. 3: Bestimmte Bindungsenergien für $\mathrm{CN}$ bzw. $\mathrm{O}$ von 2c

Anhand der berechneten Bindungsenergien stellt man fest, dass die Bindungsspaltung bei den angebundenen $\mathrm{CN}^{-}$-Ionen leichter vonstattengehen sollte, als es bei dem Sauerstoff der Fall ist. Somit sollte das Durchführen der Reaktion bei leicht sauren Bedingungen die Abspaltung des Sauerstoffs als $\mathrm{H}_{2} \mathrm{O}$ bzw. $\mathrm{OH}^{-}$erleichtern. Da die $\mathrm{Mo}=\mathrm{O}$ Bindung in der Regel stärker ist, als die $\mathrm{W}=\mathrm{O}$ Bindung, kann man das Produkt mit Molybdän gerade noch erhalten. Mit Wolfram kommt es dagegen zur Abtrennung der Sauerstoffatome. Hieran kann man den unterschiedlichen Ausgang dieser Reaktionen bei pH-Wert-Änderung feststellen.

\subsubsection{Geometrische Betrachtungen von $\left[\mathrm{W}(\mathrm{CN})_{2}(\mathrm{tdt})_{2}\right]^{2-}(2 \mathrm{c})$}

Von der erhaltenen Verbindung (2c) konnten auch Einkristalle mit den TetraethylammoniumGegenion erhalten werden. Die Auswertung der Kristallstruktur zeigte einige Schwierigkeiten, da die vorhandenen Kationen sich auf einer „Speziellen Lage“ im Kristallgitter befinden. Aufgrund des Vorhandenseins dieser nicht translatierenden Spiegelebene können sich die schon festgelegten Gitterpunkte nicht mehr durch das Symmetrieelement verdoppeln, wodurch es zu einer Verminderung der Lage kommt. Da sich 
beide vorhandenen Stickstoffatome des Tetraethylammoniumions auf einer Speziellen Lage befinden, war es schwer die vorhandenen Kationen eindeutig in der Kristallstruktur zu charakterisieren. Beim $\mathrm{R}_{1}$ bzw. $\mathrm{R}_{2}$ konnten gute Werte erzielt werden, doch es war nicht vollkommen möglich ein Tetraethylammonium mit allen Ethylketten $\mathrm{zu}$ verfeinern. Im Folgenden ist die Kristallstruktur des anionischen Parts gezeigt.

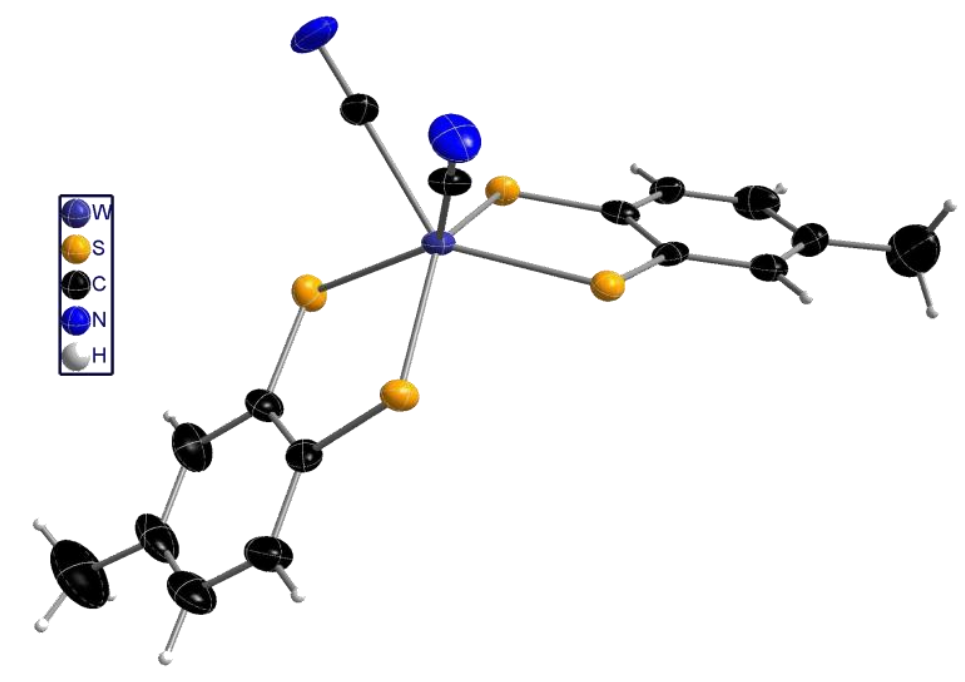

Abb. 35: Einkristallstruktur von Verbindung 2c, Kationen sind nicht gezeigt

Da es wie bei der Verbindung 2a in der trans-Anordnung zwei verschiedene Rotationsiosmere gibt, links und rechtsgängige Drehachse, ist wieder eine Fehlordnung in den möglichen Positionen am Aromaten vorhanden. Betrachtet man nun die Bindungslängen, so stellt man einen Bindungsabstand vom zentralen Wolframatom $\mathrm{zu}$ den CN-Fragmenten von 2.1314 (4) $\AA$ bzw. 2.1319 (4) $\AA$ fest. Dies stellt eine Verkürzung der Bindungslänge um $0.0734 \AA$ im Vergleich zum Ausgangsmaterial dar. Die Bindungsverkürzung kommt wahrscheinlich durch die fehlende Verbrückung zu Kaliumionen, die im Ausgangsprodukt vorhanden ist, zustande. Da beiden $\mathrm{CN}^{-}$-Liganden auf einer Seite des Metallzentrums koordiniert sind und auch keine gegenüberliegenden Schwefelatome ( $S^{1}=2.3606(7)$, $S^{2}=2.3564(6), S^{3}=2.3518(4), S^{4}=2.3649$ (5)) eine ähnliche Bindungslängen aufweisen, kann bei dieser Struktur nicht mehr von einer verzerrten oktaedrischen geometrischen Anordnung ausgegangen werden. Deshalb sollte es sich ähnlich, wie es bei Trisdithiolenverbindungen der Fall ist ${ }^{[49]}$ um eine trigonal-prismatische Koordinationsumgebung beim Wolframzentrum handeln. 


\subsubsection{Folgereaktionen von $\left[\mathrm{W}(\mathrm{CN})_{2}(\mathrm{tdt})_{2}\right]^{2-}(2 \mathrm{c})$}

Diese Verbindung ermöglichte es nun vielfältige Substitutionen an den Positionen der

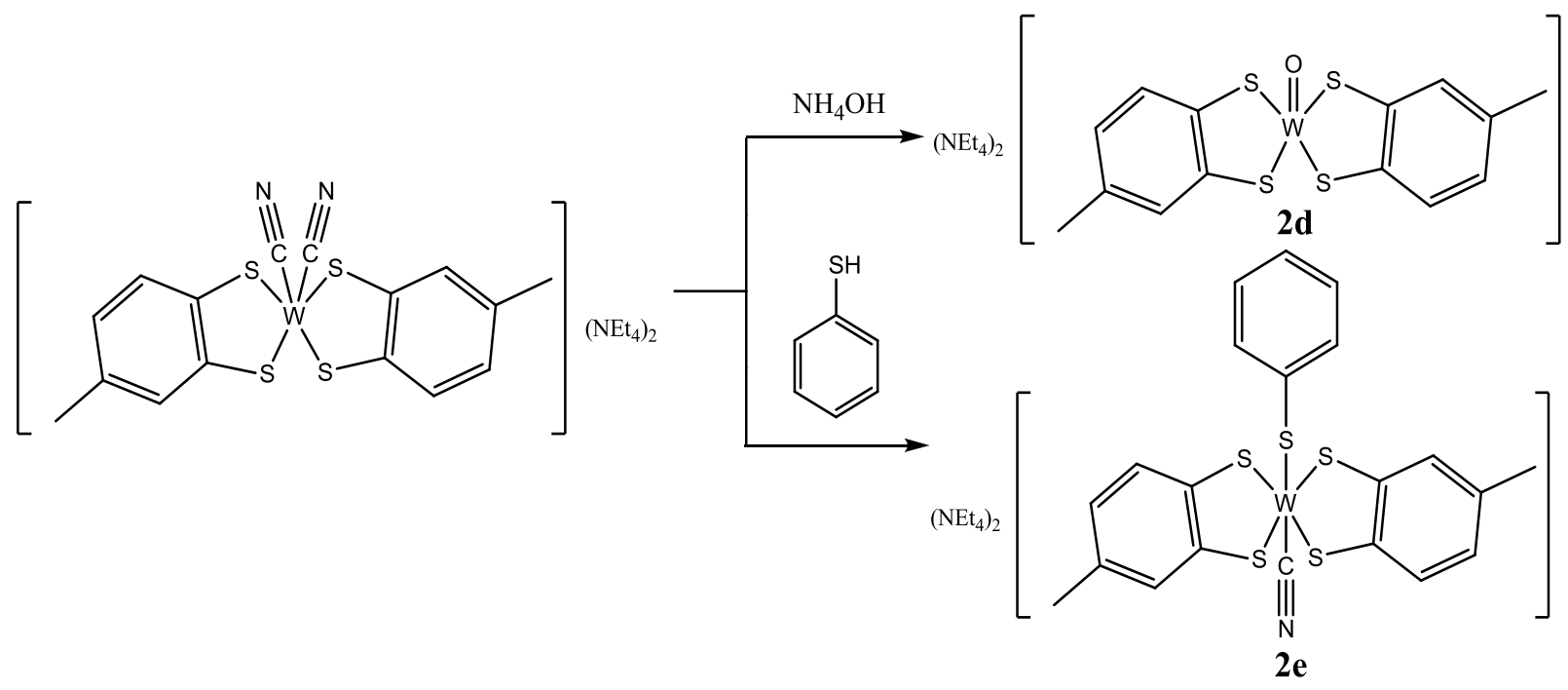

Abb. 36: Substitutionsreaktionen an $\left[\mathrm{WCN}_{2}(\mathrm{tdt})_{2}\right]^{2-}$

Cyanoliganden durchzuführen. Mit Hilfe von $\mathrm{NH}_{4} \mathrm{OH}$ können beide $\mathrm{CN}$-Liganden entfernt und durch eine Oxofunktion ersetzt werden. Hierbei findet ein Angriff von zwei $\mathrm{OH}^{-}$am Metallzentrum statt und es kommt zur Abspaltung von Wasser unter Bildung einer OxoFunktion. Die Substitution beider $\mathrm{CN}^{-}$durch einen Oxo-Liganden wird durch das IR bestätigt, indem die $\mathrm{CN}^{-}$- Signale nicht mehr zu beobachten sind, jedoch die neuere $\mathrm{Mo}=\mathrm{O}-$ Valenzschwingung.

Weitere Versuche gingen dahin, die Natriumsalze von z.B. Thiolphenol mit diesen Verbindungen umzusetzen. Hierbei konnte die Substitution der $\mathrm{CN}^{-}$-Liganden durch Thiophenolat erreicht werden. Problematisch war die Abtrennung von nicht reagiertem Natriumsalz des Thiophenols, da sowohl Ausgangsverbindung als auch Produkte ähnliche Löslichkeiten aufweisen. Deshalb bot es sich an, direkt die Thiophenolverbindung zu benutzen, um somit als Nebenprodukt gasförmiges HCN zu bilden. Durch Trocknung des Endprodukts im Vakuum konnte nicht reagiertes Thiophenol aus dem Gemisch entfernt werden. Nach Reinigung mit Hexan wurde eine dunkelrote Verbindung (2e) erhalten. Diese Verbindung stellt ein Modell für das in Enzymen vorkommende $\mathrm{Mo}\left(\mathrm{S}_{\mathrm{Cys}}\right)(\mathrm{mpt})_{2}$-Zentrum dar. Die Verbindung konnte durch Elementaranalysen, $\mathrm{FAB}^{-}$- Massenspektrometrie, sowie ${ }^{1} \mathrm{H}-\mathrm{NMR}$ identifiziert werden. Aufgrund der geringen Ausbeuten, konnte noch kein brauchbares ${ }^{13} \mathrm{C}$-NMR-Spektrum erhalten werden, um die vorhandene $\mathrm{CN}$-Gruppe eindeutig nachzuweisen. Doch im IR-Spektrum wurde eine Schwingung bei $2359 \mathrm{~cm}^{-1}$ festgestellt werden, die Hinweise auf das Vorhandensein eines $\mathrm{CN}^{-}$-Liganden gibt. 
Da die Mono-Oxo-Verbindung zunächst nur über „Umwege“ aus den Tetracyanoverbindungen erhalten werden konnte, wurde nach einer anderen Methode für diese Synthese gesucht. An der Verbindung sollten elektrochemische Untersuchungen durchgeführt werden. Deshalb war die zu erhaltene Oxidationsstufe nicht entscheidend, solange sich eine Oxofunktion und zwei der Dithiolenliganden am Metall befanden. Die Synthese wurde schließlich erfolgreich aus dem $\left[\mathrm{WOCl}_{3}(\mathrm{thf})_{2}\right]$ Ausgangsmaterial in Acetonitril durchgeführt. Hierzu wurde Triethylamin als Base verwendet und es konnte nach mehrfachem Waschen und Umkristallisieren mit Acetonitril/Diethylether ein dunkelroter mikrokristalliner Feststoff in der Oxidationsstufe $\mathrm{V}$ erhalten werden.
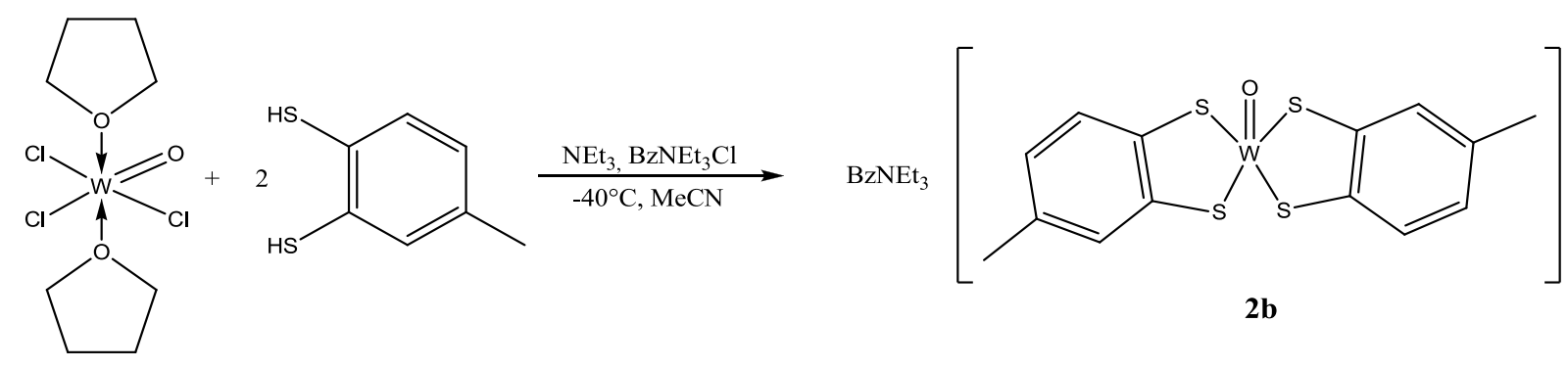

$\mathbf{2 b}$

Abb. 37: Synthese von $\left[\mathrm{WO}(\mathrm{tdt})_{2}\right]^{-}$

Aufgrund der unterschiedlichen Möglichkeit der Anordnung der Methylgruppen (cis/trans) in dem Toluolliganden, werden in der Reaktion Gemische aus beiden Verbindungspaaren entstehen. Aus thermodynamischer Sicht sollte die trans-Verbindung aber favorisiert sein. Dies konnte auch durch DFT-Rechnungen, anhand der berechneten Stabilisierungsenergien belegt werden.

Die nun erhaltenen tdt-Komplexe 2a und 2b konnten elektrochemischen Untersuchungen unterzogen werden. In den DPV-Spektren wurden reversible Signale, sowohl für den Oxidationsstufenübergang von IV nach $\mathrm{V}$, als auch von $\mathrm{V}$ nach VI erhalten. Die Molybdänkomplexe zeigten Potentiale von $-0.80 \mathrm{~V}$ bzw. $0.02 \mathrm{~V}$. Wolframverbindung zeigte wie gewohnt ein geringeres Redoxpotential gegen das Ferrocen/Ferrocenium Paar in Acetonitrillösung bei $-1.49 \mathrm{~V}$ bzw. $-0.61 \mathrm{~V}$. 


\subsubsection{Untersuchungen des Verbindungspaares $\left[\mathrm{MO}(\mathrm{vdt})_{2}\right]^{2-}$}

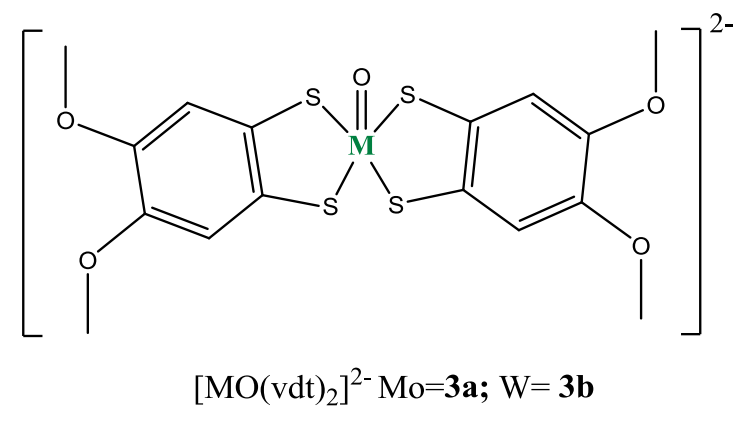

Das Verbindungspaar konnte durch NMR, MS, EA sowie IR-Spektroskopie eindeutig charakterisiert werden. Die Schwingungen der Methoxygruppen konnten im IR-Spektrum bei $2861 \mathrm{~cm}^{.1}$ (Mo) bzw. $2871 \mathrm{~cm}^{-1}$ (W) nachgewiesen werden. Beide Verbindungen konnten in den Voltammogrammen als Metallverbindungen in der Oxidaitionsstufe IV identifiziert werden. Es konnten Oxidationen von IV nach V sowie nach VI und Reduktion zurück nach IV beobachtet werden. Die Peaks zeigten annäherend reversibles Verhalten. Es konnte ein Potential für den $\mathrm{M}^{\mathrm{IV}} \leftrightarrow \mathrm{M}^{\mathrm{V}}$ Übergang von $-0.77 \mathrm{~V}(\mathrm{Mo})$ bzw. $-1.45 \mathrm{~V}$ (W) und für den Übergang $\mathrm{M}^{\mathrm{V}} \leftrightarrow \mathrm{M}^{\mathrm{VI}}$ von $-0.03 \mathrm{~V}(\mathrm{Mo})$ bzw. $-0.75 \mathrm{~V}(\mathrm{~W})$ festgestellt werden. Beim Vergleich der Potentiale mit den Komplexpaaren $\mathbf{1}$ und 2, mit dem Toluol- und dem Xyloldithiolliganden fallen ähnliche Potentiale bei allen Molybdänverbindungen $(\mathbf{a}-0.85 \mathrm{~V}, \mathbf{2 a}-0.80 \mathrm{~V})$ auf. Nur bei der Wolframverbindung ist ein Unterschied der Potentiale vom Toluol- zum Xyloldithiolliganden festzustellen. (1b -1.03 V, 2b-1.49 V)

\subsubsection{Geometrische Betrachtungen von [MO(vdt) $]^{2-}$}

Sowohl von der Molybdän- als auch von der Wolframverbindung konnten Kristalle erhalten werden, die durch Röntgenbeugung untersucht wurden. Dabei zeigte sich, dass bei Verwendung von Methanol als Lösungsmittel eine veränderte Struktur, im Vergleich zu den aus Acetonitril erhaltenen Kristallen, auftrat.

\subsection{Strukturanalyse von [MoO(vdt) $\left.{ }_{2}\right]^{2-}(3 a)$}

Bei der Molybdänverbindung wurden Kristalle durch „Vapor Diffusion“ aus Methanol/Diethylether erhalten. Es findet eine Verbrückung der Oxo-Gruppen des Molybdäns über zwei Natriumionen, einmal zu dem Sauerstoffatom eines Methanolmoleküls und einmal zu den Sauerstoffatomen der Methoxygruppen des vdt-Ligandens statt. Diese Verbrückung ist ziemlich ungewöhnlich und zeigt die Möglichkeit dieser Gruppen mit z.B. Substraten zu interagieren. 


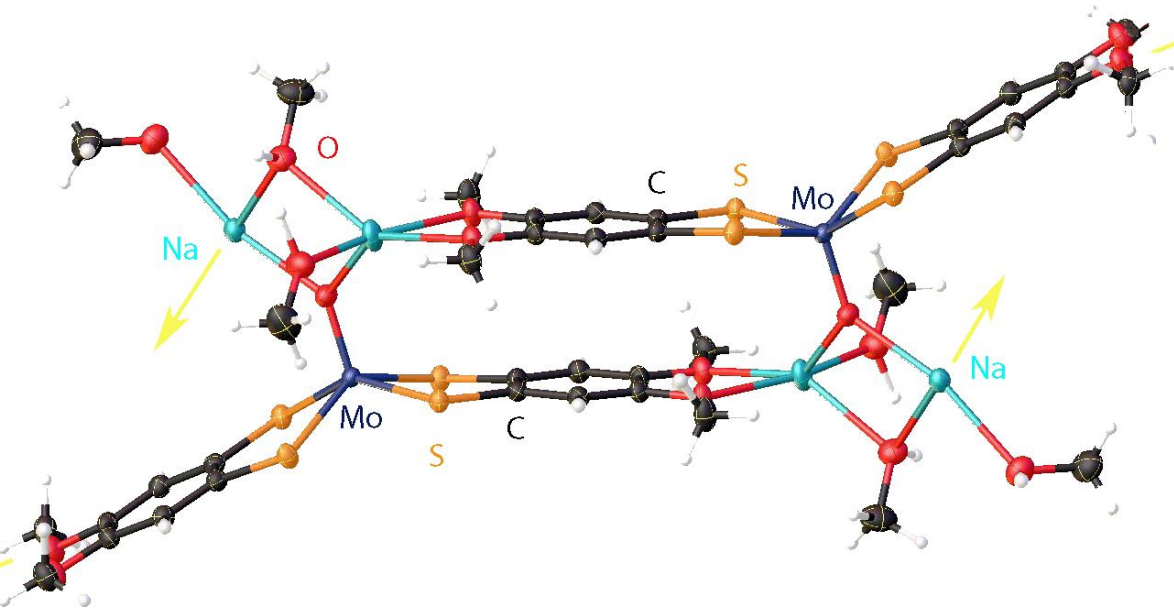

Abb. 38: Kristallstruktur von $\left[\mathrm{MoO}(\mathrm{vdt})_{2}\right]^{2-}$ in $\mathrm{MeOH}$ mit Verbrückung über die Natriumionen

Betrachtet man nun mehrere dieser Moleküle so fällt auf, dass dieser Strukturtyp sich in Strängen anordnet. Siehe dazu $A b b .39$.

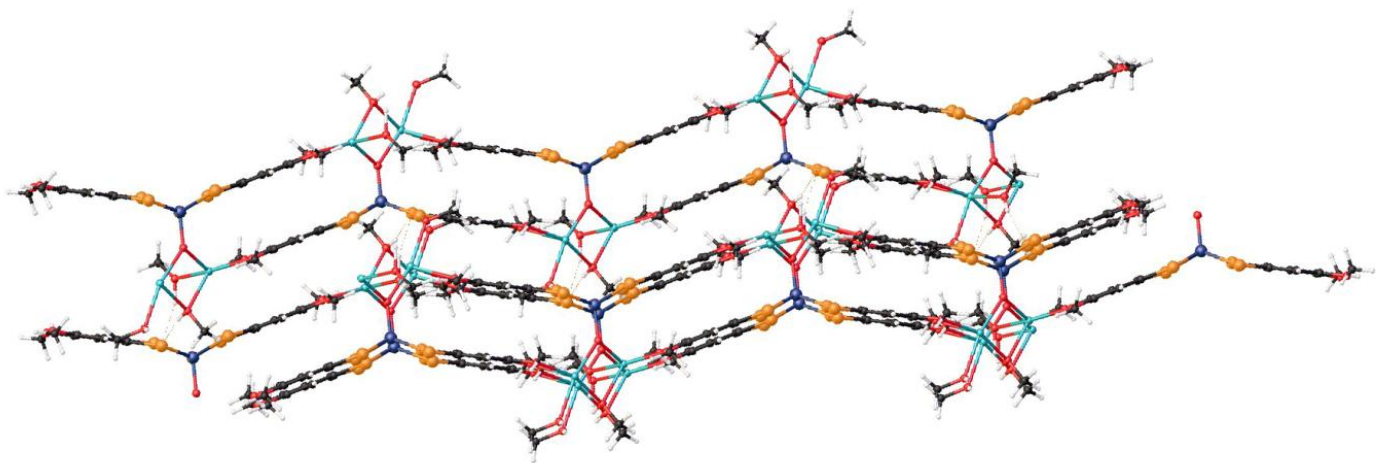

Abb. 39: Anordnung des Strukturtyps in einer strängenartigen Struktur

Im unteren Teil der Struktur sind mögliche weiter Stränge dargestellt, zu denen aber keine weiteren Wechselwirkungen bestehen. Der Hohlraum (hellgelb markiert), der sich zwischen den Strängen ausbildet, ist aufgrund der großen Natrium- und Molybdänatome sehr klein (Höhe $3.701 \AA$, Breite $10.414 \AA$ bei Annahme einer quadratischen Fläche), was auch im Spacefillingmodell beobachtet werden kann. (Abb.40) Es kommt deshalb zur Ausbildung von kleinen „Löchern“ in den einzelnen Strängen, die aber nicht groß genug sind, um andere Metallionen im Inneren aufzunehmen.

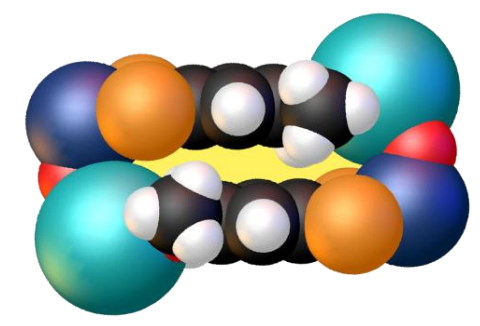


Betrachtet man nun mehrere dieser Stränge nebeneinander gelegt, so ist eine Zusammenlagerung der Moleküle zu sehen.
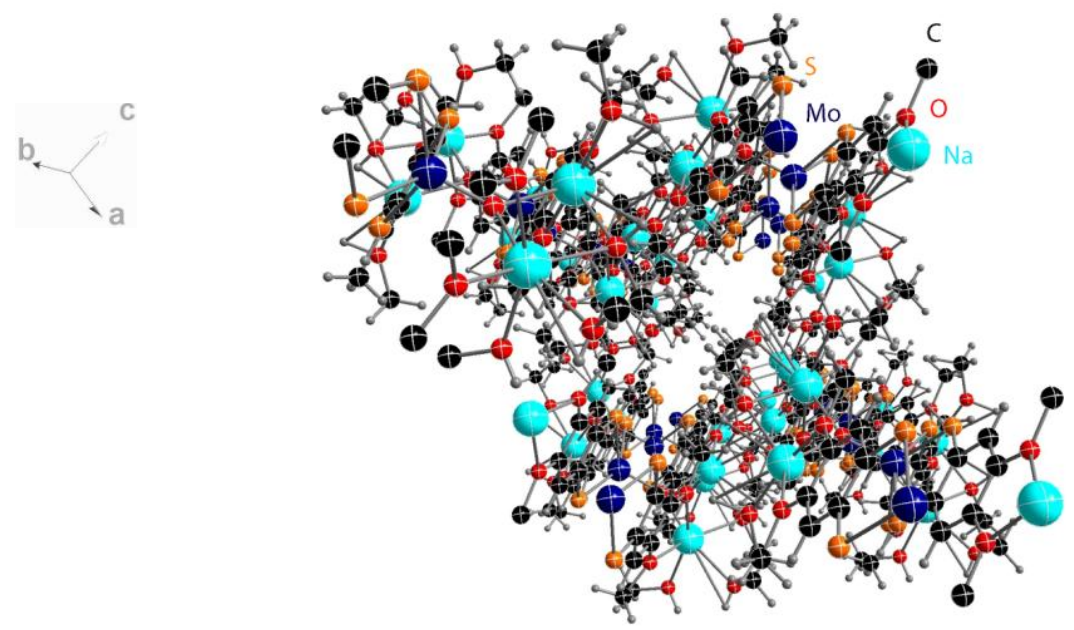

Abb. 41: Darstellung von mehreren Strängen

\subsection{Strukturanalyse von [WO(vdt $\left.)_{2}\right]^{2-(3 b)}$}

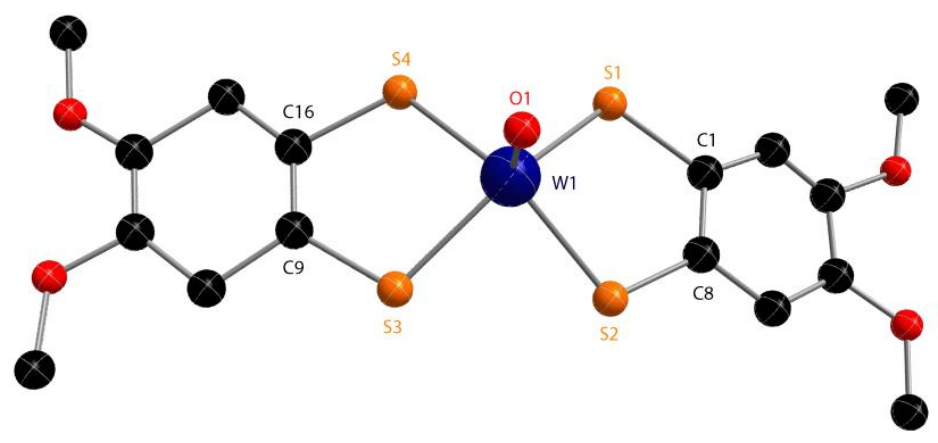

Abb. 42: Kristallstruktur von $\left(\mathrm{BzNEt}_{3}\right)_{2}\left[\mathrm{WO}(\mathrm{vdt})_{2}\right]$, die Kationen nicht sind gezeigt

Da die Kristalle aus Acetonitril erhalten wurden, ist die beim Molybdän auftretende Verbrückung, durch die Natrium und Methanol-Fragmente nicht festzustellen. Im Folgenden sind die wichtigsten Bindungslängen und Bindungswinkel der Verbindungen aufgeführt. 


\begin{tabular}{|cc|cc|}
\hline \multicolumn{2}{|c|}{ Molybdänstruktur } & \multicolumn{2}{c|}{ Wolframstruktur } \\
\hline Bindung & Bindungslängen/winkel & Bindung & Bindungslängen/winkel \\
\hline Mo1=01 & $1.7116(4) \AA$ & W1=01 & $1.7313(4) \AA$ \\
\hline Mo1-S1 & $2.3670(1) \AA$ & W1-S1 & $2.3848(6) \AA$ \\
\hline Mo1-S2 & $2.3728(6) \AA$ & W1-S2 & $2.3900(5) \AA$ \\
\hline Mo1-S3 & $2.3678(10) \AA$ & W1-S3 & $2.3839(5) \AA$ \\
\hline Mo1-S4 & $2.3837(7) \AA$ & W1-S4 & $2.3946(7) \AA$ \\
\hline Mo1-S & $2.3728(6) \AA$ & W1-S & $1.3883(6) \AA$ \\
\hline C1=C8 & $1.3876(5) \AA$ & C1-C8 & $1.3495(5) \AA$ \\
\hline C9=C16 & $1.3901(5) \AA$ & C9=C16 & $107.997(26)^{\circ}$ \\
\hline 01-Mo1-S1 & $105.885(32)^{\circ}$ & O1-W1-S1 & $110.766(41)^{\circ}$ \\
\hline O1-Mo1-S2 & $110.461(27)^{\circ}$ & O1-W1-S2 & $108.186(26)^{\circ}$ \\
\hline 01-Mo1-S3 & $111.433(29)^{\circ}$ & O1-W1-S3 & $105.790(37)^{\circ}$ \\
\hline O1-Mo1-S4 & $106.406(28)^{\circ}$ & O1-W1-S4 & \\
\hline
\end{tabular}

Tab. 4: Vergleich der Bindungsanstände und -winkel der Molybdän- und Wolframverbindung

Vergleicht man die Metallsauerstoffbindungen in beiden Komplexen, wird eine kürzere Bindung bei der Molybdänverbindung festgestellt. In den zuvor betrachteten Komplexpaaren 1 und 2 wurden immer längere Metallsauerstoffbindungen aufgrund des geringfügig größeren Atomradius des Wolframatoms, gefunden. Somit sollte diese unterschiedliche Tendenz auf die Verbrückung des Sauerstoffatoms mit den Natriumatomen zustande kommen. Da es durch die in Abb.38 gezeigten Atome $\mathrm{zu}$ einer Ringbildung kommt, sollte bei der Molybdänverbindung eine stärkere Fixierung im Vergleich $\mathrm{zu}$ der Wolframverbindung stattfinden. Vergleicht man nun die Metall-Schwefelbindungen, so stellt man im Mittel kürzere Bindungslängen bei der Molybdänverbindung fest. Dies ist auch auf die Wechselwirkung mit den umgebenden ringbildenen Atomen zurückzuführen. Es werden längere Dithiolendoppelbindungen in der Wolframkristallstruktur gefunden [Mittelwerte $1.3889 \AA$ (Mo), $1.3845 \AA$ (W)]. Bei der Wolframverbindung werden die kürzeren und elektronenreicheren Doppelbindungen gefunden. Deshalb sollte bei der Molybdänverbindung die größere „non innocene“ des Ligandens vorhanden sein. Werden die O-M-S Bindungswinkel betrachtet, so fallen die größeren Winkel bei der Molybdänverbindung auf. Wahrscheinlich ist diese Beobachtung auf die Verbrückung der Natriumionen und der daraus resultierenden Ringbildung mit einem anderen Molybdänzentrum zurückzuführen. 


\subsection{Herstellung von Verbindungen in Oxidationsstufe V}
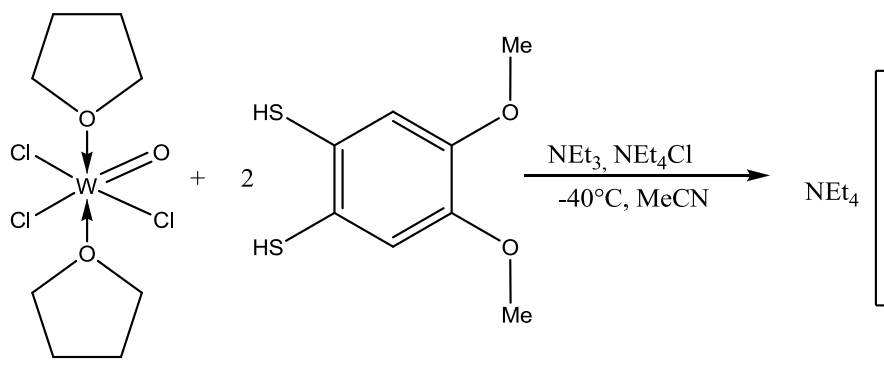<smiles>COCC1(C)Sc2cc(OC)c(OC)cc2Sc2cc(OC)c(OC)cc2S1</smiles>

Abb. 43: Reaktion zur Bildung von Verbindung $3 c$

Analog zu der Darstellung der Verbindung 2b konnte eine Komplexbildung zwischen $\left[\mathrm{WOCl}_{3}(\mathrm{thf})_{2}\right]$ mit zwei äquivalenten des vdt-Liganden erhalten werden. Ein rotbrauner kristalliner Feststoff (3c) in der Oxidationsstufe V wurde erhalten. Die Verbindung wurde durch FAB -Massenspektrometrie und Elementaranalysen charakterisiert. Es konnten leider keine Kristalle dieser Verbindung erhalten werden, um einen Vergleich der Bindungsparameter mit der Oxidationsstufe IV anzustellen.

\subsubsection{Untersuchung des Verbindungspaars [MO(ndt) ${ }_{2}^{2-}$}

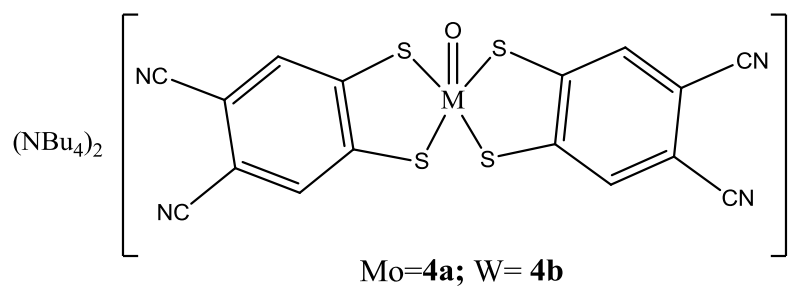

Abb. 44: Verbindungspaar $4 a$ und $4 b$

Beide Verbindungen konnten bei Verwendung des Tetrabutylammonium-Ions als kationisches als orange/rotes mikrokristallines Pulver erhalten werden. Dabei zeigte die Wolframverbindung einen stärker rötlichen Farbton. Die Verbindungen konnten durch NMR, IR, MS und Elementaranalyse nachgewiesen werden. $\operatorname{Im}{ }^{13} \mathrm{C}-\mathrm{NMR}$ können die $\mathrm{CN}$ Substituenten gut bei einer Verschiebung von 105.3 ppm beim Molybdän und bei 108.2 ppm beim Wolfram identifiziert werden. Den CN-Gruppen konnten außerdem in den IR-Spektren den entsprechenden Schwingungen zugeordnet werden, Mo: $1510 \mathrm{~cm}^{-1}$; W: $1509 \mathrm{~cm}^{-1}$ Außerdem ist der Wegfall der Bande bei $2536 \mathrm{~cm}^{-1}$ festzustelllen, die aus der SH-Schwingung des freien Liganden stammen würde. 
Im Folgenden ist das Massenspektrum des Molybdänkomplexes 4a aufgezeigt. Eine Besonderheit bei diesen beiden Spektren, im Vergleich zu den anderen angefertigten Spektren dieses Strukturtyps ist, dass im $\mathrm{FAB}^{+}$-Spektrum der anionische Komplexteil gemeinsam mit einem Kation als Molekularpeak zu erkennen ist. Normalerweise werden in den $\mathrm{FAB}^{+}$- Spektren nur die Kationen-Komplexfragmente gefunden, während der anionische Teil im $\mathrm{FAB}^{-}$erhalten wird.
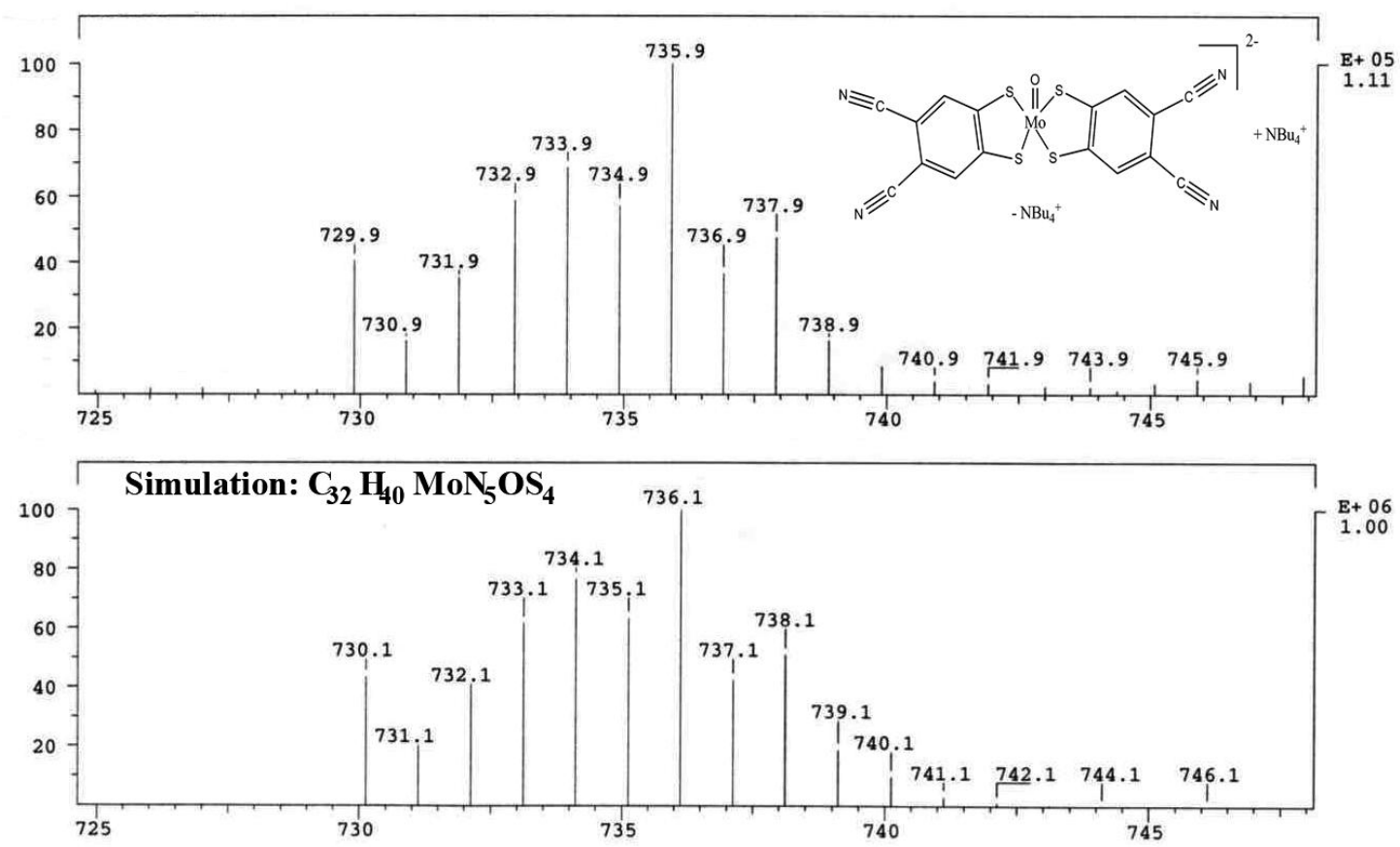

Abb. 45. Massenspektrum von 4a; oben experimentelle Messung; unten simuliertes Spektrum

Es konnten von beiden Verbindungen CV-Voltammogrammen aufgenommen werden. Es wurden reversibele Signale bei $-0.28 \mathrm{~V}(\mathrm{Mo})$ bzw. $-0.57 \mathrm{~V}(\mathrm{~W})$ gegen $\mathrm{Fc} / \mathrm{Fc}^{+}$festgestellt.

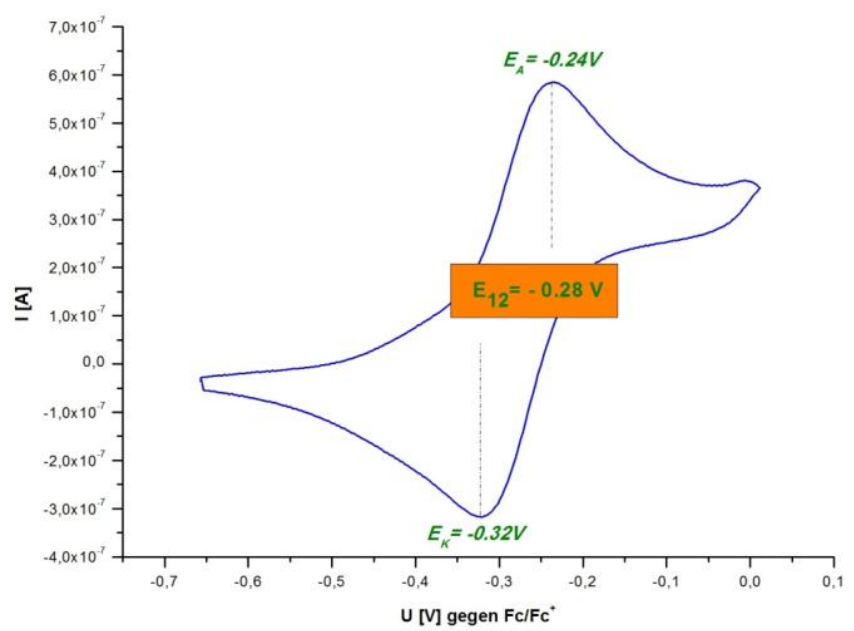

Abb. 46: CV-Spektrum aus MeCN von 4a 
Eine Oxidation zur Oxidationsstufe VI wurde nicht erreicht. Betrachtet man das CVVoltammogramm (Abb.46) genau, so stellt man eine leichte Verbreiterung des reduktiven Signals fest. Dies weist auf einen etwas langsameren Reduktionsvorgang hin. Im Vergleich zu den anderen vermessenen Komplexen (1-3), mit ähnlichen Ligandensystemen, kann hier eine stärkere Verschiebung in den positiven Potentialbereich festgestellt werden, was auf eine elektronenziehende Wirkung des Ligandenssystems hinweist.

\subsubsection{Untersuchungen des Verbindungspaars [MO(qdt)2 ${ }^{2-}$}<smiles>O=[W]1Sc2nc3ccccc3nc2S12Sc1nc3ccccc3nc1S2</smiles>

$\mathrm{Mo}=\mathbf{5 a} ; \mathrm{W}=\mathbf{5 b}$

Abb. 47: Verbindungen $5 a$ und $5 b$

Aufgrund der großen Instabilität zeigte sich, dass bei der Herstellung der Wolframverbindung nur moderate Wärme für kurze Zeit zur Darstellung zugeführt werden darf. Es ist zwar eine gewisse Wärme notwendig um die Aktivierungsenergie zu liefern, allerdings konnte bei der Herstellung bei Temperaturen über $40^{\circ} \mathrm{C}$ ein Zerfall der Verbindung beobachtet werden. Nach Erhalt der Wolframverbindung zeigt sich eine sehr starke Empfindlichkeit gegen Luftsauerstoff, sowie gegen starke Temperaturänderungen. Acetonitrillösungen dieser Verbindungen die zur Kristallisation bei $-35^{\circ} \mathrm{C}$ über Nacht aufbewahrt wurden, zeigten vollständigen Zerfall und es kam zur Bildung eines gelben Feststoffs, der auch durch Röntgenbeugung am Einkristall charakterisiert werden konnte.

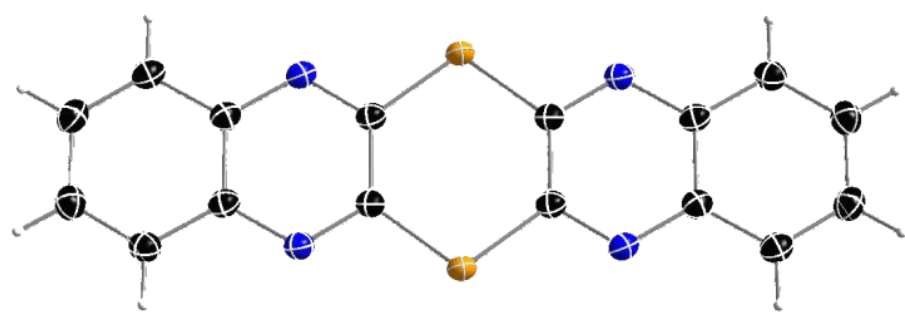

Abb. 48: Kristallstruktur vom Zerfallsprodukt 5c

Somit kommt es zum kompletten Zerfall der Wolframverbindung und zu einer Dimerbildung zwischen den abgespalten Liganden. Es ist wahrscheinlich, dass sich zuvor ein Ligandengerüst mit Disulfidbrücke gebildet hat, welches sich dann aufgrund der Instabilität 
zu der in Abb. 48 gezeigten Struktur umwandelt. Die beste Aufbewahrungsmethode dieser Verbindungen stellt das Lagern bei $1^{\circ} \mathrm{C}$ im Kühlschrank dar.

Trotzdem konnten beide Verbindungen, sowohl durch NMR, IR, MS, UV-Vis als auch durch Elementaranalyse charakterisiert werden. Bei der Wolframkomponente wurde im UV-Vis eine intensive Absorption bei $628 \mathrm{~nm}\left(2150 \mathrm{M}^{-1} \cdot \mathrm{cm}^{-1}\right)$ und bei der Molybdänverbindung bei $562 \mathrm{~nm}\left(6730 \mathrm{M}^{-1} \cdot \mathrm{cm}^{-1}\right)$ beobachtet. Diese Absorption sollten durch einen Ligand-MetallCharge-Transferübergang vom Schwefel-p-Orbital des qdt-Ligandens zum $d_{x y}$-Orbital des Metalls zustande kommen. ${ }^{[50]} \mathrm{Da}$ die Übergänge quantenmechanisch erlaubt sind, ergeben sich sehr intensive Banden, was an den relativ großen molaren Extinktionskoeffinzenten zu sehen ist. Elektrochemische Untersuchungen dieser Verbindungen zeigten beim Molybdän schmale Signale der Elektronenübergänge im DP-Voltammogrammen bei $-0.31 \mathrm{~V}$ gegen $\mathrm{Fc} / \mathrm{Fc}^{+}$. Die Wolframverbindung dagegen konnte nur durch breite Signale im DPV-Spektrum bei $-0.67 \mathrm{~V}$ gegen $\mathrm{Fc} / \mathrm{Fc}^{+}$nachgewiesen werden. Die Breite der Signale konnte sowohl durch Verminderung der Konzentration, als auch durch Veränderungen der Vorschubgeschwindigkeit nicht verändert werden (Spektrum im Anhang unter Abschnitt 11.1). An diesem Beispiel kann man erkennen, dass bei manchen Wolframverbindungen die geometrische Veränderung, welche Oxidation und Reduktion begleitet, langsamer zwischen den Elektronenübergängen stattfindet, als die eigentliche Elektronenübertragung zwischen der Elektrode und dem Komplex. Hierzu mehr in Kapitel 4.

\subsubsection{Geometrische Untersuchungen der Verbindungen 5a und 5b}

Von beiden Verbindungen konnten Einkristalle erhalten werden, die für eine Strukturanalyse durch Röntgenbeugung geeignet waren. Die Molybdänverbindung wurde durch „VaporDiffusion“ aus Methanol und Diethylether erhalten. Wie am Anfang dieses Abschnittes bereits erwähnt, zeigt die Verbindung $\mathbf{5 b}$ Zerfall bei niedrigen Temperaturen über längere Zeit. Schlussendlich konnten die Kristalle von $\mathbf{5 b}$ aus Methanol bei $1^{\circ} \mathrm{C}$ erhalten werden. Doch die Kristalle zeigten schnelle Ölbildung bei Temperaturveränderung um ca. $5^{\circ} \mathrm{C}$. Während der röntgenspektroskopischen Untersuchung bei $-140^{\circ} \mathrm{C}$ konnte jedoch für die notwendige Dauer der Messzeit keine Veränderung der Kristalle beobachtet werden. 


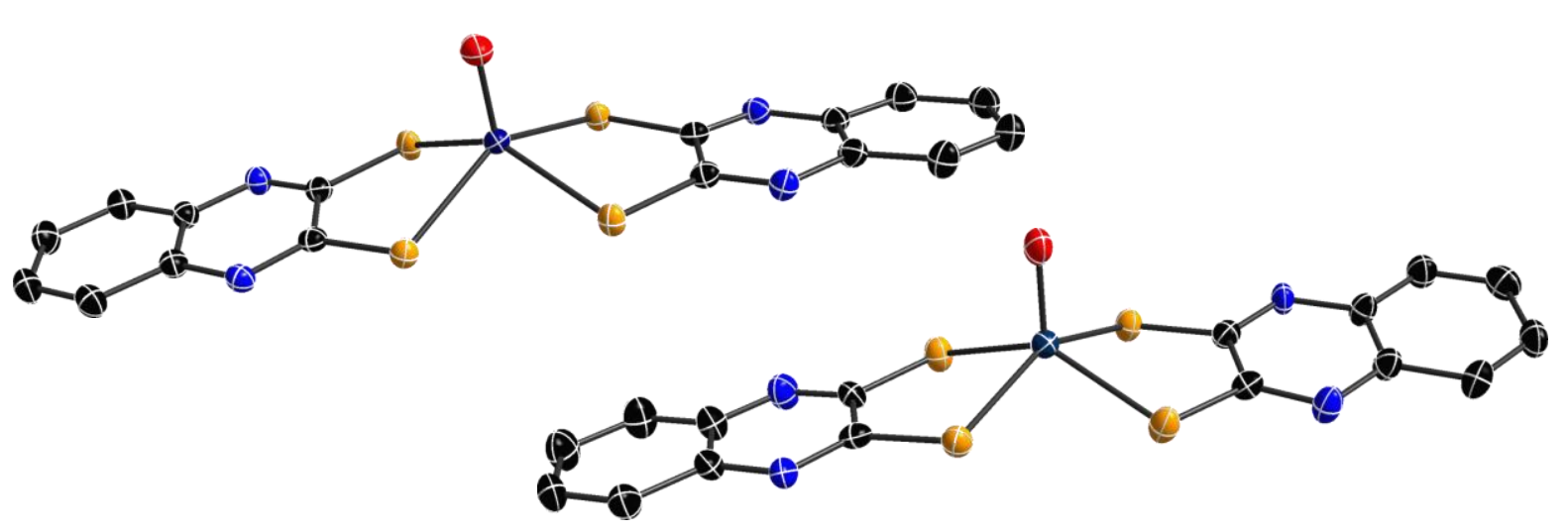

Abb. 49: Kristallstruktur von $\left(\mathrm{BzNEt}_{3}\right)_{2}\left[\mathrm{MoO}(\mathrm{qdt})_{2}\right] 5 \mathrm{a}$ (links) und $\left(\mathrm{Bu}_{4} \mathrm{~N}\right)_{2}\left[\mathrm{WO}(q d t)_{2}\right] 5 \mathrm{~b}$ (rechts) zur Übersichtlichkeit wurden die Kationen nicht gezeigt

Bei der vergleichenden Betrachtung der Bindungslängen und -winkel wurde folgende Nummerierung der Atome benutzt.

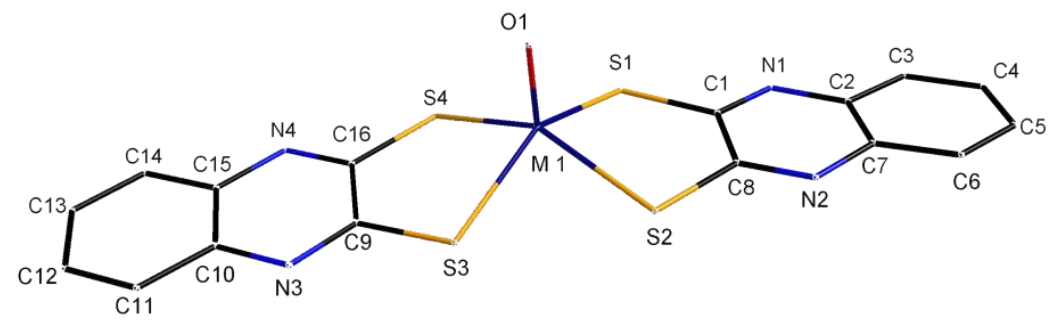

Abb. 50: Verwendete Nummerierungen der Verbindungen

\begin{tabular}{|c|c|c|}
\hline Atome & Molybdän & Wolfram \\
\hline M1-01 & $1.6814(2) \AA$ & $1.6790(4) \AA$ \\
\hline M1-S1 & $2.4045(5) \AA$ & $2.3854(4) \AA$ \\
\hline M1-S2 & $2.3898(4) \AA$ & $2.3795(9) \AA$ \\
\hline M1-S3 & $2.3918(5) \AA$ & $2.3807(4) \AA$ \\
\hline M1-S4 & $2.4037(5) \AA$ & $2.3666(6) \AA$ \\
\hline S1-C1 & $1.7579(3) \AA$ & $1.7420(5) \AA$ \\
\hline S2-C8 & $1.7591(3) \AA$ & $1.7485(3) \AA$ \\
\hline S3-C9 & $1.7565(2) \AA$ & $1.7489(4) \AA$ \\
\hline S4-C16 & $1.7519(3) \AA$ & $1.7565(3) \AA$ \\
\hline C1-C8 & $1.4507(5) \AA$ & $1.4563(4) \AA$ \\
\hline C9-C16 & $1.4524(5) \AA$ & $1.4370(4) \AA$ \\
\hline S2-M1-01 & $109.809^{\circ}(12)$ & $105.240^{\circ}(14)$ \\
\hline S3-M1-01 & $108.159^{\circ}(13)$ & $110.728^{\circ}(12)$ \\
\hline S4-M1-01 & $108.108^{\circ}(11)$ & $105.452^{\circ}(14)$ \\
\hline M1-S1-C1 & $108.670^{\circ}(11)$ & $107.595^{\circ}(12)$ \\
\hline M1-S2-C8 & $105.737^{\circ}(12)$ & $103.702^{\circ}(17)$ \\
\hline M1-S3-C9 & $105.299^{\circ}(12)$ & $105.228^{\circ}(14)$ \\
\hline M1-S4-C16 & $105.160^{\circ}(11)$ & $106.020^{\circ}(17)$ \\
\hline
\end{tabular}


So zeigen beide Verbindungen eine annährend quadratisch pyramidale geometrische Anordnung. In $A b b .51$ und $A b b .52$ wurde ein pyramidales strukturelles Modell, in die von dem Metall umgebenden Atome, eingelegt. Die Dreiecksflächen, welche die einzelnen Dithiolenliganden und den Sauerstoff miteinander verbinden, weisen Winkel von etwa 60-62 ${ }^{\circ}$ auf. Betrachtet man nun aber die Winkel der Dreiecksflächen, die sich auf der Seite eines Dithiolenligandens mit dem Sauerstoffatom ergeben, so sind die Winkel um die $80^{\circ}$ groß. Somit kann bei beiden Strukturen von einer pyramidalen Geometrie ausgegangen werden, die eine nicht quadratische Grundfläche aufweist.

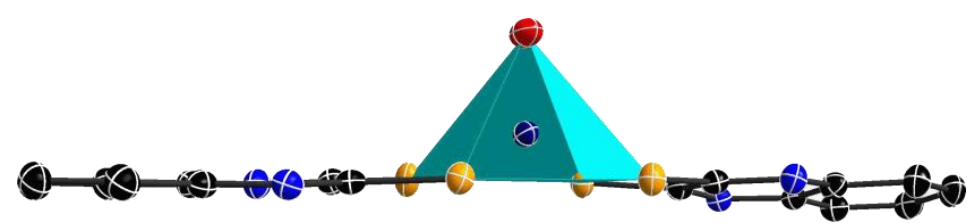

Abb. 51: Strukturelles Modell zur geometrischen Evaluierung am Beispiel von Verbindung 5a (Mo)

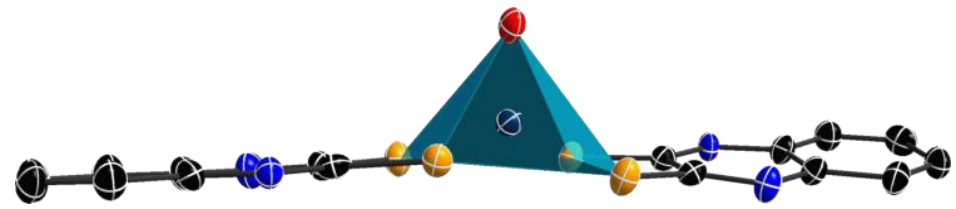

Abb. 52: Strukturelles Modell zur geometrischen Evaluierung am Beispiel von Verbindung 5b (W)

Außerdem ist ersichtlich, dass die beiden Dithiolenliganden im Mittel um einen Winkel von $9.33^{\circ}(\mathrm{W})$ bzw. $14.85^{\circ}(\mathrm{Mo})$ gegeneinander verdreht sind.

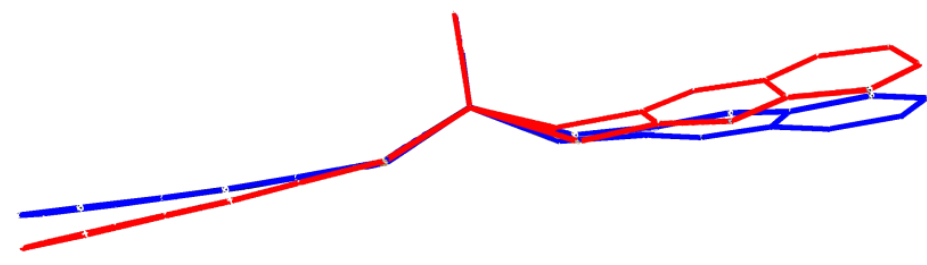

Abb. 53: Übereinandergelegte Strukturen Mo(blau), W (rot)

Das in den Abbildungen auf der linken Seite gezeigte Ligandensystem weist über das gesamte System eine relativ planare Struktur auf. An der Seite des verdrehten Dithiolenliganden (im Vergleich zu dem anderen Liganden), stellt man auch eine leichte Verdrillung des Liganden in sich selbst fest. Dabei befinden sich die Stickstoffatome nicht mehr in einer Ebene. Bei der 
Betrachtung der Bindungslängen fällt auf, dass bei der Wolframverbindung kürzere WolframOxobindungen im Vergleich zum Molybdän festzustellen (0.24 pm) sind. Der Unterschied ist auf den geringfügigen Größenunterschied der beiden Zentralmetalle in der Oxidationsstufe IV zurückzuführen. Somit ergeben sich Ionenradien von $65 \mathrm{pm}$ beim Molybdän und $66 \mathrm{pm}$ bei Wolfram. ${ }^{[51]}$ Bei diesen Werten handelt es sich um die Annahme, einer sechsfach Koordination zum Metall. Aufgrund von relativistischen Effekten ergeben sich annährend gleiche Ionengrößen, obwohl beim Wolframatom 32 Elektronen mehr vorhanden sind. Bei der Wolframverbindung werden kürzere Bindungen zu den Schwefelatomen gefunden. Dies ist darauf zurückzuführen, dass das Wolframatom sowie das Schwefelatom nach Pearson, die weicheren und leichter polarisierbaren Atome im Vergleich zum Molybdän sind. Hierdurch bildet sich eine stabilere und kürzere Bindung bei der Kombination W-S. Durch die angesprochene Verdrillung, kommt es bei der Wolframverbindung zu einer stärkeren Verkürzung der Dithiolendoppelbindung des geneigten Dithiolenliganden. Somit ist in der Kohlenstoffdoppelbindung der Wolframbindung eine größere Elektronendichte vorhanden, wodurch sich die non-innocence des Ligandens verringert.

\subsubsection{ESR-Untersuchungen}

Bei Betrachtung des qdt-Ligandens kann man erkennen, dass durch die direkte Nachbarschaft zu den Stickstoffatomen eine Konjugation der Doppelbindungssysteme auftreten kann.

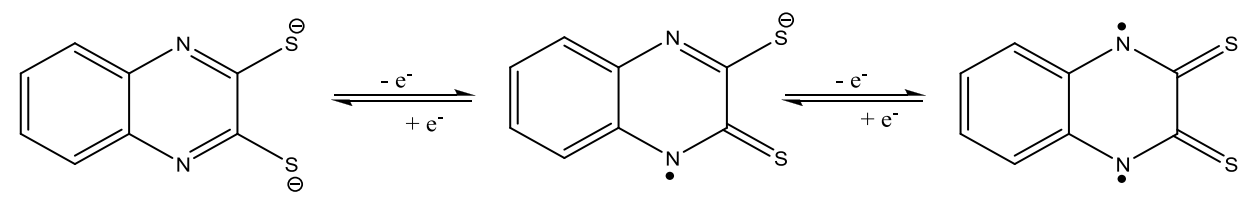

Abb. 54: Non-Innocence des qdt-Ligandens

Aufgrund der Non-Innocence des Ligandens, kann es auch zu radikalen Zwischenstufen im Ligandensystem kommen. Um die Position der Radikale in den Komplexen näher zu erforschen, wurden sowohl ESR-Messungen des Ligandenssystems, als auch der Komplexe bei $-140^{\circ} \mathrm{C}$ in DMF durchgeführt. Im Folgenden ist das ESR-Spektrum des freien Ligandens und der diamagnetischen Wolframverbindung gezeigt. (Abb.55) 


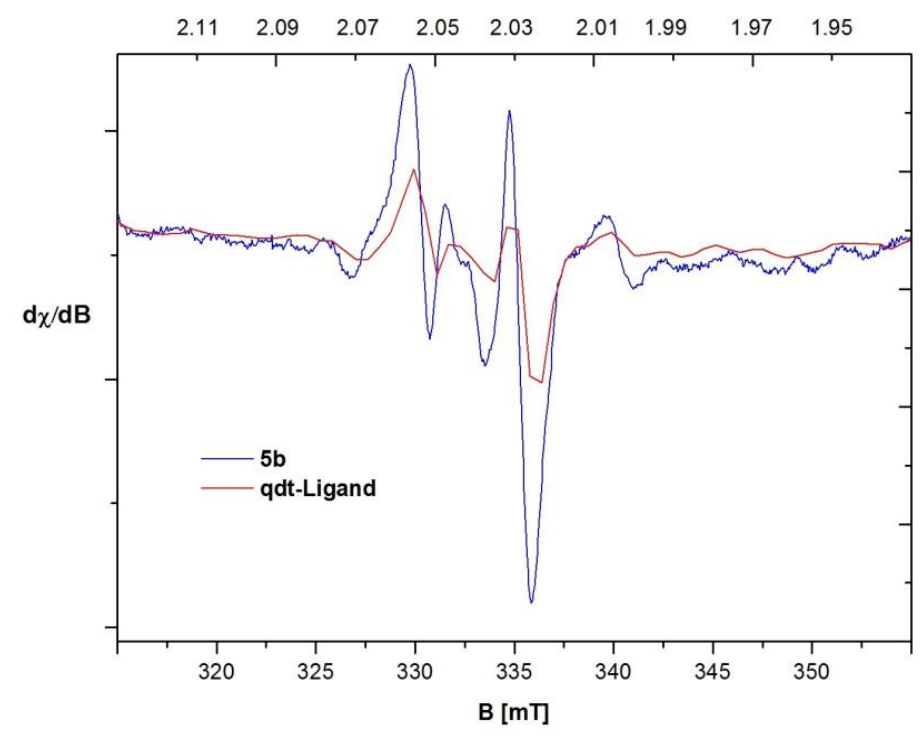

Abb. 55: ESR-Spektrum des Liganden und der Verbindung 5b (IV) in DMF bei $-140^{\circ} \mathrm{C}$

Es ist festzustellen, dass der freie Ligand ein Signal im ESR-Spektrum zeigt (rote Linie). Nun ergeben sich zwei Möglichkeiten für den Aufenthalt des ungepaarten Elektrons, der Aufenthalt an einem Stickstoffatom oder an einem Schwefelatom. Es zeigen nur das ${ }^{33} \mathrm{~S}-$ Isotop ( $\mathrm{I}=3 / 2)$ und das ${ }^{14} \mathrm{~N}$-Isotop ( $\left.\mathrm{I}=1\right)$, sowie das ${ }^{15} \mathrm{~N}$-Isotop ( $\left.\mathrm{I}=1 / 2\right)$ einen Kernspin der größer als Null ist. Beim Vorhandensein des ungepaarten Elektrons am Schwefel sollten vier Linien im Spektrum beobachtet werden. Beim Aufenthalt des Elektrons am ${ }^{14} \mathrm{~N}$-Isotop sollten sich drei Linien ergeben und zusätzlich sollten noch zwei Linien des ${ }^{15} \mathrm{~N}$-Isotops auftauchen, die aber aufgrund der geringen natürliche Häufigkeit mit sehr geringer Intensität zu sehen sein sollten. Die erhaltenen Signale des Ligandens sind nicht vollkommen aufgelöst, und mit eindeutiger Sicherheit kann nicht auf ein Atom geschlossen werden. Da aber die natürliche Häufigkeit des Schwefelisotops als gering einzustufen ist, sollte die Aufenthaltswahrscheinlichkeit am Schwefelatom als sehr gering eingeschätzt werden. Es kann sich bei dem erhaltenen Signalmuster (Intenstitätsverhältnis 1:1:1) um ein ungepaartes Elektron am Stickstoffatom handeln (mittlere Struktur in Abb.54)

Außerdem wurde die Wolframverbindung in Oxidationsstufe IV untersucht. Da das eigentliche Metallzentrum diamagnetisch ist, sollte kein Signal durch das vorhandene Metalllzentrum auftreten. Das erhaltene Spektrum ist in Abb.55 (blaue Linie) gezeigt. Es wird ein deutlicheres Signal im Vergleich zum freien Liganden beobachtet, dass das gleiche Signalmuster wie das des Ligandens aufweist. Somit kann erneut von einem Vorhandensein eines ungepaarten Elektrons am Stickstoffatom des Ligandens ausgegangen werden. Um einen Aufschluss darüber zu bekommen, inwieweit sich das Spektrum ändert, wenn auch ein 
ungepaartes Elektron am Metallzentrum vorhanden ist, wurden beide Metallkomplexe zur Oxidationsstufe V oxidiert.

\subsubsection{Oxidation der Komplexe}

Beide Verbindungen konnten durch die Verwendung von $I_{2}$, sowie durch Ferroceniumhexafluorophosphat von der Oxidationsstufe IV nach V oxidiert werden. Die dabei entstandenen Reduktionsprodukte Iodid bzw. Ferrocen, konnten durch Extraktion mit entgastem Wasser bzw. mit Hexan entfernt werden.

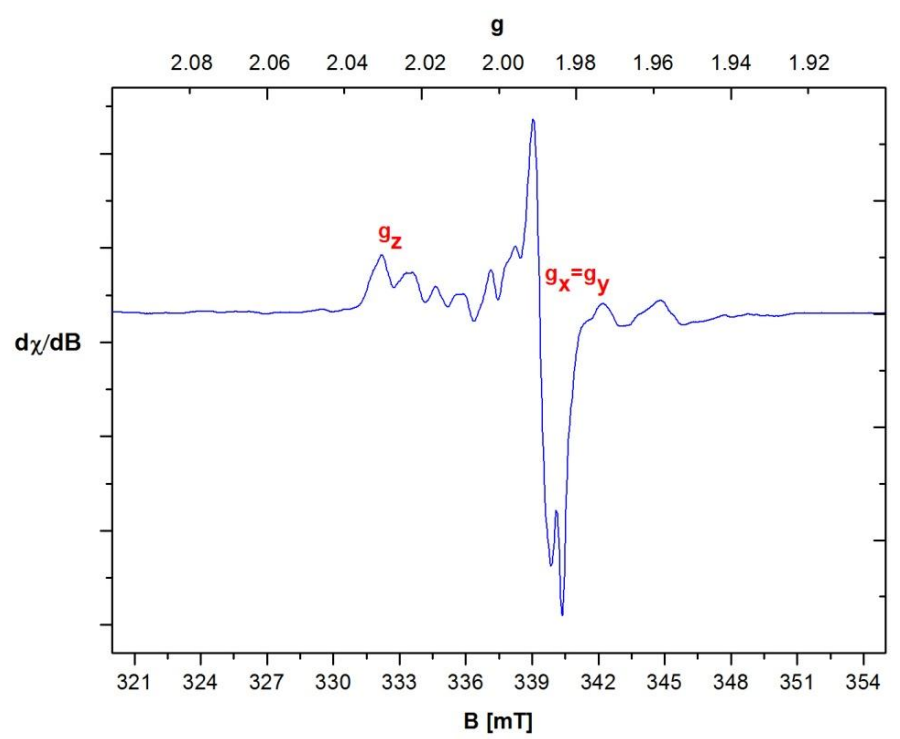

Abb. 56: ESR-Spektrum von $5 a(V)$ in DMF bei $-140^{\circ} \mathrm{C}$

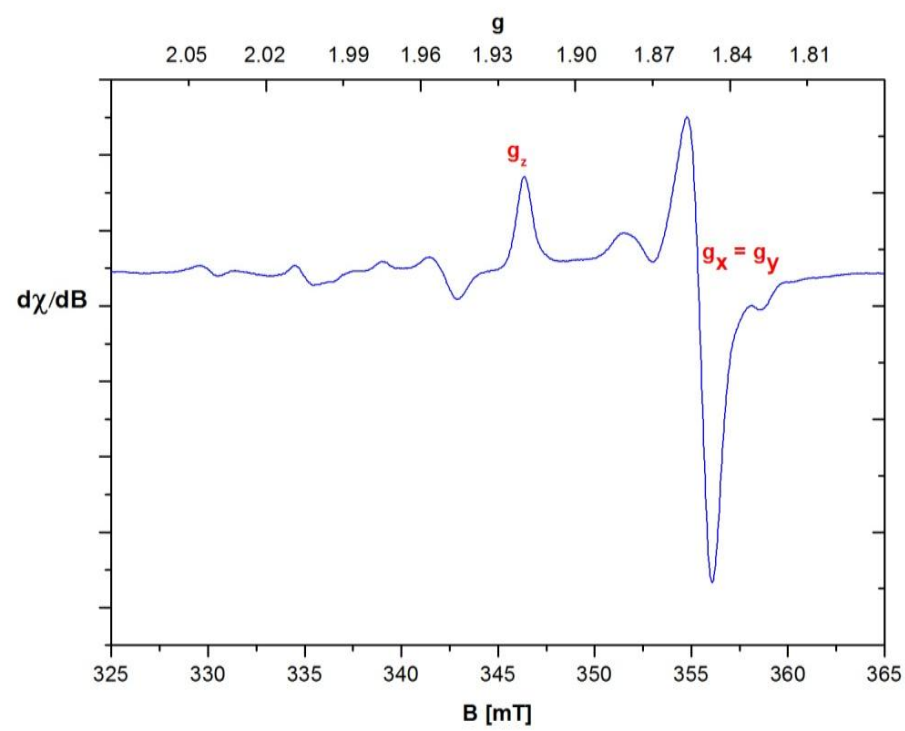


Die ESR-Spektren beider Verbindungen zeigen eine axiale Symmetrie. Somit ergibt sich folgende Beziehung zwischen den anisotropen Landé-Faktoren $\mathrm{g}_{\mathrm{x}}=\mathrm{g}_{\mathrm{y}}<\mathrm{g}_{\mathrm{z}}$.

\begin{tabular}{|c|c|c|c|}
\hline & $\mathbf{g}_{\mathbf{x}}$ & $\mathbf{g}_{\mathbf{y}}$ & $\mathbf{g}_{\mathbf{z}}$ \\
\hline $\mathbf{5 a}$ & 1.98 & 1.98 & 2.03 \\
$\mathbf{5 b}$ & 1.85 & 1.85 & 1.92 \\
\hline
\end{tabular}

Bei den Molybdän- bzw. Wolframverbindungen handelt es sich um $\mathrm{d}^{1}$-Systeme, wodurch sich ein Gesamtspin des Systems von $S=1 / 2$ ergibt. Aufgrund der Relativbewegung von Kern und Elektronen wird am Ort der Elektronen ein zusätzliches (stabilisierendes) lokales Magnetfeld (abhängig von der Kernladung) erzeugt. Da die Geometrien der Strukturen am meisten Ähnlichkeit mit quadratisch pyramidalen Strukturen aufweisen, ergibt sich als niedrigstes Orbital das $\mathrm{d}_{\mathrm{xy}}$-Orbital. Somit wird das $\mathrm{d}_{\mathrm{xy}}$-Orbital in die folgenden Orbitale rotiert.

$$
\begin{gathered}
d_{x y \stackrel{g z}{\longrightarrow}} d_{x^{2}-y^{2}} \\
d_{x y \stackrel{g y}{\longrightarrow}} d_{y z} \\
d_{x y \stackrel{g x}{\longrightarrow}} d_{x z}
\end{gathered}
$$

Da die Orbitale $d_{y z}$ und $d_{x z}$ energetisch gleich sind, zeigt die Auswirkung der Rotation um die $\mathrm{x}$ - bzw. y-Achse den gleichen Landé-Faktor. Die größten g-Werte wurden für die zKomponente erhalten, da dies einer Rotation vom $d_{x y}$ zum energetisch höchst gelegenen $\mathrm{d}_{\mathrm{x}}{ }^{2} \mathrm{y}^{2}$ entspricht. Da aber noch weitere Peaks in den Spektren zu erkennen sind, sollte es noch $\mathrm{zu}$ weiteren anisotropen Hyperfeinkopplung mit den Liganden kommen. Generell kann aber festgestellt werden, dass das ungepaarte Elektron das sich am Metallzentrum, befindet in viel größerer Intensität vorhanden ist, als die Signale die durch das ungepaarte Elektron des Ligandenszentrums ausgelöst werden. Das immer noch ein Radikal im Ligandensystem vorhanden ist, kann an einem Signal im Hochfeld bei ca. $155 \mathrm{mT}$ festgestellt werden, was auf die Wechselwirkung von zwei Ligandenteilen hinweist. Diese Untersuchungen müssen noch durch Simulationen weiter analysiert werden, um einen genaueren Aufschluss zwischen den Wechselwirkungen der Radikale zu verifizieren. 


\subsubsection{Vergleich der Verbindungen mit aromatischen Dithiolenligandensystemen}

Um die Unterschiede zwischen den elektronischen Effekten herauszustellen werden die strukturell relativ ähnlichen Verbindungen miteinander verglichen

\begin{tabular}{|c|c|c|c|c|}
\hline & $\begin{array}{c}v(M=O) \\
\exp .\left[\mathrm{cm}^{-1}\right]\end{array}$ & $\begin{array}{c}v(M=O) \\
\text { berechn. }\left[\mathrm{cm}^{-1}\right]\end{array}$ & $\begin{array}{c}\mathrm{M}=\mathbf{O} \\
\text { Kristalls.[̊] }\end{array}$ & $\begin{array}{l}\mathrm{E}_{12} \text { gegen } \\
\mathrm{Fc} / \mathrm{Fc}^{+}[\mathrm{V}]\end{array}$ \\
\hline $1 \mathrm{a}$ & 913 & 936 & 1.7548 & -0.85 \\
\hline $1 b$ & 911 & 936 & 1.7190 & -1.03 \\
\hline $2 a$ & 904 & 966 & 1.7021 & -0.80 \\
\hline $2 c$ & 898 & 964 & - & -1.49 \\
\hline $\mathbf{3 a}$ & 891 & 935 & 1.7116 & -0.77 \\
\hline $3 b$ & 904 & 933 & 1.7313 & -1.45 \\
\hline $\mathbf{4 a}$ & 916 & 950 & - & -0.28 \\
\hline $4 b$ & 919 & 948 & - & -0.57 \\
\hline $5 a$ & 941 & 955 & 1.6814 & -0.31 \\
\hline $5 b$ & 918 & 953 & 1.6790 & -0.67 \\
\hline
\end{tabular}

Tab. 7: Vergleich der $\mathrm{M}=0$ Schwingungsfrequenzen, exp. und berechnet,

$M=0$ Bindungslängen aus Kristallstrukturen, Redoxpotentiale

Aufgrund der größeren Masse des Wolframs gegenüber der des Molybdäns sollten die Schwingungen durch die Vergrößerung der Kraftkonstante, sowie der reduzierten Masse zu kleineren Wellenzahlen verschoben sein, diese Tendenz konnte nur bei dem Verbindungspaar 1, 2 und 5 festgestellt werden. Dies kann durch die vielfach beobachtete Oxophilie des Molybdäns gegenüber dem Wolfram erklärt werden. Außerdem wurden die Schwingungen mit DFT-Rechnungen berechnet, doch die hieraus erhaltenen Schwingungswerte liegen deutlich über den experimentellen Werten, was wahrscheinlich an der Vernachlässigung der anharmonischen Schwingungsbeiträge zurückzuführen ist. Durch die Rechnung konnten aber die $\mathrm{W}=\mathrm{O}-\mathrm{Sch} w i n g u n g e n$ bei kleinen Wellenzahlen im Vergleich zum Molybdän nachgewiesen werden.

Da nicht alle IR-Presslinge von Kristallen angefertigt wurden, konnte nur bei den Verbindungspaaren $\mathbf{1 b}$ und $\mathbf{3 b}$ die Tendenz festgestellt werden, dass zur Anregung der kürzeren Wolframsauerstoffbindung mehr Energie benötigt wird, was an den größeren Wellenzahlenen zu erkennen sein sollte. Bei den anderen Verbindungen konnte diese Tendenz nicht gefunden werden, weil es vielfach auch in den Kristallstrukturen zu Fehlordnungen an der Metallsauerstoffbindung kam, wodurch die Bindungslängen nicht exakt angegeben 
werden konnte. Als weitere Möglichkeit können die verschiedenen vorhandenen Kationen zu einer Beeinflussung der Metallsauerstoffbindung führen. Generell sind die Unterschiede bei den IR-Schwingung gering, wodurch es bei auftretenden Schwankungen der Messwerte zur Verschiebung der Tendenzen kommen kann.

Beim Vergleich der bestimmten Redoxpotentiale werden bei den Verbindungen mit elektronenschiebenden Substituenten ähnliche Potentiale, um die $-0.80 \mathrm{~V}$ (gegen $\mathrm{Fc} / \mathrm{Fc}^{+}$) bei Molybdän und um die $-1.50 \mathrm{~V}$ bei Wolfram gefunden. Eine Ausnahme stellt das bestimmte Potential der Verbindung 1b dar, da nur ein Potential von $1.03 \mathrm{~V}$ gefunden wird. Bei den Verbindungspaaren 4 und $\mathbf{5}$ kommt es zur Verschiebung in Richtung des positiven Potentialbereichs, was auf die elektronenziehende Wirkung der CN-Substituenten am aromatischen Liganden, sowie durch das Quinoxlinsystem zurückzuführen ist. Aufgrund des Potentialunterschieds zwischen der Molybdän- und Wolframverbindungen wird abgesehen, von 1, die größte non-innocene des Ligandensystems bei dem Verbindungspaar $\mathbf{4}$ gefunden.

\subsubsection{Untersuchungen des Verbindungspaars [MO(ntdt) $]^{2-}$}

Um der Natur des Molybdopterinligandens mehr gerecht zu werden und die Verknüpfung zum Metallzentrum nicht direkt über die Dithioleneinheit als Teil eines aromatischen System durchzuführen, wurden 1-(Naphtalen-2-yl)ethen-1,2-dithiol-Liganden mit den Metallprecursorn reagieren gelassen.

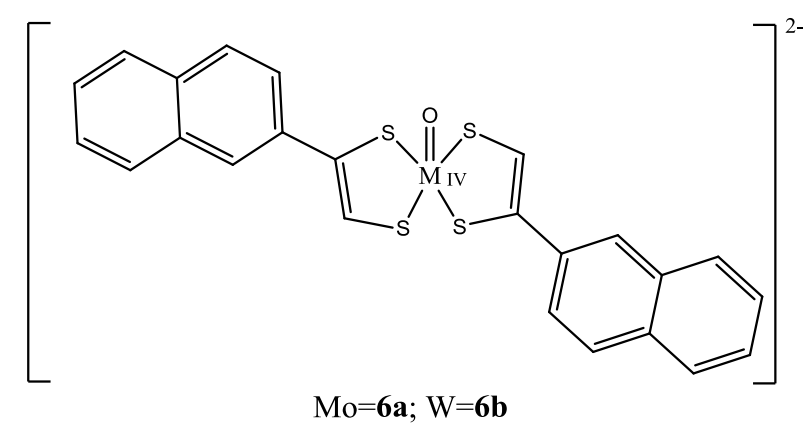

Abb. 58: Verbindungspaar $6 a$ bzw. $6 b$

Aufgrund der unsymmetrischen Substitution an der Doppelbindung des Dithiolens, können bei der Herstellung der Komplexe sowohl die cis- als auch die trans-Isomere entstehen. Die Verbindungen konnten durch ${ }^{1} \mathrm{H},{ }^{13} \mathrm{C}-\mathrm{NMR}$, IR, MS sowie Elementaranalyse identifiziert werden. Beide Verbindungen zeigen bei Verwendung des Tetrabutylammoniumions gute 
Löslichkeit in Acetonitril und Methanol, aber vollständige Unlöslichkeit in entgastem Wasser und unpolaren Lösungsmitteln. Bei der Herstellung der beiden Verbindungen fiel auf, dass sich die Wolframverbindung sehr viel empfindlicher gegenüber Sauerstoff verhielt, als die analoge Molybdänverbindung. Bei der Zersetzung trat eine sofortige Farbveränderung von dunkelrot nach grün auf. Es wurden elektrochemische Untersuchungen dieser Verbindungspaare in Acetonitrillösung durchgeführt, wobei reversible Signale $[\mathrm{Mo}=-0.88 \mathrm{~V} ; \mathrm{W}=-0.92 \mathrm{~V}]$ festgestellt wurden .

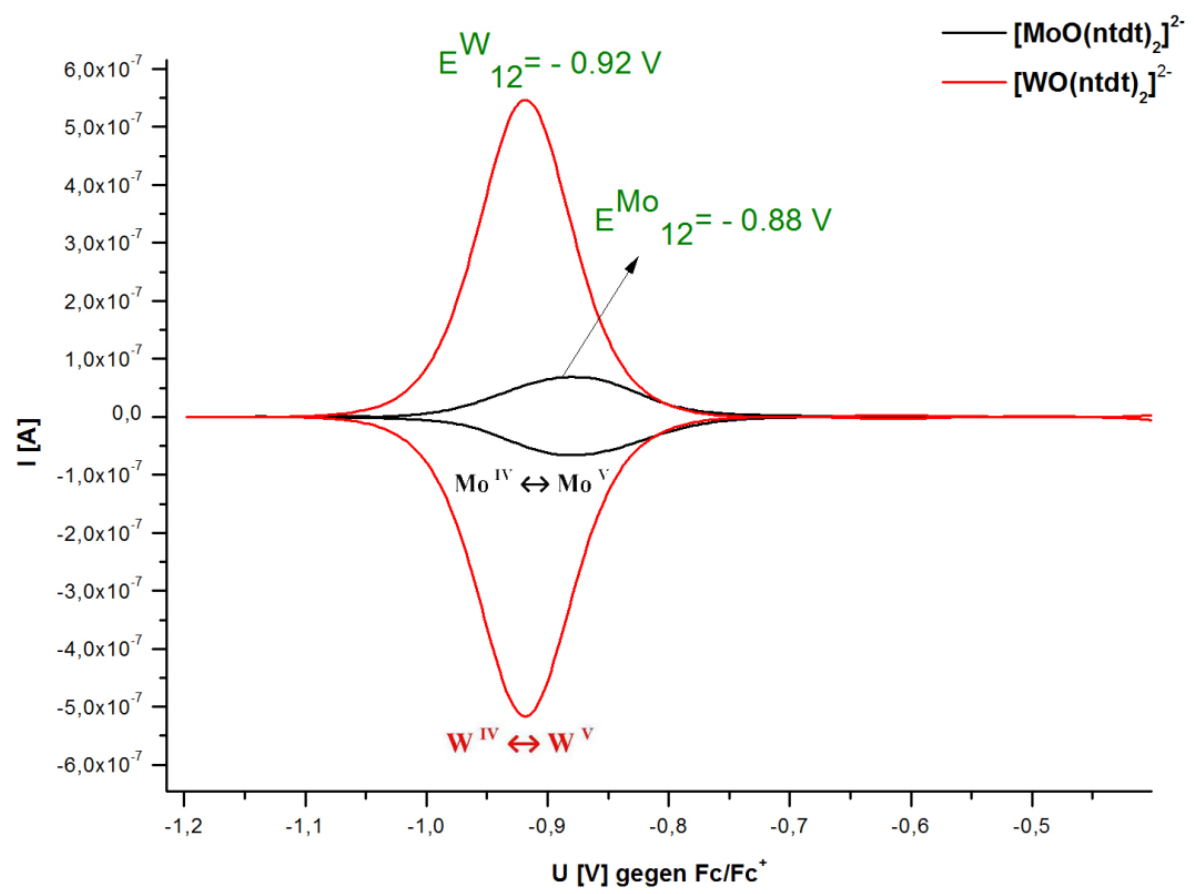

Abb. 59: DPV-Spektrum der Verbindungen 6a und 6b

\subsubsection{Geometrische Analyse von [MoO(ntdt) $]^{2-}$}

Von der Molybdänverbindung konnten Einkristalle erhalten werden, die zur

Röntgenkristallographischen Analyse geeignet waren. Hierbei konnte die aus kristallographischer Sicht eher ungewöhnliche cis-Anordnung der Liganden gefunden werden.

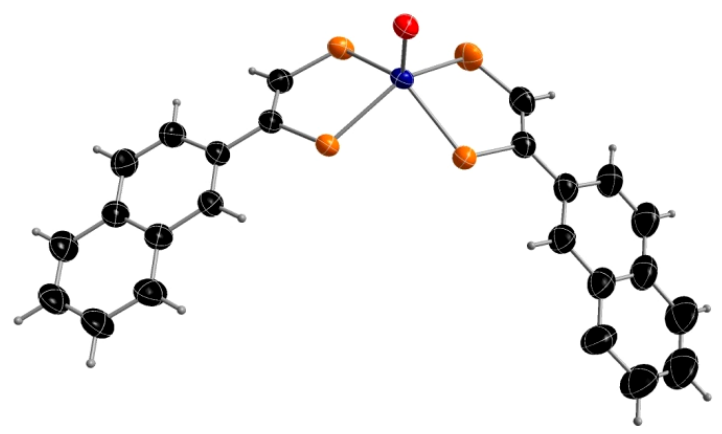


Es konnte eine quadratisch pyramidale Koordinationsumgebung des Molybdänzentrums beobachtet werden. Dabei ist der Naphtanyl-Ligand fast vollkommen linear $\left(172.5^{\circ}\right)$ an die Dithioldoppelbindung gebunden. Im Vergleich zu den zuvor diskutierten Systemen, kann die größte Ähnlichkeit zu einer vollkommen quadratischen Pyramide gefunden werden. Aufgrund der besseren Lokalisation der Doppelbinding in diesen Systemen, kommt es im Vergleich zu aromatischen Dithiolenliganden, zu einer Verkürzung der Dithiolendoppelbindung, wobei Bindungslängen von $1.335 \AA$ bzw. $1.319 \AA$ gefunden werden. Die Molybdänsauerstoffbindung zeigt keine übermäßige Verlängerung (1.702 Å) bei der Betrachtung zu anderen Strukturen mit quadratisch planarer Umgebungsgeometrie. Aufgrund der cisAnordnung des Ligandensystems sollte die zur Ligandensubstituenten abgewandte Seite leichter zugänglich sein. Somit kann ein direkter Angriff des Metallzentrums durch andere Reaktanden leichter durchgeführt werden.

\subsubsection{Verbindungspaare mit unterschiedlichen Ringatomen in Nachbarschaft zur Dithioleneinheit}

Die ersten Dithiolenkomplexe von Molybdän und Wolfram, die einen Pyranring trugen, wurden von unserer Arbeitsgruppe im Jahre 2005 synthetisiert. ${ }^{[4]}$ Durch elektrochemische Untersuchungen gab es Hinweise darauf, dass es einen Einfluss des Pyranrings auf das Oxidations- bzw. Reduktionsverhalten der entsprechenden Komplexe gibt.

Folgende zwei Verbindungspaare wurden synthetisiert, um einen Vergleich mit den entsprechenden Pyranhaltigen Verbindungen und somit die Wirkung eines Sauerstoffatoms im Ringsystem näher zu untersuchen.

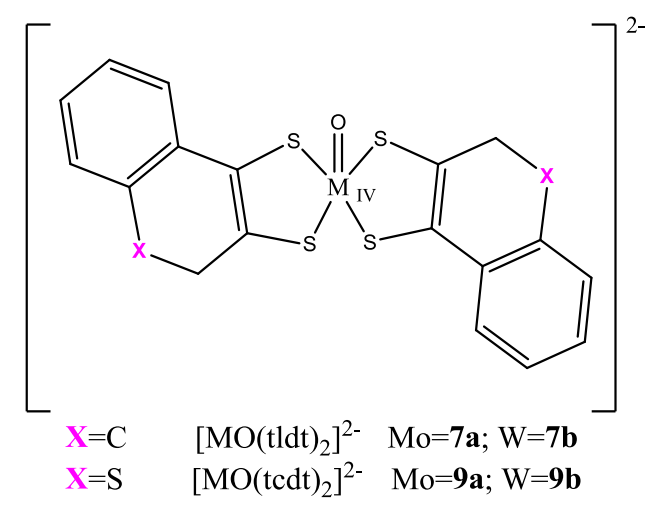

Abb. 61: Verbindungspaare zur Untersuchung Wirkung verschiedener Atome im Ligandensystem in Nachbarschaft zur Dithioleneinheit

Die Pyransysteme dieser Komplexe wurden erstmals von Dr. Kerstin Starke in unserem Arbeitskreis synthetisiert $(\mathrm{Mo}=\mathbf{8 a}, \mathrm{W}=\mathbf{8 b})^{[52]}$ und wurde mir von Prinson P. Samuel für diese 
Kooperationsstudie bereitgestellt. Die analogen Verbindungspaare mit sowohl Schwefel als auch nur Kohlenstoffatomen im Ringsystem, wurden von mir neu entwickelt.

Alle vier Verbindungen wurden mit dem Tetrabutylammoniumkation hergestellt, wodurch sie in Wasser vollkommen unlöslich wurden. Nach mehrfacher Umkristallisation der Verbindungen mit Acetonitril bzw. Methanol und Dithethylether konnten dunkelrote mikrokristalline Pulver erhalten werden. Bei Betrachtung der beiden Verbindungspaare wurde festgestellt, dass die Wolframverbindungen wieder eine viel größere Empfindlichkeit gegenüber Sauerstoff aufwiesen, als die analogen Molybdänverbindungen. Alle Verbindungen wurden durch $\mathrm{FAB}^{+/-}$-Massenspektrometrie durch die jeweiligen Molmassenpeaks für den Kationenanteil im $\mathrm{FAB}^{+}$, sowie für den anionischen Part im $\mathrm{FAB}^{-}$ identifiziert. Elementaranalysen, IR sowie NMR-Spektroskopische ${ }^{1} \mathrm{H}-,{ }^{13} \mathrm{C}$ - Spektren wiesen die Verbindungen in hoher Reinheit nach. Mehrfache Versuche Einkristalle dieser Verbindungen zu erhalten scheiterten. Obwohl von Verbindung 7a und 9a Kristalle erhalten wurden, waren diese aufgrund der ungenügenden Qualität für Röntgendiffraktometrische Untersuchungen ungeeignet. Die Verbindungen wurden sowohl elektrochemisch, als auch auf ihre katalytische Aktivität hin untersucht, um den Einfluss dieser Atome im Ringsystem zu studieren. (siehe hierzu Kapitel 4 bzw. 7 für die katalytischen Untersuchungen)

\subsubsection{Synthese von Verbindungen mit nicht aromatischen Dithiolensystemen}

Um $\mathrm{zu}$ evaluieren in wieweit aromatische Dithiolenliganden einen Einfluss auf elektrochemische und katalytische Eigenschaften der Komplexe haben, wurden die nicht aromatischen Cyclohexandithiolenverbindungen für vergleichende Untersuchungen synthetisiert. Die Molybdän- und die Wolframverbindung mit dem Cyclohexanliganden sind in der Literatur bekannt und wurden von Sugimto et al. im Jahre 2005 bzw. 2008 publiziert. ${ }^{[30,}$ ${ }^{53]}$ Diese Arbeitsgruppe verwendete für die Darstellung der Verbindungen eine aufwändige Syntheseroute mit Ausbeuten von ca. 10\%, für die jeweiligen Metallkomplexe. Dabei wurden zuerst die Nickeldithiolenkomplexe synthetisiert und diese dann auf Mo bzw. W übertragen. Dazu wurde Ni(dithiolen $)_{2}$ mit Precursorn wie $\left[\mathrm{M}(\mathrm{CO})_{3}(\mathrm{MeCN})_{3}\right]$ umgesetzt. Nach Oxidation konnten $\left[\mathrm{M}(\mathrm{CO})_{2}(\text { dithiolen })_{2}\right]$-Verbindungen erhalten werden, die dann mit $\mathrm{NH}_{4} \mathrm{OH}$ weiter zur $\left[\mathrm{MO}(\text { dithiolen })_{2}\right]^{2-}$-Verbindung umgesetzt wurden. Um genügende Mengen an Komplexen nach dieser Syntheseroute zu erhalten, muss mit relativ großen Ausgangsmengen des Ligandens (>25 g) gearbeitet werden, um genügend Material für nachfolgende Reaktionen zu synthetisieren. 
Deshalb wurde versucht die Darstellung dieser Verbindungen zu optimieren, wofür die entsprechenden Liganden direkt mit dem Ausgangsmaterial $\left[\mathrm{MO}_{2}(\mathrm{CN})_{4}\right]^{4-}$ umgesetzt wurden.

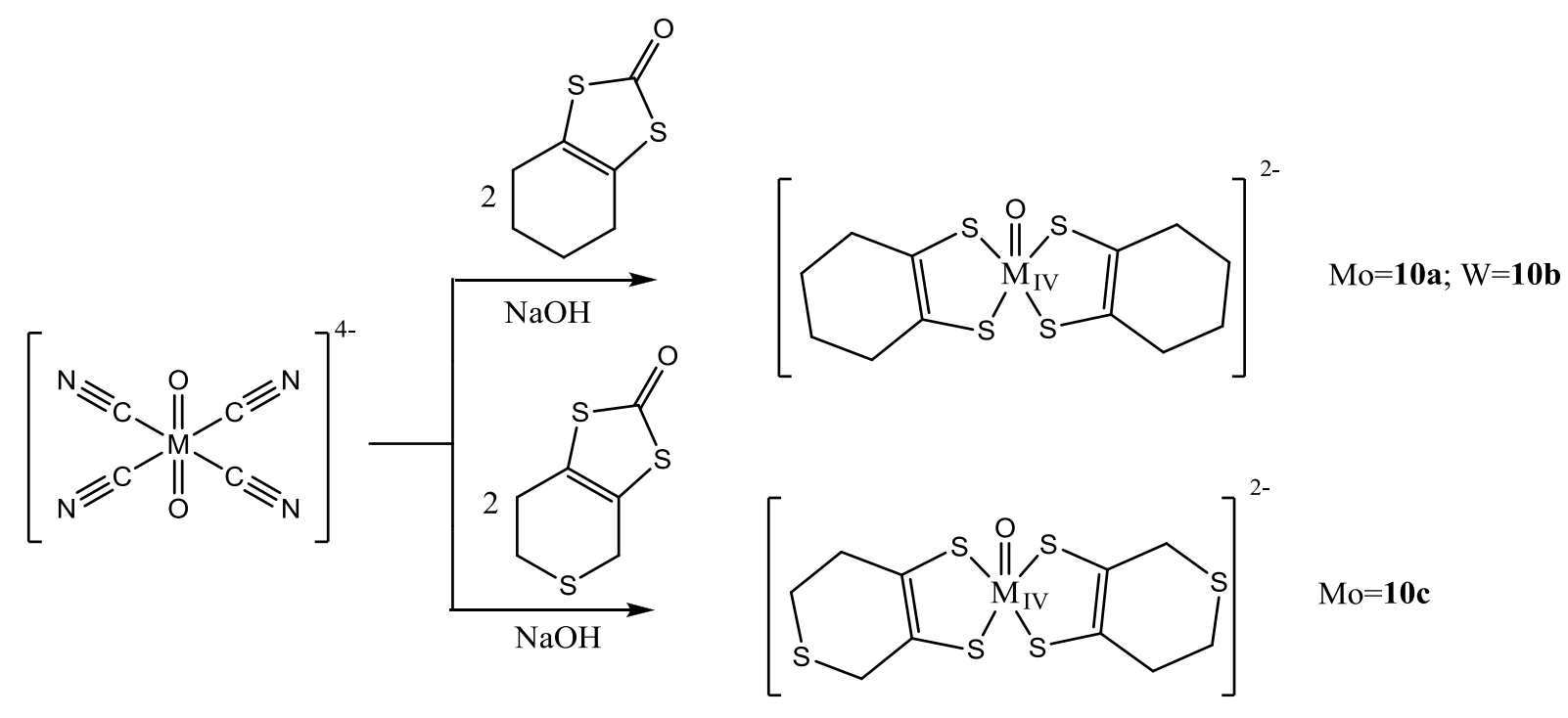

Abb. 62: Syntheseschema der Komplexe 10a, 10b, 10c

Durch diese Synthesemethode konnte sowohl die Molybdän- als auch die Wolframverbindung mit dem Cyclohexandithiolenliganden erhalten werden 10a und 10b. Die Koordination an das Wolframzentrum benötigte längere Reaktionszeiten sowie höhere Reaktionstemperaturen, als das bei der analogen Molybdänverbindung der Fall war. Bei der Thiopyranverbindung konnte nur der Komplex mit dem Molybdänzentrum erhalten werden. Trotz vielfacher Veränderung der Reaktionsbedingungen erfolgte keine Koordination an den Wolframprecursor. Die Verbindungen wurden elektrochemisch untersucht. Es wurden Potentiale in Acetonitril gegen das Ferrocen/Ferrocenium Paar von -0.98 V (10a), -1.39 V (10b) und -0.88 V (10c) erhalten. Im Vergleich der Redoxpotentiale mit den bdt-Komplexen (-0.78 V (Mo); -1.30 V (W)), die eine gleiche Zahl an Ringatomen aufweisen wie die Cyclohexandithiolenliganden, stellt man bei den cydt-Systemen eine deutlich negativeres Redoxpotential fest. Diese Wirkung ist bei den Molybdänzentren etwas stärker ausgeprägt, als das bei den Wolframsystemen der Fall ist. Somit stellt man bei den bdt-Systemen eine stärker elektronenziehende Wirkung im Vergleich zu den Cyclohexanverbindungen fest. Durch den Einbau eines Schwefelatoms in den Cyclohexanring kommt es zu einem leicht positiveren Redoxpotential $(+10 \mathrm{mV})$. Von der Molybdänverbindung mit dem Cyclohexanliganden konnte ähnlich wie von Sugimoto et al. ${ }^{[30 \text {, }}$ 54] eine Kristallstruktur erhalten werden. Im Folgenden sind die Ellipsoide mit einer Aufenthaltswahrscheinlichkeit von 50\% aufgezeigt. 


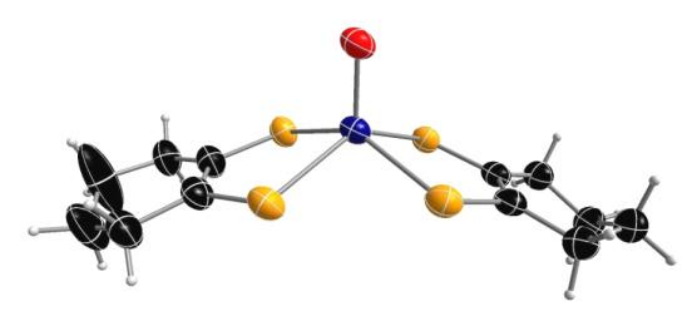

Abb. 63: Kristallstruktur von $\left[\mathrm{MoO}(\mathrm{cydt})_{2}\right]^{2-}$ (Kationen nicht gezeigt)

Hierbei ist die Abwinkelung der einzelnen Kohlenstoffatome in Cyclohexanring um ca. $15^{\circ}$ zu erkennen. Es ergibt sich im Mittel ein Mo-S-Bindungsabstand von $2.3795 \AA$, was nur eine geringfügig längere Bindungslänge $\mathrm{zu}$ einer Molybdänverbindungen mit einem Benzoldithiolenliganden entspricht $\left(2.3881 \AA\right.$ ̊). ${ }^{[28]}$ Die Kohlenstoffdoppelbindung (1.3391 ̊̊) sowie die Metallsauerstoffbindung (1.7122 $)$ weisen im Mittel längere Bindungsabstände auf, als es bei den zuvor diskutierten Systemen der Fall war. 


\subsubsection{Modellkomplexe für die reduzierte Form der DMSO-Reduktase Familie}

\subsubsection{Synthese von Non-0xo-Verbindungen}

Modellkomplexe, die eine Ähnlichkeit zur reduzierten Form der DMSO-Reduktase Familie aufweisen, wurden durch einfache Substitution an $\mathrm{MoCl}_{4}$-Lösungsmitteladdukten, mit den Natriumsalzen der Dithiolenverbindugen, erhalten.

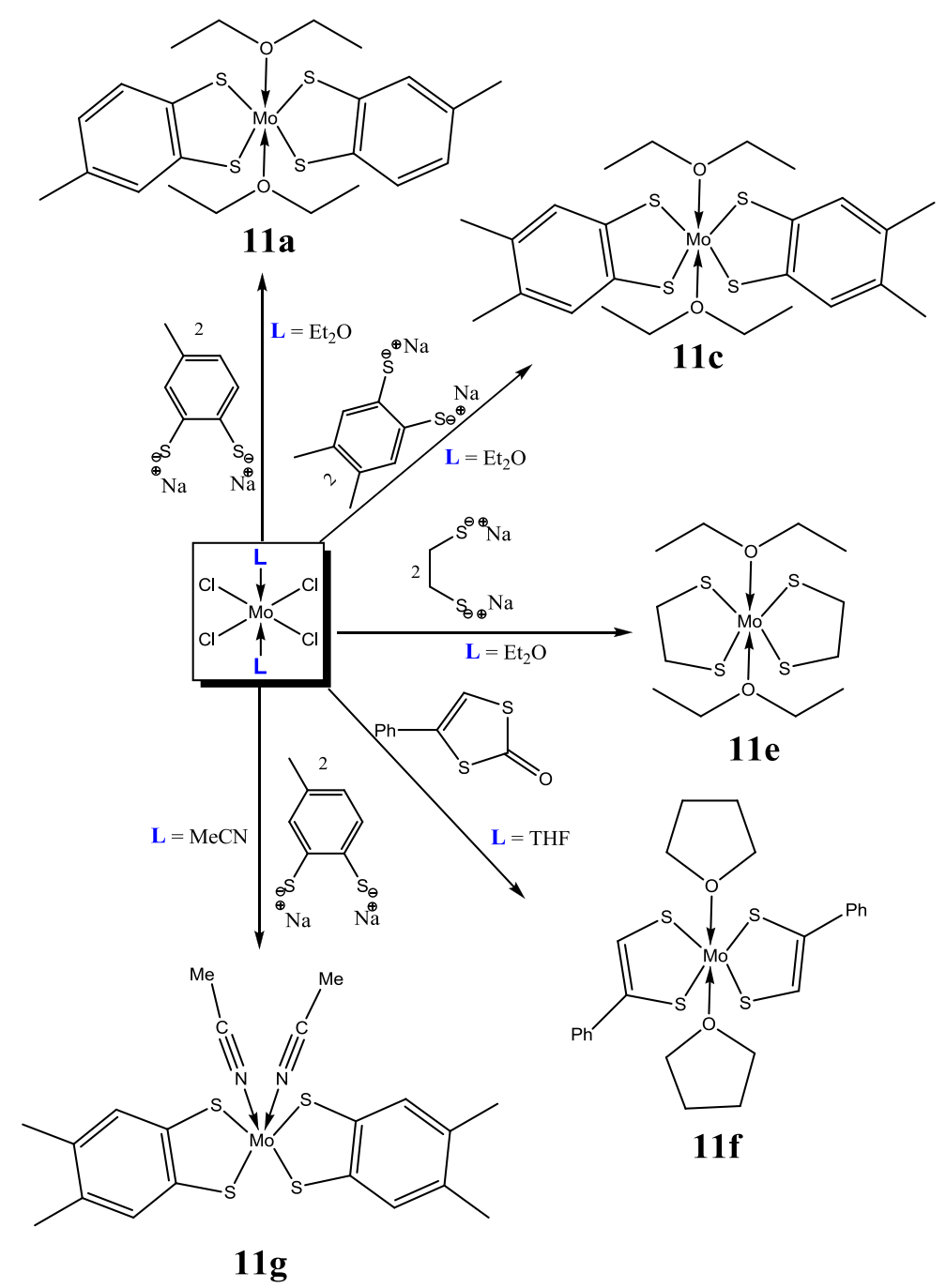

Abb. 64: Syntheseweg zur Darstellung von Non-Oxo-Molybdänverbindungen

Da die Lösungsmitteladduktkomplexe zwei gute Abgangsgruppen besitzen, besteht die Gefahr einer Bildung von Trisdithiolenkomplexen. Die Bildung von $\mathrm{NaCl}$ ist im Vergleich zu der Ablösung des koordinierten Lösungsmittels bevorzugt. Die Produkte wurden dadurch erhalten, dass das Natriumsalz des Dithiolenligandens mit dem Molybdänkomplex unter Stickstoffatmosphäre ohne Lösungsmittel bei RT gerührt wurde. Nach ca. 2 h Rühren konnte die ersten Verfärbung von orange nach dunkelgrün beobachtet werden. Anfängliche Reaktionen in Lösungen ergaben vielfach eine Koordination von drei Dithiolenen. Da die 
erhaltenen Komplexe sehr instabil sind, wurde das entstandene Natriumchlorid durch Lösen der Verbindungen in nichtkoordinierenden Lösungsmitteln bei $-60^{\circ} \mathrm{C}$ gelöst. Anschließend wurde durch einen mit Trockeneis gekühlten Filter über Celite filtriert. Um immer noch anhaftende Reste des nicht reagierten Liganden zu entfernen, wurde die Lösung durch einen Filter mit eingefülltem Kieselgel filtriert. Die erste anfallende hellgelbe Fraktion wurde verworfen. Nach erneutem Waschen mit Diethylether wurde das Lösungsmittel des Filtrats unter reduziertem Druck und bei niedrigen Temperaturen entfernt. Die Verbindung 11a wurde als grüner Feststoff mit einer Ausbeute von $\sim 40 \%$ erhalten.

Um die analoge Wolframverbindung $11 b$ zu erhalten, wurde die Verbindung $\left(\mathrm{WCl}_{4}\right)_{\mathrm{x}^{-}}$ Verbindung zwei Tage ohne Lösungsmittel mit dem Natriumsalz des Dithilolenliganden gerührt. Dann wurde die Verbindung mit Diethylether bei $-30^{\circ} \mathrm{C}$ versetzt. Nach Filtrieren konnte eine Abtrennung des Natriumchlorids und nicht reagiertem Liganden erreicht werden. Die Verbindungen konnten als dunkelgrüne Feststoffe erhalten werden.

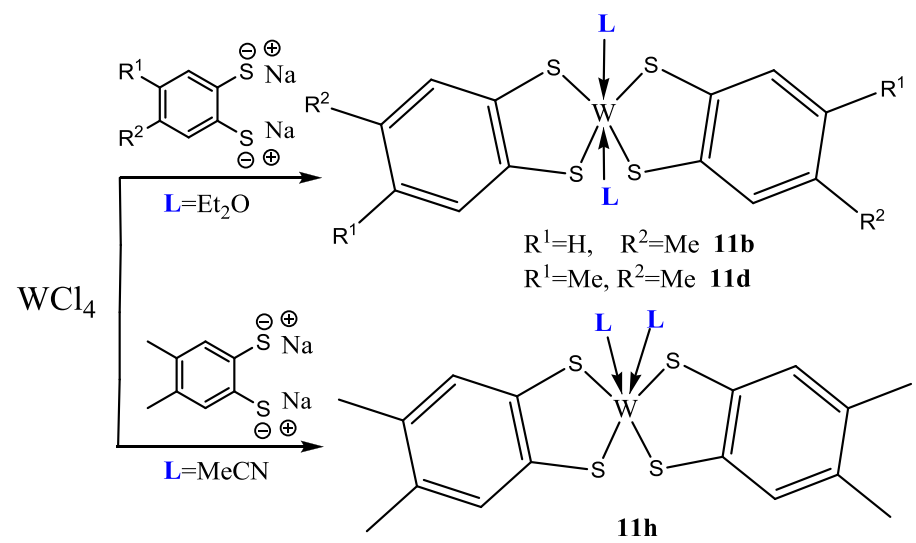

Abb. 65: Syntheseweg zur Darstellung von Non-Oxo-Wolframverbindungen

Um eine bessere Aussage über die tatsächliche Molekülstruktur zu bekommen, wurden DFTRechnungen an diesen Komplexen durchgeführt. Untersucht wurde, ob die vorhandenen Diethylether Liganden sich eher in einer trans- oder in einer cis-Anordnung befinden. Hierzu wurden die beiden vorhandenen Anordnungen geometrieoptimiert.

Berechnet wurde die Stabilisierungsenergie der cis- und der trans-Anordnung. Anhand der erhaltenen Werte konnte festgestellt werden, dass ein trans-Anordnung der Diethylethermoleküle um $6.18 \mathrm{kcal} / \mathrm{mol}$ favorisiert wird. 
In Abb.66 ist ersichtlich, dass es bei einer cis-Anordnung der Etherliganden zu einer viel größeren Abstoßung zwischen den Molekülen kommt. Im Mittel wird in der trans-Anordnung ein Abstand der Sauerstoffatome von $2.28 \AA$ zum Molybdänatom festgestellt.
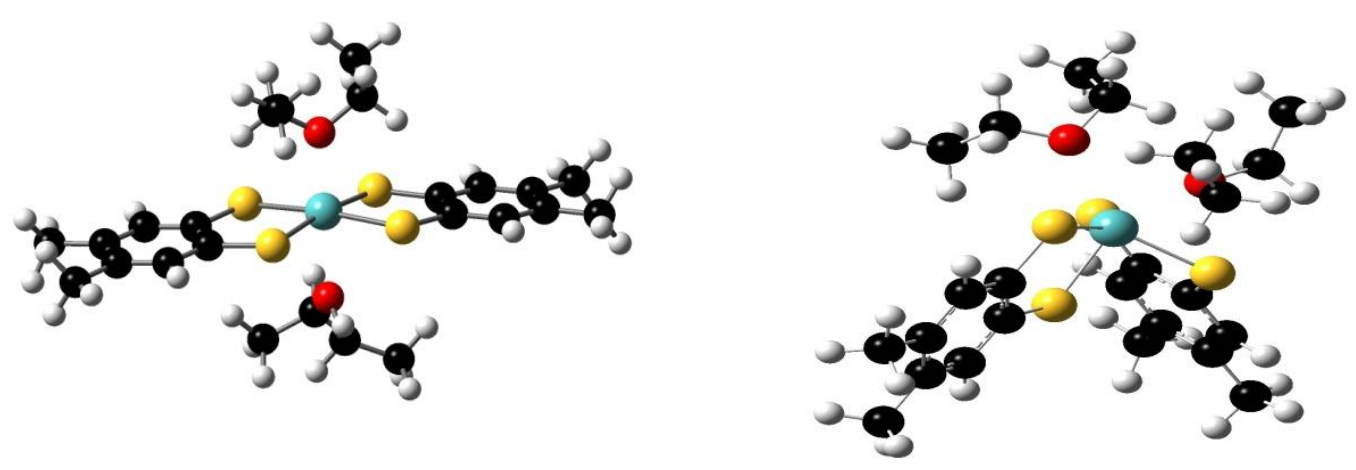

Abb. 66: Darstellung der trans und der cis-Anordnung der Non-Oxo-Verbindungen

Da es bei der verzerrt oktaedrischen Anordnung dieser Molybdän(IV)-Verbindung in transAnordnung zu einem Jahn-Teller-Effekt kommt, stellt man ein energetisches Absenken aller Orbitale mit z-Komponente fest. Somit sollten jeweils zwei ungepaarte Elektronen im $d_{x z}$ bzw. $d_{y z}-$ Orbital vorhanden sein, wodurch ein paramagnetischer Charakter dieser Moleküle auftaucht. Doch NMR-Messungen dieser Verbindungen sind ohne Weiteres in normalen diamagnetischen Verschiebungen möglich. Anzunehmen ist, dass es beim Lösen in z.B. deuteriertem Chloroform, aufgrund der sehr viel größeren Anzahl an anderen Lösungsmittelmolekülen, zu einer Bindungsverlängerung zu den Ethermolekülen kommt. In diesem Fall würde man eher eine quadratisch planare Struktur dieser Verbindungen in Lösung erwarten. Somit sollte das energetisch am niedrigsten liegende $\mathrm{d}_{\mathrm{z}}{ }^{2}$-Orbital voll besetzt sein und eine negative Suszeptibilität bei diesen Verbindungen in Lösung auftreten.

Da bei Verwendung des tdt-Liganden zusätzlich zwei verschiedene Isomere (cis/trans) bezüglich der Orientierung der Liganden möglich sind, wurde der analoge symmetrische xdtLigand mit zwei Metylgruppe in meta-Position zu den Dithiolengruppen verwendet. Es konnten die analogen Reaktionen durchgeführt werden, wodurch die Verbindungen 11c und 11d als dunkelgrüne Verbindung erhalten wurden. Hierbei konnte in den Massenspektren nur das Molekül ohne die koordinierten Lösungsmoleküle im EI-Spektrum beobachtet werden (Abb.67), da es bei der Erwärmung der Verbindungen und unter Hochvakuum zur schnellen Entfernung der Lösungsmittelmoleküle kommt. 

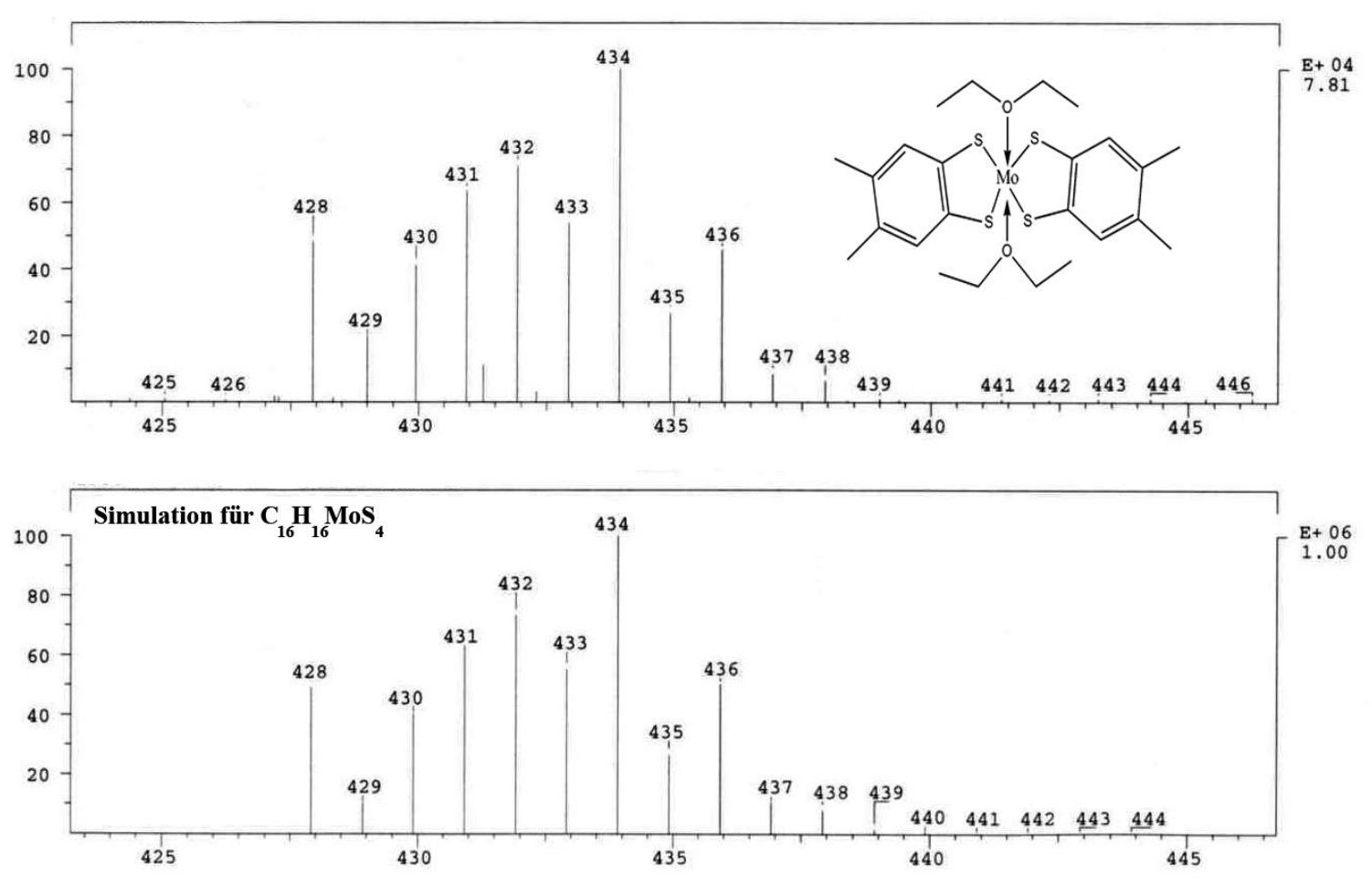

Abb. 67: El-Massenspektrum der Verbindung 11c

Um einen genauen Einblick in den Oxidationszustand des Metalls zu bekommen, wurden elektrochemische Untersuchungen durchgeführt. Aufgrund der verhältnismäßig schwachen koordinativen Bindung der beiden Ethermoleküle an das Metallzentrum, lassen sich diese leicht verdrängen. Dies ist insbesondere relevant für die elektrochemischen Untersuchungen im Standardlösungsmittel Acetonitril, welches in großem Überschuss vorhanden, die Ethermoleküle vollständig verdrängt. Um zu Überprüfen, ob die an den Verbindungen 11c und 11d durchgeführten elektrochemischen Untersuchungen tatsächlich die Diethyletheraddukte oder vielmehr die Acetonitriladdukte beschreiben, wurden letztere in analoger Weise 11g und 11h synthetisiert. Die elektrochemischen Resultate für die Verbindungen 11c und 11d in Acetonitril lieferten ähnliche Potentiale wie die hergestellten Verbindungspaare (Mo: 11c: -0.34 V/11g: -0.38 V); W: 11d: -0.51 V/11h: -0.67) mit direkt angelagerten Acetonitril wodurch die Vermutung bestätigt wurde, dass es zu einer Verdrängung der Diethylethermoleküle kommt.

Um nicht jedes Mal eine Veränderung der Verbindung durch einen Wechsel des koordinierenden Lösungsmittelligandens festzustellen, wurden die Verbindungspaare 11a und 11b in Dichlormethan vermessen, wodurch stabile Potentiale erhalten werden konnten. Reversible Signale wurden in den Cyclovoltammogrammen bei $-0.11 \mathrm{~V}$ (Mo) und $-0.29 \mathrm{~V}$ (W) festgestellt. 
Außerdem konnte an das $\left[\mathrm{MoCl}_{4}\left(\mathrm{OEt}_{2}\right)_{2}\right]$-Ausgangsmaterial die Koordination des Natriumsalzes von Ethan-1,2-dithiol erreicht werden 11e. Die Verbindung konnte als hellgrüner Feststoff erhalten werden, der durch das Auftreten des Signals bei $282 \mathrm{amu}$ $\left[\mathrm{Mo}(\mathrm{edt})_{2}-2 \mathrm{Et}_{2} \mathrm{O}\right]$ im EI-Massenspektrum nachgewiesen wurde. Auch Dithiocarbonatliganden konnten zu diesen Metallzentren koordiniert werden. Das Ringsystem von 4-Phenyl1,3-dithiol-2-on wurde mit Natriummethanolat geöffnet und an $\left[\mathrm{MoCl}_{4}(\text { thf })_{2}\right]$ koordiniert, wodurch ein dunkelgrüner Feststoff $11 f$ erhalten wurde und durch Elementaranalyse, EI-MS und ${ }^{1} \mathrm{H}-\mathrm{NMR}$ charakterisiert werden konnte.

Von keiner der Verbindungen 11a-11h wurden Kristalle in genügender Qualität für die Röntgenstrukturanalyse erhalten.

\subsection{Synthese von Modellsystemen für die reduzierte Form der Xanthin-Oxidase Familie}

\subsubsection{Synthese von Monodithiolenverbindungen}

Um den Einfluss eines Dithiolenligandens auf die Redoxpotentiale der Metallzentren genauer untersuchen zu können, wurden Mono-Dithiolenkomplexe hergestellt, die als Modelle für die aktiven Zentren der Enzyme der Xanthin-Oxidase Familie aufgefasst werden können. Aufgrund der entropischen Begünstigung der di- und trisubstituierten Dithiolenkomplexe, ist es nicht trivial monosubstituierte Verbindungen mit nur einer Dithioleneinheit zu erhalten. In der Literatur sind aktuell zwei Methoden zur Darstellung von monosubstituierten Dithiolenverbindungen verfügbar. Nach der Methode von Holm et al. kann durch die Abspaltung einer Dithioleneinheit von einem Bisdithiolen Molybdän(V)-Komplex mit $\mathrm{PhSeCl}$, eine Monosubstituierte Verbindung erhalten werden. ${ }^{[18]}$ Umgesetzt wurde die $\left[\mathrm{MoO}(\mathrm{xdt})_{2}\right]^{-}$- Verbindung zu $\left[\mathrm{MoO}(\mathrm{xdt}) \mathrm{Cl}_{2}\right]^{-}$. Nachfolgend angefertigtes Cyclovoltammogramm der $\left[\mathrm{MoO}(\mathrm{xdt}) \mathrm{Cl}_{2}\right]^{-}$- Verbindung zeigte nur einen irreversibles Signal, das für die nötigen Untersuchungen nicht verwendet werden konnte.

Eine weitere Methode wurde von Sugimoto et al. publiziert, die sich nach Modifikation als nützlich herausstellte. ${ }^{[55]}$ Hier wird $\left[\mathrm{MoOS}_{8}\right]^{2-}$ (17a) mit nur einem Äquivalent an Dithiolen umgesetzt (Abb.68). Da der von mir verwendete xdt-Ligand (aufgrund der elektronenschiebenden Eigenschaft der Methylgruppen) aktivierter ist, als der von Sugimoto et al. verwendete bdt-Ligand, liefert die Umsetzung in refluxierendem Acetonitril nur die unerwünschte Bisdithiolenmetallverbindung. 


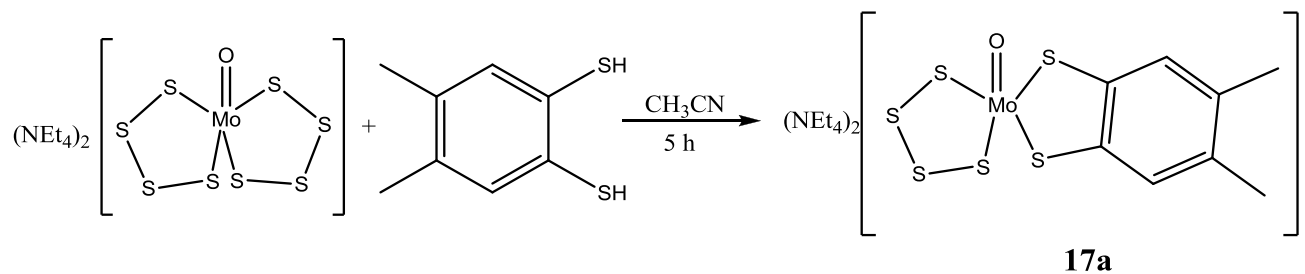

Abb. 68: Reaktionsweg zur Synthese von Molybdänsystemen mit nur einer Dithioleneinheit

Daher wurde auf eine schonendere Methode durch Rühren bei Raumtemperatur zurückgegriffen. Auf diese Weise konnte die monosubstituierte DithiolenMolybdänverbindung synthetisiert werden. Im Folgenden sind die erhaltenen Kristallstrukturen der Ausgangsverbindung und des Produktes dargestellt.
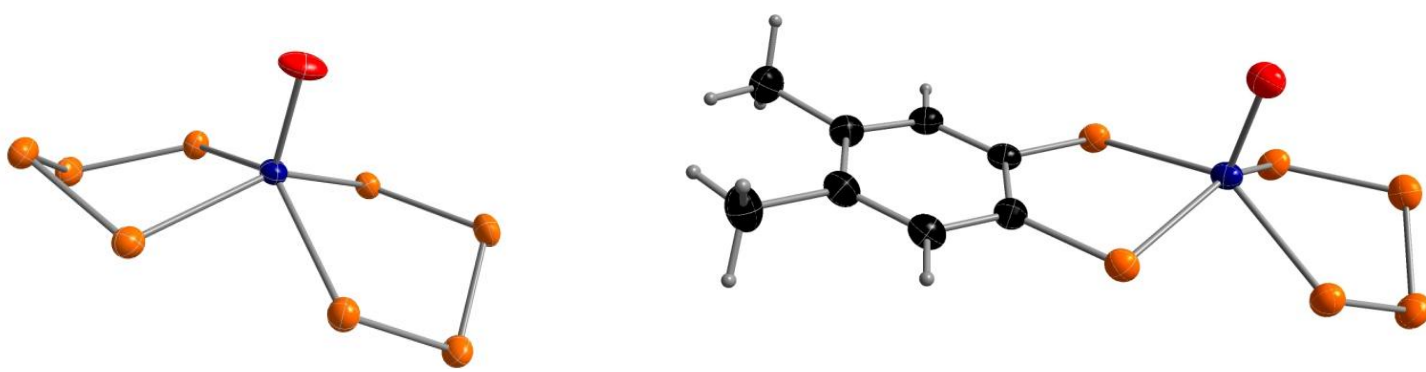

Abb. 69: Kristallstruktur von $\left[\mathrm{MoOS}_{8}\right]^{2-}$ (17a) (links); $\left(\mathrm{NEt}_{4}\right)_{2}\left[\mathrm{MoOS}_{4} \mathrm{xdt}\right]$ (17b) (rechts), Kationen sind nicht dargestellt

Vergleich man nun beide Strukturen miteinander, so stellt man fest, dass es zu keiner Veränderung der geometrischen Umgebung kommt. Der koordinierende $\mathrm{S}_{4}$-Ring zeigt wie in der Ausgangsstruktur eine gefaltete Anordnung. Um den Einfluss eines Dithiolenliganden auf das Redoxpotential des Metallzentrums untersuchen zu können, wurden sowohl das Ausgangsmaterial $\left[\mathrm{MoOS}_{8}\right]^{2-}(\mathbf{1 7 a})$, als auch die Verbindung $\left[\mathrm{MoO}(\mathrm{xdt}) \mathrm{S}_{4}\right]^{2-} \quad(\mathbf{1 7 b})$ elektrochemisch und auch in Hinblick auf die Abhängigkeit der Redoxpotentale bei Temperaturänderung untersucht. Siehe dazu Kapitel 4.

Da mit der oben geschilderten Methode die Synthese der Monodithiolenverbindung erfolgreich verlief, wurde versucht die auch auf Ligandenprecursorn mit einer DithiocarbonatEinheit anzuwenden. Hierzu wurde der Ligandenprecursor in methanolischer Lösung mit Kaliumhydroxid versetzt, um eine Ringöffnung und Entfernung der Schutzgruppe des Liganden $\mathrm{zu}$ erreichen. Das nun erhaltene Kaliumsalz der Liganden wurde direkt mit $\left[\mathrm{MoOS}_{8}\right]^{2-}$ umgesetzt. Dabei konnte festgestellt werden, dass das Vorhandensein von Alkalimetallionen in Lösung $\mathrm{zu}$ einer verstärkten Ablösung der $\mathrm{S}_{4}{ }^{2-}$ - Ringe am Ausgangsmaterial führte. 
Als Hauptprodukt 17c dieser Reaktion wurde eine $\mathrm{S}_{4}{ }^{2-}$-Verbindung isoliert. Die beiden negativen Ladungen wurden durch ein Tetraethylammoniumion und ein Kaliumionen ausgeglichen. Im Folgenden ist die erhaltene Struktur aufgezeigt.
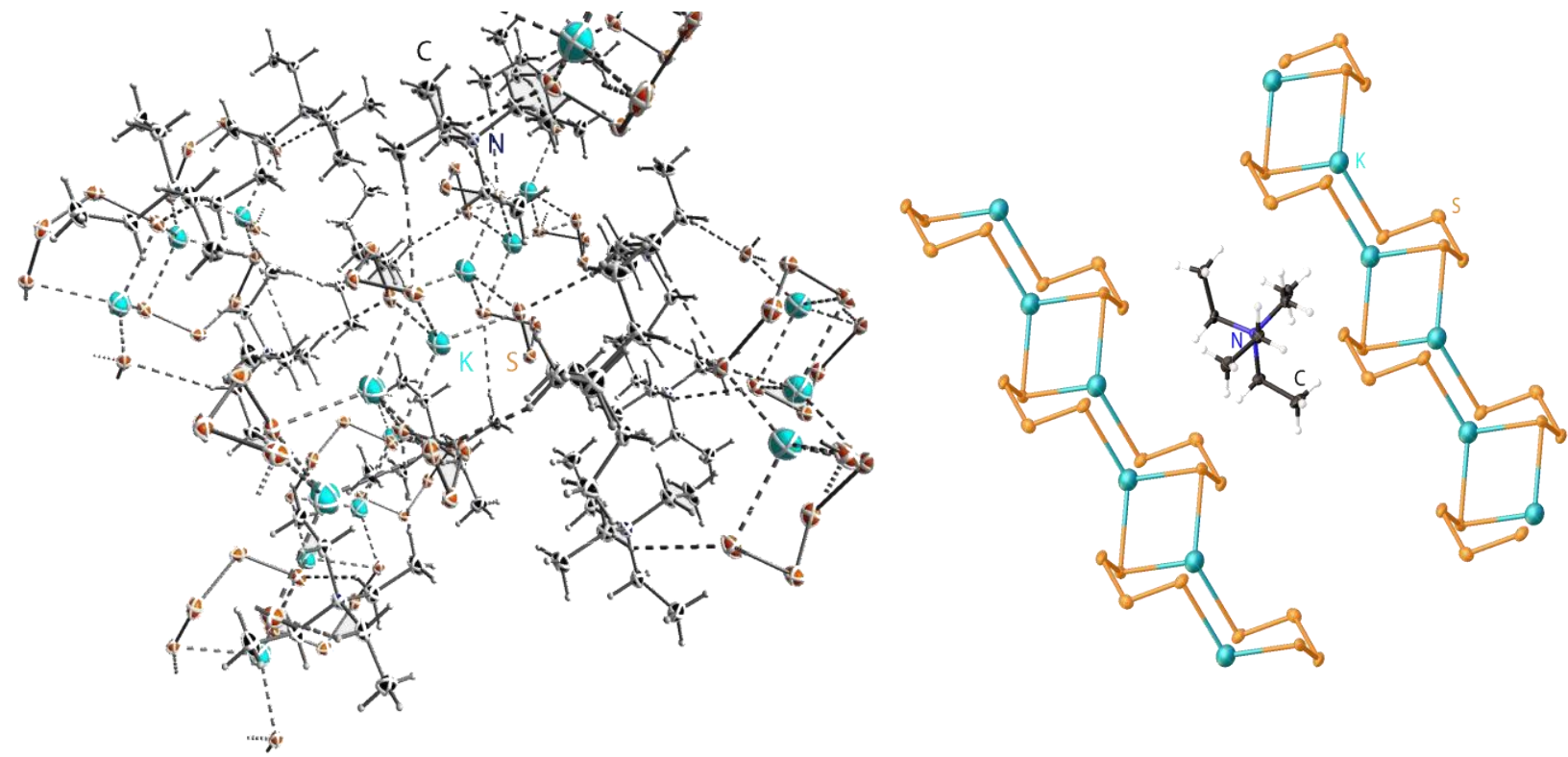

Abb. 70: Packung (links) und kettenartige Anordnung des $\mathrm{S}_{4}{ }^{2-}$-Systems (rechts)

In Abb.70 kann man die Schwefelkette mit der Verbrückung zu Kaliumionen sehen, wodurch es zur Bildung von 10-gliedrigen Ringen kommt. Es kommt zur Zusammenlagerung von zwei $\mathrm{S}_{4}{ }^{2-}$-Einheiten durch die Kaliumionen. Die Kaliumionen bilden wieder eine VierringStruktur. Zwischen den Schwefelketten sind die Tetraethylammoniumionen lokalisiert. In der oben gezeigten Verbindung wurde nur ein Molekül eingefügt, um eine bessere Übersichtlichkeit zu gewährleisten.

Als Nebenprodukt dieser Reaktionen werden in geringen Ausbeuten $(<10 \%)$, der Bisdithiolenkomplex gefunden.

\subsubsection{Herstellung von unsymmetrischen Dithiolenkomplexen}

Die monosubstituierten Dithiolen-Verbindungen konnten dazu benutzt werden, unsymmetrische Monooxobisdithiolen Molybdänkomplexe zu synthetisieren. Dazu wurde $\left[\mathrm{MoO}(\mathrm{xdt}) \mathrm{S}_{4}\right]^{2-}(\mathbf{1 7 b})$ sowohl mit dem vdt, als auch mit dem ndt-Liganden umgesetzt. 


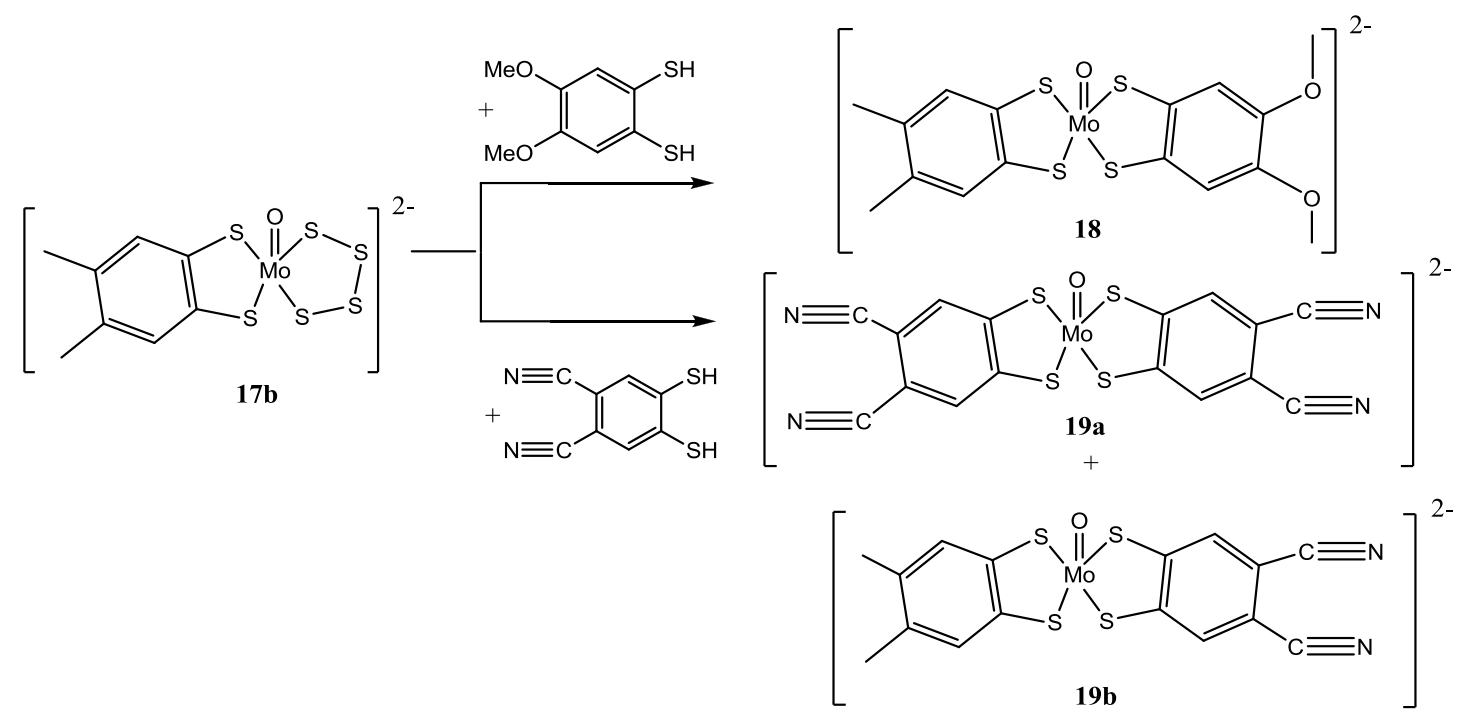

Abb. 71: Bildung von unsymmetrischen Dithiolenkomplexen

Durch Reaktion der Verbindung 17b mit dem vdt-Liganden konnte die Synthese der dunkelgrünen gemischten aus Dithiolen-Molybdänverbindung erreicht werden. Im Folgenden ist das erhaltene $\mathrm{FAB}^{-}$-Massenspektrum der Verbindung gezeigt.
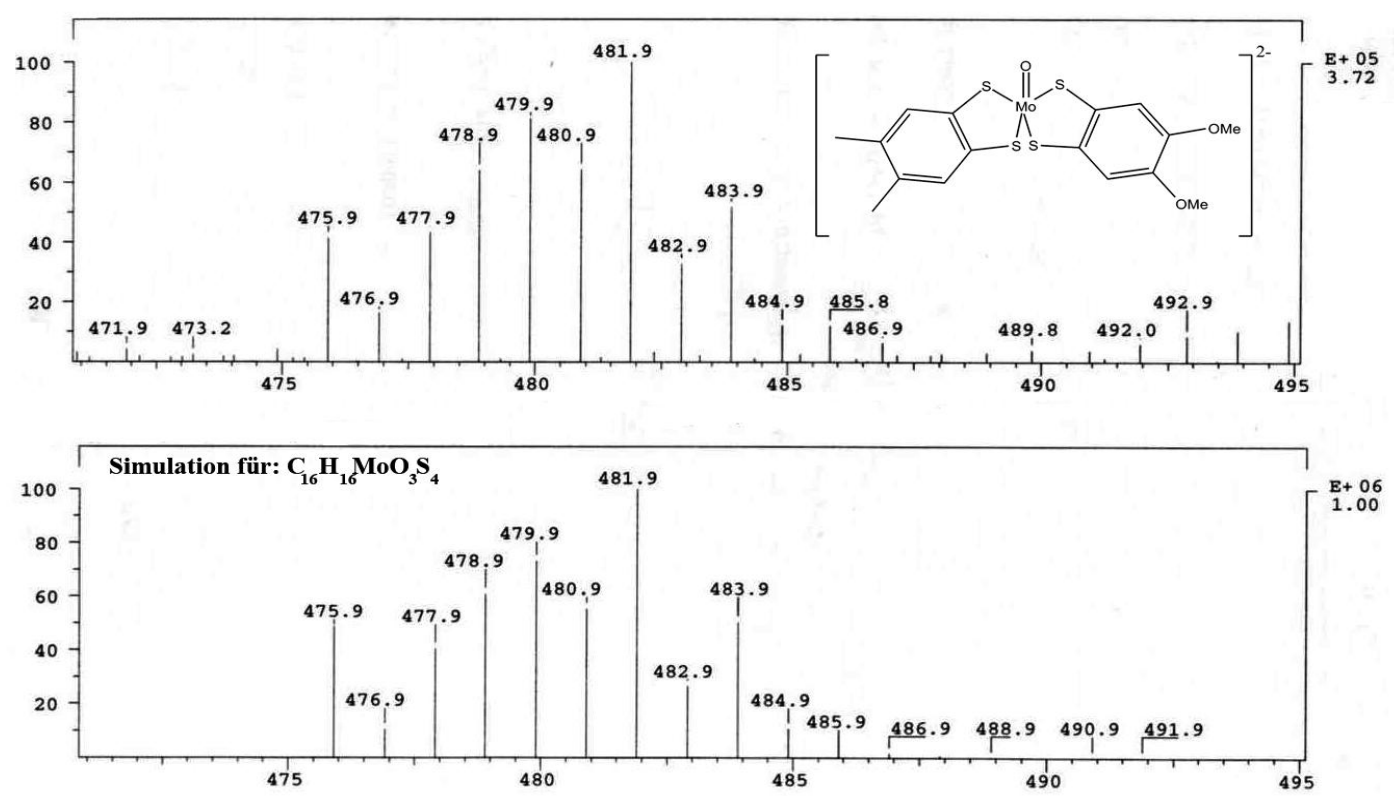

Abb. 72: Erhaltenes FAB' (3-NBA)-Spektrum von 18

Im Fall des ndt-Liganden kam es zu einer Mischung aus dem doppelt substituierten und dem monosubstituierten Produkt. Da beide Verbindungen ähnliche Löslichkeiten aufweisen, konnten diese Verbindung nicht effizent voneinander getrennt werden. Chromatographische Aufarbeitungen unter Stickstoffatmosphäre brachte keine merkliche Veränderung der Produktverhältnisse. Da die Schwefelatome des ndt-Liganden aufgrund der elektronen- 
ziehenden CN-Liganden mehr polarisiert werden, sollten diese Liganden reaktiver sein, wodurch es zur vermehrten Bildung von Bisdithiolenkomplexen kommt.

Da die Potentiale von beiden entstandenen Produkten 19a und 19b um ca. $40 \mathrm{mV}$ auseinander lagen, konnten auch elektrochemische Untersuchung dieser Verbindungen durchgeführt werden.

\subsection{Bildung von dimeren Verbindungen}

Um zu untersuchen, ob auch die Bildung von Dimer-Verbindungen möglich ist, wurde das Ausgangsmaterial $\left[\mathrm{MoO}_{2} \mathrm{~S}_{2}\right]^{2-}$ mit nur einem Äquivalent von Benzoldithiol versetzt. Durch die am Anfang des Kapitels beschriebene Reaktion zur Bildung von 1a konnte gezeigt werden, dass bei der Umsetzung von 2 Äquivalenten Ligand mit einem Teil Metallprecursor, die monomeren Verbindungen synthetisiert werden konnten.
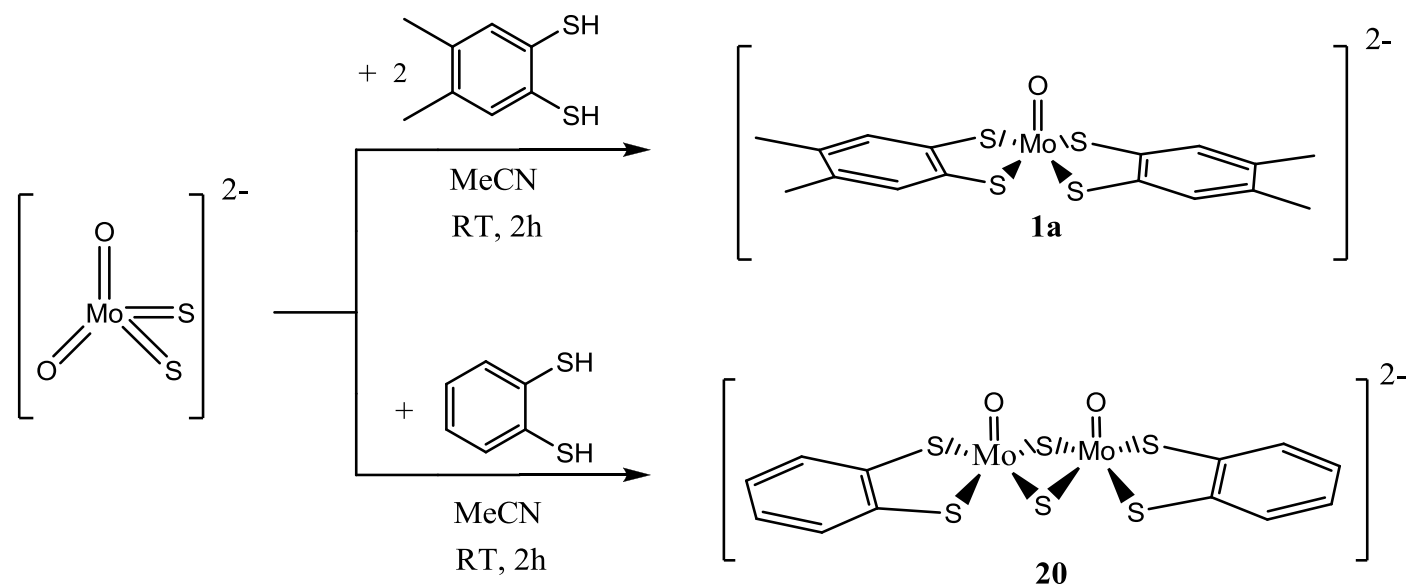

Abb. 73: Reaktionen zur Bildung einer monomeren und einer dimeren Verbindung

Durch Variation der Menge an Dithiolen unter gleichen Reaktionsbedingungen wurden unterschiedliche Produkte erhalten. Kristalle der dimeren Molybdänverbindung konnten kristallographisch untersucht werden. (Abb.74)

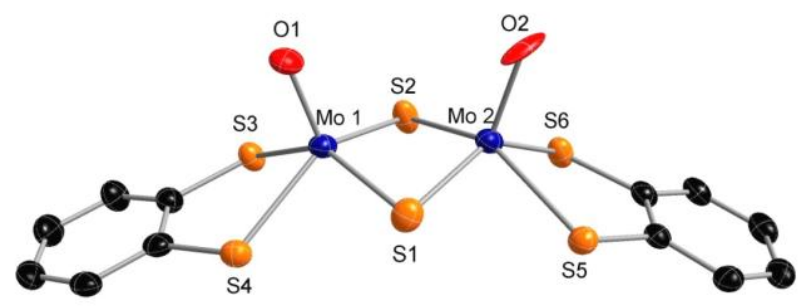

Abb. 74: Kristallstruktur von $\left(\mathrm{NEt}_{4}\right)_{2}\left[\mathrm{Mo}_{2} \mathrm{O}_{2}(\mu-\mathrm{S})_{2}(\mathrm{bdt})_{2}\right](20)$ 
Eine der Zusammensetzung nach identische Verbindung konnte über einen anderen Syntheseweg von Holm et al. bereits synthetisiert und die entsprechende Struktur publiziert werden. Bei der Synthesemethode von Holm et al. wurde das Ausgangsmaterial $\left[\mathrm{MoO}_{3}(\mathrm{bdt})\right]$ mit $\mathrm{H}_{2} \mathrm{~S}$ begast und so wurde eine Substitution von zwei Oxo-Liganden durch verbrückende Sulfide am Metallzentrum erzielt. ${ }^{[56]}$ Der Unterschied zwischen der von Holm et al. erhaltenen Struktur und der neuen Struktur 20 ${ }^{[57]}$ ist, dass in unserem Fall das syn-Produkt erhalten wurde, während im anderen Fall die anti-Verbindung gefunden wurde.

\begin{tabular}{|c|c|c|c|}
\hline & & Mo (syn) & Mo $(a n t i)^{[56]}$ \\
\hline M-M & & $2.8905(4)$ & $2.9018(13)$ \\
\hline \multirow[t]{2}{*}{$M=0$} & Mo1 & $1.699(2)$ & \\
\hline & Mo2 & $1.784(2)$ & \\
\hline Mittelwert $(M=0)$ & & 1.7415 & 1.697 \\
\hline \multirow[t]{4}{*}{$M-\mu S$} & Mo1 & $2.3314(9)$ & \\
\hline & Mo1 & $2.3332(9)$ & \\
\hline & Mo2 & $2.3344(9)$ & \\
\hline & Mo2 & $2.3395(9)$ & \\
\hline Mittelwert (M- $\mu \mathrm{S})$ & & 2.3346 & 2.3075 \\
\hline \multirow[t]{4}{*}{$M-S(b d t)$} & Mo1 & $2.4050(8)$ & \\
\hline & Mo1 & $2.4220(8)$ & \\
\hline & Mo2 & $2.4059(9)$ & \\
\hline & Mo2 & $2.4129(8)$ & \\
\hline Mittelwert (M-S(bdt)) & & 2.411 & 2.485 \\
\hline \multirow[t]{2}{*}{ M-(S4-Fläche) } & Mo1 & 0.7060 & \\
\hline & Mo2 & 0.7143 & \\
\hline Mittelwert(M-(S4-Fläche)) & & 0.7102 & 0.6242 \\
\hline \multirow[t]{2}{*}{$\mu S-M-\mu S$} & Mo1 & 100.68 & \\
\hline & Mo2 & 100.40 & \\
\hline Mittelwert( $\mu \mathrm{S}-\mathrm{M}-\mu \mathrm{S})$ & & 100.54 & 102.10 \\
\hline \multirow[t]{2}{*}{ S-M-S } & Mo1 & 81.29 & \\
\hline & Mo2 & 81.57 & \\
\hline Mittelwert (S-M-S) & & 81.43 & 81.02 \\
\hline \multirow[t]{4}{*}{$\mu S-M-S($ cis) } & Mo1 & 78.85 & \\
\hline & Mo1 & 78.55 & \\
\hline & Mo2 & 78.32 & \\
\hline & Mo2 & 78.52 & \\
\hline Mittelwert ( $\mu \mathrm{S}-\mathrm{M}-\mathrm{S}(\mathrm{cis})$ ) & & 78.56 & 80.23 \\
\hline \multirow[t]{4}{*}{$\mu \mathrm{S}-\mathrm{M}-\mathrm{S}(\operatorname{trans})$} & Mo1 & 145.81 & \\
\hline & Mo1 & 141.63 & \\
\hline & Mo2 & 143.21 & \\
\hline & Mo2 & 143.64 & \\
\hline Mittelwert ( $\mu \mathrm{S}-\mathrm{M}-\mathrm{S}(\operatorname{trans}))$ & & 143.57 & 147.22 \\
\hline
\end{tabular}

Tab. 8: Vergleich der Bindungslängen des syn und anti-Isomers, Bindungslängen in [Å], Bindungswinkel in [ $\left.{ }^{\circ}\right]$

Der größte Unterschied zwischen der syn- und der anti-Struktur ist im Unterschied der $\mathrm{Mo}=\mathrm{O}$-Bindungslängen zu sehen. Beim anti-Isomer werden hier Bindungslängen mit $1.697 \AA$ und beim syn-Produkt mit einem Abstand von $1.7415 \AA$ gefunden. Da in der von mir erhaltenen Struktur eine Oxogruppe fehlgeordnet ist, ergeben sich ungewöhnlich größe Abstände zum Molybdänatom. Somit konnte angenommen werden, dass es sich bei dem vorhandenem Sauerstoffatom um eine OH-Gruppe handeln könnte. Allerdings wurde diese 
Annahme durch Messen von ${ }^{1} \mathrm{H}-\mathrm{NMR}-$ Spektren ausgeschlossen werden. Betrachtet man nun die Abstände des Zentralmetalls zu der aufgespannten Schwefel-Fläche aus den Schwefelatomen eines Dithiolenliganden und den verbrückenden Schwefelatomen, so fällt ein signifikant kürzer Abstand von $0.62 \AA$ im Vergleich zu $0.71 \AA$ bei dem syn-Isomer auf. Auch die Bindungswinkel von den schwefelverbrückten Atomen zu dem jeweiligen gegenüberliegenden Schwefel der Dithioleneinheit, zeigen beim anti-Isomer größere Werte, als beim syn-Produkt $\left(147.22^{\circ} \mathrm{zu} 143.57^{\circ}\right)$. Generell erlaubt die anti-Konfiguration dem Metall sich in einer weniger verzerrten quadratisch pyramidalen Geometrie zu befinden, als es bei der synKonfiguration der Fall ist. In der Literatur sind verschiedene theoretische Studien über den syn und anti Isomorphismus von Verbindungen des Typs $\left[\mathrm{M}_{2} \mathrm{O}_{2} \mathrm{~S}_{2}\right]$ bekannt. Es wurde gefunden, dass das syn-Isomer thermodynamisch favorisiert ist, wobei aber nur ein energetischer Unterschied von $1-4 \mathrm{kcal} / \mathrm{mol}$ auftritt. Der Unterschied wird auf eine $\sigma$-Wechselwirkung von jeweils einem d-Orbtial der beiden Metalle zurückgeführt, die in einer syn-Konfiguration stark gebogen ist, während es in der anti-Konfiguration zu einer seitlichen Überlappung der Orbitale kommt. ${ }^{[58]}$ Um eine bessere Orbitalüberlappung zu erreichen, werden die Metalle in der syn-Konfiguration stärker aus der Schwefelebene herausgehoben, worin der größere Abstand des Zentralatoms von der aufgespannten Schwefelebene begründet ist. Außerdem wird in der theoretischen Studie behauptet, dass die Bildung eines syn- oder eines anti-Isomers durch die Verwendung von unterschiedlichen Kationen beeinflusst wird. Dieses konnten wir nun wiederlegen, da sowohl in der Synthese von Holm et al. als auch in unserer das gleiche Kation verwendet wurde. Die Ursache für die Bildung eines syn- oder anti-Isomers liegt vielmehr im Reaktionsmechanismus bzw. den verwendeten Metallprecursorn. Normalerweise sollte immer das syn-Produkt bevorzugt werden, wenn kleine nicht so sterisch anspruchsvolle Reaktionsfragmente sich zusammen lagern, da es hierbei zu der besten Überlappung der Orbitale kommt und somit das thermodynamisch stabilere Produkt gebildet wird. 


\section{Kapitel 4}

\section{Dalton Transactions}

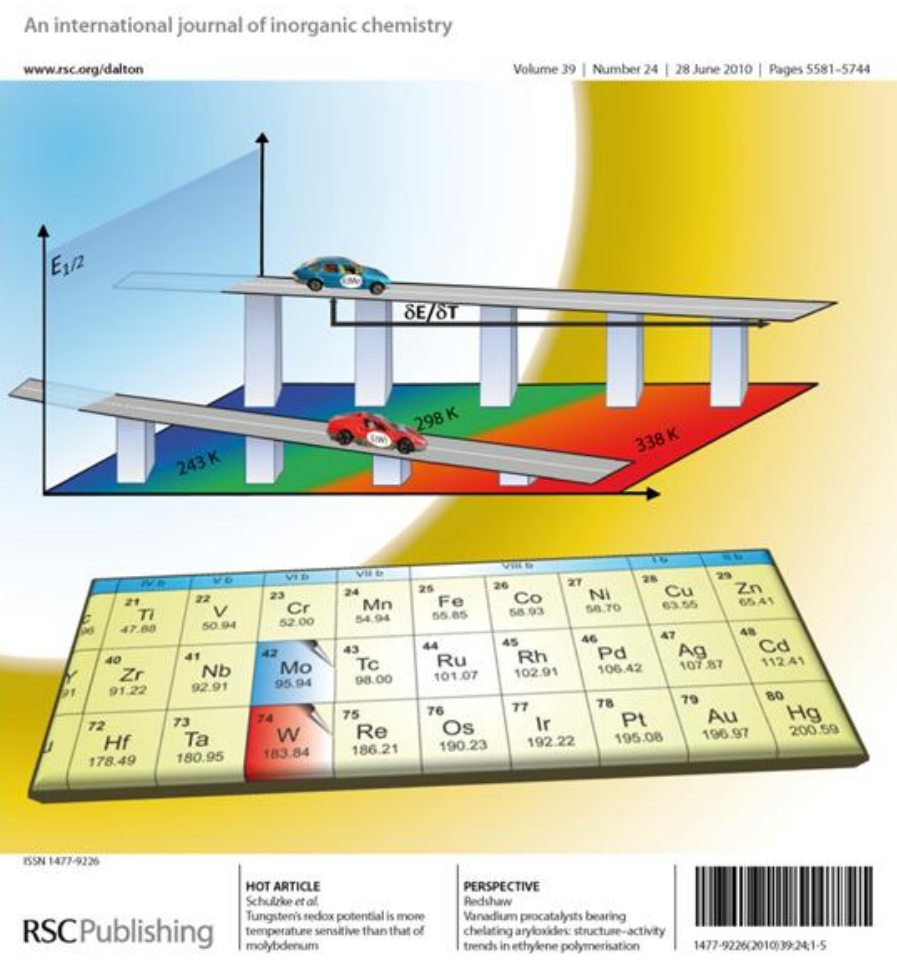

\section{Elektrochemische Untersuchungen ${ }^{[59]}$}

\section{Zusammenfassung}

In diesem Kapitel werden zunächst die für die elektrochemischen Untersuchungen verwendeten Messtechniken erläutert. Des Weiteren werden die temperaturabhängigen Messungen der Verbindungspaare vorgestellt. Außerdem werden Methoden vorgestellt, um Fehlerquellen zu vermindern. 


\subsection{Einleitung}

Das Auftreten von Elektronentransferprozessen in großen Teilen der Naturwissenschaften hat der Elektrochemie einen festen Platz in Forschungsbereichen der Chemie, Biologie, Physik und vielen weiteren Bereichen eingeräumt. Ein alltägliches Leben ohne elektrochemische Prozesse ist in unserer heutigen Zeit nicht mehr wegzudenken. Die Elektrochemie zeigt eine vielfältige Nutzung. Erwähnenswert sind in der Analytischen Chemie die Bestimmung kleinster Konzentrationen an Metallverbindungen in den verschiedensten Materialen, sowie die großtechnisch angewandten elektrochemischen Synthesemethoden, wie etwa bei der Reduktion von Metallsalzen zur Herstellung unedler Metalle wie Lithium, Natrium, Magnesium und viele mehr. Des Weiteren ist die Herstellung von Halogenen möglich durch Oxidation der Halogenide zu Chlor und Fluor. Mit Hilfe des elektrischen Stroms sind Reduktionen von Verbindungen möglich, die durch normale Reduktionsmittel in der chemischen Synthese nicht möglich wären, sowie die Veredelung (z.B. Versilbern) von Gebrauchsgegenständen und Schmuck.

Auch in vielen biologischen Systemen spielen Redoxreaktionen eine große Rolle. Deshalb wird es immer wichtiger, sich mit der Bestimmung von Redoxpotentialen zu beschäftigten.

\subsection{Untersuchungstechniken}

\subsubsection{Cyclovoltammetrie}

Es haben sich in den letzten Jahrzehnten zahlreiche elektroanalytische Methoden entwickelt unter denen die Cyclovoltammetrie besonders große Popularität erlangt hat. ${ }^{[53 b]}$ Sie hat die klassischen Methoden wie die Polarographie, außer bei analytischen Problemen, weitgehend verdrängt. Die Anwendung von cylclovoltammometrischen Untersuchungen erstreckt sich in alle Gebieten der Anorganischen und Organischen Chemie, bis zur Charakterisierung von Multielektronentransferprozessen in der Biochemie und Makromolekularen Chemie.

Aus Cyoclovoltammogrammen können, neben Informationen über die Thermodynamik von Redoxprozessen, auch Kenntnisse über die Kinetik von heterogenen Elektronentransferreaktionen, so wie von angekoppelten Reaktionen erhalten werden. Die charakteristische Form der Cyclovoltammogramme und die eindeutige Potentiallage der Peaks ermöglicht die Bestimmung der nahezu ,fingerprint“-artigen elektrochemischen Eigenschaften von Redoxsystemen.

Bei cyclovoltammetrischen Experimenten wird üblicherweise eine stationäre Arbeitselektrode verwendet, die in einen ruhenden Elektrolyten eintaucht. Diese Elektrode wird nun vom Potentiotaten, ein sich linear änderndes Potential, bis zu einem eingestellten Umkehrpunkt, 
angelegt. Nach Erreichen dieses Umkehrpunkts wird das Potential linear zum Ausgangspunkt zurückgeführt. Die Geschwindigkeit mit der das Potential geändert wird, bezeichnet man als Vorschubgeschwindigkeit. Da der Massentransport der elektroaktiven Spezies, die an der Elektrode reduziert werden, ausschließlich durch Diffusion erfolgt, wird zur Sicherung der Leitfähigkeit ein Elektrolyt ( $\sim 0.1 \mathrm{~mol} / \mathrm{l})$ zugesetzt. Hier wird hauptsächlich das Tetra- $n$-butylammoniumhexafluorophosphat-Salz verwendet, da es sich durch außerordentlich hohe Zersetzungsspannungen auszeichnet. Befinden sich in dem gemessenen Potentialbereich der untersuchten Lösung elektroaktive Spezies, so kann das Fließen eines Faradayschen Stroms beobachtet werden.

\subsubsection{Differenzpulsvoltammetrie (DPV)}

Bei der DPV-Messtechnik handelt es sich um eine Pulstechnik die gleichermaßen für irreversible, als auch für reversible Systeme benutzt werden kann. Ein Puls mit konstanter Amplitude wird mit einer linear ansteigenden Gleichspannung überlagert. Dabei ist der Anstieg der Gleichspannung sehr gering. Die Messung des Stroms I als Funktion der Spannung erfolgt unmittelbar vor dem Puls und am Ende des Pulses. Da die Modulationsamplitude während des gesamten Messvorgangs konstant bleibt, ändert sich das Untergrundsignal nur geringfügig. Somit können mit dieser Methode Messungen bis zu Konzentrationen von etwa $10^{-8} \mathrm{M}$ durchgeführt werden.

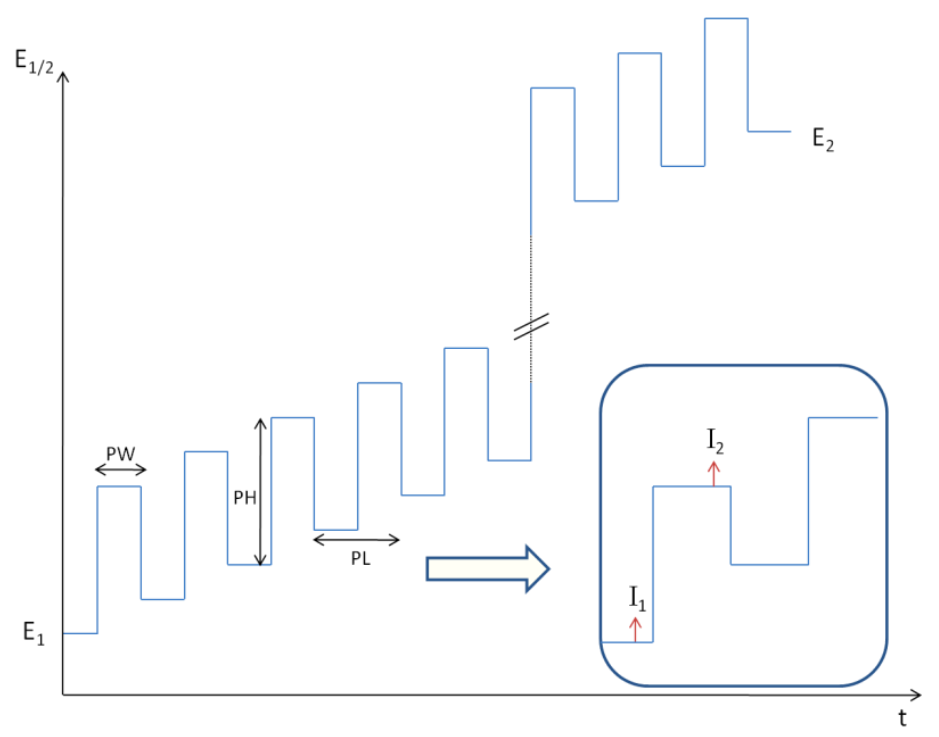

Abb. 75: Messtechnik der DPV, hierbei entspricht $E_{1}$ der Anfangsspannung und $E_{2}$ der Endspannung, PW-Pulsweite; PHHöhe der Pulsamplitude, PL-Pulslänge

\subsubsection{Square-Wave Voltammometrie (SWV)}

Bei der SWV-Messtechnik handelt es sich ebenfalls um eine Pulstechnikmethode. Sie wird häufig verwendet, wenn es bei CV-Messungen zur Überlagerung von Signalen kommt und sie 
deshalb nicht mehr vollständig aufgelöst werden können. Außerdem wird diese Methode verwendet, wenn die Lösungseigenschaften der zu untersuchenden Verbindung nicht sehr gut sind, oder die erhaltenen Signale schlecht von den Hintergrundströmen (kapazitve Ströme) unterschieden werden können.

Bei der SWV-Methode wird durch den Potentiostaten eine Serie von Potentialpulsen zwischen Arbeitselektrode und Referenzelektrode angelegt. Mit einem Startpotential $\mathrm{E}_{1}$ wird begonnen und bis zu einem Endpotential von $\mathrm{E}_{2}$ variiert.

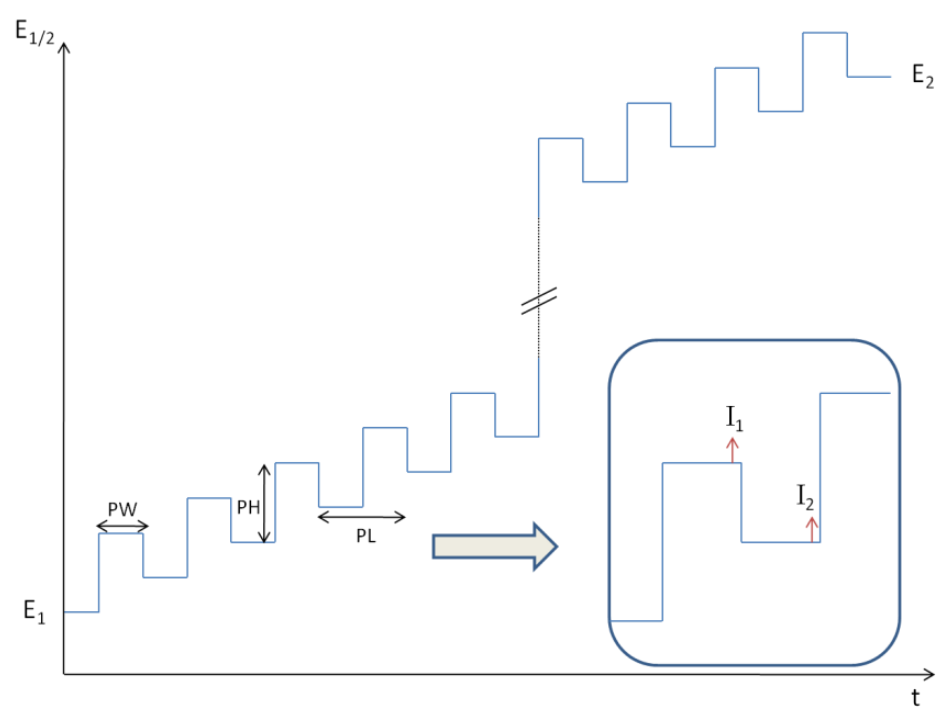

Abb. 76: Messtechnik der SWV, Messtechnik der DPV, hierbei entspricht $E_{1}$ der Anfangsspannung und $E_{2}$ der Endspannung; PW-Pulsweite; PH-Höhe der Pulsamplitude, PL-Pulslänge

Die Geschwindigkeit der Messung, ähnlich der Vorschubgeschwindigkeit bei CV Messungen, wird durch die Länge des Pulses und durch die Größe der Potentialänderung nach einem solchen Puls bestimmt. Jeweils am Ende eines Strompulses werden die Strompaare $\mathrm{I}_{1}$ und $\mathrm{I}_{2}$ gemessen und die Differenz dann aus beiden gegen das Potential aufgetragen. Die Aufnahme der Stromstärke am Ende des Pulses ermöglicht es lediglich den aus dem Elektronentransfer resultierenden Faradayschen Strom zu messen. Der Anteil des kapaziativen Stroms ist sehr klein, wodurch die Messung sehr empfindlich wird und geringste Konzentrationen vermessen werden können. Die Peakbreite von reversiblen Signalen im SWV sollte theoretisch $90.5 \mathrm{mV}$ betragen. Bei quasireversiblen sowie irreversiblen Elektronenübergängen kommt es zu einer Verbreiterung der Peaks. 


\subsection{Temperaturabhängige elektrochemische Messungen}

\subsubsection{Einleitung zur Tieftemperaturvoltammetrie}

Van Duyne und Reilley konnten 1972 erstmals zeigten, das Messungen bei tiefen Temperaturen es ermöglichen reaktive Zwischenstufen zu untersuchen, die normalerweise bei Messungen bei Raumtemperatur nicht mehr nachgewiesen werden konnten. ${ }^{[60]}$ Die so erhaltenen Möglichkeiten eröffneten neue Untersuchungsgebiete, wie z.B. genaue Untersuchungen von Konformationsgleichgewichten, ${ }^{[61]}$ Bestimmungen von Aktivierungsenergien und ausgiebige kinetische Untersuchungen verschiedenster Reaktionen. ${ }^{[62]}$

\subsubsection{Beeinflussende Faktoren der elektrochemischen Messungen}

\subsubsection{Zeitraum in dem der Messzyklus aufgenommen wird}

Da es während des Messzyklus zu einer Veränderung des Probeansatzes durch z.B. Belegung der Elektrodenoberfläche, sowie zum Verdunsten des Lösungsmittels kommen kann, wurde für die Messungen ein spezieller Messzyklus entwickelt. Hierbei sind die Temperaturen auf diesem Messzyklus so gewählt, dass Temperaturen im Abstand von $10^{\circ} \mathrm{C}$ gemessen werden. Beim Erreichen der Umkehrpunkte $-20^{\circ} \mathrm{C}$ bzw. $+50^{\circ} \mathrm{C}$ wird als nächster Temperaturschritt nur ein $5^{\circ} \mathrm{C}$ Unterschied eingefügt. Dies führt dazu, dass die Messpunkte bei Temperaturerhöhung und Temperaturerniedrigung ineinander greifen, so dass benachbarte Werte zu ganz unterschiedlichen Zeiten aufgenommen werden. Auf diese Weise lassen sich zeitabhängige Einflüsse auf das Redoxpotential nivellieren. Gestartet wurde bei einem frei gewählten Temperaturstartpunkt auf diesem Zyklus, der dann im oder gegen den Uhrzeigersinn durchlaufen wurde. Es wurden mindestens fünf Messzyklen von zuvor frisch angesetzten Proben durchgeführt. Bei zwei Messungen wurde im Bereich von $30-50^{\circ} \mathrm{C}$ gestartet, sowie bei zwei Messungen im Bereich von $-20-0^{\circ} \mathrm{C}$. Die letzte Messung wurde bei $25^{\circ} \mathrm{C}$ gestartet. Durch das verwendete Silikonöl im Thermostaten wurde die Obergrenze für den Temperaturbereich festgelegt. Aufgrund der verwendeten Glaselektroden konnten keine Messungen bei Temperaturen unter $-20^{\circ} \mathrm{C}\left(-30^{\circ} \mathrm{C}\right)$ durchgeführt werden, wodurch die Untergrenze des Messzyklus bestimmt wurde. Im Folgenden ist der verwendete Messzyklus aufgeführt. 


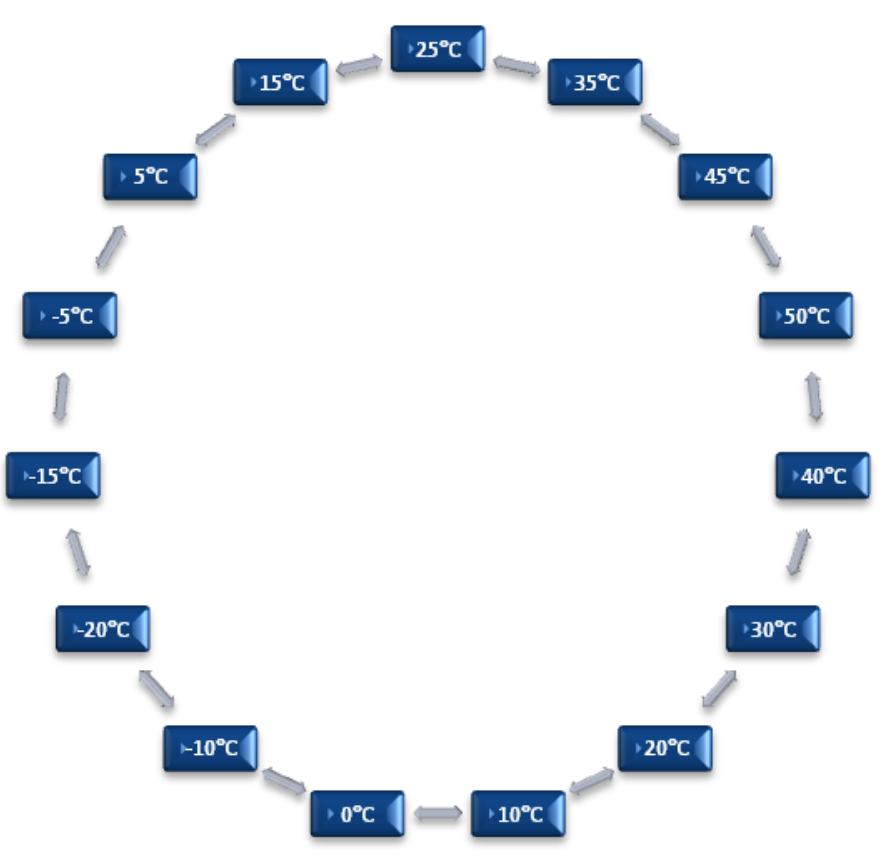

Abb. 77: Verwendeter Temperaturmesszyklus

Am Ende jeder Messreihe wurde eine Referenzierung gegen Ferrocen bzw. Decamethylferrocen durchgeführt. ${ }^{[29 i]}$ Hierbei ist es von Wichtigkeit die Referenzierung bei $25^{\circ} \mathrm{C}$ durchzuführen, da das $\mathrm{Fc} / \mathrm{Fc}^{+}$Potential sonst nicht zur Referenzierung zulässig ist. Um die Veränderung der Potentiale bei verschiedenen Temperaturen von Ferrocen/Ferrocenium zu untersuchen, wurde dieser Elektronenübergang ebenfalls temperaturabhängig bestimmt. Im Abb.78 ist die Abhängigkeit gezeigt.

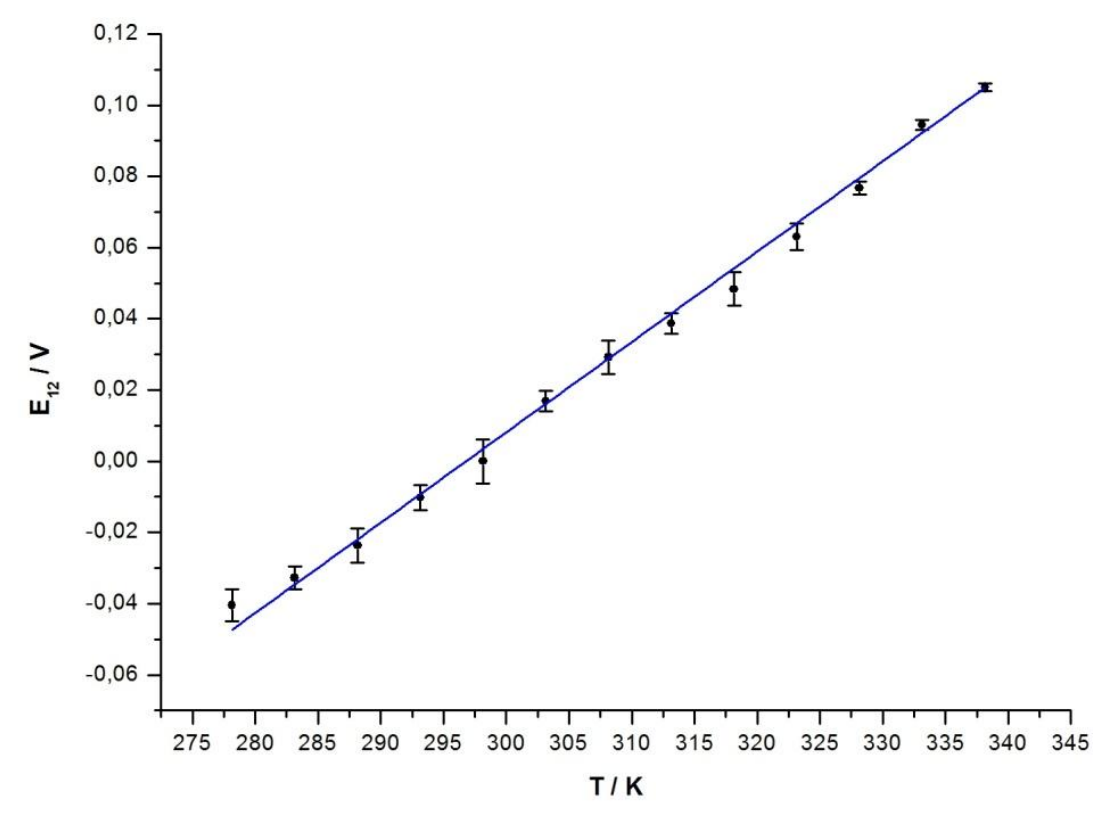

Abb. 78: Temperaturabhängigkeit der Ferrocen/Ferroceniumkopplung 
Hieran ist zu erkennen, dass es von äußerster Wichtigkeit ist, die Referenzierung immer bei $25^{\circ} \mathrm{C}$ durchzuführen, da in dem Temperaturbereich von $5^{\circ} \mathrm{C}$ bis $\mathrm{zu} \quad 65^{\circ} \mathrm{C}$ ein Potentialunterschied von ca. $150 \mathrm{mV}$ festgestellt werden konnte.

Mit Hilfe der in der Tabelle 9 enthaltenen Werte kann eine Umrechnung zwischen den verwendeten Referenzierungssystemen durchgeführt werden. Dabei ist die Wahl der Referenzelektrode bzw. Referenzsubstanz von den untersuchten Systemen abhängig. Hierbei bietet sich in wässrigen Lösungen die Verwendung einer Silber/Silberchlorid (gefüllt mit $3 \mathrm{M}$ $\mathrm{KCl})$ an. Für nichtwässrige Systeme haben sich interne Referenzsubstanzen wie Ferrocen, Decamethylferrocen oder Cobaltocen als sehr nützlich erwiesen. Des Weiteren ist die Verwendung einer Ag/AgCl-Elektrode auch in nicht wässrigen Lösungsmitteln, gefüllt mit z.B. $\mathrm{LiCl}$ in EtOH (abs.), möglich. Die Verwendung von Elektroden 2. Art bieten einen großen Vorteil, da das Potential nur noch indirekt von der umgebenden Elektrolytlösung abhängig ist, weil die Metallelektrode mit einer schwerlöslichen Verbindung des Metallions im Gleichgewicht steht. Doch es tritt eine stärkere Temperaturabhängigkeit auf, als es bei normalen nicht redoxaktiven Elektroden der Fall ist. Des Weiteren ist für die Wahl der Referenz auch das Potential der Referenzsubstanz, im Vergleich zur untersuchten Substanz, entscheidend. Bei Überlagerung von Referenz und Probensignal ist keine genaue Bestimmung des Redoxpotentials möglich.

\begin{tabular}{cccccc}
\hline & NHE & SCE & $\mathrm{Ag} / \mathrm{AgCl}$ & $\mathrm{Fc} / \mathrm{Fc}^{+}$ & $\mathrm{dmFc} / \mathrm{dmFc}^{+}$ \\
\hline NHE & - & -0.241 & -0.197 & -0.548 & -1.036 \\
$\mathrm{SCE}$ & +0.241 & - & 0.042 & -0.307 & -0.795 \\
$\mathrm{Ag} / \mathrm{AgCl}$ & +0.197 & -0.042 & - & -0.352 & -0.840 \\
$\mathrm{Fc} / \mathrm{Fc}^{+}$ & +0.548 & +0.307 & +0.352 & - & $+0.488^{\mathrm{a}}$ \\
$\mathrm{DMFc}^{+}$ & +1.036 & +0.795 & +0.840 & $-0.488^{\mathrm{a}}$ & - \\
\hline
\end{tabular}




\subsection{Temperaturabhängigkeit der Elektroden}

Da die verwendeten Referenzelektroden (Platinstab, $\mathrm{Ag} / \mathrm{AgCl}$ ) auch eine Temperaturabhängigkeit zeigen, wurde nach einer Methode zur Messung der Potentiale bei Verwendung einer temperaturabhängigen Redoxelektrode gesucht. Es wurde hierfür eine „non isothermale"-Zelle verwendet. Bei dieser Zelle sind die Arbeits- und Gegenelektroden räumlich von der Referenzelektrode getrennt. Die einzelnen Elektrodenräume können unabhängig voneinander temperiert werden. Mit konstanter Temperatur wurde die Referenzelektrode bei $25^{\circ} \mathrm{C}$ temperiert, während der größere Zellraum die einzelnen Temperaturbereiche durchläuft.

Im Abb.79 ist ein Bild der verwendeten ,non-isothermalen“ Zelle gezeigt.

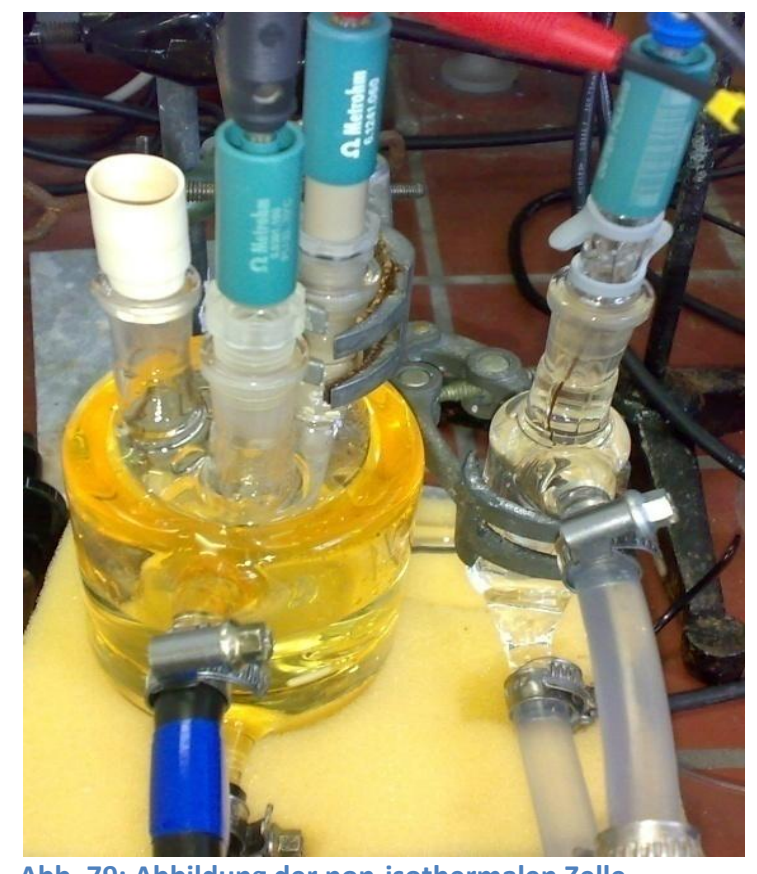

Abb. 79: Abbildung der non-isothermalen Zelle

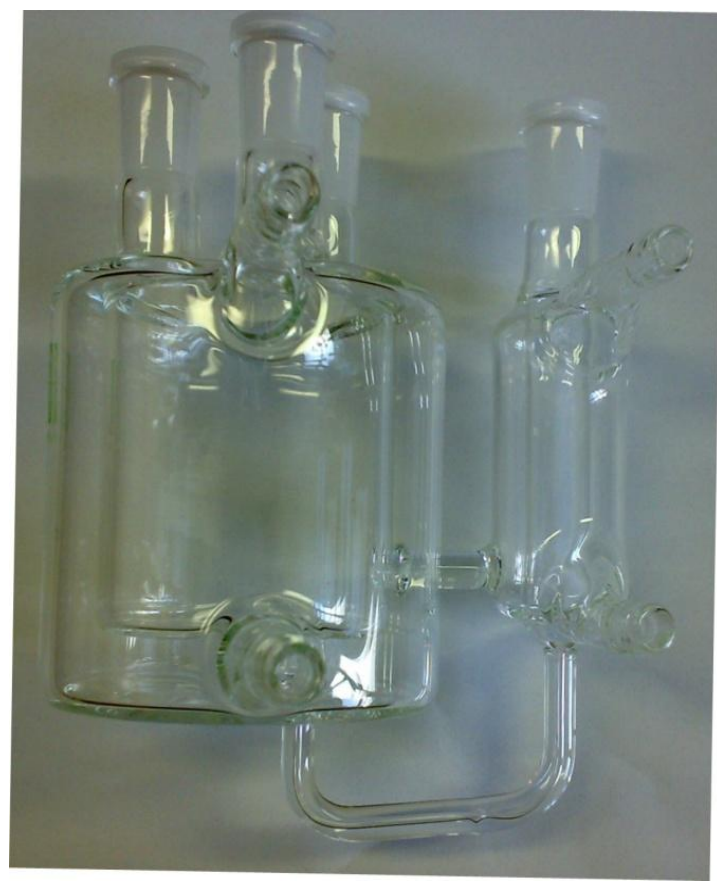

Um eine elektrische Verbindung der Referenzelektrode mit der Probelösung zu gewährleistenden sind die beiden Räume über eine dünne Kapillare miteinander verbunden, wie in Abb.79 rechts zu sehen.

Nachteil bei Messungen mit non-isothermalen Zellen ist, dass es in der dünnen Kapillare zwischen den unterschiedlich temperierten Reservoirs zu einem Diffusionskoeffizienten kommt. Dadurch bildet sich zwischen dem heißen und dem kalten Ende eine Potentialdifferenz, die auch als Soret Potential bezeichnet wird. ${ }^{[63]}$ Das Soret-Potential ändert sich mit der Zeit, bis es seinen endgültigen Wert erreicht, was allerdings nur bei stationären Messungen möglich ist und abhängig von der Temperaturdifferenz ist. In den durchgeführten Messungen kann das Soret Potential als sehr gering und vernachlässigbar klein angesehen 
werden und es wurden keine weiteren Korrekturen durchgeführt, um diesen Faktor in der Auswertung zu berïcksichtigen.

\subsubsection{Temperaturabhängigkeit der Silber/Silberchlorid-Elektrode}

Die verwendete $\mathrm{Ag} / \mathrm{AgCl}$-Elektrode wurde mit $3 \mathrm{M}$ wässriger Kaliumchloridlösung befüllt. Da sich die Löslichkeit des Salzes bei Temperaturänderung ständig ändert, kann die Elektrode bei Temperaturveränderungen kein stabiles Potential liefern. Bei steigender Temperatur kommt es zu stärkerer Löslichkeit und somit steigt die Aktivität der Chloridionen in der Elektrode. Eine Referenzelektrode muss ein stabiles Potential über einen längeren Zeitraum liefern, um die Nernstgleichung zu erfüllen. Das Potential muss nach mehreren Stromdurchgängen immer noch den Anfangswert zeigen und es darf keine Hysterese auftreten. Bei der Ag/AgCl-Elektrode handelt es sich um eine der meist genutzten Referenzelektroden in wässrigen Lösungen. Die Löslichkeit des Silberchlorids in Wasser beträgt bei RT etwa $10^{-5} \mathrm{M}$. Außerdem wird die Diffusion durch den vorhandenen Magnesiastift am Ende der Elektrode ebenfalls durch die Temperatur beeinflusst. Bei einer Bezugselektrode muss die Aktivität des potentialbestimmenden Ions konstant gehalten werden. Hier geschieht das durch Zugabe eines Elektrolyten $(\mathrm{KCl})$ in sehr hoher Konzentration, der mit den Silber-Ionen zu einem schwer löslichen Niederschlag ausfällt. Das Potential der Elektrode ist allein von der Aktivität der Chlorid-Ionen abhängig. Verbrauchtes dissoziiertes Chlorid wird durch sofortige Dissoziation des noch nicht dissoziertem $\mathrm{KCl}$ ersetzt. Dadurch sinkt zwar die Konzentration der KCl-Lösung, nicht jedoch die Aktivität des Chlorids. Um die Temperaturabhängigkeit der Silber/Silberchloridelektrode zu bestimmen, wurde die Verbindung $\left[\mathrm{Mo}(\mathrm{CN})_{8}\right]^{4-}(0.01 \mathrm{~mol} / \mathrm{l})$ in $0.1 \mathrm{M} \mathrm{KCl-Lösung} \mathrm{(wässrig)} \mathrm{mit} \mathrm{Hilfe}$ einer non-isothermalen Zelle bestimmt. Die Verbindung wurde einmal non-isothermal und einmal isothermal temperaturabhängig untersucht. Die Messung besteht aus einer Summe der Temperaturabhängigkeiten der Redoxpotentiale der Probe und der Referenzelektrode. Bei der non-isothermalen Messung hingegen, spielt nur die Temperaturabhängigkeit der Redoxpotentiale eine Rolle. Somit kann durch Subtraktion der beiden Temperaturabhängigkeiten die Temperaturabhängigkeit der Referenzelektrode bestimmt werden.

$$
\text { Temp.abhängigkeit der Referenzelektrode }=\quad \text { Potential }_{\text {Isothermal }}-\text { Potential }_{\text {Non-Isotherma }}
$$

Hierdurch konnte eine Temperaturabhängigkeit der Ag/AgCl-Referenzelektrode von $0.67 \mathrm{mV} / \mathrm{K}$ ermittelt werden, was auch eine gute Übereinstimmung mit den Herstellerangaben von $0.60 \mathrm{mV} / \mathrm{K}$ liefert. Aufgrund dieser bestimmten Faktoren wurden alle Messwerte, die mit 
der Silber/Silberchlorid-Elektrode in einem isothermalen Setup bestimmt wurden, durch diesen Wert korrigiert.

\subsubsection{Temperaturabhängigkeit des Platinstabes}

Da die Austrittsarbeit für den Strom aus dem Platinstab auch temperaturabhängig sein sollte, wurde auch dessen Temperaturabhängigkeit bestimmt. Hierzu wurde der Elektronenübergang von Ferrocen/Ferrocenium in Acetonitrillösung untersucht. Da eine Vielzahl von Messungen mit Hilfe des Platinstabes als Referenz durchgeführt wurde und hierzu keine Literatur bekannten Messdaten vorhanden waren, wurde die Temperaturabhängigkeit über zwei verschiedene Methoden bestimmt. Zuerst wurde die Methode angewendet, die schon bei der Untersuchung der Ag/AgCl-Referenzelektrode benutzt wurde. In Abb.80 sind die erhaltenen Messgeraden aufgezeigt.
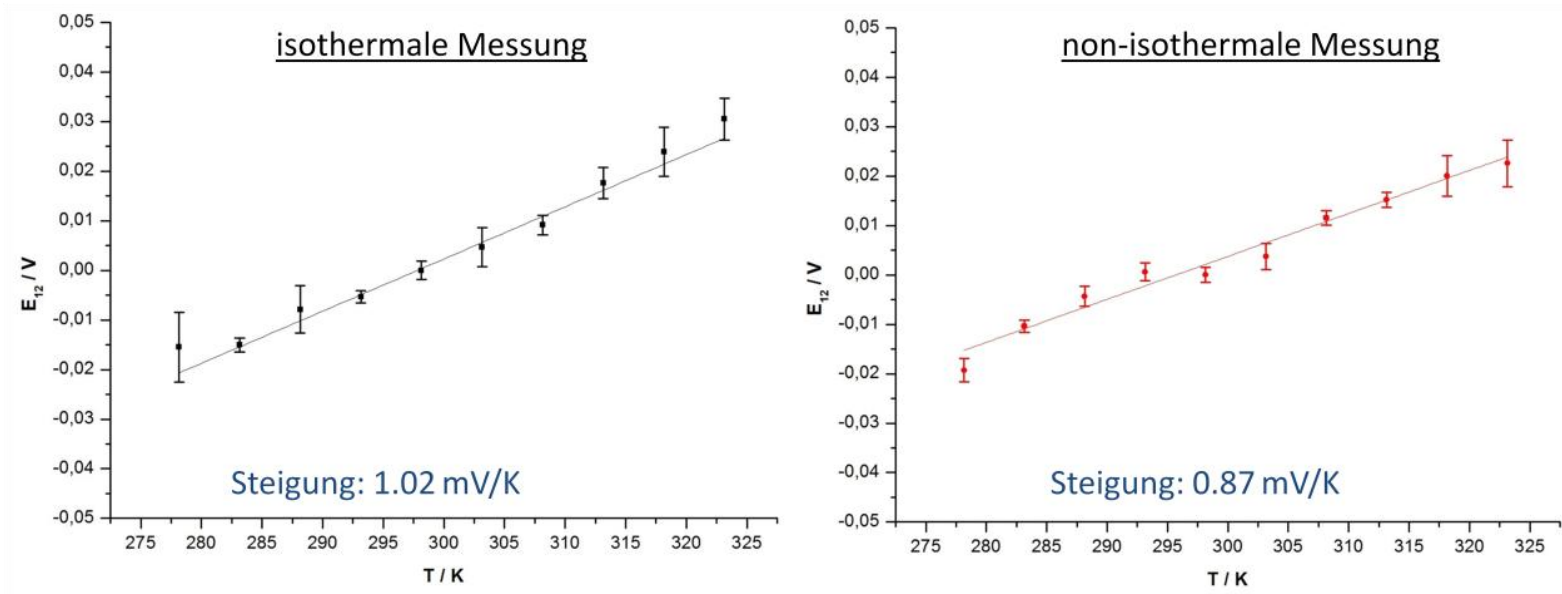

Abb. 80: Erhaltene Temperaturabhängigkeiten für die isothermale- sowie non-isothermale Messung von $\mathrm{Fc}_{\mathrm{Fc}}{ }^{+}$

Die Oxidation von Ferrocen zum Ferrocenium-Kation wurde je durch fünf Messreihen isothermal sowohl non-isothermal bestimmt. Nach Substraktion konnte ein Steigungswert für die Temperaturabhängigkeit des Platinstabes von $0.148 \mathrm{mV} / \mathrm{K}$ ermittelt werden.

Um noch eine weitere Untersuchung der Abhängigkeiten durchzuführen, wurde diesmal die Untersuchungslösung in der non-isothermalen Zelle bei $25^{\circ} \mathrm{C}$ temperiert, während der sich im Referenzraum befindende Platinstab bei den jeweiligen Messtemperaturen eingestellt wurde. Die Untersuchung wurde in fünf Messreihen mit dem Ferrocen/Ferrocenium-Paar in Acetonitril durchgeführt. Dabei konnte eine Abhängigkeit des Platinstabes von $0.080 \mathrm{mV} / \mathrm{K}$ ermittelt werden. Die Ergebnisse stimmen nicht überein, liegen aber in derselben Größenordnung. Da insgesamt die Temperaturabhängigkeiten als sehr gering anzusehen sind, wurde der Mittelwert aus beiden Messungen verwendet. Für alle Messungen wurde deshalb ein Korrekturfaktor für die Temperaturabhängigkeit des Platinstabes von $0.114 \mathrm{mV} / \mathrm{K}$ angesetzt. 


\subsection{Temperaturabhängige Untersuchungen}

Um Unterschiede bei den Temperaturabhängigkeiten der Redoxpotentiale von Molybdän- und Wolframpaaren aufzuzeigen, wurden Verbindungspaare hergestellt, welche die gleichen Ligandensysteme tragen. Somit bestand der einzige Unterschied im Metallzentrum. Um weitere Fehlerquellen zu minimieren, wurde jedes Verbindungspaar mit der gleichen elektrochemischen Untersuchungsmethode, den Messparametern sowie in den gleichen Lösungsmitteln vermessen. In vorangegangen Messungen $^{[4]}$ konnte durch unsere Arbeitsgruppe gezeigt werden, dass es bei der Verwendung von Dithiolenverbindungen mit Molybdän und Wolfram, bei den Redoxpotentialen der Wolframverbindungen, zu einer stärkeren Temperaturabhängigkeit kam, als es bei den analogen Molybdänverbindungen der Fall war. Ziel dieser Arbeit war, die vorherigen Ergebnisse mit Dithiolenverbindungen zu untermauern oder zu widerlegen und festzustellen, ob diese Abhängigkeit der Redoxpotentiale von der Temperatur auch bei nicht-Dithiolenverbindungen auftritt. Hierzu wurde eine Vielzahl von weiteren Dithiolenverbindungen sowie verschiedenster Molybdän- und Wolframverbindungen untersucht. Im Folgenden alle die untersuchten Paare aufgeführt.

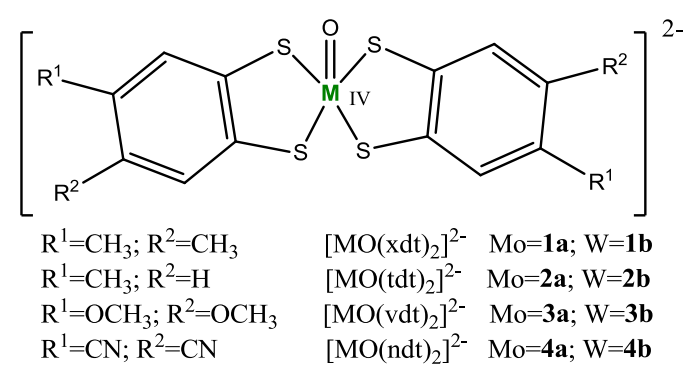<smiles></smiles>

$\left[\mathrm{MO}(\mathrm{qdt})_{2}\right]^{2-} \quad \mathrm{Mo}=\mathbf{5 a} ; \mathrm{W}=\mathbf{5 b}$ $\mathrm{R}^{1}=\mathrm{CN} ; \mathrm{R}^{2}=\mathrm{CN} \quad\left[\mathrm{MO}(\mathrm{ndt})_{2}\right]^{2-} \quad \mathrm{Mo}=\mathbf{4 a} ; \mathrm{W}=\mathbf{4 b}$

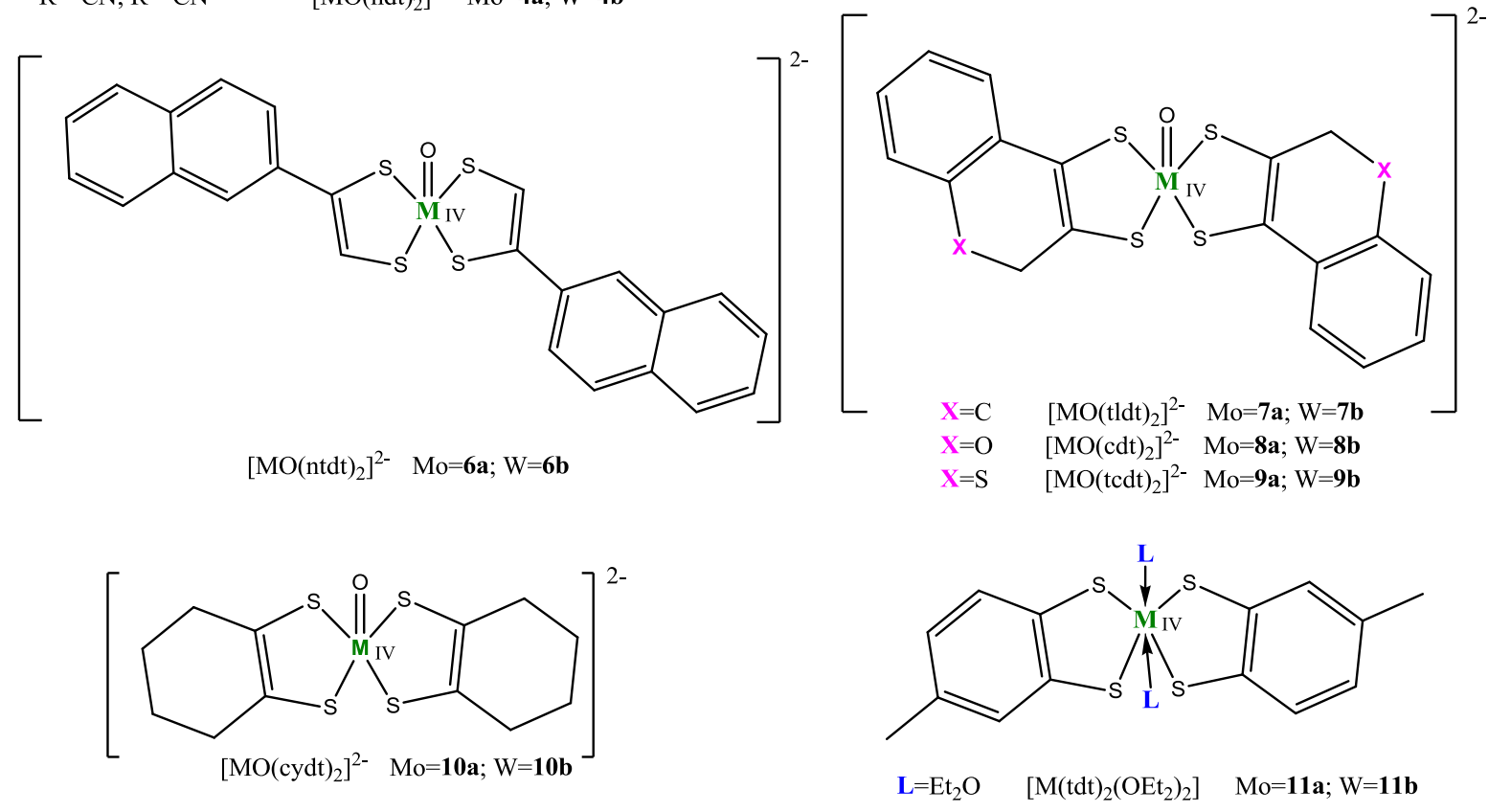




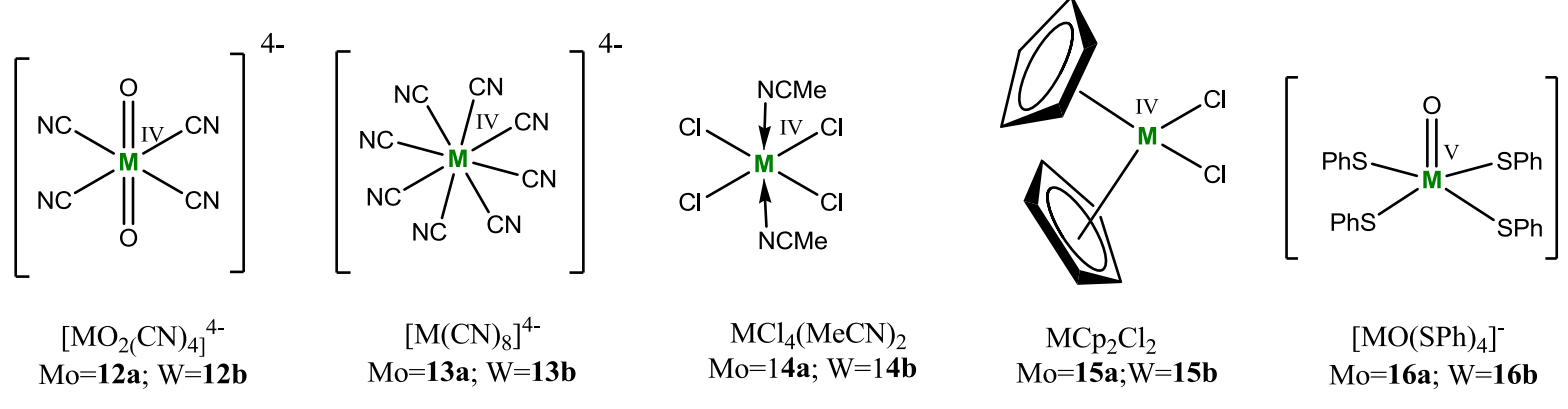

Abb. 82: Untersuchte Nicht-Dithiolenverbindungen

Die meisten der hier gezeigten Verbindungen konnten im Lösungsmittel Acetonitril in einem Temperaturbereich von $-20^{\circ} \mathrm{C}$ bis $+50^{\circ} \mathrm{C}$ auf die Veränderung der Redoxpotentiale hin untersucht werden. Verbindungspaar 11 wurde aufgrund der vorhandenen Koordination durch zwei Diethylethermoleküle in einem nicht koordinierenden Lösungsmittel vermessen, da die Etherliganden sonst durch die Lösungsmittelmoleküle substituiert werden würden. (siehe Kap.3 Seite 65) In diesem Fall wurde auf Dichlormethan als Lösungsmittel zurückgegriffen, wodurch der messbare Temperaturbereich zwar verkleinert wurde, doch es konnte ein stabiles Signal über einen langen Messzeitraum erhalten werden. Diese Substitution der koordinierten Lösungsmoleküle konnte auch bei Verbindungen des Typs 14 beobachtet werden. Deshalb wurde direkt das Addukt mit koordinierten Acetonitril verwendet. Die Verbindungspaare 12 und 13 wurden in entgasten wässrigen $0.1 \mathrm{M}$ KCl-Lösung mit einer Silber/Silberchloridelektrode als Referenzelektrode vermessen. Hier konnte ein Temperaturbereich von $5^{\circ} \mathrm{C}$ bis $\mathrm{zu} 85^{\circ} \mathrm{C}$ untersucht werden. In nachfolgender Tabelle 10 auf Seite 86 sind die gemessenen Redoxpotentiale und die Temperaturabhängigkeiten der jeweiligen Verbindungspaare aufgeführt.

Um weitere thermodynamische Parameter aus den bestimmten Redoxpotentialen zu erhalten kann man durch folgende Beziehungen die Gibbs-Energie, die Entropie und auch die Reaktionsenthalpie erhalten. ${ }^{[64]}$

$$
\Delta G=-Z \cdot F \cdot E
$$

Mit Hilfe der thermodynamischen Beziehung für die Entropie kann durch Einsetzen von Glg.5 für die Gibbs-Energie Gleichung Glg.6 erhalten werden.

$$
\Delta S=z \cdot F \cdot\left(\frac{\delta E}{\delta T}\right)_{p}
$$

Hierbei entspricht der Quotient $\delta E / \delta T$ dem erhaltenen Steigungswert der Temperaturabhängigkeit der Redoxpotentiale, z stellt die Anzahl der übertragenen Elektronen 
dar und F steht für die Farraday-Konstante. Aus den erhaltenen entropischen Parametern können nun Werte für die Enthalpie ermittelt werden.

$$
\Delta H=Z \cdot F \cdot\left[T \cdot\left(\frac{\delta E}{\delta T}\right)-E\right]
$$

Die hier berechneten thermodynamischen Parameter außer der Entropie stellen keine absoluten Werte dar, weshalb die Werte nur in Relation zu einander diskutiert werden. Die berechneten Entropiewerte sind in Tabelle 10 angegeben.

Mit steigender Temperatur ist eine Zunahme oder Abnahme der Redoxpotentiale mit der Temperatur festzustellen. Beispielhaft ist hier die Temperaturabhängigkeit des $\left[\mathrm{MO}(\mathrm{vdt})_{2}\right]^{2-}$ Verbindungspaares aufgezeigt. Bei diesem Paar war es möglich sowohl den Oxidationsstufenübergang von IV nach V, sowie von V nach VI zu untersuchen. Die Temperaturabhängigkeiten der Verbindungspaare sowie die manche erhaltenen Spektren bei jeweils $25^{\circ} \mathrm{C}$ sowie bei der niedrigen und höchsten Temperatur sind im Anhang unter Abschnitt $11.1 \mathrm{zu}$ finden.

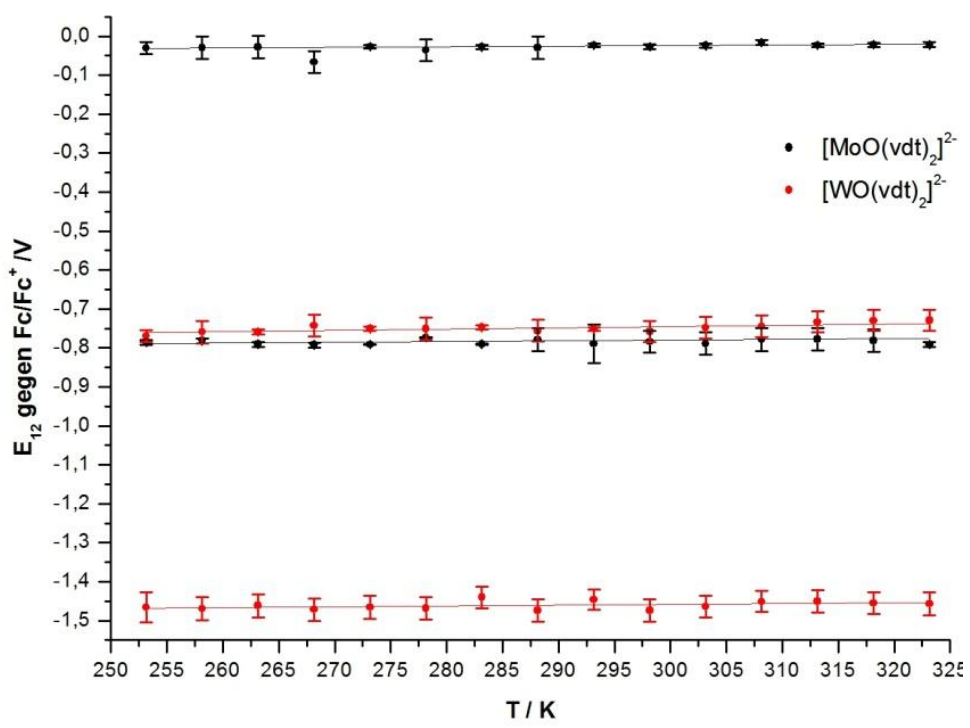

Abb. 83:Temperaturabhängigkeit von $\left[\mathrm{MO}(\mathrm{vdt})_{2}\right]^{2}$ 


\begin{tabular}{|c|c|c|c|c|}
\hline & $\begin{array}{l}\mathrm{E}_{1 / 2}{ }^{0} \text { gegen } \\
\mathrm{Fc} / \mathrm{Fc}^{+}[\mathrm{V}]\end{array}$ & $\mathrm{E}_{1 / 2}{ }^{\mathrm{W}}-\mathrm{E}_{1 / 2}{ }^{\mathrm{Mo}}[\mathrm{V}]$ & $\begin{array}{c}\text { Steigungswert } \\
{[\mathrm{mV} / \mathrm{K}]}\end{array}$ & $\begin{array}{c}\Delta S \\
{\left[\mathbf{J} \cdot \mathbf{m o l}^{-1} \cdot \mathbf{K}^{-1}\right]}\end{array}$ \\
\hline $1 \mathrm{a}(\mathrm{IV} \leftrightarrow \mathrm{V})$ & -0.85 & \multirow{2}{*}{0.18} & $0.52 \pm 0.075$ & $50.17 \pm 7.24$ \\
\hline $1 \mathrm{~b}(\mathrm{IV} \leftrightarrow \mathrm{V})$ & -1.03 & & $2.13 \pm 0.070$ & $205.5 \pm 6.75$ \\
\hline $2 \mathbf{a}(\mathrm{IV} \leftrightarrow \mathrm{V})$ & -0.80 & \multirow{2}{*}{0.69} & $0.46 \pm 0.081$ & $44.78 \pm 7.79$ \\
\hline $2 b(I V \leftrightarrow V)$ & -1.49 & & $0.91 \pm 0.072$ & $87.35 \pm 6.99$ \\
\hline $2 \mathbf{a}(\mathrm{V} \leftrightarrow \mathrm{VI})$ & -0.02 & \multirow{2}{*}{0.59} & $0.68 \pm 0.061$ & $65.24 \pm 5.89$ \\
\hline $2 b(V \leftrightarrow V I)$ & -0.61 & & $1.33 \pm 0.126$ & $128.3 \pm 12.1$ \\
\hline 3a $(I V \leftrightarrow V)$ & -0.77 & \multirow{2}{*}{0.68} & $0.05 \pm 0.075$ & $5.09 \pm 7.22$ \\
\hline $3 b(I V \leftrightarrow V)$ & -1.45 & & $0.20 \pm 0.118$ & $19.19 \pm 11.4$ \\
\hline $3 a(V \leftrightarrow V I)$ & -0.03 & \multirow{2}{*}{0.72} & $0.25 \pm 0.115$ & $24.27 \pm 11.1$ \\
\hline $3 b(V \leftrightarrow V I)$ & -0.75 & & $0.41 \pm 0.088$ & $39.40 \pm 8.49$ \\
\hline $4 \mathrm{a}(\mathrm{IV} \leftrightarrow \mathrm{V})$ & -0.28 & \multirow{2}{*}{0.29} & $-0.38 \pm 0.056$ & $36.66 \pm 5.40$ \\
\hline $4 b(I V \leftrightarrow V)$ & -0.57 & & $-0.48 \pm 0.080$ & $46.31 \pm 7.72$ \\
\hline $5 a(I V \leftrightarrow V)$ & -0.31 & \multirow{2}{*}{0.36} & $-0.33 \pm 0.025$ & $-32.03 \pm 2.37$ \\
\hline $5 b(I V \leftrightarrow V)$ & -0.67 & & $-1.79 \pm 0.066$ & $-172.7 \pm 6.37$ \\
\hline $\mathbf{6 a}(\mathrm{IV} \leftrightarrow \mathrm{V})$ & -0.88 & \multirow{2}{*}{0.04} & $-0.76 \pm 0.038$ & $-73.33 \pm 3.67$ \\
\hline $6 b(I V \leftrightarrow V)$ & -0.92 & & $-0.78 \pm 0.044$ & $-75.26 \pm 4.25$ \\
\hline $7 \mathbf{a}(\mathrm{IV} \leftrightarrow \mathrm{V})$ & -0.95 & \multirow{2}{*}{0.26} & $-0.56 \pm 0.033$ & $-54.03 \pm 3.18$ \\
\hline $7 b(I V \leftrightarrow V)$ & -1.21 & & $-0.98 \pm 0.061$ & $-94.56 \pm 5.89$ \\
\hline $8 \mathbf{a}(\mathrm{IV} \leftrightarrow \mathrm{V})$ & -0.72 & \multirow{2}{*}{0.17} & $-0.34 \pm 0.062$ & $-32.80 \pm 5.98$ \\
\hline $8 b(I V \leftrightarrow V)$ & -0.89 & & $-0.46 \pm 0.045$ & $-44.38 \pm 4.34$ \\
\hline 9a $(\mathbf{I V} \leftrightarrow \mathrm{V})$ & -0.87 & \multirow{2}{*}{0.32} & $-0.26 \pm 0.004$ & $-25.09 \pm 0.39$ \\
\hline $9 \mathrm{~b}(\mathrm{IV} \leftrightarrow \mathrm{V})$ & -1.19 & & $-0.66 \pm 0.008$ & $-63.68 \pm 0.77$ \\
\hline $10 \mathrm{a}(\mathrm{IV} \leftrightarrow \mathrm{V})$ & -0.98 & \multirow{2}{*}{0.41} & $0.24 \pm 0.015$ & $23.35 \pm 1.45$ \\
\hline $10 \mathrm{~b}(\mathrm{IV} \leftrightarrow \mathrm{V})$ & -1.39 & & $1.29 \pm 0.074$ & $124.5 \pm 7.14$ \\
\hline $11 \mathrm{a}(\mathrm{IV} \leftrightarrow \mathrm{V})^{\mathrm{e}}$ & -0.11 & \multirow{2}{*}{0.18} & $0.36 \pm 0.032$ & $34.73 \pm 3.09$ \\
\hline $11 b(I V \leftrightarrow V)^{e}$ & -0.29 & & $1.02 \pm 0.071$ & $98.41 \pm 6.85$ \\
\hline $12 \mathrm{a}(\mathrm{IV} \leftrightarrow \mathrm{V})^{\mathbf{a}, \mathbf{b}, \mathbf{d}}$ & -0.26 & \multirow{2}{*}{0.33} & $-1.41 \pm 0.080$ & $-136.0 \pm 7.70$ \\
\hline $12 b(I V \leftrightarrow V)^{a, b, d}$ & -0.59 & & $-2.13 \pm 0.049$ & $-205.5 \pm 4.70$ \\
\hline $13 a(I V \leftrightarrow V)^{a, b, d}$ & -0.56 & \multirow{2}{*}{0.25} & $-0.84 \pm 0.111$ & $-81.43 \pm 10.7$ \\
\hline $13 b(I V \leftrightarrow V)^{a, b, d}$ & -0.81 & & $-1.33 \pm 0.069$ & $-128.3 \pm 6.70$ \\
\hline $14 a(I V \leftrightarrow V)^{d}$ & 0.00 & \multirow{2}{*}{0.67} & $-1.13 \pm 0.106$ & $-109.0 \pm 10.2$ \\
\hline $14 b(I V \leftrightarrow V)^{d}$ & -0.67 & & $-1.27 \pm 0.078$ & $-122.5 \pm 7.50$ \\
\hline $15 \mathrm{a}(\mathrm{IV} \leftrightarrow \mathrm{V})^{\mathrm{d}}$ & 0.00 & \multirow{2}{*}{0.07} & $0.31 \pm 0.060$ & $29.50 \pm 5.60$ \\
\hline $15 b(I V \leftrightarrow V)^{d}$ & -0.07 & & $0.79 \pm 0.080$ & $76.22 \pm 7.72$ \\
\hline $15 \mathrm{a}(\mathrm{IV} \leftrightarrow \mathrm{V})^{\mathrm{c}, \mathrm{d}}$ & 0.04 & \multirow{2}{*}{0.14} & $0.28 \pm 0.046$ & $27.02 \pm 4.45$ \\
\hline $15 b(I V \leftrightarrow V)^{c, d}$ & -0.10 & & $0.86 \pm 0.133$ & $83.26 \pm 12.8$ \\
\hline $16 \mathrm{a}(\mathrm{V} \leftrightarrow \mathrm{IV})$ & -1.22 & \multirow{2}{*}{0.17} & $-0.73 \pm 0.085$ & $-70.85 \pm 8.25$ \\
\hline $16 b(V \leftrightarrow I V)$ & -1.39 & & $-1.01 \pm 0.070$ & $-97.45 \pm 6.80$ \\
\hline
\end{tabular}

Tab. 10: Bestimmte Potentiale und deren Temperaturabhängigkeit,

a berechnet von $\mathrm{Ag} / \mathrm{AgCl}-$ Referenzierten Potentialen; ${ }^{\mathrm{b}}$ Messung in Wasser; ${ }^{\mathrm{c}}$ Messung in DMF;

${ }^{d}$ Cyclovoltammometrische Untersuchung; ${ }^{e}$ Messung in $\mathrm{CH}_{2} \mathrm{Cl}_{2}$ 
Vergleicht man nun die Unterschiede zwischen den Molybdän- und Wolframkomplexen, so stellt man bei ausnahmslos allen untersuchten Verbindungspaaren fest, dass die Redoxpotentiale der Wolframverbindungen stärker von der Temperatur abhängig sind, als die der analogen Molybdänverbindungen. Aufgrund der stabileren Redoxpotentiale die bei Temperaturveränderungen bei der Verwendung von Molybdän vorhanden sind, kann dies als ein weiterer Grund für den Wechsel, von Wolfram zu Molybdän in den aktiven Zentren, während der evolutionären Entwicklung der frühen Organismen, gedeutet werden. Nachdem dies zunächst nur für Dithiolenverbindungen beobachtet worden war, kann es nun als ein genereller Unterschied zwischen Molybdän und Wolframverbindungen angesehen werden. Um die Unterschiede zwischen den Steigungen $\mathrm{zu}$ verdeutlichen, wurde folgendes Balkendiagramm angefertigt.

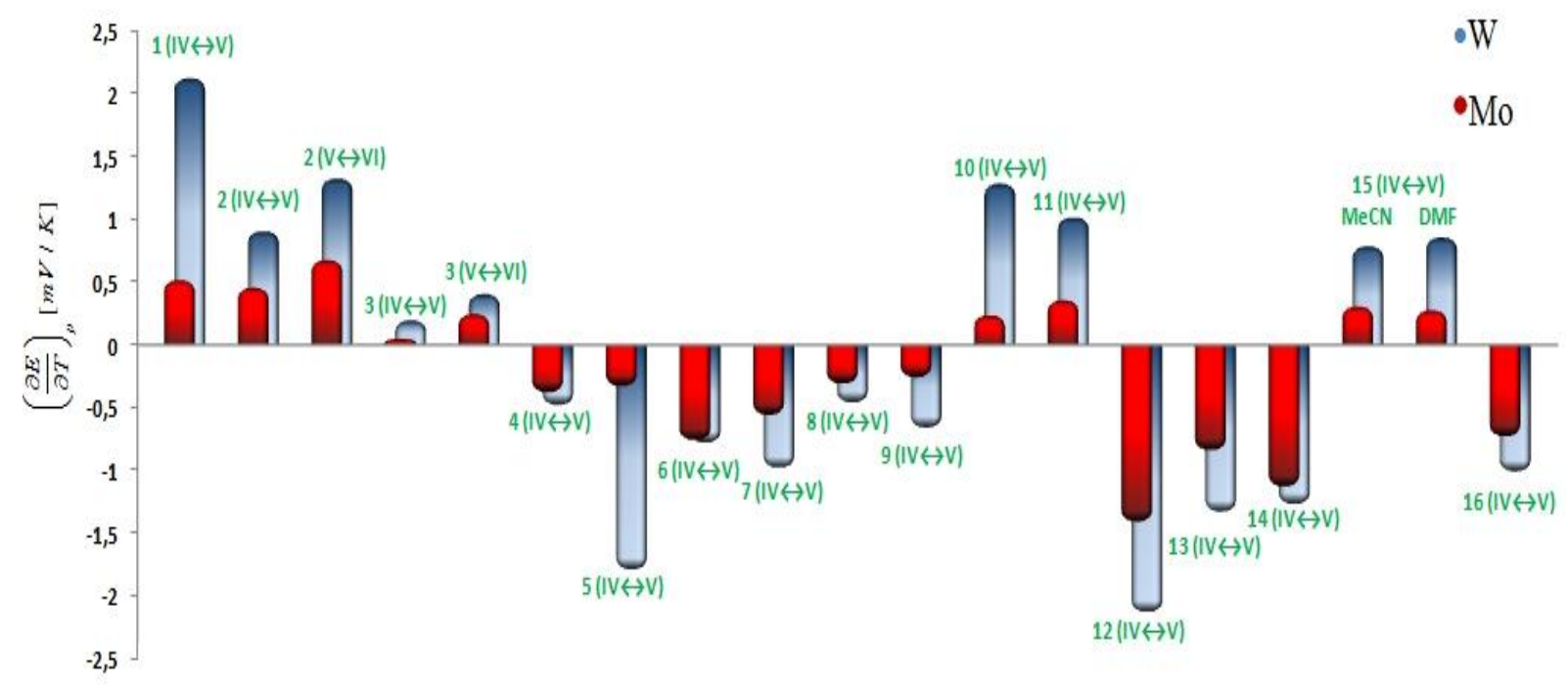

Abb. 84: Erhaltene Steigungswerte im Vergleich

Abhängig von dem Ligandensystem am Metallzentrum kam es zu einer Abnahme (negative Steigung) oder einer Zunahme (positive Steigung) der Redoxpotentiale mit der Temperatur. Es konnte beobachtet werden, dass es bei Verwendung von elektronenziehenden Substituenten am Liganden nur zu einer Abnahme der Redoxpotentiale mit steigender Temperatur kam. Dieses geht einher mit einem Entropienverlust für den Reduktionsvorgang. Das bedeutet, dass bei den Verbindungen mit einer negativen Entropie die Oxidation aus entropischer Sicht favorisiert werden sollte. Dieselben Verbindungspaare zeigen aber auch ein positiveres Potential im Vergleich zu den anderen und haben entsprechend ein negativeres $\Delta \mathrm{G}$ für den Reduktionsvorgang. entropische und enthalpische Beträge in Kombination bewirken also folglich gemeinsam eine Stabilisierung der reduzierten Form. Diese Beobachtung stimmt 
auch damit überein, dass elektronenziehende Liganden Elektronendichte vom Metallzentrum entfernen und somit die Einführung von zusätzlichen Elektronen erleichtern.

\subsection{Unterschiede zwischen Molybdän und Wolframverbindungen}

Da die Redoxpotentiale der Wolframverbindungen immer eine stärkere Temperaturabhängigkeit zeigten, als die der analogen Molybdänverbindung, egal ob redoxaktive Ligandensysteme am Metallzentrum angebunden sind oder nicht, sollte dies einen fundamentalen Unterschied zwischen den zwei Metallen darstellen. Chemisch verhalten sich die beiden Metalle ausgesprochen ähnlich. Der größte Unterschied zwischen diesen beiden Metallen ist, dass die Molybdänverbindung eine höhere Oxidationsfähigkeit mit einem höheren Redoxpotential aufweist. Ein weiterer wichtiger Unterschied ist, dass Wolfram das relativistischere Element der beiden Metalle darstellt. Aufgrund der höheren Ladung des Kerns beim Wolframatom, müssen die umgebenden Elektronen sich schneller im Vergleich zum Molybdän bewegen, um nicht in den Atomkern zu stürzen. Da sich die umgebenden Elektronen mit annährender Lichtgeschwindigkeit bewegen, kommt es zu einer Zunahme der Masse der Elektronen und damit zu einer Abnahme des effektiven Bohr-Radius. ${ }^{[65]}$ Durch diesen Effekt kommt es zu einer Kontraktion der s-Orbitale sowie einiger p-Orbitale. Diese kontrahierten Orbitale können nun die Kernladung effektiv gegen die anderen Orbitale abschirmen, was insbesondere für die d-Orbitale in der Valenzschale von Bedeutung ist. Somit werden die d-Elektronen schwächer an das Metall gebunden und der energetische Abstand zwischen den p und d-Orbitalen wird größer. Dieser Effekt tritt beim Wolfram stärker als beim Molybdän auf, wodurch sich die Valenzelektronen des Wolframs freier bewegen können, im Vergleich zu den kernnahen Elektronen. Dieses wirkt sich zum Beispiel in einer stärkeren Beeinflussbarkeit durch äußere Faktoren (z.B. Temperaturveränderungen) aus und einer größeren Flexibilität bei der Koordinationsgeometrie. Aufgrund des höheren energetischen Niveaus der d-Orbitale des Wolframs, kann auch das kleinere Redoxpotential beim Wolfram erklärt werden, weil die äußeren Elektronen leichter entfernt werden können. Die größere geometrische Flexibilität zeigte sich bei der stärkeren geometrischen Veränderung bei der Oxidation oder Reduktion. Dies wird deutlich, durch die größeren entropischen Werte für die Reduktionsprozesse, die mit einer stärkeren Änderung der Freiheitsgerade einhergehen. 


\subsection{Geometische Änderungen während des Redoxprozesses}

In einigen Fällen werden im Vergleich zu den anderen Messungen beim Wolfram vor allem zu Molybdän relativ breite Signale sowohl für die Oxidation als auch für die Reduktion gefunden. Breite Signale können z.B. durch sehr hohe Probenkonzentrationen entstehen. Durch Messungen in Konzentrationsbereichen von $10^{-4}-10^{-7} \mathrm{~mol} / \mathrm{l}$ konnte allerdings keine Verschmälerung der Signale beobachtet werden. Somit sollte die Verbreiterung nicht auf die Verbindungskonzentration zurückzuführen sein. Speziell bei diesen Verbindungstypen (wie z.B. $\left[\mathrm{MO}(\mathrm{qdt})_{2}\right]^{2-}$ ist ein sehr starker Unterschied zwischen der Temperaturabhängigkeit der Mo- und W-Verbindung festzustellen. Wird eine große Entropieveränderung (eine Zunahme oder eine Abnahme an Freiheitsgeraden) festgestellt, weist dies auf eine große geometrische Veränderung hin. Es ist dabei möglich, dass die geometrische Veränderung langsamer stattfindet, als der eigentliche Redoxübergang, wodurch es zu einer Verbreiterung der Signale kommt. Ein extremes Beispiel, ebenfalls für eine Wolframverbindung, wurde im Jahre 1995 von Lerke und Evans beschrieben. Dabei wurden zwei separate Signale jeweils für die Oxidation und für die Reduktion festgestellt, da sich oxidierte und reduzierte Spezies in unterschiedlichen Geometrien befanden und der Übergang verhältnismäßig langsam stattfand. ${ }^{[66]}$ Im Cyclovoltammogramm wurde durch Veränderung der Vorschubgeschwindigkeiten schließlich doch ein reversibles Signal gefunden. Dies zeigt, dass es sich im Ganzen um einen reversiblen Redoxprozess handelt, auch wenn es bei schnellen Vorschubgeschwindigkeiten nicht so aussieht. Diese Veränderung des irreversiblen Verhaltens konnte bei der Molybdänverbindung $\mathrm{MoCp}^{*} \mathrm{Me}_{4}$ nicht gefunden werden. ${ }^{[67]} \mathrm{Um}$ sich die Redoxchemie dieser Verbindung noch genauer anzuschauen, wurde die Verbindung nach Literaturangaben synthetisiert. Nach genaueren Untersuchungen dieser Verbindung, konnte ein reversibles Signal (siehe $A b b .85$ ) erhalten werden. Dies wiederrum steht im Wiederspruch zu den zuvor publizierten Ergebnissen. Zur weiteren Bestätigung der Struktur wurden Kristalle dieser Verbindung röntgenographisch untersucht und damit konnte belegt werden, dass es sich bei der hergestellten und elektrochemisch untersuchten Verbindung um die zuvor publizierte Struktur handelt. 


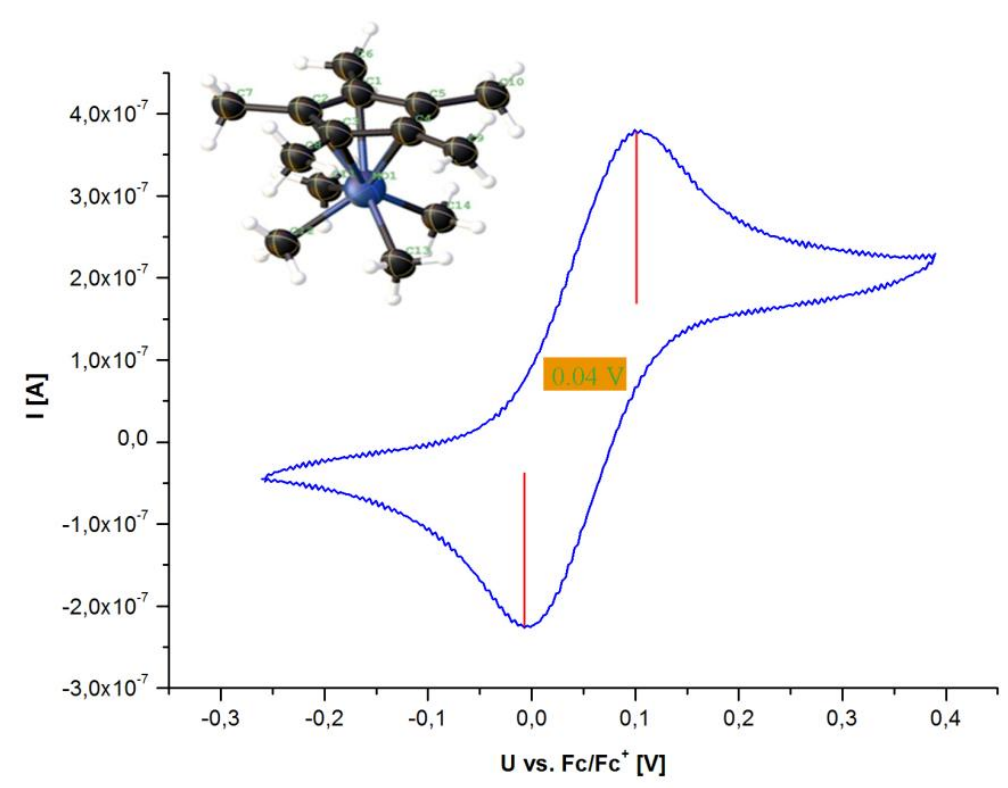

Abb. 85: Reversibles CV-Signal von $\mathrm{MoCp}^{*} \mathrm{Me}_{4}$

\subsection{Weitere Beeinflussungen der Redoxpotentiale}

\subsubsection{Beeinflussung der Potentiale durch das Lösungsmittel}

Um zu untersuchen, ob die verwendeten Lösungsmittel einen Einfluss auf die elektrochemischen Messwerte haben, wurde ein Verbindungspaar $\left(\mathrm{MCp}_{2} \mathrm{Cl}_{2}\right)$ sowohl in Acetonitril als auch in DMF vermessen. Das Ergebnis der beiden Untersuchungen zeigte, dass eine Verschiebung der Potentiale von $60 \mathrm{mV}$ bzw. $70 \mathrm{mV}$, beim Wechsel des Lösungsmittels durch den unterschiedlichen Einfluss der Solvathülle der Proben, auftrat. Wichtig ist hier, dass keine großen Veränderungen bei den Temperaturabhängigkeiten der Redoxpotentiale beobachtet werden, d.h. die ermittelten Entropiewerte sind nicht von den verwendeten Lösungsmitteln beeinflusst.

\subsubsection{Beeinflussung durch den Umgebungsdruck}

Da es durch die Erwärmung der Temperierflüssigkeit und der Probenlösung zu einem Anstieg des Füllgasdrucks in der Glovebox kommt, wurde auch der Einfluss dieses Druckunterschieds näher untersucht. Hierbei wurde Ferrocen in Acetonitril gelöst und das sich ergebene Potential bei Drücken von 0.5 mbar bis 6.0 mbar im Vergleich zum gegenwertigen Außendruck untersucht. In diesem relevanten engen Druckbereich wurden dabei keine Veränderungen der Potentiale beobachtet.

Eine Veränderung der Potentiale durch Druck sollte dementsprechend erst bei sehr großen Drücken zum Tragen kommen, die im Inneren einer Glovebox nicht erreicht werden. Ein 
Einfluss des Druckes auf die Messergebnisse kann im Rahmen dieser Studie ausgeschlossen werden.

\subsubsection{Beeinflussung durch Messgeschwindigkeit und Konzentrationen}

Da die Vorschubgeschwindigkeit einen Einfluss auf das Erscheinungsbild der Cyclovoltammogramme und Differenzpulsvoltammogramme hat und es somit auch zur geringfügigen Veränderung des Potentials während der Redoxprozesse kommen kann, wurden alle vergleichende Paare mit der gleichen Messmethode und den gleichen Messparametern vermessen (siehe dazu Abschnitt 9.8).

Die Intensität sowie die Breite der Messsignale erhöhen sich bei Verwendung von hohen Konzentrationen, wodurch sich der Schwerpunkt des Signals in den einzelnen Spektren auch geringfügig verändern kann. Es wurden daher stets frisch angesetzte Probenlösungen in ausrechender Verdünnung und gleichen Konzentrationen für ein Molybdän-/Wolframpaar verwendet.

\subsection{Einfluss einer Dithioleneinheit}

Um den Einfluss eines Dithiolenligandens im Vergleich zur unsubstituierten Form auf das Redoxpotential des Metallzentrums näher untersuchen $\mathrm{zu}$ können, wurde das Ausgangsmaterial $\left[\mathrm{MoOS}_{8}\right]^{2-}$ (17a) und die hergestellte Monodithiolenverbindung 17b $\left[\mathrm{MoO}(\mathrm{xdt}) \mathrm{S}_{4}\right]^{2-}$ auf ihre Redoxpotentiale hin untersucht. Für die Verbindung 17a konnte ein Potential von $-0.26 \mathrm{~V}$ und für $\mathbf{1 7 b}$ von $-0.53 \mathrm{~V}$ aus Cyclovoltammogrammen bei $25^{\circ} \mathrm{C}$ gegen $\mathrm{Fc} / \mathrm{Fc}^{+}$ermittelt werden. Beim Vergleich der Redoxpotentiale fällt auf, dass es durch das Hinzufügen eines Dithiolenligandens zu einer Abnahme des Redoxpotentials um $0.27 \mathrm{~V}$ kommt. Setzt man das Potential des monosubstituierten Dithiolenliganden 17b in Relation zu dem des symmetrischen $\left[\mathrm{MoO}(\mathrm{xdt})_{2}\right]^{2-}$ - Komplex mit zwei koordinierten xdt-Liganden mit einem Redoxpotential von $-0.85 \mathrm{~V}\left(\mathrm{Fc} / \mathrm{Fc}^{+}\right)$, so ist ein weiteres Absinken des Redoxpotentials um $0.32 \mathrm{~V}$ vorhanden. Da der Unterschied der Redoxpotentiale zum doppelt substituierten Komplex ähnlich zur Koordination eines Dithiolenligandens an ein $\left[\mathrm{MoOS}_{8}\right]^{2-}$ ausfällt, sollte den vorhandenen $\mathrm{S}_{4}$-Ringen nur ein geringfügiger Einfluss zugesprochen werden.

Um auch den Einfluss des Dithiolenliganden auf die Temperaturabhängigkeit der Redoxpotentiale $\mathrm{zu}$ untersuchen, wurden von beiden Verbindungen $\left[\mathrm{MoOS}_{8}\right]^{2-}$ und $\left[\mathrm{MoO}(\mathrm{xdt}) \mathrm{S}_{4}\right]^{2-}$ temperaturabhängige Messungen in einem Temperaturbereich von -20 bis $50^{\circ} \mathrm{C}$ durchgeführt. 


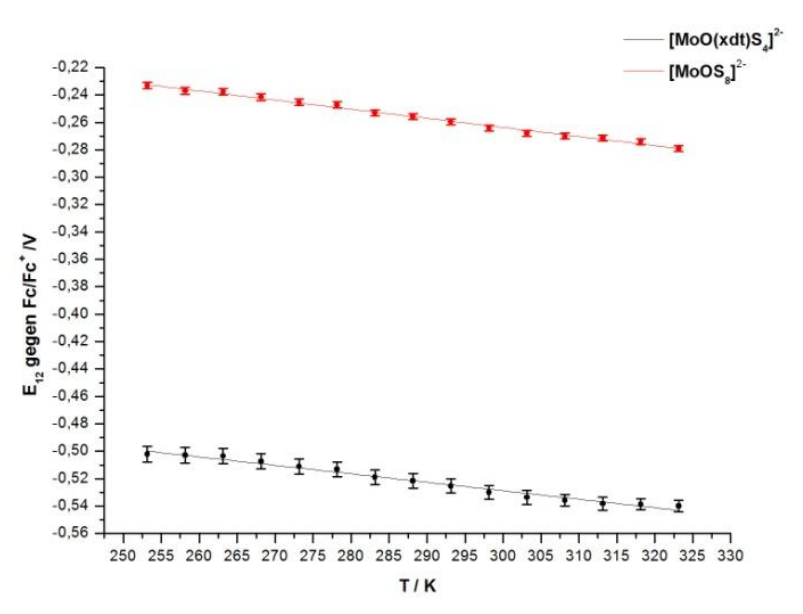

Abb. 86: Temperaturabhängigkeit von $17 \mathrm{a}$ und $17 \mathrm{~b}$

Durch Hinzufügen des Dithiolenligandes an die Verbindung 17a konnte die Abnahme der Redoxpotentiale mit steigender Temperatur, die bei $\left[\mathrm{MoOS}_{8}\right]^{2-}$ mit $-0.665 \mathrm{mV} / \mathrm{K}$ vorhanden war, verringert werden. Somit konnte bei der Verbindung 17b ein stabileres Redoxpotential (-0.617 mV/K) über den untersuchten Temperaturbereich beobachtet werden. Vergleicht man das Ergebnis mit der Temperaturabhängigkeit der Redoxpotentiale des Molybdänsystems mit zwei koordinierten Dithiolenliganden 1a, so wird bei diesem eine deutlich positivere Temperaturabhängigkeit mit $0.52 \mathrm{mV} / \mathrm{K}$ gefunden. Die Wirkung sollte durch nur einen Dithiolenliganden auf die Veränderung der Redoxpotentiale bei Temperaturänderungen nicht so ausgeprägt sein, wie es bei der Koordination durch zwei Dithiolenliganden der Fall ist. Daher sollte die Abhängigkeit auf ein Zusammenspiel von beiden Dithiolenliganden mit dem Metallzentrum zurückzuführen sein.

\subsection{Wirkung von unsymmetrisch substituierten Dithiolenkomplexen auf die Redoxpotentiale}

Um zu untersuchen, ob die Koordination von zwei auch elektronisch unterschiedlichen Dithiolenliganden einen Einfluss auf die Temperaturabhängigkeit der Redoxpotentiale hat, wurde die Verbindung $18\left([\mathrm{MoO}(\mathrm{xdt})(\mathrm{vdt})]^{2-}\right)$ und 19b $\left([\mathrm{MoO}(\mathrm{xdt})(\mathrm{ndt})]^{2-}\right)$ elektrochemisch gemessen. Im Folgenden ist die Abhängigkeit der Potentiale bei Temperaturänderung gezeigt. 


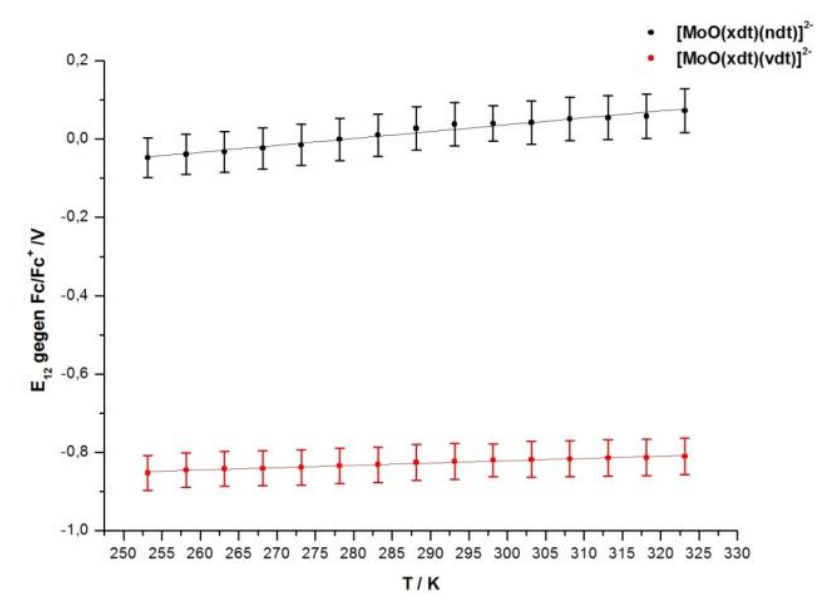

Abb. 87: Temperaturabhängigkeit der Verbindungspaare 18 und 19b

Um den Einfluss durch zwei unterschiedliche Dithiolenliganden auf das unsymmetrische Metallzentrum $\mathrm{zu}$ evaluieren, werden diese mit den symmetrisch substituierten Dithiolenkomplexen verglichen.

\begin{tabular}{|c|c|c|}
\hline Verbindung & $\begin{array}{c}\text { Potentiale } \\
\text { gegen } \mathbf{F c} / \mathbf{F c}^{+}[\mathbf{V}]\end{array}$ & $\begin{array}{c}\text { Temperaturabhängigkeit } \\
{[\mathbf{m V} / \mathbf{K}]}\end{array}$ \\
\hline$\left[\mathbf{M o O}(\mathbf{x d t})_{2}\right]^{2-}(1 \mathrm{a})$ & -0.85 & $0.52 \pm 0.075$ \\
\hline$\left[\mathbf{M o O}(\mathbf{v d t})_{2}\right]^{2-}(3 \mathrm{a})$ & -0.77 & $0.05 \pm 0.075$ \\
\hline$\left[\mathbf{M o O}(\mathbf{n d t})_{2}\right]^{2-}(4 \mathrm{a})$ & -0.28 & $-0.38 \pm 0.056$ \\
\hline$[\mathbf{M o O}(\mathbf{x d t})(\mathbf{v d t})]^{2-}(18)$ & -0.82 & $1.76 \pm 0.078$ \\
\hline$[\mathbf{M o O}(\mathbf{x d t})(\mathbf{n d t})]^{2-}(19 \mathrm{a})$ & 0.04 & $0.59 \pm 0.024$ \\
\hline
\end{tabular}

Tab. 11: Vergleich der Potentiale und Steigungswerte von symmetrisch und unsymmetrisch substituierten Verbindungen

Betrachtet man die Potentiale so ist bei der unsymmetrischen Verbindung 18 keine unerwartete Änderung der Redoxpotentiale im Vergleich zu den symmetrisch substituierten Verbindungen 1a und 3a zu erkennen. Es wird eine Potential gefunden, dass sich genau zwischen den Potentialen der symmetrisch substituierten Verbindungen befindet. Bei der Temperaturabhängikeit der Redoxpotentiale fällt aber auf, dass sich ein sehr viel größerer Steigungswert ergibt, als es bei den Verbindungen 1a und 3a der Fall ist. Die Tendenz einer positiven Steigung blieb aber erhalten. Aufgrund des unterschiedlichen Elektronenschubs der Ligandenssysteme, von beiden Seiten zu dem Metallzentrum, wird es zu einer stärkeren geometrischen Flexibilität kommen, die sich in einer stärkeren Abhängigkeit der Redoxpotentiale bei Temperaturänderung und damit der Entropie widerspiegelt. Bei der Verbindung 19a stellt man hingegen eine größere Veränderung $(0.32 \mathrm{~V})$ der Potentiale im 
Vergleich zu den symmetrischen Verbindungen fest. Diese Verschiebung kann nicht durch die einzelnen elektronischen Einflüsse der Ligandensysteme erklärt werden. Deshalb muss es an der Kombination aus einem elektronenziehenden und einem elektronenschiebenden Ligandensystem am Metallzentrum liegen. Insgesamt wird offenbar die Elektronendichte effizienter vom Metallzentrum abgezogen, als es bei den symmetrischen Komplexen der Fall ist. Unter Umständen sind hier auch Ligandenorbitale stärker an dem Redoxprozess beteiligt, als es bei symmetrischen Dithiolenkomplexen üblich ist. Die deutlich positivere Steigung der Redoxpotentiale ist überraschend, die hier auch die Reduktion entropisch begünstigt ist, was letztlich auch für das verhältnismäßig positive Redoxpotential verantwortlich sein mag. Vorausgesetzt, dass sich $\Delta \mathrm{H}$ nur wenig ändert bei der Verwendung zweier unterschiedlicher Dithiolene.

\subsection{Wirkung von wechselnden Ringatomen im Ligandensystem}

Um die Wirkung von verschiedenen Ringatomen im Ligandensystem in Nachbarschaft $\mathrm{zu}$ der Dithiolenheit $\mathrm{zu}$ untersuchen, wurden die Verbindungspaare $\quad 7 \quad(\mathrm{C}), \quad 8 \quad(\mathrm{O})$ und $\mathbf{9} \quad(\mathrm{S})$ temperaturabhängigen Messungen unterzogen.

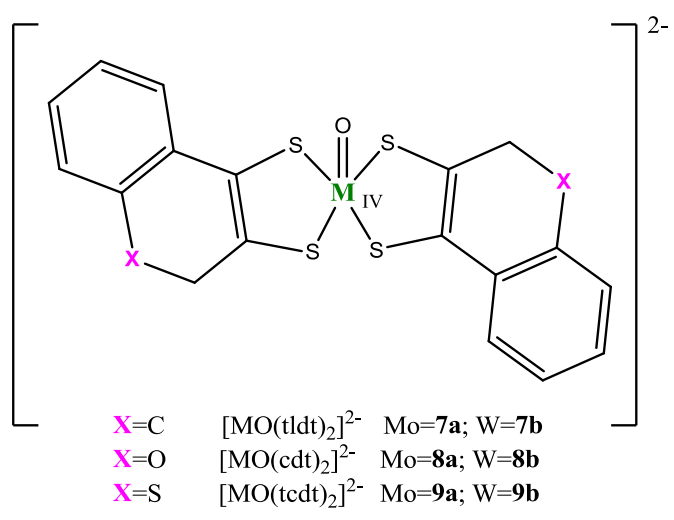

\begin{tabular}{|c|c|c|c|}
\hline Verbindungen & $\begin{array}{c}\mathbf{E}_{\mathbf{1 / 2}}{ }^{\mathbf{0}} \text { gegen } \\
\mathbf{F c}_{\mathbf{F} \mathbf{F c}^{+}}[\mathbf{V}]\end{array}$ & $\begin{array}{c}\mathbf{E}_{\mathbf{1 / 2}}{ }^{\mathrm{W}}-\mathbf{E}_{\mathbf{1 / 2}}{ }^{\mathbf{M o}} \\
{[\mathbf{V}]}\end{array}$ & $\begin{array}{c}\text { Steigungswert } \\
{[\mathbf{m V} / \mathbf{K}]}\end{array}$ \\
\hline $\mathbf{7 a}(\mathbf{I V} \leftrightarrow \mathbf{V})$ & -0.95 & 0.26 & $-0.56 \pm 0.033$ \\
$\mathbf{7 b}(\mathbf{I V} \leftrightarrow \mathbf{V})$ & -1.21 & & $-0.98 \pm 0.061$ \\
$\mathbf{8 a}(\mathbf{I V} \leftrightarrow \mathbf{V})$ & -0.72 & 0.17 & $-0.31 \pm 0.062$ \\
$\mathbf{8 b}(\mathbf{I V} \leftrightarrow \mathbf{V})$ & -0.89 & & $-0.46 \pm 0.045$ \\
$\mathbf{9 a}(\mathbf{I V} \leftrightarrow \mathbf{V})$ & -0.87 & 0.32 & $-0.34 \pm 0.004$ \\
$\mathbf{9 b}(\mathbf{I V} \leftrightarrow \mathbf{V})$ & -1.19 & & $-0.66 \pm 0.008$ \\
\hline
\end{tabular}

Tab. 12: Vergleich der elektrochemischen Messdaten der Verbindungspaare 7, 8, 9

Vergleicht man die erhaltenen elektrochemischen Messdaten, so stellt man fest, dass es bei Verwendung von Ligandenssystemen mit Sauerstoffatomen im Ringsystem zu einem kleineren Abstand, zwischen den Potentialen der Molybdän bzw. der Wolframverbindung, kommt. Somit sollte der cdt-Ligand hier die stärkste non-innocence aufweisen. Da die im Ringsystem eingefügten Atome über unterschiedliche Elektronegativitäten verfügen, sollten diese in unterschiedlichem Maße Elektronendichte von der Dithioleneinheit abziehen, was die 
non-innocence unterstützt durch Stabilisierung radikalischer Resonanzstrukturen. Der Effekt sollte beim Sauerstoffatom, mit der größten Elektronegativität in dieser Reihe, am stärksten ausgeprägt sein, was mit den beobachteten Potentialen übereinstimmt, die für die cdt am positivsten sind. Werden die erhaltenen Temperaturabhängigkeiten der Redoxpotentiale verglichen, so können die größten negativen Steigungen bei den Komplexen 7 mit nur Kohlenstoffatomen im Ringsystem beobachten werden. Die Komplexe 9 mit einem eingebundenen Schwefelatom im Ringsystem zeigen positivere Steigungswerte im Vergleich zu dem Verbindungspaar 7. Bei den Chromanonsystemen 8 sind die am wenigsten negativen Steigungswerte im Vergleich zu den anderen Paaren vorhanden. Das bedeutet, dass die Verwendung des Chromonliganden (cdt) im Vergleich zu einer tendenziellen Stabilisierung der Redoxpotentiale bei Temperaturänderungen führt. Abschließend kann zur Untersuchung dieser sechs Komplexe gesagt werden, dass ein Einfluss auf die Redoxpotentiale, durch die Verwendung von Pyransystemen in Nachbarschaft zur Dithioleneinheit, durchaus nicht zu vernachlässigen ist. Deshalb sollte man bei der Herstellung von Modellsystemen ein Ligandensystem mit ähnlichen elektronischen Eigenschaften verwenden. Ob es wirklich nur der Einfluss des Sauerstoffs, oder am elektronischen Effekt des gesamten Liganden liegt, kann an dieser Stelle nicht abschließend geklärt werden. 


\section{Kapitel 5}

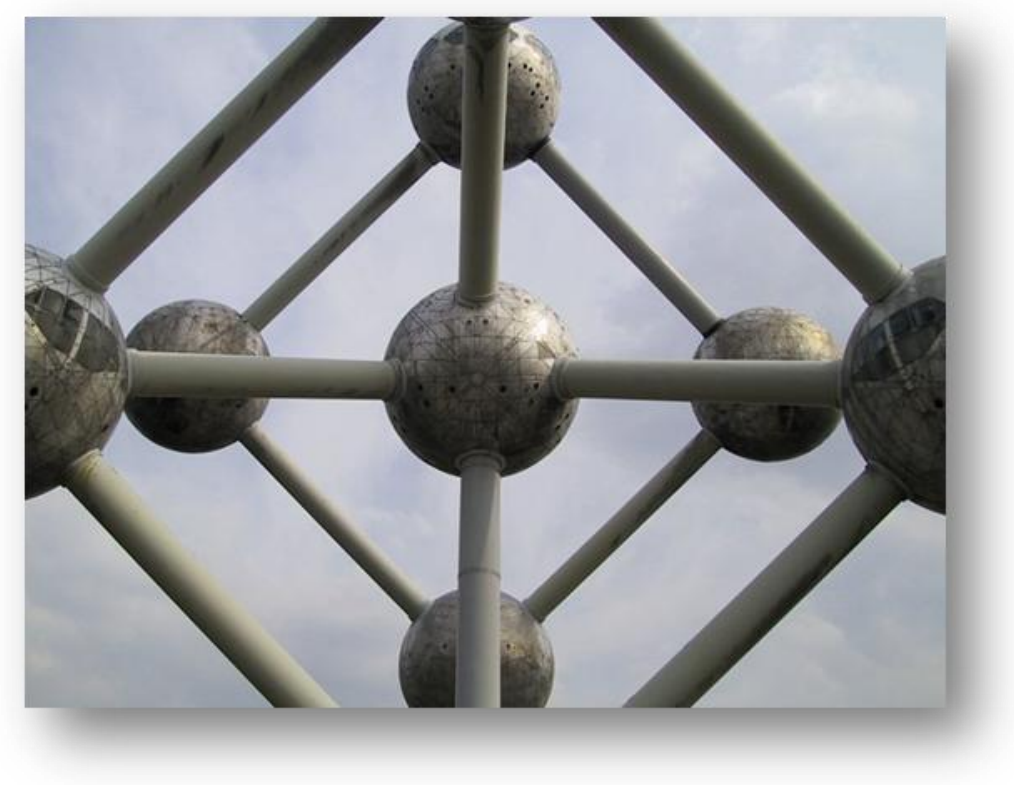

\section{Theoretische Untersuchungen}

\section{Zusammenfassung}

In diesem Kapitel wird mit Hilfe von Dichte-Funktionaltheoretischen-Studien untersucht, welche funktionellen Gruppen in den Ligandensystemen einen Einfluss auf Bindungs/ängen und -winkel um das Metallzentrum herum haben. Außerdem werden die HOMOs und LUMOs bestimmt, um eine Verbindung zu den elektronischen Gegebenheiten in den biologischen Systemen zu ziehen. Das Kapitel schließt mit den von mir durch DFT-Rechnungen bestimmten Redoxpotentialen im Vergleich zu den experimentellen Messungen. Außerdem wird ein Vergleich zu den temperaturabhängigen elektrochemischen Untersuchungen gezogen. 


\subsection{Geometrische Untersuchungen}

Die Geometrie des Metalls beim Entfernen oder Hinzufügen eines Elektrons bei quadratischpyramidalen Oxo-Komplexen von Molybdän und Wolfram ändert sich drastisch. ${ }^{[52]}$ Werden die berechneten Geometrien von $\left[\mathrm{MoO}(\mathrm{xdt})_{2}\right]^{\mathrm{x}-}$ mit $\mathrm{X}=-2,-1,0$ exemplarisch betrachtet, so erkennt man, dass die Dithiolenliganden sich bei Oxidation von $(+\mathrm{IV} \rightarrow+\mathrm{VI})$ aus der Ebene entfernen.

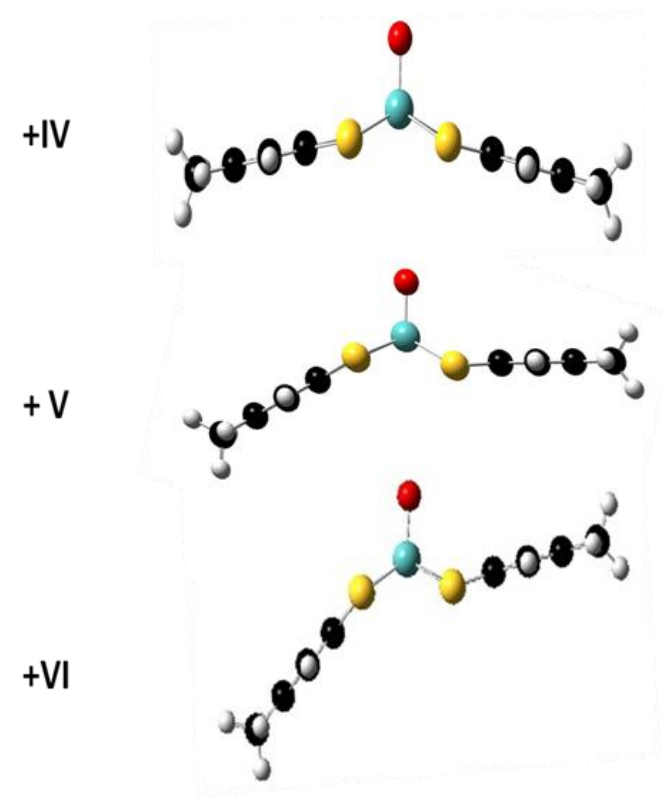

Abb. 88: Berechnete Geometrieveränderungen bei der Oxidation der Vebindung $\left[\mathrm{MoO}(\mathrm{xdt})_{2}\right]^{\mathrm{x}-} ; \mathrm{x}=2,1,0$

\subsection{Faltungswinkel}

Das Abknicken eines Dithiolenligandens bei Oxidationsstufenveränderungen des Metallzentrums wurde bei verschiedenen Komplexen beobachtet. ${ }^{[68]}$ Der Faltungswinkel als struktureller Parameter wurde erstmals von Lauher und Hoffmann bei der Untersuchung von Dithiolenverbindungen eingeführt. ${ }^{[69]}$ Er ist definiert durch den Winkel, der sich zwischen der M-S-S-Fläche und der abgeknickten $\mathrm{S}-\mathrm{C}=\mathrm{C}-\mathrm{S}-$ Fläche ergibt. Während sich der Dithiolenligand auf einer Seite zum Metall neigt, kommt es auf der anderen Seite des Metalls zum Abknicken des zweiten Liganden vom Metall weg. Daher ergeben sich zwei Faltungswinkel $\eta$ und $\chi$. Im Folgenden sind die definierten Faltungswinkel angegeben.
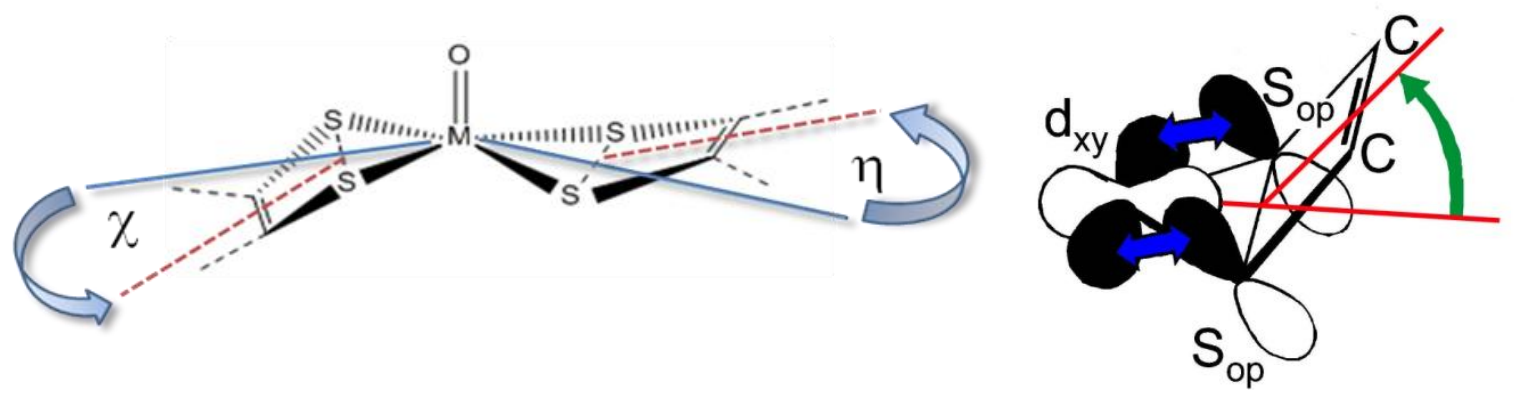

Abb. 89: Definition des Faltungswinkels (links) und Wechselwirkung der Orbitale (rechts) 
Der Faltungswinkel ist bei Bisdithiolenkomplexen mit quadratisch pyramidaler Geometrie von der OS des Metallzentrums abhängig. ${ }^{[70]}$ Das Abknicken des Dithiolenliganden zum Metallzentrum bewirkt eine bessere $\pi$-Wechselwirkung des Metall-d-Orbitals mit den p-Orbitalen der Schwefelatome. Durch diese stärkere Überlappung wird eine günstigere Verteilung der Elektronendichte ermöglicht, der besonders bei hohen Oxidationsstufen $(+\mathrm{V}$ $\left[\mathrm{d}^{1}\right]$ und $\left.+\mathrm{VI}\left[\mathrm{d}^{0}\right]\right)$ zu einer größeren Stabilisierung führt. In $A b b .90$ sind die bestimmten Faltungswinkel $\eta$ von zwanzig Modellverbindungen, im Vergleich mit den entsprechenden Komplexen des biologischen Molybdopterinligandens [(Mo)-mpt] (oberes Bild) bzw. [(W)-mpt] (unteres Bild) Zentrum, dargestellt. Die berechneten Bindungsparameter aller untersuchten Verbindungen sind im Anhang unter Abschnitt $11.3 \mathrm{zu}$ finden.
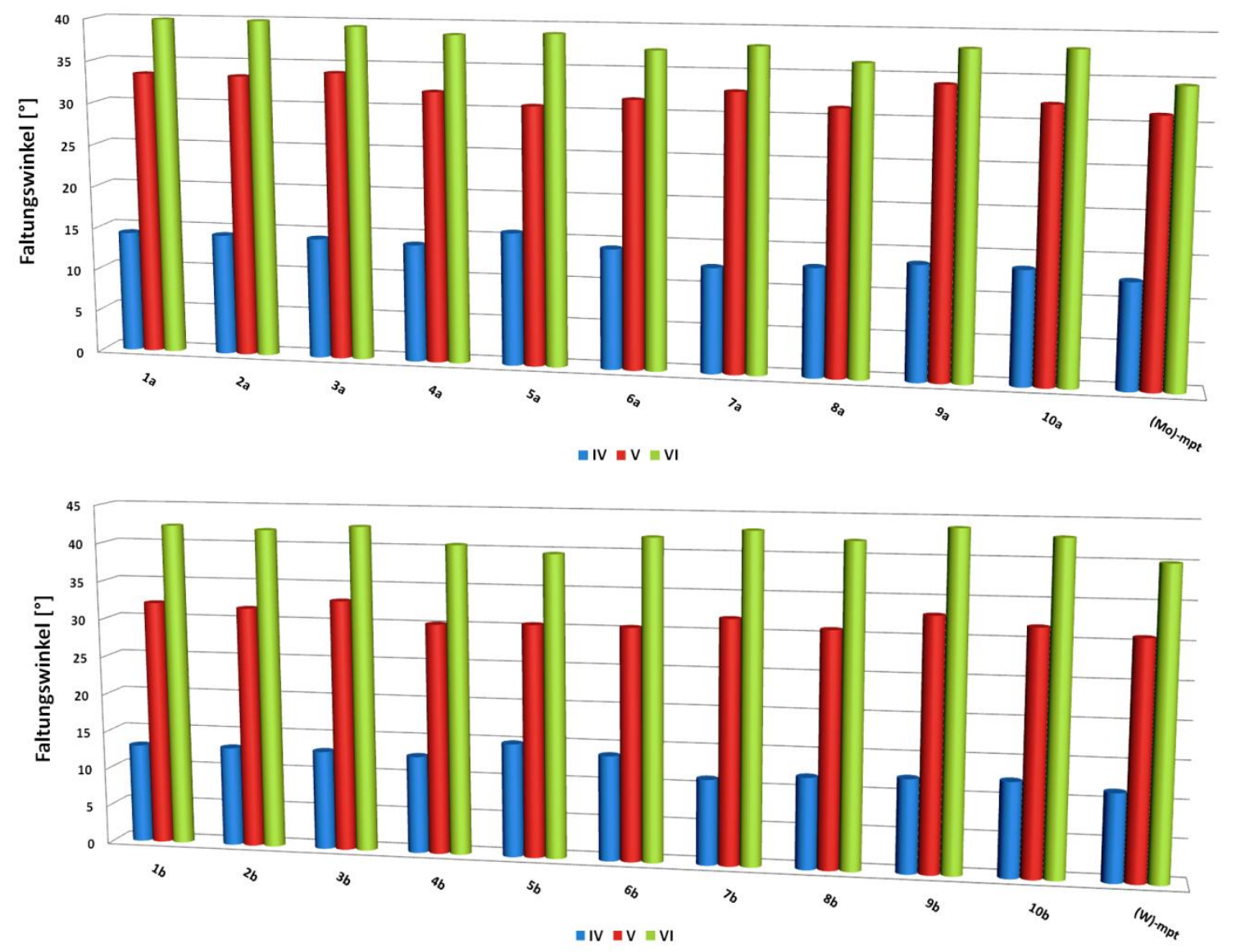

Abb. 90: Berechnete Faltungswinkel hin zur $M=0$ Einheit; Mo-Verbindungen im Vergleich zu Mo-mpt (oben); W-Verbindungen im Vergleich $\mathbf{W}$-mpt (unten)

Hierzu wurde folgende Anordnung des Molybdopterinligandes für die Berechnungen verwendet, auf die Anbindung von Phosphat oder ähnlichen Gruppen wurde verzichtet. 


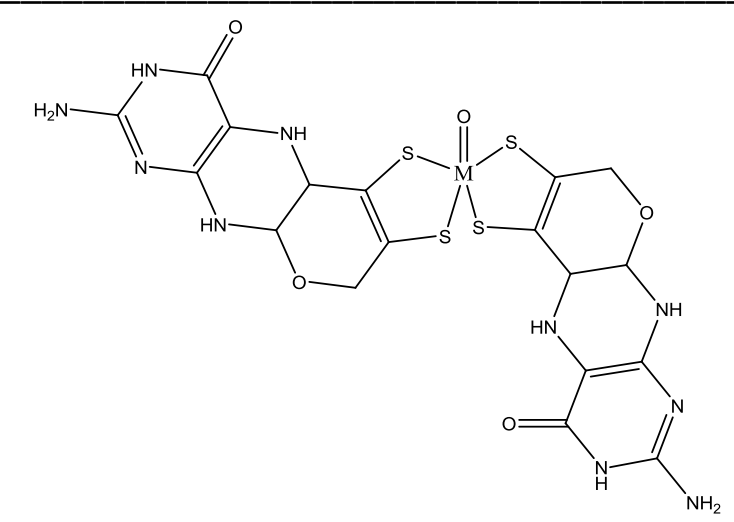

Abb. 91: Verendete Struktur zur Simulation des Molybdopterin-Kofaktors

Vergleicht man nun die Faltungswinkel der einzelnen Verbindungen, so kann bei allen Verbindungen eine ähnliche Faltung beobachtet werden. In Oxidationsstufe IV werden Winkel von $10-16^{\circ}$ gefunden. Beim Übergang $\mathrm{zu}$ Oxidationsstufe $\mathrm{V}$ kommt es $\mathrm{zu}$ Abwinkelungen um $27-33^{\circ}$, bis bei Oxidationsstufe VI Winkel von $35-43^{\circ}$ gefunden werden. Es konnte ähnlich wie in vorangegangen Untersuchungen ${ }^{[71]}$ gezeigt werden, dass bei Ligandensystemen bei denen die Dithioleneinheit Teil eines Pyransystems 8a ist, es zu den größten Ähnlichkeiten zu den mpt-Komplexen, besonders bei den Molybdänkomplexen kommt. Bei Verwendung von aromatischen Dithiolenliganden bei den Molybdänverbindungen werden besonders in der OS VI große Abweichungen zu den mpt-Systemen festgestellt. Somit sollten die aromatischen Liganden nicht die beste Nachbildung des Molybdopterinligandes für Molybdänverbindungen in hohen OS darstellen. Doch bei den Wolframverbindungen zeigte sich, dass besonders die Verbindungen $\mathbf{4 b}\left[\mathrm{WO}(\mathrm{ndt})_{2}\right]^{\mathrm{x}-}$ und $\mathbf{5 b}$ $\left[\mathrm{WO}(\mathrm{qdt})_{2}\right]^{\mathrm{x}-}$ eine bessere Nachbildung als die Pyranringsysteme mit Dithioleneinheit zeigen. Da aber die aromatischen Ligandensysteme mit elektronenschiebenden Substituenten zu größeren Abweichungen führen, ist der elektronenziehende Charakter der CN-Liganden mit entscheidend. Durch den Elektronenzug sollte es zur Verringerung der Ladung in den pOrbitalen der Schwefelatome kommen, wodurch gerade der bestmögliche Faltungswinkel erzielt wird. Weiterhin wurden Untersuchungen $\mathrm{zu}$ dem elektronischen Einfluss der Substituenten an den aromatischen Dithiolenliganden durchgeführt, die unter Punkt 5.3.1 zu finden sind.

\subsection{Elektronische Verhältnisse in den Komplexen}

Mit Hilfe der „Natural population analysis“ (NPA) wurde die Elektronendichte in den einzelnen Komplexen mit aromatischen Dithiolenliganden näher untersucht. Die Veränderung der elektronischen Zustände wurde bei der Oxidation von +IV nach +VI betrachtet. 
Insbesondere wurden die Veränderungen der Ladungen am Metallzentrum, dem Sauerstoffatom und den Schwefelatomen betrachtet.

\subsubsection{Vergleich der Verbindungen mit aromatischen Dithiolenverbindungen}

Durch Vergleich dieser relativ ähnlichen Verbindungen soll der Einfluss der am aromatischen

System substituierten Reste (Methyl, Methoxy, Nitril) untersucht werden.

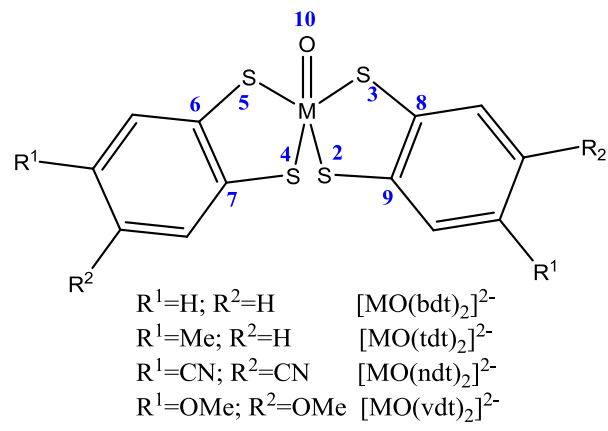

Abb. 92: Nummerierung der Atome

\begin{tabular}{|c|c|c|c|c|c|c|c|c|c|c|c|c|}
\hline & \multicolumn{3}{|c|}{$\left[\mathrm{MoO}(x d t)_{2}\right]^{2-}$} & \multicolumn{3}{|c|}{$\left[\mathrm{WO}(\mathrm{xdt})_{2}\right]^{2-}$} & \multicolumn{3}{|c|}{$\left[\mathrm{MoO}(\mathrm{tdt})_{2}\right]^{2-}$} & \multicolumn{3}{|c|}{$\left[\mathrm{WO}(\mathrm{tdt})_{2}\right]^{2-}$} \\
\hline & OS4 & OS5 & OS6 & OS4 & OS5 & OS6 & OS4 & OS5 & OS6 & OS4 & OS5 & OS6 \\
\hline$M(1)$ & 0.54 & 0.75 & 0.61 & 0.69 & 0.97 & 0.89 & 0.55 & 0.75 & 0.66 & 0.69 & 0.98 & 0.90 \\
\hline $0(10$ & -0.57 & -0.52 & -0.45 & -0.66 & -0.62 & -0.55 & -0.57 & -0.52 & -0.44 & -0.66 & -0.62 & -0.55 \\
\hline$S(2)$ & -0.18 & -0.08 & 0.08 & -0.20 & -0.12 & 0.04 & -0.18 & -0.08 & 0.07 & -0.20 & -0.12 & 0.06 \\
\hline$s(3)$ & -0.18 & -0.08 & 0.08 & -0.20 & -0.12 & 0.04 & -0.18 & -0.08 & 0.07 & -0.20 & & 0.05 \\
\hline$S(4)$ & -0.18 & -0.0 & 0.10 & -0.20 & -0.12 & 0.05 & -0.18 & -0.08 & 0.09 & -0.20 & -0 & 0.04 \\
\hline$S(5)$ & -0.18 & -0.0 & 0.10 & -0.20 & -0.12 & 0.05 & -0.18 & -0.08 & 0.10 & -0.20 & -0 & 0.04 \\
\hline$C(6)$ & -0.18 & -0.19 & -0.19 & -0.18 & -0.18 & -0.19 & -0.18 & -0.20 & -0.20 & -0.18 & -0.19 & -0.20 \\
\hline$C(7)$ & -0.18 & -0.19 & -0.19 & -0.18 & -0.18 & -0.19 & -0.17 & -0.19 & -0.19 & -0.17 & -0.18 & -0.19 \\
\hline$C(8)$ & -0.18 & -0.19 & -0.19 & -0.18 & -0.18 & -0.20 & -0.18 & -0.20 & -0.21 & -0.18 & -0.19 & -0.19 \\
\hline$c(9)$ & -0.18 & -0.19 & -0.19 & -0.18 & -0.18 & -0.20 & -0.17 & -0.19 & -0.19 & -0.17 & -0.18 & -0.20 \\
\hline
\end{tabular}

\begin{tabular}{|c|c|c|c|c|c|c|c|c|c|c|c|c|}
\hline & \multicolumn{3}{|c|}{$\left[\mathrm{MoO}(\mathrm{ndt})_{2}\right]^{2-}$} & \multicolumn{3}{|c|}{$\left[\mathrm{WO}(\mathrm{ndt})_{2}\right]^{2-}$} & \multicolumn{3}{|c|}{$\left[\mathrm{MoO}(\mathrm{vdt})_{2}\right]^{2-}$} & \multicolumn{3}{|c|}{$\left[\mathrm{WO}(\mathrm{vdt})_{2}\right]^{2-}$} \\
\hline & OS4 & OS5 & OS6 & OS4 & OS5 & OS6 & OS4 & OS5 & OS6 & OS4 & OS5 & OS6 \\
\hline$M(1)$ & $\overline{0.52}$ & $\overline{0.77}$ & $\overline{0.64}$ & $\overline{0.69}$ & $\overline{0.98}$ & $\overline{0.92}$ & $\overline{0.54}$ & $\overline{0.73}$ & $\overline{0.58}$ & $\overline{0.68}$ & $\overline{0.97}$ & $\overline{0.87}$ \\
\hline$O(10)$ & -0.53 & -0.49 & -0.41 & -0.63 & -0.59 & -0.52 & -0.56 & -0.53 & -0.46 & -0.66 & -0.62 & -0.56 \\
\hline$S(2)$ & -0.12 & -0.04 & 0.14 & -0.14 & -0.08 & 0.10 & -0.19 & -0.08 & 0.08 & -0.20 & -0.12 & 0.03 \\
\hline$S(3)$ & -0.12 & -0.04 & 0.14 & -0.14 & -0.08 & 0.10 & -0.19 & -0.08 & 0.08 & -0.20 & -0.12 & 0.03 \\
\hline$S(4)$ & -0.12 & -0.04 & 0.11 & -0.14 & -0.07 & 0.07 & -0.19 & -0.08 & 0.06 & -0.20 & -0.13 & 0.04 \\
\hline$S(5)$ & -0.12 & -0.04 & 0.11 & -0.14 & -0.07 & 0.07 & -0.19 & -0.08 & 0.06 & -0.20 & -0.13 & 0.04 \\
\hline$C(6)$ & -0.17 & -0.18 & -0.19 & -0.17 & -0.18 & -0.19 & -0.18 & -0.20 & -0.19 & -0.19 & -0.18 & -0.19 \\
\hline$C(7)$ & -0.17 & -0.18 & -0.19 & -0.17 & -0.18 & -0.19 & -0.18 & -0.20 & -0.19 & -0.19 & -0.19 & -0.19 \\
\hline$C(8)$ & -0.17 & -0.19 & -0.19 & -0.17 & -0.18 & -0.19 & -0.18 & -0.20 & -0.19 & -0.19 & -0.19 & -0.19 \\
\hline$C(9)$ & -0.17 & -0.19 & -0.19 & -0.17 & -0.18 & -0.19 & -0.18 & -0.20 & -0.19 & -0.19 & -0.19 & -0.19 \\
\hline
\end{tabular}

Tab. 13: Bestimmte NPA-Ladung für Komplexe mit aromatischen Ligandensystemen

Die Änderung der Ladung ist besonders am jeweiligen Metall, sowie den direkt gebundenen Atomen stark ausgeprägt, während die an die C-C-Doppelbindung angrenzenden Atome weniger in den Ladungstransfer involviert sind. Die Metalle weisen eine positive Ladung im 
Bereich von 0.5 bis 1 auf. Beide Metalle unterscheiden sich durch alle OS hindurch in der Ladung um etwa 0.2 atomare Einheiten, zugunsten einer positiveren Ladung beim Wolfram. Dies ist auf die stärkere Abschirmung der Valenzorbitale bei Wolfram anhand von relativistischen Effekten zu erklären.

Bei der Oxidation des Metallzentrums von Oxidationsstufe IV zu V wird eine Zunahme der Ladung am Metallzentrum festgestellt. Beim Übergang zu der Oxidationsstufe VI, kommt es wieder zur Verringerung der Ladung. Dies ist auf eine stärkere Verschiebung der Elektronendichte von den Liganden auf das Metallzentrum zurückzuführen und steht im Einklang mit den berechneten Faltungswinkeln. Formal werden von dem Ligandensystem im Durchschnitt 3.5 Elektronen (Oxidationsstufe +IV), 4.5 (Oxidationsstufe +V) bzw. 5.5 Elektronen in der Oxidationsstufe +VI übertragen. Dieser Effekt ist beim Wolframatom stärker als beim Molybdänatom ausgeprägt. Analog dazu stellt man eine Abnahme der negativen Ladung an den Schwefelatomen fest, wenn es zur Oxidation des Metallzentrums kommt. Werden die Ladungen der vier Schwefelatome im Vergleich zu denen der Sauerstoffatome betrachtet, wird beim Sauerstoff nur einer Veränderung von ca. 0.1 bis $0.15 \mathrm{au}$ im Zuge der Oxidation festgestellt. Bei den Schwefelatomen kommt es zu einer ausgeprägten Veränderung der Ladung, die in einem Bereich von etwa $0.2-0.3$ au pro Schwefel liegt. Wird berücksichtigt, dass pro Molekül vier Schwefelatome, jedoch nur ein Sauerstoff beteiligt sind, ergibt sich über die Dithioleneinheit ein recht hoher Ladungstransfer. Dieser Transfer ist auch auf der auf die non-innocence dieses Ligandensystems zurückzuführen. Weiterhin ist in Oxidationsstufe VI festzustellen, dass die Ladungen an den Schwefelatomen nicht mehr gleich erscheinen wie es durchgängig in der Oxidationsstufe IV und V gewesen ist. Diese Beobachtung ist auf die stärkere Faltung des Moleküls in der Oxidationsstufe VI zurückzuführen. Aufgrund der stärkeren Überlappung mit den $\pi$-Orbitalen kommt es zur Veränderung der Elektronendichte an diesen Zentren. Bei der Betrachtung der erhaltenen Bindungslängen der einzelnen Verbindungen in den verschiedenen Oxidationsstufen und der Veränderung der Elektronendichte beim Oxidationsprozess, kann festgestellt werden, dass beim Abzug von Elektronen aus dem Molekül die direkt an das Zentralmetall gebunden Bindungen kürzer werden. Dadurch wird die verbleibende Elektronendichte besser genutzt. Elektronen aus dem $\pi$-Rückgrat der C-C-Doppelbindung werden dabei mit einbezogen. Der angebundene Dithiolenligand, wirkt als $\pi$-Donator. Diese Wirkung wird außerdem durch die leicht verfügbaren $\pi$-Elektronen der C-C-Doppelbindung verstärkt. 
Betrachtet werden nun die Auswirkungen auf die Elektronendichte durch die Substitution des aromatischen Dithiolensystems durch verschiedene elektronenziehende, sowie elektronenschiebende Substituenten. So ist festzustellen, dass die elektronische Wirkung dieser Gruppen nur über wenige Atome im Ringsystem beobachtet werden kann. Die Substitution durch Methylgruppen (mit einen + I-Effekt) am aromatischen System zeigt nur geringfügig höhere Ladungen an den Schwefelatomen, als es bei den unsubsituierten bdt-Komplexen der Fall ist. Es sind Veränderungen der Ladungen an den Schwefelatomen im Durchschnitt von 0.03 au zu finden. Die Auswirkung der Substituenten nimmt bei höheren OS ab, wobei nur noch kleine Änderungen der Ladungen zu beobachten sind.

\begin{tabular}{|c|c|c|c|c|c|c|}
\hline & \multicolumn{3}{|c|}{$\left[\mathrm{MoO}(\mathrm{bdt})_{2}\right]^{2-}$} & \multicolumn{3}{|c|}{$\left[\mathrm{WO}(\mathrm{bdt})_{2}\right]^{2-}$} \\
\hline & $\underline{\text { OS4 }}$ & $\underline{\text { OS5 }}$ & $\underline{\text { OS6 }}$ & $\underline{\text { OS4 }}$ & $\underline{\text { OS5 }}$ & $\underline{0 S 6}$ \\
\hline$M(1)$ & 0.54 & 0.75 & 0.61 & 0.69 & 0.97 & 0.90 \\
\hline$O(10)$ & -0.57 & -0.52 & -0.44 & -0.67 & -0.62 & -0.55 \\
\hline$S(2)$ & -0.15 & -0.07 & 0.09 & -0.18 & -0.11 & 0.06 \\
\hline$S(3)$ & -0.16 & -0.07 & 0.08 & -0.19 & -0.10 & 0.06 \\
\hline$S(4)$ & -0.16 & -0.07 & 0.10 & -0.19 & -0.10 & 0.04 \\
\hline$S(5)$ & -0.15 & -0.07 & 0.11 & -0.18 & -0.11 & 0.05 \\
\hline$C(6)$ & -0.19 & -0.19 & -0.20 & -0.19 & -0.19 & -0.20 \\
\hline$C(7)$ & -0.19 & -0.20 & -0.20 & -0.19 & -0.19 & -0.20 \\
\hline$C(8)$ & -0.19 & -0.19 & -0.20 & -0.19 & -0.19 & -0.20 \\
\hline$C(9)$ & -0.19 & -0.20 & -0.20 & -0.19 & -0.19 & -0.20 \\
\hline
\end{tabular}

Tab. 14: Bestimmte NPA-Ladungen für die $\left[\mathrm{MO}(\mathrm{bdt})_{2}\right]^{\mathrm{X}-}-$ Komplexe

Unabhängig davon, ob ein oder zwei Methylgruppen am aromatischen System substituiert wurden, konnten keine Veränderungen der Ladungen an den Schwefelatomen beobachtet werden. Es sind lediglich, aufgrund der unsymmetrischen Substitution am Aromaten, bei den Ladungen an der $\mathrm{C}=\mathrm{C}$-Doppelbindung zwei unterschiedliche Ladungswerte für die Kohlenstoffatome festzustellen, die bei den symmetrisch substituierten Systemen nicht auftreten. Gleiche Tendenzen konnten bei den aromatischen Systemen mit Methoxy-Gruppen beobachtet werden. Aufgrund des + M-Effekt dieser Gruppe ist die elektronische Wirkung stärker im Ringsystem ausgeprägt, wodurch geringfügig größere Ladungswerte bei den Schwefelatomen $(0.01 \mathrm{au})$ bei der Molybdänverbindung im Vergleich zu den methylsubstituierten Liganden beobachtet werden. Bei den aromatischen Systemen mit Nitrilen, kommt es aufgrund des -I und -M-Effekts zu einer Verringerung der Ladung an den Schwefelatomen (um ca. $0.03 \mathrm{au}$ ). Außerdem kommt es zur Verminderung der negativen Ladung an der Doppelbindung der Dithioleneinheit. Somit treten durch die verschiedenen Substitutionen des aromatischen Dithiolensystems hauptsächlich nur Veränderung an den 
Schwefelatomen auf, wodurch die unterschiedlichen Faltungswinkel in den Systemen, als auch die unterschiedliche Reaktivität zustande kommen.

\subsection{Untersuchung der redoxaktiven Orbitale}

Um das Redoxverhalten von Verbindungen zu untersuchen, müssen die relevanten Energien von HOMO, SOMO und LUMO bestimmt werden. Diese Orbitale interagieren mit redoxaktiven Spezies im Zuge von Redoxreaktionen. Der energetische Unterschied zwischen HOMO und LUMO bestimmt die unterschiedliche Redoxaktivität dieser Verbindungen. Die erhaltenen Orbitalenergien, sowie die energetischen Unterschiede zwischen dem HOMO und dem LUMO sind im Anhang im Abschnitt $11.3 \mathrm{zu}$ finden. Zur besseren Übersicht werden hier nur die erhaltenen Energiewerte im folgenden Balkendiagramm dargestellt. Die Bezeichnungen wurden analog $\mathrm{zu}$ den in den vorherigen Kapiteln definierten Nummerierungen verwendet. Dabei weisen alle Verbindungen mit einem „a“ als Index auf Molybdänverbindungen hin, die mit „, b“ versehenden auf Wolfram.

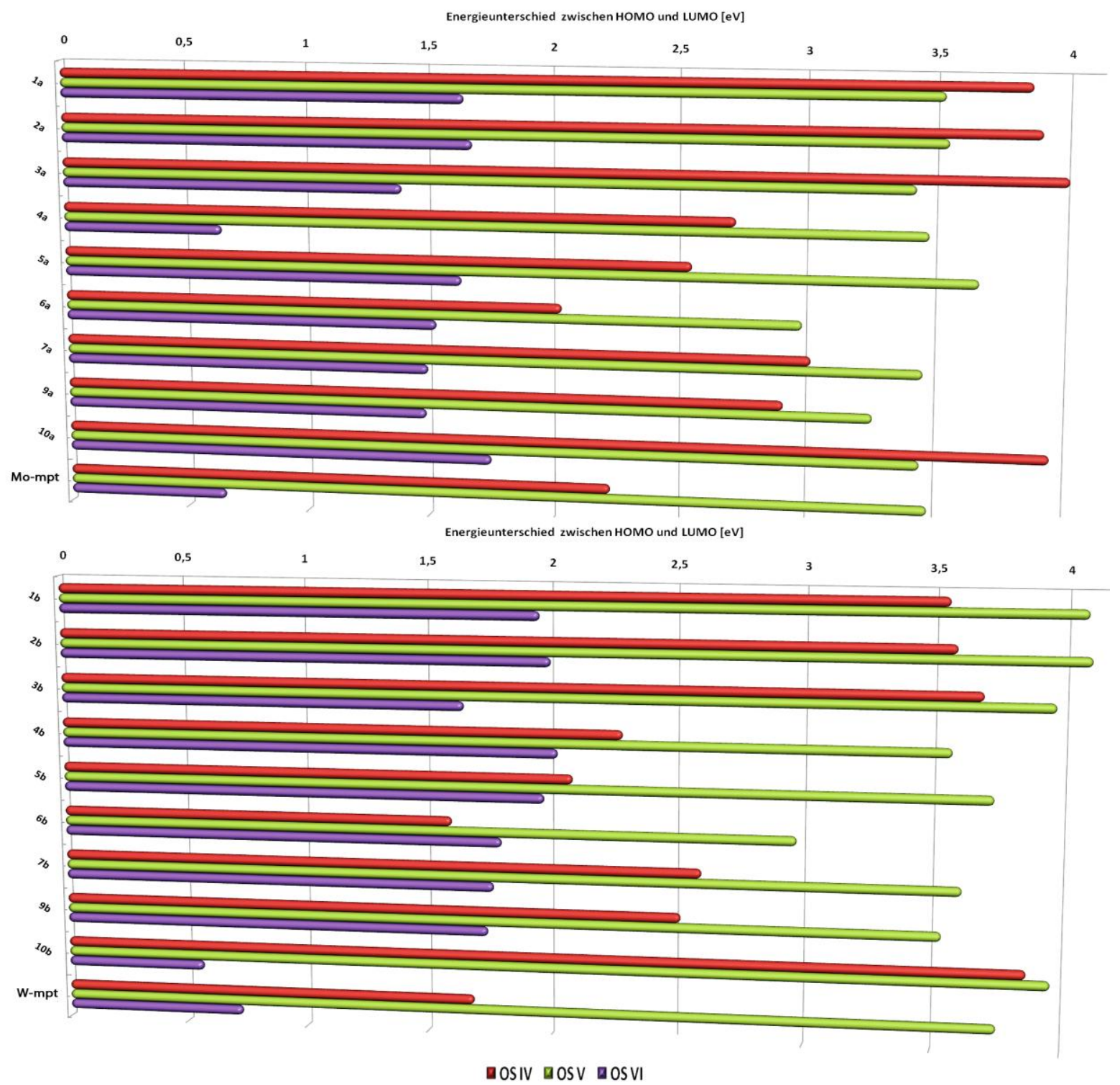


Werden die Orbitalenergien der HOMOs und LUMOs betrachtet, so fällt auf, dass die hier berechneten Orbitalenergien wesentlich größer ausfallen, als die in vergleichbaren Nickelkomplexen mit Dithiolenliganden $\left[\mathrm{Ni}\left(\mathrm{S}_{2} \mathrm{C}_{2} \mathrm{Me}_{2}\right]\right.$ die sich überlicherweise im Bereich von $~ 0.2$ Hartree befinden. ${ }^{[72]}$ Bei der Aufspaltung zwischen dem HOMO und LUMONiveau bei den Molybdän- und Wolframverbindungen der OS IV, treten generell kleinere Werte bei den Wolframverbindungen im Vergleich zu dem Molybdänverbindungen auf. Der Wechsel zu den OS V und VI führt zu einer Abnahme der Aufspaltung zwischen dem HOMO

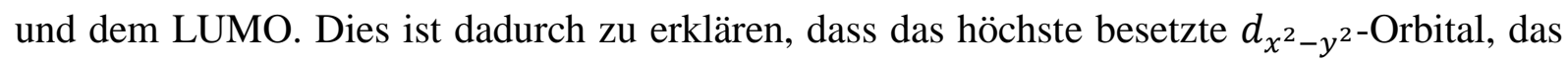
in der OS IV und V mit zwei bzw. einem Elektron besetzt ist, nach der Abgabe aller Elektronen zum LUMO wird. Durch die größere Kernladung wird das LUMO energetisch abgesenkt. Vielfach wurde etwa die halbe Orbitalaufspaltungsenergie bei OS VI im Vergleich zu OS IV gefunden.

Im Hinblick auf die energetischen Unterschiede zwischen HOMO und LUMO werden die theoretischen Werte der mpt-Verbindungen beim Molybdän am ehesten durch $\left[\mathrm{MoO}(\mathrm{ndt})_{2}\right]^{\mathrm{x}-}$ (4a) und beim Wolfram durch $\left[\mathrm{WO}(\mathrm{qdt})_{2}\right]^{\mathrm{X}-}(\mathbf{5 b})$ wiedergegeben. Die Zuordnung ist bei den Wolframverbindungen nicht einfach zu treffen, da auch bei der $\left[\mathrm{WO}(\mathrm{qdt})_{2}\right]^{\mathrm{x}-}-$ Unterschiede in der OS VI auftreten. Bei diesen Systemen 4a $\left(-0.28 \mathrm{~V}\right.$ gegen $\left.\mathrm{Fc} / \mathrm{Fc}^{+}\right)$und $\mathbf{5 b}(-0.67 \mathrm{~V}$ gegen $\mathrm{Fc} / \mathrm{Fc}^{+}$) sollte folglich das Redoxverhalten des freien Kofaktors am besten wiedergegeben werden. Auch schon durch die Bestimmung der Faltungswinkel für die Wolframverbindungen konnte gezeigt werden, dass die $\left[\mathrm{WO}(\mathrm{qdt})_{2}\right]^{\mathrm{x}-}$ ein gutes Modell zur Nachbildung des biologischen Molybdopterinligandens darstellt. Durch die Anbindung des QuinoxalinSystems an die Dithiolenbindung sollten die elektronischen Gegebenheiten gut nachbilden werden können, da sich hierdurch eine ähnliche energetische Abfolge der Redoxorbitale ergibt. Auch die Verwendung des qdt-Ligandens bei Molybdänsystemen zeigt im Vergleich zu den Orbitalaufspaltungen des Mo-mpt-Systems nicht sehr große Abweichungen, wodurch ein weiterer Hinweis für ein gutes Ligandenmodell für Molybdopterinhaltige Enzymsysteme gegeben ist. Aufgrund der ähnlichen Energieunterschiede zwischen HOMO und LUMO bei den Systemen mit qdt- 5 zu den mit ndt-Liganden $\mathbf{4}$ ergeben sich annährend gleiche Redoxpotentiale von $-0.31 \mathrm{~V}[\mathrm{Mo}]$ und $-0.67 \mathrm{~V}[\mathrm{~W}]\left(\right.$ gegen $\mathrm{Fc} / \mathrm{Fc}^{+}$).

Beim Vergleich der mit Donorgruppen substituierten Dithiolenkomplexe (1-3) ist keine große Änderung bei den Aufspaltungen zwischen HOMO und LUMO festzustellen. So lassen sich auch die sehr ähnlichen Redoxpotentiale (etwa $-0.8 \mathrm{~V}$ [Mo] und $-1.4 \mathrm{~V}$ [W] gegen $\mathrm{Fc} / \mathrm{Fc}^{+}$) dieser Verbindungen erklären. Beim Wechsel zu den ndt-Systemen mit elektronenziehenden 
Substituenten stellt man eine Abnahme der Aufspaltung fest, wodurch es zur besseren Reduzierbarkeit der Verbindungen kommt.

Bei der vergleichenden Betrachtung der Komplexe mit nichtaromatischen Dithiolenligandensystemen (7-tldt, 9-tcdt, 10-cydt) fällt auf, dass die größte Aufspaltung bei den $\left[\mathrm{MO}(\mathrm{cydt})_{2}\right]^{\mathrm{x}-}$ gefunden wird, bei dem nur Kohlenstoffatome im Ringsystem zu finden sind. Kommt es nun zur Koordination eines weiteren aromatischen Systems an den Cyclohexanring, das bei den tldt-Liganden vorliegt, so wird eine Verkleinerung des Abstandes zwischen HOMO und LUMO beobachtet. Dies kann auch an dem positiv werdenden Redoxpotential um $0.03 \mathrm{~V}$ [Mo] und $0.18 \mathrm{~V}$ [W] erkannt werden. Wird nun noch das Heteroatom Schwefel in das Ringsystem eingebaut, so wird aufgrund des elektronenziehenden Charakters ein weiter positiv werdendes Redoxpotential festgestellt (-0.87 V [Mo] bzw. -1.19 V [W]). Dadurch wird der kleinste Abstand zwischen beim HOMO und dem LUMO bei diesen drei Paaren gefunden.

Abschließend kann festgestellt werden, dass aufgrund dieser theoretischen Betrachtungen eine Klassifizierung der jeweiligen Liganden nach der Qualität als Modellverbindungen für Molybdopterinhaltige Kofaktoren gemacht werden konnte. Aufgrund der erhaltenen „energygap“-Werte konnten Verknüpfungen $\mathrm{zu}$ den experimentell gemessenen Redoxpotentialen gezogen werden. Allerdings wurden die gemessenen Potentiale in Acetonitril-Lösung bestimmt und so können die erhaltenen Redoxpotentiale nicht exakt, auf die sich in wässrigen Medien befindlichen biologischen Enzyme übertragen werden. Aber es konnte zumindest ein möglicher Potentialbereich für das Enzym bestimmt werden. 


\subsection{Bestimmung von Redoxpotentialen mit DFT-Methoden}

Um die experimentell erhalten Redoxpotentiale durch berechnete Potentiale zu verifizieren, wurden mit Hilfe des Programmpakets Gaussian03 ${ }^{[73]}$ Untersuchungen durchgeführt. Die Bestimmung von Redoxpotentialen stellt immer noch eine große Herausforderung an die Theoretische Chemie dar, wodurch die Bestimmung auf die jeweiligen Systeme genau angepasst werden muss.

\subsubsection{Möglichkeiten zur Bestimmungen von Redoxpotentialen}

Um Redoxpotentiale mit Hilfe von DFT-Rechnungen bestimmen zu können, ergeben sich hauptsächlich zwei Möglichkeiten.

Würde die zu bestimmende Reaktion in der Gasphase ablaufen, so müsste man nur die Ionisierungsenergie des Moleküls berechnen und es ggf. durch rovibronische Beiträge (Nullpunktsenergie, Entropiebeiträge) korrigieren. Möchte man nun die ,vertikale Ionisierungsenergie" berechnen, so bietet sich das Koopmans-Theorem an. Hierbei wird angenommen, dass das Ionisierungspotential nährungsweise gleich der negativen Energie des HOMOs ist. Das Problem bei dieser Methode ist, dass sie eine Nährung ist und nur auf einfache Systeme angewendet werden kann. In der Gasphase werden darüber hinaus keine relevanten Lösungsmitteleinflüsse berücksichtigt, was zwangsläufig $\mathrm{zu}$ nicht realen Potentialen führt. Darüber hinaus sollen bei den berechneten Potentialen auch Korrekturen der Nullpunktsschwingungsenergie mit eingehen, weil reale Messungen nicht bei $0 \mathrm{~K}$ durchgeführt werden können. Um eine genauere Bestimmung durchzuführen, wurden sowohl die Lösungsmitteleinflüsse, als auch die Temperaturbedingungen in die Berechnungen einbezogen.

\subsubsection{Einfluss des Lösungsmittels}

Bei Lösungsmitteln die keine Wasserstoffbrückenbindungen zu den gelösten Molekülteilen ausbilden können, werden relativ ähnliche Geometrieoptimierte Strukturen wie in der Gasphase gefunden. Probleme treten besonders bei polaren Lösungsmitteln auf, da es hier aufgrund von verschiedensten Wechselwirkungen zu starken Beeinflussungen der Geometrie kommt. Um die Lösungsmitteleffekte berücksichtigen zu können, bietet sich die Verwendung von Reaktionsfeldern an. Die populärsten Modelle sind das Onsager-Modell ${ }^{[74]}$, das COSMOModell $^{[75]}$ und das PCM-Modell. ${ }^{[76]}$ Weil im Folgenden ausschließlich eine modifizierte Form des PCM-Modells (polarisable continuum model) verwendet wurde, wird nur dieses Modell hier näher erläutert. 
Zunächst wird um die Atome des Moleküls eine virtuelle Hülle gelegt. Dazu werden für die jeweiligen Moleküle als Kugeln konstruiert, deren Durchmesser den Van-der-Waalsradien entsprechen. Um das gesamte Molekül wird eine Oberfläche konstruiert. Innerhalb dieser Oberfläche ist der Aufenthalt von Lösungsmittelmolekülen nicht erlaubt.

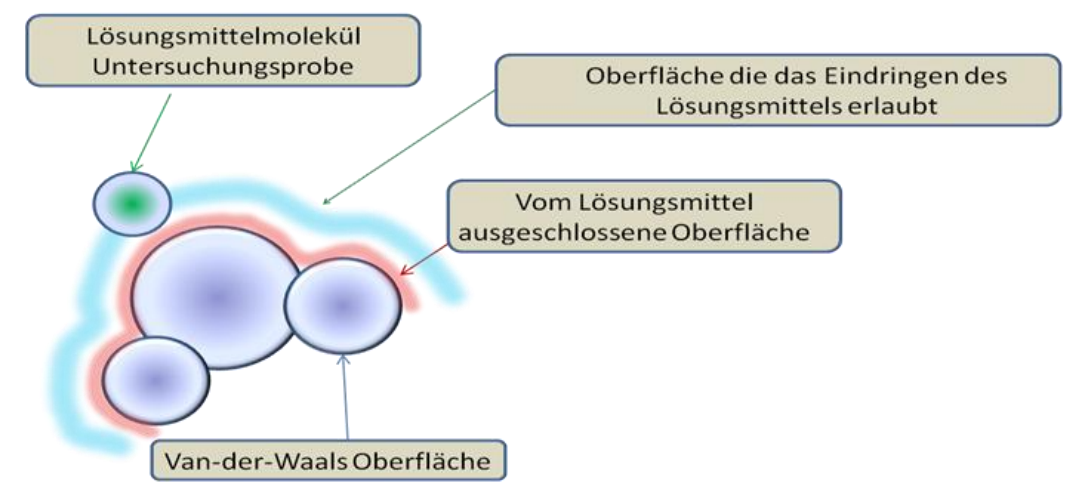

Abb. 94: Schematische Darstellung des IEFPCM-Modells

Nun wird abhängig vom jeweils verwendeten Lösungsmittel eine in einem bestimmten Abstand vorhandene weitere Oberfläche konstruiert. Innerhalb dieser Schicht ist nur der Aufenthalt von Lösungsmittelmolekülen erlaubt. Die Solvatationsenthalpie enthält nun drei Beiträge: $\quad \Delta G_{S}^{O}=\Delta G_{E S}^{O}+\Delta G_{\text {disp }}^{O}+\Delta G_{c a v}^{O}, \quad\left(\Delta G_{E S}^{O}=\right.$ elektrostatische Wechselwirkungen, $\Delta G_{\text {disp }}^{O}=$ Dispersionsbeiträge, $\Delta G_{\text {cav }}^{O}=$ "cavity"-Beitrag) die alle semi-empirisch berechnet werden können. Hierbei wird ein Netz aus Punktladungen (Dichte und Betrag sind abhängig vom Lösungsmittel) auf die Oberfläche gelegt, die von den Lösungsmittelmolekülen besetzt werden kann. Diese Ladungen werden im Hamilton-Operator des Molekülteils berücksichtigt und haben somit einen direkten Einfluss auf alle Eigenschaften des gelösten Moleküls, die nun mit Hilfe der Erwartungswerte berechnet werden können. Aufgrund des Einflusses auf den Hamilton Operator des Systems, wird von der Methode des selbstkonsistenen Reaktionsfeldes (SCRF) gesprochen.

\subsubsection{Referenzierung der berechneten Parameter}

Wie auch bei der Bestimmung von Redoxpotentialen mit elektrochemischen Untersuchungsmethoden wie z.B. Cyclovoltammetrie, bei der die Potentiale relativ zu einer Referenz angegeben werden ( $0 \mathrm{~V}$ für die Standardwasserstoffelektrode), wird bei Berechnungen der Redoxpotentiale mit theoretischen Methoden ebenfalls ein „Nullpunkt“ der Potentiale benötigt. Hierfür bietet sich die Oxidation von Ferrocen zum Ferroceniumkation an, die auch bei experimentellen Studien als Referenz genutzt wird. 
Um diese Referenz einzuführen wurde das Molekül Ferrocen sowie das Ferrocenion-Kation auf B3LYP-Level mit Basissätzen von LANL2Dz für Eisen sowie 6-311G mit einer d sowie p- Polarisationsfunktion für Kohlenstoff und Wasserstoff in der Gasphase und in einer Acetonitrilumgebung (IEFPCM) berechnet. Nach abschließender geometrischer Optimierung wurde eine Frequenzrechnung der Moleküle durchgeführt, wodurch folgende Gibbsenergien erhalten werden konnten.

\begin{tabular}{lcc}
\hline & $\Delta \mathbf{G}($ Gasphase) [Hartreé] & $\Delta \mathbf{G}$ (in Acetonitril) [Hartreé] \\
\hline Ferrocen & -510.392039 & -510.401798 \\
Ferroceniumkation & -510.130863 & -510.215756 \\
$\Delta(\Delta \mathbf{G})$ & 0.261176 & 0.186042 \\
\hline
\end{tabular}

Tab. 15: Bestimmte Gibbs-Energien von Ferrocen/Ferrocenium auf B3LYP-Level

Im Vergleich der Berechnungen in Gasphase und im Lösungsmittel wird der Einfluss durch das Vorhandensein eines Lösungsmittels deutlich. Auch experimentell hat die Wahl des Lösungsmittels einen Einfluss auf das gemessene Redoxpotential, wie bereits in Kapitel 4 bei dem Verbindungspaar $\left[\mathrm{MCp}_{2} \mathrm{Cl}_{2}\right]$ gezeigt werden konnte.

\subsubsection{Bestimmung der thermodynamischen Parameter}

Um die Gibbs-Energien der synthetisierten Verbindungspaare dieser Arbeit zu bestimmen, wurde deren jeweilige Struktur sowohl in der Gasphase, als auch im Lösungsmittel Geometrieoptimiert. Hierbei wurden die Übergänge von Oxidationsstufe IV und V betrachtet. Nach erhalten der jeweiligen Gibbsenergien für die Verbindungen, wurde die Differenz zwischen den benachbarten Oxidationsstufen IV und V gebildet. Dann wurde die Differenz zum virtuellen Referenzsystem Ferrocen/Ferrocenium, das auf ein Potential von $0 \mathrm{~V}$ gelegt wurde, gebildet. Nach Umrechnung der Energien in Joule und Bezugnehmen auf molare Einheiten, kann man das Redoxpotential nach folgender Gleichung berechnen.

$$
\Delta E=-\frac{\Delta G}{z \cdot F}
$$

Da immer nur Ein-Elektronenübergange untersucht werden, ergibt sich die Anzahl der übertragenen Elektronen $\mathrm{z}$ ) $\mathrm{zu}$ 1. Hieraus ergeben sich folgende mit Hilfe der B3LYPHybridfunktion berechnete Redoxpotentiale. 


\begin{tabular}{|c|c|c|c|c|}
\hline & $\begin{array}{c}\text { Potential } \\
\text { IV } \leftrightarrow V \\
{[V]^{*}}\end{array}$ & $\begin{array}{l}\text { exp. Potential } \\
{[\mathrm{V}]^{*}}\end{array}$ & $\begin{array}{c}\Delta \mathbf{E}_{\text {calc }}-\Delta \mathbf{E}_{\text {exp }} \\
{[\mathbf{V}]}\end{array}$ & $\begin{array}{c}\Delta \mathbf{E} / \Delta \mathrm{T} \\
{[\mathrm{mV} / \mathrm{K}]^{* *}}\end{array}$ \\
\hline $\begin{array}{c}{\left[\mathrm{MoO}(\mathbf{x d t})_{2}\right]^{2-}} \\
(\mathbf{1 a})\end{array}$ & -1.04 & -0.85 & 0.19 & -0.13 \\
\hline $\begin{array}{c}{\left[\mathrm{WO}(\mathrm{xdt})_{2}\right]^{2-}} \\
(\mathbf{1 b})\end{array}$ & -1.34 & -1.03 & 0.31 & -0.16 \\
\hline $\begin{array}{c}{\left[\mathrm{MoO}(\mathrm{tdt})_{2}\right]^{2-}} \\
(\mathbf{2 a})\end{array}$ & -1.13 & -0.80 & 0.33 & -0.37 \\
\hline $\begin{array}{c}{\left[\mathrm{WO}(\mathbf{t d t})_{2}\right]^{2-}} \\
(\mathbf{2 b})\end{array}$ & -1.41 & -1.49 & 0.08 & -0.41 \\
\hline $\begin{array}{c}{\left[\mathrm{MoO}(\mathrm{vdt})_{2}\right]^{2-}} \\
(3 \mathrm{3a})\end{array}$ & -0.90 & -0.77 & 0.13 & -0.09 \\
\hline $\begin{array}{c}{\left[\mathrm{WO}(\mathrm{vdt})_{2}\right]^{2-}} \\
(\mathbf{3 b})\end{array}$ & -1.33 & -1.45 & 0.12 & -0.13 \\
\hline $\begin{array}{c}{\left[\mathrm{MoO}(\mathbf{n d t})_{2}\right]^{2-}} \\
(\mathbf{4 a})\end{array}$ & -0.54 & -0.28 & 0.26 & -0.07 \\
\hline $\begin{array}{c}{\left[\mathrm{WO}(\mathrm{ndt})_{2}\right]^{2-}} \\
(\mathbf{4 b})\end{array}$ & -0.83 & -0.57 & 0.26 & -0.18 \\
\hline $\begin{array}{c}{\left[\mathrm{MoO}(\mathbf{q d t})_{2}\right]^{2-}} \\
(5 \mathrm{a})\end{array}$ & -0.71 & -0.31 & 0.40 & -0.20 \\
\hline$\underset{(5 \mathrm{~b})}{\left[\mathrm{WO}(\mathbf{q d t})_{2}\right]^{2}}$ & -0.84 & -0.67 & 0.17 & -0.33 \\
\hline $\begin{array}{c}{\left[\mathrm{MoO}(\mathrm{ntdt})_{2}\right]^{2-}} \\
(\mathbf{6 a})\end{array}$ & -1.16 & -0.88 & 0.28 & -0.15 \\
\hline $\begin{array}{c}{\left[\mathrm{WO}(\mathrm{ntdt})_{2}\right]^{2-}} \\
(\mathbf{6 b})\end{array}$ & -0.83 & -0.92 & 0.09 & -0.18 \\
\hline $\begin{array}{c}{\left[\mathrm{MoO}(\mathrm{tldt})_{2}\right]^{2-}} \\
(\mathbf{7 a})\end{array}$ & -1.18 & -0.95 & 0.23 & -0.12 \\
\hline $\begin{array}{c}{\left[\mathrm{WO}(\mathrm{tldt})_{2}\right]^{2-}} \\
(7 \mathbf{b})\end{array}$ & -1.43 & -1.21 & 0.22 & -0.16 \\
\hline $\begin{array}{c}{\left[\mathrm{MoO}(\mathrm{tcdt})_{2}\right]^{2-}} \\
(9 \mathrm{aa})\end{array}$ & -1.04 & -0.87 & 0.17 & -0.14 \\
\hline $\begin{array}{c}{\left[\mathrm{WO}(\mathrm{tcdt})_{2}\right]^{2-}} \\
(9 \mathrm{~b})\end{array}$ & -1.27 & -1.19 & 0.08 & -0.23 \\
\hline $\begin{array}{c}{\left[\mathrm{MoO}(\mathrm{cydt})_{2}\right]^{2-}} \\
(\mathbf{1 0 a})\end{array}$ & -1.28 & -0.98 & 0.31 & -0.15 \\
\hline $\begin{array}{c}{\left[\mathrm{WO}(\mathrm{cydt})_{2}\right]^{2-}} \\
(\mathbf{1 0 b})\end{array}$ & -1.52 & -1.39 & 0.13 & -0.17 \\
\hline $\begin{array}{c}{\left[\mathrm{MoO}\left(\mathrm{C}_{2} \mathrm{~S}_{2} \mathbf{M e}_{2}\right)_{2}\right]^{2-}} \\
(\mathbf{2 1 a})\end{array}$ & -1.29 & -0.74 & 0.55 & -0.16 \\
\hline $\begin{array}{c}{\left[\mathrm{WO}\left(\mathrm{C}_{2} \mathrm{~S}_{2} \mathrm{Me}_{2}\right)_{2}\right]^{2-}} \\
(\mathbf{2 1 b})\end{array}$ & -1.51 & -1.33 & 0.18 & -0.18 \\
\hline
\end{tabular}

Tab. 16: Potentiale die mit der B3LYP-Hybridfunktion erhalten wurden, * gegen $\mathrm{Fc} / \mathrm{Fc}^{+}$;

** berechnete Temperaturabhängigkeit

Die Abweichungen der berechneten von den experimentellen Werten, ist bei den Molybdänverbindungen etwa doppelt so groß (durchschnittlich $0.29 \mathrm{~V}$ ), wie bei den Wolframverbindungen (durchschnittlich $0.16 \mathrm{~V}$ ). Um auch die Temperaturabhängigkeit der bestimmten Redoxpotentiale zu erhalten wurden diese Potentiale bei fünf verschiedenen Temperaturen $(253,273,298,323,338 \mathrm{~K})$ berechnet. Auffällig ist, dass wie bei den experimentellen Bestimmungen, immer die größere Temperaturabhängigkeit bei den 
Wolframverbindungen festzustellen wurde. Allerdings konnten durch die berechneten Steigungen nicht die genauen Steigungswerte der experimentellen Untersuchungen erhalten werden. Bei den berechneten Steigungen wurde bei allen untersuchten Verbindungspaaren nur eine Abnahme der Redoxpotentiale mit der Temperatur erhalten. Somit sollte bei den Berechnungen die Temperaturkomponente im Vergleich zu der Reaktionsentropie größere Werte im Faktor $\Delta G=\Delta H-T \Delta S$ liefen, wodurch bei allen Berechnungen eine Abnahme der Potentiale mit der Temperatur festzustellen ist.

Um die theoretischen und experimentellen Werte besser in Einklang zu bekommen, wurde mit verschiedensten Hybridfunktionen versucht die Rechnungen zu optimieren. Als Modellsystem wurde hier das rechnerisch unaufwendigste $\left[\mathrm{MoO}\left(\mathrm{C}_{2} \mathrm{~S}_{2} \mathrm{Me}_{2}\right)_{2}\right]^{\mathrm{X}-}$ gewählt. Hierbei wurden zusätzlich zu den in Tab.16 aufgeführten Werten, die mit der B3LYP-Funktion berechnet wurden, folgende Hybridbasisfunktionen verwendet: O3LYP ${ }^{[77]}$ B3PW91 ${ }^{[78]}$, MPW1PW91 ${ }^{[78 b, 79]}$, PBE1PBE $^{[80]}$, PBE1W ${ }^{[81]}$, PBELYP1W $^{[82]}, \mathrm{BHandH}^{[78 c]}$

Die Verbindung $\left[\mathrm{MoO}\left(\mathrm{C}_{2} \mathrm{~S}_{2} \mathrm{Me}_{2}\right)_{2}\right]^{\mathrm{x}-}$ wurde in der OS +IV und $\mathrm{V}$ als auch das $\mathrm{Fc} / \mathrm{Fc}^{+}-\mathrm{Paar}$ mit der jeweiligen Hybridfunktionen im Lösungsmittel Acetonitril geometrieoptimiert. Nach der oben erläuterten Differenzbildung der Gibbsenergien wurden die in Tab. 17 angegebenen Potentiale bestimmt, sowie die Differenz zu den experimentellen Werten.

\begin{tabular}{ccc}
\hline Funktion & $\mathbf{E}_{\text {cal }}[\mathbf{V}]^{*}$ & $\Delta \mathbf{E}=\mathbf{E}_{\text {cal }}-\mathbf{E}_{\exp }[\mathbf{V}]$ \\
\hline O3LYP & -1.365 & 0.625 \\
B3PW91 & -1.257 & 0.517 \\
MPW1PW91 & -1.185 & 0.445 \\
PBE1PBE & -1.180 & 0.440 \\
PBE1W & -1.133 & 0.393 \\
PBELYP1W & -1.001 & 0.261 \\
BHandH & -0.992 & 0.252
\end{tabular}

Tab. 17: Vergleich der verwendeten Funktionen für die Berechnung des Potentials von $\left[\mathrm{MoO}\left(\mathrm{C}_{2} \mathrm{~S}_{2} \mathrm{Me}_{2}\right)_{2}\right]^{2-}$; ${ }^{*}$ gegen Fc/Fc Im Vergleich der benutzten Hybridfunktionen kann festgestellt werden, dass die beste Übereinstimmung von experimentellen und theoretischen Werten mit der Funktion BHandH erzielt wurde. Die Unterschiede der einzelnen Funktionen bestehen vorwiegend in einer abweichenden Mischung des sogenannten Austauschterms mit dem Korrelationsterm. Bei einigen Funktionen kommen noch andere Funktionsteile hinzu. Generell kann festgestellt werden, dass die Funktionen mit einem Korrelationsanteil von größer als $75 \%$ zu besseren 
Ergebnissen führen. Bei Verwendung eines 100\%igen Anteils des Korrelationsterms, wie bei der PBE1PBE-Hybridfunktion, werden die Ergebnisse wieder schlechter.

Deshalb wurden mit Hilfe der BHandH Funktionen erneut die Redoxpotentiale, der in der Arbeit verwendeten Verbindungspaare, berechnet und die erhaltenen Werte sind mit den jeweiligen Abweichungen zu den experimentell bestimmten Potentialen in Tabl8 aufgeführt.

\begin{tabular}{|c|c|c|c|}
\hline & $\begin{array}{c}\text { Potential } \\
\text { IV } \leftrightarrow \text { V BHandH } \\
{[V]^{*}}\end{array}$ & $\begin{array}{l}\text { exp. Potential } \\
{[\mathrm{V}]^{*}}\end{array}$ & $\begin{array}{c}\Delta \mathbf{E}_{\text {calc }}-\Delta \mathbf{E}_{\text {exp }} \\
{[\mathbf{V}\}}\end{array}$ \\
\hline $\begin{array}{c}{\left[\mathrm{MoO}(\mathrm{xdt})_{2}\right]^{2-}} \\
(\mathbf{1 a})\end{array}$ & -0.75 & -0.85 & 0.10 \\
\hline $\begin{array}{c}{\left[\mathrm{WO}(\mathbf{x d t})_{2}\right]^{2-}} \\
(\mathbf{1 b})\end{array}$ & -1.15 & -1.03 & 0.12 \\
\hline $\begin{array}{c}{\left[\mathrm{MoO}(\mathbf{t d t})_{2}\right]^{2-}} \\
(\mathbf{2 a})\end{array}$ & -0.72 & -0.80 & 0.08 \\
\hline $\begin{array}{c}{\left[\mathrm{WO}(\mathrm{tdt})_{2}\right]^{2-}} \\
(\mathbf{2 b})\end{array}$ & -1.14 & -1.49 & 0.35 \\
\hline $\begin{array}{c}{\left[\mathrm{MoO}(\mathbf{v d t})_{2}\right]^{2-}} \\
(\mathbf{3 a})\end{array}$ & -0.75 & -0.77 & 0.02 \\
\hline $\begin{array}{c}{\left[\mathrm{WO}(\mathrm{vdt})_{2}\right]^{2-}} \\
(\mathbf{3 b})\end{array}$ & -1.14 & -1.45 & 0.31 \\
\hline $\begin{array}{c}{\left[\mathrm{MoO}(\mathrm{ndt})_{2}\right]^{2-}} \\
(\mathbf{4 a})\end{array}$ & -0.34 & -0.28 & 0.06 \\
\hline $\begin{array}{c}{\left[\mathrm{WO}(\mathrm{ndt})_{2}\right]^{2-}} \\
(\mathbf{4 b})\end{array}$ & -0.66 & -0.57 & 0.09 \\
\hline$\underset{(5 a)}{\left[\mathrm{MoO}(\mathrm{qdt})_{2}\right]^{2-}}$ & -0.41 & -0.31 & 0.10 \\
\hline $\begin{array}{c}{\left[\mathrm{WO}(\mathrm{qdt})_{2}\right]^{2}} \\
(5 \mathrm{~b})\end{array}$ & -0.76 & -0.67 & 0.09 \\
\hline $\begin{array}{c}{\left[\mathrm{MoO}(\mathrm{ntdt})_{2}\right]^{2-}} \\
(\mathbf{6 a})\end{array}$ & -0.83 & -0.88 & 0.05 \\
\hline $\begin{array}{c}{\left[\mathrm{WO}(\mathrm{ntdt})_{2}\right]^{2-}} \\
(6 \mathrm{~b})\end{array}$ & -1.00 & -0.92 & 0.08 \\
\hline $\begin{array}{c}{\left[\mathrm{MoO}(\mathrm{tldt})_{2}\right]^{2-}} \\
(\mathbf{7 a})\end{array}$ & -0.87 & -0.95 & 0.08 \\
\hline $\begin{array}{c}{\left[\mathrm{WO}(\mathrm{tldt})_{2}\right]^{2-}} \\
(7 \mathrm{bb})\end{array}$ & -1.26 & -1.21 & 0.05 \\
\hline $\begin{array}{c}{\left[\mathrm{MoO}(\mathrm{tcdt})_{2}\right]^{2-}} \\
(\mathbf{9 a})\end{array}$ & -0.74 & -0.87 & 0.13 \\
\hline $\begin{array}{c}{\left[\mathrm{WO}(\text { tcdt })_{2}\right]^{2-}} \\
(9 \mathrm{~b})\end{array}$ & -1.10 & -1.19 & 0.09 \\
\hline $\begin{array}{c}{\left[\mathrm{MoO}(\mathrm{cydt})_{2}\right]^{2-}} \\
(\mathbf{1 0 a})\end{array}$ & -0.98 & -0.98 & 0.00 \\
\hline $\begin{array}{c}{\left[\mathrm{WO}(\mathrm{cydt})_{2}\right]^{2-}} \\
(\mathbf{1 0 b})\end{array}$ & -1.36 & -1.39 & 0.03 \\
\hline $\begin{array}{c}{\left[\mathrm{MoO}\left(\mathrm{C}_{2} \mathrm{~S}_{2} \mathbf{M e}_{2}\right)_{2}\right]^{2-}} \\
(\mathbf{2 1 a})\end{array}$ & -0.99 & -0.74 & 0.25 \\
\hline $\begin{array}{c}{\left[\mathrm{WO}\left(\mathrm{C}_{2} \mathbf{S}_{2} \mathbf{M e}_{2}\right)_{2}\right]^{2-}} \\
(21 b)\end{array}$ & -1.32 & -1.33 & 0.01 \\
\hline
\end{tabular}

Tab. 18: Berechnete Potentiale mit der BHandH - Hybridfunktion, * gegen Fc/Fc ${ }^{+}$ 
Durch die Benutzung der BHandH-Hybridfunktion wurden deutlich bessere Übereinstimmungen mit den experimentell bestimmten Redoxpotentialen gefunden. Es werden im Durchschnitt Abweichungen von 0.09 V [Mo] bzw. $0.12 \mathrm{~V}$ [W] gefunden. Diese Differenzen liegen in den normalen Fehlergrenzen, die bei experimentellen elektrochemischen Messungen auftreten. Bei der $\left[\mathrm{MoO}(\mathrm{cydt})_{2}\right]^{2-}-$ Verbindung konnte sogar exakt der experimentelle Potentialwert berechnet werden. Generell wurden die Potentiale bis auf drei Aussnahmen mit einer Genauigkeit von $\pm 0.13 \mathrm{~V}$ (größte Abweichung) bestimmt. Nur bei den Wolframverbindungen mit den aromatischen vdt und tdt-Liganden zeigten sich noch große Abweichungen um die $\sim 0.3 \mathrm{~V}$. Außerdem konnte das Potential des $\left[\mathrm{MoO}\left(\mathrm{S}_{2} \mathrm{C}_{2} \mathrm{Me}_{2}\right)_{2}\right]^{\mathrm{x}-}$ mit einer Abweichung von $0.25 \mathrm{~V}$ berechnet werden, dass aber eine Verbesserung von $200 \mathrm{mV}$ im Vergleich zu den Berechnungen mit der B3LYP-Funktion darstellt.

Mit Hilfe der Methode können nun Redoxpotentiale berechnet werden, die mit einer ausreichenden Genauigkeit bestimmt werden können, die speziell auf die geladenen Dithiolenkomplexe und Molybdän- und Wolframverbindungen, die zuvor noch nicht in dieser Genauigkeit bei anderen Molybdän- und Wolframverbindungen von anderen Arbeitsgruppen bestimmt werden konnten. In der Literatur wird von Berechnungen der Redoxpotentiale für Molybdän- und Wolframverbindungen berichtet bei denen Abweichungen um die $300 \mathrm{mV} z u$ den experimentell erhaltenen Redoxpotentialen auftreten. ${ }^{[71]}$ 


\section{Kapitel 6}

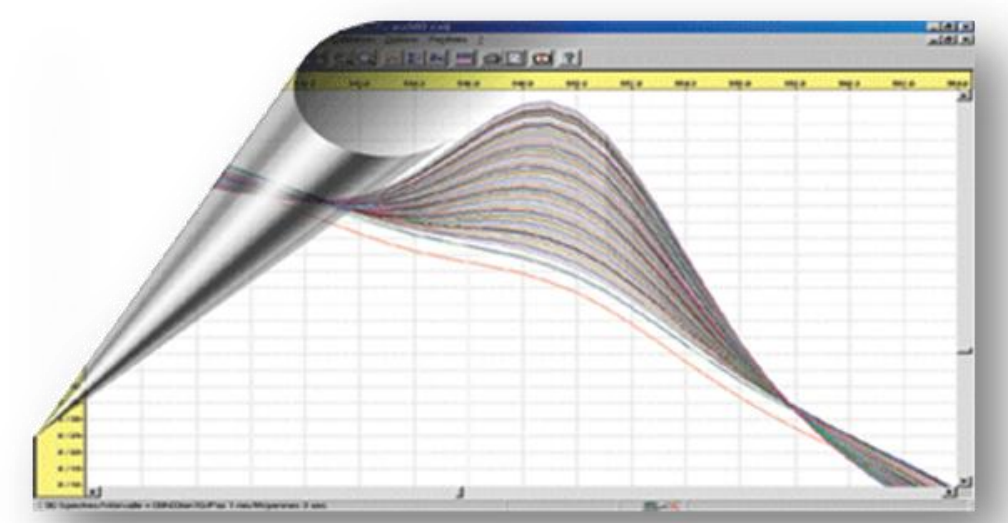

\section{Katalytische Untersuchungen}

\section{Zusammenfassung}

In diesem Kapitel werden vergleichende katalytische Untersuchungen an den zuvor hergestellten Verbindungspaaren beschrieben. Hiermit soll der Einfluss der verschiedenen Ligandensysteme auf die Oxo-Transfer-Eigenschaft evaluiert werden und Rückschlüsse auf die Relevanz der verschiedenen Modelle für die biologischen Cofaktoren gezogen werden. Es wurden sowohl temperaturabhängige als auch konzentrationsabhänige Oxo-Transfer Katalysen durchgeführt. 


\subsection{Einleitung}

Mit Ausnahme der Nitrogenase katalysieren die molybdän- und wolframabhängigen Enzyme Sauerstoffübertragungenreaktion des folgenden Typs.

$$
\mathrm{X}+\mathrm{H}_{2} \mathrm{O} \leftrightarrows \mathrm{XO}+2 \mathrm{H}^{+}+2 \mathrm{e}^{-}
$$

Dieser Reaktionstyp wird als Sauerstoffatomtransfer (SAT) bezeichnet. Die ersten Sauerstoffübertragungen von Molybdänverbindungen in einer Koordinationsumgebung die den aktiven Zentren ähnelt, wurden von Holm et al. im Jahre 1990 durchgeführt. ${ }^{[83]}$ Durch Isotopenmarkierungsexperimente mit ${ }^{18} \mathrm{O}$ im Substrat $\mathrm{Ph}_{2} \mathrm{~S}^{18} \mathrm{O}$, konnte ein Transfer auf die Metallkomplexe nachgewiesen werden. ${ }^{[84]}$ In einer späteren Studie von Holm et al. wurde das isolierte Enzym von $R$. sphaerodes aus der DMSO-Reduktase Familie ebenfalls mit $\mathrm{Me}_{2} \mathrm{~S}^{18} \mathrm{O}$ umgesetzt. Es konnte wieder eine Übertragung des Sauerstoffatoms auf das Metallzentrum beobachtet werden und durch Absorptionsspektroskopie sowie CI-Massenspektrometrie bestätigt werden. ${ }^{[85]}$ Die Gruppe von Lyon konnte weiterhin zeigen, dass es bei manchen SAT-Reaktionen zur Bildung von $\mu$-Oxo $\mathrm{Mo}(\mathrm{V})$-Verbindungen während des katalytischen Zykluses kommen kann. ${ }^{[83,86]}$ Es wird angenommen, dass das Dimer durch nicht reagiertes Monooxomolybdän, das mit der noch nicht umgesetzten Dioxo-Verbindung reagiert, gebildet wird. ${ }^{[29 b]}$

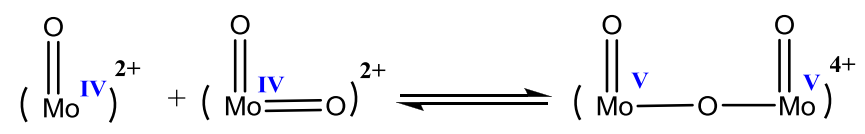

Abb. 95: Dimerbildung

Alle drei Reaktionsfragmente stehen miteinander im Gleichgewicht ${ }^{[87]}$ und es wurde beobachtet, dass die Lage des Gleichgewichts durch die Verwendung von sterisch anspruchsvollen Liganden nach links verschoben werden kann. ${ }^{[29 b, 88]}$ Außerdem wurde festgestellt, dass die Verwendung von Donorlösungsmitteln wie DMF, MeCN oder THF zu einer bevorzugten Dimerbildung führt. ${ }^{[88 \mathrm{~b}]}$

Bei der Sauerstoffübertragung kommt es in Modellsystemen während der Übertragung des Sauerstoffs auf das Metall zu einer cis/trans Umlagerung der Ligandensysteme. Dies konnte durch ESR und Absorptionsuntersuchungen nachgewiesen werden. Der vorgeschlagene Mechanismus am Beispiel mit Trimethyl- $N$-oxid ist im Folgenden gezeigt. ${ }^{\text {[29b] }}$ 


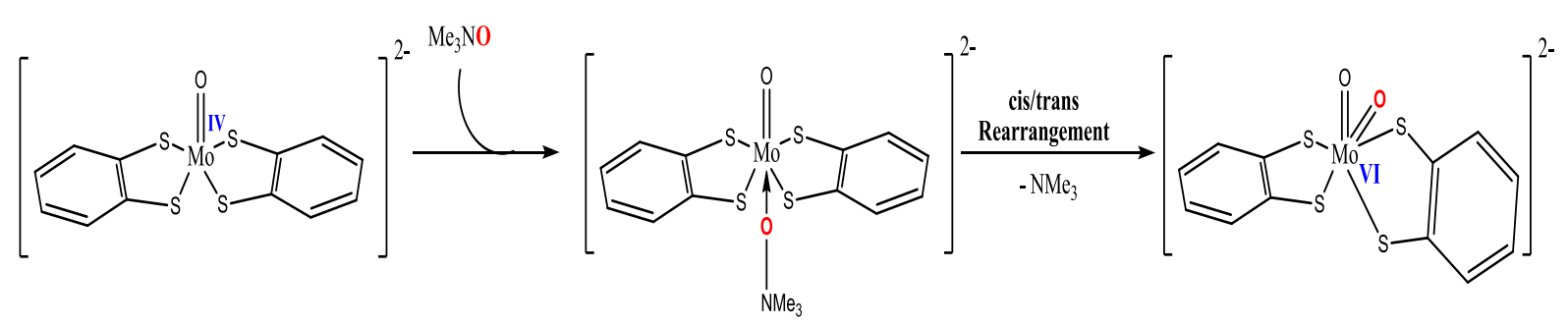

Abb. 96: Vorgeschlagener Mechanismus für die Sauerstoffübertragung auf [MoO(bdt $\left.)_{2}\right]^{2-[29 b]}$

Die Dimerbildung kann durch den Angriff eines Sauerstoffatomes der Mo(VI) $\mathrm{O}_{2}$ Verbindung in der trans Position an einen Mo(IV)O-Kern erfolgen, der dann über die cis/trans Umverteilung und einen Elektronentransfer zur biomolekularen Verbrückung führt. Nakamura und Mitarbeiter beschäftigten sich speziell mit der Möglichkeit diese Bildung von Dimeren und deren Unterdrückung durch elektronische Kontrolle über die Ligandensysteme. Dazu wurden $\left[\mathrm{MoO}(\mathrm{bdt})_{2}\right]^{2-}$ Verbindungen in der Oxidationsstufe IV mit Verbindungen $\left[\mathrm{MoO}_{2}\left(\mathrm{~S}_{2} \mathrm{CNEt}_{2}\right)_{2}\right]$ in Oxidationsstufe VI umgesetzt. Es kam zur Bildung von $\left[\mathrm{Mo}_{2} \mathrm{O}_{3}\left(\mathrm{~S}_{2} \mathrm{CNEt}_{2}\right)_{2}\right]$ in der Oxidationstufe $\mathrm{V}$ unter Verlust jeweils eines Dithiolenliganden. Im Gegensatz dazu wurden keine Dimer-Verbindungen der bdt-Verbindung beobachtet. Dies zeigt, dass der bdt-Ligand im Vergleich zu dem $\mathrm{S}_{2} \mathrm{CNEt}_{2}$ stärker an das Metall gebunden ist. Somit konnte belegt werden, dass eine Verminderung der Dimer-Bildung nicht nur durch sterisch anspruchsvolle Liganden, sondern nämlich auch, durch die Wahl der Liganden im Hinblick auf elektronische Effekte erzielt werden kann.

\subsection{Herstellung einer Dioxoverbindung}

Da die von mir hergestellten Verbindungen nur eine Oxo-Gruppe am Metall aufweisen und in Oxidationstufe IV vorliegen, muss der erste Schritt im Zyklus die Übertragung eines Sauerstoffatoms auf das Metallzentrum sein. Hierzu sind verschiedene Möglichkeiten in der Literatur bekannt. ${ }^{[83]}$ Darüber hinaus besteht die Möglichkeit die Verbindungen gleich mit zwei Oxo-Gruppen am Metall aus Verbindungen wie $\mathrm{MoO}_{2}(\mathrm{acac})_{2}$ herzustellen.

Um eine Sauerstoffübertragung auf die Monooxometallzentren $\mathrm{zu}$ erreichen wurden verschiedene Substrate (DMSO, $\mathrm{Bu}_{4} \mathrm{NNO}_{3}, \mathrm{Me}_{3} \mathrm{NO}$ ) zur Oxidation eingesetzt. Der Reaktionsverlauf wurde durch UV/VIS- spektrometrische Untersuchungen verfolgt, um einen Eindruck von der Geschwindigkeit dieser Übertragung zu bekommen. Dazu wurde die jeweilige Verbindungsprobe, gelöst in frisch entgastem und trockenem Methanol, durch das aufgesetzte Septum der Küvette direkt mit dem Oxidationsreagenz im 20fachen Überschuss zur Metallverbindung versetzt. Der Reaktionsverlauf wurde im Bereich von $800 \mathrm{~nm}$ bis 
$380 \mathrm{~nm}$ verfolgt und ist für die drei verwendeten Oxidationsreagenzien im Folgenden aufgeführt.

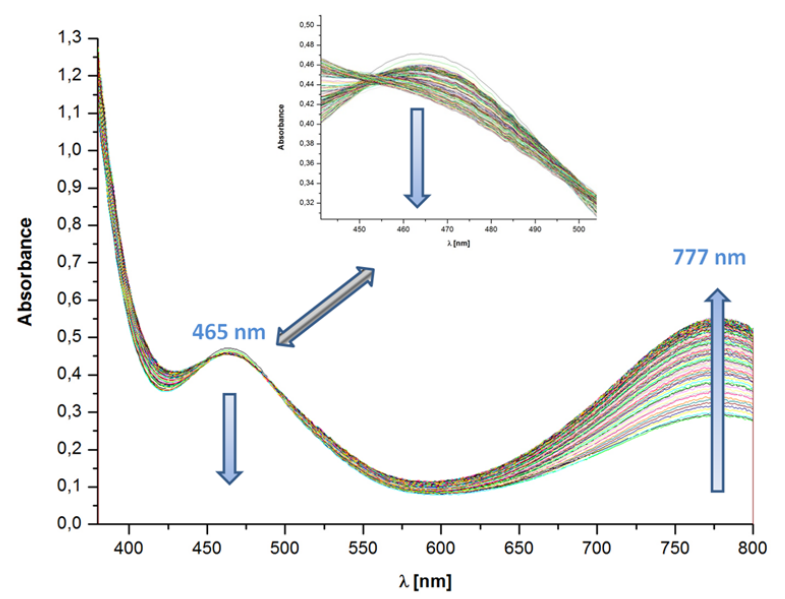

Abb. 97: $\left.\left(\mathrm{PPh}_{4}\right)_{2}[\mathrm{MoOxdt})_{2}\right](0.173 \mathrm{mmol} / \mathrm{l})$ in $\mathrm{MeOH}(\mathrm{abs}$.$) nach Zugabe von DMSO (im 20$ fachen Überschuss)

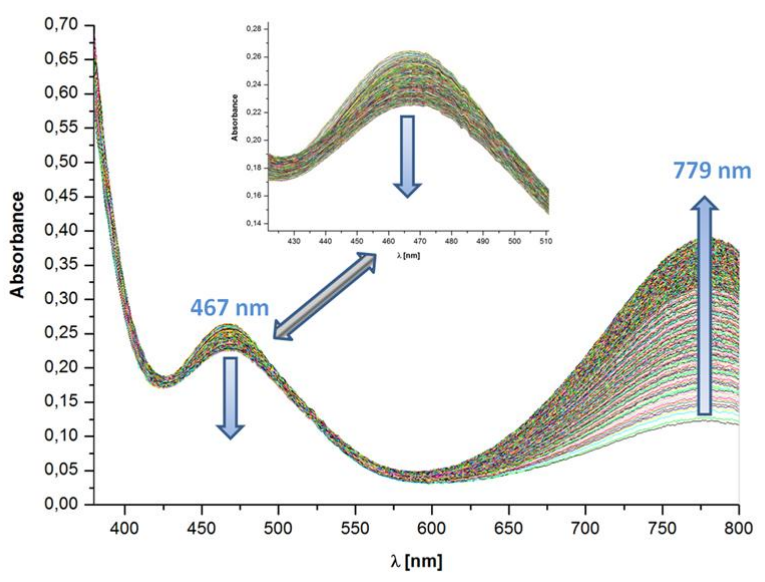

Abb. 98: $\left.\left(\mathrm{PPh}_{4}\right)_{2}[\mathrm{MoOxdt})_{2}\right](0.173 \mathrm{mmol} / \mathrm{I})$ in $\mathrm{MeOH}\left(\mathrm{abs}\right.$.) nach Zugabe von $\mathrm{Bu}_{4} \mathrm{NNO}_{3}$ in $\mathrm{MeOH}(\mathrm{abs}$.) (im 20 fachen Überschuss)

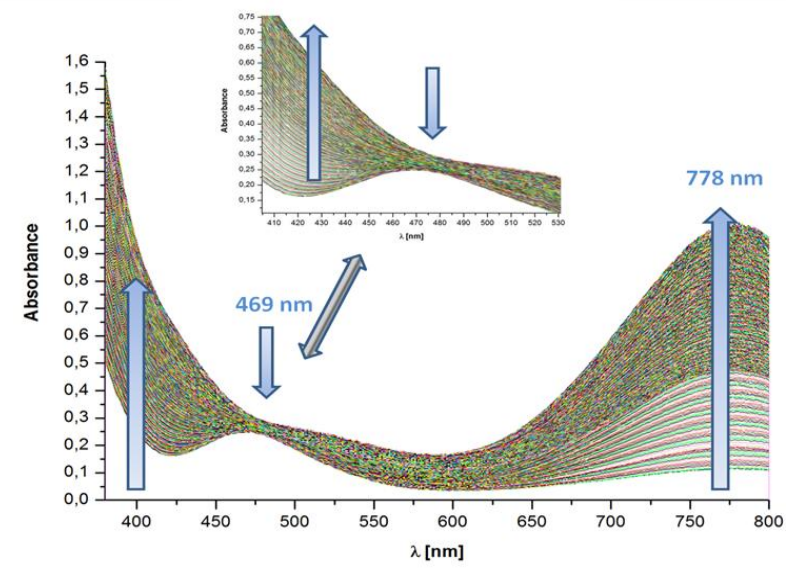

Abb. 99: $\left.\left(\mathrm{PPh}_{4}\right)_{2}[\mathrm{MoOxdt})_{2}\right](0.173 \mathrm{mmol} / \mathrm{I})$ in $\mathrm{MeOH}\left(\right.$ abs.) nach Zugabe von $\mathrm{Me}_{3} \mathrm{NO}$ in $\mathrm{MeOH}(\mathrm{abs})$. (im 20 fachen Überschuss) 
In allen erhaltenen Spektren konnte eine Abnahme des Peaks bei $\sim 67 \mathrm{~nm}$ und eine Zunahme eines Peak bei $\sim 778 \mathrm{~nm}$ mit der Zeit festgestellt werden. Die Spektren wurden in einem Abstand von 20 Sekunden aufgenommen. Entsprechend kann der Peak bei $467 \mathrm{~nm}$ den Elektronenübergängen der Molybdänverbindungen in der Oxidationsstufe IV zugeordnet werden. Der zunehmende Peak bei $\sim 778 \mathrm{~nm}$ weist auf die entstehende Dioxoverbindung hin. Es trat bereits in dem Spektrum im frisch entgastem Methanol ein kleiner Peak bei $\sim 778 \mathrm{~nm}$ auf, somit reichen schon geringe Spuren an Sauerstoff aus, um diese Verbindung zu oxidieren. Dieser Peak konnte auch trotz mehrerer Versuche nicht komplett entfernt werden. Somit entstehen bei der Synthese der Verbindungen schon geringe Spuren der Dioxoverbindung. Bei der Oxidationsreagenz $\mathrm{Me}_{3} \mathrm{NO}$ konnte die Bildung eines starken Signals bei ca. $400 \mathrm{~nm}$ beobachtet werden, somit sollte es auch zur Bildung von anderen Spezies kommen. Es ist unklar durch welche Spezies dieses Signal verursacht wird. Um das Ende der Sauerstoffübertragung zu bestimmen, wurde die Veränderung des Peaks der Dioxoverbindung gegen die Zeit aufgetragen.
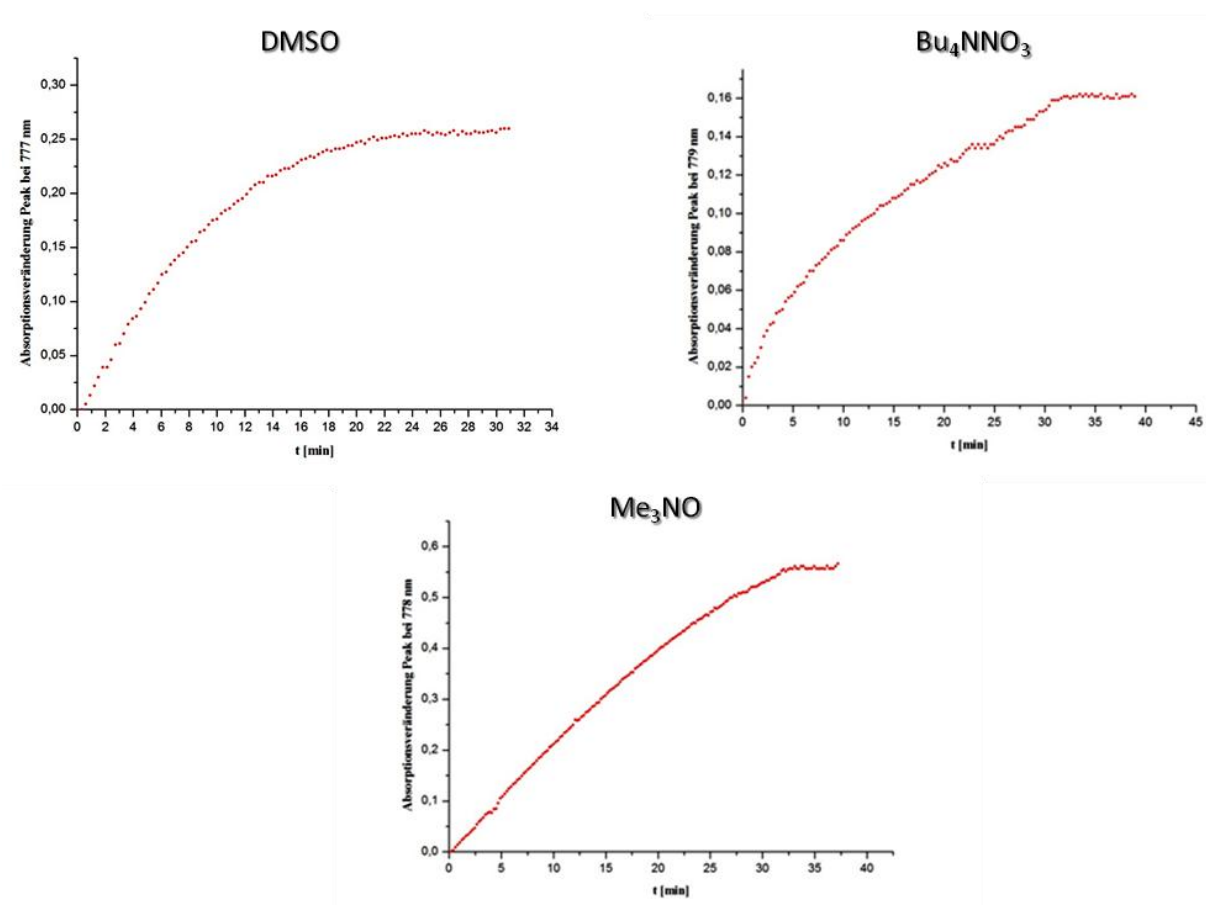

Abb. 100: Verlauf der Sauerstoffübertragung durch die jeweiligen Oxidationsreagenzien (Zunahme Absorption 778 nm)

Festgestellt werden konnte, dass nach einer Zeit von ca. 40 Minuten die Peakintensität relativ konstant bleibt, wurde für die Sauerstoffübertragungen bei den folgenden katalytischen Experimenten die Reaktionslösung 60 Min rühren gelassen. Da innerhalb einer UV-VisKüvette die Durchmischung der Reaktionslösung lediglich durch Diffusion erfolgt, ist 
anzunehmen, dass durch Rühren eine Beschleunigung dieser Reaktion zu erreichen ist. Deshalb sollte eine Vorlaufzeit von 60 Min lang genug sein. Um die im Reaktionskolben entstehenden gasförmigen Nebenprodukte (DMS bzw. $\mathrm{Me}_{3} \mathrm{~N}$ ) zu entfernen wurde die Lösung im Reaktionskolben mit einem leichten Stickstoffstrom durchströmt. Die Löslichkeit der gasförmigen Nebenprodukte in polaren Lösungsmitteln ist als gering einzustufen. Somit sollten die Nebenprodukte leicht durch den Stickstoffstrom aus der Lösung ausgetrieben werden. Sarkar et. al. stellte bei seinen Modellsystemen für die Nitratreduktase fest, dass eine Inhibierung der Katalyse durch das in der Reaktion entstandene Nebenprodukt DMS auftrat. $^{[89]}$

\subsubsection{Vergleich der verschiedenen Sauerstoffübertragungen}

Es kann abschließend gesagt werden, dass die Übertragungen mit DMSO und Tetrabutylammoniumnitrat gut zur Sauerstoffübertragung genutzt werden können. Die isobestischen Punkte sind nicht immer vollkommen klar aufgelöst, doch es konnte keine Bildung von anderen Nebenprodukten festgestellt werden.

Die Oxidation mit Trimethyl- $N$-oxid stellt die stärkste Oxidationsmethode unter den hier vorgestellten Methoden dar. Man erkennt, dass bei RT eine gute Übertragung vorhanden ist, allerdings werden jedoch während der Oxidation auch einige Nebenprodukte gebildet. Führt man die Reaktionen bei niedrigeren Temperaturen $\left(-30^{\circ} \mathrm{C}\right)$ durch, so ist der prozentuale Anteil an Nebenprodukten geringer. Doch es konnte keine vollkommene selektive Oxidation der Monooxoverbindung zur Dioxoverbindung erzielt werden.

Um bei den NMR spektroskopischen Untersuchungen einen Nachweis für die Sauerstoffübertragung durch DMSO zu bekommen und nicht jedes Mal eine photometrische Bestimmung durchzuführen, wurde zur Kontrolle aus einer kleinen Probe der Lösung das entstandene DMS mit Hilfe von Quecksilberchlorid als $\left[\left(\mathrm{CH}_{3}\right)_{2} \mathrm{~S}_{2} 3 \mathrm{HgCl}_{2}\right.$ ausgefällt. ${ }^{[90]}$ Bei nicht vollständig abgelaufener Sauerstoffübertragungen kommt es zu einer Reaktion zwischen Quecksilberchlorid und dem Metallkomplex in der Oxidationsstufe IV, wobei eine Verbindung in Oxidationsstufe $\mathrm{V}$ gebildet wird, bei der ein Cl-Atom am Metallzentrum angelagert ist. ${ }^{[91]}$ Diese Reaktion kann durch eine schlagartige Farbveränderung beobachtet werden. Bei einer schlagartigen Farbveränderung zu dunkelbraun kann die Probe nicht zur katalytischen Sauerstoffübertragungsreaktionen eingesetzt werden. Die Reaktion wurde bei den von mir durchgeführten Katalysen nur weiter überwacht, wenn dieser Test in einer 
separaten Probe erfolgreich verlief. Ansonsten konnte nicht von den gleichen stöchiometrischen Verhältnissen ausgegangen werden.

\subsection{Geometrische Aspekte von Dioxo-Verbindungen}

Ob eine cis- oder trans-Anordnung der zwei Oxo-Gruppen am Metallzentrum vorliegt, wurde durch Kristallstrukturen, ${ }^{[92]}$ sowie durch theoretische Berechnungen untersucht. Sakar et al. konnte bei $\left[\mathrm{MoO}(\mathrm{mnt})_{2}\right]^{2-}$ zeigen, dass ein Winkel von $107^{\circ}$ zwischen den zwei Oxogruppen im Molekül auftritt. ${ }^{[29 \mathrm{~h}]}$ Die Gruppe von Hoffmann fand durch theoretische Untersuchungen heraus, dass das 4 d Orbital des Molybdäns eine entscheidende Rolle bei der Stabilisierung der cis-Konfiguration spielt. ${ }^{[93]}$ Bei der Bindungsanalyse der $\mathrm{M}=\mathrm{O}$ Bindung stellt man fest, dass es zu einer Interaktion des valenten d-Orbitals des Metalls und zur Ausbildung einer $\pi$ Bindung zum Sauerstoffatoms kommt. Wäre eine trans-Position des Sauerstoffatoms bevorzugt, so könnten die d-Orbitale mit je zwei p-Orbitalen des Sauerstoffs wechselwirken. Bei der cis- Anordnung hingegen kommt es zu Wechselwirkungen mit drei p-Orbitalen, wodurch eine gewisse Favorisieren dieser Anordnung auftritt. Im Folgenden sind die relevanten Orbitale gezeigt.

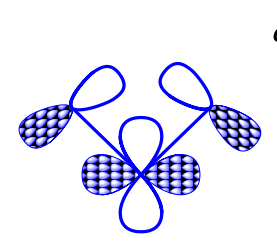

$\mathrm{a}_{1}$
cis-Anordnung

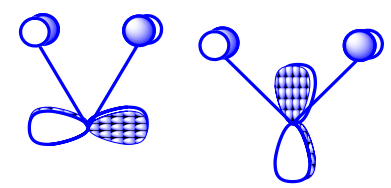

$\mathrm{a}_{2}$ $\mathrm{b}_{2}$ trans-Anordnung

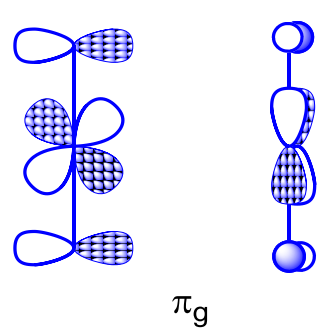

Abb. 101: Wechselwirkung der Metall-d-Orbitale mit den $\pi$-Orbitalen der Sauerstoffatome

\subsection{Entfernung des angelagerten Sauerstoffatoms}

Als Sauerstoffakzeptoren und Reduktionsmittel, haben sich tertiäre Phosphine wie z.B. $\mathrm{PMe}_{3}$ oder $\mathrm{PPh}_{3}$ als Verbindungen der Wahl herausgestellt. Sie weisen eine hohe Löslichkeit in organischen Lösungsmitteln auf und können durch Modifikation der Substituenten in ihrer Reaktivität beeinflusst werden. Im Verlauf der Reaktion kommt es zum nukleophilen Angriff des Phosphoratoms auf das leere $\mathrm{Mo}=\mathrm{O} \pi^{*}$ Orbital. ${ }^{[94]}$ Durch das freie Elektronenpaar des 
Sauerstoffs kommt es zum simultanen nukleophilen Angriff an dem P-C $\sigma^{*}$-Orbital. Dieser Schritt ist aufgrund des hypervalenzen Charakters des Phosphoratoms am Übergangszustand möglich, wodurch es letztlich zur Zweielektronenreduktion des Metalls kommt.

Im Folgenden ist ein Vorschlag für den kompletten katalytischen Zyklus aufgeführt.

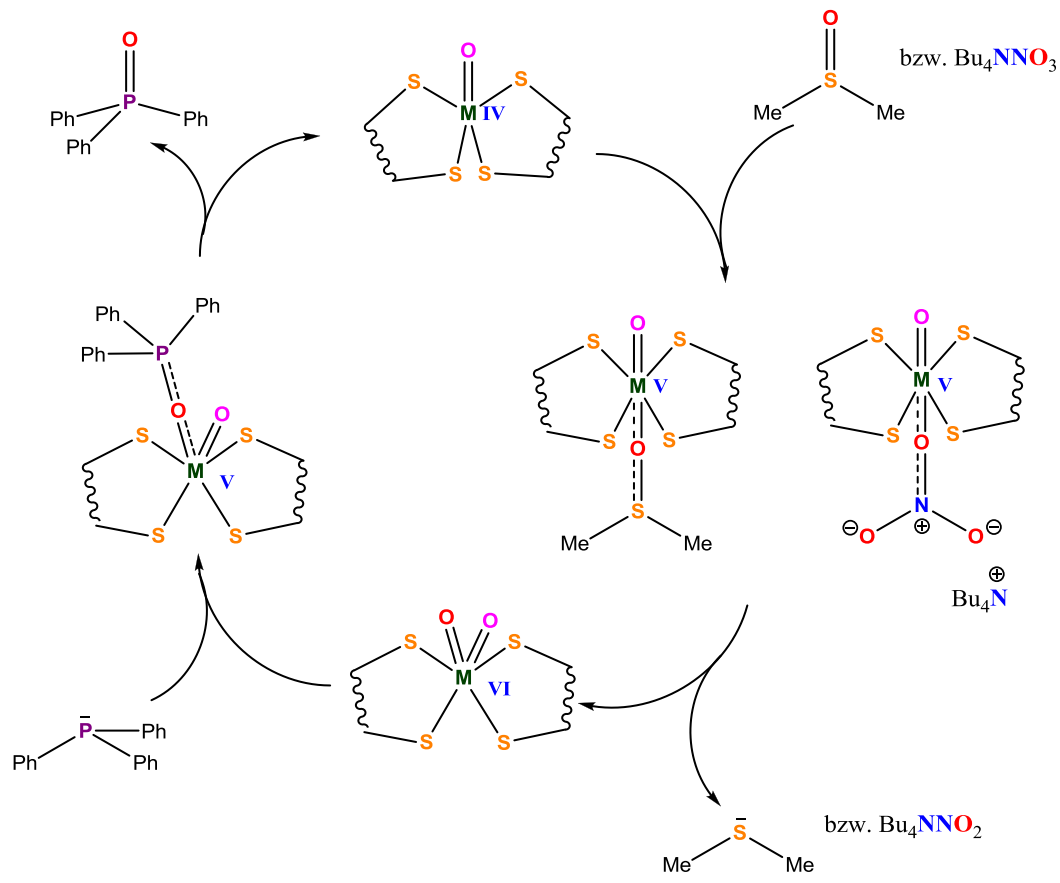

Abb. 102: Ablauf der katalytischen Reaktion

Der Fortschritt der Katalyse kann gut durch ${ }^{31} \mathrm{P}-\mathrm{NMR}$ verfolgt werden, da Substrat $\left(\mathrm{PPh}_{3}\right)$ und Produkt $\left(\mathrm{PPh}_{3} \mathrm{O}\right)$ gut separierte Signale erzeugen. Im Folgenden sind die Phosphor-NMRSpektren beispielhaft gezeigt, die den Umsatz von $\mathrm{PPh}_{3} \mathrm{zu} \mathrm{PPh}_{3} \mathrm{O}$ belegen.

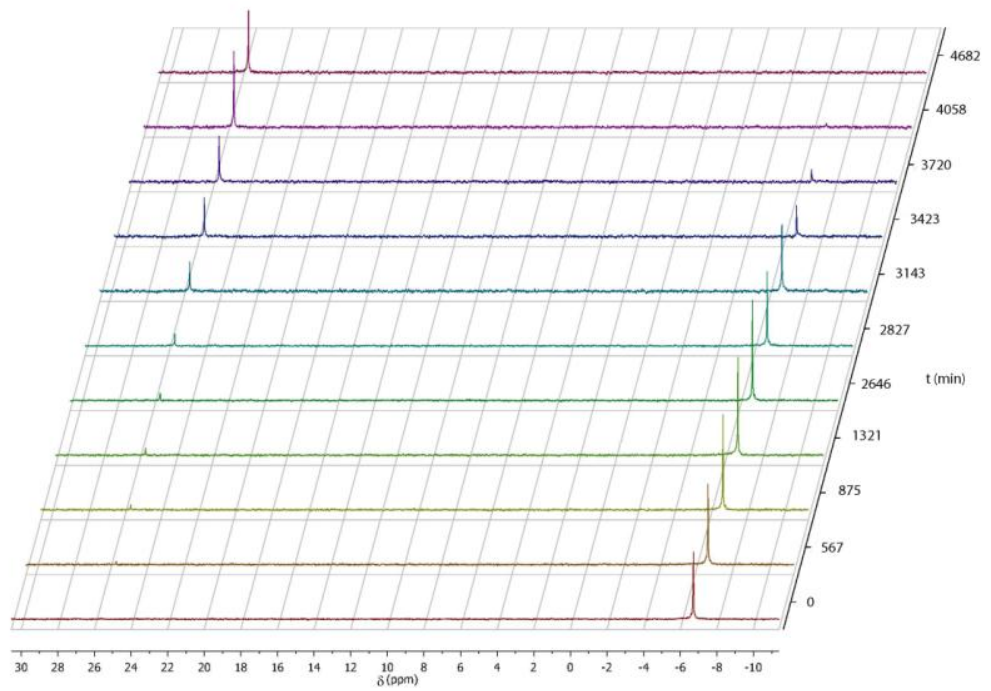

Abb. 103: Beispiel für die Verfolgung des Reaktionsfortschritts durch ${ }^{31} \mathrm{P}-\mathrm{NMR}$-Spektroskopie der Verbindung [WO(ntdt) $\left.{ }_{2}\right]^{2-}$ mit einem Verhältnis von 1 : 2.5 Katalysator/PPh 
Am Anfang der Katalyse ist ausschließlich $\mathrm{PPh}_{3}$ als Phosphorspezies vorhanden, wie am Singulett $\sim-6$ ppm zu sehen. Im Laufe der Reaktion ist eine Entstehung und eine Zunahme eines Singuletts bei 26 ppm zu beobachten, bis am Ende des katalytischen Zyklus nur noch $\mathrm{PPh}_{3} \mathrm{O}$ vorhanden ist.

\subsection{Temperaturabhängige katalytische Untersuchungen}

Es wurden Katalysen bei $25^{\circ} \mathrm{C}, 35^{\circ} \mathrm{C}, 45^{\circ} \mathrm{C}, 55^{\circ} \mathrm{C}$ und $65^{\circ} \mathrm{C}$ durchgeführt, um den Einfluss der Temperatur auf die Reaktionsgeschwindigkeiten $\mathrm{zu}$ untersuchen und die Aktivierungsenergie zu bestimmen. Der Katalysator wurde zu $\mathrm{PPh}_{3}$ im Verhältnis 1:10 verwendet. Da das Oxidationsmittel im Überschuss im Vergleich zum Katalysator verwendet wurde, handelt es sich bei den folgenden Katalysen um Reaktionen, welche zur pseudo-erster Ordnungs Reaktionsart zugeordnet werden können.

\subsubsection{Untersuchung der Paare $\left[\mathrm{MO}(\mathrm{xdt})_{2}\right]^{2-;}\left[\mathrm{MO}(\mathrm{vdt})_{2}\right]^{2-}$ und $\left[\mathrm{MO}(\mathrm{qdt})_{2}\right]^{2-}$}
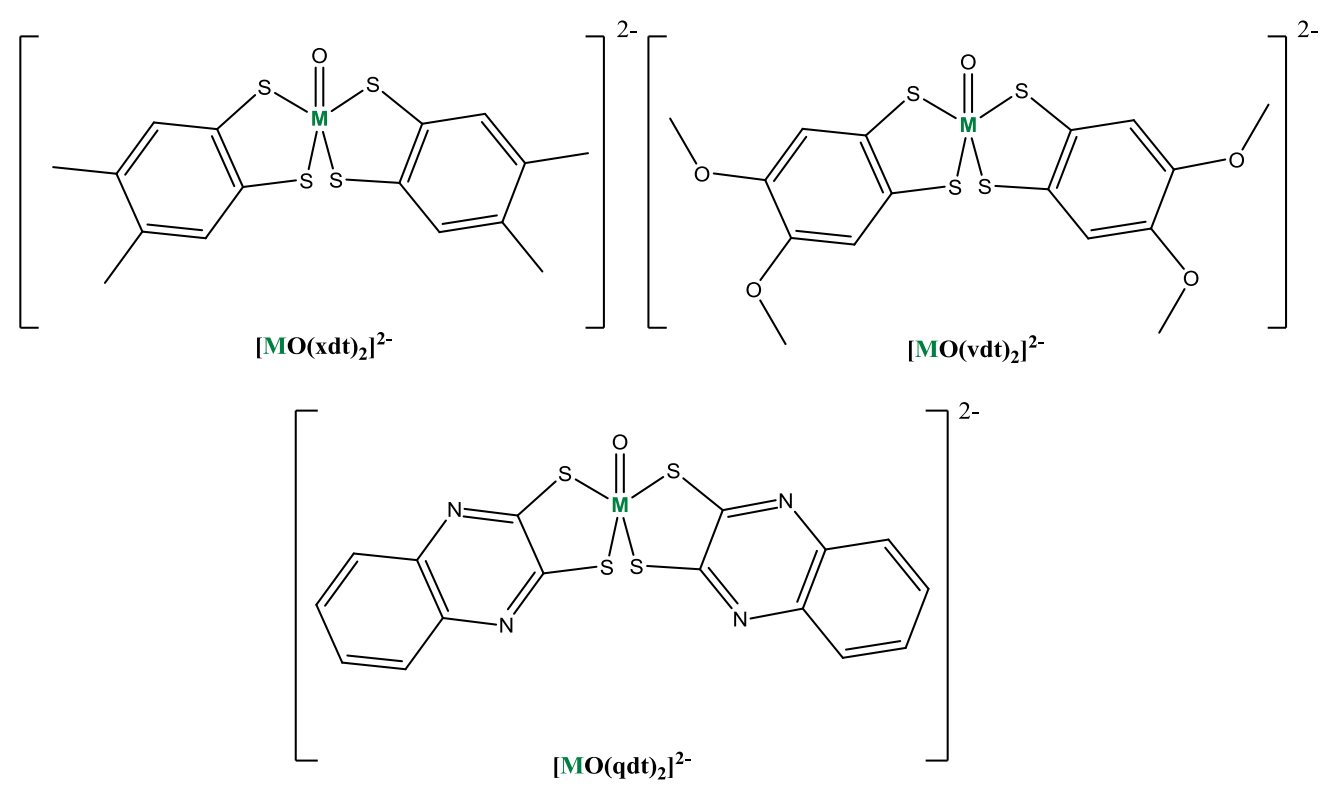

Abb. 104: Untersuchte Verbindungspaare

Der Fortschritt der Katalyse wurde mit Hilfe von ${ }^{31} \mathrm{P}-\mathrm{NMR}-$ Spektroskopie verfolgt und der sich daraus ergebende Umsatz $\mathrm{PPh}_{3}$ zu $\mathrm{PPh}_{3} \mathrm{O}$ wurde gegen die Reaktionszeit aufgetragen. 
$\left[\mathrm{MoO}(\mathrm{xdt})_{2}\right]^{2-}$

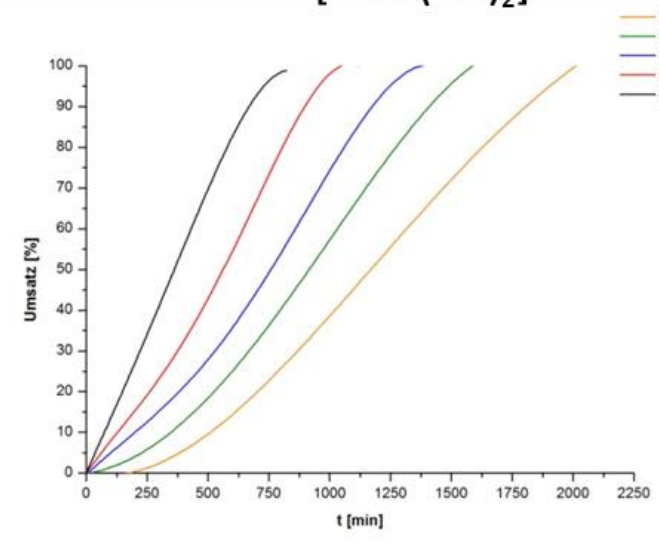

$\left[\mathrm{WO}(\mathrm{xdt})_{2}\right]^{2-}$

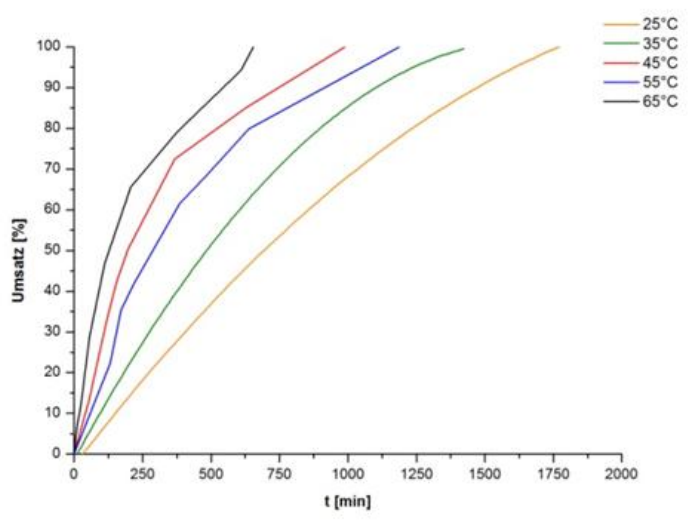

Abb. 105: Umsatzdiagramme für das Paar $\left[\mathrm{MO}(\mathrm{xdt})_{2}\right]^{2-}$

Die Umsatzdiagramme der Verbindungspaare $\left[\mathrm{MO}(\mathrm{vdt})_{2}\right]^{2-}$ und $\left[\mathrm{MO}(\mathrm{qdt})_{2}\right]^{2-}$ sind im Anhang im Abschnitt $11.2 \mathrm{zu}$ finden. Bei allen katalytischen Reaktionen erkennt man deutlich den Anstieg der Reaktionsgeschwindigkeit (Steigung der Kurven) und eine Verkürzung der Gesamtreaktionszeiten durch die Erhöhung der Reaktionstemperatur. In der folgenden Tabelle sind die Reaktionszeiten bis zum vollständigem Umsatz gezeigt.

\begin{tabular}{|c|c|c|c|c|c|c|}
\hline & Oxidationsmittel & $25^{\circ} \mathrm{C}[\mathrm{h}]$ & $35^{\circ} \mathrm{C}[\mathrm{h}]$ & $45^{\circ} \mathrm{C}[\mathrm{h}]$ & $55^{\circ} \mathrm{C}[\mathrm{h}]$ & $65^{\circ} \mathrm{C}[\mathrm{h}]$ \\
\hline$\left[\mathrm{MoO}(x d t)_{2}\right]^{2-}$ & DMSO & 33.72 & 26.85 & 23.13 & 18.62 & 13.72 \\
\hline$\left[\mathrm{WO}(\mathrm{xdt})_{2}\right]^{2-}$ & DMSO & 30.48 & 23.72 & 19.78 & 16.47 & 10.92 \\
\hline$\left[\mathrm{MoO}(\mathrm{vdt})_{2}\right]^{2}$ & DMSO & 106.7 & 58.60 & 52.03 & 41.78 & 34.68 \\
\hline$\left[\mathrm{WO}(\mathrm{vdt})_{2}{ }^{2-}\right.$ & DMSO & 101.7 & 53.53 & 51.03 & 39.95 & 31.53 \\
\hline$\left[\mathrm{MoO}(q d t)_{2}\right]^{2-}$ & DMSO & 121.7 & 97.93 & 89.33 & 48.00 & 39.82 \\
\hline$\left[\mathrm{WO}(\mathrm{qdt})_{2}\right]^{2-}$ & DMSO & 84.35 & 51.47 & 41.90 & 29.73 & 24.15 \\
\hline
\end{tabular}

Tab. 19: Reaktionszeiten bis zum vollständigen Umsatz des Substrats (in grau: die jeweils kürzere Reaktionszeit der Katalysatorpaare)

Werden nun die Reaktionsgeschwindigkeiten im Hinblick auf die Molybdän- und Wolframkatalysatoren verglichen, so stellt man fest, dass die Wolframverbindung immer die kürzeren Umsatzzeiten (Zeiten grau markiert) liefern, als die analoge Molybdänverbindung.

\subsubsection{Bestimmung der kinetischen Parameter}

Um die Geschwindigkeitskonstante der Reaktionen zu bestimmen, wurde unter der Annahme einer Reaktion 1. Ordnung bezüglich $\left[\mathrm{PPh}_{3}\right]$ eine Auftragung $\ln [\mathrm{S}]$ gegen $\mathrm{t}$ angefertigt. Aus der Steigung der resultierenden Gerade ergibt sich die Geschwindigkeitskonstante siehe Tab.20. 


\begin{tabular}{|c|c|c|c|c|c|c|}
\hline & Oxidationsmittel & $25^{\circ} \mathrm{C}\left[\mathrm{s}^{-1}\right]$ & $35^{\circ} \mathrm{C}\left[\mathrm{s}^{-1}\right]$ & $45^{\circ} \mathrm{C}\left[\mathrm{s}^{-1}\right]$ & $55^{\circ} \mathrm{C}\left[\mathrm{s}^{-1}\right]$ & $65^{\circ} \mathrm{C}\left[\mathrm{s}^{-1}\right]$ \\
\hline$\left[\mathrm{MoO}(\mathrm{xdt})_{2}\right]^{2-}$ & DMSO & $2.18 \cdot 10^{5}$ & $2.80 \cdot 10^{5}$ & $3.36 \cdot 10^{5}$ & $4.48 \cdot 10^{5}$ & $6.21 \cdot 10^{5}$ \\
\hline$\left[\mathrm{WO}(x d t)_{2}\right]^{2-}$ & DMSO & $2.53 \cdot 10^{5}$ & $2.93 \cdot 10^{5}$ & $4.18 \cdot 10^{5}$ & $5.20 \cdot 10^{5}$ & $7.31 \cdot 10^{5}$ \\
\hline$\left[\mathrm{MoO}(\mathrm{vdt})_{2}\right]^{2-}$ & DMSO & $0.83 \cdot 10^{5}$ & $1.27 \cdot 10^{5}$ & $1.38 \cdot 10^{5}$ & $2.05 \cdot 10^{5}$ & $2.82 \cdot 10^{5}$ \\
\hline$\left[\mathrm{WO}(\mathrm{vdt})_{2}^{2-}\right.$ & DMSO & $1.04 \cdot 10^{5}$ & $1.36 \cdot 10^{5}$ & $1.95 \cdot 10^{5}$ & $2.22 \cdot 10^{5}$ & $3.23 \cdot 10^{5}$ \\
\hline$\left[\mathrm{MoO}(\mathrm{qdt})_{2}\right]^{2-}$ & DMSO & $0.53 \cdot 10^{5}$ & $0.66 \cdot 10^{5}$ & $0.83 \cdot 10^{5}$ & $1.05 \cdot 10^{5}$ & $1.68 \cdot 10^{5}$ \\
\hline$\left[\mathrm{WO}(\mathrm{qdt})_{2}\right]^{2-}$ & DMSO & $1.01 \cdot 10^{5}$ & $1.52 \cdot 10^{5}$ & $1.98 \cdot 10^{5}$ & $2.75 \cdot 10^{5}$ & $2.99 \cdot 10^{5}$ \\
\hline
\end{tabular}

Tab. 20: Geschwindigkeitskonstanten für die Oxo-Transfer Katalysen mit $\mathrm{PPh}_{3}$ als Reduktionsmittel

An den Geschwindigkeiten kann gesehen werden, dass immer die größeren Geschwindigkeitskonstanten bei den Wolframverbindungen im Vergleich zu den analogen Molybdänverbindungen vorhanden sind.

Um die Aktivierungsenergien zu bestimmen, bedient man sich der Arrhenius-Gleichung und kann durch eine Auftragung von $\ln (\mathrm{k})$ gegen $1 / \mathrm{T}$ aus der Steigung der linearen Regressionsgeraden den Faktor $-\mathrm{E}_{\mathrm{A}} / \mathrm{R}$ ermitteln.

\begin{tabular}{lccc} 
& Oxidationsmittel & $\mathrm{E}_{\mathrm{A}}[\mathrm{kJ} / \mathrm{mol}]$ & $\mathrm{k} / \mathrm{T}\left[10^{-8} \mathrm{~s}^{-1} \mathrm{~K}^{-1}\right]$ \\
\hline$\left[\mathrm{MoO}(\mathbf{x d t})_{2}\right]^{2-}$ & DMSO & 21.05 & 1.03 \\
\hline$\left[\mathrm{WO}(\mathbf{x d t})_{2}\right]^{2-}$ & DMSO & 20.44 & 3.07 \\
\hline$\left[\mathrm{MoO}(\mathbf{v d t})_{2}\right]^{2-}$ & DMSO & 24.77 & 0.14 \\
\hline$\left[\mathrm{WO}(\mathbf{v d t})_{2}{ }^{2-}\right.$ & DMSO & 23.00 & 52.3 \\
\hline$\left[\mathrm{MoO}(\mathbf{q d t})_{2}\right]^{2-}$ & DMSO & 23.08 & 0.13 \\
\hline$\left[\mathrm{WO}(\mathbf{q d t})_{2}\right]^{2-}$ & DMSO & 20.30 & 0.29
\end{tabular}

Tab. 21: Bestimmte Aktivierungsenergien und die Temperaturabhangigkeiten der Geschwindigkeitskonstanten

Im Einklang mit den Reaktionsgeschwindigkeiten sind die Aktivierungsenergien für die wolframkatalysierten Reaktionen kleiner als für die molybdänkatalysierten. Weiterhin wurden die Geschwindigkeitskonstanten $(\mathrm{k})$ gegen $\mathrm{T}$ aufgetragen. Dadurch kann die Veränderung der Geschwindigkeit mit der Temperatur dargestellt werden. Weil die Reaktionsgeschwindigkeit exponentiell mit der Temperatur zunehmen muss, wurden exponentielle Funktionen in die entsprechenden Kurven gefittet. Die erhaltenen Steigungswerte der exponentiellen Funktionen wurden in Tab.21 aufgezeigt und die Auftragungen für die xdt-Komplexe sind in Abb.106 dargestellt. 


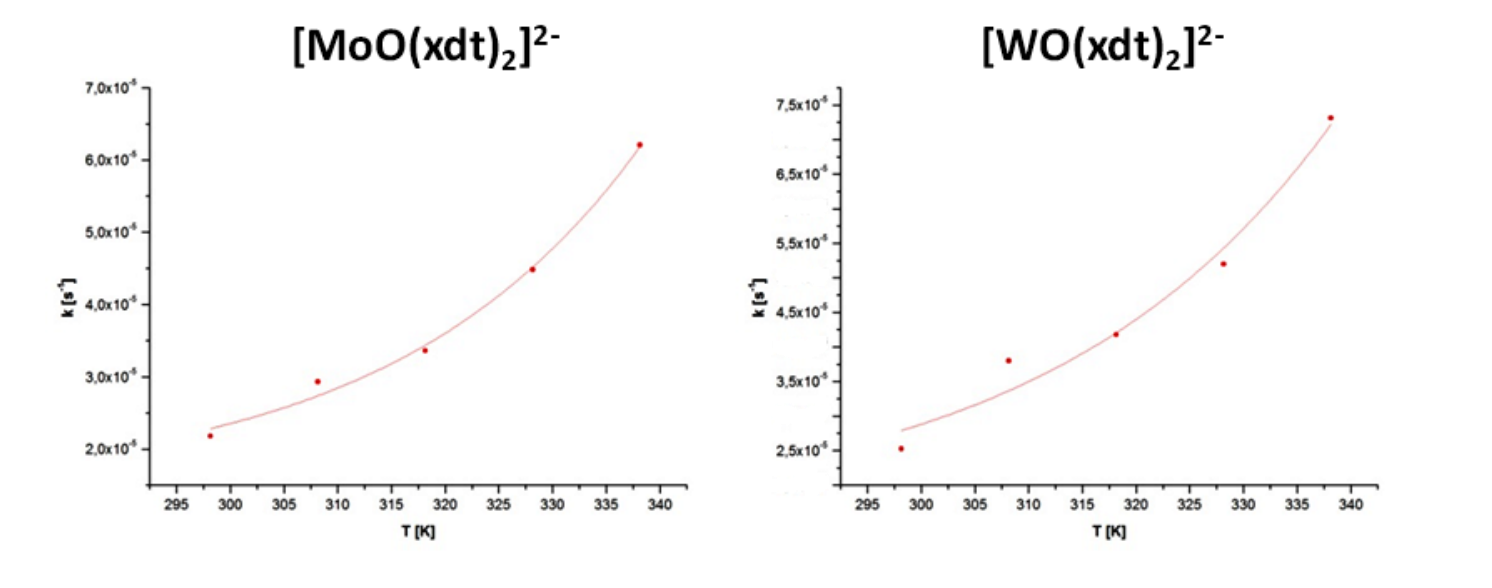

Abb. 106: Temperaturabhängigkeit der Geschwindigkeitskonstanten des Paares $\left[\mathrm{MO}(\mathrm{xdt})_{2}\right]^{2-}$

Die Faustregel, die als RGT-Regel oder Van`t-Hoff-Regel bezeichnet wird, sagt grob voraus, dass eine Erhöhung der Reaktionstemperatur um $10 \mathrm{~K}$ zu einer Verdoppelung bis Vervierfachung der Reaktionsgeschwindigkeit führt. Mit den hier diskutierten Katalysen konnte diese Faustregel nicht bestätigt werden. Lediglich die Beschleunigung der Reaktion um etwa die Hälfte der Reaktionsgeschwindigkeit wurde bei Erhöhung um $10^{\circ} \mathrm{C}$ festgestellt. Interessanterweise ist die Geschwindigkeit der Reaktion bei den Wolframkatalysatoren stärker von der Temperatur abhängig, als bei den Molybdänkatalysatoren. Ähnliche Beobachtungen wurden auch von Nakamura et al. und Holm et al. gemacht. ${ }^{[95]}$ Dies untermauert die Ergebnisse und Interpretationen der temperaturabhängigen elektrochemischen Untersuchungen (Kapitel 4) mit einem stärkeren Einfluss der Temperatur auf wolframhaltige Verbindungen in vielerlei Hinsicht.

\subsection{Untersuchungen des katalytischen Zyklus mit Hilfe von DFT-Rechnungen}

Um die experimentell bestimmten Parameter mit DFT-Rechnungen zu verifizieren, wurde versucht, den katalytischen Zyklus des $\left[\mathrm{MO}(\mathrm{xdt})_{2}\right]^{2-}-$ Paares durch theoretische Rechnungen nachzuvollziehen.

\subsubsection{Untersuchungen zur Anlagerung des Sauerstoffatoms}

Ausgehend von der Anfangsverbindung mit nur einer Oxogruppe wurde versucht, ein Dimethylsulfoxid-Molekül an den Komplex zu koordinieren. Durch Geometrieoptimierungen der Zustände dieses Prozesses konnte ein Übergangszustand dieses Zyklus gefunden werden (Verbindung Ib [Mo] bzw. IIb [W]). Zunächst wurde untersucht, ob ein Angriff des DMSOs von der Grundfläche der pyramidalen Geometrie stattfindet, oder ob ein Seitenangriff 
bevorzugt ist. Nach vergleichenden Untersuchung zeigte sich, dass ein Angriff des DMSOs von der Seite energetische Vorteile mit sich bringt. In der geometrieoptimierten Struktur ist das Abknicken eines Dithiolenliganden zum gegenüberliegenden Dithiolenliganden festzustellen, wodurch sich eine verzerrt-oktaedrische Anordnung ergibt. Die erhaltene Struktur ist im Folgenden für die Wolframverbindung gezeigt.

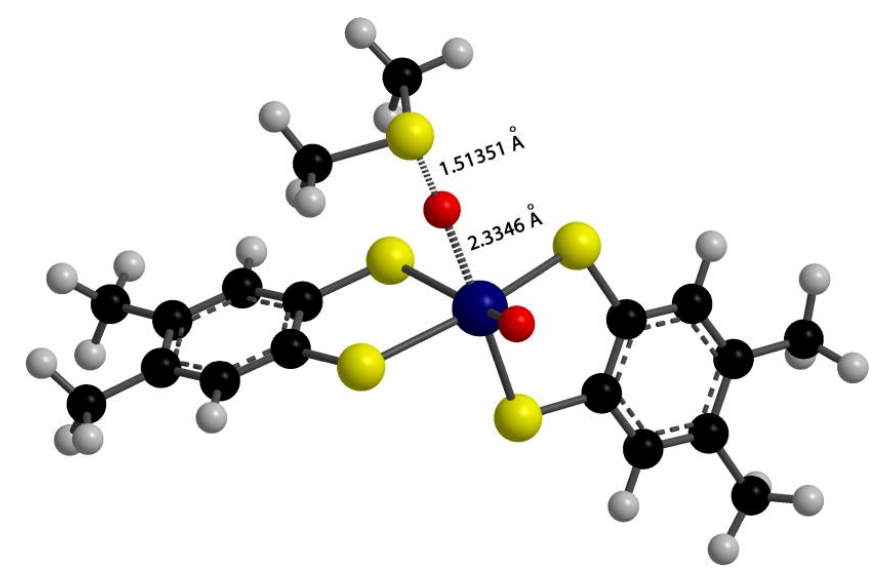

Abb. 107: Struktur des Übergangszustandes von $\left[\mathrm{WO}(\mathrm{xdt})_{2}\right]^{2-}(\mathrm{Ilb})$ bei der Reaktion mit DMSO

Bei der Analyse der Bindungslängen ist ein Abstand des Sauerstoffatoms des DMSOs von $2.3346 \AA$ zum Wolframzentrum zu beobachten. Diese Bindungslänge weist auf eine Mischung aus Doppel- bzw. Einfachbindungen hin. Durch die Wechselwirkung mit dem Metallzentrum kommt es außerdem zu einer Verlängerung der Schwefel-Sauerstoffbindung im DMSO-Molekül, die hier mit 1.51351 Å gefunden wurde und somit $0.17834 \AA$ länger als im freien DMSO-Molekül erscheint. Bei der Betrachtung der Winkel in dem Molekül kann in axialer Position $(\mathrm{O}=\mathrm{W}-\mathrm{S})$ ein Winkel von $160.49^{\circ}$ festgestellt werden, was auf eine trigonal prismatische Geometrie hinweist. Die äquatorialen Winkel zeigen ähnliche Abweichungen, die hier mit $163.80^{\circ}$ gefunden werden. Die Tendenzen bei den Molybdänverbindungen sind ähnlich und werden deshalb nicht mehr explizit aufgeführt.

Um den genauen Abstand zu bestimmen, bei dem die $\mathrm{S}_{\mathrm{DMSO}}=\mathrm{O}$ Bindung bricht, und die $\mathrm{M}=\mathrm{O}-$ Bindung gebildet wird, wurde das koordinierte DMS-Molekül von der M-O-Einheit in Schritten von $0.01 \AA$ entfernt. Die M-O Bindung wurde dabei als starr angesehen. Nach jeder Abstandsvergrößerung wurde das komplette Molekül einer Geometrieoptimierung unterzogen. Auf diese Weise konnte die energetische Veränderung des Moleküls bei Veränderung der spezifischen Bindungen analysiert werden. Nach Identifizierung einer drastischen Energieveränderung wurde, ausgehend von den Punkten vor den Energieeanstieg zu einem Punkt nach dem Anstieg eine Sattelpunktssuche durchgeführt. Es wurde außerdem eine Frequenzrechnung angeschlossen, bei der durch Bildung von imaginären Frequenzen ein 
Übergangszustand ausgemacht werden konnte, weil es am Übergangszustand zum Wegfall von Freiheitsgraden kommt. Durch diese Untersuchungen wurde ein einziger Übergangszustand (ÜZ) gefunden. Analoge Ergebnisse wurden bereits im Jahre 2002 von Nordlander et al. bei Verwendung eines mnt-Ligandens erhalten. ${ }^{[96]}$ Manche Arbeitsgruppen, die mit DFT-Rechnungen Katalysezyklen nachvollzogen haben, verwendeten am Metallzentrum zusätzlich eine OMe-Gruppe, um die Anbindung von Amminosäureresten in den Enzymen simulieren zu können. Unter Einsatz dieser zusätzlichen Gruppe am Metallzentrum wurden weitere Übergangszustände gefunden. ${ }^{[96-97]}$ In den hier untersuchten Katalysen kommt ein solcher Ligand nicht vor. Um die Ausgangsverbindungen $\left[\mathrm{MO}\left(\mathrm{xdt}_{2}\right)_{2}\right]^{2-}+$ DMSO (Ia) und die Endprodukte $\left[\mathrm{MoO}_{2}(\mathrm{xdt})_{2}\right]+$ DMS (Ic) nachzubilden, wurde ausgehend von dem Übergangszustand, die Metall-Sauerstoffbindung als eingefroren betrachtet und dann die Geometrieoptimierung durchgeführt. Außerdem wurde die $\mathrm{S}=\mathrm{O}$-Bindung des DMSOs als starr angesehen, um die Ausgangsverbindungen zu erhalten. Mit Hilfe dieser Techniken wurden alle möglichen Reaktionsintermediate für die Sauerstoffübertragung durch DMSO erhalten. In $A b b .108$ ist das berechnete Energiediagramm für die Oxidation der Molybdänverbindung gezeigt. Es ist zu beachten, dass es während des Katalysezyklus zum Wechsel der Oxidationsstufe von IV über V nach VI kommt, was zu Geometrieänderungen aufgrund des Oxidationswechsels führt.

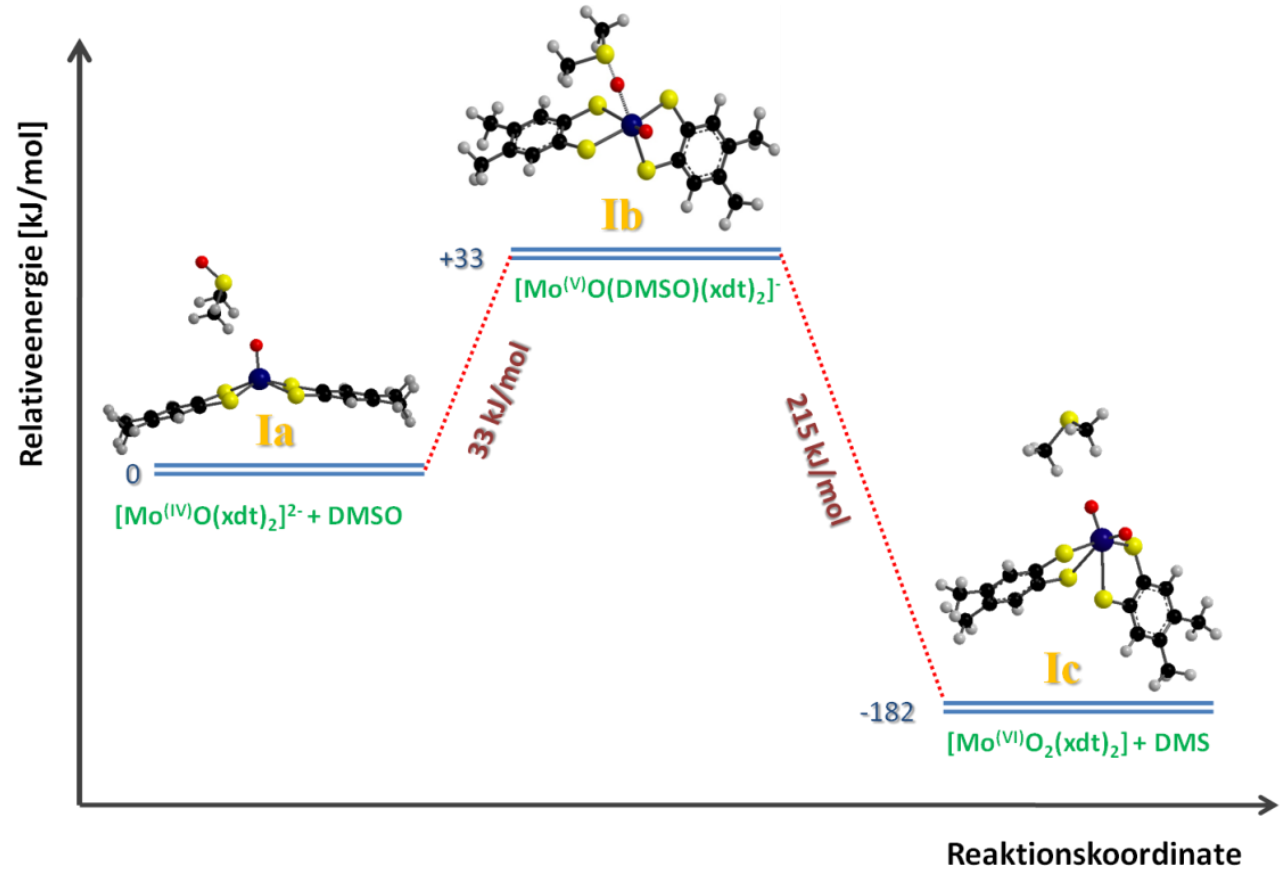

Abb. 108: Berechneter Energien für die $\left[\mathrm{MoO}\left(\mathrm{xdt}_{2}\right]^{2-}{ }^{2-} \mathrm{DMSO}\right.$

Durch Differenzbildung der Energien der jeweiligen Intermediate konnten die Aktivierungsenergie und die Reaktionsenthalpie bestimmt werden. Es wurde eine 
Aktivierungsenergie von $33 \mathrm{~kJ} / \mathrm{mol}$ bestimmt. Da ein $\Delta \mathrm{H}$ von $-182 \mathrm{~kJ} / \mathrm{mol}$ auftritt, kann davon ausgegangen werden, dass die Reaktion selbständig abläuft ohne zu Hilfenahme von anderen äußeren Faktoren. Zusammengefasst ergeben sich folgende Energiewerte:

\begin{tabular}{|c|c|}
\hline & Relative Energien [kJ/mol] \\
\hline$\left[\mathrm{Mo}^{(\mathrm{IV})} \mathrm{O}(\mathrm{xdt})_{2}\right]^{2-}+$ DMSO (Ia) & 0 \\
\hline$\left[\mathbf{M o}^{(\mathrm{V})} \mathbf{O}(\mathrm{DMSO})(\mathbf{x d t})_{2}\right]^{-}(\mathrm{Ib})$ & +32.78 \\
\hline$\left[\mathrm{Mo}^{(\mathrm{VI})} \mathrm{O}_{2}(\mathrm{xdt})_{2}\right]+\mathrm{DMS}(\mathrm{Ic})$ & -182.35 \\
\hline$\left[\mathrm{W}^{(\mathrm{IV})} \mathrm{O}(\mathrm{xdt})_{2}\right]^{2-}+$ DMSO (IIa) & 0 \\
\hline$\left[\mathrm{W}^{(\mathrm{V})} \mathbf{O}(\mathrm{DMSO})(\mathbf{x d t})_{2}\right]^{-}(\mathrm{IIb})$ & +80.76 \\
\hline$\left[\mathrm{W}^{(\mathrm{VI})} \mathrm{O}_{2}(\mathbf{x d t})_{2}\right]+\mathbf{D M S}(\mathrm{IIc})$ & -3.07 \\
\hline
\end{tabular}

Tab. 22: Energiewerte der Sauerstoffübertragung durch DMSO

Für die Oxidation mit DMSO ergibt sich für die Wolframverbindung eine höhere Aktivierungsenergie und nur leicht negativer Wert für $\Delta \mathrm{H}$. Die Oxidation ist für Molybdän gesamtenergetisch günstiger. Aufgrund der kleineren Aktivierungsenergie läuft diese auch schneller ab.

\subsubsection{Untersuchungen der Übertragung des Sauerstoffatoms auf $\mathrm{PR}_{3}$}

Bei den experimentellen Untersuchungen wurde $\mathrm{PPh}_{3}$ zur Reduktion genutzt. Da die Optimierung für $\mathrm{PPh}_{3}$ sehr groß ist, benötigen theoretische Untersuchungen des Moleküls enorm viel Rechenzeit. Deshalb wurde auf das ähnliche aber handlichere Teilchen $\mathrm{PMe}_{3}$ zurückgegriffen. Das Intermediat sowie die Strukturen der Anfangs- und Endprodukte wurden nach dem gleichen Schema, wie für die Berechnung des ersten Teilzyklus mit DMSO durchgeführt. Im Folgenden ist der berechnete Übergangszustand von $\left[\mathrm{MoO}\left(\mathrm{OPMe}_{3}\right)(\mathrm{xdt})_{2}\right]^{-}$ (Ie) gezeigt.

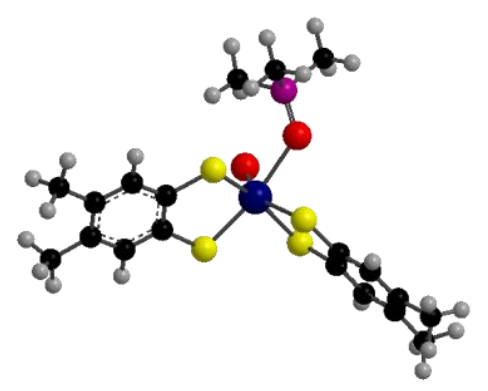

Abb. 109: Struktur des Übergangszustandes mit angelagertem $\mathrm{PMe}_{3}$

Es ergibt sich ein Abstand der Molybdänsauerstoff-Bindung mit angelagertem $\mathrm{PMe}_{3}$ von $2.4977 \AA$ A. Das stellt eine starke Verlängerung im Vergleich zu der anderen Mo=O Bindung mit $1.72528 \AA$ dar. Wie oben bereits beschrieben wurden sowohl die Edukte, als auch die 
Produkte der Reduktion des Molybdänkomplexes und Oxidation von $\mathrm{PMe}_{3}$ zu $\mathrm{OPMe}_{3}$ berechnet und es ergibt sich das Energiediagramm aus Abb.111

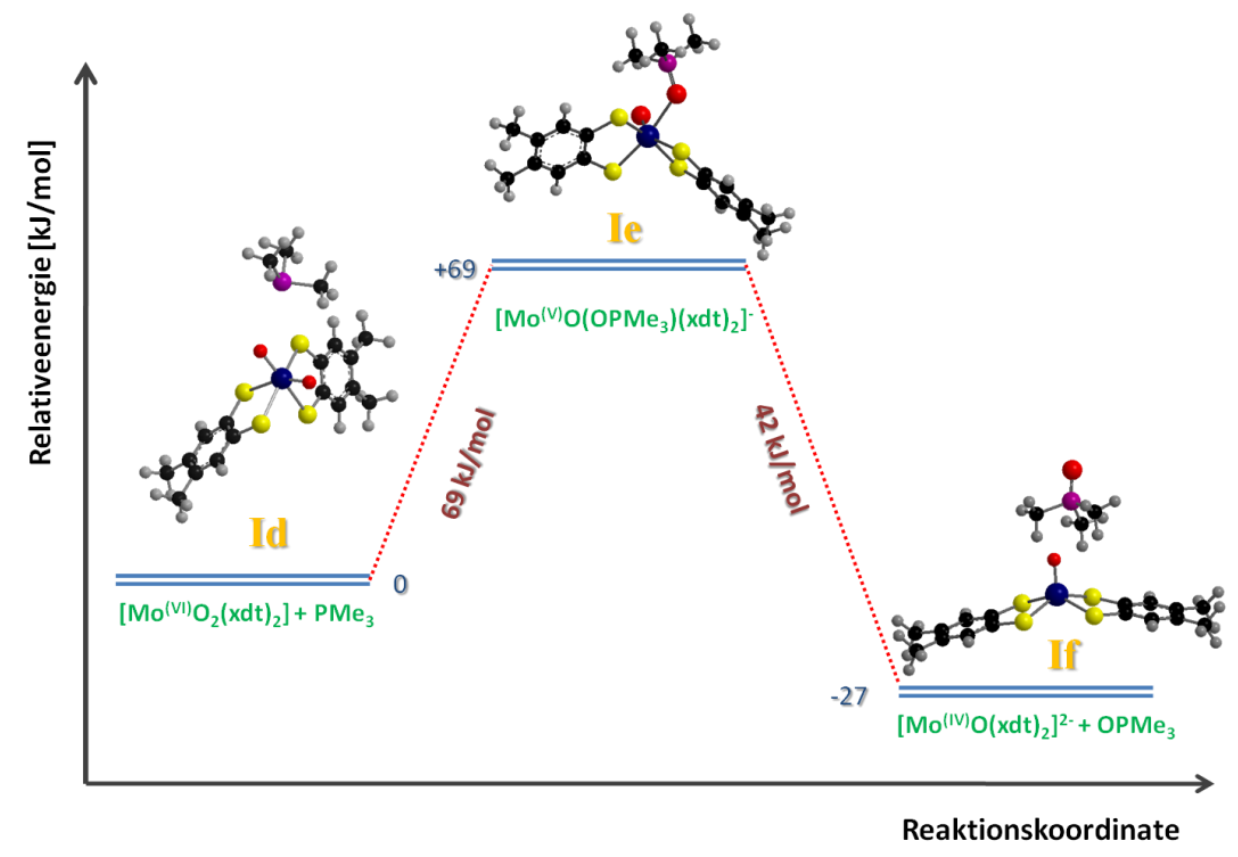

Abb. 110: Berechnete Energien der katalytische Halbreaktion

Es ergeben sich bei der Molybdän und der Wolframverbindung folgende Energiewerte:

\begin{tabular}{|c|c|}
\hline & Relative Energien [kJ/mol] \\
\hline$\left[\mathrm{Mo}^{(\mathrm{VI})} \mathrm{O}_{2}(\mathrm{xdt})_{2}\right]+\mathrm{PMe}_{3}(\mathrm{Id})$ & 0 \\
\hline$\left[\mathrm{Mo}^{(\mathrm{V})} \mathrm{O}\left(\mathrm{OPMe}_{3}\right)(\mathrm{xdt})_{2}\right]^{-}(\mathrm{Ie})$ & +69.24 \\
\hline$\left[\mathrm{Mo}^{(\mathrm{IV})} \mathrm{O}(\mathrm{xdt})_{2}\right]^{2-}+\mathrm{PMe}_{3} \mathrm{O}($ If $)$ & -27.42 \\
\hline$\left[\mathrm{W}^{\mathrm{VI})} \mathrm{O}_{2}(\mathrm{xdt})_{2}\right]+\mathrm{PMe}_{3}(\mathrm{IId})$ & 0 \\
\hline$\left[\mathrm{W}^{\mathrm{V})} \mathrm{O}\left(\mathrm{OPMe}_{3}\right)(\mathrm{xdt})_{2}\right]^{-}(\mathrm{IIe})$ & +45.64 \\
\hline$\left[\mathrm{W}^{\mathrm{IV})} \mathrm{O}(\mathrm{xdt})_{2}\right]^{2-}+\mathrm{PMe}_{3} \mathrm{O}$ (IIf) & -36.45 \\
\hline
\end{tabular}

Tab. 23: Berechnete Energiewerte für die Reduktion mit $\mathrm{PMe}_{3}$

Vergleicht man diese Werte mit den experimentellen Werten $(21.05 \mathrm{~kJ} / \mathrm{mol}$ [Mo] und 20.44 kJ/mol [W]), stellt man eine größere Abweichung fest. Doch die Tendenz der größeren Aktivierungsenergie bei der Molybdänverbindung, konnte auch durch die theoretischen Berechnungen erhalten werden. Als Folge der erhaltenen größeren Werte für die Reaktionsenthalpie bei der Wolframverbindung, kann der schnellere Ablauf der Reaktion erklärt werden. Die Abweichungen zwischen den theoretischen und experimentellen Werten lassen sich durch das Rechnen in der Gasphase, sowie den Ersatz des $\mathrm{PPh}_{3}$ durch $\mathrm{PMe}_{3}$ erklären. 


\subsection{Konzentrationsabhänge katalytische Untersuchungen}

Um einen Einblick in mechanistischen Verlauf dieser Katalysen zu bekommen, wurden katalytische Untersuchungen mit verschiedenen Substratkonzentrationen durchgeführt. Mit Hilfe der Michaelis-Menten-Beziehung können daraus Rückschlüsse über Affinitäten der einzelnen Reaktionspartner zueinander gezogen werden. Durch die Michaelis-MentenGleichung wird der Zusammenhang zwischen Anfangsgeschwindigkeit und Substratkonzentration beschrieben.

\subsubsection{Untersuchungen der katalytischen Sauerstoffübertragung des [MO(ntdt) $\left.{ }_{2}\right]^{2-}$ -}

\section{Paares}

Von den folgenden Verbindungen wurden katalytische Untersuchungen mit vier verschiedenen Substratkonzentrationen ([Kat]:[S]=1:2.5; $1: 5 ; 1: 7.5 ; 1: 10$ ) bei $60^{\circ} \mathrm{C}$ durchgeführt.

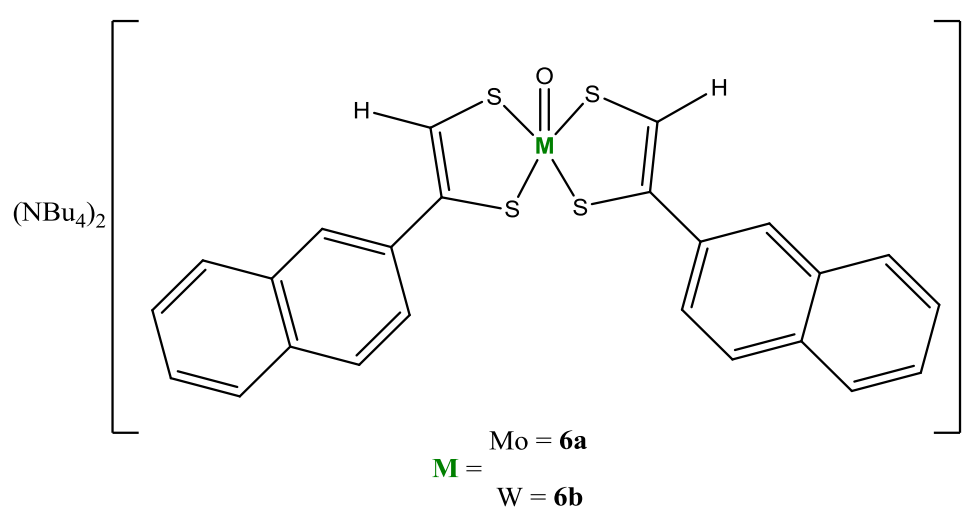

Abb. 111: $\left[\mathrm{MO}(\mathrm{ntdt})_{2}\right]^{2-}$-Verbindungspaar

Bei diesem Verbindungspaar wurden zuerst mit Hilfe von DMSO die Dioxokomplexe hergestellt und diese zur Oxidation von $\mathrm{PPh}_{3}$ verwendet. Die Übertragung des Sauerstoffatoms kann durch folgendes Reaktionsschema veranschaulicht werden.

$$
\begin{aligned}
& {\left[\mathrm{MoO}(n t d t)_{2}\right]^{2-}+\mathrm{DMSO} \underset{k_{-1}}{\stackrel{k_{1}}{\rightleftarrows}}\left[\mathrm{MoO}\left(\mathrm{OSMe}_{2}\right)(n t d t)_{2}\right]^{-} \stackrel{k_{2}}{\longrightarrow}\left[\mathrm{MoO}_{2}(n t d t)_{2}\right]+\mathrm{DMS}} \\
& {\left[\mathrm{MoO}_{2}(n t d t)_{2}\right]+\mathrm{PPh}_{3} \underset{k_{-3}}{\stackrel{k_{3}}{\rightleftarrows}}\left[\mathrm{MoO}\left(\mathrm{OPPh}_{3}\right)(n t d t)_{2}\right]^{-} \stackrel{k_{4}}{\longrightarrow}\left[\mathrm{MoO}(n t d t)_{2}\right]^{2-}+\mathrm{PPh}_{3} \mathrm{O}}
\end{aligned}
$$


Im Folgenden ist der Verlauf der Reaktion anhand der Konzentration von $\mathrm{OPPh}_{3}$ aufgeführt.
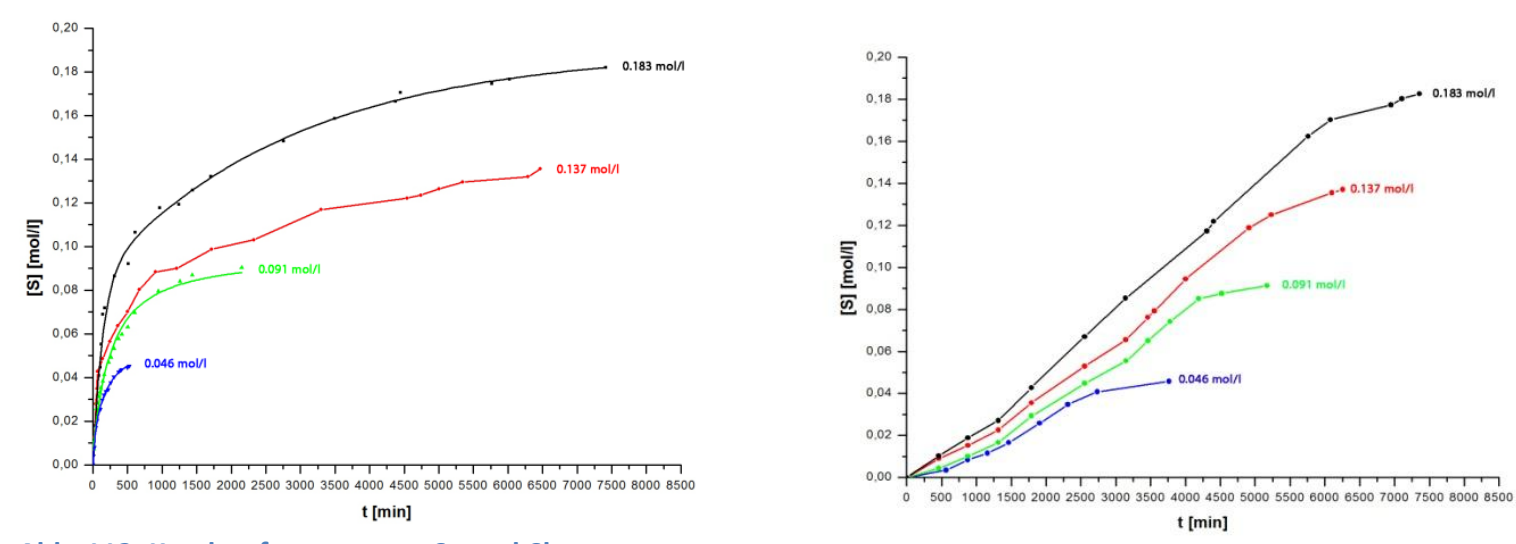

Abb. 112: Katalysefortgang von $6 \mathrm{a}$ und $6 \mathrm{~b}$

$\mathrm{Zu}$ Beginn der Katalyse ist die Reaktionsgeschwindigkeit relativ hoch, solange die Konzentration an freiem $\mathrm{PPh}_{3}$ hoch ist. In diesem Bereich ist die Reaktionsgeschwindigkeit von der Substratkonzentration abhängig. Nach einiger Zeit nimmt die Reaktionsgeschwindigkeit ab und nähert sich einem Plateau an, bei der Konzentration an Substrat abgenommen hat. Um die Anfangsgeschwindigkeit $\mathrm{zu}$ bestimmen, werden Regressionsgeraden in den Bereich gelegt, der als annährend linear betrachtet werden kann. Die hieraus erhaltenen Geschwindigkeiten werden für die weiteren auf Michaelis-Menten Kinetik beruhenden Berechnungen verwendet. Die Bestimmung von kinetischen Parametern nach Methoden wie z.B. dem Lineweaver-Burk Plot sind für Enzymsysteme entwickelt worden. Da es sich bei den verwendeten anorganischen Katalysatoren um ähnliche Modelle handelt, kann diese Methode auch hier angewendet werden.

Die Lineweaver Burk-Plots stellen die gängigste Methode zur Darstellung der Messwerte dar. Jedoch ist diese Methode bei bestimmten Wertebereichen nicht sehr zuverlässig. Kleine Fehler der Geschwindigkeit v ergeben bei kleinen [S]-Werten eine große Abweichung in 1/v, bei großen [S]-Werten ist dies jedoch eher zu vernachlässigen. Die Autoren dieser Methode wiesen darauf hin, dass bei dieser Auftragung eine gewisse Unsicherheit bei großen 1/vWerten auftritt. ${ }^{[98]}$ Vielfach wurden diese entstehenden Abweichungen bei den meisten Anwendungen jedoch vernachlässigt. Parallel zu der Lineweaver-Burk Methode wurde eine große Anzahl an weiteren Auswertungsmöglichkeiten für die Enzymkinetik entwickelt. Die aktuell hauptsächlich verwendete lineare Auftragungsart stellt das Hanes-Woolf-Diagramm dar. Die Methode wurde von Charles Samuel Hanes und Barnet Woolf im Jahre 1932 
entwickelt. ${ }^{[99]}$ Eine Auftragung von $[\mathrm{S}] / \mathrm{v}$ wird als eine Funktion von [S] aufgetragen. Der Zusammenhang ergibt sich aus folgender Gleichung:

$$
\frac{[S]}{v}=\frac{1}{v_{\max }}[S]+\frac{K_{m}}{v_{\max }}
$$

Da der Quotient aus Substatkonzentration und Reaktionsgeschwindigkeit verwendet wird, kommt es zu einer Spreizung der Werte, wodurch Ausreisser das Endergebnis nur wenig verfälschen. Die Steigung dieser linearen Regressionsgeraden liefert einen Wert von $1 / v_{\max }$. Der Schnittpunkt mit der y-Achse entspricht einem Wert von $\mathrm{K}_{\mathrm{M}} / \mathrm{v}_{\max }$. Der Schnittpunkt mit der $\mathrm{X}$-Achse gibt den negativen Wert der Michalis-Konstante wieder.

Nach Auftragung der Messwerte ergibt sich folgendes Hanes-Woolf-Diagramm:

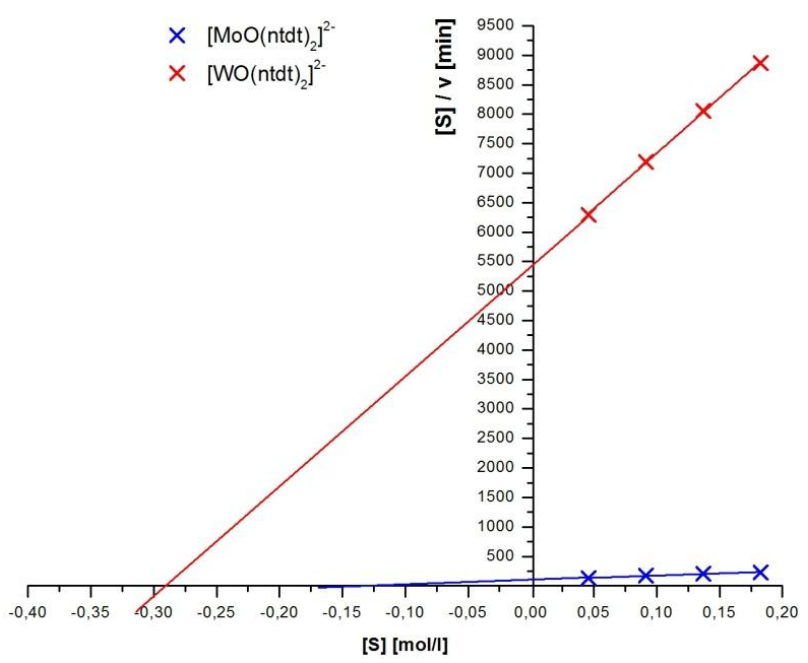

Abb. 113: Hanes-Woolf-Diagramm des Paares $\left[\mathrm{MO}(\mathrm{ntdt})_{2}\right]^{2}$

Hieraus ergeben sich folgende Werte:

\begin{tabular}{ccccccc}
\hline Verbindung & $\begin{array}{c}{[\mathrm{S}]} \\
{[\mathrm{mol} / \mathrm{l}]}\end{array}$ & $\begin{array}{c}{[\mathrm{S}] / \mathrm{v}} \\
{[\mathrm{min}]}\end{array}$ & $\begin{array}{c}\mathrm{k}_{\mathrm{cat}} \\
{\left[\mathrm{s}^{-1}\right]}\end{array}$ & $\begin{array}{c}\mathrm{k}_{\mathrm{cat}} / \mathrm{K}_{\mathrm{M}} \\
{\left[\mathrm{s}^{-1} \cdot \mathrm{mol}^{-1} \cdot \mathrm{l}\right]}\end{array}$ & $\begin{array}{c}\mathrm{v}_{\max } \\
{[\mathrm{mol} / \mathrm{l} \cdot \mathrm{min}]}\end{array}$ & $\begin{array}{c}\mathrm{K}_{\mathrm{M}} \\
{[\mathrm{mol} / \mathrm{l}]}\end{array}$ \\
\hline Mo & 0.0457 & $1.32 \cdot 10^{2}$ & & & & \\
& 0.0909 & $1.71 \cdot 10^{2}$ & & & & \\
& 0.1370 & $2.06 \cdot 10^{2}$ & 4.60 & 41.8 & $1.4 \cdot 10^{-3}$ & 0.11 \\
& 0.1825 & $2.33 \cdot 10^{2}$ & & & & \\
\hline $\mathbf{W}$ & 0.0457 & $6.29 \cdot 10^{3}$ & & & & \\
& 0.0909 & $7.19 \cdot 10^{3}$ & & & & \\
& 0.1370 & $8.06 \cdot 10^{3}$ & 0.17 & 0.60 & & \\
& 0.1825 & $8.86 \cdot 10^{3}$ & & & & \\
\end{tabular}

Tab. 24: Berechnete kinetische Parameter für das Paar [MO(ntdt) $\left.{ }_{2}\right]^{2}$ 
In Tabelle 24 wurde außerdem die „Turn over number“ (TON) $\mathrm{k}_{\text {cat }}$ und die katalytische Effizenz $\mathrm{k}_{\mathrm{cat}} / \mathrm{K}_{\mathrm{M}}$ angegeben. Man erhält $\mathrm{k}_{\mathrm{cat}}$, indem man die Aktivität $\left(\mathrm{V}_{\text {max }}\right)$ durch die Katalysatorkonzentration dividiert. Die TON für Enzymreaktionen beschreibt die Anzahl an Formelumsätzen, die durch ein Katalysatormolekül pro Sekunde umgesetzt werden.

Vergleich man nun die katalytische Aktivität der Molybdän- und Wolframverbindung, so stellt man einen sehr viel stärkeren Anstieg der Reaktionsgeschwindigkeit bei der Molybdänverbindung fest. Die kleinere Michaelis-Konstante für den Molybdänkatalysator, weist auf eine stärkere Affinität zwischen Metallzentrum und Substrat, zur Bildung des Triphenylposphinoxidmetallkomplexes, hin. Auch an den berechneten Maximalgeschwindigkeiten ist $\mathrm{zu}$ erkennen, dass eine deutlich schnellere Reaktion bei der Molybdänverbindung im Vergleich zur Wolframverbindung auftritt.

Das Ergebnis, dass die Molybdänverbindung bei diesem Verbindungspaar die bessere katalytische Aktivität zeigt, steht im Gegensatz zu den untersuchten Verbindungen 1, 3 und 5 mit aromatischem Ligandensystem, wobei die Wolframverbindungen die besten Aktivitäten zeigten. Somit muss der elektronische Einfluss des Ligandensystems eine entscheidende Rolle auf den katalytischen Zyklus haben.

\subsubsection{Einfluss auf die katalytische Aktivität durch bestimmte Ringatome}

Um zu untersuchen, ob verschiedene Atome im, zur Dithioleneinheit angrenzenden, Ringsystem einen Einfluss auf die katalytische Sauerstoffübertragung haben, wurden die Verbindungspaare $\left[\mathrm{MO}(\mathrm{tldt})_{2}\right]^{2-}$ sowie $\left[\mathrm{MO}(\mathrm{tcdt})_{2}\right]^{2-}$ untersucht. Bei allen vier Verbindungen konnte die Dioxoverbindung in der Hinreaktion durch die Zugabe von DMSO hergestellt werden. Anschließend wurde der koordinierte Oxo-Ligand mit Hilfe von $\mathrm{PPh}_{3}$ katalytisch bei $60^{\circ} \mathrm{C}$ wieder vom Metallzentrum entfernt. Um die Konzentrationsabhängigkeit dieser Katalysen zu untersuchen, wurden jeweils vier verschiedene Katalysator-SubstratVerhältnisse $(1: 2.5 ; 1: 5 ; 1: 7.5 ; 1: 10)$ eingesetzt.

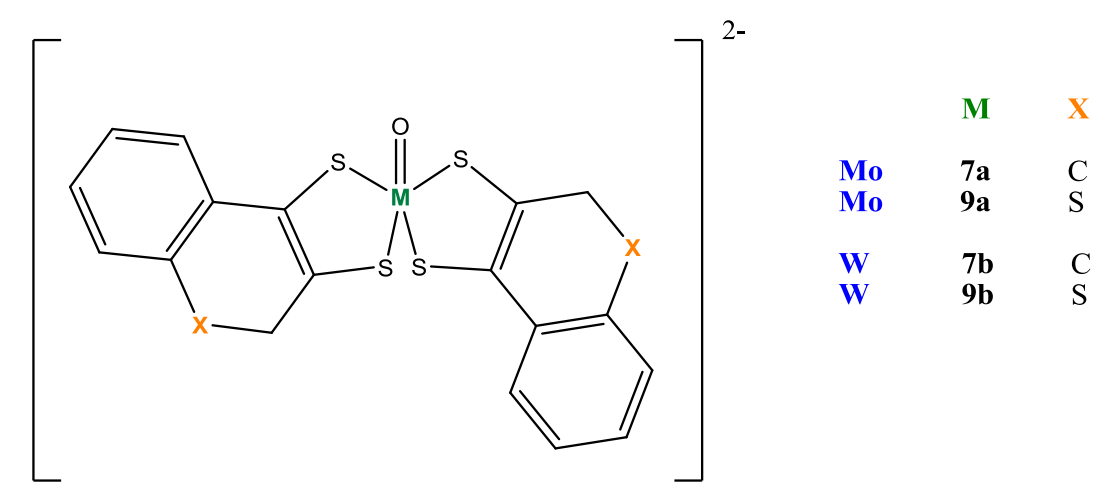

Abb. 114: Katalytische Untersuchungen der Paare $\left[\mathrm{MO}(\mathrm{tldt})_{2}\right]^{2-}[7] \mathrm{bzw} .\left[\mathrm{MO}(\mathrm{tcdt})_{2}\right]^{2-}[9] ; \mathrm{M}=\mathrm{Mo}, \mathrm{W}$ 


\subsubsection{Untersuchung der katalytischen Sauerstoffübertragung durch das}

\section{$\left[\mathrm{MO}(\mathrm{tld} \mathrm{dt})_{2}\right]^{2-}$-Paar}

Im Folgenden sind die katalytischen Verläufe bzgl. [OPPh$\left.{ }_{3}\right]$ mit der Molybdän und Wolframverbindung dargestellt.
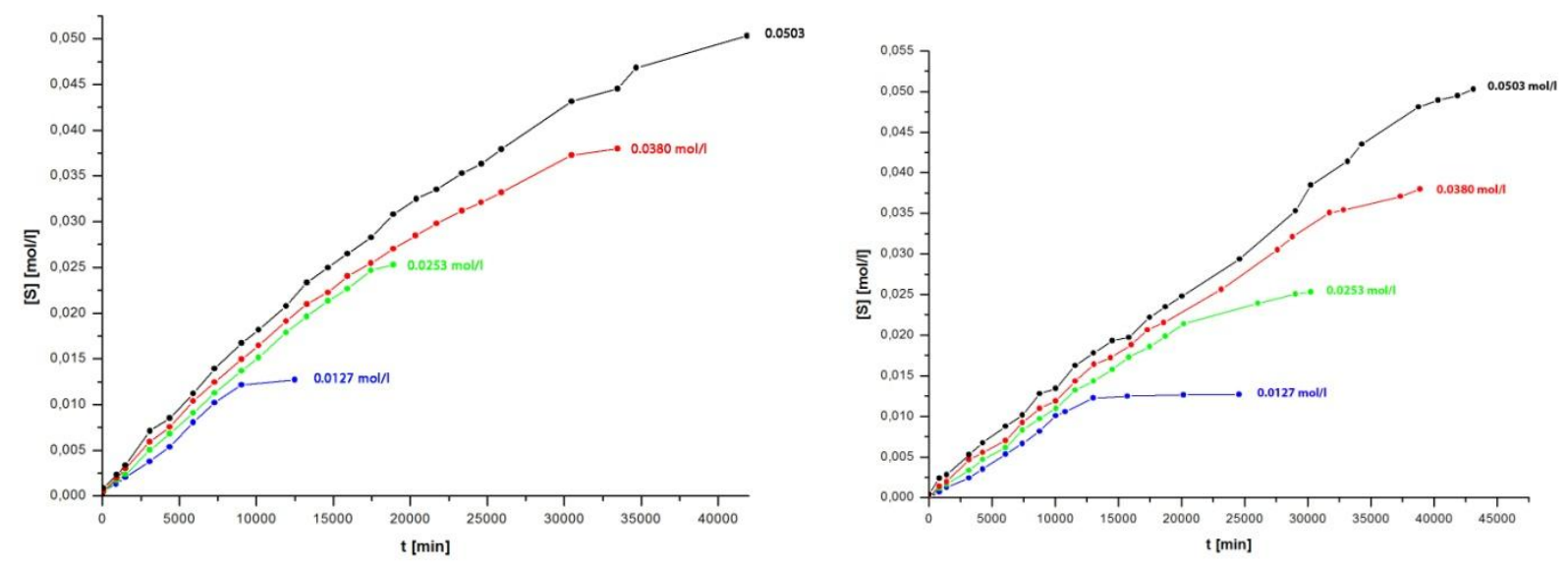

Abb. 115: Verlauf der katalytischen Reaktion, bzgl [OPPh 3 ] mit den Kat.[MoO(tldt) $]^{2-}$ (links), [WO(tldt) $]^{2-}($ rechts)

Im Folgenden ist die Reaktionszeit, die zu einem vollständigen Umsatz benötigt wurde, vergleichend von beiden Verbindungen aufgeführt.

\begin{tabular}{|c|c|c|c|c|}
\hline [Kat] : [PPh $]$ & $1: 2.5$ & 1:5 & $1: 7.5$ & $1: 10$ \\
\hline & $0.0127 \mathrm{~mol} / \mathrm{l}$ & $0.0253 \mathrm{~mol} / \mathrm{l}$ & $0.0380 \mathrm{~mol} / \mathrm{l}$ & $0.0503 \mathrm{~mol} / \mathrm{l}$ \\
\hline$\left[\mathrm{MoO}(\mathrm{t} / \mathrm{dt})_{2}\right]^{2-}$ & $208.2 \mathrm{~h}$ & $315.1 \mathrm{~h}$ & $557.7 \mathrm{~h}$ & $698.1 \mathrm{~h}$ \\
\hline$\left[\mathrm{WO}(\mathrm{t} / \mathrm{dt})_{2}\right]^{2-}$ & $409.0 \mathrm{~h}$ & $503.6 \mathrm{~h}$ & $647.8 \mathrm{~h}$ & $718.5 \mathrm{~h}$ \\
\hline
\end{tabular}

Tab. 25: Reaktionszeiten bis zum vollständigen Umsatz des Substrates

Auch in diesem Fall ist die Molybdänverbindung der bessere Katalysator, es finden schnellere Katalysen statt, wobei der Unterschied zwischen den Katalysatoren kleiner wird, je mehr Substrat verwendet wird.

Beide Verbindungen benötigen bei einem Katalysator-Substratverhältnis von 1:10 eine Umsatzzeit von ca. 30 Tagen bei $60^{\circ} \mathrm{C}$, um einen vollständigen Umsatz des Substrats zu erreichen.

Kinetische Parameter aus dem Reaktionsverlauf wurden mit Hilfe des Hanes-WoolfDiagramm angefertigt. Die berechneten Werte sind in Tab.26 zu finden. 


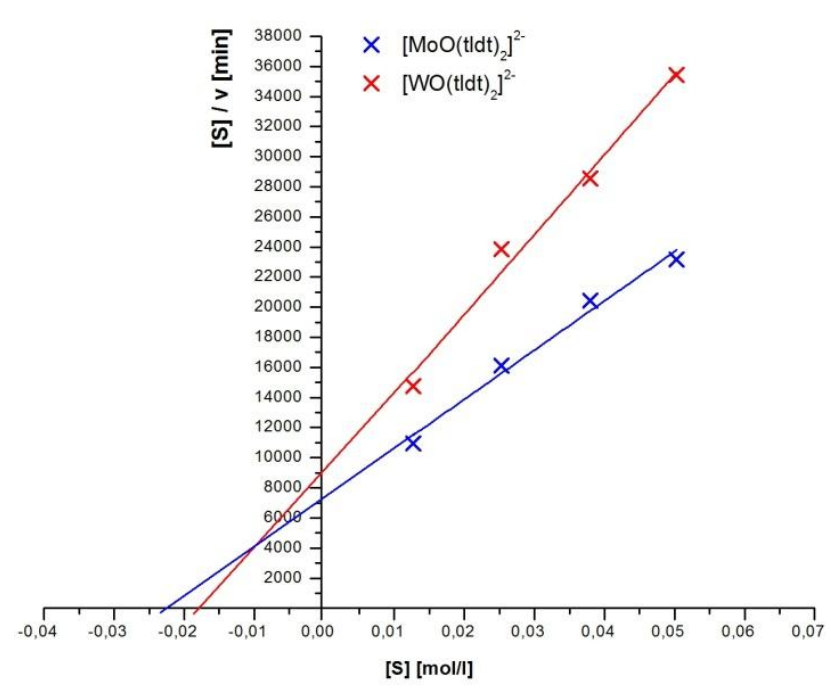

Abb. 116: Hanes-Woolf-Diagramm des Paares $\left[\mathrm{MO}(\mathrm{t} / \mathrm{dt})_{2}\right]^{2-}$

\begin{tabular}{cccccccc} 
Verbindung & $\begin{array}{c}{[\mathrm{S}]} \\
{[\mathrm{mol} / \mathrm{l}]}\end{array}$ & $\begin{array}{c}\mathrm{v} \\
{[\mathrm{mol} / \mathrm{l} \cdot \mathrm{min}]}\end{array}$ & $\begin{array}{c}{[\mathrm{S}] / \mathrm{v}} \\
{[\mathrm{min}]}\end{array}$ & $\begin{array}{c}\mathrm{k}_{\mathrm{cat}} \\
{\left[\mathrm{s}^{-1}\right]}\end{array}$ & $\begin{array}{c}\mathrm{k}_{\mathrm{cat}} / \mathrm{K}_{\mathrm{M}} \\
{\left[\mathrm{s}^{-1} \cdot \mathrm{mol}^{-1} \cdot 1\right]}\end{array}$ & $\begin{array}{c}\mathrm{V}_{\text {max }} \\
{[\mathrm{mol} / \mathrm{l} \cdot \mathrm{min}]}\end{array}$ & $\begin{array}{c}\mathrm{K}_{\mathrm{M}} \\
{[\mathrm{mol} / \mathrm{l}]}\end{array}$ \\
\hline Mo & 0.0127 & $1.16 \cdot 10^{-6}$ & $1.09 \cdot 10^{4}$ & & & & \\
& 0.0253 & $1.57 \cdot 10^{-6}$ & $1.61 \cdot 10^{4}$ & & & & \\
& 0.0380 & $1.86 \cdot 10^{-6}$ & $2.04 \cdot 10^{4}$ & $3.61 \cdot 10^{-2}$ & 1.57 & $3.06 \cdot 10^{-6}$ & $2.3 \cdot 10^{-2}$ \\
& 0.0503 & $2.17 \cdot 10^{-6}$ & $2.32 \cdot 10^{4}$ & & & & \\
\hline $\mathbf{W}$ & 0.0127 & $8.60 \cdot 10^{-7}$ & $1.48 \cdot 10^{4}$ & & & & \\
& 0.0253 & $1.06 \cdot 10^{-6}$ & $2.39 \cdot 10^{4}$ & & & & \\
& 0.0380 & $1.33 \cdot 10^{-6}$ & $2.86 \cdot 10^{4}$ & $2.22 \cdot 10^{-2}$ & 1.23 & & \\
& 0.0503 & $1.42 \cdot 10^{-6}$ & $3.54 \cdot 10^{4}$ & & & & \\
\end{tabular}

Tab. 26: Berechnete kinetische Parameter für das Verbindungspaar [MO(tldt $\left.)_{2}\right]^{2}$

Hier ist der Unterschied zwischen der katalytischen Aktivität beim Molybdän- und WolframKatalysator erheblich kleiner, als bei dem zuvor vorgestellten Verbindungspaar $\left[\mathrm{MO}(\mathrm{ntdt})_{2}\right]^{2-}$. 


\subsubsection{Untersuchung der katalytischen Sauerstoffübertragung durch das}

[MO(tcdt) 2$]^{2--P a a r}$

Dieses Verbindungspaar wurde analog $\mathrm{zu}$ dem $\left[\mathrm{MO}(\mathrm{tldt})_{2}\right]^{2-}-$ Paar in einer katalytischen Sauerstoffübertragungsreaktion eingesetzt. Dabei konnte folgender Verlauf bzgl. $\left[\mathrm{OPPh}_{3}\right]$ bei der Reaktion beobachtet werden.
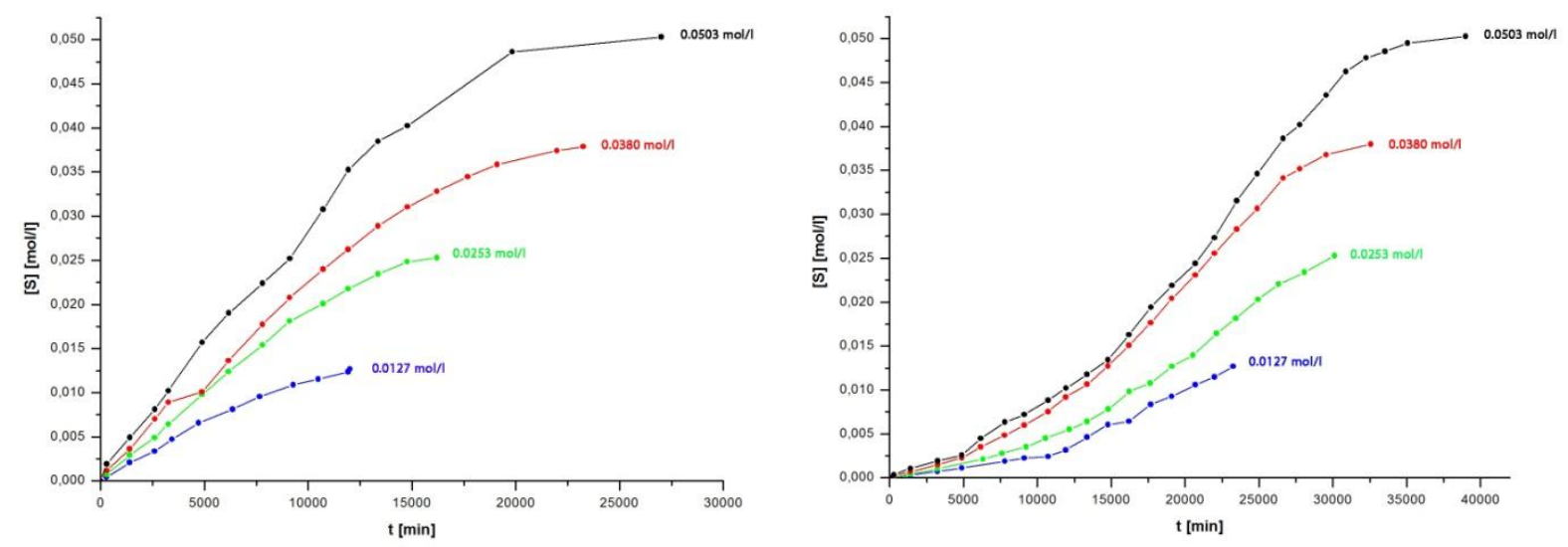

Abb. 117: Verlauf der katalytischen Reaktion, bzgl. [OPPh $\left.{ }_{3}\right]$ mit den Katalysatoren $\left[\mathrm{MoO}(\mathrm{tcdt})_{2}\right]^{2-}\left(\right.$ links) und $\left[\mathrm{WO}(\mathrm{tcdt})_{2}\right]^{2-}$ (rechts)

Im Folgenden sind die Umsatzzeiten bis zum 100\% Umsatz des Substrats aufgeführt.

\begin{tabular}{|c|c|c|c|c|}
\hline [Kat] : [PPh $]$ & $1: 2.5$ & 1:5 & $1: 7.5$ & $1: 10$ \\
\hline & $0.0127 \mathrm{~mol} / \mathrm{l}$ & $0.0253 \mathrm{~mol} / \mathrm{l}$ & $0.0380 \mathrm{~mol} / \mathrm{l}$ & $0.0503 \mathrm{~mol} / \mathrm{l}$ \\
\hline$\left[\mathrm{MoO}(\mathrm{tcdt})_{2}\right]^{2-}$ & $200.2 \mathrm{~h}$ & $269.9 \mathrm{~h}$ & $387.5 \mathrm{~h}$ & $450.2 \mathrm{~h}$ \\
\hline$\left[\mathrm{WO}(\mathrm{tcdt})_{2}\right]^{2}$ & $387.5 \mathrm{~h}$ & $501.9 \mathrm{~h}$ & $542.8 \mathrm{~h}$ & $649.7 \mathrm{~h}$ \\
\hline
\end{tabular}

Tab. 27: Reaktionszeiten bis zum vollständigen Umsatz des Substrats durch das Verbindungspaar [MO(tcdt) $\left.{ }_{2}\right]^{2-}$

Beim Vergleich der beiden Katalysatoren fällt auf, dass die Molybdänverbindung wieder einen schnelleren Umsatz der Substrate liefert. Bei der höchsten Menge an Substrat ist eine Gesamtzeit für die Katalyse mit Molybdän von ca. 19 Tagen festzustellen, während für die gleiche Substratmenge mit Wolfram eine Reaktionszeit von ca. 27 Tagen nötig ist.

Wiederrum wurden Hanes-Woolf-Diagramme (Abb.118) angefertigt, um kinetische Parameter zu bestimmen. 


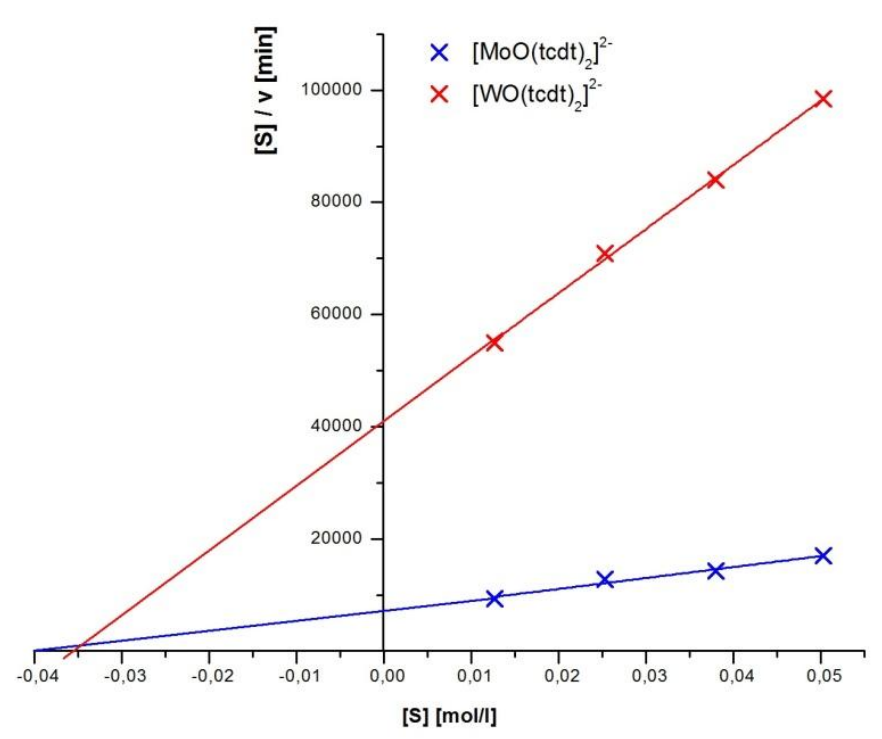

Abb. 118: Hanes-Woolf-Diagramm des Paares $\left[\mathrm{MO}(\mathrm{tcdt})_{2}\right]^{2-}$

Hieraus ergeben sich den in Tab.28 enthaltenen kinetischen Parameter

\begin{tabular}{cccccccc} 
Verbindung & $\begin{array}{c}{[\mathrm{S}]} \\
{[\mathrm{mol} / \mathrm{l}]}\end{array}$ & $\begin{array}{c}\mathrm{v} \\
{[\mathrm{mol} / 1 \cdot \mathrm{min}]}\end{array}$ & $\begin{array}{c}{[\mathrm{S}] / \mathrm{v}} \\
{[\mathrm{min}]}\end{array}$ & $\begin{array}{c}\mathrm{k}_{\mathrm{cat}} \\
{\left[\mathrm{s}^{-1}\right]}\end{array}$ & $\begin{array}{c}\mathrm{k}_{\mathrm{cat}} / \mathrm{K}_{\mathrm{M}} \\
{\left[\mathrm{s}^{-1} \cdot \mathrm{mol}^{-1} \cdot 1\right]}\end{array}$ & $\begin{array}{c}\mathrm{v}_{\max } \\
{[\mathrm{mol} / \mathrm{l} \cdot \mathrm{min}]}\end{array}$ & $\begin{array}{c}\mathrm{K}_{\mathrm{M}} \\
{[\mathrm{mol} / \mathrm{l}]}\end{array}$ \\
\hline Mo & 0.0127 & $1.37 \cdot 10^{-6}$ & $9.27 \cdot 10^{4}$ & & & & \\
& 0.0253 & $1.97 \cdot 10^{-6}$ & $1.28 \cdot 10^{4}$ & & & & \\
& 0.0380 & $2.66 \cdot 10^{-6}$ & $1.43 \cdot 10^{4}$ & $6.07 \cdot 10^{-2}$ & 1.52 & $5.14 \cdot 10^{-6}$ & $4.0 \cdot 10^{-2}$ \\
& 0.0503 & $2.97 \cdot 10^{-6}$ & $1.69 \cdot 10^{4}$ & & & & \\
\hline $\mathbf{W}$ & 0.0127 & $2.31 \cdot 10^{-7}$ & $5.50 \cdot 10^{4}$ & & & & \\
& 0.0253 & $3.57 \cdot 10^{-7}$ & $7.09 \cdot 10^{4}$ & & & & \\
& 0.0380 & $4.52 \cdot 10^{-7}$ & $8.41 \cdot 10^{4}$ & $1.03 \cdot 10^{-2}$ & 0.29 & & \\
& 0.0503 & $5.11 \cdot 10^{-7}$ & $9.84 \cdot 10^{4}$ & & & & \\
\end{tabular}

Tab. 28: Berechnete kinetische Parameter für das Verbindungspaar [MO(tcdt $\left.)_{2}\right]^{2}$

Bei diesen zwei Katalysatoren ist der Unterschied in der Katalysatoreffizenz stärker ausgeprägt, als es bei den Katalysatorpaar $\left[\mathrm{MO}(\mathrm{tldt})_{2}\right]^{2-}$ der Fall war. Da der Kurvenverlauf bei der Wolframverbindung anfangs flacher verläuft, ergeben sich geringere Geschwindigkeitswerte. 


\subsubsection{Vergleichende Betrachtungen der Katalysen durch die $\left[\mathrm{MO}(\mathrm{tld} \mathrm{t})_{2}\right]^{2-}$ und}

\section{$\left[\mathrm{MO}(\mathrm{tcdt})_{2}\right]^{2-}-$ Verbindungen}

Betrachtet man nun alle Katalysen im Vergleich, so stellt man fest, dass die Molybdänverbindungen $\left[\mathrm{Mo}(\mathrm{tldt})_{2}\right]^{2-}$ und $\left[\mathrm{Mo}(\mathrm{tcdt})_{2}\right]^{2-}$ eine bessere katalytische Aktivität, im Vergleich zu den wolframhaltigen Verbindungen, aufweisen.

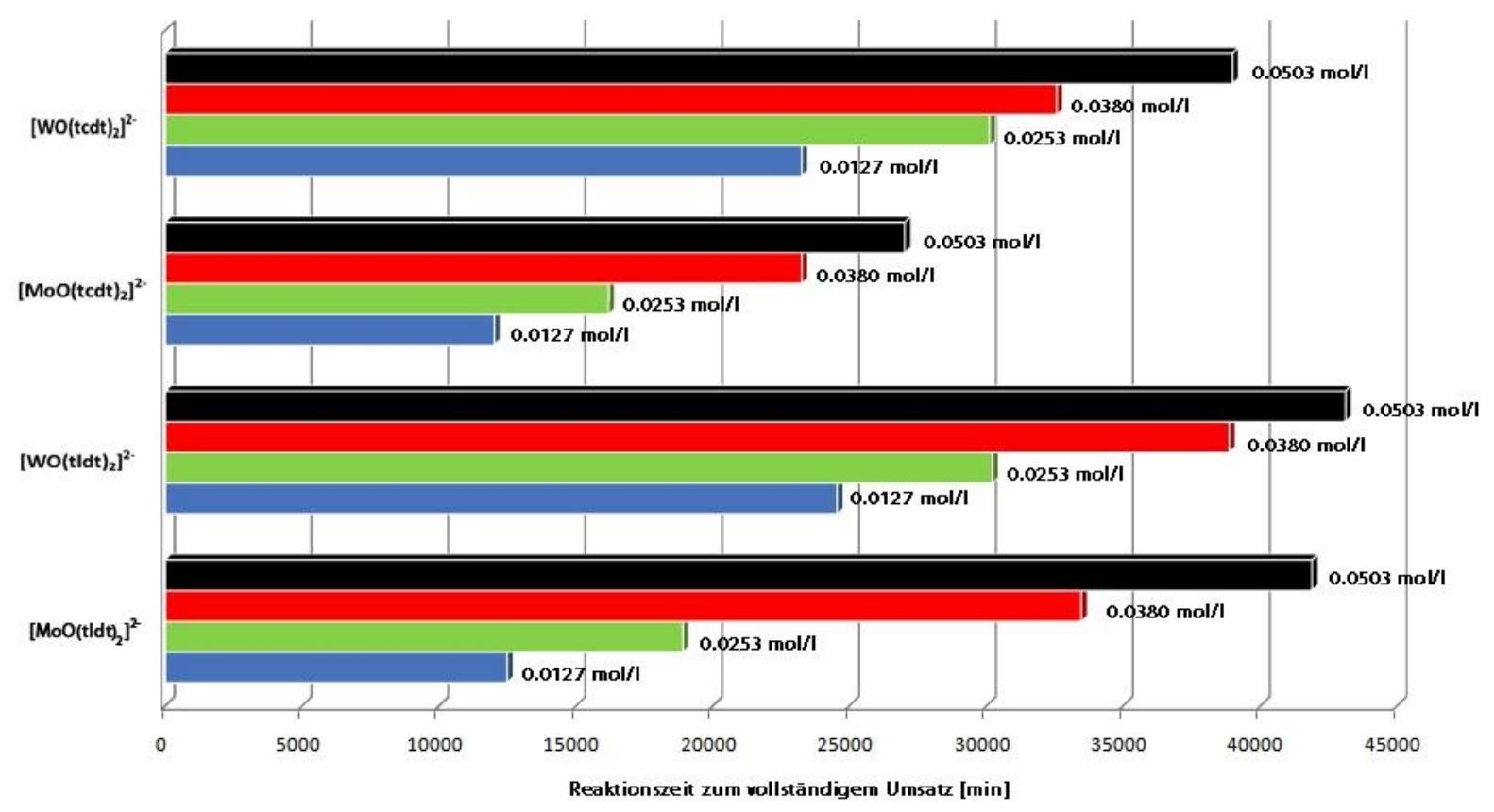

Abb. 119: Vergleich der Zeiten bis zum vollständigen Umsatz

Interessant ist jedoch, dass die Verbindung mit dem tcdt-Liganden, also mit einem Heteroatom (S) im an die Dithioleneinheit angrenzenden 6-Ring, die besseren Katalysatoren sind, als die ohne Heteroatom. Da auch in den natürlichen Ligandensystemen an dieser Stelle ein Heteroatom (Sauerstoff) vorkommt, ist möglicherweise eine Optimierung der Katalyse erfolgt. Die katalytischen Untersuchungen mit Sauerstoff im Ringsystem werden in einer Kooperationstudie durch andere Mitarbeiter durchgeführt und liegen zum gegenwertigen Zeitpunkt noch nicht vor. 


\subsection{Katalytische Untersuchungen von Non-Oxo-Metall(IV)komplexen}

Um auch die katalytische Sauerstoffübertragung von Metallkomplexen zu untersuchen, die in der reduzierten Form keine Oxo-Gruppe am Metallzentrum tragen und somit ein besseres Modell für die reduzierte Form der DMSO-Reduktase Familie darstellen, wurden die Verbindungen 11a und 11b katalytischen Studien unterzogen.

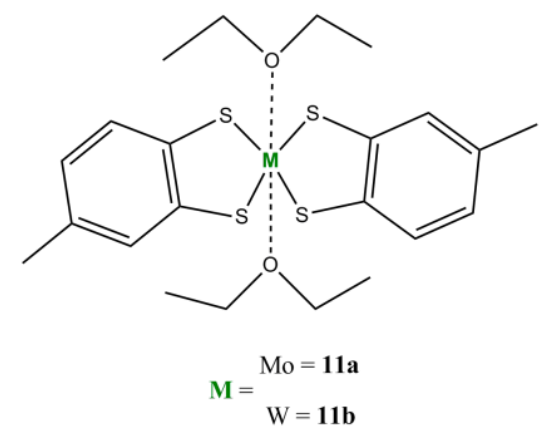

Abb. 120: Verwendete Non-Oxo-Komplexe

Da die Verbindungen zwei angelagerte Diethylethermoleküle tragen, sollte es durch das besser koordinierende DMSO $\mathrm{zu}$ einer Verdrängung der angelagerten Lösungsmittelmolekülen kommen, so dass die koordinative Absättigung der Komplexe kein Problem darstellen sollte. Weil die Verbindungen nicht sehr gut in DMSO löslich sind, wurden sie in $\mathrm{CDCl}_{3}-\mathrm{d}_{1}$ gelöst und mit einem 100fachen Überschuss des Oxidationssubstrats DMSO versetzt. Als Sauerstoffakzeptor und Reduktionsmittel wurde wiederrum $\mathrm{PPh}_{3}$ verwendet. Der mögliche katalytische Reaktionsmechanismus ist im Folgenden aufgeführt.

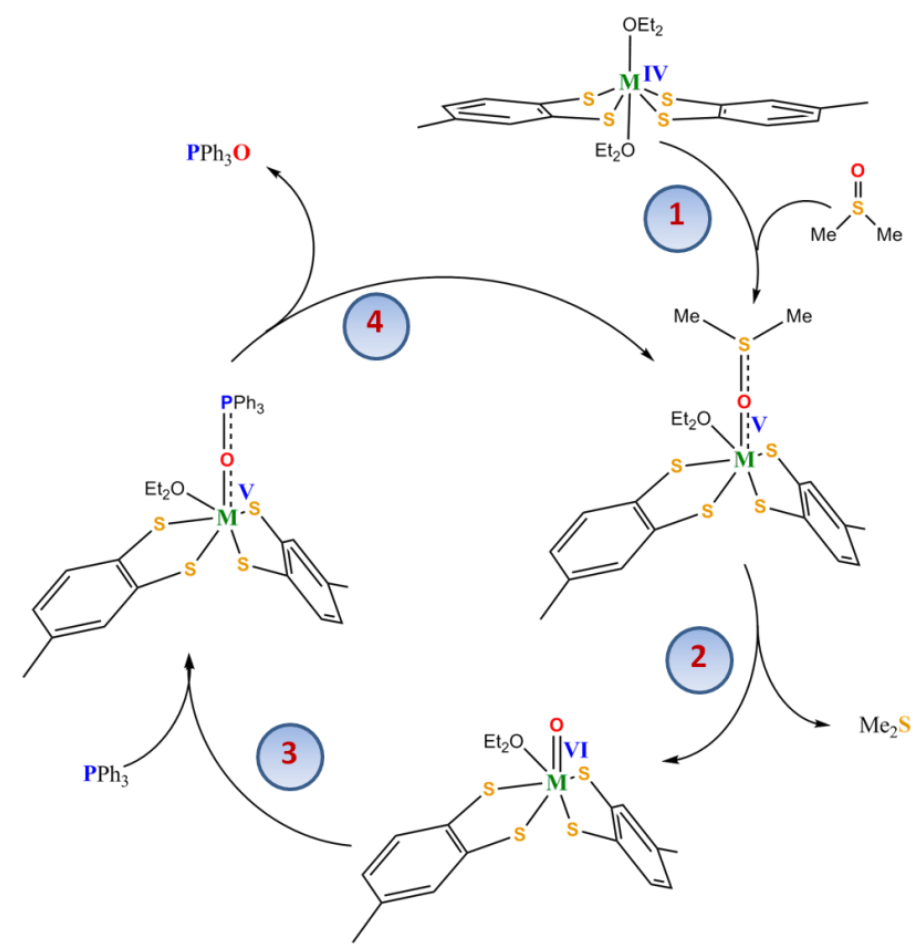


Im ersten katalytischen Schritt sollte es zu einer Verdrängung eines oder beider DiethyletherLiganden kommen und zur Koordination des DMSOs. Somit sollte sich ein Übergangszustand bilden, beim dem es zu einer Wechselwirkung zwischen dem Sauerstoffatom des DMSOs und dem Metallzentrum kommt. Es folgt die Oxidation des Metallzentrums zur Oxidationsstufe V (Schritt 1). Nach Übertragung des Sauerstoffatoms (Schritt 2) liegt das Metallzentrum in Oxidationsstufe VI vor (Schritt 3). Ein Ethermolekül sollte immer noch koordiniert sein, um die freie Koordinationsstelle zu besetzen. Durch die Zugabe von $\mathrm{PPh}_{3}$ kommt es nun zum Angriff des freien Elektronenpaars des Phosphors auf das Sauerstoffatom (Schritt 3). Dadurch bildet sich wieder ein Übergangszustand, bei dem sich das Metall in der Oxidationsstufe V befindet. Durch das Abtrennen von Triphenylphosphinoxid kommt es zur Rückreduktion des Metallzentrums zur Oxidationsstufe IV. Wahrscheinlich sollte es aufgrund des Überschusses an DMSO nicht zur erneuten Anlagerung des Diethylethers kommen, so eine direkte Weiterreaktion zur Verbindung in der Oxidationsstufe VI erfolgt (Schritt 2 und 4). Damit schließt sich der Katalysezyklus.

Es besteht generell die Möglichkeit der direkten Koordination des Triphenylphosphins über das vorhandene freie Elektronenpaar an das Metall. Entsteht eine stabile Verbindung, würde dies zu einem weiteren Phosphorsignal im ${ }^{31} \mathrm{P}-\mathrm{NMR}$ führen, das aber in keiner unserer Studien beobachtet werden konnte.

Im Folgenden ist der Fortgang der Katalysen ermittelt durch ${ }^{31} \mathrm{P}-\mathrm{NMR}$ gezeigt. Es wurden Konzentrationen von $1: 2.5 ; 1: 5 ; 1: 7.5 ; 1: 10$ von Katalysator $z u \mathrm{PPh}_{3}$ verwendet.
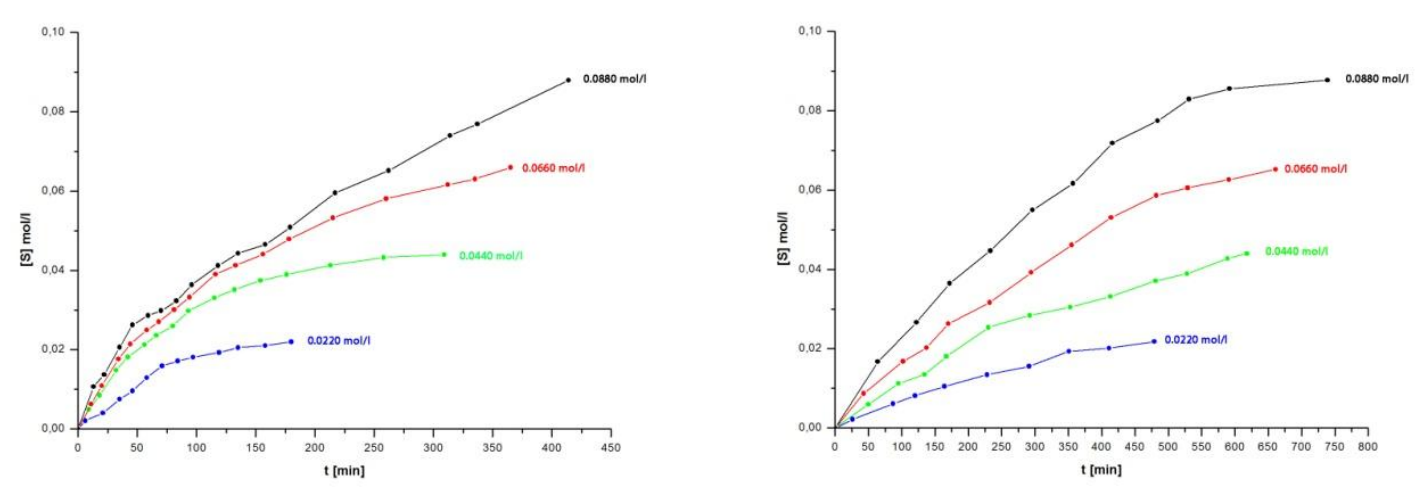

Abb. 122: Verlauf der katalytischen Reaktion bzgl. [OPPh${ }_{3}$ ] mit den Katalysatoren, $\left[\mathrm{Mo}\left(\mathrm{OEt}_{2}\right)_{2}(\mathrm{tdt})_{2}\right]$ (links), [W(OEt $\left.)_{2}(\mathrm{tdt})_{2}\right]$ (rechts) 
Nach Anfertigung eines Hanes-Woolf-Diagrammes (Abb.123) konnten kinetische Parameter erhalten werden die in Tab.29 aufgeführt sind.

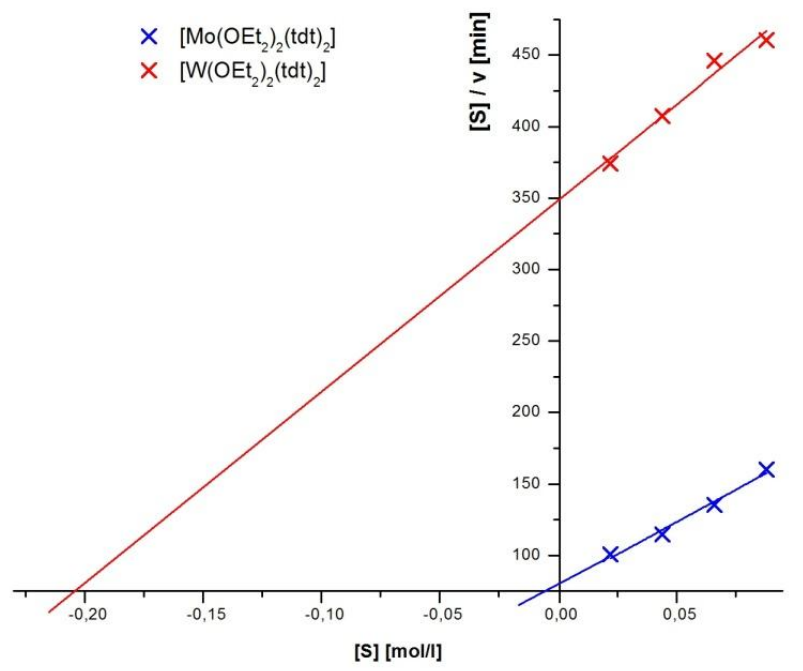

Abb. 123: Hanes-Woolf-Diagramm des Paares $\left[\mathrm{M}\left(\mathrm{OEt}_{2}\right)_{2}(\mathrm{tdt})_{2}\right]$

\begin{tabular}{cccccccc} 
Verbindung & $\begin{array}{c}{[\mathrm{S}]} \\
{[\mathrm{mol} / \mathrm{l}]}\end{array}$ & $\begin{array}{c}\mathrm{v} \\
{[\mathrm{mol} / 1 \cdot \mathrm{min}]}\end{array}$ & $\begin{array}{c}{[\mathrm{S}] / \mathrm{v}} \\
{[\mathrm{min}]}\end{array}$ & $\begin{array}{c}\mathrm{k}_{\mathrm{cat}} \\
{\left[\mathrm{s}^{-1}\right]}\end{array}$ & $\begin{array}{c}\mathrm{k}_{\mathrm{cat}} / \mathrm{K}_{\mathrm{M}} \\
{\left[\mathrm{s}^{-1} \cdot \mathrm{mol}^{-1} \cdot \mathrm{l}\right]}\end{array}$ & $\begin{array}{c}\mathrm{v}_{\max } \\
{[\mathrm{mol} / \mathrm{l} \cdot \mathrm{min}]}\end{array}$ & $\begin{array}{c}\mathrm{K}_{\mathrm{M}} \\
{[\mathrm{mol} / 1]}\end{array}$ \\
\hline Mo & 0.0220 & $2.18 \cdot 10^{-4}$ & $1.01 \cdot 10^{2}$ & & & & \\
& 0.0440 & $3.84 \cdot 10^{-4}$ & $1.15 \cdot 10^{2}$ & & & & \\
& 0.0660 & $4.88 \cdot 10^{-4}$ & $1.35 \cdot 10^{2}$ & 7.57 & 1168 & $1.11 \cdot 10^{-3}$ & $6.48 \cdot 10^{-3}$ \\
& 0.0880 & $5.49 \cdot 10^{-4}$ & $1.60 \cdot 10^{2}$ & & & & \\
\hline $\mathbf{W}$ & 0.0220 & $5.88 \cdot 10^{-5}$ & $3.74 \cdot 10^{2}$ & & & & \\
& 0.0440 & $1.08 \cdot 10^{-4}$ & $4.07 \cdot 10^{2}$ & & & & \\
& 0.0660 & $1.48 \cdot 10^{-4}$ & $4.46 \cdot 10^{2}$ & 5.03 & 25.2 & & \\
& 0.0880 & $1.91 \cdot 10^{-4}$ & $4.61 \cdot 10^{2}$ & & & & \\
\end{tabular}

Tab. 29: Erhaltene kinetische Parameter

Wiederrum ist die Molybdänverbindung im Vergleich zu der Wolframverbindung der bessere Katalysator. Das ist auch anhand der Katalysatoreffizenz ersichtlich. Beim Wolfram wird nur eine Effizienz von 2\% im Vergleich zum Molybdän gefunden, was gut an den Werten von $\mathrm{k}_{\mathrm{cat}} / \mathrm{K}_{\mathrm{M}} \mathrm{zu}$ erkennen ist.

Bei den zuvor durchgeführten Katalysen mit den Paaren $\left[\mathrm{MO}(\mathrm{xdt})_{2}\right]^{2-}$, $\left[\mathrm{MO}(\mathrm{vdt})_{2}\right]^{2-}$ und $\left[\mathrm{MO}(\mathrm{qdt})_{2}\right]^{2-}$ zeigten die Wolframverbindungen immer die größere katalytische Aktivität zum Oxo-Transfer. Es wurde angenommen, dass die elektronischen Gegebenheiten des aromatischen Dithiolenliganden einen Einfluss im katalytischen Zyklus zeigen. Da in den hier 
untersuchten $\left[\mathrm{M}(\mathrm{tdt})_{2}\left(\mathrm{OEt}_{2}\right)_{2}\right]$ - Verbindungen auch ein aromatischer Dithiolenligand vorhanden ist, könnte es daran liegen, dass ohne die Oxo-Gruppe mehr Elektronendichte am Metall vorhanden ist, was die Reduktion erschwert, die für Wolfram ohnehin schon ungünstig ist.

\subsection{Katalytische Einfluss durch eine Dithioleneinheit im Vergleich zu einer Nicht-}

\section{Dithioleneinheit}

Um zu untersuchen, wie sich die Koordination einer Dithioleneinheit auf die katalytische Aktivität auswirkt, wurden die Verbindungen $\left[\mathrm{MoOS}_{4} \mathrm{xdt}\right]^{2-}$ und $\left[\mathrm{MoOS}_{8}\right]^{2-}$ bei verschiedenen Konzentrationen ([Kat]:[S] $=1: 2.5 ; 1: 5 ; 1: 7.5 ; 1: 10)$ bezüglich der Oxo-Transfer-Katalyse nach vorheriger Oxidation durch DMSO untersucht.
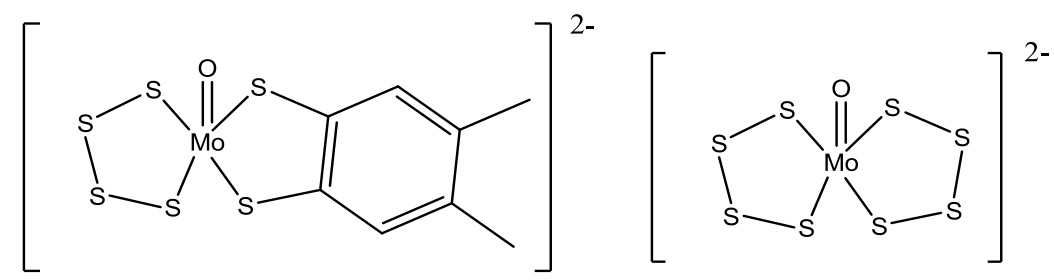

Abb. 124:Untersuchungen an den Paaren $\left[\mathrm{MoOS}_{8}\right]^{2-}$ und $\left[\mathrm{MoOS}_{4} \mathrm{xdt}\right]^{2-}$
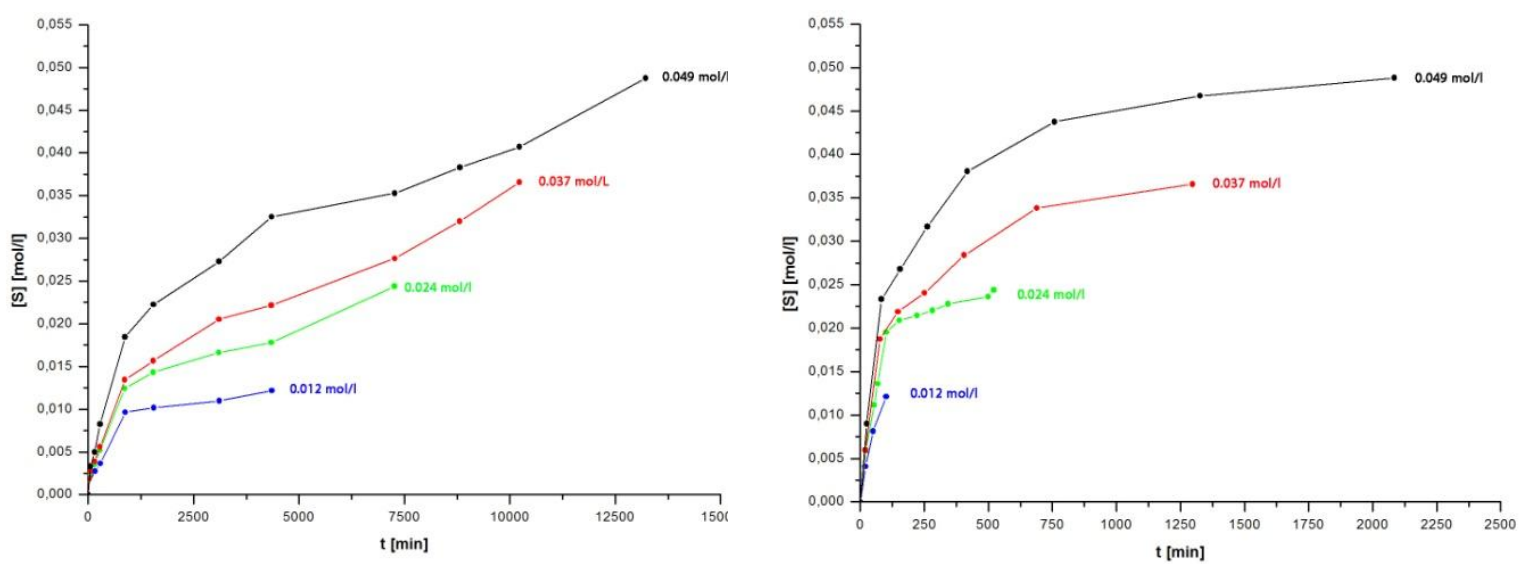

Abb. 125: Verlauf der katalytischen Reaktion bzgl. [OPPh ${ }_{3}$ mit den Katalysatoren $\left[\mathrm{MoOS}_{4} \times \mathrm{dt}\right]^{2-}$ (links) und $\left[\mathrm{MoOS}_{8}\right]^{2-}$ (rechts)

Im Vergleich der beiden Katalysen stellt wurde festgestellt, dass die katalytische Aktivität ohne das Vorhandensein einer Dithioleneinheit viel schneller stattfindet, als es bei der unsymmetrischen Dithiolenverbindung der Fall ist. Wenn ein Dithiolenligand am Metall vorhanden ist, kommt es bis zum vollständigen Umsatz zu einer Verlängerung der Reaktionszeit um ca. das 6-fache. 
Kinetische Parameter der beiden Reaktionen wurden durch ein Hanes-Woolf-Diagramm (Abb.126) bestimmt und sind in Tab.30 dargestellt.

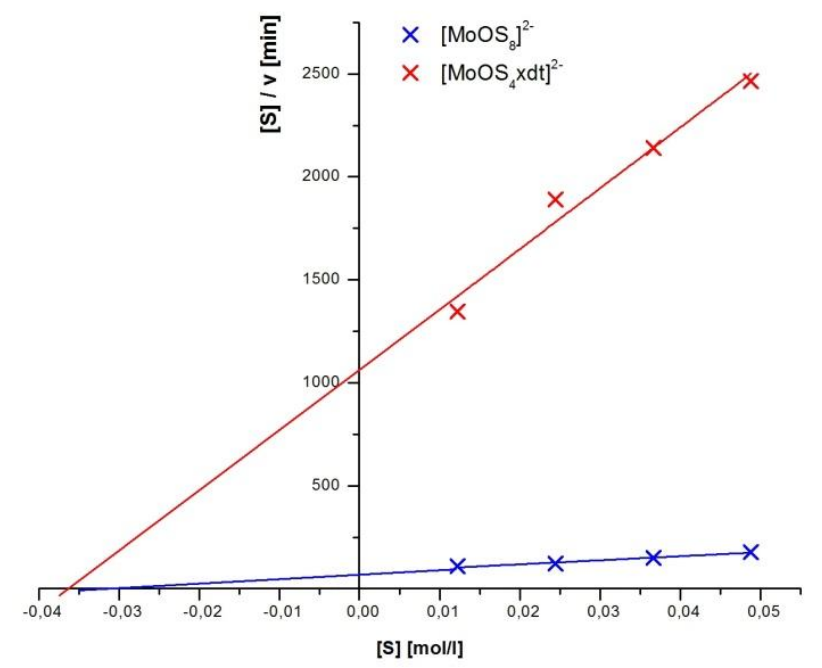

Abb. 126: Hanes-Woolf-Diagramm von $\left[\mathrm{MoOS}_{8}\right]^{2-}$ und $\left[\mathrm{MoOS}_{4} \mathrm{xdt}\right]^{2-}$

\begin{tabular}{|c|c|c|c|c|c|c|c|}
\hline Verbindung & $\begin{array}{c}{[\mathrm{S}]} \\
{[\mathrm{mol} / \mathrm{l}]}\end{array}$ & $\begin{array}{c}\mathrm{v} \\
{[\mathrm{mol} / \mathrm{l} \cdot \mathrm{min}]}\end{array}$ & $\begin{array}{l}{[\mathrm{S}] / \mathrm{v}} \\
{[\mathrm{min}]}\end{array}$ & $\begin{array}{l}\mathrm{k}_{\mathrm{cat}} \\
{\left[\mathrm{s}^{-1}\right]}\end{array}$ & $\begin{array}{c}\mathrm{k}_{\mathrm{cat}} / \mathrm{K}_{\mathrm{M}} \\
{\left[\mathrm{s}^{-1} \cdot \mathrm{mol}^{-1} \cdot \mathrm{l}\right]}\end{array}$ & $\begin{array}{c}\mathrm{v}_{\max } \\
{[\mathrm{mol} / \mathrm{l} \cdot \min ]}\end{array}$ & $\begin{array}{c}\mathrm{K}_{\mathrm{M}} \\
{[\mathrm{mol} / \mathrm{l}]}\end{array}$ \\
\hline \multirow[t]{4}{*}[\mathrm{MoOS}_{4}\mathrm{xdt}]{$^{2-}$} & 0.0122 & $9.06 \cdot 10^{-6}$ & $1.35 \cdot 10^{3}$ & \multirow{4}{*}{0.42} & \multirow{4}{*}{11.58} & \multirow{4}{*}{$3.39 \cdot 10^{-5}$} & \multirow{4}{*}{0.036} \\
\hline & 0.0244 & $1.29 \cdot 10^{-5}$ & $1.89 \cdot 10^{3}$ & & & & \\
\hline & 0.0366 & $1.71 \cdot 10^{-5}$ & $2.14 \cdot 10^{3}$ & & & & \\
\hline & 0.0488 & $1.98 \cdot 10^{-5}$ & $2.46 \cdot 10^{3}$ & & & & \\
\hline \multirow[t]{4}{*}[\mathrm{MoOS}_{8}]{$^{2-}$} & 0.0122 & $1.13 \cdot 10^{-4}$ & $1.08 \cdot 10^{-2}$ & \multirow{4}{*}{6.28} & \multirow{4}{*}{232.7} & \multirow{4}{*}{$5.11 \cdot 10^{-4}$} & \multirow{4}{*}{0.027} \\
\hline & 0.0244 & $2.03 \cdot 10^{-4}$ & $2.44 \cdot 10^{-2}$ & & & & \\
\hline & 0.0366 & $2.43 \cdot 10^{-4}$ & $3.66 \cdot 10^{-2}$ & & & & \\
\hline & 0.0488 & $2.75 \cdot 10^{-4}$ & $4.88 \cdot 10^{-2}$ & & & & \\
\hline
\end{tabular}

Tab. 30: Erhaltene kinetische Parameter für $\left[\mathrm{MoOS}_{8}\right]^{2-}$ und $\left[\mathrm{MoOS}_{4}(\mathrm{xdt})\right]^{2-}$

Die bessere Aktivität der $\left[\mathrm{MoOS}_{8}\right]^{2-}$ - Moleküle kann an dem viel größeren $\mathrm{k}_{\text {cat }}$-Wert sowie den größeren $\mathrm{V}_{\max }-$ Werten beobachtet werden. 


\subsection{Erkenntnisse aus den katalytischen Untersuchungen}

Durch temperaturabhängige Oxo-Transfer Katalysen bei fünf verschiedenen Temperaturen von drei Verbindungspaaren $\left(\left[\mathrm{MO}(\mathrm{xdt})_{2}\right]^{2-},\left[\mathrm{MO}(\mathrm{vdt})_{2}\right]^{2-}\right.$ und $\left.\left[\mathrm{MO}(\mathrm{qdt})_{2}\right]^{2-}\right)$ konnte festgestellt werden, dass die Reaktionsgeschwindigkeit der wolframhaltigen Katalysatoren stärker von der Temperatur abhängig ist, als die der analogen Molybdänkatalysatoren.

Außerdem konnte ein unterschiedlich starker Einfluss auf die katalytische Aktivität, durch die Wahl des Ligandensystems (aromatisch oder nicht aromatisch) beobachtet werden. Bei diesen Katalysatoren mit einem aromatischen Dithiolenligandensystem und einer Oxo-Funktion am Zentralmetall konnten die schnelleren Katalysen bei den Wolframkatalysatoren beobachtet werden. Im Gegensatz dazu wurde bei Verwendung von nicht aromatischen Dithiolenliganden ein schnellerer Ablauf der Katalyse bei den Molybdänkatalysatoren festgestellt.

Durch Analysen der Sauerstoffübertragung bei Non-Oxo-Verbindung mit aromatischen Ligandensystemen konnten die besseren Katalysen mit den molybdänhaltigen Katalysatoren beobachtet werden. Wodurch es wahrscheinlich, aufgrund der größeren Elektronendichte am Wolframatom, durch die nicht vorhandene Oxofunktion zu einer schweren Reduktion dieser Verbindungen kommt.

Untersuchungen von Verbindungspaaren, die ein Schwefel-Heteroatom im angrenzenden 6Ring zur Dithioleneinheit tragen, weisen eine bessere katalytische Aktivität auf, als Ringsysteme die nur Kohlenstoffe beinhalten. Da in den biologischen Ligandensystemen auch ein Sauerstoffatom im Ringsystem vorhanden ist, konnte gezeigt werden, dass ein Heteroatom (Schwefel) in dieser Position des Ringsystems, einen möglichen Einfluss während der Katalyse mit sich bringt. Dabei werden die Untersuchungen der Verbindungspaare mit Pyranringsystem an der Dithioleneinheit von anderen Mitarbeiten durchgeführt. Die Ergebnisse liegen zum gegenwertigen Zeitpunkt noch nicht vor.

Abschließend wurde noch der Einfluss eines Dithiolenligandens auf die katalytische Sauerstoffübertragung untersucht. Beobachtet wurde, dass nach Koordination eines Dithiolenligandens etwa die 6-fach längere Reaktionszeit bis zum vollständigen Umsatz nötig gewesen ist, als bei einem Nicht-Dithiolensystem. 


\section{Kapitel 7}

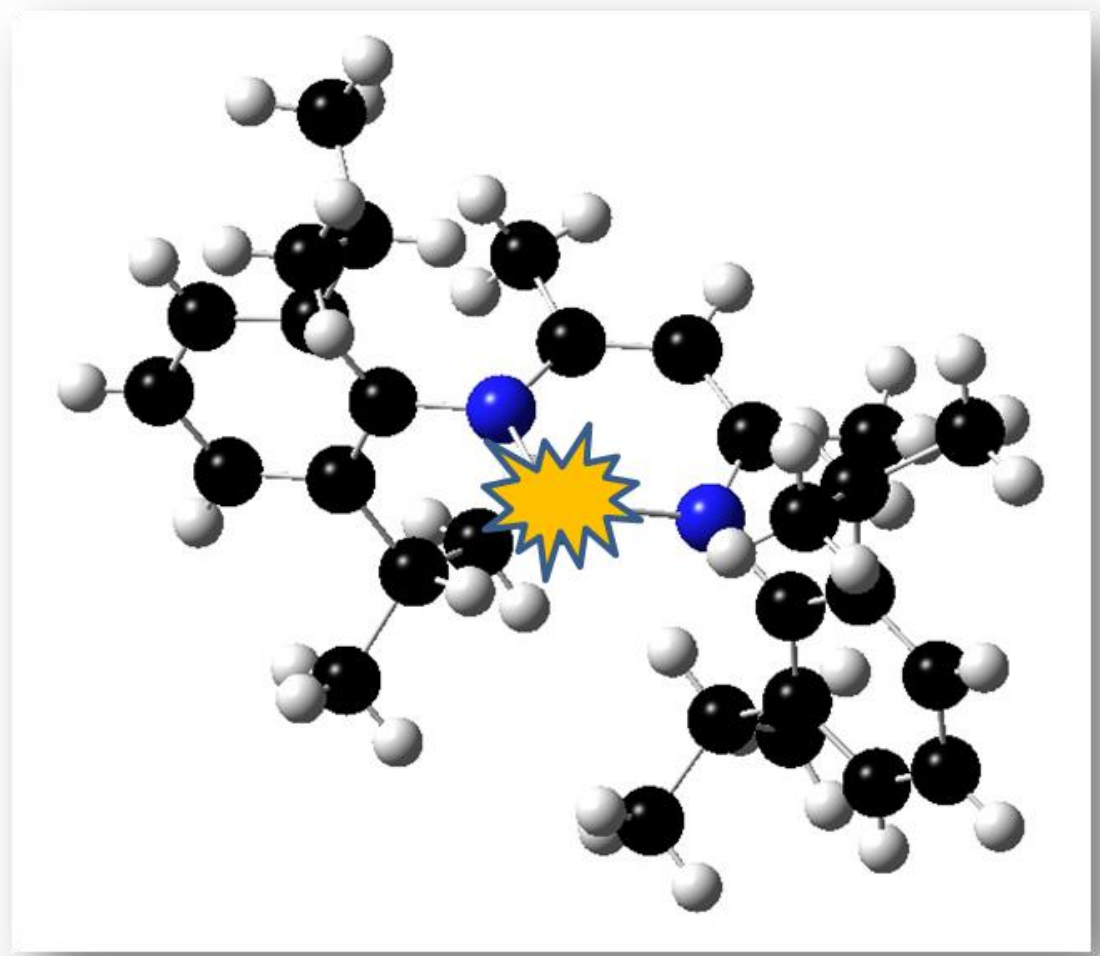

\section{Reaktionen mit $\beta$-Diketiminen}

\section{Zusammenfassung}

Um bei der Herstellung von Monodithiolenkomplexen Mehrfachsubstitutionen durch Dithiolene zu vermeiden, wurden sterisch anspruchsvolle $\beta$-Diketimin-Liganden verwendet. Nach Erhalt von Molybdän- und Wolframmono-dithiolenkomplexen wurden vergleichende elektrochemische und ESRUntersuchungen durchgeführt. 


\subsection{Einleitung}

$\beta$-Diketaminliganden die auch als „nacnac“ bezeichnet werden, stellen sich als ein nützliches Ligandenssystems heraus. Aufgrund ihrer starken Bindung an Metallzentren und durch ihre sterischen und elektronischen Einflüsse könne sie in vielen Feldern der Koordinationschemie verwendet werden. ${ }^{[100]}$<smiles>[R]N/C([R])=C\C([R])N[R]</smiles>

Abb. 127: „nacnac“-System, hierbei $\mathbf{R}^{1}=$ Alkylgruppe; $\mathrm{R}^{2}=$ Alkyl, Silyl oder Aryl-gruppe

Komplexe mit $\beta$-Ketaminligandensystemen wurden erstmals in den späten 60er Jahren des 20. Jahrhunderts synthetisiert. Es handelte sich um homoleptische Komplexe von $\mathrm{Co}, \mathrm{Ni}, \mathrm{Cu}$ und Zn. ${ }^{[101]}$ Dieser Ligandentyp wird vielfach auch bei der Synthese von Modellsystemen für kupferhaltige Enzyme verwendet. ${ }^{[102]}$

\subsection{Reaktionen mit $\beta$-Ketimin-Komplexen mit Molybdän- und}

\section{Wolframverbindungen}

Da diese Ligandensysteme sterisch anspruchsvoll sind und üblicherweise starke koordinative Bindungen an Metallzentren ausbilden, wurde dieses System dazu verwendet Monodithiolenverbindungen herzustellen. Durch die gute Abschirmung des Metallzentrums sollte eine Mehrfachsubstitution durch Dithiolene vermindert werden können. Für die Koordination an Metalle ist die Substitution von Chloridliganden an Metallprecursoren durch das als Lithiumsalz eingesetzte nacnac eine vorteilhafte Strategie. Um zu untersuchen, welche Precursor geeignet sind, wurden verschiedene chlorierte Molybdän- und Wolframverbindungen mit dem Li-nacnac-Salz umgesetzt.

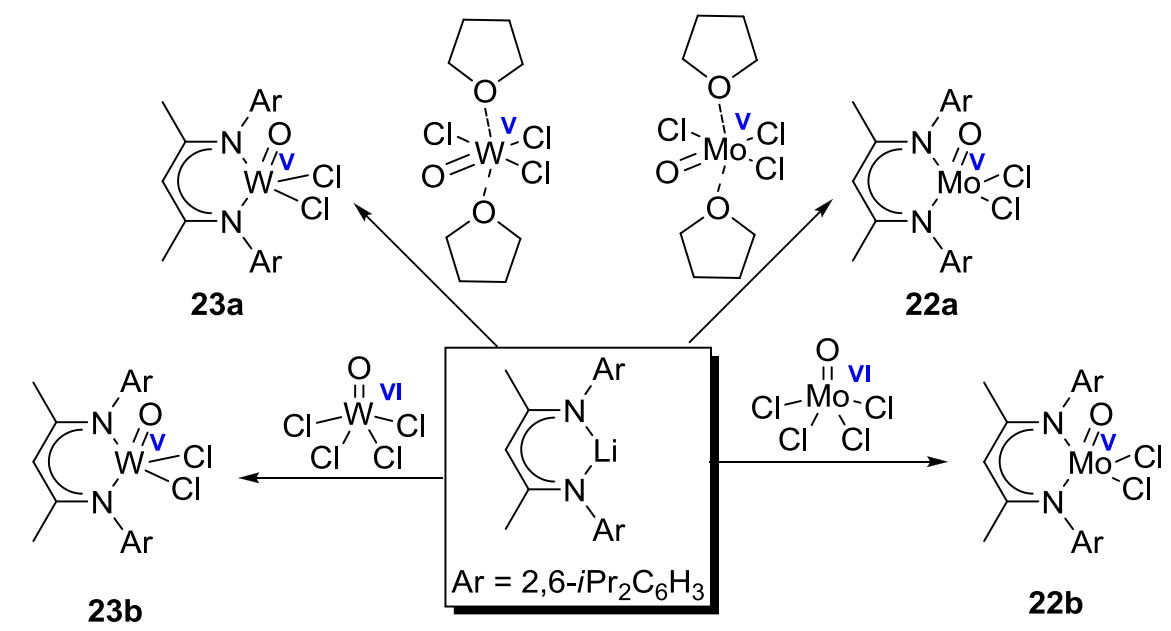


Die Reaktionen mit je einem Äquivalent Li-nacnac zu den Produkten 22a und 23a verliefen wie erwartet, wobei jeweils ein Chloroligand der Molybdän- bzw. Wolframverbindung ausgetauscht wurde. Wegen des enormen Platzbedarfs des nacnac-Liganden sind im Produkt keine Lösungsmittelmoleküle mehr koordiniert.

Bei der Synthese von 22b bzw. 23b kam es unerwarteter Weise während der Reaktion zum Oxidationsstufenwechsel von VI zu V. Der Raumbedarf des nacnac dürfte hierfür verantwortlich sein. Auch wenn nur zwei koordinierende Atome im nacnac vorhanden sind, so werden effektiv noch Koordinationsstellen einer geometrischen Struktur besetzt. Dadurch wird ein weiterer Chloro-Ligand abgespalten. Denkbar ist eine Oxidation von $\mathrm{Cl}^{-} \mathrm{zu} \mathrm{Cl}_{2}$ unter Bildung der Metallverbindung in Oxidationsstufe V. Die erhaltene Verbindung wurde sowohl durch massenspektrometrische Untersuchungen als auch das paramagnetische Verhalten im NMR bestätigt. Von den Molybdänverbindungen 22a und 23a konnten Einkristalle erhalten werden, die kristallographisch untersucht wurden. Im Folgenden ist nur die Kristallstruktur der Verbindung 22a gezeigt.

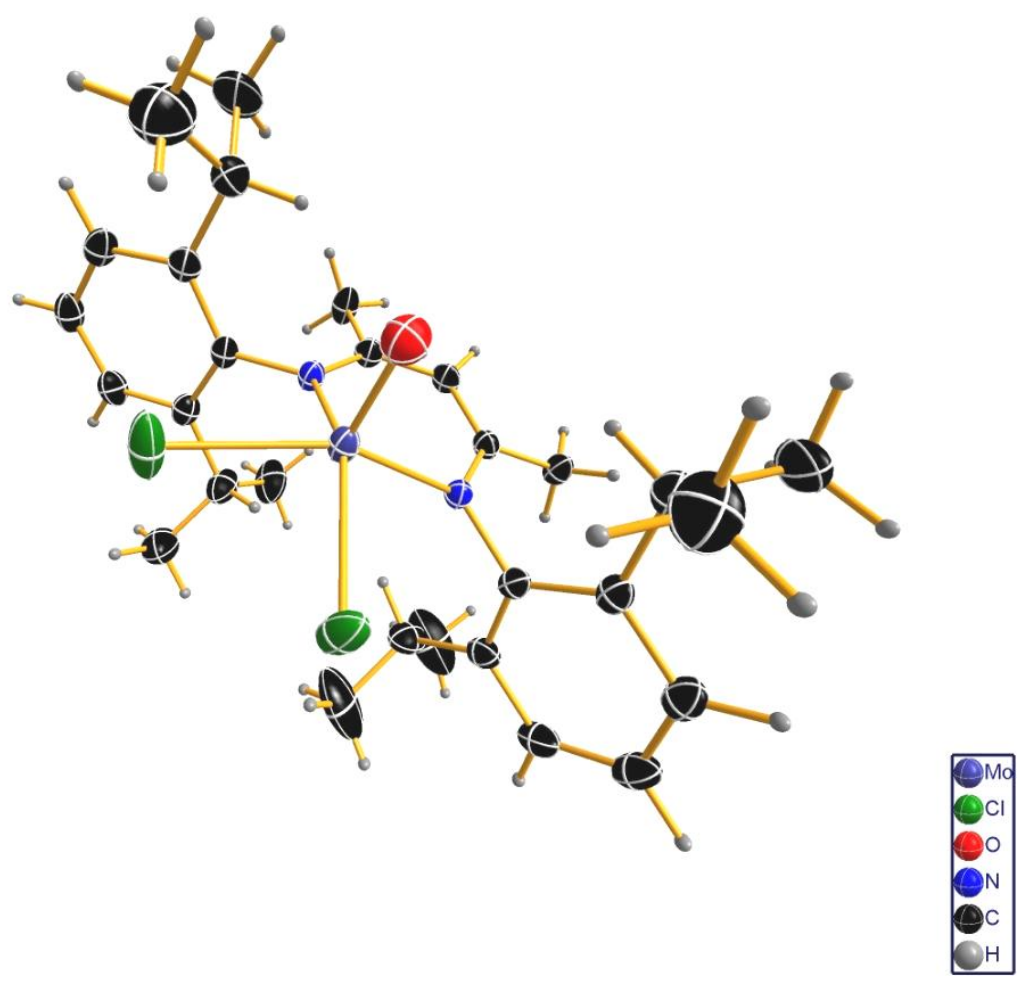

Abb. 129: Kristallstruktur von Verbindung 22a

Molybdän liegt in einer quadratisch pyramidalen Koordinationsumgebung vor. Es ergeben sich Bindungslängen vom Mo zum $\mathrm{Cl}$ im Durchschnitt von $2.3463 \AA$ und zu den Stickstoffatomen des nacnac-Ligandens von 2.0974 A. Die Oxidationsstufe dieser Verbindung ergibt sich formal $\mathrm{zu} \mathrm{V}$, da keine zusätzliche Anionen oder Kationen in der 
Struktur vorhanden sind. Dies kann auch an der Molybdänsauerstoffbindung mit einer Bindungslänge von $1.6528 \AA$ beobachtet werden, die hier kleiner als bei den Verbindungen mit zwei koordinierten Dithiolenliganden in OS IV $\left(\mathrm{M}^{\mathrm{IV}}=\mathrm{O} \sim 1.7 \AA\right)$ ist. Die Verbindungen wurden außerdem elektrochemischen Untersuchungen unterzogen. Dazu ist im Folgenden das Cyclovoltammogramm dieser Verbindung in THF mit einer Vorschubgeschwindigkeit von $0.1 \mathrm{~V} / \mathrm{s}$ gezeigt.

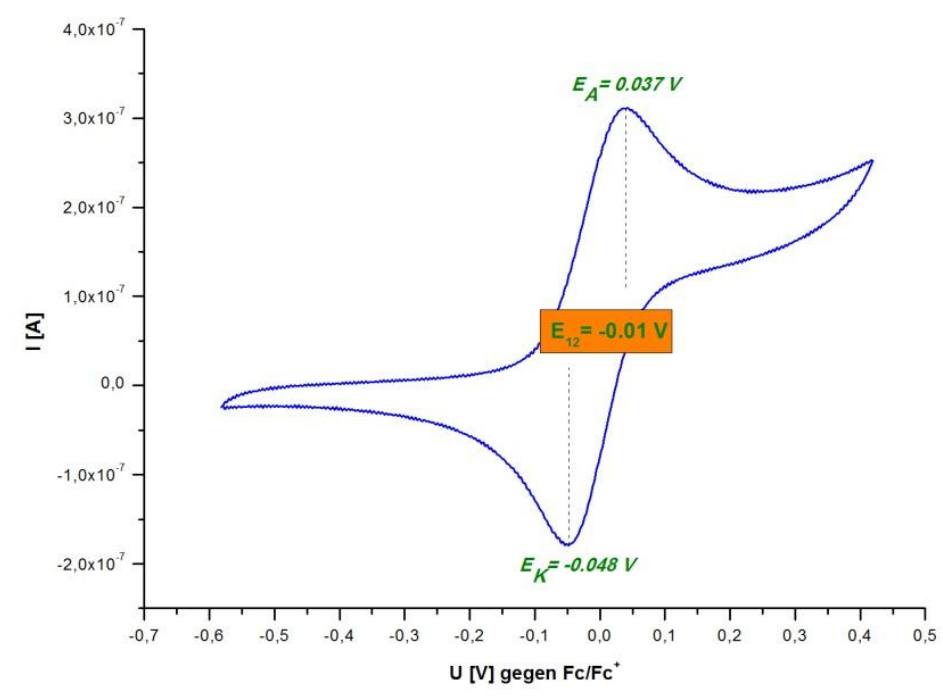

Abb. 130: CV von 22a in THF

Es ergibt sich ein Potential für die Molybdänverbindung gegen das Ferrocen/FerroceniumPaar von $-0.01 \mathrm{~V}$ und bei der Wolframverbindung von $-0.04 \mathrm{~V}$.

\subsection{Substitutionsreaktionen mit Dithiolenverbindungen}

Da die Verbindungen 22 und $\mathbf{2 3}$ jeweils zwei Chloroliganden am Molybdän- bzw. Wolframzentrum tragen, ist der Austausch durch verschiedene Substituenten möglich. Im Rahmen dieser Arbeit ist vor allem die Koordination von Dithiolens interessant.<smiles>CC1=CC(C)=C(Cl)N(Cl)N1[Al]</smiles>

22, 23<smiles></smiles>

$$
\mathrm{Ar}=2,6-\mathrm{Pr}_{2} \mathrm{C}_{6} \mathrm{H}_{3}
$$

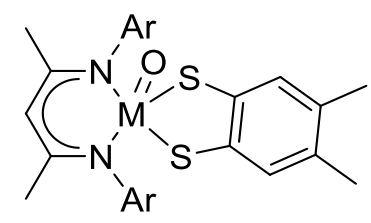

$M o=24 ; W=25$

Abb. 131: Substitutionsreaktion dem xdt-Liganden

Bei Reaktionen mit dem Natriumsalz des xdt-Ligandens konnte eine Substitution bei $-60^{\circ} \mathrm{C}$ in Diethylether erreicht werden. Für beide Metalle wurde ein brauner Feststoff nach Abtrennen 
des Natriumchlorids durch Filtration erhalten. Die Verbindungen konnte durch infrarotspektroskopische, Elementar- und massenspektrometrische Analysen eindeutig nachgewiesen werden. Im Folgenden ist das Massenspektrum der Verbindung $\mathbf{2 4}$ gezeigt.
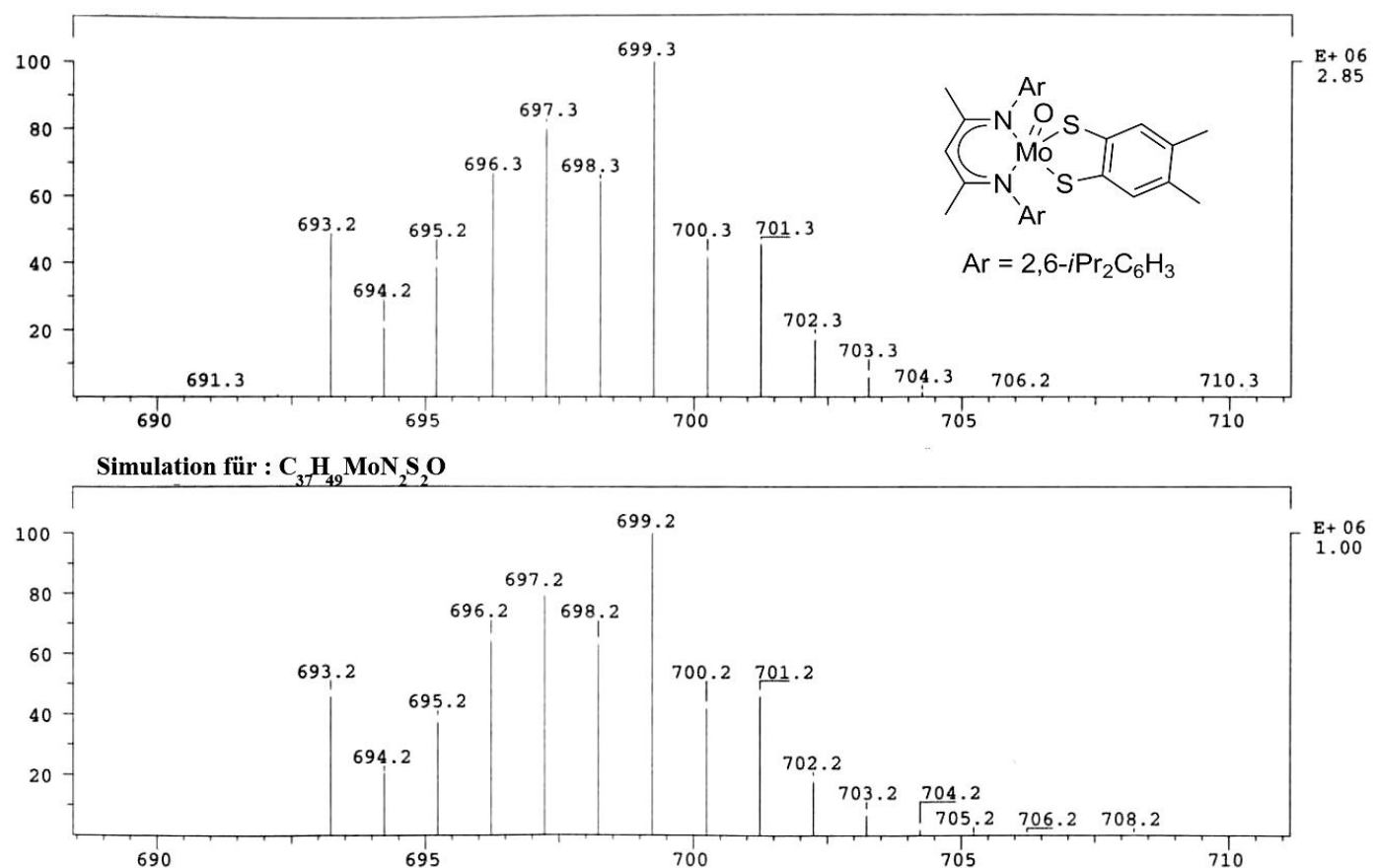

Abb. 132: El-Massenspektrum von Verbindung 24

\subsection{Vergleichende Elektrochemische Untersuchungen}

Es wurden sowohl Cyclovoltammogramme von den Komplexen 22 und 23 ohne Dithiolenligand, sowie von den substituierten Komplexen 24 und 25 mit Dithiolenliganden, aufgenommen.

\begin{tabular}{|c|c|c|}
\hline Verbindung & Potential in V gegen Fc/Fc & Differenz zwischen Mo und W [V] \\
\hline $\mathbf{2 2}$ & -0.01 & 0.03 \\
\hline $\mathbf{2 3}$ & -0.04 & \multirow{2}{*}{0.09} \\
\hline $\mathbf{2 4}$ & -1.04 & \\
\hline $\mathbf{2 5}$ & -1.13 & \\
\hline
\end{tabular}

Tab. 31: Redoxpotentiale von Verbindungen 22-25 in THF-Lösungen aus CVs

Durch diese Verbindungspaare wird der Einfluss der Dithioleneinheit deutlich. Zu beobachten ist eine drastische Verringerung der Potentiale um ca. 1 V. Nach der Substitution kann der Komplex besser oxidiert werden, weil es zum Wechsel von zwei stark elektronenziehenden Liganden $\left(\mathrm{Cl}^{-}\right)$, zu einem Dithiolenliganden kommt. 


\subsection{ESR-Untersuchungen}

Da alle synthetisierten Molybdän bzw. Wolfram nacnac-Verbindungen in der Oxidationsstufe V $\left(d^{1}\right)$ vorliegen, konnte das ungepaarte Elektron mittels Elektronenspinresonanz Spektroskopie untersucht werden. Hierzu wurde die Ausgangsverbindung $\mathrm{MoOCl}_{2}$ (nacnac) 22 und die substituierte Verbindung 24 [MoO(xdt)(nacnac)] untersucht.

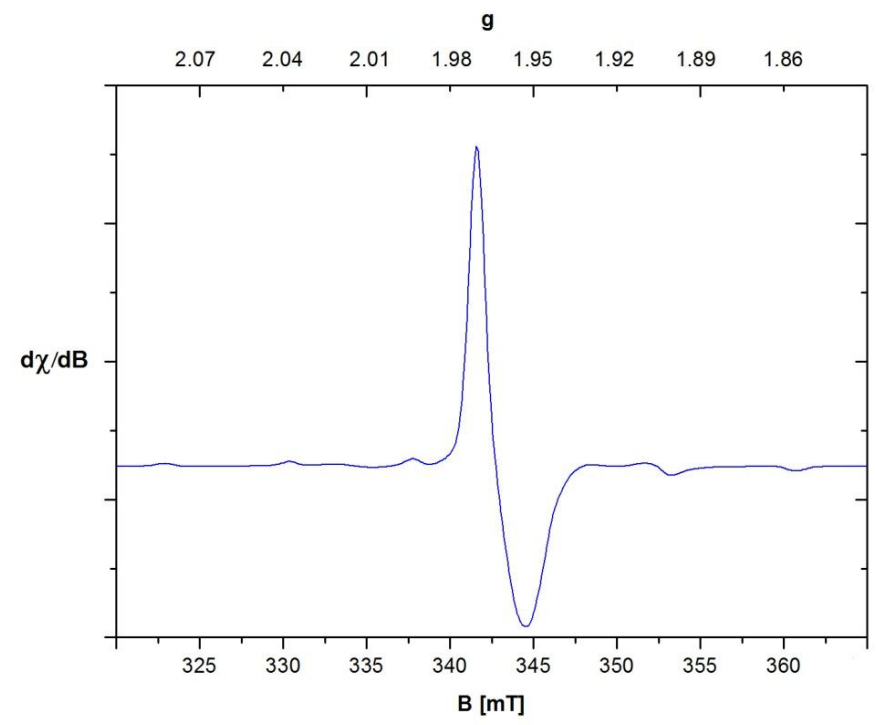

Abb. 133: ESR-Spektrum von $\mathrm{MoOCl}_{2}$ (nacnac) 22 in THF bei $-140^{\circ} \mathrm{C}$

Nach Messungen bei einer Radiofrequenz von $9.436072 \mathrm{GHz}$ konnte für $\left[\mathrm{MoCl}_{2}(\right.$ nacnac] 22 nur ein isotropes Signal mit einem Landé - Faktor von 1.97 beobachtet werden. Die kleinen Signale bei den Magnetfeldstärken von 330, 337, 353 und $361 \mathrm{mT}$ kommen durch das ${ }^{97}$ MoIsotop $(\mathrm{I}=5 / 2, \mathrm{NH}=9.6 \%)$, das neben dem ${ }^{95} \mathrm{Mo}(\mathrm{I}=5 / 2, \mathrm{NH}=16 \%)$ vorliegt, zustande. Dies zeigt, dass das ungepaarte Elektron, ausschließlich am Metall lokalisiert ist. Es findet kein Eindringen des Elektrons in das nacnac-Ligandensystem statt. Die Einführung des Dithiolenliganden in Verbindung $\mathbf{2 4}$ bewirkte eine starke Veränderung des ESR-Spektrums (Abb.135).

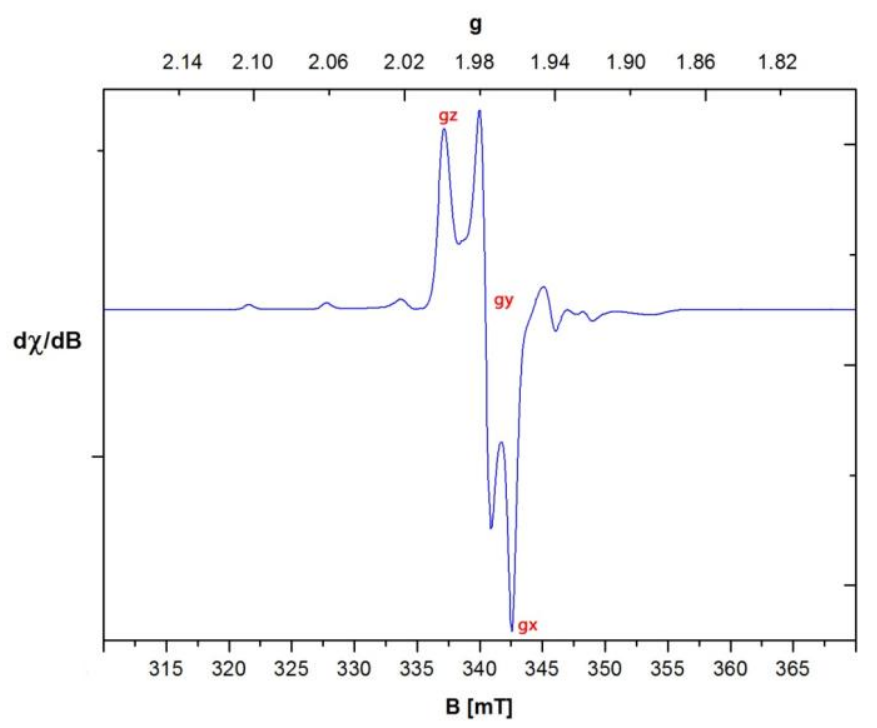


Es wird ein rhombisches Signal mit drei verschiedenen Landé Faktoren $g_{x}=1.97, g_{y}=1.98$, $\mathrm{g}_{\mathrm{z}}=2.00$ gefunden. Da sich die Geometrie bei beiden Verbindungen nicht stark änderte, kann das erhaltene Spektrum nicht auf Geometrieänderungen zurückzuführen sein. Bei Verbindung 22 konnte kein Aufenthalt des ungepaarten Elektrons im nacnac-System festgestellt werden und es wurden nur zwei Chloro-Liganden substituiert. Folglich muss eine Wechselwirkung zwischen den Schwefelatomen der Dithioleneinheit, mit dem ungepaarten Elektron stattgefunden haben. Aufgrund der Tatsache, dass bei Verbindung 1c mit zwei koordinierten xdt-Liganden zum Metallzentrum, nur ein metallzentriertes Signal im ESR-Spektrum festgestellt werden konnte, muss die Veränderung der elektronischen Gegebenheiten durch das Vorhandensein eines Dithiolenligandens ausgelöst worden sein. Im erhaltenen Spektrum von 24 bei $\mathrm{g}=2.11,2.07,2.03,1.93$ und 1.88 tauchen immer noch kleine Signale des ${ }^{97}$ MoIsotops auf. Somit sollte noch eine Aufenthaltswahrscheinlichkeit am Metall möglich sein. Für eine Wechselwirkung mit dem magnetischen Moment des Schwefels kann nur das ${ }^{33} \mathrm{~S}$ Isotop mit einem Kernspin von 3/2 relevant sein. Da es aber mit einer natürlichen Häufigkeit von $0.75 \%$ vorkommt, sollten die entstehenden Signale geringer als bei den Molybdänsignalen ausfallen. Bei der Verbindung 24 kann von einer teilweisen Lokalisation des ungepaarten Elektrons am Schwefel und am Molybdän ausgegangen werden. Hierzu müssen noch theoretische Berechnungen für die Aufenthaltswahrscheinlichkeiten folgen. Allerdings konnte mit diesem Beispiel gezeigt werden, dass die Dithiolenkoordination einen dramatischen Einfluss auf die elektronische Struktur am Metall übernimmt. 


\section{Kapitel 8}

\section{Zusammenfassung}


Das Ziel der Arbeit war, die Synthese von Modellverbindungen für molybdän- und wolframhaltige- Kofaktoren, die in fast allen Organismen, angefangen von den ArchaeaBakterien bis hin zu menschlichen Lebewesen vorkommen. Um die Funktions- und Wirkungsweise dieser Enzyme besser verstehen zu können und zu hinterfragen, weshalb in manchen aktiven Zentren Molybdän und in anderen Wolfram verwendet wird, wurde eine Vielzahl von Molybdän- und Wolframpaaren synthetisiert. An diesen wurden vergleichende Studien (katalytische Sauerstoffübertragungen, elektrochemische Untersuchungen, ESRMessungen, kristallographische Strukturbestimmungen sowie DFT-Berechnungen) durchgeführt, um Unterschiede und Gemeinsamkeiten herauszustellen.

Da in den biologischen Systemen zu den Molybdän- und Wolframzentren eine Koordination über eine Dithiolenfunktion durch den sogenannten Molybdopterinliganden vorhanden ist, wurden im Laufe dieser Arbeit Verbindungspaare hergestellt, die hinsichtlich bestimmter Aspekte Ähnlichkeit mit den biologischen Systemen der DMSO-Reduktase Familie aufweisen. Es konnten folgende Verbindungspaare erhalten werden.

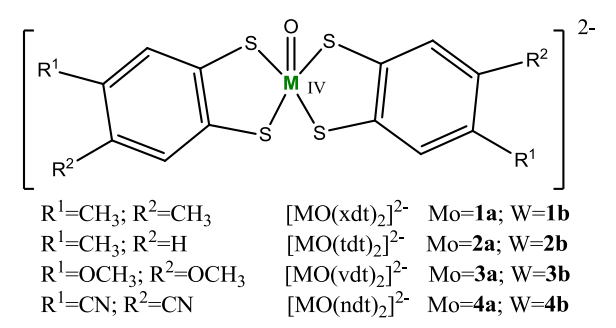<smiles>O=[W]1Sc2nc3ccccc3nc2S1</smiles>

$\left[\mathrm{MO}(\mathrm{qdt})_{2}\right]^{2-} \quad \mathrm{Mo}=\mathbf{5 a} ; \mathrm{W}=\mathbf{5 b}$ $\mathrm{R}^{1}=\mathrm{CN} ; \mathrm{R}^{2}=\mathrm{CN} \quad\left[\mathrm{MO}(\mathrm{ndt})_{2}\right]^{2-} \mathrm{Mo}=\mathbf{4 a} ; \mathrm{W}=\mathbf{4 b}$

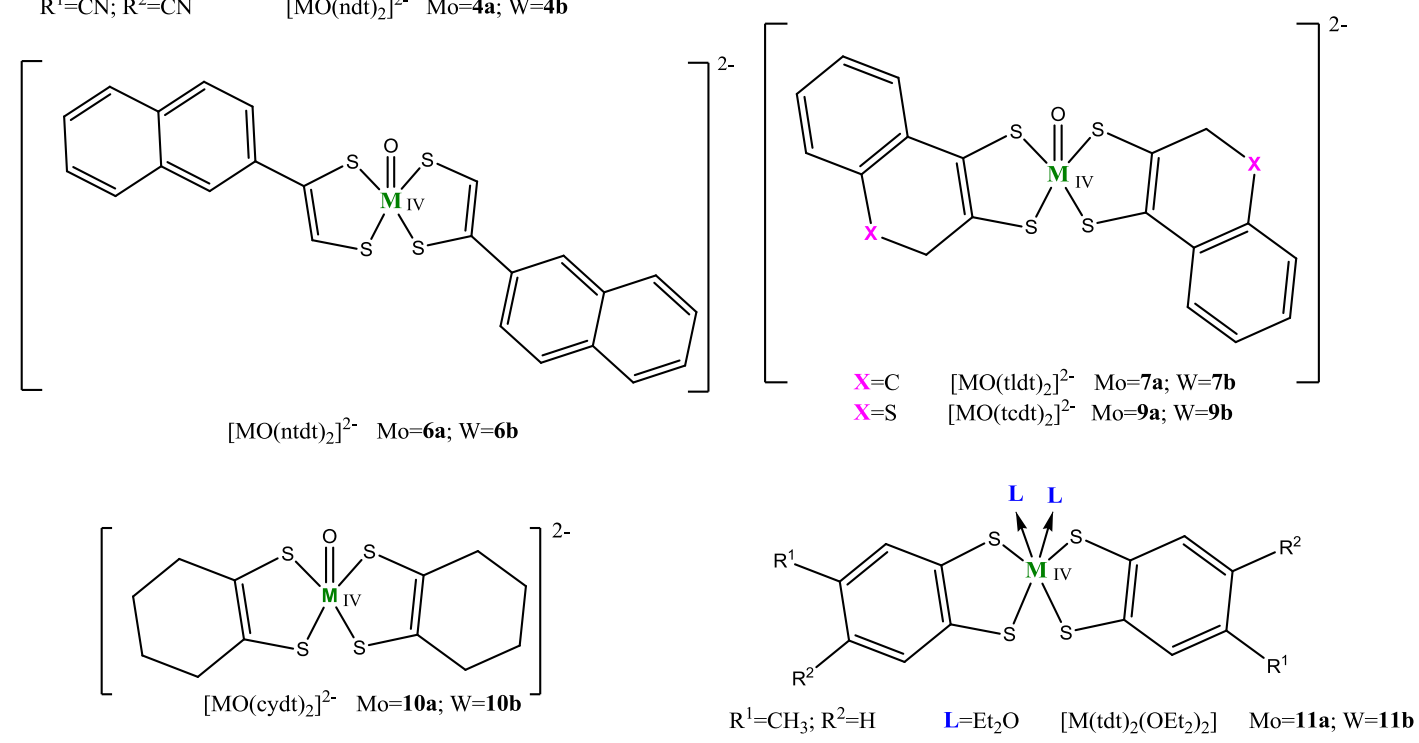

Abb. 135: Hergestellte Verbindungspaare als Modellverbindungen für die DMSO-Reduktase-Familie

Alle Verbindungen konnten eindeutig durch NMR-, infrarotspektroskopische, massenspektrometrische sowie Elementaranalysen charakterisiert werden. Außerdem konnten von den meisten der Verbindungen Einkristalle erhalten werden, die durch Röntgenbeugung 
auch strukturell aufgeklärt wurden. Manche unerwartete Produkte, konnten bei der Herstellung der Modellsysteme erhalten werden. So wurde z.B. bei der Synthese der Verbindung $\left[\mathrm{WO}(\mathrm{tdt})_{2}\right]^{2-}(\mathbf{2 b})$, durch Arbeiten unter leicht sauren Bedingungen, die eigentlich unübliche Abspaltung von zwei Oxoliganden aus dem Ausgangsmaterial $\left[\mathrm{WO}_{2}(\mathrm{CN})_{4}\right] \cdot 6 \mathrm{H}_{2} \mathrm{O}$ erhalten. Daraus entstand eine Verbindung mit zwei $\mathrm{CN}^{-}$Liganden (2c), welche durch andere Substituenten ausgetauscht werden konnten.

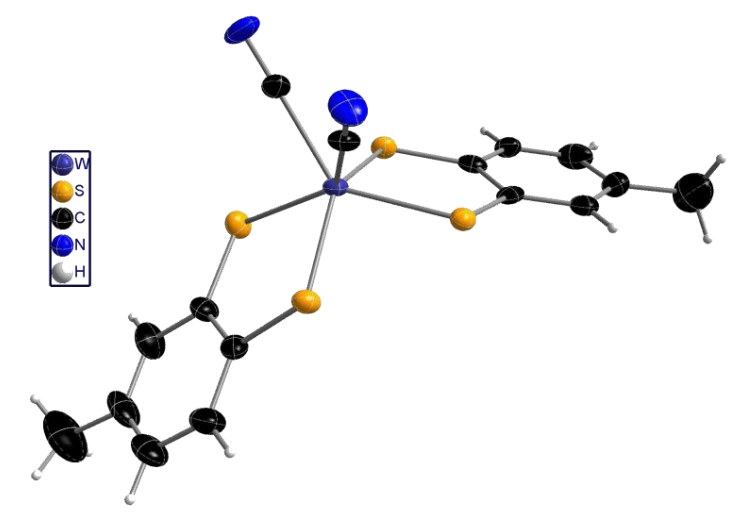

Abb. 136: Kristallstruktur der Verbindung 2c, Kationen nicht gezeigt

Ein weiteres interessantes Strukturmotiv konnte bei dem Verbindungspaar 3a nach Kristallisation aus Methanol beobachtet werden, da es $\mathrm{zu}$ einer Verbrückung über Natriumionen sowie der Oxogruppen im Komplex kam, bildeten sich kettenartige Strukturen aus.

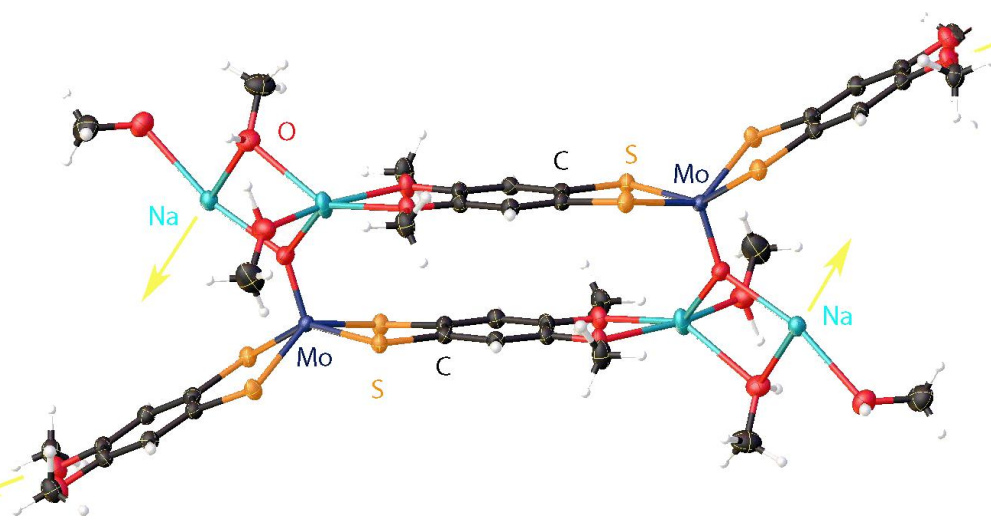

Abb. 137: Kristallstruktur von 3a aus Methanol 
Mit Ausnahme der Nitrogenase katalysieren alle Molybdän- und Wolframenzyme die folgende Sauerstoffübertragung, deshalb ist es von großem Nutzen diese Katalyse im Detail verstehen zu können.

$$
\mathrm{X}+\mathrm{H}_{2} \mathrm{O} \leftrightarrows \mathrm{XO}+2 \mathrm{H}^{+}+2 \mathrm{e}^{-}
$$

Dazu wurden katalytische Sauerstoffübertragungsuntersuchungen bei verschiedenen Temperaturen, sowie bei unterschiedlichen Substratkonzentrationen durchgeführt. Bei drei verschiedenen Molybdän- und Wolframpaaren konnte festgestellt werden, dass sich die Geschwindigkeit der katalytischen Reaktion bei Temperaturveränderungen der wolframhaltigen Verbindungen stärker änderte, als es bei den analogen Molybdänverbindungen der Fall war. Dies könnte ein Indiz dafür sein, dass in vielen Enzymsystemen der frühen Evolution Wolfram im Gegensatz zu Molybdän in den aktiven Zentren eingebaut wurde. Aufgrund der Bestimmung der katalytischen Oxotransferaktivität bei verschiedenen Temperaturen, konnten Aktivierungsenergien berechnet werden. Der Katalysezyklus wurde zusätzlich mit einer Molybdän- und einer Wolframverbindung durch DFT-Rechnungen nachvollzogen, wodurch die theoretischen und experimentellen Energiewerte miteinander verglichen werden konnten.

Außerdem konnte beobachtet werden, dass die Wahl des verwendeten Dithiolenligandens (aromatisches oder nichtaromatisch) als Modell für den biologischen Molybdopterinliganden eine Wirkung auf die katalytische Aktivität der Verbindung hat. Somit zeigte sich, dass bei Verwendung von aromatischen Dithiolenliganden, die katalytische Aktivität der Wolframverbindung stärker, als bei den Molybdänkatalysatoren ist. Im Gegensatz dazu, konnte bei Modellverbindungen welche die Dithioleneinheit nicht direkt an ein aromatisches Ligandensystem gebunden hatten, die bessere Katalysatoraktivität bei den Molybdänverbindungen beobachtet werden. Da in dem biologisch vorkommenden Molybdopterinliganden ein Pyranring direkt an die Dithiolenbindungen gebunden ist, wurde untersucht welchen Einfluss dieses Sauerstoffatom im Ringsystem auf das Metallzentrum hat.

Dazu wurden Modellsysteme die ein Sauerstoffatom im Ringsystem trugen, vergleichend zu Paaren untersucht, die andere Atome (C, S) im Austausch zum Sauerstoffatom in Ligandensystem aufwiesen. Folgende Versuchspaare (Abb.138) wurden sowohl auf ihre katalytische Aktivität für Sauerstoffübertragungen, als auch auf die Veränderbarkeit der Redoxpotentiale bei Temperaturänderungen hin untersucht. 


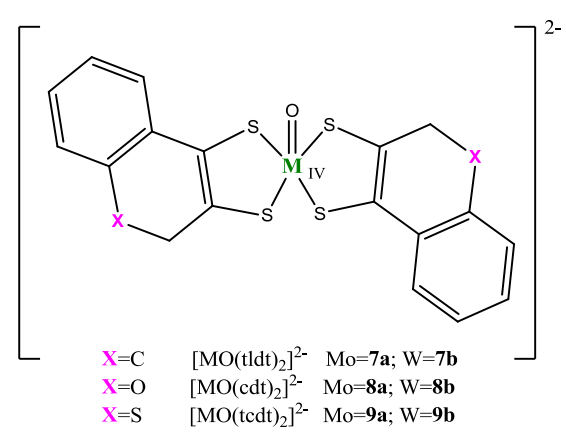

Abb. 138: Verbindungspaare zur Untersuchung des Einflusses durch verschiedene Ringatome

Durch elektrochemische Untersuchungen dieser Verbindungspaare, konnte eine Abnahme des Redoxpotentialunterschieds zwischen der Molybdän- bzw. Wolframverbindung vom eingelagerten Schwefelatom über Kohlenstoff zum Sauerstoffatom beobachtet werden. Es konnte gezeigt werden, dass bei Verwendung eines Sauerstoffatoms im Ringsystem die Redoxpotentiale bei Temperaturänderungen ein stabileres Potential im Vergleich zu den anderen zwei Paaren darstellen. Somit sollte sich die katalytische Aktivität über einen gewissen Temperaturbereich relativ stabil verhalten. Durch katalytische Untersuchungen, konnte bei diesen Verbindungspaaren eine Verbesserung der katalytischen Sauerstoffübertragung, bei Verwendung von Schwefel im Vergleich zu Kohlenstoff, beobachtet werden. Folgendes Balkendiagramm veranschaulicht die Reaktionszeiten bis zum vollständigen Umsatz des Substrats. (Vergleichende Studien mit den Pyranringsystemen (8) werden in einer kooperativen Zusammenarbeit, von einem anderen Mitarbeiter durchgeführt und liegen noch nicht vor)

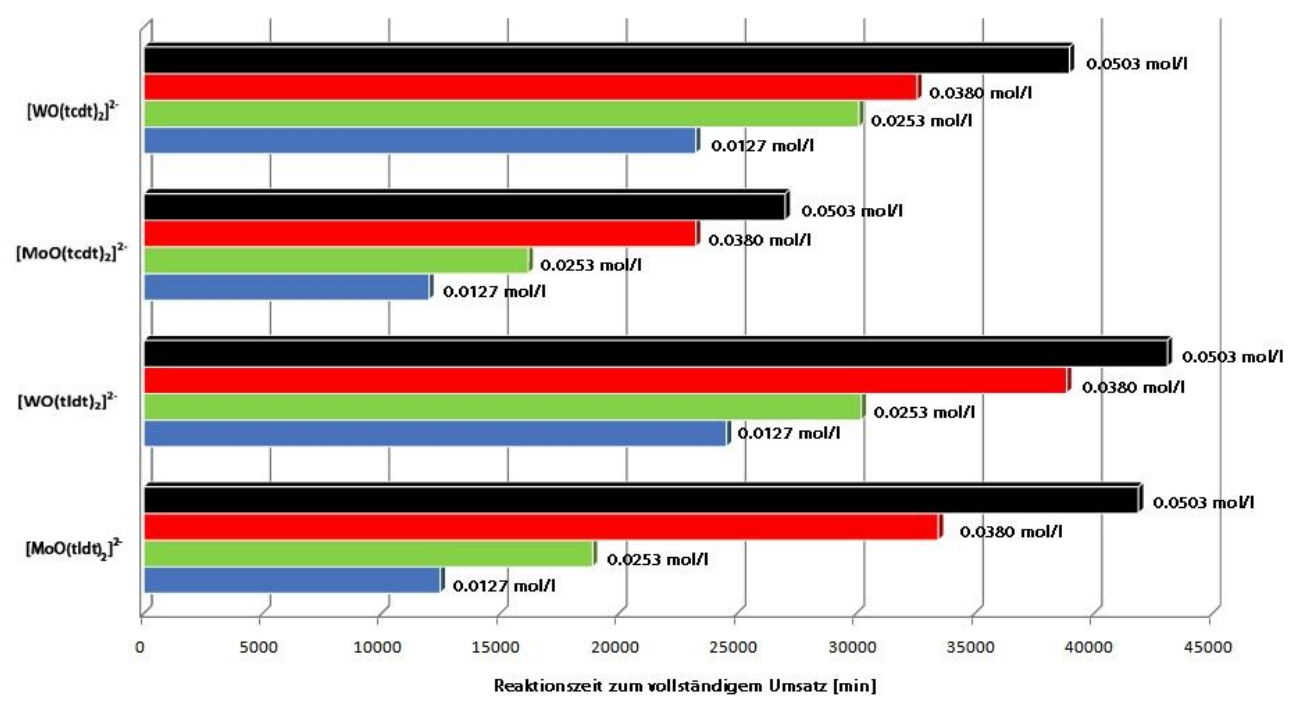


Mit dieser Studie konnte gezeigt werden, dass das Vorhandensein eines Heteroatoms im Ringsystem entscheidenden Einfluss auf die katalytische Aktivität nimmt und dass bei der Verwendungen von Dithiolenmodellliganden generell auf dieses Merkmal geachtet werden sollte, um eine gute Nachbildung der biologischen Systeme zu erhalten.

Um eine Beurteilung der verwendeten Ligandensysteme im Vergleich zu den biologischen Systemen als Modellliganden durchzuführen, wurden DFT-Rechnungen an den hergestellten Komplexverbindungen durchgeführt. Dabei wurden die Bindungslängen und -winkel, die Faltungswinkel (Abknicken des Dithiolenligandens aus der durch die Schwefelatome aufgespannten Ebene), sowie die Redoxorbitale der Komplexe mit den jeweiligen Liganden in verschiedenen Oxidationsstufen betrachtet. Alle berechneten Einflüsse der Liganden wurden mit den berechneten Parametern für die natürlichen Kofaktoren verglichen. Es konnte beobachtet werden, dass die Verwendung des Quinoxalin-2,3-dithiol-Ligandens, sowohl vom elektronischen Verhalten, als auch von seiner geometrischen Anordnung an die Metallzentren, besonders beim Wolfram, zu guten Übereinstimmungen mit den biologischen Systemen führt. Als Folge daraus, sollte dieses Ligandensystem auch als ein guter Modellligand für die Nachbildung des Molybdopterinliganden Anwendung finden.

Aufgrund der non-innocence der Dithiolenliganden sollte ein gewisser Einfluss dieses Ligandens auf das Metallzentrum vorhanden sein. Um die Wirkung eines einzelnen Dithiolenligandens auf die Redoxpotentiale und auch auf die katalytischen Eigenschaften hin $\mathrm{zu}$ untersuchen, wurde die Verbindung $\left[\mathrm{MoOS}_{8}\right]^{2-} \mathbf{1 7 a}$ und die durch Austausch eines $\mathrm{S}_{4}$-Ring erhaltene $\left[\mathrm{MoO}(\mathrm{xdt}) \mathrm{S}_{4}\right]^{2-} \mathbf{1 7 b}$ vergleichend untersucht.
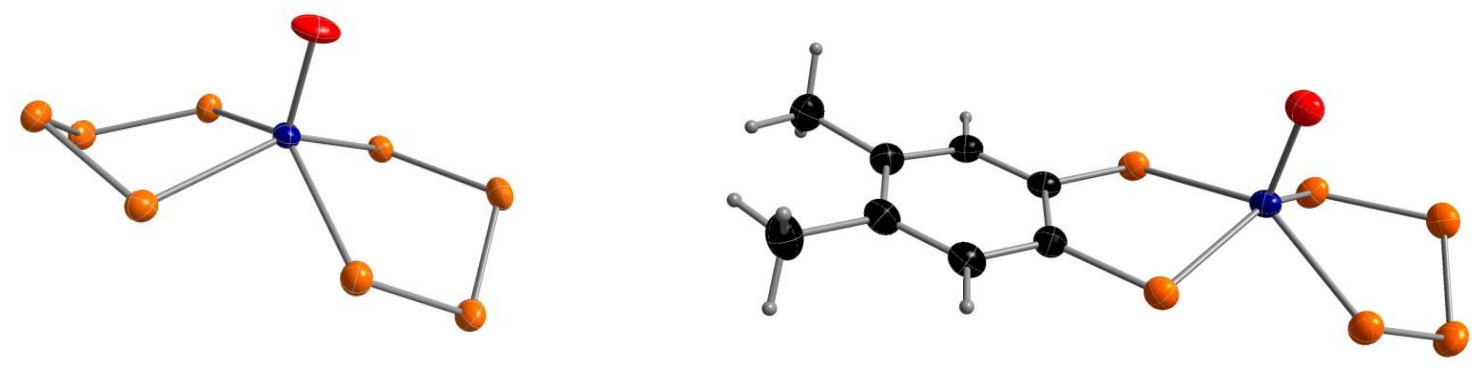

Abb. 140: Verbindungspaare $\left[\mathrm{MoOS}_{8}\right]^{2-}$ und $\left[\mathrm{MoO}(\mathrm{xdt}) \mathrm{S}_{4}\right]^{2-}$

Beobachtet wurde, dass es nach Koordination eines Dithiolenligandens an Verbindung 17a zu einer Abnahme des Redoxpotentials um $0.27 \mathrm{~V}$ kam. Durch vergleichende katalytische Untersuchungen der Sauerstoffübertragung beider Verbindungen, konnte eine Verminderung der katalytischen Aktivität, nach Koordination des Dithiolenligandens, festgestellt werden. 
Um die Wirkung eines Dithiolenligandes auf das Metallzentrum noch mit anderen Verbindungen zu untersuchen, wurde zur Verminderung von Mehrfachsubstitutionen ein sterisch sehr anspruchsvolles Ligandensystem benutzt. Deshalb beschäftigte ich mich mit der Reaktion von $\beta$-Diketiminen mit Molybdän- und Wolframverbindungen. Besonders bei Verwendung von Metallpresursorn in Oxidationsstufe VI wurde entdeckt, dass es zur Reduktion des Metalllzentrums unter Abspaltung eines Chloroligandens kam. Wahrscheinlich führte die Oxidation zu Chlor zu einer Reduktion des Metalls nach Oxidationsstufe V. Nachdem Komplexe erhalten wurden, die noch über Chloro-Liganden am Metallzentrum verfügten, konnte durch einfache Substitutionsreaktionen ein Austausch durch einen Dithiolenliganden erreicht werden.

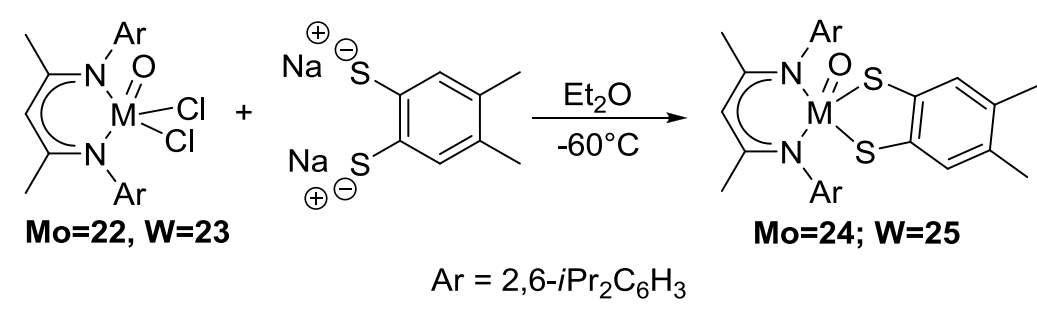

Abb. 141: Substitutionsreaktionen mit Dithiolenen

Da sowohl die Ausgangsprodukte, als auch die Verbindungen mit koordinierten Dithiolenliganden am Metallzentrum ein ungepaartes Elektron besaßen, konnten ESRUntersuchungen durchgeführt werden. Bei der Ausgangsverbindungen 22 wurde eine Lokalisation des ungepaarten Elektrons am Metallzentrum beobachtet. Nach Analyse der Verbindung mit einen koordinierten Dithiolenliganden $\mathbf{2 4}$ kam es zur teilweisen Lokalisation am Metall und an den Schwefelatomen des Dithiolenligandens. Hierzu müssen noch weitere theoretische Berechnungen durchgeführt werden, aber durch diese Untersuchungen konnte gezeigt werden, dass die Dithiolenkoordination einen dramatischen Einfluss auf die elektronische Struktur am Metall übernimmt.

Thermophile und hyperthermophile Mikroorganismen bauen im Gegensatz zu mesophilen Lebewesen Wolfram statt Molybdän in die aktiven Zentren ihrer molybdopterinhaltigen Oxidasen ein. $\mathrm{Ob}$ dies durch die Veränderungen der Umweltbedingungen hervorgerufen wurde oder aufgrund des Redoxpotentials erfolgt, ist bis heute noch nicht vollkommen geklärt. Um der Frage nachzugehen, ob es Vorteile mit sich bringt, wenn Enzyme Wolfram statt Molybdän bei höheren Temperaturen verwenden, wurden temperaturabhängige Bestimmungen der Redoxpotentiale an Verbindungspaaren, die über gleiche Ligandensysteme verfügten, durchgeführt. Fehlerquellen wurden bei diesen Messungen minimiert, indem durch Messungen in isothermalen Zellen, die Temperaturabhängigkeit der verwendeten Elektroden (Platinstab, Ag/AgCl-Elektrode) bestimmt wurde, um die Ergebnisse entsprechen korrigieren 
zu können. Außerdem wurden mögliche beeinflussende Faktoren wie die Messzeit, die Konzentration der Untersuchungslösungen, sowie der Umgebungsdruck auf die Redoxpotentiale untersucht und sofern vorhanden durch Modifikation der Messbedingungen eliminiert. Diese temperaturabhängigen elektrochemischen Messungen zeigten, dass die Redoxpotentiale der Wolframverbindungen eine stärkere Temperaturabhängigkeit aufwiesen, als die der analogen Molybdänverbindungen. Durch weitere Untersuchungen von Verbindungspaaren ohne Dithiolenkoordination konnte nachgewiesen werden, dass dieser Trend eine generelle Gesetzmäßigkeit für Molybdän- und Wolframverbindungen darstellt. Im nachfolgenden Balkendiagramm sind die erhaltenen Temperaturabhängigkeiten gegenübergestellt. In meinen Studien konnte keine einzige Ausnahme dieses Verhaltens festgestellt werden.

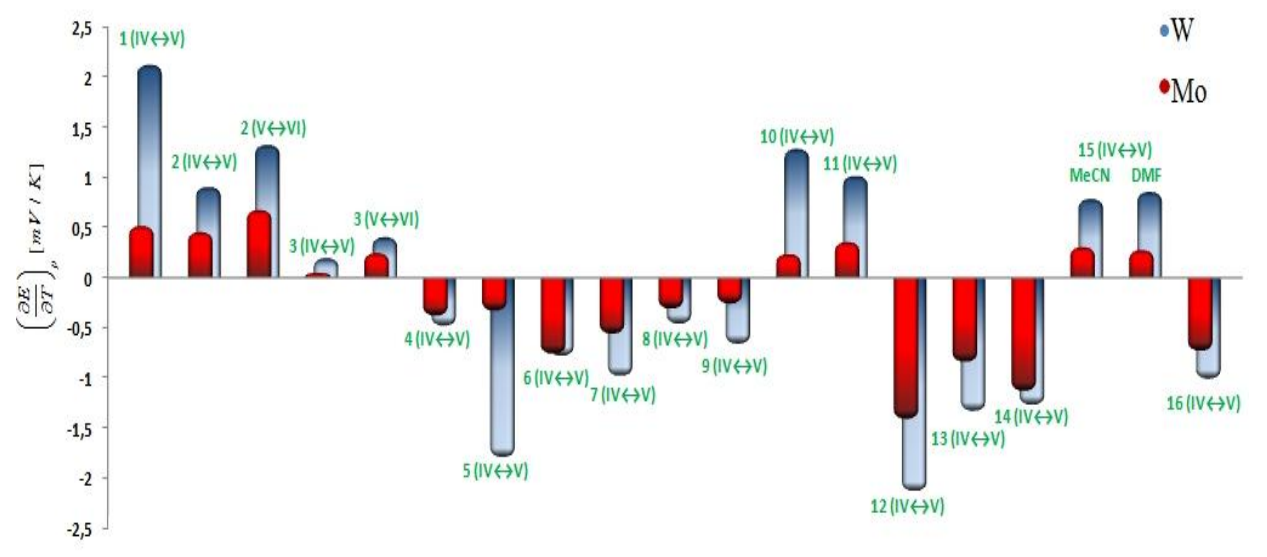

Abb. 142. Gemessene Temperaturabhängigkeit der Redoxpotentiale

Diese fundamentale Gesetzmäßigkeit zwischen diesen zwei relativ ähnlichen Metallen zeigt Wichtigkeit in vielerlei Bereichen, wie auch z.B. bei der industriellen Prozessführung von Katalysen mit Molybdän- oder Wolframverbindungen. Da Molybdän bei Temperaturschwankungen ein stabileres Redoxpotential bereitstellen kann, liefert dies einen weiteren Hinweis auf den Wechsel von Wolfram zu Molybdän in den aktiven Zentren während der Erdentstehung.

Um die gemessenen Redoxpotentiale auch durch theoretische Untersuchungen zu verifizieren, wurden Untersuchungen mit Hilfe des Programmpakets Gaussian03 durchgeführt. Da die Bestimmung von Redoxpotentialen immer noch eine große Herausforderung an die theoretische Chemie stellt, wurden die Berechnungen speziell auf die untersuchten Verbindungspaare abgestimmt. Somit konnten bis auf wenige Ausnahmen die Redoxpotentiale mit einer Genauigkeit von etwa $100 \mathrm{mV}$ zu den experimentell bestimmten Werten erreicht werden. 


\section{Kapitel 9}

Materialien und Methoden 


\subsection{Ausgangsverbindungen}

$\underline{\text { Folgende Metallverbindungen wurden nach literaturbekannten Vorschriften hergestellt: }}$

$\mathrm{K}_{3} \mathrm{Na}\left[\mathrm{MoO}_{2}(\mathrm{CN})_{4}\right] \cdot 6 \mathrm{H}_{2} \mathrm{O},{ }^{[103]} \quad \mathrm{K}_{3} \mathrm{Na}\left[\mathrm{WO}_{2}(\mathrm{CN})_{4}\right] \cdot 6 \mathrm{H}_{2} \mathrm{O},{ }^{[104]} \quad \mathrm{K}_{4}\left[\mathrm{Mo}(\mathrm{CN})_{8}\right] \cdot 2 \mathrm{H}_{2} \mathrm{O},{ }^{[105]}$

$\mathrm{K}_{4}\left[\mathrm{~W}(\mathrm{CN})_{8}\right] \cdot 2 \mathrm{H}_{2} \mathrm{O},{ }^{[106]} \quad\left[\mathrm{MoCl}_{4}\left(\mathrm{OEt}_{2}\right)_{2}\right],{ }^{[107]}, \quad\left[\mathrm{MoCl}_{4}(\text { thf })_{2}\right],{ }^{[107]} \quad\left[\mathrm{WCl}_{4}(\text { thf })_{2}\right],{ }^{[108]}$

$\left[\mathrm{MoCl}_{4}(\mathrm{dme})\right],{ }^{[108]} \quad\left[\mathrm{WCl}_{4}(\mathrm{dme})\right],{ }^{[108]} \quad\left[\mathrm{MoCl}_{4}(\mathrm{MeCN})_{2}\right],{ }^{[109]} \quad\left[\mathrm{WCl}_{4}\left(\mathrm{MeCN}_{2}\right],{ }^{[110]}\right.$

$\left[\mathrm{MoO}(\mathrm{SPh})_{4}\right]^{-[111]}\left[\mathrm{WO}(\mathrm{SPh})_{4}\right]^{-},{ }^{[27]} \quad\left[\mathrm{MoO}_{2} \mathrm{~S}_{2}\right]^{2-,},{ }^{[112]} \quad\left[\mathrm{WO}_{2} \mathrm{~S}_{2}\right]^{2-,[112]} \quad\left[\mathrm{WOCl}_{4}\right]^{[113]}$

$\left[\mathrm{MoOCl}_{4}\right],{ }^{[114]}\left[\mathrm{MoOCl}_{3}(\mathrm{thf})_{2}\right],{ }^{[115]}\left[\mathrm{WOCl}_{3}(\mathrm{thf})_{2}\right],{ }^{[115]} \quad\left[\mathrm{MoOS}_{8}\right]^{2-[29 \mathrm{c}]} \quad\left[\mathrm{MoSS}_{8}\right]^{2-[116]}$

$\left[\mathrm{Mo}(\mathrm{CO})_{3}(\mathrm{MeCN})_{3}\right],{ }^{[117]} \quad\left[\mathrm{W}(\mathrm{CO})_{3}(\mathrm{MeCN})_{3}\right],{ }^{[118]} \quad\left(\mathrm{WCl}_{4}\right)_{\mathrm{x}},{ }^{[110]} \quad\left[\mathrm{MoO}(\mathrm{cdt})_{2}\right]^{2-}(8 \mathrm{a})^{[52]}$, $\left[\mathrm{WO}(\mathrm{cdt})_{2}\right]^{2-}(8 \mathrm{~b})^{[52]}, \mathrm{CuSBu},{ }^{[119]} \mathrm{Cp}^{*}{ }^{[120]} \mathrm{MoCp}^{*} \mathrm{Me}_{4}{ }^{[67]}, \mathrm{HClO}_{4}-\mathrm{SiO}_{2}{ }^{[121]}$, nacnac-Li ${ }^{[122]}$, $\mathrm{KSC}(\mathrm{S})-\mathrm{O} i$ Pr bzw. $-\mathrm{OEt}^{[42]}$

$\underline{\text { Folgende Verbindungen wurden von kommerziellen Herstellern bezogen: }}$

[Mo(CO) $\left.)_{6}\right],\left[\mathrm{W}(\mathrm{CO})_{6}\right], \mathrm{NiCl}_{2} \cdot 6 \mathrm{H}_{2} \mathrm{O}, \mathrm{MoO}_{3}, \mathrm{WO}_{3}, \mathrm{Na}_{2}\left[\mathrm{MoO}_{4}\right] \cdot 2 \mathrm{H}_{2} \mathrm{O}, \mathrm{Na}_{2}\left[\mathrm{WO}_{4}\right] \cdot 2 \mathrm{H}_{2} \mathrm{O}$, $\mathrm{H}_{2}\left[\mathrm{MoO}_{4}\right], \mathrm{H}_{2}\left[\mathrm{WO}_{4}\right], \mathrm{WCl}_{6}, \mathrm{MoCl}_{5}, \mathrm{MoCp}_{2} \mathrm{Cl}_{2}, \mathrm{WCp}_{2} \mathrm{Cl}_{2}, 3,4-$ Toluoldithiol

Natriumhydrid wurde in Mineralöl bezogen und vor Verwendung mit Hexan gewaschen und im HV getrocknet.

\subsection{Trocknen von Lösungsmitteln}

Beim Trocknen mit metallischem Natrium bzw. Kalium wurde der Trocknungszustand durch Zugabe von Benzophenon überprüft. Vor Zugabe des Alkalimetalls wurden die Lösungsmittel 2 d über Kaliumhydroxid refluxiert und dann mit dem Trocknungsmittel versetzt. Folgende Trocknungsmittel kamen zum Einsatz:

$\begin{array}{ll}\text { Aceton } & \text { Calciumchlorid gefolgt von Molsieb } 3 \AA \\ \text { Acetonitril } & \text { Calciumhydrid, gefolgt von Phosphorpentoxid } \\ \text { Chloroform } & \text { Phosphorpentoxid } \\ \text { Dichlormethan } & \text { Calciumhydrid } \\ \text { Diethylether } & \text { Natrium/Kalium } \\ \text { Dimethoxyethan } & \text { Natrium } \\ \text { DMF } & \text { Calciumhydrid, Vakuumdestillation } \\ \text { DMSO } & \text { Calciumhydrid, Vakuumdestillation } \\ \text { Ethanol } & \text { Natrium/Phtalsäurediethylester } \\ \text { Hexan } & \text { Natrium/Diphenylether }\end{array}$


Methanol

Pentan

Petrolether

Pyridin

Tetrachlorkohlenstoff

Tetrahydrofuran

Toluol

Triethylamin
Magnesiumspäne (Aktiviert durch Zugabe von Iod)

Natrium/Diphenylether

Calciumhydrid

Calciumhydrid

Calciumhydrid

Natrium/Kalium

Natrium

Natrium

Die destillierten Lösungsmittel wurden über Molekularsieb 3 bzw. $4 \AA$ bzw. die mit Alkalimetallen getrockneten Lösungsmittel über verflüssigtem Natrium in Schlenkgefäßen unter Stickstoff- bzw. Argon- Atmosphäre aufbewahrt.

\subsection{Entgaste Lösungsmittel}

Demineralisiertes Wasser, Methanol, Ethanol wurden für die Verwendung bei Atmosphärendruck destilliert und dann bei $50^{\circ} \mathrm{C}$ im Ultraschallbad mit Stickstoff durchströmt. Alle verwendeten absolutierten Lösungsmittel wurden vor der Verwendung durch mehrfaches Einfrieren unter Vakuum vom Sauerstoff befreit.

\subsection{Inertgasatmosphäre}

Um Feuchtigkeits sowie Sauerstofffreiheit des Gasstroms zu gewährleisten wurde Stickstoff bzw. Argon in hoher Reinheit 5.0 (nach DIN EN 437 99.9990\%) von den Herstellern Air Liquid und Linde Gas bezogen. Der Schutzgasstrom wurde zuerst über einen Kupferkatalysator der Firma BASF mit der Bezeichnung R3/11 bei einer Reaktionstemperatur von $200^{\circ} \mathrm{C}$ geleitet. Nachfolgende wurde der Gasstrom über mehrere $\mathrm{P}_{4} \mathrm{O}_{10^{-}}$und $\mathrm{KOH}-$ Kolonnen geleitet. Abschließend wurde der Inertgasstrom durch eine Molsieb-Kolonne ( $3 \AA$ und $4 \AA$ ) getrocknet. 


\subsection{Geräte Spezifikationen}

\subsubsection{Kernresonanzspektroskopische Untersuchungen}

Die NMR-Spektren wurden mit Geräten des Typs Bruker Avance $500\left({ }^{1} \mathrm{H}: 500.13 \mathrm{MHz},{ }^{13} \mathrm{C}\right.$ : 125.77 MHz), $300\left({ }^{1} \mathrm{H}: 300.13 \mathrm{MHz},{ }^{13} \mathrm{C}: 75.47 \mathrm{MHz}\right)$ und $200\left({ }^{1} \mathrm{H}: 200.13 \mathrm{MHz},{ }^{13} \mathrm{C}\right.$ : $50.32 \mathrm{MHz}$ ) aufgenommen. Die angegebenen Werte der chemischen Verschiebung $\delta$ beziehen sich auf den internen Standard durch die Lösungsmittel $\mathrm{CDCl}_{3}\left({ }^{1} \mathrm{H}: \delta=7.24 \mathrm{ppm}\right.$, $\left.{ }^{13} \mathrm{C}: 77.0 \mathrm{ppm}\right), \mathrm{d}_{6}$-Dimethylsulfoxid $\left({ }^{1} \mathrm{H}: \delta=2.49 \mathrm{ppm},{ }^{13} \mathrm{C}: 39.5 \mathrm{ppm}\right)$, und $\mathrm{d}_{3}$-Acetonitril $\left({ }^{1} \mathrm{H}: \delta=1.94 \mathrm{ppm},{ }^{13} \mathrm{C}: 1.3 \mathrm{ppm}\right)$ relativ zu externem Tetramethylsilan (jeweils $\delta=0 \mathrm{ppm}$ ). Die ${ }^{13} \mathrm{C}$-NMR-Spektren wurden ${ }^{1} \mathrm{H}$-entkoppelt aufgenommen. Bei der Zuordnung der Signale wurden die Abkürzungen: $\mathrm{s}=$ Singulett, $\mathrm{d}=$ Dublett, $\mathrm{t}=$ Triplett, quar $=$ Quartett, quin $=$ Quintett, sech $=$ Sechstett, Sept $=$ Septett, $\mathrm{m}=$ Multiplett, $\mathrm{br}=$ breites Singulett, verwendet .

\subsubsection{Massenspektrometrische Untersuchungen}

Die Massenspektren wurden mit den Geräten Finnigan MAT 8200 und Finnigan MAT 95 aufgenommen. Ionisierungsmethode für die EI-Massenspektren war Ionenstoß-Ionisation bei 70 eV. Bei Fast-Atom-Bombardement-(FAB)-Experimenten wurde die zu messende Substanz in eine Matrix aus 3-Nitrobenzylalkohol (3-NBA) eingelagert und durch Beschuss mit Cäsium-Kationen ionisiert. Für Elektronen-Spray-Ionisations-(ESI)-Messungen wurden die Proben im jeweils angegebenen Lösungsmittel gelöst und anschließend mit einem Finnigan MAT LCQ ESI-Spektren oder Applied Biosystems API 2000 aufgenommen.

\subsubsection{Elementaranalysen}

Elementaranalysen wurden vom Analytischen Labor des Anorganischen Instituts Göttingen mit einem 4.1 vario EL 3 der Firma Elementar durchgeführt.

\subsubsection{Infrarotspektroskopie}

Die Infrarotspektren wurden auf einem Digilab FTS 1000 aufgenommen. Feststoffe wurden meistens als $\mathrm{KBr}$ Presslinge gemessen. Zur genauen Blindwerteichung wurden zuvor frisch hergestellte KBr-Presslinge verwendet. Außerdem wurde eine Nujol-Verreibung zwischen KBr-Platten durchgeführt. Die Lage der Bande ist in Wellenzahlen $\left(\mathrm{cm}^{-1}\right)$ angegeben. Es wurden Spektren von $4000-400 \mathrm{~cm}^{-1}$ aufgenommen. Die Intensitäten sind wie folgt abgekürzt: $\mathrm{w}=$ schwach, $\mathrm{m}=$ mittel, $\mathrm{s}=$ stark, $\mathrm{vs}=$ sehr stark, $\mathrm{br}=$ breit .

\subsubsection{UV/VIS-Spektroskopie}

Die UV/VIS-Spektren wurden an einem Virian Cary 50 Spektrometer in Küvetten aus Quarzglas mit einer Schichtdicke von $1 \mathrm{~cm}$ aufgenommen. 


\subsubsection{Röntgenstrukturanalysen}

Die Bestimmung der Elementarzellen und die Sammlung der Messdaten erfolgte an einem STOE/IPDS II - Gerät mit graphitmonochromatisierter Mo-K $\alpha$-Strahlung $(\lambda=0.711 \AA$ ) bei $-140^{\circ} \mathrm{C}$. Die Messwerte wurden bezüglich Lorentz- und Polarisationseffekten korrigiert. Zusätzlich erfolgten Absorptionskorrekturen bei vorhandenen Schweratomen in der Verbindung. Lösung und Verfeinerung wurden mit den Programmen SHELXS-97 ${ }^{[123]}$ und SHELXL-97 ${ }^{[124]}$ durchgeführt ${ }^{[125]}$.

\subsubsection{Dünnschichtchromatographie}

Dünnschichtchromatische Trennungen wurden auf Fertigfolien „Polygram Sil/UV254“ der Firma Machery, Nagel \& Co. verwendet. Die Detektion erfolgte unter UV-Licht (bei $254 \mathrm{~nm}$ ).

\subsubsection{ESR-Spektroskopische Untersuchungen}

X-Band ESR-Spektren wurde auf einem Bruker ELEXSYS E500 Spektrometer aufgenommen. Die Mikrowellenfrequenz wurde mit einem Hewlett-Packard „frequenz counter“ (HP5352B) und die Feldkontrolle wurde durch eine Bruker field probe (ER035M) kalibriert. Alle Untersuchungen wurden aus gelösten Proben aufgenommen.

\subsection{Abfallentsorgung}

Die verwendeten Lösungsmittel wurden destillativ entfernt oder in Kühlfallen einkondensiert. Falls keine Möglichkeit der Wiederverwertung besteht, wurden sie getrennt und nach halogenierten und nicht halogenierten Lösungsmittelabfällen in die dafür vorgesehenen Sammelbehälter gegeben und der zentralen Entsorgung zugeführt. Aceton, das zur Reinigung von Glasgeräten diente, wurde gesammelt und destillativ zurückgewonnen. Zum Trocknen von Lösungsmitteln verwendetes Natrium wurde mit iso-Propanol oder Ethanol zersetzt und dem alkalischen Reinigungsbad zugeführt. Andere Trockenmittelreste wie $\mathrm{KOH}$ und $\mathrm{P}_{4} \mathrm{O}_{10}$ wurden in die Basen- bzw. Säureabfälle überführt. Säurebäder wurden mit $\mathrm{Na}_{2} \mathrm{CO}_{3}$ neutralisiert und der zentralen Entsorgung zugeführt. Das zur Säuberung von Glasgeräten verwendete ethanolische Kaliumhydroxidbad wurde durch Eindampfen reduziert und anschließend in die zentrale Sammelstelle gegeben.

\subsection{DFT-Rechnungen}

Dichtefunktionaltheoretische Berechnungen wurden mit dem Programmpaket Gaussian03 ${ }^{[73]}$ durchgeführt. Für alle Rechnungen wurde das Drei-Parameter-Hybridfunktional von Becke $(B 3)^{[78 c]}$ und das Korrelationsfunktional von Lee, Yang und Parr (LYP) ${ }^{[126]}$ angewendet. Mit Ausnahme der Berechnungen der Redoxpotentiale, aber hierbei sind alle veränderten 
Parameter im dazugehörigen Kapitel zu finden. Für die Metallatome Molybdän und Wolfram wurde der Basisatz LANL2DZ ${ }^{[127]}$ und für alle übrigen Atome $(\mathrm{C}, \mathrm{H}, \mathrm{N}, \mathrm{O}, \mathrm{S}, \mathrm{P})$ wurde 6$311 \mathrm{G}(\mathrm{d}, \mathrm{p})^{[127]}$ verwendet. Die Basis LAN2DZ beinhaltet die effektiven Kernpotentiale (Los Alamos) mit DZ-Funktionen für Metalle ab der zweiten Übergangsmetallreihe. Die Basis 6311G(d,p) besteht aus der 6-311G-Basis erweitert um zusätzliche Polarisationsfunktionen.

\subsection{Elektrochemische Messungen}

Die elektrochemischen Messungen wurden mit einem AUTOLAB PGSTAT12 Potentiostat/Galvanostat mit einer Glassy Carbon oder mit einem Platinelektrode mit einer diffinierten Fläche von $1 \mathrm{~mm}^{2}$ als Arbeitselektrode durchgeführt. Als Referenzelektrode diente ein Platinstab und als Hilfselektrode eine Platinelektrode. Die Messungen fanden, wenn es nicht explizit andere Bedingungen beschrieben werden, in einer „Glovebox“ unter Argonatmosphäre statt. Für Untersuchungen in wässrigen Lösungen wurde eine $0.1 \mathrm{~mol} / 1 \mathrm{KCl}$ Lösung verwendet. In nicht-wässrigen Untersuchungslösungen (DMF, MeCN, THF, DCM) wurde $\mathrm{Bu}_{4} \mathrm{NPF}_{6}$ in einer Konzentration von $0.1 \mathrm{~mol} / \mathrm{l}$ als Elektrolyt verwendet. Zur Referenzierung in nicht-wässrigen Lösungsmitteln nur die Ferreocen/Ferrocenium-Kopplung verwendet. In wässrigen Lösungen wurde auf die Verwendung einer Silber/SilberchloridElektrode befüllt mit einer $3 \mathrm{M}$ KCl-Lösung zurückgegriffen. Für die temperaturabhängigen Messungen wurden jeweils $5 \mathrm{ml}$ der zu untersuchenden Lösungen in eine doppelwandige elektrochemische Zelle gegeben. Die Temperatur der Zelle wurde durch eine Julabo FP50MV Thermostaten kontrolliert und eingestellt. Um die Temperaturkonstanz zu gewährleisten, wurde vor jeder Messung $10 \mathrm{Min}$ bei der jeweiligen Temperatur gerührt. Für Cyclovoltammometrische Messungen wurde mit Vorschubgeschwindigkeiten von 0.05 $0.3 \mathrm{~V} / \mathrm{s}$ gearbeitet. Differenzpulsvoltammometrische Untersuchungen wurden mit einer Modulationszeit von $0.05 \mathrm{~s}$ einer Intervallzeit von $0.5 \mathrm{~s}$, einem Stufenpotential von $2.55 \mathrm{mV}$ und einer Modulationsamplitude von $25.05 \mathrm{mV}$ durchgeführt. Für Untersuchungsmethoden mit der Square Wave Untersuchungsmethode wurden mit einem Stufenpotential von $2.55 \mathrm{mV}$, einer Modulationsamplitude von $19.95 \mathrm{mV}$ sowie einer Frequenz von $25 \mathrm{~Hz}$ durchgeführt.

\subsection{Katalytische Untersuchungen}

Triphenylphoshin wurde vor Verwendung in Methanol umkristallisiert und die erhaltene Qualität wurde durch Schmelzpunktanalysen in offenen Kapillaren bestimmt. Es wurde $\mathrm{PPh}_{3}$ Kristalle verwendet die einen Schmelzpunkt von $80-81^{\circ} \mathrm{C}$ aufwiesen. 
Die Temperaturabhängigen Messungen wurde entweder mit Hilfe eines Trockenschrank des Herstellers Heraeus klevitron®t mit digitaler Temperatureinstellung oder einem Heizblock der Firma Fischer FV15101 konstant gehalten. Da die Wege zum NMR-Geräte nicht sehr weit waren wurden die NMR-Röhrchen in zuvor temperierten Wasserbädern zum NMR-Gerät transportiert.

\subsubsection{Nachweis der Sauerstoffübertragung}

Eine wässrige Lösung aus $\mathrm{HgCl}_{2}$ wurde jeweils zu den Komplexverbindung (1:20) gelöst in DMSO gegeben. Bei Sauerstoffübertagung konnte die Bildung des $\left(\mathrm{Me}_{2} \mathrm{~S}\right)_{2} 3 \mathrm{HgCl}_{2}$ beobachtet werden. Nach trocknen im Vakuum konnten Schmelzpunkte im Bereich von $146^{\circ} \mathrm{C}$ bis $155^{\circ} \mathrm{C}$ festgestellt werden. 


\section{Kapitel 10}

\section{Experimentelles}




\subsection{Experimentelles}

\subsection{Ligandensynthese}

\subsection{1 xdt-Ligand (L1) ${ }^{[44]}$}

\subsection{1a Bromierung (L1a)}

In einem 11 2-Halskolben wurden $0.4 \mathrm{~g}$ Iod $(1.58 \mathrm{mmol})$-Kristalle zusammen mit $92 \mathrm{ml}$ (0.66 mol) $o$-Xylol versetzt. Nun wurde langsam Brom zu der Reaktionslösung getropft, hierbei wurde die Temperatur bei $0^{\circ} \mathrm{C}$ konstant gehalten. Nach einer Zutropfzeit von ca. $3 \mathrm{~h}$ wurde die Reaktionslösung über Nacht rühren gelassen. Am darauffolgenden Tag wurde der Rückstand in $300 \mathrm{ml}$ Diethylether gelöst und dann mit $2 \mathrm{~N} \mathrm{NaOH}(2 \times 150 \mathrm{ml})$ sowie $\mathrm{H}_{2} \mathrm{O}$ ( 2 x $100 \mathrm{ml}$ ) extrahiert. Nach Trocknen der organischen Phase über $\mathrm{MgSO}_{4}$ wurde das Lösungsmittel im Vakuum entfernt. Der nun erhaltene Feststoff wurde aus Methanol umkristallisiert und es konnten $98.32 \mathrm{~g}, 0.37 \mathrm{~mol}, 56 \%$ eines weißen kristallinen Feststoffs

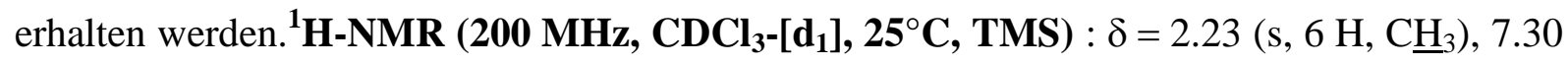
(s, $\left.2 \mathrm{H}, \underline{\mathrm{H}}_{\text {Arom }}\right) \mathrm{ppm}$.

\subsection{1b Reaktion mit CuSBu (L1b)}

Die bromierte $o$-Xylolverbindung $(7.4 \mathrm{~g}, 0.025 \mathrm{~mol})$ wurde in einer Mischung aus Quinolin $(25 \mathrm{ml})$ und Pyridin $(8 \mathrm{ml})$ gelöst. Nun wurde die frisch hergestellt CuSButyl-Verbindung $(8.4 \mathrm{~g}, 0.055 \mathrm{~mol})$ zugegeben und für $3.5 \mathrm{~h}$ unter Rückfluss erhitzt. Dann wurde die Reaktionsmischung auf ca. $100^{\circ} \mathrm{C}$ abgekühlt und in ein Eisbad (150 g) mit $40 \mathrm{ml} \mathrm{HCl}$ (konz.) gegossen. Die nun flüssige braune Phase wurde vom Gummiartigen Rückstand abdekantiert. Der Rückstand wurde mehrmals mit Diethylether versetzt und filtriert. Die zusammengefügten Etherphasen wurden nun 2x mit 10\% $\mathrm{HCl}, 1 \times 10 \mathrm{ml} 10 \%$ ig $\mathrm{HCl}, 1 \times \mathrm{H}_{2} \mathrm{O}$ $(100 \mathrm{ml})$ und 2x mit konz. $\mathrm{NH}_{4} \mathrm{OH}$ gewaschen. Nach erneuter Extraktion mit Wasser wurde über $\mathrm{K}_{2} \mathrm{CO}_{3}$ getrocknet und das Lösungsmittel am Rotationsverdampfer entfernt. Es konnte ein dunkelbraunes Öl erhalten werden, dass Säulenchromatographisch an Kieselgel gereinigt wurde $\left(\mathrm{Hexan}_{\mathrm{Et}} \mathrm{O}\right)(10: 1) \quad \mathrm{R}_{\mathrm{f}}=0.67$ wodurch $4.10 \mathrm{~g}, 0.015 \mathrm{~mol}$, 58\% eines orangen Öls erhalten wurden. ${ }^{1} \mathbf{H}-\mathbf{N M R}\left(\mathbf{2 0 0} \mathbf{M H z}, \mathbf{C D C l}_{3}-\left[\mathbf{d}_{1}\right], \mathbf{2 5}^{\circ} \mathbf{C}, \mathbf{T M S}\right): \delta=0.96\left(\mathrm{t}, 6 \mathrm{H}, \mathrm{CH}_{3^{-}}\right.$ Buthyl), 1.48 (sech, $4 \mathrm{H}, \mathrm{C}_{2}-\mathrm{CH}_{3}$ ), 1.65 (quin, $4 \mathrm{H}, \underline{\mathrm{C}}_{2}-\mathrm{CH}_{2}-\mathrm{CH}_{3}$ ), 2.28 (s, $6 \mathrm{H}, \underline{\mathrm{C}}_{3}$ ), 3.06 (t, $\left.4 \mathrm{H}, \mathrm{S}-\mathrm{C}_{2}{ }^{-}\right), 7.32$ (s, $2 \mathrm{H}, \underline{\mathrm{H}}_{\text {Arom }}$ ) ppm. 


\subsection{1c Birch-Reduktion (L1c)}

L1b $4.10 \mathrm{~g}$, (0.015 mol) wurde gelöst in Petrolether $(10 \mathrm{ml})$ zu verflüssigtem Ammoniak bei $-40^{\circ} \mathrm{C}$ gegeben. Nun wurde Portionsweise Natrium (1.50 g, $\left.0.065 \mathrm{~mol}\right)$ unter Stickstoffstrom zugegeben. Es konnte die charakteristische Blaufärbung der Lösung beobachtet werden. Diese wurde nun für weitere 20-30 Min gerührt. Dann wurde zum Abbrechen der Reaktion 0.22 g (4.11 mmol) $\mathrm{NH}_{4} \mathrm{Cl}$ zugegeben und versucht das $\mathrm{NH}_{3}$ durch langsames Erwärmen auf RT auszutreiben. Es konnte die Bildung eines weißen Feststoffs beobachtet werden, dieser wurde in $\mathrm{MeOH}$ (abs) $1.5 \mathrm{ml}$ gelöst. Gefolgt von der Zugabe von $50 \mathrm{ml}$ entgastem Wasser. Nun wurde die Lösung unter Stickstoff filtriert und das Produkt konnte durch tropfenweise Zugabe von $\mathrm{HCl}$ (konz.) zum Filtrat als weißer Feststoff erhalten werden. Nach Filtration und Trocknen im HV konnten $2.01 \mathrm{~g}, 0.012 \mathrm{~mol}$, 79\% farbloser leicht weißer Nadeln erhalten werden. IR (KBr) [cm ${ }^{-1}$ ]: 438 (s), 491 (w), 584 (w), 675 (w), 867 (s), 940 (s), 997 (m), 1020 (m), 1134 (m), 1164 (m), 1245 (w), 1270 (m), 1348 (m), 1381 (m), 1444 (s), 1474 (s), 1525 (w), 1588 (w), 2343 (w), 2361 (w), 2522 (s), 2609 (w), 2736 (w), 2853 (w), 2912 (w), 2937

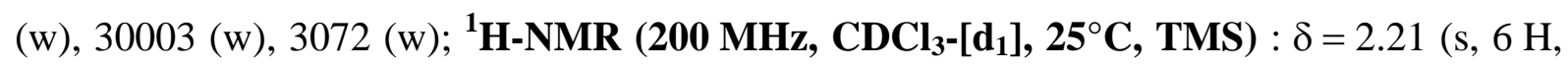
$\left.\mathrm{C}_{3}\right), 3.67$ (s, $\left.2 \mathrm{H}, \mathrm{S} \underline{\mathrm{H}}\right), 7.30$ (s, $\left.2 \mathrm{H}, \underline{\mathrm{H}}_{\text {Arom }}\right) \mathrm{ppm}$.

\subsection{2 vdt-Ligand (L2)[128]}

\subsection{2a Bromierung (L2a)}

Die Bromierung wurde analog zur Reaktionsführung von L1a durchgeführt, dabei wurden $40.0 \mathrm{~g}, 0.29 \mathrm{~mol}$ 1,2-Dimethoxybenzol eingesetzt und es konnten $51.23 \mathrm{~g}$ (0.17 mol, 59\%) farblose Nadeln erhalten werden. ${ }^{\mathbf{1}} \mathbf{H}-\mathbf{N M R}\left(\mathbf{2 0 0} \mathbf{M H z}, \mathbf{C D C l}_{\mathbf{3}}-\left[\mathbf{d}_{\mathbf{1}}\right], \mathbf{2 5}^{\circ} \mathbf{C}, \mathbf{T M S}\right): \delta=3.83$ (s, $\left.6 \mathrm{H}, \mathrm{OC}_{3}\right), 7.03$ (s, $\left.2 \mathrm{H}, \underline{\mathrm{H}}_{\text {Arom }}\right) \mathrm{ppm}$.

\subsection{2 b Reaktion mit CuSBu (L2b)}

Die Reaktionsschritte wurden analog zur Synthese von L1b durchgeführt. Hierbei konnte nach einer säulenchromatographischen Reinigung an Kieselgel mit (Hexan/Et $2 \mathrm{O})(10: 1) \mathrm{R}_{\mathrm{f}}$ $=0.54 ; 4.47 \mathrm{~g}, 0.014 \mathrm{~mol}, 61 \%$ eines orangen Öls erhalten werden. ${ }^{\mathbf{H}} \mathbf{H}-\mathbf{N M R}(\mathbf{2 0 0} \mathbf{~ M H z}$, $\left.\mathbf{C D C l}_{3}-\left[\mathbf{d}_{1}\right], \mathbf{2 5}^{\circ} \mathbf{C}, \mathbf{T M S}\right): \delta=0.98$ (t, $\left.6 \mathrm{H}, \mathrm{CH}_{3}-\mathrm{Buthyl}\right), 1.51$ (sech, $4 \mathrm{H}, \mathrm{CH}_{2}-\mathrm{CH}_{3}$ ), 1.66 (quin, $4 \mathrm{H}, \underline{\mathrm{CH}}_{2}-\mathrm{CH}_{2}-\mathrm{CH}_{3}$ ), 3.06 (t, $\left.4 \mathrm{H}, \mathrm{S}-\underline{\mathrm{CH}}_{2}-\right), 3.92$ (s, $\left.6 \mathrm{H}, \mathrm{OC}_{3}\right), 7.32$ (s, $2 \mathrm{H}, \underline{\mathrm{H}}_{\text {Arom }}$ ) ppm.

\subsection{2c Birch-Reduktion (L2c)}

Die Reduktion wurde analog zur Synthese von L1c durchgeführt, hierbei wurde $9.95 \mathrm{~g}$ (0.032 mol) von L2b für die Reaktion eingesetzt. Einzige Veränderung stellt die Aufarbeitung 
bei dieser Reaktion dar, da sich hier nach Entfernen des flüssigen Ammoniaks und lösen in Wasser ein grünliche Lösung bildete. Diese wurde unter Stickstoffatomosphäre mit Diethylether extahiert. Die Wasserphase wurde filtriert und das Filtrat wurde mit $\mathrm{HCl}$ (konz.) versetzt wodurch der Ausfall eines grünen Öls beobachtet werden konnte. Diese Mischung wurde nun erneut mit Dichlormethan extrahiert und nach Entfernen des Dichlormethans konnte aus dieser Phase $2.13 \mathrm{~g}, 0.011 \mathrm{mmol}, 34 \%$ eines dunkelgrünes Öl erhalten werden. ${ }^{1} \mathbf{H}-\mathbf{N M R}\left(200 \mathrm{MHz}, \mathbf{C D C l}_{3}-\left[\mathbf{d}_{1}\right], \mathbf{2 5}^{\circ} \mathbf{C}, \mathbf{T M S}\right): \delta=2.41(\mathrm{~s}, 2 \mathrm{H}, \mathrm{S} \underline{\mathrm{H}}), 4.08\left(\mathrm{~s}, 6 \mathrm{H}, \mathrm{OC}_{\underline{H}}\right)$, $5.62\left(\mathrm{~s}, 2 \mathrm{H}, \underline{\mathrm{H}}_{\text {Arom }}\right) \mathrm{ppm}$.

\subsection{3 ndt-Ligand (L3)}

Eine Suspension aus 4,5-Bis(benzylthio)phthalonitril ${ }^{[129]}(2.65 \mathrm{~g}, 7.12 \mathrm{mmol})$ in Toluol $200 \mathrm{ml}$ wurde mit $\mathrm{AlCl}_{3}(3.80 \mathrm{~g}, 0.028 \mathrm{~mol})$ versetzt. Diese Suspension wurde für $4 \mathrm{~d}$ bei RT gerührt. Die Lösung wurde unter Stickstoffatmosphäre in eine Eislösung geben. Die erhaltene organische Phase wurde mehrere Mal mit $\mathrm{H}_{2} \mathrm{O}$ extrahiert. Die Wasserphase wurde mit konz. $\mathrm{HCl}$ versetzt, wobei der Ausfall eines weißen Feststoffs beobachtet wurde. Nach Filtration unter Stickstoff und Trocknen im HV konnten $1.24 \mathrm{~g}, 6.45$ mol, 91\% des Liganden erhalten werden. IR (KBr) [ $\mathbf{c m}^{-1}$ ]: 472 (w), 463 (w), 493 (w), 530 (m), 648 (w), 674 (w), 732 (w), 803 (w), 893 (m), 934 (w), 950 (w), 985 (w), 1136 (m), 1221 (m), 1246 (w), 1269 (w), 1346 (m), 1385 (w), 1469 (s), 1526 (w), 1570 (s), 1637 (w), 1718 (w), 1773 (w), 2231 (vs), 2345 (w), 2361 (w), 2536 (s), 2854 (w), 2924 (w), 3008 (w), 3081 (w); ${ }^{1}$ H-NMR (300 MHz, DMSO-

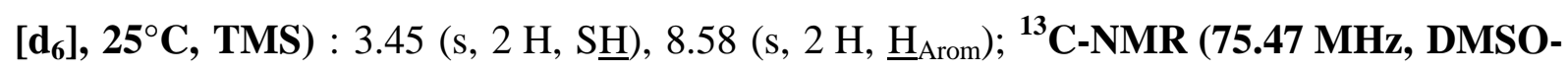

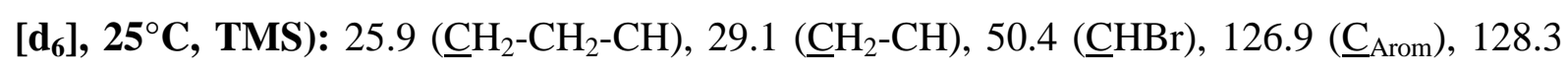
$\left(\underline{\mathrm{C}}_{\text {Arom }}\right), 128.6$ ( $\left.\underline{\mathrm{C}}_{\text {Arom }}\right), 129.7$ ( $\left.\underline{\mathrm{C}}_{\text {Arom }}\right), 129.7\left(\underline{\mathrm{C}}_{\text {quart }} \mathrm{C}=\mathrm{C}\right), 142.8\left(\underline{\mathrm{C}}_{\text {quart }}-\mathrm{CH}_{2}\right), 190.2$ ( $\left.\underline{\mathrm{C}}=\mathrm{O}\right)$ ppm.

\subsection{4 qdt-Ligand (L4) ${ }^{[47]}$}

Eine Lösung aus $3.70 \mathrm{~g}$ (0.023 mol) 2,3-Dihydroxyquinoxalin und $17 \mathrm{~g} \quad(0.076 \mathrm{~mol})$ Phosphorpentasulfid in $80 \mathrm{ml}$ Pyridin (abs) wurde für $2 \mathrm{~h}$ unter Rückfluss erhitzt. Nachdem die Lösung auf RT abgekühlt war, wurde das vorhandene Pyridin im HV entfernt. Der Rückstand wurde vorsichtig unter Kühlung mit 100 ml Wasser versetzt und dann langsam für $1 \mathrm{~h}$ in einem Wasserbad erhitzt. Die Lösung wurde dann durch Zugabe von $100 \mathrm{ml}$ Ammoniak alkalisiert, wodurch sich die Lösung rötlich verfärbte. Die Lösung wurde dann über Nacht gerührt und nachfolgend filtriert. Das Filtrat wurde mit Essigsäure auf $\mathrm{pH}=5$ 
eingestellt. Der dabei ausfallende dunkle Niederschlag wurde mit Wasser gewaschen und erneut in Ammoniaklösung gelöst. Der entstehende gelbliche Niederschlag wurde wieder entfernt und das Filtrat wurde erneut auf einen pH von 5 mit Essigsäure eingestellt. Der dabei entstehende rotbraune Feststoff wurde abfiltriert und im HV getrocknet. Es wurde $2.6 \mathrm{~g}$, $0.013 \mathrm{~mol}, 58 \%$ erhalten. MS (EI, 70 eV) e/z (\%): 194(100) [qdt]; EA: Berechnet für $\mathrm{C}_{8} \mathrm{H}_{6} \mathrm{~N}_{2} \mathrm{~S}_{2}(\%): \mathrm{C}: 49.46, \mathrm{H}: 3.11, \mathrm{~N}: 14.42 ; \mathrm{S}: 33.01$; Gefunden (\%) C: 49.25, H: 2.99; N: 14.88; S: 33.36

\subsection{5 tcdt-Ligand (L5)}

\subsection{5a Bromierung (L5a) Methode A Vorsicht:}

Bei der Darstellung der bromierten Zwischenstufen ist erhöhte Vorsicht geboten, da sowohl die bei der Reaktion erstehenden Dämpfe sowie die Substanzen schwere Irritationen an Schleimhäuten und der Haut hervorrufen.

$4.0 \mathrm{~g}, 24.36 \mathrm{mmol}$ Thiochromanon wurde in $100 \mathrm{ml} \mathrm{CHCl} \mathrm{CH}_{3}$ gelöst. Nun wurde bei RT $\mathrm{Br}_{2}$ $(1.26 \mathrm{ml}, 15.49 \mathrm{mmol})$ tropfenweise zugegeben. Hierbei konnte eine Verfärbung von hellgelb nach orange beobachtet werden. Es wurde nun unter Lichtabschluss für $3 \mathrm{~h}$ gerührt. Nun wurde mit $50 \mathrm{ml} \mathrm{NaHCO}_{3}$-Lsg. (10\%ig) gewaschen. Nach einer weiteren Extrahieren mit Wasser, Trocknen über $\mathrm{MgSO}_{4}$ und entfernen des Lösungsmittels konnte ein oranges Öl erhalten werden. Nun wurde mit Hexan überschichtet und bei $1^{\circ} \mathrm{C}$ über Nacht aufbewahrt. Am nächsten Tag konnte ein Niederschlag von gelben Kristallen beobachtet werden. Nach Filtration und Trocknen im HV konnten $5.25 \mathrm{~g}, 21.59 \mathrm{mmol}$, 89\% der gelben Kristalle erhalten werden. ${ }^{1} \mathbf{H}-\mathbf{N M R}\left(200 \mathbf{~ M H z}, \mathbf{C D C l}_{3}-\left[\mathbf{d}_{1}\right], \mathbf{2 5}^{\circ} \mathbf{C}, \mathbf{T M S}\right): \delta=4.49-4.68(\mathrm{~m}, 2 \mathrm{H}, \mathrm{S}-$ $\left.\mathrm{C}_{2}\right)$, 5.32 (t, $\left.1 \mathrm{H}, \mathrm{C} \underline{\mathrm{HBr}}\right), 6.99-7.08$ (m, $1 \mathrm{H}, \underline{\mathrm{H}}_{\text {Arom }}$ ), 7.12-7.23 (m, $1 \mathrm{H}, \underline{\mathrm{H}}_{\text {Arom }}$ ), 7.46-7.50 (m, $\left.1 \mathrm{H}, \underline{\mathrm{H}}_{\text {Arom }}\right)$, 7.89-7.96 (m, $\left.1 \mathrm{H}, \underline{\mathrm{H}}_{\text {Arom }}\right)$ ppm.

\subsection{5a Bromierung (L5a) Methode B}

$\mathrm{Zu}$ einer Mischung aus $4.0 \mathrm{~g}, 24.36 \mathrm{mmol}$ Thiochromanon, $4.87 \mathrm{~g} \mathrm{HClO}_{4}-\mathrm{SiO}_{2}$ und $31.67 \mathrm{mmol}$ NBS wurden $170 \mathrm{ml}$ Hexan gegeben. Die Mischung wurde nun bei RT für $15 \mathrm{~h}$ rühren gelassen. Nach Filtration konnte nach Entfernen des Lösungsmittels das Produkt als gelber Feststoff erhalten werden. Nach Umkristallisation aus Hexan konnten $5.77 \mathrm{~g}$, $23.73 \mathrm{mmol}, 97 \%$ als gelbe Kristalle erhalten werden. 
${ }^{1} \mathbf{H}-\mathrm{NMR}\left(200 \mathrm{MHz}, \mathbf{C D C l}_{3}-\left[\mathbf{d}_{\mathbf{1}}\right], \mathbf{2 5}^{\circ} \mathbf{C}, \mathbf{T M S}\right): \delta=4.49-4.68\left(\mathrm{~m}, 2 \mathrm{H}, \mathrm{S}-\underline{\mathrm{H}}_{2}\right), 5.32(\mathrm{t}$, C $\underline{H B r}), 6.99-7.08$ (m, $\left.1 \mathrm{H}, \underline{\mathrm{H}}_{\text {Arom }}\right), 7.12-7.23$ (m, 1H, $\left.\underline{\mathrm{H}}_{\text {Arom }}\right), 7.46-7.50$ (m, 1H, $\underline{\mathrm{H}}_{\text {Arom }}$ ), 7.89$7.96\left(\mathrm{~m}, 1 \mathrm{H}, \underline{\mathrm{H}}_{\text {Arom }}\right) \mathrm{ppm}$.

\subsection{5b Xanthogenat-Schritt (L5b)}

Die Bromierte Verbindung $(5.25 \mathrm{~g}, 21.59 \mathrm{mmol})$ aus L5a wurde in einer Mischung aus $\mathrm{MeOH}(40 \mathrm{ml})$ und Aceton $(40 \mathrm{ml})$ gelöst. Nun wurde das Iso-propylxanthogenatsalz gelöst in Aceton zu der Lösung gegeben. Es konnte eine Verfärbung ins orange beobachtet werden. Die Reaktionslösung wurde für $12 \mathrm{~h}$ bei RT gerührt. Nach einer Stunde rühren konnte eine Trübung der Lösung beobachtet werden. Darauf wurde für $30 \mathrm{Min}$ auf $50^{\circ} \mathrm{C}$ erhitzt, wodurch nach dem Abkühlen der Lösung auf RT eine große Menge an weißem Feststoff ausfiel. Das komplette Lösungsmittel wurde von der Reaktionslösung entfernt und der Rückstand wurde dann mit $10 \% \mathrm{HCl}$ versetzt. Die Lösung wurde nun für $15 \mathrm{Min}$ bei RT gerührt, gefolgt von der Zugabe an Diethylether $100 \mathrm{ml}$. Nach weiteren $15 \mathrm{Min}$ rühren bei RT wurde die Etherphase 3x mit $100 \mathrm{ml}$ Wasser extrahiert. Nach Trocknen über $\mathrm{MgSO}_{4}$ und entfernen des kompletten Lösungsmittels konnte ein orangenes Öl erhalten werden. Nach Umkristallisation aus Ethanol konnten $5.83 \mathrm{~g}, 19.53 \mathrm{mmol}$, 90\% eines gelben Feststoffs isoliert werden. ${ }^{1} \mathbf{H}$ -

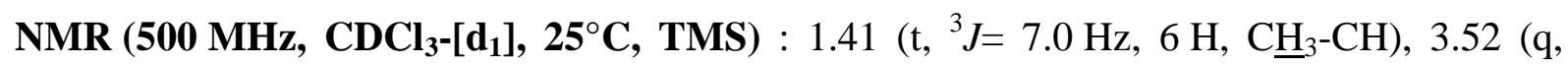
$\left.{ }^{3} J=7.0 \mathrm{~Hz}, \mathrm{CH}-\underline{\mathrm{H}}_{3}\right), 3.58\left(\mathrm{t},{ }^{3} \mathrm{~J}=11 \mathrm{~Hz}, 1 \mathrm{H}, \mathrm{C} \underline{\mathrm{H}}-\mathrm{S}\right), 5.10\left(\mathrm{~m}, 1 \mathrm{H}, \mathrm{CH}_{2}-\mathrm{CH}\right), 5.73(\mathrm{~m}, 1 \mathrm{H}$, $\underline{\mathrm{C}}_{2}-\mathrm{CH}$ ), 7.02 (m, $\left.1 \mathrm{H}, \underline{\mathrm{H}}_{\text {Arom }}\right), 7.17$ (m, $\left.1 \mathrm{H}, \underline{\mathrm{H}}_{\text {Arom }}\right), 7.41$ (m, $\left.1 \mathrm{H}, \underline{\mathrm{H}}_{\text {Arom }}\right), 8.07$ (m, $1 \mathrm{H}$, $\left.\underline{\mathrm{H}}_{\text {Arom }}\right)$ ppm; ${ }^{13} \mathbf{C}-\mathbf{N M R}\left(\mathbf{1 2 5 . 7} \mathbf{~ M H z}, \mathbf{C D C l}_{3}-\left[\mathbf{d}_{1}\right], \mathbf{2 5}^{\circ} \mathbf{C}, \mathbf{T M S}\right): 21.2\left(\underline{\mathrm{CH}}_{3}\right), 32.1\left(\underline{\mathrm{CH}}_{2}-\mathrm{CH}\right)$,

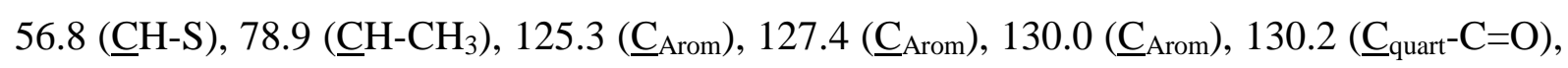
133.7 ( $\left.\underline{\mathrm{C}}_{\text {Arom }}\right), 141.4$ ( $\left.\underline{\mathrm{C}}_{\text {quart }} \mathrm{S}\right), 189.4(\mathrm{C}=\mathrm{O}), 210.9(\mathrm{C}=\mathrm{S}) \mathrm{ppm}$.

\subsection{5c Ringschluss (L5c)}

$5.50 \mathrm{~g}, 18.43 \mathrm{mmol} \mathbf{L 5 b}$ wurde mit $101 \mathrm{ml} \mathrm{HBr} / \mathrm{CH}_{3} \mathrm{COOH}$ (33\%) versetzt. Es konnte eine sofortige Verfärbung nach orange beobachtet werden. Die Reaktionslösung wurde über Nacht gerührt. Am nächsten Tag wurde die Reaktionslösung auf Eis gegossen und es fiel ein brauner Feststoff aus. Diese Eislösung wurde nun $12 \mathrm{~h}$ bei $1^{\circ} \mathrm{C}$ aufbewahrt. Nach Filtration und waschen mit $600 \mathrm{ml}$ Wasser und Trocknen im HV konnten $3.60 \mathrm{~g}, 15.10 \mathrm{~mol}$, 82\% eines

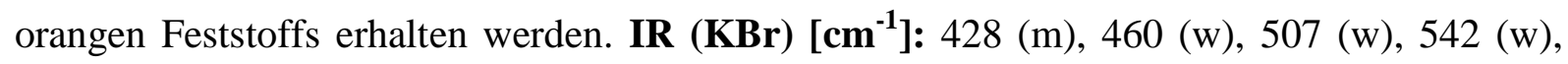
$564(\mathrm{w}), 590(\mathrm{w}), 636(\mathrm{w}), 669$ (w), 679 (m), $701(\mathrm{w}), 720$ (m), 744 (m), 799 (m), 873 (w), $905(\mathrm{w}), 935$ (w), $981(\mathrm{w}), 1019$ (w), 1062 (w), 1098 (w), $1151(\mathrm{w}), 1124(\mathrm{w}), 1155(\mathrm{w}), 1250$ (s), 1311 (w), 1412 (w), 1431 (w), 1522 (w), 1607 (w), 1541 (w), 1559 (w), 1576 (w), 1636 (s), 1683 (m), 1701 (w), 1742 (w), 1773 (w), 1943 (w), 2340 (m), 2343 (m), 2361 (m), 2517 (w), 2852 (w)m 2924 (w), 2964 (w), 3055 (w), 3115 (w), 3150 (w); ${ }^{1}$ H-NMR (200 MHz, 
$\left.\mathbf{C D C l}_{3}-\left[\mathbf{d}_{\mathbf{1}}\right], \mathbf{2 5}^{\circ} \mathbf{C}, \mathbf{T M S}\right): 3.78$ (s, 2 H, C $\left.\underline{\mathrm{H}}-\mathrm{S}-\right), 7.10$ (m, $\left.2 \mathrm{H}, \underline{\mathrm{H}}_{\text {Arom }}\right), 7.20$ (m, $\left.2 \mathrm{H}, \underline{\mathrm{H}}_{\text {Arom }}\right)$, 7.33 (m, $\left.2 \mathrm{H}, \underline{\mathrm{H}}_{\text {Arom }}\right), 7.39$ (m, $\left.2 \mathrm{H}, \underline{\mathrm{H}}_{\text {Arom }}\right)$ ppm; ${ }^{13} \mathbf{C}$-NMR (75.47 $\mathbf{M H z}, \mathbf{C D C l}_{\mathbf{3}}-\left[\mathbf{d}_{\mathbf{1}}\right], \mathbf{2 5}^{\circ} \mathbf{C}$, TMS): 25.3 ( $\left.\underline{\mathrm{CH}}_{2}-\mathrm{S}-\right), 120.6$ ( $\underline{\mathrm{C}}^{2}-$ Thiochromanon), 126.7 ( $\underline{\mathrm{C}}^{6}$-Thiochromanon), 126.7 ( $\underline{\mathrm{C}}^{9}-$ Thiochromanon), 127.2 ( $\underline{\mathrm{C}}^{8}$-Thiochromanon), 127.9 ( $\underline{\mathrm{C}}^{7}$-Thiochromanon), 128.1 ( $\underline{\mathrm{C}}_{\text {quart }}=\underline{\mathrm{C}}_{\text {quart }}{ }^{-}$ $\mathrm{S}), 128.5\left(\mathrm{C}_{\text {quart }}=\underline{\mathrm{C}}_{\text {quart }} \mathrm{S}\right), 130.5\left(\mathrm{C}_{\text {quart }}-\underline{\mathrm{C}}-\mathrm{S}\right), 189.2(\underline{\mathrm{C}}=\mathrm{O}) \mathrm{ppm}$.

\subsection{6 tldt-Ligand (L6)}

Vorsicht:

Bei der Darstellung der bromierten Zwischenstufen ist erhöhte Vorsicht geboten, da sowohl die bei der Reaktion erstehenden Dämpfe sowie die Substanzen schwere Irritationen an Schleimhäuten und der Haut hervorrufen.

\subsection{6a Bromierung (L6a)}

Eine Suspension aus $\mathrm{K}_{2} \mathrm{CO}_{3}(10.40 \mathrm{~g}, 75.25 \mathrm{mmol})$ in $50 \mathrm{ml} \mathrm{CCl} 4$ wurde mit $\alpha$-Tetralon $(10 \mathrm{~g}$, $0.068 \mathrm{~mol}$ ) bei $-20^{\circ} \mathrm{C}$ versetzt. Die Reaktionslösung wurde für $5 \mathrm{~h}$ bei $-20^{\circ} \mathrm{C}$ sowie $5 \mathrm{~h}$ bei RT gerührt. Die erhaltene Lösung wurde filtriert und das Filtrat wurde vom kompletten Lösungsmittel im Vakuum entfernt. Der braune Feststoff wurde in $\mathrm{CH}_{2} \mathrm{Cl}_{2}$ gelöst und wurde 3x mit $100 \mathrm{ml}$ Wasser extrahiert. Anschließend wurde mit gesättigter $\mathrm{NaHCO}_{3}$-Lsg., $\mathrm{NaCl}$ sowie erneut mit Wasser gewaschen. Trocknen über $\mathrm{MgSO}_{4}$ und Entfernung des Lösungsmittels lieferte ein dunkelbraunes Öl. Nach Umkristallisation aus Ethanol konnten $11.67 \mathrm{~g}, 51.84 \mathrm{mmol}, 69 \%$ eines hellbraunen Öls isoliert werden. ${ }^{\mathbf{1}} \mathbf{H}-\mathbf{N M R}$ (300 MHz, $\left.\mathbf{C D C l}_{3}-\left[\mathbf{d}_{1}\right], \mathbf{2 5}^{\circ} \mathbf{C}, \mathbf{T M S}\right): 2.35-2.52\left(\mathrm{~m}, 2 \mathrm{H}, \mathrm{CH}-\underline{\mathrm{H}}_{2}\right), 2.82-3.05\left(\mathrm{~m}, 1 \mathrm{H}, \mathrm{CH}_{2}-\mathrm{CH}_{2}-\mathrm{CH}\right)$, 3.19-3.29 (m, $\left.1 \mathrm{H}, \underline{\mathrm{C}}_{2}-\mathrm{CH}_{2}-\mathrm{CH}\right), 4.68$ (t, $\left.1 \mathrm{H}, \mathrm{C} \underline{\mathrm{H}}-\mathrm{Br}\right), 7.24-7.35$ (m, $1 \mathrm{H}, \underline{\mathrm{H}}_{\text {Arom }}$ ), 7.44-7.52 (m, $1 \mathrm{H}, \mathrm{H}_{\text {Arom }}$ ), 8.02 (m, $1 \mathrm{H}, \mathrm{H}_{\text {Arom }}$ ), 8.10 (m, $\left.1 \mathrm{H}, \mathrm{H}_{\text {Arom }}\right)$ ppm; ${ }^{13} \mathbf{C}-\mathbf{N M R}(\mathbf{7 5 . 4 7} \mathbf{~ M H z}$,

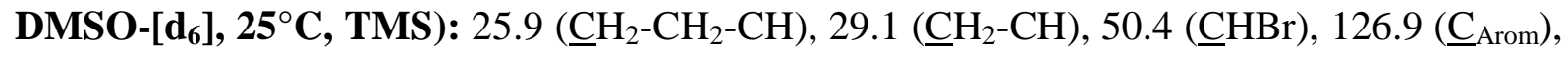
128.3 ( $\left.\underline{\mathrm{C}}_{\text {Arom }}\right), 128.6$ ( $\left.\underline{\mathrm{C}}_{\text {Arom }}\right), 129.7$ ( $\left.\underline{\mathrm{C}}_{\text {Arom }}\right), 129.7\left(\underline{\mathrm{C}}_{\text {quart }}-\mathrm{C}=\mathrm{O}\right), 142.8\left(\underline{\mathrm{C}}_{\text {quart }}-\mathrm{CH}_{2}\right), 190.2$ $(\underline{\mathrm{C}}=\mathrm{O}) \mathrm{ppm}$.

\subsection{6b Xanthogenat-Schritt (L6b)}

Dieser Reaktionsschritt wurde analog zu L5b durchgeführt, hierbei konnte aber nur ein braunes Öl nach der Aufarbeitung erhalten werden. Deshalb wurde eine säulenchromatographische Reinigung an Kieselgel (Hexan/Et $\left.{ }_{2} \mathrm{O}\right)(8: 1) \mathrm{R}_{\mathrm{f}}=0.41$ durchgeführt, wodurch ein hellbraunes Öl erhalten wurde, das dann erneut aus $\mathrm{Et}_{2} \mathrm{O}$ umkristallisiert wurde, wodurch $4.21 \mathrm{~g}, \quad 15.01 \mathrm{mmol}, 39 \%$ eines weißen Feststoffs erhalten wurden. ${ }^{\mathbf{1}} \mathbf{H}$ NMR (200 MHz, $\left.\mathbf{C D C l}_{3}-\left[\mathbf{d}_{1}\right], \mathbf{2 5}^{\circ} \mathbf{C}, \mathbf{T M S}\right): 0.89$ (d, $\left.{ }^{3} J=1.5 \mathrm{~Hz}, 6 \mathrm{H}, \mathrm{C}_{3}-\mathrm{CH}\right), 2.51-2.78$ (t, 
$2 \mathrm{H}, \underline{\mathrm{CH}}_{2}-\mathrm{CH}-\mathrm{S}$ ), 3.15-3.25 (t, $2 \mathrm{H}, \mathrm{CH}_{2}-\mathrm{CH}_{2}-\mathrm{CH}$ ), 4.73 (t, ${ }^{3} \mathrm{~J}=1.5 \mathrm{~Hz}, 1 \mathrm{H}, \mathrm{C} \underline{H}-\mathrm{S}$ ), 4.88 (sep, $\left.1 \mathrm{H}, \mathrm{C} \underline{\mathrm{H}}-\mathrm{CH}_{3}\right), 7.30-7.42$ (m, $\left.1 \mathrm{H}, \underline{\mathrm{H}}_{\text {Arom }}\right), 7.52-7.60$ (m, $\left.1 \mathrm{H}, \underline{\mathrm{H}}_{\text {Arom }}\right), 8.08$ (d, ${ }^{3} J=1.4 \mathrm{~Hz}, 1 \mathrm{H}$, $\left.\underline{\mathrm{H}}_{\text {Arom }}\right), \quad 8.12 \quad\left(\mathrm{~d}, \quad{ }^{3} J=1.5 \mathrm{~Hz}, \quad 1 \mathrm{H}, \quad \underline{\mathrm{H}}_{\text {Arom }}\right) \quad$ ppm; $\quad$ MS (EI, $\left.70 \mathrm{eV}\right) \quad$ e/z (\%): 266.0 (100) $\left[\mathrm{C}_{13} \mathrm{H}_{14} \mathrm{O}_{2} \mathrm{~S}_{2}\right]$

\subsection{6c Ringschluss (L6c)}

Die Ringschlussreaktion wurde analog zu der Reaktion L5c durchgeführt, hierbei wurden $1.51 \mathrm{~g}, 6.85 \mathrm{mmol}, 84 \%$ eines rosa/gelblichen Feststoffs erhalten. IR (KBr) [ $\left.\mathbf{c m}^{-1}\right]$ : 430 (m), $471(\mathrm{w}), 507(\mathrm{w}), 517(\mathrm{w}), 560(\mathrm{w}), 604(\mathrm{w}), 640(\mathrm{w}), 669(\mathrm{w}), 679(\mathrm{w}), 713(\mathrm{w}), 747(\mathrm{~s}), 798$ (m), 861 (w), 900 (w), 931 (w), 989 (m), 1021 (w), 1090 (s), 1154 (m), 1246 (s), 1310 (w), 1385 (s), 1435 (w), 1484 (m), 1507 (w), 1636 (vs), 1669 (s), 1699 (w), 1717 (w), 1752 (w), 1792 (w), 1830 (w), 1900 (w), 2340 (m), 2342 (m), 2361 (m), 2852 (w), 2905 (w), 2942 (w), 3032 (w), 3286 (w), 3416 (m), 3428 (m); MS (EI,70 eV) e/z (\%): 220 (100) [tldt]; ${ }^{1} \mathbf{H}-\mathbf{N M R}\left(300 \mathrm{MHz}, \mathbf{C D C l}_{3}-\left[\mathbf{d}_{1}\right], 2^{\circ} \mathbf{C}\right.$, TMS): 2.64 (t, $\left.2 \mathrm{H}, \mathrm{CH}_{2}-\mathrm{CH}-\mathrm{S}\right), 3.11$ (t, $2 \mathrm{H}, \mathrm{CH}_{2}-$ S), 7.35-7.46 (m, $\left.1 \mathrm{H}, \underline{\mathrm{H}}_{\text {Arom }}\right), 7.58-7.62\left(\mathrm{~m}, 1 \mathrm{H}, \underline{\mathrm{H}}_{\text {Arom }}\right), 8.12\left(\mathrm{~d},{ }^{3} \mathrm{~J}=1.8 \mathrm{~Hz}, 1 \mathrm{H}, \underline{\mathrm{H}}_{\text {Arom }}\right), 8.22$ (d, ${ }^{3} J=1.8 \mathrm{~Hz}, 1 \mathrm{H}, \underline{\mathrm{H}}_{\text {Arom }}$ ) ppm; ${ }^{13} \mathbf{C}-\mathbf{N M R}\left(\mathbf{7 5 . 4 7} \mathbf{M H z}, \mathbf{D M S O}-\left[\mathbf{d}_{6}\right], \mathbf{2 5}^{\circ} \mathbf{C}\right.$, TMS): 32.7 (SC- $\left.\mathrm{CH}_{2}-\underline{\mathrm{CH}}_{2}\right), 34.8\left(\mathrm{~S}-\mathrm{C}-\underline{\mathrm{CH}}_{2}\right), 119.2\left(\mathrm{~S}-\underline{\mathrm{C}}-\mathrm{CH}_{2}\right), 122.1$ ( $\left.\underline{\mathrm{C}}_{\text {Arom }}\right), 122.7$ ( $\left.\underline{\mathrm{C}}_{\text {Arom }}\right), 126.8$ ( $\underline{\mathrm{C}}_{\text {quart- }}$ Arom), 134.2 ( $\left.\underline{\mathrm{C}}_{\text {Arom }}\right), 134.8$ ( $\left.\underline{\mathrm{C}}_{\text {Arom }}\right), 140.4$ ( $\left.\underline{\mathrm{C}}_{\text {quart-Arom }}\right), 142.8\left(\underline{\mathrm{C}}_{\text {quart }} \mathrm{S}\right), 189.1(\underline{\mathrm{C}}=\mathrm{O}) \mathrm{ppm}$.

\subsection{7 ntdt-Ligand (L7)}

\subsection{7a Xanthogenat-Schritt (L7a)}

$\mathrm{Zu}$ einer Lösung aus 2-Brom-1(naphtylen-2-yl)ethanon (3.00 g $12.0 \mathrm{mmol})$ in $30 \mathrm{ml} \mathrm{MeOH}$ wurde das Kaliumisopropylxanthogenat $(2.10 \mathrm{~g}, 12.0 \mathrm{mmol})$ gelöst in $20 \mathrm{ml} \mathrm{MeOH}$ langsam bei RT zugegeben. Es konnte eine schlagartige Aufhellung der Lösung beobachtet werden. Nun wurde über Nacht bei RT gerührt. Anschließend wurde für $1 \mathrm{~h}$ auf $80^{\circ} \mathrm{C}$ erhitzt. Es konnte der Ausfall von $\mathrm{KBr}$ beobachtet werden. Beim Abkühlen der Lösung konnte der Ausfall von rötlichen Kristallen beobachtet werden. Die Kristalle wurden zusammen mit dem vorhandenen Feststoff durch Filtration entfernt. Dieser wurde in $\mathrm{Et}_{2} \mathrm{O}(40 \mathrm{ml})$ gelöst und erneut mit 3 x $(60 \mathrm{ml})$ Wasser extrahiert. Die Etherphasen wurden über $\mathrm{MgSO}_{4}$ getrocknet und das Lösungsmittel wurde im HV entfernt. Es konnten $3.34 \mathrm{~g}, 11.0 \mathrm{mmol}, 91 \%$ eines hellroten kristallinen Feststoffs erhalten werden. ${ }^{\mathbf{1}} \mathbf{H}$-NMR $\left(\mathbf{2 0 0} \mathbf{M H z}, \mathbf{C D C l}_{\mathbf{3}}\right.$-[ $\left.\mathbf{d}_{\mathbf{1}}\right], \mathbf{2 5}^{\circ} \mathbf{C}$, TMS) : 1.13 (d, ${ }^{3} J=6.2 \mathrm{~Hz}, 6 \mathrm{H}, \mathrm{CH}_{3}-\mathrm{CH}$ ), 4.55 (s, $2 \mathrm{H}, \mathrm{S}-\mathrm{C}_{2}$ ), 5.51 (quin, ${ }^{3} J=6.2 \mathrm{~Hz}, \mathrm{C} \underline{\mathrm{H}}-$ $\mathrm{CH}_{3}$ ), 7.35 (m, $2 \mathrm{H}, 6^{\prime}, 7^{\prime}$-Naphtalin), 7.38-7.42 (m, $3 \mathrm{H}, 4^{\prime}, 5^{\prime}, 8^{\prime}$-Naphtalin), 7.68 (m, $1 \mathrm{H}$, 3'-Naphtalin), 8.33 (m, 1 H, 1'-Naphtalin) ppm. 


\subsection{7c Ringschluss (L7b)}

Die Ringschlussreaktion wurde analog zu der Methode L5c durchgeführt, hierbei konnten 2.54 g, 10.40 mmol, $94 \%$ eines hellbraunen Feststoffs erhalten werden. IR (KBr) $\left[\mathbf{c m}^{-1}\right]$ : 435 (m), $482(\mathrm{~m}), 541(\mathrm{w}), 565(\mathrm{w}), 625(\mathrm{w}), 662(\mathrm{w}), 679(\mathrm{w}), 755(\mathrm{~m}), 777(\mathrm{~m}), 794(\mathrm{~m}), 818$ (m), 861 (m), 889 (m), 926 (w), 966 (w), 1096 (w), 1128 (w), 1150 (w), 1206 (m), 1243 (w), 1275 (w), 1362 (w), 1385 (w), 1435 (w), 1504 (w), 1534 (w), 1633 (s), 1708 (w), 1742 (w), 1773 (w), 2340 (w), 2343 (w), 2362 (w), 2929 (w), 3052 (w), 3090 (w); ${ }^{\mathbf{1} H-N M R}$ (300 MHz,

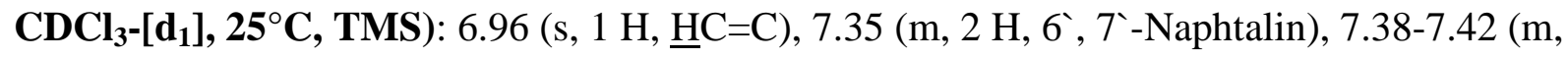
$3 \mathrm{H}, \quad 4^{\prime}, 5^{\prime}, 8^{\prime}$-Naphtalin), 7.68 (m, 1 H, 3'-Naphtalin), 8.33 (m, $1 \mathrm{H}, \quad 1^{\prime}$-Naphtalin);

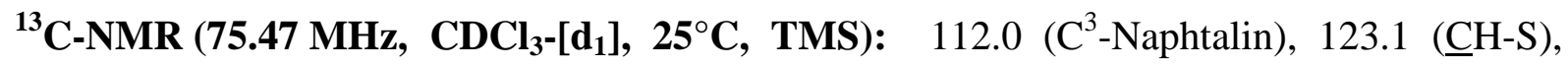
126.2 ( $\mathrm{C}^{1}$-Naphtalin), 127.1 ( $\mathrm{C}^{6}$-Naphtalin), 127.1 ( $\mathrm{C}^{7}$-Naphtalin), 127.8 ( $\mathrm{C}^{5}$-Naphtalin), 128.3 ( $\mathrm{C}^{8}$-Naphtalin), 129.1 ( $\mathrm{C}^{4}$-Naphtalin), 129.9 ( $\mathrm{C}^{10}$-Naphtalin), 133.2 ( $\mathrm{C}^{9}$-Naphtalin), 133.3 (C Naphtalin), $135.0(\mathrm{C}=\underline{\mathrm{C}}-(\mathrm{C})-\mathrm{S}), 192.3(\underline{\mathrm{C}}=\mathrm{O}) \mathrm{ppm}$.

\subsection{8 cydt-Ligand (L8)}

\subsection{8a Bromierung (L8a) ${ }^{[43]}$}

$30 \mathrm{~g}, 0.31 \mathrm{~mol}$ Cyclohexanon und $90 \mathrm{ml}$ Wasser wurden unter Eiskühlung tropfenweise mit Brom über einen Zeitraum von 2 h versetzt, gefolgt von einer weiteren Stunde rühren bei RT. Dann wurde die untere leicht gelbliche Phase mit einem Scheidetrichter abgetrennt. Die Wasserphase wurde mit $\mathrm{Et}_{2} \mathrm{O}(100 \mathrm{ml})$ extrahiert. Die vereinigten org. Phasen wurde mit Wasser und $\mathrm{NaCl}$ extrahiert. Abschließend wurde die Etherphase über $\mathrm{Na}_{2} \mathrm{SO}_{4}$ getrocknet und eine Destillation unter reduziertem Druck durchgeführt. Die Produktfraktion $24.65 \mathrm{~g}$, $0.14 \mathrm{~mol} 45 \%$ konnte bei 13 mbar und bei $90^{\circ} \mathrm{C}$ als farblose Flüssigkeit erhalten werden. ${ }^{1} \mathbf{H}-\mathrm{NMR}\left(200 \mathbf{M H z} \mathbf{C D C l}_{3}-\left[\mathbf{d}_{1}\right], \mathbf{2 5}^{\circ} \mathbf{C}\right.$, TMS): 1.72-1.78(m, $2 \mathrm{H}, \mathrm{C}_{2 \mathrm{Ring}}$ ), 1.81-1.94 (m, $4 \mathrm{H}, \underline{\mathrm{C}}_{2 \mathrm{Ring}}$ ), 2.20-2.31 (m, $2 \mathrm{H}, \underline{\mathrm{C}}_{2 \mathrm{Ring}}$ ), 4.43 (t, $1 \mathrm{H}, \mathrm{C} \underline{\mathrm{HBr}}$ ) ppm.

\subsection{8b Xanthogenat-Schritt (L8b)}

Dieser Reaktionsschritt wurde analog nach der Methode L5b durchgeführt. Die Reaktionslösung wurde $2 \mathrm{~d}$ bei RT rühren gelassen. Nach der üblichen Aufarbeitungen konnten 12 g, $55.0 \mathrm{mmol}, 92 \%$ eines hellgelben Öls erhalten werden. ${ }^{\mathbf{1}} \mathbf{H}-\mathbf{N M R}$ (200 MHz,

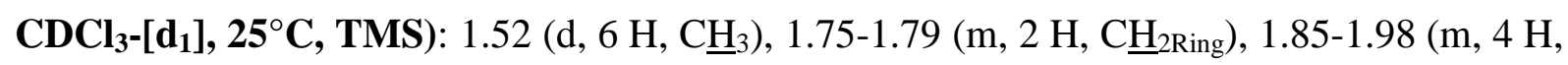
$\mathrm{C}_{2} \underline{\mathrm{Ring}}_{\text {) }}$, 2.21-2.28 (m, $2 \mathrm{H}, \underline{\mathrm{CH}}_{2 \mathrm{Ring}}$ ), 3.21 (t, $1 \mathrm{H}, \underline{\mathrm{C}} \underline{\mathrm{H}} \mathrm{S}$ ), 4.41 (quin, $1 \mathrm{H}, \mathrm{C} \underline{\mathrm{H}}-\mathrm{CH}_{3}$ ) ppm. 


\subsection{8c Ringschluss (L8c) [130]}

$\mathrm{Zu}$ einer Lösung aus (L8b) (12.0 g, $55.0 \mathrm{mmol}, 1.0$ eq.) in $\mathrm{Et}_{2} \mathrm{O}(60 \mathrm{ml})$ und DCM $(60 \mathrm{ml})$ wurde langsam Perchlorsäure $(16 \mathrm{ml})$ getropft. Nach $18 \mathrm{~h}$ Rühren bei RT wurde das Reaktionsgemisch auf Eis $(100 \mathrm{ml})$ gegeben. Das Gemisch wurde mit $\mathrm{Et}_{2} \mathrm{O}(2 \times 70 \mathrm{ml})$ extrahiert. Die vereinigten organischen Phasen wurden mit Wasser $(2 \times 50 \mathrm{ml})$ sowie gesättigter NaCl-Lsg. (50 ml) gewaschen, über MgSO4 getrocknet und im Vakuum. eingeengt. Das Rohprodukt wurde einer säulenchromatographisch Reinigung an Kieselgel (Hexan/Et $\left.{ }_{2} \mathrm{O}, 7: 1, \mathrm{R}_{\mathrm{f}}=0.3\right)$. unterzogen. Es konnten (2.99 g, $\left.17.4 \mathrm{mmol}, 32 \%\right)$ eines farblosen Öls erhalten werden, dass bei Temperaturen um die $4^{\circ} \mathrm{C}$ als farblose Kristalle ausfiel. ${ }^{1}$ H-NMR (200 MHz, $\left.\mathbf{C D C l}_{3}-\left[\mathbf{d}_{1}\right], \mathbf{2 5}^{\circ} \mathbf{C}, \mathbf{T M S}\right)$ : 1.85 (m, $\left.4 \mathrm{H}, \underline{\mathrm{C}}_{2 \mathrm{Ring}}\right), 2.41$ (m, $4 \mathrm{H}$, $\mathrm{C}_{2} \underline{H}_{2 i n g}$ ), $1.85-1.98$ (m, $4 \mathrm{H}, \underline{\mathrm{CH}}_{2 \text { Ring }}$ ), 2.21-2.28 (m, $2 \mathrm{H}, \underline{\mathrm{CH}}_{2 \text { Ring }}$ ), 3.21 (t, $1 \mathrm{H}, \mathrm{CH} S$ ), 4.41 (quin, $\left.1 \mathrm{H}, \mathrm{CH}-\mathrm{CH}_{3}\right)$ ppm; ${ }^{13} \mathbf{C}-\mathrm{NMR}\left(\mathbf{7 5 . 4 7} \mathbf{M H z}, \mathbf{C D C l}_{3}-\left[\mathbf{d}_{\mathbf{1}}\right], \mathbf{2 5}^{\circ} \mathbf{C}, \mathbf{T M S}\right): 22.7\left(\underline{\mathrm{CH}}_{2 \mathrm{Ring}}\right)$, $25.6\left(\underline{\mathrm{CH}}_{2 \text { Ring }}\right), 125.6(\underline{\mathrm{C}}=\mathrm{C}), 192.6(\underline{\mathrm{C}}=\mathrm{O}) \mathrm{ppm}$.

\subsection{9 tpdt-Ligand (L9)}

\subsection{9a Bromierung (L9a)[131]}

Dieser Reaktionsschritt wurde analog zur Reaktion L6a durchgeführt. Es konnten $3.03 \mathrm{~g}$, $15.53 \mathrm{mmol}, 82 \%$ eines gelben Öls erhalten werden. Aufgrund der Instabilität wurde die Verbindung ohne weitere Analysen direkt zur nächsten Stufe umgesetzt.

\subsection{9b Xanthogenat-Schritt (L9b)}

Dieser Reaktionsschritt wurde analog zu L5b durchgeführt, doch hierbei wurde das Ethanxanthogenatsalz verwendet und es konnten $4.28 \mathrm{~g}, 18.11 \mathrm{mmol}, 76 \%$ eines orangen Feststoffs erhalten werden. ${ }^{1} \mathbf{H}-\mathbf{N M R}\left(\mathbf{2 0 0} \mathbf{M H z}, \mathbf{C D C l}_{3}-\left[\mathbf{d}_{1}\right], \mathbf{2 5}^{\circ} \mathbf{C}, \mathbf{T M S}\right): 1.11$ (t, $3 \mathrm{H}$, $\mathrm{C}_{3}$ ), 2.71-2.78 (m, $4 \mathrm{H}, \underline{\mathrm{C}}_{2 \text { Ring }}$ ), 3.21-3.49 (m, $4 \mathrm{H}, \underline{\mathrm{C}}_{2 \text { Ring }}$ ), 3.48 (quart, $2 \mathrm{H}, \mathrm{C}_{2}-\mathrm{CH}_{3}$ ), 3.69 (t, $1 \mathrm{H}, \mathrm{C} \underline{\mathrm{H} S}$ ), 4.41 (quin, $1 \mathrm{H}, \mathrm{C} \underline{\mathrm{H}}-\mathrm{CH}_{3}$ ) ppm.

\subsection{9c Ringschluss (L9c)}

Die Ringschlussreaktion wurde analog zu L5c durchgeführt und es konnten $2.06 \mathrm{~g}$,

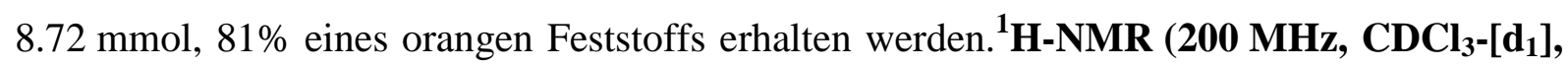
25 $^{\circ} \mathbf{C}$, TMS): 2.38 (m, $2 \mathrm{H}, \underline{\mathrm{C}}_{2 \mathrm{Ring}}$ ), 2.54 (m, $2 \mathrm{H}, \mathrm{C}_{2}$ Ring), 3.26 (s, $2 \mathrm{H}, \underline{\mathrm{C}}_{2}-\mathrm{S}$ ) ppm; MS (EI, 70 eV) e/z (\%): 190(100) [tpdt] 


\subsection{Komplexsynthese}

\subsubsection{Synthese von $\left[\mathrm{MoO}(\mathrm{xdt})_{2}\right]^{2-}$ Methode A (1a)}

Der xdt-Ligand (0.50 g, $2.94 \mathrm{mmol})$ wurde in $20 \mathrm{ml} \mathrm{MeOH}$ (entg.) gelöst und langsam bei RT $\mathrm{zu}$ einer Lösung aus $\mathrm{K}_{3} \mathrm{Na}\left[\mathrm{MoO}_{2}(\mathrm{CN})_{4}\right] \cdot 6 \mathrm{H}_{2} \mathrm{O}(0.86 \mathrm{~g}, 1.62 \mathrm{~mol})$ und $\mathrm{NaOH}(0.23 \mathrm{~g}$, $5.87 \mathrm{mmol}$ ) in $20 \mathrm{ml}$ Wasser (entg.) gegeben. Hierbei konnte eine Verfärbung von violett nach rot festgestellt werden. Die Reaktionsmischung wurde nun für $1 \mathrm{~h}$ für 20 Min refluxiert und es konnte die Bildung einer dunkelroten Lösung beobachtet werden. Nach Abkühlen der Lösung und Filtern wurde eine Lösung aus $\mathrm{PPh}_{4} \mathrm{Cl}(1.10 \mathrm{~g}, 2.94 \mathrm{mmol})$ in $5 \mathrm{ml} \mathrm{MeOH}$ zugegeben. Es wurde $1 \mathrm{~h}$ bei RT weitergerührt. Gefolgt von der Zugabe von $15 \mathrm{ml}$ Wasser (entg.) und es konnte der Ausfall eines hellbraunen Feststoffs beobachtet werden. Es wurde mit kaltem Ethanol gewaschen. Der erhaltene Feststoff wurde in $\mathrm{CH}_{3} \mathrm{CN}$ (abs.) gelöst und mit $\mathrm{Et}_{2} \mathrm{O}$ (abs.) überschichtet und es nach $3 \mathrm{~d}$ konnten orange/braune Kristalle aus der Lösung erhalten werden. Nach Filtration und Trocknung im HV konnten $0.63 \mathrm{~g}$ (0.56 mmol, 34\%) eines orange/braunen Feststoffs erhalten werden. IR (KBr) $\left[\mathbf{c m}^{-1}\right]$ : 421 (w), 447 (w), 526 (vs), 691 (s), 724 (vs), 761 (m), 802 (w), 874 (w), 913 (s), 995 (m), 1027 (w), 1108 (vs), 1166 (w), 1187 (w), 1247 (w), 1276 (w), 1315 (w), 1338 (w), 1385 (w), 1436 (s), 1481 (m), 1523 (w), 1584 (w), 1631 (w), 1776 (w), 1822 (w), 1913 (w), 2208 (s), 2852 (w), 2909 (w), 2978 (w), 3051 (w); MS (FAB $\left.{ }^{+}, \mathbf{3 - N B A}\right)$ e/z (\%): $339.1(100)\left[\mathrm{PPh}_{4}{ }^{+}\right] ; \mathbf{M S}\left(\mathbf{F A B}^{-}, \mathbf{3}-\mathbf{N B A}\right)$ e/z

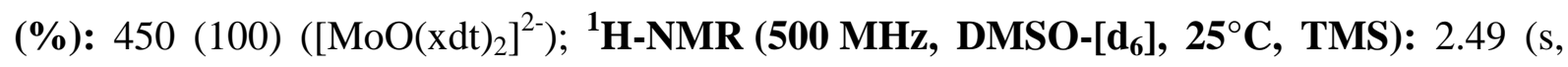
$\left.12 \mathrm{H}, \underline{\mathrm{CH}}_{3}\right), 7.18$ (s, $\left.4 \mathrm{H}, \underline{\mathrm{H}}_{\mathrm{Arom}}\right), 7.75$ (m, $\left.40 \mathrm{H}, \mathrm{P}-\underline{\mathrm{H}}_{\mathrm{Arom}}\right) \mathrm{ppm} ;{ }^{13} \mathbf{C}-\mathbf{N M R}(\mathbf{1 2 5 . 8} \mathbf{M H z}$,

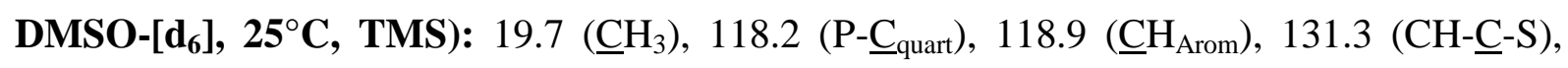

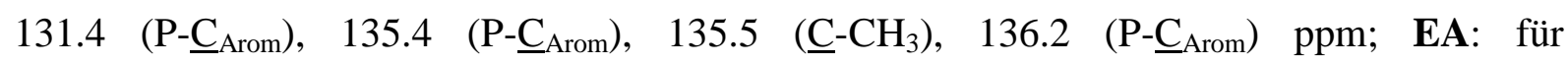
$\mathrm{C}_{64} \mathrm{H}_{56} \mathrm{MoOP}_{2} \mathrm{~S}_{4}$ Berechnet (\%):C: 68.19, H: 5.01, S: 11.38; Gefunden (\%):C: 68.77, H: 5.33; S: $11.55 ; \mathbf{E}^{\mathbf{0}}\left(\mathbf{2 5}^{\circ} \mathbf{C}, \mathbf{B u}_{4} \mathbf{N P F}_{6}, \mathbf{C V}, \mathbf{C H}_{3} \mathrm{CN}\right)$ : $-0.85 \mathrm{~V}\left(\mathrm{Fc} / \mathrm{Fc}^{+}\right) ; 0.48 \mathrm{~V}(\mathrm{SCE}) ; \mathbf{U V} / \mathrm{VIS}$ (MeOH) $\lambda_{\max }[\mathbf{n m}]\left(\varepsilon\left[\mathbf{M}^{-1} \cdot \mathbf{c m}^{-1}\right]\right): 467$ (2473), 777 (249)

\subsubsection{Synthese von $\left[\mathrm{MoO}(\mathrm{xdt})_{2}\right]^{2-}$ Methode B (1a)}

Eine Lösung von 4,5-Dimethylbenzene-1,2-dithiol (0.12 g, $0.71 \mathrm{mmol})$ in $20 \mathrm{ml}$ Acetonitril wurde zu einer Lösung aus $\left(\mathrm{NEt}_{4}\right)_{2}\left[\mathrm{MoO}_{2} \mathrm{~S}_{2}\right](0.16 \mathrm{~g}, 0.35 \mathrm{mmol})$ in $20 \mathrm{ml}$ Acetonitril bei RT gegeben. Hierbei konnte ein sofortiger Farbwechsel von orange nach rot beobachtet werden. Nach zwei Stunden rühren wurde die Lösung filtriert. Nach $10 \mathrm{~d}$ stehenlassen bei $-35^{\circ} \mathrm{C}$ konnte der Ausfall von rotbraunen Kristallen beobachtet werden, (0.14 g, $0.20 \mathrm{mmol}, 56 \%)$. Analytik siehe Methode A 


\subsubsection{Synthese von [WO(xdt) $\left.{ }^{2}\right]^{2-}(1 \mathrm{~b})$}

Die Herstellung des Komplexes wurde analog zur Methode zur Herstellung von 1a benutzt, nur das $\mathrm{K}_{3} \mathrm{Na}\left[\mathrm{WO}_{2}(\mathrm{CN})_{4}\right] \cdot 6 \mathrm{H}_{2} \mathrm{O}$ sowie $\mathrm{BzNEt}_{3} \mathrm{Cl}$ als Gegenion eingesetzt wurde und somit $0.45 \mathrm{~g}, 0.49 \mathrm{mmol}, 41 \%$ eines hellbraunen mikrokristallinen Feststoffs erhalten. IR (KBr) [cm ${ }^{-1}$ ]: 440(w) $461(\mathrm{w}), 505(\mathrm{w}), 605(\mathrm{w}), 676(\mathrm{w}), 702$ (s), $756(\mathrm{~m}), 787(\mathrm{w}), 815(\mathrm{w}), 868$ (m), 911 (s), 989 (w), 1016 (w), 1062 (w), 1102 (w), 1155 (m), 1185 (w), 1214 (w), 1244 (w), 1278 (w), 1338 (w), 1278 (w), 1338 (w), 1372 (w), 1393 (m), 1410 (w), 1446 (s), 1474 (s), 1576 (w), 2360 (w), 2875 (w), 2914 (m), 2937 (m), 2973 (m) 3035 (w); MS (FAB ${ }^{+}$, 3-NBA) e/z (\%): $192.2(100)\left[\mathrm{BzNEt}_{3}{ }^{+}\right] ; \quad \mathbf{M S}\left(\mathrm{FAB}^{-}, \mathbf{3 - N B A}\right) \mathbf{e} / \mathbf{z}(\%): 536(100) \quad\left(\left[\mathrm{WO}(\mathrm{xdt})_{2}\right]^{2-}\right)$;

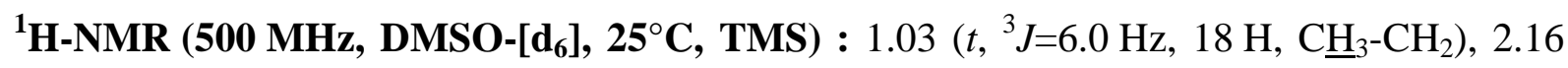
(s, $\left.12 \mathrm{H}, \mathrm{CH}_{3}\right), 2.78\left(q,{ }^{3} J=6.0 \mathrm{~Hz}, 12 \mathrm{H}, \mathrm{CH}_{3}-\underline{\mathrm{C}}_{2}\right), 3.79$ (s, $\left.4 \mathrm{H}, \mathrm{C}_{2}-\mathrm{Ph}\right), 7.15$ (s, $4 \mathrm{H}$, $\left.\underline{\mathrm{H}}_{\text {Arom }}\right), 7.29$ (m, $\left.6 \mathrm{H}, \underline{\mathrm{H}}_{\text {Arom }}\right), 7.34$ (m, $\left.4 \mathrm{H}, \underline{\mathrm{H}}_{\text {Arom }}\right)$ ppm; ${ }^{13} \mathbf{C}-\mathbf{N M R}$ (125.8 MHz, DMSO-[d $]$ ], $\left.\mathbf{2 5}^{\circ} \mathbf{C}, \mathbf{T M S}\right): 9.2\left(\underline{\mathrm{CH}}_{3}-\mathrm{CH}_{2}\right), 18.6\left(\underline{\mathrm{CH}}_{3}-\mathrm{C}_{\mathrm{Arom}}\right), 54.1\left(\underline{\mathrm{CH}}_{2}-\mathrm{CH}_{3}\right), 72.3\left(\underline{\mathrm{CH}}_{2}-\mathrm{Ph}\right), 126.9$ $\left.\left(\underline{\mathrm{CH}}_{\text {Arom }}\right), 127.3\left(\underline{\mathrm{CH}}_{\text {Arom }}\right), 128.2\left(\underline{\mathrm{CH}}_{\text {Arom }}\right), 128.6 \underline{\mathrm{CH}}_{\text {Arom }}\right) 129.1(\mathrm{CH}-\underline{\mathrm{C}}-\mathrm{S}), 147.8\left(\mathrm{C}-\underline{\mathrm{CH}}_{3}\right)$, 148.2 ( $\mathrm{C}_{\text {qart-Arom }}$ ) ppm; EA: für $\mathrm{C}_{42} \mathrm{H}_{60} \mathrm{~N}_{2} \mathrm{OS}_{4} \mathrm{~W}$ Berechnet (\%):C: 54.77, H: 6.57, N: 3.04, S: 13.93 Gefunden (\%): C: 54.11, H: 6.52; N: 2.96, S: $13.55 ; \mathbf{E}^{0}\left(\mathbf{2 5}^{\circ} \mathbf{C}, \mathbf{B u}_{4} \mathbf{N P F}_{6}, \mathbf{C V}\right.$, $\left.\mathbf{C H}_{3} \mathbf{C N}\right):-1.03 \mathrm{~V}\left(\mathrm{Fc} / \mathrm{Fc}^{+}\right) ;-0.66 \mathrm{~V}(\mathrm{SCE}) ; \mathrm{UV} / \mathrm{VIS}(\mathrm{MeOH}) \lambda_{\max }[\mathbf{n m}]\left(\varepsilon\left[\mathbf{M}^{-\mathbf{1}} \cdot \mathbf{c m}^{-1}\right]\right): 467$ (2473), 777 (249)

\subsubsection{Synthese von [MoO $\left.(\mathrm{xdt})_{2}\right]^{-(1 \mathrm{c})}$}

Triethylamin (abs.) (0.56 ml, $4.04 \mathrm{mmol}$ ) wurde zu einer Lösung des 4,5-Dimthylbenzol-1,2dithiol Ligandens $(0.17 \mathrm{~g}, 1.01 \mathrm{mmol}, 1.9 \mathrm{eq})$ gelöst in $10 \mathrm{ml} \mathrm{CH}_{3} \mathrm{CN}$ (abs) gegeben. Es konnte eine leichte Verfärbung nach gelb festgestellt werden. Nun wurde diese Lösung zu einer Acetonitrillösung von Trichlorooxobis(tetrahydrofuran)molybdän(V) $(0.19 \mathrm{~g}$, $0.53 \mathrm{mmol}$ ) bei $-40^{\circ} \mathrm{C}$ gegeben. Hierbei konnte eine Verfärbung nach dunkelrot beobachtet werden. Die Lösung wurde nun für 30 Min bei RT gerührt, gefolgt von einer Filtration. Das Volumen der Lösung wurde auf ein Volumen von ca. $2 \mathrm{ml}$ reduziert. Dann wurde eine Lösung aus $\mathrm{NBzEt}_{3} \mathrm{Cl}(0.12 \mathrm{~g}, 0.53 \mathrm{mmol})$ gelöst in $\mathrm{EtOH}$ (abs.) zugegeben und weitere $30 \mathrm{Min}$ bei RT gerührt. Ein Feststoff fiel nach Lagerung bei $1^{\circ} \mathrm{C}$ über Nacht aus. Zur weiteren Reinigung wurde erneut in $\mathrm{CH}_{3} \mathrm{CN}$ (abs.) gelöst und mit Hilfe von $\mathrm{Et}_{2} \mathrm{O}$ (abs.) erneut wieder ausgefällt. Es konnten $0.21 \mathrm{~g}, 0.33 \mathrm{mmol}, 62 \%$ eines rot schwarzen kristallinen Feststoffs erhalten werden. MS [FAB $\left.{ }^{+}, \mathbf{3 - N B A}\right] \mathbf{e} / \mathbf{z}(\boldsymbol{\%}): 192.2(100)\left[\mathrm{NBzEt}_{3}{ }^{+}\right]$; MS [FAB $\left.{ }^{-}, \mathbf{3 - N B A}\right] \mathbf{e} / \mathbf{z}(\boldsymbol{\%})$ : 450.0 (100) ([MoO(xdt) $\left.\left.]_{2}\right]^{-}\right)$; EA: für $\mathrm{C}_{29} \mathrm{H}_{38} \mathrm{MoNOS}_{4}$ : Berechnet (\%):C: 54.35, H: 5.98, N: 2.19, S: 20.02 Gefunden (\%): C: 54.88, H: 6.12; N: 2.31, S: 20.11 
10.2.5 Synthese von $\left[\mathrm{MoO}(\mathrm{tdt})_{2}\right]^{2-}(2 \mathrm{a})$

Toluol-3,4-dithiol (0.30 g, $1.92 \mathrm{mmol}$ ) wurde in $20 \mathrm{ml} \mathrm{EtOH} \mathrm{(entg.)} \mathrm{gelöst} \mathrm{und} \mathrm{langsam} \mathrm{bei}$ RT zu einer Lösung aus $\mathrm{K}_{3} \mathrm{Na}\left[\mathrm{MoO}_{2}(\mathrm{CN})_{4}\right] \cdot 6 \mathrm{H}_{2} \mathrm{O}(0.52 \mathrm{~g}, 1.08 \mathrm{mmol})$ in $20 \mathrm{ml}$ Wasser (entg.) gegeben. Hierbei konnte eine Verfärbung von blau nach rot beobachtet werden. Nun wurde $1 \mathrm{~h}$ bei RT weitergerührt, gefolgt von der Zugabe an $\mathrm{NEt}_{4} \mathrm{Br}(0.44 \mathrm{~g}, 2.09 \mathrm{mmol})$. Es könnte eine Verfärbung ins dunkelrot beobachtet werden. Zur Vervollständigung wurde eine weitere Stunde gerührt. Durch Filtration konnte ein roter Feststoff abgetrennt werden. Nun wurde mit kaltem Ethanol gewaschen. Der erhaltene Feststoff wurde in $\mathrm{CH}_{3} \mathrm{CN}$ (abs.) gelöst und mit $\mathrm{Et}_{2} \mathrm{O}$ (abs.) überschichtet. Nach $3 \mathrm{~d}$ wurden rote Kristalle aus der Lösung erhalten. Nach Filtration und Trocknung im HV konnten $0.48 \mathrm{~g}(0.70 \mathrm{mmol}, 65 \%)$ eines hellroten kristallinen Feststoffs erhalten werden. IR (KBr) [ $\left.\mathbf{c m}^{-1}\right]$ : 418 (w), 440 (w), 471 (w), 507 (w), $556(\mathrm{w}), 648(\mathrm{w}), 669(\mathrm{w}), 679(\mathrm{w}), 686(\mathrm{w}), 714$ (w), 808 (s), 839 (s), 860 (s), 904 (s), 984 (m), 999 (m), 1052 (w), 1092 (m), 1128 (w), 1154 (w), 11721242 (vs), 1310 (m), 1392 (m), 1448 (s), 1481 (s), 1541 (w), 1559 (w), 1576 (w), 1654 (w), 1701 (w), 1718 (w), 1734 (w), $1793(\mathrm{w}), 2083$ (w), 2340 (m), 2343 (m), 2343 (m), 2361 (m), 2858 (w), 2918 (w), 2976 (m), 3179 (w); $\quad \mathbf{M S}\left(\mathbf{E S I}^{+}, \mathbf{M e O H}\right) \mathbf{e} / \mathbf{z}(\%): 130.2(80)\left[\mathrm{NEt}_{4}{ }^{+}\right], \quad 682.3 \quad\left[\left(\mathrm{NEt}_{4}\right)_{2}\left[\mathrm{MoO}(\mathrm{tdt})_{2}\right] \quad\right]$

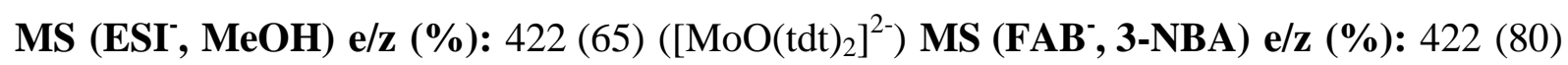
$\left(\left[\mathrm{MoO}(\mathrm{tdt})_{2}\right]^{2-}\right) ;{ }^{1} \mathbf{H}-\mathbf{N M R}\left(\mathbf{5 0 0} \mathbf{M H z}, \mathbf{C D}_{\mathbf{3}} \mathrm{OD}-\left[\mathbf{d}_{4}\right], \mathbf{2 5}^{\circ} \mathbf{C}\right.$, TMS $): 1.06\left(t,{ }^{3} \mathrm{~J}=6.0 \mathrm{~Hz}, 24 \mathrm{H}\right.$, $\left.\mathrm{C}_{3}-\mathrm{CH}_{2}\right), 2.24\left(\mathrm{~s}, 6 \mathrm{H}, \underline{\mathrm{C}}_{3}\right), 2.98\left(q,{ }^{3} \mathrm{~J}=6.0 \mathrm{~Hz}, 16 \mathrm{H}, \mathrm{CH}_{3}-\underline{\mathrm{C}}_{2}\right), 6.91\left(\mathrm{~m} 2 \mathrm{H}, \underline{\mathrm{H}}_{\text {Arom }}\right), 7.12$ (m, $\left.2 \mathrm{H}, \underline{\mathrm{H}}_{\text {Arom }}\right), 7.23$ (m, $\left.2 \mathrm{H}, \underline{\mathrm{H}}_{\text {Arom }}\right)$ ppm; ${ }^{13} \mathrm{C}-\mathbf{N M R}\left(\mathbf{1 2 5 . 8} \mathbf{M H z}, \mathbf{C D}_{\mathbf{3}} \mathrm{OD}-\left[\mathbf{d}_{\mathbf{4}}\right], \mathbf{2 5}^{\circ} \mathbf{C}\right.$, TMS): $9.4\left(\underline{\mathrm{CH}}_{3}-\mathrm{CH}_{2}\right), 21.3\left(\underline{\mathrm{CH}}_{3}-\mathrm{C}_{\text {Arom }}\right), 54.4\left(\underline{\mathrm{CH}}_{2}-\mathrm{CH}_{3}\right), 126.2\left(\underline{\mathrm{CH}}_{\text {Arom }}\right), 127.4\left(\underline{\mathrm{C}}_{\text {Arom }}\right)$, $\left.128.6\left(\underline{\mathrm{CH}}_{\text {Arom }}\right), 130.6 \underline{\mathrm{CH}}_{\text {Arom }}\right) 133.5(\mathrm{CH}-\underline{\mathrm{C}}-\mathrm{S}), 137.8\left(\mathrm{C}-\underline{\mathrm{CH}}_{3}\right)$, ppm; EA:Berechnet für $\mathrm{C}_{30} \mathrm{H}_{52} \mathrm{MoN}_{2} \mathrm{OS}_{4}(\%)$ : C: 52.91, H: 7.70, N: 4.11; S: 18.84; Gefunden (\%):C: 52.66, H: 7.44; N: $3.88 ; S: 18.66 ; \mathbf{E}^{\mathbf{0}}\left(\mathbf{2 5}^{\circ} \mathbf{C}, \mathbf{B u}_{4} \mathbf{N P F}_{6}, \mathbf{C V}, \mathbf{C H}_{3} \mathbf{C N}\right):-0.80 \mathrm{~V} /-0.02 \mathrm{~V}\left(\mathrm{Fc} / \mathrm{Fc}^{+}\right) ; 0.43 \mathrm{~V} /$ $0.35 \mathrm{~V}(\mathrm{SCE})$

\subsubsection{Synthese von [WO(tdt $\left.)_{2}\right]^{-}(2 \mathrm{~b})$}

Die Reaktion wurde analog zu der Methode zur Darstellung von Verbindungspaar 1c durchgeführt, nur das in dieser Synthese der 3,4-Toluoldithiolligand sowie Trichlorooxobis(tetrahydrofuran)-wolfram(V) benutzt wurden. Es konnten $0.19 \mathrm{~g}, 0.28 \mathrm{mmol}, 45 \%$ eines rot schwarzen kristallinen Feststoffs erhalten werden. IR(KBr) [ $\left.\mathbf{c m}^{-\mathbf{1}}\right]$ : 411(w), 503(w), 548(w), 689(w), 805(s), 869(w), 912(s), 945(w), 1031(m), 1104(s), 1156(m), 1208 (m), 1247(s), 1259(s), 1307(w), 1391(w), 1455(s), 1541(w), 1560(w), 1578(w), 1637(w), 2365(w), 2497(w), 2605(w), 2861(w), 2915(w), 2966(w), 3036(w); MS [ESI $\left.{ }^{+} \mathbf{C H}_{3} \mathbf{C N}\right]$ e/z (\%): 192 
(100) $\left[\mathrm{BzNEt}_{3}{ }^{+}\right], \mathbf{M S}\left[\mathbf{E S I}{ }^{-}, \mathbf{C H}_{3} \mathbf{C N}\right] \mathbf{e} / \mathbf{z}(\%): 508$ (71) [WO(tdt $\left.)_{2}\right]^{-}$; EA: Berechnet für $\mathrm{C}_{27} \mathrm{H}_{34} \mathrm{NOS}_{4} \mathrm{~W}(\%)$ : C: 46.28, H: 4.98, N: 2.00; S: 18.31; Gefunden (\%): C: 45.98, H: 4.73; N: 1.74; S: $18.23 ; \mathbf{E}^{\mathbf{0}}\left(\mathbf{2 5}^{\circ} \mathbf{C}, \mathbf{B u}_{4} \mathbf{N P F}_{6}, \mathbf{C V}, \mathbf{C H}_{3} \mathbf{C N}\right):-1.49 \mathrm{~V} /-0.61 \mathrm{~V}\left(\mathrm{Fc} / \mathrm{Fc}^{+}\right) ;-1.12 \mathrm{~V} /-$ $0.24 \mathrm{~V}(\mathrm{SCE}) ; \mathrm{UV} / \mathrm{VIS}(\mathbf{M e O H}) \boldsymbol{\lambda}_{\max }[\mathbf{n m}]\left(\varepsilon\left[\mathbf{M}^{-1} \cdot \mathbf{c m}^{-1}\right]\right): 524(1911)$

\subsubsection{Synthese von $\left[\mathrm{W}(\mathrm{CN}) 2(\mathrm{tdl})_{2}\right]^{2-}(2 \mathrm{c})$}

Toluol-3,4-dithiol (0.30 g, $1.92 \mathrm{mmol}$ ) wurde in $20 \mathrm{ml} \mathrm{EtOH}$ (entg.) gelöst und langsam bei RT zu einer Lösung aus $\mathrm{K}_{3} \mathrm{Na}\left[\mathrm{WO}_{2}(\mathrm{CN})_{4}\right] \cdot 6 \mathrm{H}_{2} \mathrm{O}(0.55 \mathrm{~g}, 0.97 \mathrm{mmol})$ in $20 \mathrm{ml}$ Wasser (entg.) gegeben. Hierbei konnte eine Verfärbung von violett nach rot beobachtet werden. Nun wurde $1 \mathrm{~h}$ bei RT weitergerührt, gefolgt von der Zugabe an $\mathrm{NEt}_{4} \mathrm{Br}(0.41 \mathrm{~g}, 1.95 \mathrm{mmol})$. Die Lösung färbte sich dunkelrot. Zur Vervollständigung wurde eine weitere Stunde gerührt. Durch Filtration konnte ein roter Feststoff abgetrennt werden. Nun wurde mit kaltem Ethanol gewaschen. Der erhaltene Feststoff wurde in $\mathrm{CH}_{3} \mathrm{CN}$ (abs.) gelöst und mit $\mathrm{Et}_{2} \mathrm{O}$ (abs.) überschichtet. Nach 10 Tagen bildeten sich rote Kristalle aus in der Lösung. Es konnten $0.48 \mathrm{~g}$ (0.60 mmol, 62\%) eines hellroten kristallinen Feststoffs erhalten werden. IR (KBr) [cm ${ }^{-1}$ ]: $354(\mathrm{~m}), 379(\mathrm{w}), 447(\mathrm{w}), 481(\mathrm{w}), 554(\mathrm{w}), 643$ (w), 691(w), $713(\mathrm{w}), 784(\mathrm{~m}), 800$ (m), 872 (w), 1000 (m), 1035 (w), 1052 (w), 1092 (w), 1138 (w), 1172 (m), 1183 (w), 1205 (w), 1244 (m), 1297 m), 1369 (m), 1391 (m), 1437 (m), 1451 (s), 1479 (s), 1582 (w), 1632 (w), 2083 (ws), 2862 (w), 2917 (w), 2945 (w), 2978 (m), 3030 (w); MS (FAB ${ }^{+}, 3-$

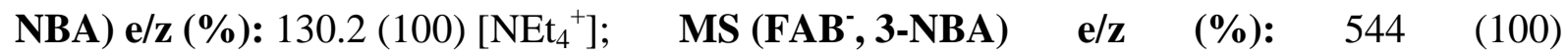
$\left(\left[\mathrm{W}(\mathrm{CN})_{2}(\mathrm{tdt})_{2}\right]^{2-}\right) ; \quad$ MS (ESI', $\quad$ MeCN) e/z (\%): $543.8(18) \quad\left(\left[\mathrm{W}(\mathrm{CN})_{2}(\mathrm{tdt})_{2}\right]^{2-}\right)$; ${ }^{1} \mathbf{H}-\mathrm{NMR}\left(300 \mathrm{MHz}, \mathrm{CD}_{\mathbf{3}} \mathrm{CN}-\left[\mathbf{d}_{3}\right], \mathbf{2 5}^{\circ} \mathbf{C}\right.$, TMS): 1.02 (t, ${ }^{3} \mathrm{~J}=6.0 \mathrm{~Hz}, 24 \mathrm{H}, \mathrm{CH}_{2} \mathrm{CH}_{3}$ ), 2.14 (s, $6 \mathrm{H}, \mathrm{C}-\mathrm{CH}_{3}$ ), 2.92 (quart, $\left.{ }^{3} \mathrm{~J}=6.0 \mathrm{~Hz}, 16 \mathrm{H}, \mathrm{C}_{2}-\mathrm{CH}_{3}\right), 6.80$ (m, $2 \mathrm{H}, \underline{\mathrm{H}}_{\text {Arom }}$ ), 7.74 (m, $2 \mathrm{H}$, $\left.\underline{\mathrm{H}}_{\text {Arom }}\right), 7.81$ (m, $\left.2 \mathrm{H}, \underline{\mathrm{H}}_{\text {Arom }}\right)$ ppm; ${ }^{13} \mathrm{C}-\mathbf{N M R}\left(\mathbf{1 2 5 . 8} \mathbf{M H z}, \mathbf{C D}_{\mathbf{3}} \mathbf{C N}-\left[\mathbf{d}_{3}\right], \mathbf{2 5}^{\circ} \mathbf{C}, \mathbf{T M S}\right)$ : $\left.7.5\left(\underline{\mathrm{CH}}_{3}\right), 18.7 \mathrm{C}-\underline{\mathrm{C}} \mathrm{H}_{3}\right), 52.9\left(\underline{\mathrm{CH}}_{2} \mathrm{CH}_{3}\right), 124.3(\underline{\mathrm{CN}}), 128.5$ ( $\left.\underline{\mathrm{C}}_{\text {Arom }}\right), 129.1$ ( $\left.\underline{\mathrm{C}}_{\text {Arom}}\right), 132.4$ $(\mathrm{C}=\underline{\mathrm{C}}), 132.4\left(\underline{\mathrm{C}}_{\mathrm{Arom}}\right), 152.8(\underline{\mathrm{C}}=\mathrm{C}), 155.8\left(\underline{\mathrm{C}}-\mathrm{CH}_{3}\right)$ ppm. EA: Berechnet für $\mathrm{C}_{32} \mathrm{H}_{52} \mathrm{~N}_{4} \mathrm{~S}_{4} \mathrm{~W}$ (\%): C: 47.75, H: 6.51, N: 6.96; S: 15.94; Gefunden (\%):C: 47.63, H: 6.42; N:6.88; S:15.67 $\mathbf{E}^{\mathbf{0}}\left(\mathbf{2 5}^{\circ} \mathbf{C}, \mathbf{B u}_{4} \mathbf{N P F}_{6}, \mathbf{C V}, \mathbf{C H}_{3} \mathrm{CN}\right):-0.51 \mathrm{~V} / 0.03 \mathrm{~V}\left(\mathrm{Fc} / \mathrm{Fc}^{+}\right) ;-0.14 \mathrm{~V} / 0.40 \mathrm{~V}(\mathrm{SCE}) ; \mathbf{U V} / \mathrm{VIS}$ (MeOH) $\lambda_{\max }[\mathbf{n m}]\left(\varepsilon\left[\mathbf{M}^{-1} \cdot \mathbf{c m}^{-1}\right]\right): 478.9$ (899), 393.0 (1889), 358.9 (2349)

\subsubsection{Synthese von [WO $\left.(\mathrm{tdt})_{2}\right]^{2-}(2 \mathrm{~d})$}

$0.20 \mathrm{~g}, 0.25 \mathrm{mmol}$ von $\left(\mathrm{NEt}_{4}\right)_{2}\left[\mathrm{~W}(\mathrm{CN})_{2}(\mathrm{tdt})_{2}\right]$ wurden in $30 \mathrm{ml}$ Acetontril gelöst und mit $0.08 \mathrm{ml} \mathrm{Et}{ }_{4} \mathrm{NOH}(25 \%$ in Methanol) versetzt. Es konnte eine schlagartige Farbveränderung von rot nach orange beobachtet werden. Nun wurde für weitere $2 \mathrm{~h}$ bei RT gerührt. Nach 
Filtration wurde das Lösungsmittel im HV vollständig vom Filtrat entfernt. Nach erneutem Lösen in Acetonitril wurde mit Diethylether im Überschuss versetzt und es konnte der Ausfall eines orangen Feststoffs beobachtet werden. Nach Filtration wurde noch zwei weitere Male aus Acetonitril/Ditethylether umkristallisiert. Nach Trocknung im HV konnten $0.07 \mathrm{~g}$, 0.09 mmol, 36\% eines orangen Feststoffs erhalten werden. IR (KBr) [ $\mathbf{c m}^{-\mathbf{1}}$ ]: 446 (w), 480 (w), $504(\mathrm{w}), 554(\mathrm{w}), 641(\mathrm{w}), 692(\mathrm{w}), 747(\mathrm{w}), 799(\mathrm{~m}), 898(\mathrm{~s}), 985(\mathrm{~m}), 1000(\mathrm{~m}), 1026$ (w), 1154 (m), 1209 (w), 1244 (vs), 1310 (m), 1385 (m), 1458 (m), 1480 (m), 1544 (w), 1578 (w), 1629 (w), 1655 (w), 2345 (w), 2364 (w), 2466 (w), 2857 (w), 2922 (w), 2972 (w), 3135 (w); ${ }^{1} \mathbf{H}$-NMR (500 MHz, $\left.\mathbf{C D}_{3} \mathbf{C N}-\left[\mathbf{d}_{3}\right], \mathbf{2 5}^{\circ} \mathbf{C}, \mathbf{T M S}\right): 1.07\left(t,{ }^{3} \mathrm{~J}=6.0 \mathrm{~Hz}, 24 \mathrm{H}, \mathrm{CH}_{3}-\mathrm{CH}_{2}\right)$, 2.27 (s, $\left.6 \mathrm{H}, \mathrm{C}_{3}\right), 3.03\left(q,{ }^{3} \mathrm{~J}=6.0 \mathrm{~Hz}, 16 \mathrm{H}, \mathrm{CH}_{3}-\underline{\mathrm{C}}_{2}\right), 6.93\left(\mathrm{~m} 2 \mathrm{H}, \underline{\mathrm{H}}_{\mathrm{Arom}}\right), 7.17$ (m, $2 \mathrm{H}$, $\left.\underline{\mathrm{H}}_{\text {Arom }}\right), 7.29$ (m, $2 \mathrm{H}, \underline{\mathrm{H}}_{\text {Arom }}$ ) ppm; EA: Berechnet für $\mathrm{C}_{30} \mathrm{H}_{52} \mathrm{~N}_{2} \mathrm{OS}_{4} \mathrm{~W}(\%)$ : C: 46.87, H: 6.82, $\mathrm{N}: 3.64$; S: 16.68; Gefunden (\%): C: 45.77, H: 6.66; N: 3.23; S: 16.07; UV/VIS (MeOH) $\lambda_{\max }$ $[\mathrm{nm}]\left(\varepsilon\left[\mathbf{M}^{-1} \cdot \mathbf{c m}^{-1}\right]\right): 461.2(846)$

\subsubsection{Synthese von $\left[\mathrm{W}(\mathrm{CN})(\mathrm{SPh})(\mathrm{tdt})_{2}\right]^{2-}(2 \mathrm{e})$}

$0.013 \mathrm{ml}, 0.13 \mathrm{~mol}$ Thiophenol wurde $\mathrm{zu}$ einer Lösung aus $0.20 \mathrm{~g}, 0.25 \mathrm{mmol}$ von $\left(\mathrm{NEt}_{4}\right)_{2}\left[\mathrm{~W}(\mathrm{CN})_{2}(\mathrm{tdt})_{2}\right]$ in $30 \mathrm{ml}$ Acetontril gelöst. Es wurde bei RT für $12 \mathrm{~h}$ gerührt, wobei eine Farbänderung von dunkelrot nach braun eintrat. Nach Filtration wurde mit $20 \mathrm{ml}$ Diethylether versetzt, wobei eine der Ausfall eines brauen Feststoffs beobachtet werden konnte. Der erneut abfiltrierte Feststoff wurde wiederum aus Acetonitril/Et ${ }_{2} \mathrm{O}$ umkristallisiert. Hierbei konnten $0.06 \mathrm{~g}, 0.07 \mathrm{mmol}, 27 \%$ erhalten werden. MS (FAB $\left.\mathbf{F}^{-}, 3-\mathrm{NBA}\right)$ e/z (\%): $627.9(17)\left(\left[\mathrm{W}(\mathrm{CN}) \mathrm{SPh}(\mathrm{tdt})_{2}\right]^{2-}\right)$; IR (KBr) $\left[\mathbf{c m}^{-1}\right]$ : $442(\mathrm{w}), 504(\mathrm{w}), 572(\mathrm{~m}), 621(\mathrm{~m}), 692$ (s), $810(\mathrm{w}), 842(\mathrm{w}), 980(\mathrm{~m}), 1029(\mathrm{~s}), 1085(\mathrm{w}), 1134(\mathrm{~m}), 1156(\mathrm{w}), 1198$ (s), 1224 (s), 1236 (s), 1307 (w), 1445 (s), 1638(w), 2359 (s), 2855 (w), 2924 (w), 3060 (w);

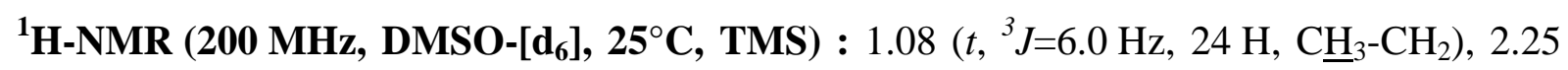
(s, $\left.6 \mathrm{H}, \mathrm{CH}_{3}\right), 2.98\left(q,{ }^{3} J=6.0 \mathrm{~Hz}, 16 \mathrm{H}, \mathrm{CH}_{3}-\underline{\mathrm{C}}_{2}\right), 6.83$ (m $\left.2 \mathrm{H}, \underline{\mathrm{H}}_{\text {Arom }}\right), 7.09$ (m, $2 \mathrm{H}, \underline{\mathrm{H}}_{\text {Arom }}$ ), $7.18\left(\mathrm{~m}, 2 \mathrm{H}, \underline{\mathrm{H}}_{\text {Arom}}\right), 7.48$ (m, $\left.5 \mathrm{H}, \underline{\mathrm{H}}-\mathrm{Ph}\right) \mathrm{ppm}$; EA: Berechnet für $\mathrm{C}_{30} \mathrm{H}_{52} \mathrm{~N}_{2} \mathrm{OS}_{4} \mathrm{~W}(\%)$ : C: 46.87, H: 6.82, N: 3.64; S: 16.68; Gefunden (\%): C: 45.57, H: 6.66; N: 3.23; S: 15.99

\subsubsection{Synthese von $\left[\mathrm{MoO}(\mathrm{vdt})_{2}\right]^{2-}(3 \mathrm{a})$}

$\mathrm{Zu}$ einer Lösung von $\mathrm{K}_{3} \mathrm{Na}\left[\mathrm{MoO}_{2}(\mathrm{CN})_{4}\right] .6 \mathrm{H}_{2} \mathrm{O}(0.44 \mathrm{~g}, 0.77 \mathrm{mmol})$ und $\mathrm{NaOH}(0.6 \mathrm{~g})$ in Wasser (entg. $20 \mathrm{ml}$ ) wurde eine Lösung von 4,5-Dimethoxy-benzol-1,2-dithiol (0.26 g, $1.55 \mathrm{mmol})$ in Methanol $(10 \mathrm{ml})$ gegeben und $15 \mathrm{~min}$ auf $45^{\circ} \mathrm{C}$ erwärmt. Die Farbe der 
Lösung veränderte sich hierbei von blau nach rot. Nach Abkühlen wurde $\left[\mathrm{NEt}_{3}\left(\mathrm{PhCH}_{2}\right)\right] \mathrm{Cl}$ $(0.3 \mathrm{~g}, 0.8 \mathrm{mmol})$ in $5 \mathrm{ml}$ Methanol zugegeben und über Nacht bei $1^{\circ} \mathrm{C}$ aufbewahrt. Durch Filtration wurde das Produkt als orangeroter Feststoff erhalten $(0.17 \mathrm{~g}, 0.33 \mathrm{mmol}, 43 \%)$. Anschließendes Umkristallisieren aus Acetonitril/Diethylether lieferte analysenreines Produkt. IR (KBr) [cm ${ }^{-1}$ ]: 454(w), 540(w), 679(w), 708(w), 788(m), 843(m), 891(s), 1032(s), 1102(w), 1177(s), 1196(m), 1246(s), 1311(w), 1353(m), 1442(s), 1469(s), 1495(s), 1587(w), 1627(w), 2085(w), 2845(w), 2947(w), 3008(w), 3071(w); MS (ESI', CH $\mathbf{3}$ CN): 514.0 (15),

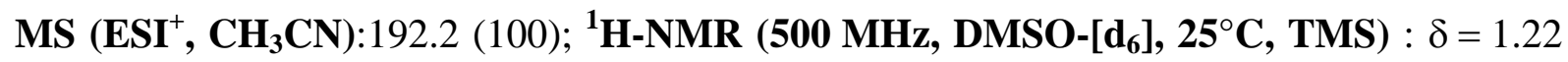
(t, $\left.{ }^{3} \mathrm{~J}=2.7 \mathrm{~Hz}, 18 \mathrm{H}, \mathrm{CH}_{3}-\mathrm{CH}_{2}-\mathrm{N}\right), 3.14$ (q, $\left.{ }^{3} \mathrm{~J}=2.7 \mathrm{~Hz}, 12 \mathrm{H}, \mathrm{CH}_{3}-\underline{\mathrm{C}}_{2}-\mathrm{N}\right), 4.47$ (s, $12 \mathrm{H}$,

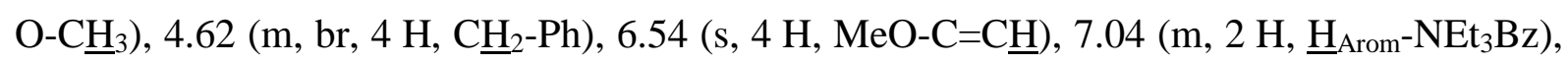
$7.51\left(\mathrm{~m}, 4 \mathrm{H}, \underline{\mathrm{H}}_{\mathrm{Arom}}-\mathrm{NEt}_{3} \mathrm{Bz}\right), 8.33$ (m, $\left.4 \mathrm{H}, \underline{\mathrm{H}}_{\mathrm{Arom}}-\mathrm{NEt}_{3} \mathrm{Bz}\right) \mathrm{ppm} ;{ }^{13} \mathrm{C}-\mathrm{NMR}$ (125.8 MHz,

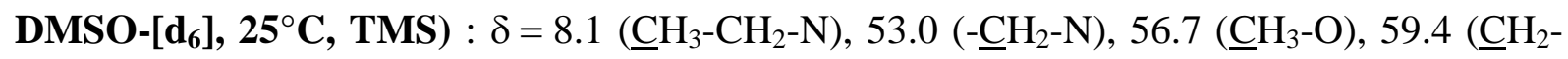

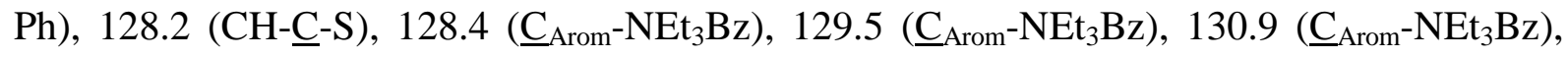
$131.3 \quad\left(\underline{\mathrm{C}}_{\mathrm{Arom}}-\mathrm{NEt}_{3} \mathrm{Bz}\right), \quad 132.4 \quad(\underline{\mathrm{C}}=\underline{\mathrm{C}}), \quad 207.1 \quad\left(\underline{\mathrm{C}}_{\text {quart }} \mathrm{O}\right) \quad$ ppm; $\mathbf{E A}$ : Berechnet für $\mathrm{C}_{42} \mathrm{H}_{60} \mathrm{MoN}_{2} \mathrm{O}_{5} \mathrm{~S}_{4}: \mathrm{C}: 56.23 \%$; $\mathrm{H}: 6.74 \%$; N: 3.12\%; S: 14.30\%; Gefunden: C: 56.02\%; H: 6.59\%; N: 3.03\%; S: 14.17\%; $\mathbf{E}^{\mathbf{0}}\left(\mathbf{2 5}^{\circ} \mathbf{C}, \mathbf{B u}_{4} \mathbf{N P F}_{6}, \mathbf{C V}, \mathbf{C H}_{3} \mathbf{C N}\right):-0.77 \mathrm{~V} /-0.03\left(\mathrm{Fc} / \mathrm{Fc}^{+}\right)$; $-0.40 \mathrm{~V} / 0.34 \mathrm{~V}(\mathrm{SCE})$ :

\subsubsection{Synthese von [WO(vdt) $]^{2-}(3 \mathrm{~b})$}

Die Darstellung erfolgte analog zu 3a mit $\mathrm{K}_{3} \mathrm{Na}\left[\mathrm{WO}_{2}(\mathrm{CN})_{4}\right] \cdot 6 \mathrm{H}_{2} \mathrm{O}(0.94 \mathrm{~g}, 1.65 \mathrm{mmol})$ als Ausgangsmaterial. Es wurden $(0.32 \mathrm{~g}, 0.6 \mathrm{mmol}, 36 \%)$ eines hellbraunen kristallinen Feststoffs erhalten. Abschließendes Umkristallisieren aus Acetonitril/Diethylether lieferte orange Kristalle. IR (KBr) [ $\mathbf{c m}^{-1}$ ]: 461(w), 538(w), 608(w), 673(w), 709(m), 753(m), 773(m), 851(m), 904(s), 1038(m), 1086(w), 1154(m), 1172(m), 1199(s), 1241(s), 1342(m), 1392(m), 1436(s), 1465(s), 1558(w), 1578(w), 2243(w), 2830(w), 2900(w), 2939(w), 2985(w); MS (ESI', $\left.\mathbf{C H}_{3} \mathbf{C N}\right): 600.0$ (8) $\left[\mathrm{WO}(\mathrm{vdt})_{2}\right]^{2-} ; \mathbf{M S}\left(\mathbf{E S I}^{+}, \mathbf{C H}_{3} \mathbf{C N}\right): 192.2\left[\mathrm{NEt}_{3}\left(\mathrm{PhCH}_{2}\right)\right]$

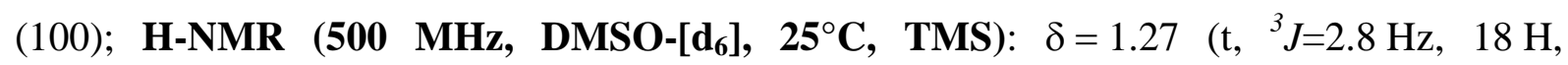
$\mathrm{CH}_{3}-\mathrm{CH}_{2}-\mathrm{N}$ ), 3.13 (q, $\left.{ }^{3} \mathrm{~J}=2.8 \mathrm{~Hz}, \mathrm{CH}_{3}-\underline{\mathrm{CH}}_{2}-\mathrm{N}\right), 4.43$ (s, $\left.12 \mathrm{H}, \mathrm{O}-\underline{\mathrm{H}}_{3}\right), 4.61$ (m-br, $4 \mathrm{H}, \mathrm{CH}_{2}-$ $\mathrm{Ph}), 6.51$ (s, $4 \mathrm{H}, \mathrm{MeO}-\mathrm{C}=\mathrm{C} \underline{\mathrm{H}}), 7.02\left(\mathrm{~m}, 2 \mathrm{H}, \underline{\mathrm{H}}_{\mathrm{Arom}}-\mathrm{NEt}_{3} \mathrm{Bz}\right), 7.50$ (m, 4 H, $\left.\underline{\mathrm{H}}_{\mathrm{Arom}}-\mathrm{NEt}_{3} \mathrm{Bz}\right)$,

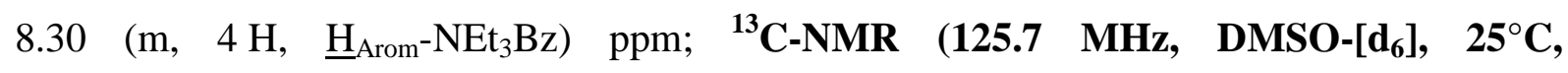
TMS): $\delta=7.4\left(\underline{\mathrm{CH}}_{3}-\mathrm{CH}_{2}-\mathrm{N}\right), 52.0\left(-\underline{\mathrm{CH}}_{2}-\mathrm{N}\right), 55.9\left(\underline{\mathrm{CH}}_{3}-\mathrm{O}\right), 59.6\left(\underline{\mathrm{CH}}_{2}-\mathrm{Ph}\right), 127.8(\mathrm{CH}-\underline{\mathrm{C}}-\mathrm{S})$, 128.9 ( $\left.\underline{\mathrm{C}}_{\mathrm{Arom}}-\mathrm{NEt}_{3} \mathrm{Bz}\right), 129.3$ ( $\left.\underline{\mathrm{C}}_{\text {Arom }}-\mathrm{NEt}_{3} \mathrm{Bz}\right), 130.1$ ( $\left.\underline{\mathrm{C}}_{A r o m}-\mathrm{NEt}_{3} \mathrm{Bz}\right), 130.6\left(\underline{\mathrm{C}}_{\mathrm{Arom}^{-}}-\mathrm{NEt}_{3} \mathrm{Bz}\right)$, $132.4(\underline{\mathrm{C}}=\underline{\mathrm{C}}), 206.2$ ( $\left.\underline{\mathrm{C}}_{\text {quart }} \mathrm{O}\right)$ ppm; EA: Berechnet für $\mathrm{C}_{42} \mathrm{H}_{60} \mathrm{~N}_{2} \mathrm{O}_{5} \mathrm{~S}_{4} W: \mathrm{C}: 51.21 \%$; 
$\mathrm{H}: 6.14 \% ; \mathrm{N}: 2.84 \%$; S:13.02\%; Gefunden: C: 50.89\%; H: 5.98\%; N:2.79\%; S:12.98\%;

$\mathbf{E}^{\mathbf{0}}\left(\mathbf{2 5}^{\circ} \mathbf{C}, \mathbf{B u}_{4} \mathbf{N P F}_{6}, \mathbf{C V}, \mathbf{C H}_{3} \mathrm{CN}\right):-1.45 \mathrm{~V} /-0.75 \mathrm{~V}\left(\mathrm{Fc}_{\mathrm{Fc}}{ }^{+}\right) ;-1.08 \mathrm{~V} / 0.38 \mathrm{~V}$ (SCE).

\subsubsection{Synthese von [WO(vdt) $]^{-}$(3c)}

Die Reaktion wurde analog zu der Methode zur Darstellung von Verbindungspaar 1c durchgeführt, nur das in dieser Synthese der 4,5-Dimethoxybenzol-1,2-dithiol sowie Trichlorooxobis(tetrahydrofuran)-wolfram(V) benutzt wurden. Es konnten 0.14 g, 0.19 mmol, $28 \%$ eines rot braunen kristallinen Feststoffs erhalten werden. MS (FAB ${ }^{+}, 3-$ NBA) e/z (\%): $130.2(100)\left[\mathrm{NEt}_{4}{ }^{+}\right]$; MS (FAB, $\left.\mathbf{3 - N B A}\right)$ e/z (\%): $599.2(20)\left(\left[\mathrm{WO}\left(\mathrm{vdt}_{2}\right]^{-}\right)^{-}\right)$; EA: Berechnet für $\mathrm{C}_{24} \mathrm{H}_{36} \mathrm{NO}_{5} \mathrm{~S}_{4} \mathrm{~W}(\%)$ : C: $39.45, \mathrm{H}: 4.97, \mathrm{~N}: 1.92 ; \mathrm{S}: 17.55 ;$ Gefunden (\%): C: 40.21, H: 5.23; N: 2.23; S: 18.12

\subsubsection{Synthese von $\left[\mathrm{MoO}(\mathrm{ndt})_{2}\right]^{2-}(4 \mathrm{a})$}

Die Synthese wurde analog zu Darstellung von Verbindung 3a durchgeführt, wobei hierbei 4,5-Dimercaptophtalonitril als Ligandensystem eingesetzt wurde. Hierbei konnten $0.54 \mathrm{~g}$, $0.55 \mathrm{mmol}, 47 \%$ eines orangen Feststoffs erhalten werden.

IR (KBr) [cm ${ }^{-1}$ ]: $405(\mathrm{w}), 418(\mathrm{w}), 467(\mathrm{w}), 503(\mathrm{w}), 529(\mathrm{~m}), 555(\mathrm{w}), 649(\mathrm{w}), 674(\mathrm{w})$, 744 (w), 804 (w), 885 (m), 916 (s), 985 (m), 1102 (m), 1129 (m), 1154 (m), 1211 (s), 1244 (vs), 1309 (m), 1384 (w), 1447 (m), 1473 (w), 1510 (s), 1542 (w), 1655 (w), 1774 (w), 2219 (s), 2343 (w), 2363 (w), 2610 (w), 2872 (w), 2939 (w), 2961 (w), 3032 (w); MS [FAB ${ }^{+}, 3-$

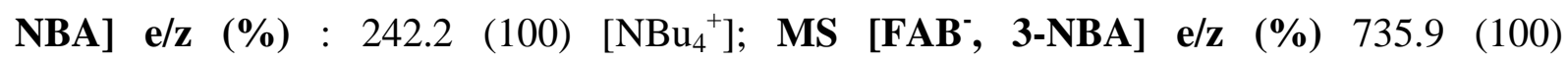

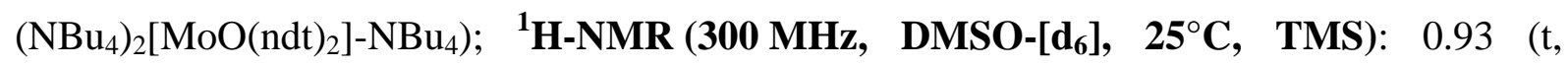
$\left.{ }^{3} J=7.0 \mathrm{~Hz}, \quad 24 \mathrm{H}, \quad \mathrm{C}_{\underline{H}_{3}}-\mathrm{CH}_{2}\right), \quad 1.24\left(\mathrm{sech}, \quad{ }^{3} J=7.0 \mathrm{~Hz}, \quad 16 \mathrm{H}, \quad \mathrm{C}_{\underline{H}_{2}}-\mathrm{CH}_{3}\right.$ ), 1.53 (quin, $\left.{ }^{3} J=7.0 \mathrm{~Hz}, 16 \mathrm{H}, \mathrm{CH}_{2}-\underline{\mathrm{C}}_{2}-\mathrm{CH}_{2}\right), 3.16\left(\mathrm{t},{ }^{3} \mathrm{~J}=8.5 \mathrm{~Hz}, 16 \mathrm{H}, \mathrm{N}-\underline{\mathrm{H}}_{2}\right), 7.36\left(\mathrm{~s}, 4 \mathrm{H}, \underline{\mathrm{H}}_{\text {Arom }}\right)$ ppm; ${ }^{13} \mathbf{C}-N M R ~\left(75.5 ~ M H z, ~ D M S O-\left[d_{6}\right], \mathbf{2 5}^{\circ} \mathbf{C}\right.$, TMS) : $12.3\left(\underline{\mathrm{CH}}_{3}\right), 18.1\left(\underline{\mathrm{CH}}_{2}-\mathrm{CH}_{3}\right), 21.9$ $\left(\underline{\mathrm{CH}}_{2}-\mathrm{CH}_{2}-\mathrm{CH}_{3}\right), 56.4\left(\mathrm{~N}-\underline{\mathrm{CH}}_{2}\right), 96.1(\underline{\mathrm{C}}-\mathrm{CN}), 105.3(\underline{\mathrm{C}} \equiv \mathrm{N}), 118.7(\mathrm{C}=\mathrm{C}-\underline{\mathrm{C}}), 131.9(\underline{\mathrm{C}}=\underline{\mathrm{C}}-\mathrm{C})$ ppm; EA: Berechnet für $\mathrm{C}_{48} \mathrm{H}_{76} \mathrm{MoN}_{6} \mathrm{OS}_{4}(\%)$ : C: 58.99; H: 7.84; N: 8.60; S:13.12; Gefunden (\%): C: 59.11; H: 7.98; N: 8.68; S: $13.22 ; \mathbf{E}^{\mathbf{0}}\left(\mathbf{2 5}^{\circ} \mathbf{C}, \mathbf{B u}_{4} \mathbf{N P F}_{6}, \mathbf{C V}, \mathbf{C H}_{3} \mathbf{C N}\right):-0.28 \mathrm{~V}$ $\left(\mathrm{Fc} / \mathrm{Fc}^{+}\right) ; 0.09 \mathrm{~V}(\mathrm{SCE})$

\subsubsection{Synthese von [WO(ndt) $\left.{ }_{2}\right]^{2-}(4 \mathrm{~b})$}

Die Darstellung erfolgte analog zu 3a mit $\mathrm{K}_{3} \mathrm{Na}\left[\mathrm{WO}_{2}(\mathrm{CN})_{4}\right] \cdot 6 \mathrm{H}_{2} \mathrm{O}(0.50 \mathrm{~g}, 0.88 \mathrm{mmol})$ als Ausgangsmaterial. Es wurden ( $0.39 \mathrm{~g}, 0.37 \mathrm{mmol}, 42 \%)$ eines dunkelroten Feststoffs erhalten. 
Abschließendes Umkristallisieren aus Acetonitril/Diethylether lieferte einen dunkelroten kristallinen Feststoff. IR (KBr) [cm ${ }^{-1}$ ]: 505 (m), 640 (w), 719 (w), 746 (w), 807 (w), 887 (w), 919 (s), 985 (m), 1154 (s), 1208 (m), 1240 (vs), 1311 (m), 1384 (w), 1509 (s), 2207 (w), 2345 (w), 2368 (w), 2871 (w), 2934 (w), 2963 (w); MS [FAB $\left.{ }^{+}, 3-\mathbf{N B A}\right]$ e/z (\%) : 242.2 (100) $\left[\mathrm{NBu}_{4}^{+}\right] ; \quad \mathbf{M S} \quad\left[\mathbf{F A B}^{-}, \quad 3-\mathrm{NBA}\right] \quad \mathbf{e} / \mathbf{z} \quad(\%): 822.0 \quad(100) \quad\left(\left(\mathrm{NBu}_{4}\right)_{2}\left[\mathrm{WO}(\mathrm{ndt})_{2}\right]-\mathrm{NBu}_{4}\right)$;

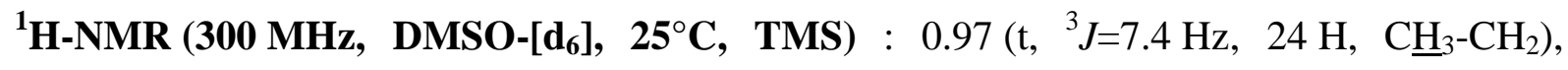
1.29 (sech, ${ }^{3} J=7.4 \mathrm{~Hz}, 16 \mathrm{H}, \underline{\mathrm{C}}_{2}-\mathrm{CH}_{3}$ ), 1.57 (quin, ${ }^{3} \mathrm{~J}=7.0 \mathrm{~Hz}, 16 \mathrm{H}, \mathrm{CH}_{2}-\mathrm{C}_{2}-\mathrm{CH}_{2}$ ), 3.22 (t,

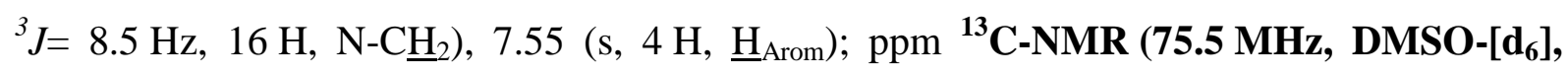
$\mathbf{2 5}^{\circ} \mathbf{C}$, TMS): $12.4\left(\underline{\mathrm{CH}}_{3}\right), 18.3\left(\underline{\mathrm{CH}}_{2}-\mathrm{CH}_{3}\right), 22.4\left(\underline{\mathrm{CH}}_{2}-\mathrm{CH}_{2}-\mathrm{CH}_{3}\right), 57.1\left(\mathrm{~N}-\underline{\mathrm{CH}}_{2}\right), 97.5(\underline{\mathrm{C}}-\mathrm{CN})$, $108.2(\underline{\mathrm{C}} \equiv \mathrm{N}), 119.6(\mathrm{C}=\mathrm{C}-\underline{\mathrm{C}}), 133.2(\underline{\mathrm{C}}=\underline{\mathrm{C}}-\mathrm{C}) \mathrm{ppm} ; \mathbf{E A}:$ Berechnet für $\mathrm{C}_{48} \mathrm{H}_{76} \mathrm{~N}_{6} \mathrm{OS}_{4} \mathrm{~W}(\%)$ : C : 54.12, H: 7.19, N: 7.89, S: 12.04; Gefunden (\%): C: 53.79, H: 6.89, N: 7.66, S: 12.12; $\mathbf{E}^{\mathbf{0}}\left(\mathbf{2 5}^{\circ} \mathrm{C}, \mathbf{B u}_{4} \mathbf{N P F}_{6}, \mathbf{C V}, \mathbf{C H}_{3} \mathrm{CN}\right):-0.57 \mathrm{~V}\left(\mathrm{Fc} / \mathrm{Fc}^{+}\right) ; 0.20 \mathrm{~V}(\mathrm{SCE})$

\subsubsection{Synthese von [MoO $\left.(\mathrm{qdt})_{2}\right]^{2-}(5 \mathrm{a})$}

Die Synthese wurde analog zu Darstellung von Verbindung 3a durchgeführt, wobei hierbei 2,3-Quinoxalin-2,3-dithiol als Ligandensystem eingesetzt wurde sowie $\mathrm{NBu}_{4} \mathrm{Br}$. Es konnten $0.55 \mathrm{~g}, \quad 0.56 \mathrm{mmol}, \quad 49 \%$ eines dunkelblauen Feststoffs nach Umkristallisation aus Acetonitril/ $/ \mathrm{Et}_{2} \mathrm{O}$ erhalten werden.

IR (KBr) [cm-1]: 404 (m), 432 (w), 469 (w), 497 (w), 545 (w), 597 (m), 704 (m), 760 (s), 788 (w), 941 (s), 984 (w), 1112 (w), 1108 (vs), 1162 (s), 1252 (s), 1302 (w), 1363 (m), 1385 (m), $1397(\mathrm{~m}), 1453$ (m), 1556 (w), 1626 (w), 2436 (w), 2943 (w), 2981 (w), 3057 (w); MS [FAB $\left.{ }^{+}, 3-\mathbf{N B A}\right]$ e/z (\%): 192.2 (100) $\left(\mathrm{NEt}_{3} \mathrm{Bz}^{+}\right) ; \mathbf{M S}$ [FAB $\left.{ }^{-}, 3-\mathrm{NBA}\right]$ e/z (\%): 497.9 (100) $\left(\left[\mathrm{MoO}(\mathrm{qdt})_{2}\right]^{2-}\right) ;{ }^{1} \mathbf{H}-\mathrm{NMR}\left(\mathbf{5 0 0} \mathbf{M H z}, \mathbf{D M S O}-\left[\mathbf{d}_{6}\right], \mathbf{2 5}^{\circ} \mathbf{C}, \mathbf{T M S}\right): \delta=1.22\left(\mathrm{t},{ }^{3} \mathrm{~J}=7.5 \mathrm{~Hz}\right.$, $\left.18 \mathrm{H}, \underline{\mathrm{C}}_{3}-\mathrm{CH}_{2}\right), 1.29$ (q, ${ }^{3} \mathrm{~J}=7.5 \mathrm{~Hz}, 12 \mathrm{H}, \underline{\mathrm{C}}_{2}-\mathrm{CH}_{3}$ ), 4.49 (s, $\left.4 \mathrm{H}, \mathrm{N}-\underline{\mathrm{H}}_{2}\right), 7.40$ (m, $10 \mathrm{H}$,

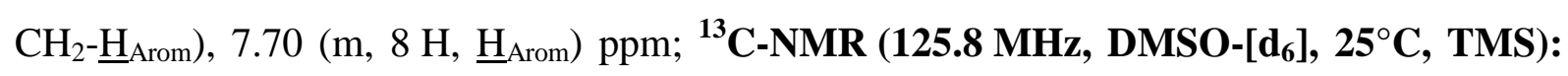
$\delta=13.2\left(\underline{\mathrm{CH}}_{3}\right), 58.3\left(\underline{\mathrm{CH}}_{2}-\mathrm{CH}_{3}\right), 68.2\left(\underline{\mathrm{CH}}_{2}-\mathrm{Ph}\right), 129.1$ ( $\underline{\mathrm{C}}_{\text {Arom }}-$ Kation$), 129.3$ ( $\underline{\mathrm{C}}_{\text {Arom }}-$ Kation$)$,

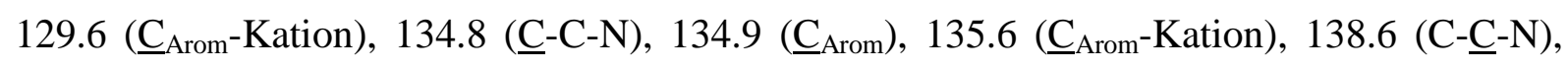
174.2 (--S-W) ppm EA: Berechnet für $\mathrm{C}_{42} \mathrm{H}_{52} \mathrm{MoN}_{6} \mathrm{OSS}_{4}(\%): \mathrm{C}: 57.25 \%, \mathrm{H}: 5.95 \%$; N: 9.54\%; S: $14.56 \%$; Gefunden: C: $57.66 \%, \mathrm{H}: 6.06 \%$; N: $9.63 \%$; S: $14.89 \%$; $\mathbf{E}^{\mathbf{0}}\left(\mathbf{2 5}^{\circ} \mathbf{C}\right.$, $\left.\mathrm{Bu}_{4} \mathrm{NPF}_{6}, \mathbf{C V}, \mathrm{CH}_{3} \mathrm{CN}\right):-0.31 \mathrm{~V}\left(\mathrm{Fc} / \mathrm{Fc}^{+}\right) ; 0.06 \mathrm{~V}(\mathrm{SCE}) ; \mathrm{UV} / \mathrm{VIS}(\mathrm{MeOH}) \boldsymbol{\lambda}_{\max }[\mathrm{nm}] \boldsymbol{\varepsilon}$ $\left.\left[\mathbf{M}^{-1} \cdot \mathbf{c m}^{-1}\right]\right): 562.0$ (6730), 636.4 (5000) [Schulter] 


\subsubsection{Synthese von [WO(qdt) $)^{2-}(5 \mathrm{~b})$}

Die Darstellung erfolgte analog zur Synthese von 3a mit $\mathrm{K}_{3} \mathrm{Na}\left[\mathrm{WO}_{2}(\mathrm{CN})_{4}\right] \cdot 6 \mathrm{H}_{2} \mathrm{O}(0.44 \mathrm{~g}$, $0.77 \mathrm{mmol})$. Hierbei wurde die Reaktionsmischung für 15 Minuten auf $50^{\circ} \mathrm{C}$ erhitzt, es konnte nach Zugabe von $\mathrm{PPh}_{4} \mathrm{Cl}$ der Ausfall eines dunkelgrünen Feststoffs (0.27 g, 0.50 mmol, 60\%) beobachtet werden. Umkristallisation aus Acetonitril/Diethylether lieferte ein

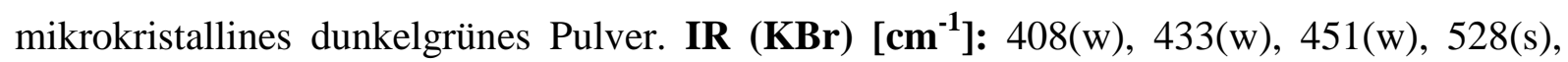
598(w), 690(m), 723(m), 756(m), 814(w), 918(s), 966(w), 1109(s), 1163(m), 1250(s), 1131(w), 1363(w), 1437(m), 1483(w), 1554(w), 1585(w), 1631(w), 1658(w), 2963(w), 3052(w), 3434(w); MS (FAB $\left.{ }^{-}, 3-N B A\right) ~ 584.0 ~\left[W O(q d t){ }_{2}\right]^{2-}(81)$; MS (FAB ${ }^{+}$, 3-NBA): 339.1

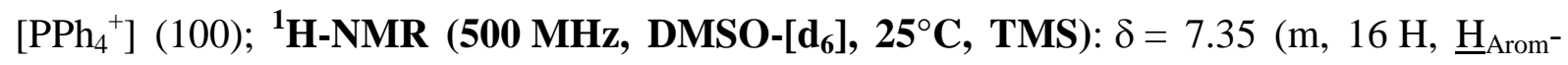
$\left.\mathrm{PPh}_{4}{ }^{+}\right), 7.43\left(\mathrm{~m}, 4 \mathrm{H}, \underline{\mathrm{H}}_{\text {Arom }}\right), 7.68\left(\mathrm{~m}, 24 \mathrm{H}, \underline{\mathrm{H}}_{\mathrm{Arom}^{-}}-\mathrm{PPh}_{4}{ }^{+}\right), 7.95$ (m, 4 H, $\left.\underline{\mathrm{H}}_{\text {Arom }}\right) \mathrm{ppm}$;

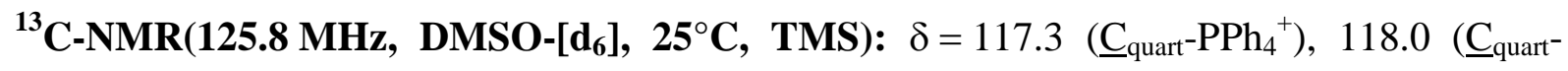
$\left.\mathrm{PPh}_{4}{ }^{+}\right), 128.7\left(\underline{\mathrm{C}}_{\mathrm{Arom}}-\mathrm{PPh}_{4}{ }^{+}\right), 128.6\left(\underline{\mathrm{C}}_{\mathrm{Arom}}-\mathrm{PPh}_{4}{ }^{+}\right), 131.9\left(\underline{\mathrm{C}}_{\mathrm{Arom}}-\mathrm{PPh}_{4}{ }^{+}\right), 131.4\left(\underline{\mathrm{C}}_{\mathrm{Arom}}-\mathrm{PPh}_{4}{ }^{+}\right)$, $130.3\left(\mathrm{C}-\underline{\mathrm{C}}_{\text {quart }}-\mathrm{PPh}_{4}{ }^{+}\right), 130.4\left(\underline{\mathrm{C}}-\mathrm{C}_{\text {quart }} \mathrm{PPh}_{4}{ }^{+}\right), 134.4(\mathrm{C}-\underline{\mathrm{C}}-\mathrm{N}), 134.5\left(\underline{\mathrm{C}}_{\text {Arom }}\right), 138.0(\mathrm{C}-\underline{\mathrm{C}}-$ N), 172.5 (C-S-W) ppm; EA: Berechnet für $\mathrm{C}_{64} \mathrm{H}_{48} \mathrm{~N}_{4} \mathrm{OP}_{2} \mathrm{~S}_{4} \mathrm{~W}: \mathrm{C}: 60.85 \% ; \mathrm{H}: 3.83 \%$; N: 4.44\% Gefunden: C: $60.57 \%$; H: $3.73 \%$; N: $4.42 \%$; $\mathbf{E}^{\mathbf{0}}\left(\mathbf{2 5}^{\circ} \mathbf{C}, \mathbf{B u}_{4} \mathbf{N P F}_{6}, \mathbf{D P V}, \mathbf{C H}_{3} \mathbf{C N}\right)$ : $0.67 \mathrm{~V}\left(\mathrm{Fc} / \mathrm{Fc}^{+}\right) ;-0.30 \mathrm{~V}(\mathrm{SCE}) ; \mathrm{UV} / \mathrm{VIS}(\mathbf{M e O H}) \lambda_{\max }[\mathbf{n m}]\left(\varepsilon\left[\mathbf{M}^{-\mathbf{1}} \cdot \mathbf{c m}^{-1}\right]\right): 628.0(2150)$, 717.6 (1710) [Schulter]

\subsubsection{Synthese von $\left[\mathrm{MoO}(\mathrm{ntdlt})_{2}\right]^{2-}(6 \mathrm{a})$}

Die Synthese wurde analog zu Darstellung von Verbindung 3a durchgeführt, wobei hier 4(Naphthalen-2-yl)-1,3-dithiol-2-on als Ligandensystem, sowie $\mathrm{NBu}_{4} \mathrm{Br}$ als Kationlieferanten eingesetzt wurde. Hierbei konnten $0.41 \mathrm{~g}, 040 \mathrm{~mol}$, 47\% eines dunkelroten Feststoffs nach Umkristallisation aus Acetonitril/ $\mathrm{Et}_{2} \mathrm{O}$ erhalten werden. IR (KBr) $\left[\mathbf{c m}^{-1}\right]$ : 472 (w), 503 (w), $546(\mathrm{w}), 651(\mathrm{w}), 679(\mathrm{w}), 669$ (w), $744(\mathrm{~m}), 787(\mathrm{~m}), 813(\mathrm{~m}), 856(\mathrm{w}), 891(\mathrm{~s}), 959(\mathrm{w}), 985$ (w), 1018 (w); 1063 (w), 1106 (w), 1123 (m), 1154 (m), 1204 (w), 1244 (s), 1309 (w), 1382 (w), 1428 (w), 1466 (m), 1478 (m), 1507 (m), 1570 (m), 1592 (m), 1622 (m), 1654 (w), 1701 (w), 1718 (w), 1734 (w), 2054 (w), 2340 (m), 2343 (m), 2361 (m), 2872 (m), 2930 (m), 2959 (m), 3051 (w), 3186 (w), 3232 (w), 3366 (w), 3398 (w), 3426 (w), 3428 (w); MS (FAB ${ }^{+}, 3-$ NBA) e/z (\%): $242.2(100)\left[\mathrm{NBu}_{4}{ }^{+}\right]$; MS (FAB, 3-NBA) e/z (\%):545.7 (100) [MoO(ntdt $\left.)_{2}\right]^{2}$;

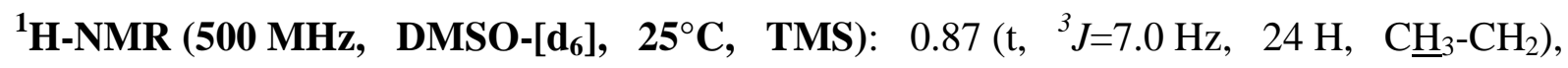
1.25 (sech, ${ }^{3} J=7.0 \mathrm{~Hz}, 16 \mathrm{H}, \underline{\mathrm{C}}_{2}-\mathrm{CH}_{3}$ ), 1.44 (quin, ${ }^{3} \mathrm{~J}=7.0 \mathrm{~Hz}, 16 \mathrm{H}, \mathrm{CH}_{2}-\mathrm{C}_{2}-\mathrm{CH}_{2}$ ), 3.06 (t, $\left.{ }^{3} J=8.5 \mathrm{~Hz}, 16 \mathrm{H}, \mathrm{N}-\underline{\mathrm{H}}_{2}\right), 6.99(\mathrm{~s}, 2 \mathrm{H}, \underline{\mathrm{HC}}=\mathrm{C}), 7.32\left(\mathrm{~d},{ }^{3} \mathrm{~J}=6.9 \mathrm{~Hz}, 2 \mathrm{H}, 3^{\prime}-\right.$ Naphtalin- $\left.\underline{\mathrm{H}}\right)$, 7.41-7.44 (m, 4 H, 6' $7^{\prime}-$ Naphtalin- $\left.\underline{\mathrm{H}}\right), 7.71-7.81$ (m, $2 \mathrm{H}, 4^{\prime}$-Naphtalin- $\left.\underline{\mathrm{H}}\right), 7.99$ (tt, 
${ }^{3} \mathrm{~J}=8.4 \mathrm{~Hz}, \quad 2 \mathrm{H}, \quad 1^{\prime}$-Naphtalin- $\left.\underline{\mathrm{H}}\right) \quad 8.35 \quad$ (m, $4 \mathrm{H}, \quad 5^{\prime}, 8^{\prime}$-Naphtalin- $\left.\underline{\mathrm{H}}\right) \quad$ ppm; $\quad{ }^{13} \mathbf{C}-\mathbf{N M R}$

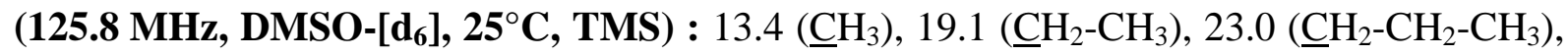
$57.4\left(\mathrm{~N}-\underline{\mathrm{CH}}_{2}\right), 118.0$ ( $\underline{\mathrm{C}}^{3}$-Naphtalin), 124.0 ( $\underline{\mathrm{C}}^{7^{\prime}}$-Naphtalin), 125.5 ( $\underline{\mathrm{C}}^{6^{\prime}}$-Naphtalin), 126.2

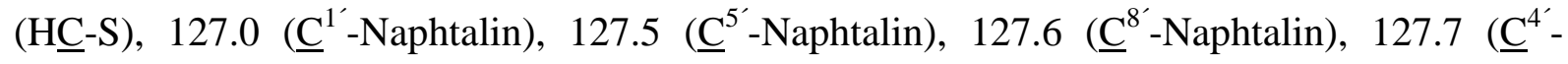
Naphtalin), 133.4 ( $\underline{\mathrm{C}}_{\text {qart }}-$ Naphtalin), 133.5 ( $\underline{\mathrm{C}}_{\text {qart }}$-Naphtalin), 133.6 ( $\left.\underline{\mathrm{C}}_{\text {qart }}-\mathrm{C}-\mathrm{S}\right), 152.6$ (C $\mathrm{C}_{\text {qart }}-\underline{\mathrm{C}}-$ S) ppm; EA: für $\mathrm{C}_{56} \mathrm{H}_{88} \mathrm{MoN}_{2} \mathrm{OS}_{4}$ Berechnet (\%): C: 65.33, H: 8.62, N: 2.72 S: 12.46; Gefunden (\%): C: $64.87, \mathrm{H}: 7.88 ; \mathrm{N}: 2.45 ; \mathrm{S}: 12.33 ; \mathbf{E}^{\mathbf{0}}\left(25^{\circ} \mathbf{C}, \mathbf{B u}_{4} \mathbf{N P F}_{6}, \mathbf{C V}, \mathbf{C H}_{3} \mathbf{C N}\right)$ : $0.88 \mathrm{~V}\left(\mathrm{Fc} / \mathrm{Fc}^{+}\right)$;

$-0.51 \mathrm{~V}(\mathrm{SCE})$

\subsubsection{Synthese von [WO(ntdt) $\left.{ }_{2}\right]^{2-}(6 \mathrm{~b})$}

Die Darstellung erfolgte analog zur Synthese von 3a mit $\mathrm{K}_{3} \mathrm{Na}\left[\mathrm{WO}_{2}(\mathrm{CN})_{4}\right] \cdot 6 \mathrm{H}_{2} \mathrm{O}(0.50 \mathrm{~g}$, $0.88 \mathrm{mmol})$. Hierbei wurde die Reaktionsmischung für $2 \mathrm{~h}$ auf $60^{\circ} \mathrm{C}$ erhitzt, es konnte nach Zugabe von $\left(\mathrm{Bu}_{4} \mathrm{~N}\right) \mathrm{Br}$ Bildung eines dunkelroten Öls beobachtet werden, dass nach Umkristallisation aus Acetonitril/Diethylether (0.38 g, $0.34 \mathrm{mmol}, 39 \%)$ einen dunkelroten

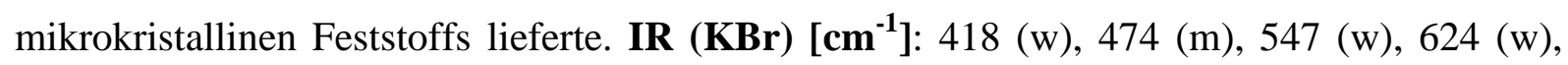
669 (w), 745 (s), 787 (s), 814 (s), 840 (m), 895 (vs), 943 (w), 983 (w), 1028 (w), 1065 (w), 1108 (w), 1123 (w), 1152 (w), 1168 (m), 1207 (w), 1243 (m), 1311 (w), 1345 (w), 1381 (s), 1471 (vs), 1501 (m), 1570 (w), 1593 (m), 1622 (m), 1671 (w), 1734 (w), 1923 (w), 2054 (m), 2340 (m), 2342 (m), 2361 (m), 2736 (w), 2873 (s), 2936 (s), 2960 (vs), 3051 (w), 3425 (w); MS [FAB $\left.{ }^{+}, 3-\mathrm{NBA}\right] \mathrm{e} / \mathbf{z}(\%): 242.2(100)\left[\mathrm{NBu}_{4}{ }^{+}\right]$; MS [FAB, 3-NBA] e/z (\%) : $631.6(95)$

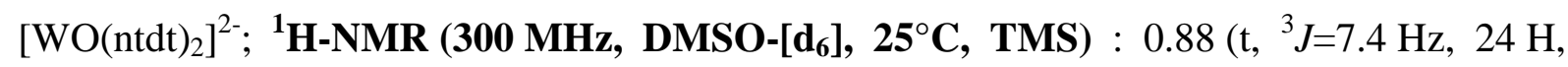
$\mathrm{CH}_{3}-\mathrm{CH}_{2}$ ), 1.28 (sech, $7.3 \mathrm{~Hz}, 16 \mathrm{H}, \underline{\mathrm{CH}}_{2}-\mathrm{CH}_{3}$ ), 1.49 (quin, ${ }^{3} \mathrm{~J}=7.4 \mathrm{~Hz}, 16 \mathrm{H}, \mathrm{CH}_{2}-\mathrm{CH}_{2}-\mathrm{CH}_{2}$ ), $3.11\left(\mathrm{t},{ }^{3} \mathrm{~J}=7.8 \mathrm{~Hz}, 16 \mathrm{H}, \mathrm{N}-\underline{\mathrm{H}}_{2}\right), 6.62(\mathrm{~s}, 2 \mathrm{H}, \underline{\mathrm{HC}}=\mathrm{C}), 7.26\left(\mathrm{~d},{ }^{3} J=2.6 \mathrm{~Hz}, 2 \mathrm{H}, 3^{\prime}-\right.$

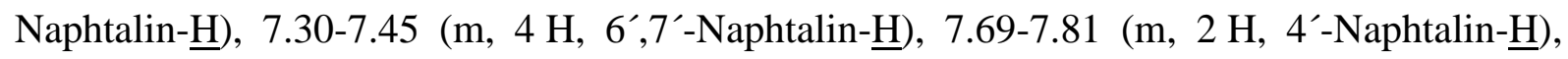
$8.02\left(\mathrm{tt}, \quad{ }^{3} \mathrm{~J}=8.7 \mathrm{~Hz}, \quad 2 \mathrm{H}, \quad 1^{\prime}\right.$-Naphtalin- $\left.\underline{\mathrm{H}}\right), \quad 8.38 \quad\left(\mathrm{~m}, \quad 4 \mathrm{H}, \quad 5^{\prime}, 8^{\prime}-\right.$ Naphtalin- $\left.\underline{\mathrm{H}}\right)$;

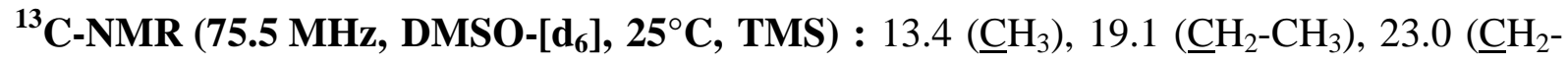
$\left.\mathrm{CH}_{2}-\mathrm{CH}_{3}\right), 57.4\left(\mathrm{~N}-\underline{\mathrm{CH}}_{2}\right), 117.9\left(\underline{\mathrm{C}}^{3}\right.$-Naphtalin), 123.1 ( $\underline{\mathrm{C}}^{7^{\prime}}$-Naphtalin), 123.6 ( $\underline{\mathrm{C}}^{6^{\prime}}$-Naphtalin), 125.5 (HC-S), 126.4 ( $\underline{\mathrm{C}}^{1^{\prime}}$-Naphtalin), 127.0 ( $\underline{\mathrm{C}}^{5}$-Naphtalin), 127.3 ( $\underline{\mathrm{C}}^{8^{\prime}}$-Naphtalin), 127.4

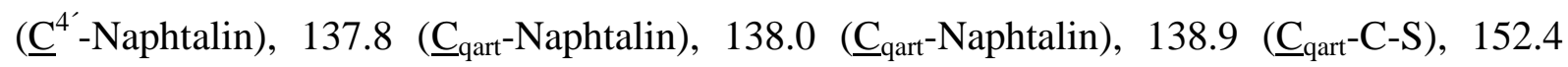
$\left(\mathrm{C}_{\text {qart-C}}-\mathrm{S}\right)$; EA: Berechnet für $\mathrm{C}_{56} \mathrm{H}_{88} \mathrm{~N}_{2} \mathrm{OS}_{4} \mathrm{~W}(\%)$ : C: 60.19, H: 7.94, N: 2.51; S: 11.48; Gefunden (\%): C: $60.38, \mathrm{H}: 8.01 ; \mathrm{N}: 2.62 ; \mathrm{S}: 11.52 ; \mathbf{E}^{\mathbf{0}}\left(\mathbf{2 5}^{\circ} \mathbf{C}, \mathbf{B u}_{\mathbf{4}} \mathbf{N P F}_{6}, \mathbf{C V}, \mathbf{C H}_{3} \mathbf{C N}\right)$ : $-0.92 \mathrm{~V}\left(\mathrm{Fc} / \mathrm{Fc}^{+}\right) ;-0.55 \mathrm{~V}(\mathrm{SCE})$ 


\subsubsection{Synthese von [MoO(tldt) 2$]^{2-}(7 \mathrm{a})$}

Die Synthese wurde analog zu Darstellung von Verbindung 3a durchgeführt, wobei hierbei 4,5-dihydronaphtho[1,2-d][1,3]dithiol-2-on als Ligandensystem sowie $\mathrm{Bu}_{4} \mathrm{NBr}$ eingesetzt wurde. Hierbei konnten $0.43 \mathrm{~g}(0.44 \mathrm{mmol}, 44 \%)$ eines dunkelroten Feststoffs nach

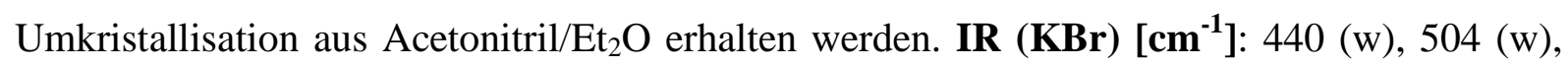
$578(\mathrm{w}), 610$ (w), 669 (w), 679 (w), 752 (s), 802 (w), 894 (s), 928 (w), $985(\mathrm{~m}), 1025(\mathrm{w})$, 1037 (w), 1063 (w), 1129 (m), 1155 (s), 1240 (vs), 1312 (w), 1383 (w), 1474 (m), 1532 (w), $1575(\mathrm{w}), 1592$ (w), 1625 (w), 2054 (m), 2340 (m), 2343 (m), 2361 (m), 2741 (w), 2813 (w),

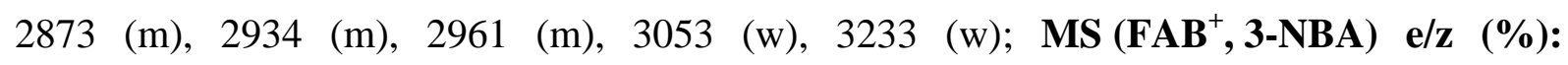
$242.3(100)\left[\mathrm{NBu}_{4}{ }^{+}\right] ; \quad \mathbf{M S}\left(\mathbf{F A B}{ }^{-}, \mathbf{3}-\mathbf{N B A}\right) \mathbf{e} / \mathbf{z}(\%): 497.7(100)\left[\mathrm{MoO}(\mathrm{tldt})_{2}\right] ; \quad{ }^{\mathbf{1}} \mathbf{H}-\mathbf{N M R}$

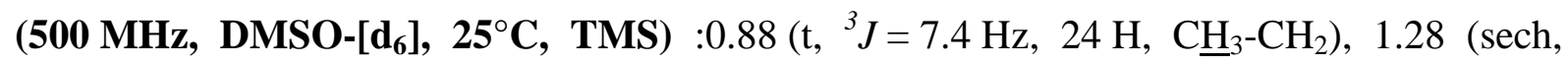
${ }^{3} J=7.4 \mathrm{~Hz}, 16 \mathrm{H}, \mathrm{CH}_{3}-\underline{\mathrm{CH}}_{2}$ ), 1.51 (quin, ${ }^{3} J=7.4 \mathrm{~Hz}, 16 \mathrm{H}, \mathrm{CH}_{2}-\mathrm{CH}_{2}-\mathrm{CH}_{3}$ ), 2.49 (t, $\left.{ }^{3} J=1.5 \mathrm{~Hz}, 4 \mathrm{H}, \mathrm{S}-\mathrm{C}-\mathrm{CH}_{2}\right), 2.69$ (m, $\left.4 \mathrm{H}, \mathrm{S}-\mathrm{C}-\mathrm{CH}_{2}-\mathrm{CH}_{2}\right), 3.13\left(\mathrm{t},{ }^{3} J=7.5 \mathrm{~Hz}, \mathrm{~N}-\underline{\mathrm{H}}_{2}\right), 6.84$ (m, $\left.2 \mathrm{H}, \underline{\mathrm{H}}_{\text {Arom }}\right), 7.04$ (m, $\left.2 \mathrm{H}, \underline{\mathrm{H}}_{\text {Arom }}\right), 7.09$ (m, $\left.2 \mathrm{H}, \underline{\mathrm{H}}_{\text {Arom }}\right), 7.89$ (m, $2 \mathrm{H}, \underline{\mathrm{H}}_{\text {Arom }}$ ) ppm;

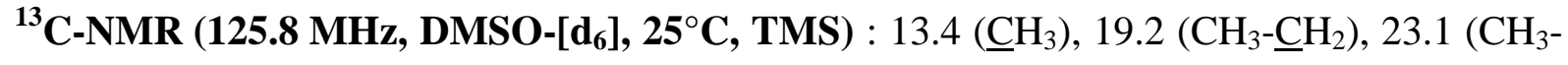
$\left.\mathrm{CH}_{2}-\underline{\mathrm{CH}}_{2}\right), 30.7\left(\mathrm{~S}-\mathrm{C}-\mathrm{CH}_{2}-\underline{\mathrm{CH}}_{2}\right), 34.2\left(\mathrm{~S}-\mathrm{C}-\underline{\mathrm{CH}}_{2}\right), 57.4\left(\mathrm{~N}-\underline{\mathrm{CH}}_{2}\right), 118.0\left(\mathrm{~S}-\underline{\mathrm{C}}-\mathrm{CH}_{2}\right), 122.4$ ( $\left.\underline{\mathrm{C}}_{\text {Arom }}\right), 122.9$ ( $\left.\underline{\mathrm{C}}_{\text {Arom }}\right), 125.6$ ( $\left.\underline{\mathrm{C}}_{\text {quart-Arom }}\right), 133.9$ ( $\left.\underline{\mathrm{C}}_{\text {Arom }}\right), 134.1$ ( $\left.\underline{\mathrm{C}}_{\text {Arom }}\right), 139.2$ ( $\left.\underline{\mathrm{C}}_{\text {quart-Arom }}\right)$, 142.5 ( $\left.\underline{\mathrm{C}}_{\text {quart }}-\mathrm{S}\right)$. ppm; EA: für $\mathrm{C}_{52} \mathrm{H}_{88} \mathrm{~N}_{2} \mathrm{OSS}_{4}$ Mo Berechnet (\%) C: 63.63, H: 9.04, N: 2.85; S: 13.07; Gefunden (\%): C: 62.88, H: 8.77; N: 2.79; S: $12.69 ; \mathbf{E}^{\mathbf{0}}\left(\mathbf{2 5}{ }^{\circ} \mathbf{C}, \mathbf{B u}_{4} \mathbf{N P F}_{6}, \mathbf{C V}\right.$, $\left.\mathbf{C H}_{3} \mathrm{CN}\right):-0.95 \mathrm{~V}\left(\mathrm{Fc} / \mathrm{Fc}^{+}\right) ;-0.58 \mathrm{~V}(\mathrm{SCE})$

\subsubsection{Synthese von [WO(tldt $\left.)_{2}\right]^{2-}(7 \mathrm{~b})$}

Die Darstellung erfolgte analog zur Synthese von 3a mit $\mathrm{K}_{3} \mathrm{Na}\left[\mathrm{WO}_{2}(\mathrm{CN})_{4}\right] \cdot 6 \mathrm{H}_{2} \mathrm{O}(0.52 \mathrm{~g}$, $0.92 \mathrm{mmol}$ ). Hierbei wurde die Reaktionsmischung für $2 \mathrm{~h}$ auf $60^{\circ} \mathrm{C}$ erhitzt, es konnte nach Zugabe von $\left(\mathrm{Bu}_{4} \mathrm{~N}\right) \mathrm{Br}$ die Bildung eines dunkelroten Öls beobachtet werden, das nach Umkristallisation aus Acetonitril/Diethylether (0.52 g, $0.49 \mathrm{mmol}, 53 \%)$ einen dunkelroten

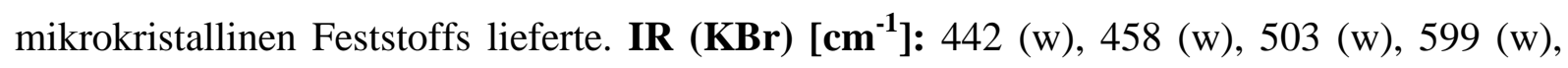
$669(\mathrm{w}), 679(\mathrm{w}), 698(\mathrm{w}), 751(\mathrm{~m}), 804(\mathrm{w}), 884(\mathrm{~s}), 985$ (s), 1033 (w), $1049(\mathrm{w}), 1065(\mathrm{w})$, 1127 (w), 1154 (m), 1245 (s), 1311 (m), 1384 (w), 1456 (m), 1478 (m), 1580 (w), 1533 (w), $1560(\mathrm{w}), 1577$ (w), 1654 (m), 1670 (w), 1701 (w), 1718 (w), 1741 (w), 2340 (m), 2343 (m),

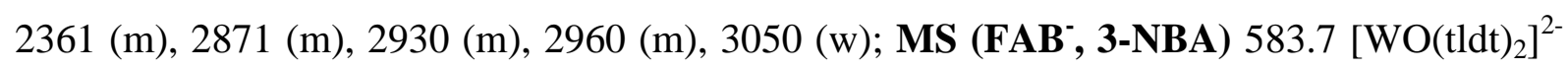
(41); MS (FAB $\left.{ }^{+}, \mathbf{3 - N B A}\right): 242.3\left[\mathrm{NBu}_{4}{ }^{+}\right](100) ;{ }^{\mathbf{1}} \mathbf{H}-\mathrm{NMR}\left(\mathbf{5 0 0} \mathrm{MHz}, \mathbf{D M S O}-\left[\mathbf{d}_{6}\right], \mathbf{2 5}^{\circ} \mathbf{C}\right.$, TMS) :0.90 (t, $\left.{ }^{3} J=7.0 \mathrm{~Hz}, 24 \mathrm{H}, \mathrm{C}_{3}-\mathrm{CH}_{2}\right), 1.27$ (sech, $\left.{ }^{3} J=7.4 \mathrm{~Hz}, 16 \mathrm{H}, \mathrm{CH}_{3}-\underline{\mathrm{H}}_{2}\right)$, 1.51 (quin, $\left.{ }^{3} \mathrm{~J}=7.5 \mathrm{~Hz}, 16 \mathrm{H}, \mathrm{C}_{2}-\mathrm{CH}_{2}-\mathrm{CH}_{3}\right), 2.05$ (m, $\left.4 \mathrm{H}, \mathrm{S}-\mathrm{C}_{\mathrm{qart}}-\mathrm{C}_{2}\right), 2.82\left(\mathrm{t},{ }^{3} \mathrm{~J}=8.5 \mathrm{~Hz}\right.$, 
$\left.4 \mathrm{H}, \mathrm{S}-\mathrm{C}_{\mathrm{qart}}-\mathrm{CH}_{2}-\mathrm{CH}_{2}\right), 3.12\left(\mathrm{t},{ }^{3} J=8.5 \mathrm{~Hz}, 16 \mathrm{H}, \mathrm{N}-\mathrm{C}_{2}\right), 6.89$ (m, $\left.2 \mathrm{H}, \underline{\mathrm{H}}_{\text {Arom }}\right), 7.09$ (m, $2 \mathrm{H}, \underline{\mathrm{H}}_{\text {Arom }}$ ), 738 (m, $2 \mathrm{H}, \underline{\mathrm{H}}_{\text {Arom }}$ ), 7.95 (m, $2 \mathrm{H}, \underline{\mathrm{H}}_{\text {Arom); }}{ }^{13}$ C-NMR (125.8 MHz, DMSO-[d 6 ], $\mathbf{2 5}^{\circ} \mathbf{C}$, TMS $): 13.3\left(\underline{\mathrm{CH}}_{3}\right), 19.1\left(\mathrm{CH}_{3}-\underline{\mathrm{CH}}_{2}\right), 23.1\left(\mathrm{CH}_{3}-\mathrm{CH}_{2}-\underline{\mathrm{CH}_{2}}\right), 30.8\left(\mathrm{~S}-\mathrm{C}_{-}-\mathrm{CH}_{2}-\underline{\mathrm{CH}}_{2}\right), 31.4$ $\left(\mathrm{S}-\mathrm{C}-\underline{\mathrm{CH}}_{2}\right), 57.5\left(\mathrm{~N}-\underline{\mathrm{CH}}_{2}\right), 117.9$ (ㄷH-S-), $123.7\left(\mathrm{CH}-\underline{\mathrm{C}}_{\text {qart }}-\mathrm{S}\right), 124.8$ ( $\left.\underline{\mathrm{C}}_{\text {Arom }}\right), 125.8$ ( $\left.\underline{\mathrm{C}}_{\text {Arom }}\right)$,

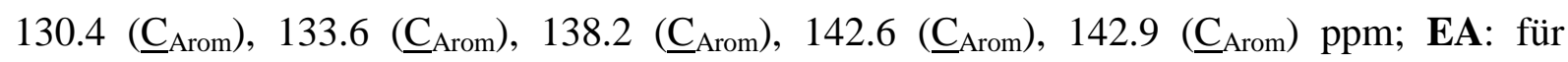
$\mathrm{C}_{52} \mathrm{H}_{88} \mathrm{~N}_{2} \mathrm{OSS}_{4} \mathrm{~W}$ Berechnet (\%): C: 58.40, H: 8.29, N: 2.62; S: 11.99; Gefunden (\%): C: 59.11, H: 8.53; N: 2.77; S: $12.55 ; \mathbf{E}^{\mathbf{0}}\left(\mathbf{2 5}^{\circ} \mathbf{C}, \mathbf{B u}_{4} \mathbf{N P F}_{6}, \mathbf{C V}, \mathbf{C H}_{3} \mathbf{C N}\right):-1.21 \mathrm{~V}\left(\mathrm{Fc} / \mathrm{Fc}^{+}\right) ;-0.84 \mathrm{~V}$ $(\mathrm{SCE})$

\subsubsection{Synthese von [MoO(tcdt) $]^{2-}(9 \mathrm{a})$}

Die Synthese wurde analog zu Darstellung von Verbindung 3a durchgeführt, wobei hierbei $4 H$-[1,3]dithiolo[4,5-c] thiochromen-2-on als Ligandensystem, sowie $\mathrm{Bu}_{4} \mathrm{NBr}$ eingesetzt wurde. Hierbei konnten nach Umkristallisation aus Acetonitril/ $\mathrm{Et}_{2} \mathrm{O} 0.49 \mathrm{~g}(0.48 \mathrm{mmol}, 48 \%)$ eines dunkelroten Feststoffs erhalten werden. IR (KBr) [ $\left.\mathbf{c m}^{-1}\right]$ : 504 (w), 607 (w), 669 (w), 753 (w), 802 (m), 896 (s), 1021 (m), 1104 (m), 1155 (m), 1242 (m), 1260 (m), 1384 (m), 1458 (w), 1474 (w), 1541 (w), $1560(\mathrm{w}), 1718$ (w), 1740 (w), 2161 (w), 2265 (w), 2298 (ws), 2343 (s), 2356 (s), 2401 (w), 2510 (m), 2632 (w, b), 2872 (m), 2929 (m), 2961 (m); MS (FAB $\left.{ }^{+}, \mathbf{3}-\mathbf{N B A}\right)$ e/z (\%): $242.3(42)\left[\mathrm{NBu}_{4}{ }^{+}\right], \quad 1018.3\left(\mathrm{NBu}_{4}\right)_{2}\left[\mathrm{MoO}(\mathrm{tcdt})_{2}\right](100) ; \quad \mathbf{M S}$

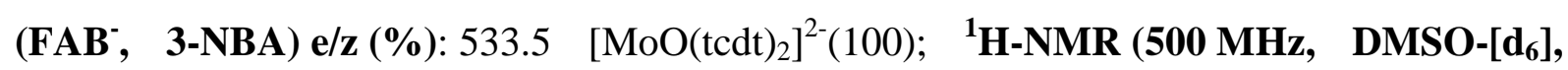
$\mathbf{2 5}^{\circ} \mathbf{C}$, TMS) :0.88 (t, $\left.{ }^{3} \mathrm{~J}=7.0 \mathrm{~Hz}, 24 \mathrm{H}, \mathrm{C}_{3}{ }^{-} \mathrm{CH}_{2}\right), 1.26$ (sech, $\left.{ }^{3} \mathrm{~J}=7.5 \mathrm{~Hz}, 16 \mathrm{H}, \mathrm{CH}_{3}-\underline{\mathrm{C}}_{2}\right)$, 1.48 (quin, ${ }^{3} J=7.5 \mathrm{~Hz}, 16 \mathrm{H}, \underline{\mathrm{CH}}_{2}-\mathrm{CH}_{2}-\mathrm{CH}_{3}$ ), 3.08 (t, ${ }^{3} \mathrm{~J}=8.5 \mathrm{~Hz}, \mathrm{~N}-\underline{\mathrm{H}}_{2}$ ), 3.79 (s, $2 \mathrm{H}, \mathrm{C} \underline{\mathrm{H}}-$ S-), 3.89 (s, 2 H, C$-\mathrm{H}-\mathrm{S}$ ), 6.88 (m, 2 H, $\left.\underline{\mathrm{H}}_{\text {Arom }}\right), 7.07$ (m, $\left.2 \mathrm{H}, \underline{\mathrm{H}}_{\text {Arom }}\right), 7.18$ (m, $2 \mathrm{H}, \underline{\mathrm{H}}_{\text {Arom }}$ ),

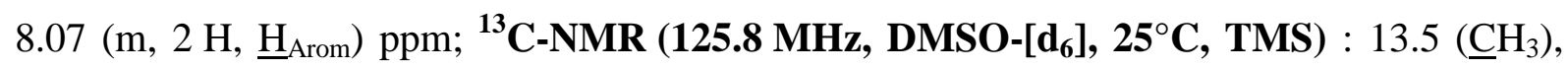
$19.1\left(\mathrm{CH}_{3}-\underline{\mathrm{CH}}_{2}\right), 23.0\left(\mathrm{CH}_{3}-\mathrm{CH}_{2}-\underline{\mathrm{CH}}_{2}\right), 57.3\left(\mathrm{~N}-\underline{\mathrm{CH}}_{2}\right), 118.0$ (ㄷH-S-), 123.4 (CH- $\left.\underline{\mathrm{Cart}}-\mathrm{S}\right)$, $124.6\left(\underline{\mathrm{C}}_{\text {Arom }}\right), 125.2$ ( $\left.\underline{\mathrm{C}}_{\text {Arom }}\right), 129.5\left(\underline{\mathrm{C}}_{\text {Arom }}\right), 135.6\left(\underline{\mathrm{C}}_{\text {Arom }}\right), 136.6\left(\underline{\mathrm{C}}_{\text {Arom }}\right), 138.1$ ( $\left.\underline{\mathrm{C}}_{\text {Arom }}\right) \mathrm{ppm}$; EA: für $\mathrm{C}_{50} \mathrm{H}_{84} \mathrm{MoN}_{2} \mathrm{OS}_{6}$ Berechnet (\%): C: 59.02; H: 8.32; N: 2.75; S: 18.91; Gefunden (\%): C: 58.77; H: 8.08; N: 2.65; S: 18.52; $\mathbf{E}^{\mathbf{0}}\left(\mathbf{2 5}^{\circ} \mathbf{C}, \mathbf{B u}_{4} \mathbf{N P F}_{6}, \mathbf{C V}, \mathbf{C H}_{3} \mathbf{C N}\right):-0.87 \mathrm{~V}\left(\mathrm{Fc}_{\mathrm{Fc}^{+}}\right)$; $-0.50 \mathrm{~V}(\mathrm{SCE})$.

\subsubsection{Synthese von [WO(tcdt) 2$]^{2-}(9 \mathrm{~b})$}

Die Darstellung erfolgte analog zur Synthese von 3a mit $\mathrm{K}_{3} \mathrm{Na}\left[\mathrm{WO}_{2}(\mathrm{CN})_{4}\right] \cdot 6 \mathrm{H}_{2} \mathrm{O}(0.60 \mathrm{~g}$, $1.06 \mathrm{mmol})$. Hierbei wurde die Reaktionsmischung für $2 \mathrm{~h}$ auf $60^{\circ} \mathrm{C}$ erhitzt. Es konnte nach Zugabe von $\left(\mathrm{Bu}_{4} \mathrm{~N}\right) \mathrm{Br}$ die Bildung eines dunkelroten Öls beobachtet werden, das nach 
Umkristallisation aus Acetonitril/Diethylether (0.46 g, $0.41 \mathrm{mmol}, 39 \%)$ einen dunkelroten mikrokristallinen Feststoff lieferte. IR (KBr) [ $\mathbf{c m}^{-1}$ ]: 504 (w), 623 (w), 669 (w), 750 (w), 802 (m), 876 (s), 985 (w), 1022 (w), 1108 (w), 1154 (w), 1260 (m), 1309 (w), 1384 (s), 1462 (m), 1537 (w), 1569 (w), 2054 (w), 2349 (s), 2382 (s), 2850 (m), 2919 (m), 2962 (m); MS (FAB ${ }^{+}$, 3-NBA) e/z (\%): $242.3(30)\left[\mathrm{NBu}_{4}{ }^{+}\right] 1120.4\left(\mathrm{NBu}_{4}\right)_{2}\left[\mathrm{WO}(\mathrm{tcdt})_{2}\right]$ (80); MS (FAB $\left.{ }^{-}, 3-\mathbf{N B A}\right)$

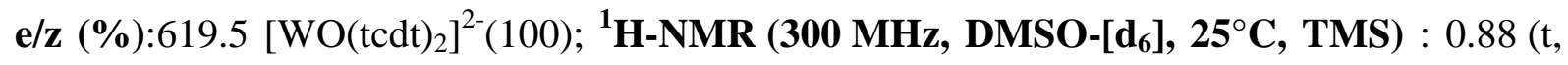
${ }^{3} J=7.4 \mathrm{~Hz}, \quad 24 \mathrm{H}, \quad \mathrm{CH}_{3}-\mathrm{CH}_{2}$ ), 1.26 (sech, ${ }^{3} J=7.2 \mathrm{~Hz}, 16 \mathrm{H}, \mathrm{CH}_{3}-\mathrm{CH}_{2}$ ), 1.48 (quin, $\left.{ }^{3} J=7.2 \mathrm{~Hz}, 16 \mathrm{H}, \mathrm{C}_{2}-\mathrm{CH}_{2}-\mathrm{CH}_{3}\right), 3.09$ (t, $\left.{ }^{3} \mathrm{~J}=8.4 \mathrm{~Hz}, 16 \mathrm{H} \mathrm{N}-\underline{\mathrm{H}}_{2}\right), 3.91$ (s, $2 \mathrm{H}, \mathrm{C} \underline{\mathrm{H}}-\mathrm{S}-$ ), 3.99 (s, 2 H, C$-S-), 6.89$ (m, 2 H, $\underline{\mathrm{H}}_{\text {Arom }}$ ), 7.07 (m, 2 H, $\underline{\mathrm{H}}_{\text {Arom }}$ ), 7.24 (m, $2 \mathrm{H}, \underline{\mathrm{H}}_{\text {Arom }}$ ), 8.11

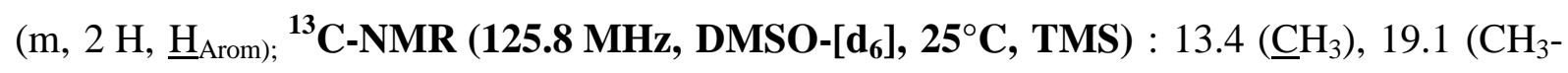
$\left.\underline{\mathrm{CH}}_{2}\right), 23.1\left(\mathrm{CH}_{3}-\mathrm{CH}_{2}-\underline{\mathrm{CH}}_{2}\right), 57.4\left(\mathrm{~N}-\underline{\mathrm{CH}}_{2}\right), 117.9$ ( $\left.\underline{\mathrm{CH}}-\mathrm{S}-\right), 123.7\left(\mathrm{CH}-\underline{\mathrm{C}}_{\mathrm{qart}}-\mathrm{S}\right), 124.8$ ( $\left.\underline{\mathrm{C}}_{\text {Arom }}\right)$, 125.8 ( $\left.\underline{\mathrm{C}}_{\text {Arom }}\right), 130.4$ ( $\left.\underline{\mathrm{C}}_{\text {Arom }}\right), 138.2$ ( $\left.\underline{\mathrm{C}}_{\text {Arom }}\right), 142.6$ ( $\left.\underline{\mathrm{C}}_{\text {Arom }}\right), 142.9$ ( $\left.\underline{\mathrm{C}}_{\text {Arom }}\right)$ ppm; EA: für $\mathrm{C}_{50} \mathrm{H}_{84} \mathrm{~N}_{2} \mathrm{OSS}_{6} \mathrm{~W}$ Berechnet (\%):C: 54.33, H: 7.66, N: 2.53; S: 17.40; Gefunden (\%): C: 53.47, H: 7.08; N: 2.25; S:17.33; $\mathbf{E}^{\mathbf{0}}\left(25^{\circ} \mathbf{C}, \mathbf{B u}_{4} \mathbf{N P F}_{6}, \mathbf{C V}, \mathbf{C H}_{3} \mathbf{C N}\right):-1.19 \mathrm{~V}\left(\mathrm{Fc} / \mathrm{Fc}^{+}\right) ;-0.82 \mathrm{~V}$ $(\mathrm{SCE})$

\subsubsection{Synthese von [MoO(cydt) $]^{2-}(10 \mathrm{a})$}

Die Synthese wurde analog zu Darstellung von Verbindung 3a durchgeführt, wobei 4,6,5,7tetrahydrobenzo[d][1,3]dithiol-2-on als Ligandensystem eingesetzt wurde. Es wurden nach Umkristallisation aus Acetonitril/ $\mathrm{Et}_{2} \mathrm{O} 0.84 \mathrm{~g}$ (1.06 mmol, 67\%) eines dunkelroten Feststoffs

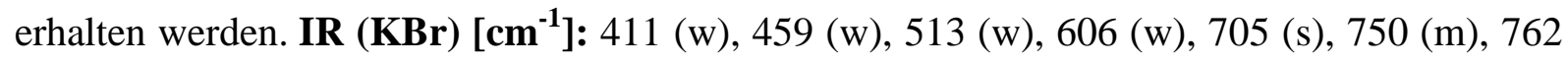
(m), 803 (m), 877 (s), 939 (w), 1024 (m), 1101 (m), 1117 (m), 1153 (s), 1211 (w), 1245 (s), 1315 (w), 1385 (s), 1471, (s), 1498 (w), 1616 (m), 2053 (w), 2223 (m), 2359 (w), 2823 (w), 2855 (w), 2923 (s), 2965 (w), 3004 (w), 3063 (w); MS (FAB ${ }^{+}$, 3-NBA) e/z (\%): 192.2 (100) $\left[\mathrm{BzNEt}_{3}{ }^{+}\right]$; MS (FAB, 3-NBA) e/z (\%): 401.9 (79) ([MoO(cydt) $\left.\left.{ }_{2}\right]^{2-}\right) ;{ }^{1} \mathbf{H}-\mathbf{N M R}$ (500 MHz, DMSO-[d $\left.\left.\mathbf{d}_{6}\right], \mathbf{2 5}^{\circ} \mathbf{C}, \mathbf{T M S}\right): \delta=1.23\left(\mathrm{t},{ }^{3} \mathrm{~J}=2.7 \mathrm{~Hz}, 18 \mathrm{H}, \mathrm{C}_{3}-\mathrm{CH}_{2}-\mathrm{N}\right), 1.76(\mathrm{~m}, 8 \mathrm{H}, \mathrm{cy}-\underline{\mathrm{H}})$,

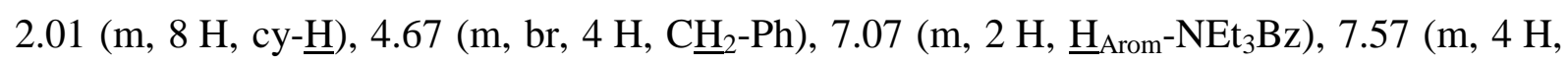
$\left.\underline{\mathrm{H}}_{\text {Arom }}-\mathrm{NEt}_{3} \mathrm{Bz}\right), 8.36\left(\mathrm{~m}, 4 \mathrm{H}, \underline{\mathrm{H}}_{\text {Arom }}-\mathrm{NEt}_{3} \mathrm{Bz}\right)$ ppm. EA: für $\mathrm{C}_{38} \mathrm{H}_{60} \mathrm{MoN}_{2} \mathrm{~S}_{4}$ Berechnet (\%):C: 58.13, H: 7.70, N: 3.57; S: 16.34; Gefunden (\%):C: 57.79; H: 7.63; N:3.45; 16.72; $\mathbf{E}^{\mathbf{0}}\left(\mathbf{2 5}^{\circ} \mathbf{C}\right.$, $\left.\mathrm{Bu}_{4} \mathrm{NPF}_{6}, \mathbf{D P V}, \mathrm{CH}_{3} \mathrm{CN}\right):-0.98 \mathrm{~V}\left(\mathrm{Fc} / \mathrm{Fc}^{+}\right) ;-0.61 \mathrm{~V}(\mathrm{SCE}) ; \mathrm{UV} / \mathrm{VIS}(\mathrm{MeOH}) \boldsymbol{\lambda}_{\max }[\mathrm{nm}](\varepsilon$ $\left.\left[\mathbf{M}^{-1} \cdot \mathbf{c m}^{-1}\right]\right): 480$ (987), 569.0 (1555) 


\subsubsection{Synthese von [WO(cydt) $]^{2-}(10 \mathrm{~b})$}

Die Darstellung erfolgte analog zur Synthese von 3a mit $\mathrm{K}_{3} \mathrm{Na}\left[\mathrm{WO}_{2}(\mathrm{CN})_{4}\right] \cdot 6 \mathrm{H}_{2} \mathrm{O}(0.42 \mathrm{~g}$, $0.74 \mathrm{mmol})$. Hierbei wurde die Reaktionsmischung für $4 \mathrm{~h}$ auf $60^{\circ} \mathrm{C}$ erhitzt, nach Zugabe des Kations konnte die Bildung eines dunkelroten Öls beobachtet werden, dass nach Umkristallisation aus Acetonitril/Diethylether (0.12 g, $0.14 \mathrm{mmol}, 19 \%)$ eines dunkelroten Feststoffs lieferte. IR (KBr) [ $\mathbf{c m}^{-1}$ ]: 418 (w), 441 (w), 506 (w), 555 (w), 626 (w), 669 (w), $679(\mathrm{w}), 741$ (m), 802 (m), 888 (s), 925 (w), 985 (w), 1023 (m), 1055 (w), 1107 (w), 1128 (w), 1154 (m), 1243 (s), 1310 (w), 1382 (m), 1466 (s), 1541 (w), 1560 (w), 1625 (w), 1670 (w), 1701 (w), 1735 (w), 2054 (s), 2340 (w), 2344 (w), 2361 (w), 2737 (w), 2873 (s), 2934

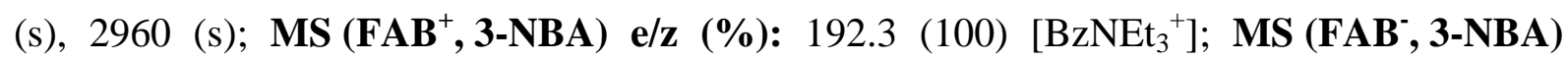

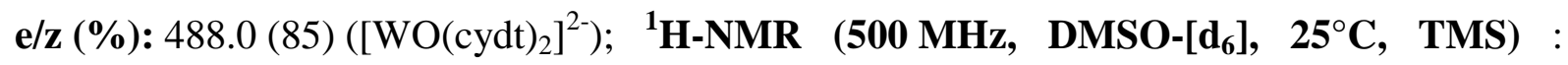
$\delta=1.25\left(\mathrm{t},{ }^{3} J=2.7 \mathrm{~Hz}, 18 \mathrm{H}, \underline{C}_{3}-\mathrm{CH}_{2}-\mathrm{N}\right), 1.78$ (m, $\left.8 \mathrm{H}, \mathrm{cy}-\underline{\mathrm{H}}\right), 2.09$ (m, $\left.8 \mathrm{H}, \mathrm{cy}-\underline{\mathrm{H}}\right), 4.68$ (m, br, $\left.4 \mathrm{H}, \underline{\mathrm{C}}_{2}-\mathrm{Ph}\right), 7.10$ (m, $\left.2 \mathrm{H}, \underline{\mathrm{H}}_{\mathrm{Arom}^{-}}-\mathrm{NEt}_{3} \mathrm{Bz}\right), 7.58$ (m, $\left.4 \mathrm{H}, \underline{\mathrm{H}}_{\mathrm{Arom}}-\mathrm{NEt}_{3} \mathrm{Bz}\right), 8.37$ (m, $4 \mathrm{H}$,

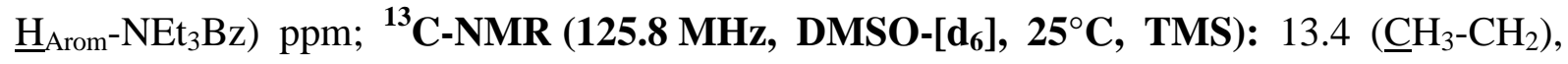
$19.1\left(\underline{\mathrm{CH}}_{2}-\mathrm{CH}_{2}-\mathrm{CH}_{2}-\underline{\mathrm{CH}}_{2}\right), 22.9\left(\mathrm{CH}_{2}-\underline{\mathrm{CH}_{2}}-\underline{\mathrm{CH}}_{2}-\mathrm{CH}_{2}\right), 57.5\left(\mathrm{CH}_{3}-\underline{\mathrm{CH}}_{2}\right), 61.5\left(\underline{\mathrm{CH}}_{2} \mathrm{Ph}\right), 97.7$ ( $\left.\underline{\mathrm{C}}_{\text {Arom}}\right), 117.9(\underline{\mathrm{C}}=\underline{\mathrm{C}}), 129.4\left(\underline{\mathrm{C}}_{\text {qart-Arom }}\right)$ ppm; EA: für $\mathrm{C}_{38} \mathrm{H}_{60} \mathrm{~N}_{2} \mathrm{OS}_{4} \mathrm{~W}$ Berechnet $(\%)$ : C: 52.28, H: 6.93 N: 3.21; S: 14.69; Gefunden (\%): C: 53.18, H: 7.43; N: 3.43; S: 14.82; $\mathbf{E}^{\mathbf{0}}\left(\mathbf{2 5}^{\circ} \mathbf{C}, \mathbf{B u}_{4} \mathrm{NPF}_{6}, \mathbf{D P V}, \mathbf{C H}_{3} \mathrm{CN}\right):-1.39 \mathrm{~V}\left(\mathrm{Fc} / \mathrm{Fc}^{+}\right) ;-1.02 \mathrm{~V}(\mathrm{SCE})$

Die nötigen Natriumsalze bzw. Lithiumsalze der Ligandensysteme wurde durch Reaktion des Dithiolenligandens mit NaH oder n-BuLi erzeugt. ${ }^{[132]}$

\subsubsection{Synthese von [Mo(tdt $\left.)_{2}\left(\mathrm{OEt}_{2}\right)_{2}\right](11 \mathrm{a})$}

$0.24 \mathrm{~g}(1.20 \mathrm{mmol})$ des Natriumsalzes vom tdt-Ligand wurde unter Stickstoffatmosphäre als Feststoff mit $0.25 \mathrm{~g}, 1.05 \mathrm{mmol}\left[\mathrm{MoCl}_{4}\left(\mathrm{OEt}_{2}\right)_{2}\right]$ vermischt. Die beiden Feststoffe wurden nun bei RT $5 \mathrm{~d}$ ohne Lösungsmittel gerührt, dabei konnte die Bildung eines dunkelgrünen Feststoffs beobachtet werden. Das erhaltene Feststoffgemisch wurde nun auf $-60^{\circ} \mathrm{C}$ herunter gekühlt und langsam mit ebenfalls auf $-60^{\circ} \mathrm{C}$ vorgekühltem Pentan (abs.) (10 ml) versetzt. Nun wurde sehr schnell durch eine mit Trockeneis gekühlte Fritte filtriert. Das Filtrat wurde in der Kälte bei $-20^{\circ} \mathrm{C}$ im HV vom Lösungsmittel entfernt. Es konnten $0.17 \mathrm{~g}(0.42 \mathrm{mmol}$, 40\%) eines dunkelgrünen Feststoffs isoliert werden. IR (KBr) [ $\left.\mathbf{c m}^{-1}\right]: 573$ (s), 690 (m), 805 (m), 869 (w), 985 (s), 1032 (w), 1077 (m), 1155 (w), 1204 (w), 1242 (m), 1308 (w), 1377 (w) 1456 (w), 1509 (w), 1636 (s), 2042 (w), 2137 (w), 2360 (w), 2929 (w), 2960 (w); MS [EI,

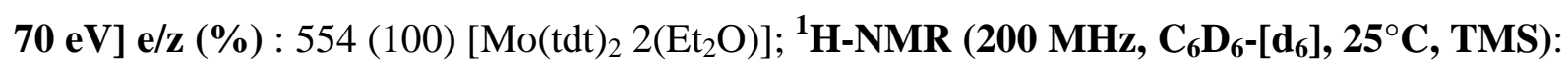
$1.13\left(\mathrm{t}, 12 \mathrm{H},{ }^{3} \mathrm{~J}=2.8 \mathrm{~Hz}, \mathrm{CH}_{2} \underline{\mathrm{CH}}_{3}\right), 2.11$ (s, $6 \mathrm{H}, \underline{\mathrm{CH}}_{3}-\mathrm{C}$ ), 3.24 (q, $8 \mathrm{H},{ }^{3} \mathrm{~J}=3.3 \mathrm{~Hz}, \mathrm{C}_{2}-\mathrm{CH}_{3}$ ), 
$6.96\left(\mathrm{~m}, 2 \mathrm{H}, \underline{\mathrm{H}}_{\text {Arom }}\right), 7.02\left(\mathrm{~m}, 2 \mathrm{H}, \underline{\mathrm{H}}_{\text {Arom }}\right), 7.06\left(\mathrm{~m}, 2 \mathrm{H}, \underline{\mathrm{H}}_{\text {Arom }}\right)$ ppm; $\mathbf{E A}:$ für $\mathrm{C}_{22} \mathrm{H}_{32} \mathrm{MoO}_{2} \mathrm{~S}_{4}$ - $\mathrm{C}_{8} \mathrm{H}_{20} \mathrm{O}_{2}$, Berechnet (\%):C: 41.58, H: 2.99; S: 31.71; Gefunden (\%): C: 41.77, H: 3.13; S: 32.08; $\mathbf{E}^{\mathbf{0}}\left(\mathbf{2 5}^{\circ} \mathbf{C}, \mathbf{B u}_{4} \mathbf{N P F}_{6}, \mathbf{C V}, \mathbf{C H}_{2} \mathbf{C l}_{2}\right):-0.11 \mathrm{~V}\left(\mathrm{Fc} / \mathrm{Fc}^{+}\right) ; 0.26 \mathrm{~V}$ (SCE)

\subsubsection{Synthese von [W(tdt $\left.)_{2}\left(\mathrm{OEt}_{2}\right)_{2}\right](11 \mathrm{~b})$}

$0.20 \mathrm{~g}, 0.61 \mathrm{mmol} \mathrm{WCl}_{4}$ wurden mit dem Natriumsalz des tdt-Ligandens $(0.24 \mathrm{~g}, 1.22 \mathrm{mmol})$ unter Argonatmosphäre ohne Lösungsmittel versetzt. Beide Feststoffe wurden für 30 Min gerührt. Nun wurde auf $-30^{\circ} \mathrm{C}$ abgekühlt und es wurde mit $10 \mathrm{ml}$ ebenfalls auf $-30^{\circ} \mathrm{C}$ gekühltem $\mathrm{Et}_{2} \mathrm{O}$ (abs.) versetzt. Es konnte die Bildung einer grünen Lösung beobachtet werden. Es wurde nun 30 Min bei dieser Temperatur gerührt. Nach Filtration wurde das Lösungsmittel des Filtrats entfernt. Dieser resultierende Feststoff wurde in einer Mischung aus Hexan und $\mathrm{Et}_{2} \mathrm{O}$ gelöst, durch einen mit Kieselgel gefüllten Filter gegeben und mit der Lösungsmittelmischung nachgewaschen. Die erste leicht gelbliche Fraktion wurde verworfen. Nachdem die filtrierende Lösung ein dunkelgrünes Erscheinungsbild bekam, wurde die Fraktion gesammelt und nach vollständigem Auffangen vom Lösungsmittel entfernt. Es konnten $0.34 \mathrm{~g}, 0.53 \mathrm{mmol}, 87 \%$ eines dunkelgrünen Feststoffs erhalten werden. IR (KBr) [cm ${ }^{-1}$ ]: $444(\mathrm{w}), 552(\mathrm{w}), 637$ (m), 689 (m), $842(\mathrm{ws}), 958$ (w), $984(\mathrm{w}), 1034(\mathrm{~m}), 1101(\mathrm{w})$, 1155 (w), 1208 (w), 1245 (m), 1308 (w), 1378 (w), 1450 (s), 1579 (w), 1637 (m), 2102 (w), 2270 (w), 2378 (w), 2855 (w), 2924 (m); MS [EI, $70 \mathbf{~ e V ] ~ e / z ~ ( \% ) ~ : ~} 492$ (65) [W(tdt) $)_{2}$ 2(Et $2 \mathrm{O})$ ]; ${ }^{\mathbf{1}} \mathbf{H}$-NMR (500 MHz, $\mathbf{C}_{\mathbf{6}} \mathbf{D}_{\mathbf{6}}-\left[\mathbf{d}_{\mathbf{6}}\right], \mathbf{2 5}^{\circ} \mathbf{C}$, TMS): $1.17\left(\mathrm{t}, 12 \mathrm{H},{ }^{3} \mathrm{~J}=3.0 \mathrm{~Hz}, \mathrm{CH}_{2} \mathrm{CH}_{3}\right)$, 2.15 (s, $6 \mathrm{H}, \underline{\mathrm{CH}}_{3}-\mathrm{C}$ ), 3.26 (q, $8 \mathrm{H},{ }^{3} \mathrm{~J}=3.0 \mathrm{~Hz}, \underline{\mathrm{C}}_{2}-\mathrm{CH}_{3}$ ), 7.04 (m, $2 \mathrm{H}, \underline{\mathrm{H}}_{\mathrm{Arom}}$ ), 7.08 (m, $2 \mathrm{H}$, $\underline{\mathrm{H}}_{\text {Arom }}$ ), $7.17\left(\mathrm{~m}, 2 \mathrm{H}, \underline{\mathrm{H}}_{\text {Arom }}\right.$ ) ppm; EA: für $\mathrm{C}_{22} \mathrm{H}_{32} \mathrm{O}_{2} \mathrm{~S}_{4} \mathrm{~W}-\mathrm{C}_{8} \mathrm{H}_{20} \mathrm{O}_{2}$, Berechnet (\%):C: 41.25 , H: 5.04; S: 20.02; Gefunden (\%): C: $41.88, \mathrm{H}: 5.42 ; \mathrm{S}: 20.41 ; \mathbf{E}^{\mathbf{0}}\left(\mathbf{2 5 ^ { \circ }} \mathbf{C}, \mathbf{B u}_{4} \mathbf{N P F}_{6}, \mathbf{C V}\right.$, $\left.\mathbf{C H}_{\mathbf{2}} \mathbf{C l}_{\mathbf{2}}\right):-0.29 \mathrm{~V}\left(\mathrm{Fc} / \mathrm{Fc}^{+}\right) ; 0.08 \mathrm{~V}(\mathrm{SCE})$

\subsubsection{Synthese von [Mo(xdt) $\left.)_{2}\left(\mathrm{OEt}_{2}\right)_{2}\right]$ (11c)}

Die Darstellung dieser Verbindung erfolgte analog zur Synthesemethode von 11a, hierbei wurde nur statt des Natriumsalzes des 3,4-Toluoldithiolligandens, der 3,4Xyloldithiolliganden verwendet. Hierbei konnten $0.11 \mathrm{~g}, 0.19 \mathrm{mmol}, 33 \%$ eines dunkelgrünen Feststoffs erhalten werden. IR (KBr) [ $\mathbf{c m}^{-1}$ ]: 423 (w), 440 (w), 503 (m), 541 (w), 645 (w), 690 (w), 747 (w), 831 (s), 857 (s), 882 (s), 994 (m), 1022 (w), 1047 (w), 1125 (w), 1155 (m), 1245 (s), 1283 (m), 1309 (m), 1342 (m), 1372 (m), 1394 (m), 1447 (s), 1502 (m), 1607 (w), 2266 (w), 2391 (w), 2391 (w), 2862 (w), 2933 (w), 2977 (w); MS [EI, 70 eV] e/z (\%) : 434 (88) $\left[\mathrm{Mo}\left(\mathrm{xdt}_{2}\right)_{2}-2\left(\mathrm{Et}_{2} \mathrm{O}\right)\right] ;{ }^{\mathbf{1}} \mathbf{H}-\mathbf{N M R}\left(\mathbf{5 0 0} \mathbf{~ M H z}, \mathbf{C}_{\mathbf{6}} \mathbf{D}_{\mathbf{6}}-\left[\mathbf{d}_{\mathbf{6}}\right], \mathbf{2 5}^{\circ} \mathbf{C}, \mathbf{T M S}\right): 1.12(\mathrm{t}, 12 \mathrm{H}$, ${ }^{3} J=2.8 \mathrm{~Hz}, \mathrm{CH}_{2} \mathrm{CH}_{3}$ ), 2.10 (s, $12 \mathrm{H}, \underline{\mathrm{CH}}_{3}-\mathrm{C}$ ), 3.25 (q, $\left.8 \mathrm{H},{ }^{3} J=3.3 \mathrm{~Hz}, \mathrm{C}_{2}-\mathrm{CH}_{3}\right), 7.12$ (s, $2 \mathrm{H}$, 
$\underline{\mathrm{H}}_{\text {Arom}}$ ) ppm; EA: für $\mathrm{C}_{24} \mathrm{H}_{36} \mathrm{MoO}_{2} \mathrm{~S}_{4}-\mathrm{C}_{4} \mathrm{H}_{10} \mathrm{O}$, Berechnet (\%):C: 47.42, H: 5.17; S: 25.32; Gefunden (\%): C: 48.39, H: 5.78; S: 25.46; $\mathbf{E}^{\mathbf{0}}\left(\mathbf{2 5}{ }^{\circ} \mathbf{C}, \mathbf{B u}_{4} \mathbf{N P F}_{6}, \mathbf{C V}, \mathbf{C H}_{3} \mathbf{C N}\right)$ : $-0.34 \mathrm{~V}\left(\mathrm{Fc} / \mathrm{Fc}^{+}\right) ; 0.03 \mathrm{~V}(\mathrm{SCE})$

\subsubsection{Synthese von [W(xdt $\left.)_{2}\left(\mathrm{OEt}_{2}\right)_{2}\right](11 \mathrm{~d})$}

Die Darstellung dieser Verbindung erfolgte analog zur Synthesemethode von 11b, hierbei wurde nur statt des Natriumsalzes des 3,4-Toluoldithiolligandens, der 3,4Xyloldithiolliganden verwendet. Hierbei konnten $0.13 \mathrm{~g}, 0.19 \mathrm{mmol}, 14 \%$ eines dunkelgrünen Feststoffs erhalten werden. IR (KBr) [ $\mathbf{c m}^{-1}$ ]: 423 (w), 440 (w), 503 (m), 541 (w), 645 (w), 690 (w), 747 (w), 831 (s), 857 (s), 882 (s), 994 (m), 1022 (w), 1047 (w), 1125 (w), 1155 (m), 1245 (s), 1283 (m), 1309 (m), 1342 (m), 1372 (m), 1394 (m), 1447 (s), 1502 (m), 1607 (w), 2266 (w), 2391 (w), 2391 (w), 2862 (w), 2933 (w), 2977 (w); MS [EI, 70 eV] e/z (\%) : 434 (55) $\left[\mathrm{Mo}\left(\mathrm{xdt}_{2}\right)_{2}-2\left(\mathrm{Et}_{2} \mathrm{O}\right)\right]$; ${ }^{1} \mathbf{H}-\mathbf{N M R}\left(\mathbf{2 0 0} \mathbf{M H z}, \mathbf{C}_{\mathbf{6}} \mathbf{D}_{\mathbf{6}}-\left[\mathbf{d}_{\mathbf{6}}\right], \mathbf{2 5}^{\circ} \mathbf{C}, \mathbf{T M S}\right): 1.06(\mathrm{t}, 12 \mathrm{H}$, ${ }^{3} J=6.0 \mathrm{~Hz}, \mathrm{CH}_{2} \mathrm{CH}_{3}$ ), 2.02 (s, $12 \mathrm{H}, \mathrm{CH}_{3}-\mathrm{C}$ ), 3.26 (q, $\left.8 \mathrm{H},{ }^{3} \mathrm{~J}=6.0 \mathrm{~Hz}, \mathrm{CH}_{2}-\mathrm{CH}_{3}\right), 7.27$ (s, $2 \mathrm{H}$, $\underline{\mathrm{H}}_{\text {Arom}}$ ) ppm; EA: für $\mathrm{C}_{24} \mathrm{H}_{36} \mathrm{O}_{2} \mathrm{~S}_{4} \mathrm{~W}-\mathrm{C}_{8} \mathrm{H}_{20} \mathrm{O}_{2}$, Berechnet (\%):C: 43.11, H: 5.43; S: 19.18; Gefunden (\%): C: 43.89, H: 5.82; S: $19.42 ; \mathbf{E}^{\mathbf{0}}\left(\mathbf{2 5 ^ { \circ }} \mathbf{C}, \mathbf{B u}_{4} \mathbf{N P F}_{6}, \mathbf{C V}, \mathbf{C H}_{3} \mathbf{C N}\right)$ : $-0.51 \mathrm{~V}\left(\mathrm{Fc} / \mathrm{Fc}^{+}\right) ; 0.14 \mathrm{~V}(\mathrm{SCE})$

\subsubsection{Synthese von [Mo(edt) $\left.{ }_{2}\left(\mathrm{OEt}_{2}\right)_{2}\right](11 \mathrm{e})$}

Die Darstellung dieser Verbindung erfolgte analog zur Synthesemethode von 11a, hierbei wurde nur statt des Natriumsalzes des 3,4-Toluoldithiolligandens, der 1,2Ethandithiolligandes verwendet. Hierbei konnten $0.19 \mathrm{~g}, 0.44 \mathrm{mmol}, 17 \%$ eines hellgrünen Feststoffs erhalten werden. IR (KBr) [ $\mathbf{c m}^{-1}$ ]: 470 (w), 505 (w), 664 (w), 732 (w), 843 (s), 878 (m), 933 (w), 987 (w), 1111 (w), 1156 (w), 1244 (s), 1309 (w), 1415 (s), 1444 (s), 1632 (s), 1657 (s), 2351 (w); MS [EI, 70 eV] e/z (\%) = 282 (40) [Mo(edt) $)_{2}-2 \quad$ Et $\left._{2} \mathrm{O}\right] ;{ }^{1} \mathbf{H}-\mathbf{N M R}$ $\left(200 \mathrm{MHz}, \mathbf{C D C l}_{3}-\left[\mathbf{d}_{1}\right], \mathbf{2 5}^{\circ} \mathbf{C}, \mathbf{T M S}\right): \delta=1.26\left(\mathrm{t},{ }^{3} J=7.5 \mathrm{~Hz}, 12 \mathrm{H}, \mathrm{CH}_{3}\right), 3.53(\mathrm{q}, 7.5 \mathrm{~Hz}$, $\left.8 \mathrm{H}, \mathrm{C}_{2}-\mathrm{CH}_{3}\right), 5.35$ (s, $\left.8 \mathrm{H}, \mathrm{CH}_{2}-\underline{\mathrm{H}}_{2}\right) ;{ }^{13} \mathbf{C}-\mathrm{NMR}\left(\mathbf{1 2 5 . 8} \mathbf{M H z}, \mathbf{C D C l}_{3}-\left[\mathbf{d}_{3}\right], \mathbf{2 5}^{\circ} \mathbf{C}\right.$, TMS) : $13.2\left(\underline{\mathrm{CH}}_{3}-\mathrm{CH}_{2}\right), 77.1\left(\mathrm{CH}_{3}-\underline{\mathrm{CH}_{2}}\right), 38.5\left(\underline{\mathrm{CH}}_{2}-\underline{\mathrm{C}} \mathrm{H}_{2}\right)$ ppm; EA: für $\mathrm{C}_{12} \mathrm{H}_{28} \mathrm{MoO}_{2} \mathrm{~S}_{4}-\mathrm{C}_{8} \mathrm{H}_{20} \mathrm{O}_{2}$, Berechnet (\%):C: 17.14, H: 2.88; S: 45.76; Gefunden (\%): C: 17.89, H: 3.05; S: 46.22 


\subsubsection{Synthese von [Mo(sdt) 2 (thf $\left.)_{2}\right]$ (11f)}

$0.20 \mathrm{~g}, 1.03 \mathrm{mmol}$ des sdt-Liganden (4-Phenyl-1,3-dithiol-2-on) wurde in $20 \mathrm{ml}$ THF (abs.) gelöst und mit $0.11 \mathrm{~g}, 0.21 \mathrm{mmol} \mathrm{NaOMe}$ bei $-20^{\circ} \mathrm{C}$ versetzt. Da keine Farbänderung zu beobachten war, wurde auf RT erwärmt und für $24 \mathrm{~h}$ weiter gerührt. Hiernach war eine leichte Orangefärbung der Reaktionslösung zu beobachten. Diese Lösung wurde nun langsam zu einer Lösung aus $0.20 \mathrm{~g}, 0.51 \mathrm{mmol}\left[\mathrm{MoCl}_{4}(\text { thf })_{2}\right]$ in $20 \mathrm{ml} \mathrm{THF}$ (abs.) bei RT gegeben, hierbei konnte eine Verfärbung nach dunkelgrün beobachtet werden. Nach 2 h Rühren bei RT wurde filtriert und das Lösungsmittel des Filtrats im HV entfernt. Der erhaltene dunkelgrüne Feststoff wurde erneut in $\mathrm{CH}_{2} \mathrm{Cl}_{2}$ gelöst und durch einen mit Kieselgel gefüllten Filter gegeben. Nach mehrfachem Waschen mit $\mathrm{CH}_{2} \mathrm{Cl}_{2}$ (abs.) konnte eine grüne Fraktion erhalten werden, die nach Entfernen des Lösungsmittels $0.07 \mathrm{~g}, 0.12 \mathrm{mmol}$, 24\% eines dunkelgrünen Feststoffs lieferte. MS [EI, 70 eV] e/z (\%)= 501 (60) [Mo(apdt) $)_{2}$ (thf)]-(thf)], 429 (9) [Mo(apdt) ${ }_{2}$ ]; ${ }^{1} \mathbf{H}-\mathbf{N M R} 200$ MHz, $\mathbf{C D C l}_{3}-\left[\mathbf{d}_{\mathbf{6}}\right], \mathbf{2 5}^{\circ} \mathbf{C}$, TMS): 1.74 (m, 4 H, THF-H), 3.59 (m, $4 \mathrm{H}, \mathrm{THF}-\underline{\mathrm{H}}), 7.13$ (s, $2 \mathrm{H}, \mathrm{C}=\mathrm{C} \underline{\mathrm{H}}), 7.56\left(\mathrm{~m}, 10 \mathrm{H}, \underline{\mathrm{H}}_{\text {Arom }}\right) \mathrm{ppm}$; EA: für $\mathrm{C}_{24} \mathrm{H}_{28} \mathrm{MoO}_{2} \mathrm{~S}_{4}-$ $\mathrm{C}_{4} \mathrm{H}_{8} \mathrm{O}_{2}$, Berechnet (\%):C: 47.99, H: 4.03; S: 25.62; Gefunden (\%): C: 48.92, H: 4.77; S: 26.02

\subsubsection{Synthese von [Mo(xdt) $\left.{ }_{2}(\mathrm{MeCN})_{2}\right](11 \mathrm{~g})$}

Die Darstellung dieser Verbindung erfolgte analog zur Synthesemethode von 11a, hierbei wurde nur statt des Ausgangsmaterials $\left[\mathrm{MoCl}_{4}\left(\mathrm{OEt}_{2}\right)_{2}\right] \quad\left[\mathrm{MoCl}_{4}(\mathrm{MeCN})_{2}\right]$ sowie das Natriumsalz des 4,5-dimethylbenzol-1,2-dithiol Liganden verwendet. Die Feststoffmischung wurde hier in Acetontril gelöst, wobei eine Verfärbung nach blau/grün auftrat. Nach den beschriebenen Reinigungsschritten konnten $0.24 \mathrm{~g}, 0.47 \mathrm{mmol}, 23 \%$ eines blaugrünen Feststoffs erhalten werden. IR (KBr) [cm $\left.{ }^{-1}\right]$ : 439 (w), 491 (w), 655 (w), 699 (m), 811 (s), 846 (m), 878 (m), 992 (w), 1047 (w), 1123 (w), 1154 (w), 1211 (w), 1251 (s), 1323 (w), 1465 (s), 1549 (w), 1582 (m), 1644 (m), 2264 (w), 2299 (w), 2393 (w), 2566 (w), 2782 (w), 2919 (m), $2977(\mathrm{w}), 3045$ (w); MS [EI, $70 \mathrm{eV}] \mathbf{e} / \mathbf{z}(\boldsymbol{\%})=516(100)\left[\mathrm{Mo}(\mathrm{xdt})_{2}(\mathrm{MeCN})_{2}\right] ;{ }^{1} \mathbf{H}-\mathbf{N M R}$ (500 MHz, $\mathbf{C}_{6} \mathbf{D}_{6}-\left[\mathbf{d}_{\mathbf{6}}\right], \mathbf{2 5}^{\circ} \mathbf{C}$, TMS) :0.59 (s, $\left.6 \mathrm{H}, \underline{\mathrm{C}}_{3}-\mathrm{CN}\right), 1.10$ (s, $12 \mathrm{H}, \mathrm{C}_{3}-\mathrm{C}$ ), 7.15 (s, $4 \mathrm{H}, \underline{\mathrm{H}}_{\text {Arom }}$ ); EA: für $\mathrm{C}_{20} \mathrm{H}_{22} \mathrm{MoN}_{2} \mathrm{~S}_{4}$, Berechnet (\%):C: 46.68, H: 4.31; S: 24.92; Gefunden (\%): C: 47.04, H: 4.52; S: 25.14; $\mathbf{E}^{\mathbf{0}}\left(\mathbf{2 5}^{\circ} \mathbf{C}, \mathbf{B u}_{4} \mathbf{N P F}_{6}, \mathbf{C V}, \mathbf{C H}_{3} \mathbf{C N}\right):-0.38 \mathrm{~V}\left(\mathrm{Fc} / \mathrm{Fc}^{+}\right)$; $-0.01 \mathrm{~V}(\mathrm{SCE})$ 


\subsubsection{Synthese von[ $\left.\mathrm{W}(\mathrm{xdt})_{2}(\mathrm{MeCN})_{2}\right](11 \mathrm{~h})$}

Die Darstellung dieser Verbindung erfolgte analog zur Synthesemethode von 11b. Hierbei wurde nur statt des Natriumsalzes des 3,4-Toluoldithiolenligandens, der 3,4Xyloldithiolenliganden verwendet. Die Feststoffmischung wurde nach dem Rühren mit Acetonitril versetzt. Hierbei konnten $0.21 \mathrm{~g}, 0.35 \mathrm{mmol}, 11 \%$ eines schwarzen Feststoffs erhalten werden. IR (KBr) [cm-1] 435 (w), 483 (w), 547 (w) 639 (w), 689 (m), 806 (s), 842 (m), 866 (m), 984 (w), 1033 (w), 1115 (w), 1134 (w), 1153 (w), 1208 (w), 1248 (s), 1308 (w), 1375 (w), 1455 (s), 1541 (w), 1580 (m), 1622 (m), 2196 (w), 2253 (w), 2290 (w), 2391 (w), 2536 (w), 2742 (w), 2861 (w), 2917 (m), 2970 (w), 3037 (w); MS [EI, 70 eV] e/z (\%)= 602 (100) $\left[\mathrm{W}(\mathrm{xdt})_{2}(\mathrm{MeCN})_{2}\right] ;{ }^{1} \mathbf{H}-\mathrm{NMR}\left(\mathbf{5 0 0} \mathbf{M H z}, \mathbf{C}_{6} \mathbf{D}_{6}-\left[\mathbf{d}_{6}\right], \mathbf{2 5}^{\circ} \mathbf{C}, \mathbf{T M S}\right): 0.89$ (s, $6 \mathrm{H}, \mathrm{C}_{3^{-}}-$ $\mathrm{CN}$ ), 1.19 (s, $\left.12 \mathrm{H}, \underline{\mathrm{CH}}_{3}-\mathrm{C}\right), 7.26$ (s, $4 \mathrm{H}, \underline{\mathrm{H}}_{\text {Arom}}$ ) ppm; EA: für $\mathrm{C}_{20} \mathrm{H}_{22} \mathrm{WN}_{2} \mathrm{~S}_{4}$, Berechnet (\%):C: 39.87, H: 3.68; S: 21.29; Gefunden (\%): C: 40.33, H: 3.98; S: $21.44 ; \mathbf{E}^{\mathbf{0}}\left(\mathbf{2 5}^{\circ} \mathbf{C}\right.$, $\left.\mathrm{Bu}_{4} \mathrm{NPF}_{6}, \mathbf{C V}, \mathrm{CH}_{3} \mathrm{CN}\right):-0.67 \mathrm{~V}\left(\mathrm{Fc} / \mathrm{Fc}^{+}\right) ;-0.30 \mathrm{~V}(\mathrm{SCE})$

\subsubsection{Synthese von $\left[\mathrm{MoO}(\mathrm{xdlt})(\mathrm{S})_{4}\right]^{2-}(17 \mathrm{~b})$}

$\mathrm{Zu}$ einer Acetonitrillösung $(50 \mathrm{ml})$ von $\left(\mathrm{Et}_{4} \mathrm{~N}\right)_{2}\left[\mathrm{MoO}\left(\mathrm{S}_{4}\right)_{2}\right](500 \mathrm{mg}, 0.795 \mathrm{mmol})$ wurde eine Lösung aus 4,5-Dimethylbenzol-1,2-dithiol (0.14 g, $0.795 \mathrm{mmol})$ ebenfalls in Acetonitril $(20 \mathrm{ml})$ bei RT gegeben. Die Lösung wurde bei RT für $5 \mathrm{~h}$ gerührt. Nachdem das Volumen der Lösung auf ca. $40 \mathrm{ml}$ reduziert wurde, konnte der Ausfall von gelbem Feststoff beobachtet werden. Nach Abtrennung des Feststoffs wurde die Lösung erneut für $12 \mathrm{~h}$ bei $-30^{\circ} \mathrm{C}$ aufbewahrt, wodurch eine erneute Menge an Schwefel ausfiel. Nach erneutem Filtrieren konnten aus dem Filtrat durch „Vapor Diffusion“ aus Acetonitril/Diethylether rotbraune Kristalle erhalten werden. Es konnten $368 \mathrm{mg}(0.57 \mathrm{mmol} ; 72 \%)$ erhalten werden. IR (KBr) [cm ${ }^{-1}$ ]: $424(\mathrm{w}), 440(\mathrm{w}), 474(\mathrm{w}), 515(\mathrm{w}), 538(\mathrm{w}), 619(\mathrm{w}), 681(\mathrm{w}), 711(\mathrm{w}), 783$ (s), 867 (m), 912 (vs), 931 (s), 999 (s), 1022 (w), 1055 (w), 1114 (w), 1172 (s), 1245 (w), 1306 (w), 1336 (w), 1364 (m), 1385 (s), 1448 (s), 1479 (vs), 1633 (m), 2239 (s), 2934 (w), 2981 (w); ${ }^{1}$ H-NMR (300 MHz, DMSO-[d 6 ], 25 ${ }^{\circ} \mathbf{C}$, TMS): $1.07\left(t,{ }^{3} \mathrm{~J}=6.0 \mathrm{~Hz}, 24 \mathrm{H}, \mathrm{C}_{3}{ }^{-} \mathrm{CH}_{2}\right), 2.26$ (s, $\left.6 \mathrm{H}, \mathrm{C}_{3}\right), 2.85\left(q,{ }^{3} \mathrm{~J}=6.0 \mathrm{~Hz}, 16 \mathrm{H}, \mathrm{CH}_{3}-\underline{\mathrm{C}}_{2}\right), 7.18$ (s, $\left.4 \mathrm{H}, \underline{\mathrm{H}}_{\mathrm{Arom}}\right) \mathrm{ppm}$; MS [ESI ${ }^{+}$, MeOH] $\mathrm{e} / \mathrm{z}(\%)=130.2(100)\left[\mathrm{NEt}_{4}{ }^{+}\right] ; \mathbf{M S}\left[\mathbf{E S I}^{-}, \mathbf{M e O H}\right] \mathbf{e} / \mathbf{z}(\%)=409.3(11)\left[\mathrm{MoO}(\mathrm{xdt})(\mathrm{S})_{4}\right]^{2-} ; \mathbf{E A}:$ Berechnet für $\mathrm{C}_{24} \mathrm{H}_{48} \mathrm{MoN}_{2} \mathrm{OS}_{6}(\%)$ : C: 43.09, H: 7.23, N: 4.19; S: 28.76; Gefunden (\%):C:43.75, H: 7.95; N: 4.35; S: 29.64; UV/VIS (MeOH) $\lambda_{\max }[\mathbf{n m}]\left(\varepsilon\left[\mathbf{M}^{-1} \cdot \mathbf{c m}^{-1}\right]\right): 295.0$ (6787) 


\section{$10.2 .34\left(\mathrm{KNEt}_{4}\right) \mathrm{S}_{4}(17 \mathrm{c})$}

Die Synthese wurde analog Synthesevorschrift von 17a durchgeführt, nur das der Ligand zuvor mit $\mathrm{KOH}$ versetzt wurde.

IR (KBr) [cm $\left.{ }^{-1}\right]$ : 473 (w), 507 (w), 669 (w), 746 (w), 797 (w), 916 (w), $986(\mathrm{~m}), 999$ (m), 1155 (m), 1243 (s), 1311 (m), 1458 (w), 1474 (w), 1741 (w), 2345 (w), 2362 (w), 2938 (w), $2976(\mathrm{w})$

\subsubsection{Synthese von $\left[\mathrm{MoO}(\mathrm{xdt})(\mathrm{vdt})_{2}\right]^{2-}(18)$}

$0.20 \mathrm{~g}, 0.30 \mathrm{mmol}\left[\mathrm{MoO}(\mathrm{xdt})\left(\mathrm{S}_{4}\right]^{2-}\right.$ wurden in $20 \mathrm{ml}$ Acetonitril gelöst, gefolgt von einer Lösung des vdt-Ligandens (0.06 g, $0.30 \mathrm{mmol})$ ebenfalls gelöst in $10 \mathrm{ml}$ Acetonitril. Hierbei konnte ein sofortiger Farbumschlag nach grün beobachtet werden. Nach 6 h Rühren bei RT wurde die Lösung bis auf ca. $10 \mathrm{ml}$ eingeengt und der ausfallende Schwefel wurde durch Filtration abgetrennt. Das Lösungsmittel des Filtrats wurde entfernt und es wurde mehrere Male mit Acetonitril aufgenommen und erneut mit Diethylether wieder ausgefällt. Es konnten 0.09 g, 0.12 mmol, $40 \%$ eines dunkelgrünen Feststoffs erhalten werden. IR (KBr) $\left[\mathbf{c m}^{-1}\right]$ : 419 (w), 443 (w), 504 (w), 678 (w), 704 (w), 748 (w), 802 (s), 872 (w), 909 (s), 985 (w), 1019 (m), 1099 (m), 1156 (w), 1245 (vs), 1261 (vs), 1381 (w), 1339 (w), 1385 (s), 1436 (w), 1457 (w), 1472 (w), 1507 (w), 1541 (w), 1559 (w), 1636 (s), 1699 (w), 1716 (w), 1733 (w), 1772 (w), 1792 (w), 1829 (w), 1868 (w), 2341 (w), 2357 (w), 2541 (w), 2926 (w), 2964 (w). ${ }^{\mathbf{1}} \mathbf{H}$ NMR (200 MHz, DMSO-[d 6 ], 25 ${ }^{\circ} \mathbf{C}$, TMS): $1.09\left(t,{ }^{3} J=6.0 \mathrm{~Hz}, 24 \mathrm{H}, \mathrm{CH}_{3}-\mathrm{CH}_{2}\right), 2.53$ (s, $\left.6 \mathrm{H}, \mathrm{C}_{3}\right), 3.10\left(q,{ }^{3} J=6.0 \mathrm{~Hz}, 16 \mathrm{H}, \mathrm{CH}_{3}-\underline{\mathrm{CH}}_{2}\right), 3.37$ (s, $\left.6 \mathrm{H}, \mathrm{O}-\underline{\mathrm{H}}_{3}\right), 6.78$ (s, $\left.2 \mathrm{H}, \underline{\mathrm{H}}_{\text {Arom }}\right)$, 7.21 (s, $\left.2 \mathrm{H}, \underline{\mathrm{H}}_{\text {Arom }}\right)$ ppm; ${ }^{13} \mathbf{C}-\mathbf{N M R}\left(\mathbf{7 5 . 5} \mathbf{M H z}, \mathbf{C D}_{\mathbf{3}} \mathbf{C N}-\left[\mathbf{d}_{3}\right], \mathbf{2 5}^{\circ} \mathbf{C}, \mathbf{T M S}\right): 6.38\left(\underline{C H}_{3}\right)$, $51.6\left(\underline{\mathrm{CH}}_{3}-\mathrm{C}\right), 51.7\left(\underline{\mathrm{CH}}_{2}-\mathrm{CH}_{3}\right), 55.4\left(\mathrm{C}-\underline{\mathrm{OCH}}_{3}\right), 121.1$ ( $\left.\underline{\mathrm{C}}_{\text {Arom }}\right), 126.4$ ( $\left.\underline{\mathrm{C}}_{\text {Arom }}\right), 126.8$ ( $\left.\underline{\mathrm{C}}_{\text {Arom }}\right)$, 129.3 ( $\left.\underline{\mathrm{C}}_{\text {Arom }}\right), 133.1$ ( $\left.\underline{\mathrm{C}}_{\text {Arom }}\right), 141.6$ ( $\left.\underline{\mathrm{C}}_{\text {Arom }}\right) ; \mathbf{M S}\left[\mathbf{F A B}{ }^{+}, \mathbf{3}-\mathbf{N B A}\right] \mathrm{e} / \mathrm{z}(\%)=130.2(100)$ $\left[\mathrm{NEt}_{4}{ }^{+}\right] ;$MS $\left[\mathbf{F A B}^{-} .3-\mathbf{N B A}\right]$ e/z $(\boldsymbol{\%})=481.9(100)[\mathrm{MoO}(\mathrm{xdt})(\mathrm{vdt})]^{2-}$ EA: für $\mathrm{C}_{32} \mathrm{H}_{56} \mathrm{MoN}_{2} \mathrm{O}_{3} \mathrm{~S}_{4}$, Berechnet (\%):C: 51.87, H: 7.62; N:3.78, S: $17.31 ;$ Gefunden $(\%)$ : C: $52.89, \mathrm{H}: 7.79 ; \mathrm{N}: 3.92 ; \mathrm{S}: 18.23$

\subsubsection{Synthese von $\left[\mathrm{MoO}(\mathrm{xdt})(\mathrm{ndt})_{2}\right]^{2-}(19 \mathrm{a} / 19 \mathrm{~b})$}

Die Verbindung wurde anlog zur Verbindung 18 hergestellt, nur das hierbei der ndt-Ligand verwendet wurde. Es konnte ein grüner Feststoff erhalten werden. Doch bei diesem handelte es sich um eine Produktmischung aus dem mono- sowie disubstituierten Produkt. Eine Säulenchromatographische Reinigung an Kieselgel unter Inertgasatmosphäre brachte keine 
genügende Abtrennung. Es konnte $0.15 \mathrm{~g}$, des grünen Produktgemischen erhalten werden. MS $\left[\mathrm{FAB}^{+}, \mathbf{3 - N B A}\right] \mathrm{e} / \mathrm{z}(\%)=130.2(100)\left[\mathrm{NEt}_{4}{ }^{+}\right] ;$MS $\left[\mathrm{FAB}^{-}\right.$. 3-NBA] e/z (\%) = $471.9(100)$ $[\mathrm{MoO}(\mathrm{xdt})(\mathrm{ndt})]^{2-}, 493.8(82)\left[\mathrm{MoO}(\mathrm{ndt})_{2}\right]^{2-}$

\subsubsection{Synthese von $\left[\mathrm{Mo}_{2} \mathrm{O}_{2}(\mu-S)_{2}(\mathrm{bdt})_{2}\right]^{2-}(20)$}

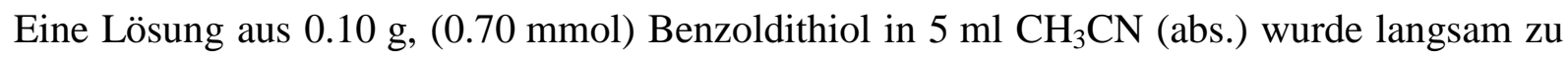
einer Lösung aus $\left(\mathrm{NEt}_{4}\right)_{2}\left[\mathrm{MoO}_{2} \mathrm{~S}_{2}\right]$ in $(0.36 \mathrm{~g}, 0.80 \mathrm{mmol}) 20 \mathrm{ml} \mathrm{CH}{ }_{3} \mathrm{CN}$ (abs.) bei RT gegeben. Hierbei konnte eine Farbveränderung von orange nach rotbraune beobachtet werden. Nun wurde $2 \mathrm{~h}$ gerührt und die erhaltene Lösung wurde filtriert. Nun wurden $5 \mathrm{ml} \mathrm{Et}_{2} \mathrm{O}$ (abs.) zum Filtrat gegeben und die Lösung bei $-35^{\circ} \mathrm{C}$ aufbewahrt. Nach $10 \mathrm{~d}$ konnten orangerote Kristalle erhalten werden. Es wurden $0.11 \mathrm{~g}, 0.13 \mathrm{mmol}, 17 \%$ erhalten. IR $(\mathbf{K B r})\left[\mathbf{c m}^{-1}\right]: 420$ (w), $472(\mathrm{w}), 518(\mathrm{w}), 665(\mathrm{w}), 750(\mathrm{w}), 797(\mathrm{~m}), 876(\mathrm{~m}), 939$ (s), 1002 (s), 1102 (s), 1153 (m), 1172 (m), 1241 (s), 1371 (w), 1385 (s), 1420 (m), 1439 (m), 1458 (s), 1474 (m), 1522 (w), 1542 (w), 1559 (w), 1637 (s), 1698 (w), 1717 (w), 1734 (w), 1749 (w), 1773 (w), 1793 (w), 1830 (w), 1845 (w), 1869 (w), 1923 (w), 2035 (m), 2047 (m), 2176 (w), 2256 (w), 2347 (m), 2361 (m), 2422 (w), 2854 (w), 2984 (w); MS [ESI ${ }^{+}, \mathbf{C H}_{3} \mathbf{C N}$ ], e/z (\%): 130.2 (100) $\left[\mathrm{NEt}_{4}{ }^{+}\right]$; MS [ESI; $\left.\mathbf{C H}_{3} \mathrm{CN}\right], \mathbf{e} / \mathbf{z}(\%): 571.5\left[\mathrm{MoO}_{2} \mathrm{~S}_{4}(\mathrm{bdt})_{2}{ }^{2-}\right] ;{ }^{1} \mathbf{H}-\mathbf{N M R}$ (200 MHz, DMSO[d $\mathbf{d}_{6}$ ], 25 $\left.{ }^{\circ} \mathbf{C}, \mathbf{T M S}\right): \delta=1.15\left(\mathrm{t}, 24 \mathrm{H},{ }^{3} \mathrm{~J}=7.2 \mathrm{~Hz}, \underline{\mathrm{CH}}_{3}\right), 3.18$ (q, $\left.16 \mathrm{H}, 7.2 \mathrm{~Hz}, \mathrm{CH}_{2}-\mathrm{CH}_{3}\right), 6.76$ (m, $\left.4 \mathrm{H}, \underline{\mathrm{H}}_{\text {Arom }}\right), 7.49$ (m, $\left.4 \mathrm{H}, \underline{\mathrm{H}}_{\text {Arom }}\right)$ ppm. EA: für $\mathrm{C}_{28} \mathrm{H}_{48} \mathrm{Mo}_{2} \mathrm{~N}_{2} \mathrm{O}_{2} \mathrm{~S}_{6}$, Berechnet (\%):C: 40.57, H: 5.84; N:3.38, S: 23.21; Gefunden (\%): C: 39.84, H: 5.62; N:3.03; S: 23.05

\subsubsection{Synthese von [ $\mathrm{MoOCl}_{2}$ (nacnac)] Methode A (22a)}

$0.50 \mathrm{~g}(1.38 \mathrm{mmol}) \mathrm{MoOCl}_{3}(\mathrm{thf})_{2}$ wurde in $20 \mathrm{ml} \mathrm{Et} 2 \mathrm{O}$ (abs.) gelöst, nun wurde bei $0^{\circ} \mathrm{C}$ eine Lösung des Lithiumnacnac-Liganden $(0.59 \mathrm{~g}, 1.38 \mathrm{mmol})$ in $\mathrm{Et}_{2} \mathrm{O}(20 \mathrm{ml})$ zugegeben. Es konnte eine Farbänderung von rot nach dunkelgrün beobachtet werden. Die Mischung wurde nun für $2 \mathrm{~h}$ bei RT gerührt, bevor durch Celite filtriert und das Lösungsmittel des Filtrats entfernt wurde. Es konnte ein dunkelgrüner schaumartiger Feststoff isoliert werden. Dieser wurde nun in Toluol (abs.) umkristallisiert, und bei $-35^{\circ} \mathrm{C}$ aufbewahrt. Nach $10 \mathrm{~d}$ konnte die Bildung von dunkelgrünen Kristallen beobachtet werden. Es wurden 0.72 g, 1.20 mol, $87 \%$ erhalten. MS [EI, 70 eV] e/z $(\%)=601.2$ (100) $\left[\mathrm{MoOCl}_{2}(\right.$ nacnac) $]$; EA: Berechnet für $\mathrm{C}_{29} \mathrm{H}_{41} \mathrm{Cl}_{2} \mathrm{MoN}_{2} \mathrm{O}(\%): \mathrm{C}: 58.00, \mathrm{H}: 6.88 ; \mathrm{N}: 4.67$ Gefunden (\%): C:58.26, H: 7.01; N: 4.75 


\subsubsection{Synthese von [ $\mathrm{MoOCl}_{2}$ (nacnac)] Methode B (22 b)}

Die Synthese wurde analog zu 22a durchgeführt, nur dass hierbei $\mathrm{MoOCl}_{4}$ verwendet wurde. Hierbei konnten 0.68 g, 1.13 mmol, 82\% von dunkelgrünen Nadeln erhalten werden.

Analytik siehe $22 a$

\subsubsection{Synthese von [WOCl 2 (nacnac)] Methode A (23a)}

Die Synthese wurde analog zu 22a durchgeführt, nur dass hierbei $\mathrm{WOCl}_{3}(\text { thf })_{2}$ verwendet wurde. Hierbei konnten 0.48 g, 0.70 mmol, 63\% von dunkelblauen Nadeln erhalten werden. MS [EI, 70 eV] e/z $(\%)=687.2(100) \quad\left[\mathrm{WOCl}_{2}\right.$ (nacnac)]; EA: Berechnet für $\mathrm{C}_{29} \mathrm{H}_{41} \mathrm{Cl}_{2} \mathrm{MoN}_{2} \mathrm{O}(\%): \mathrm{C}: 58.00, \mathrm{H}: 6.88 ; \mathrm{N}: 4.67$ Gefunden (\%): C:58.39, H: 7.06; N: 4.71

\subsubsection{Synthese von [WOCl 2 (nacnac)] Methode B (23b)}

Die Synthese wurde analog zu 22a durchgeführt, nur dass hierbei $\mathrm{WOCl}_{4}$ verwendet wurde. Hierbei konnte zuerst eine Verfärbung nach grün beobachtet werden, nach $30 \mathrm{Min}$ trat eine weitere Verfärbung nach dunkelblau auf. Nach Filtrieren wurde das Lösungsmittels des Filtrats entfernt. Der Rückstand des Filtrats wurde mit Toluol gewaschen und die grünliche Toluolphase verworfen. Nun wurde das am Boden abgesetzte Öl in THF aufgenommen. Nach Mehrfachen lösen in THF und entfernen des Lösungsmittels konnten $0.23 \mathrm{~g}, 0.33$ mmol, $72 \%$ als dunkelblauen Nadeln erhalten werden.

Analytik siehe $23 a$

\subsubsection{Synthese von [MoO(xdt)(nacnac)] (24)}

$0.30 \mathrm{~g}$, (0.50 mmol) von $\left[\mathrm{MoOCl}_{2}\right.$ (nacnac)] wurde in $10 \mathrm{ml} \mathrm{Et}_{2} \mathrm{O}$ (abs.) gelöst und wurde bei $-78^{\circ} \mathrm{C}$ zu einer Suspension des Natriumsalzes des xdt-Ligandens $(0.11 \mathrm{~g}, 0.50 \mathrm{mmol})$ in $\mathrm{Et}_{2} \mathrm{O}$ $(20 \mathrm{ml})$ zugegeben. Es konnte nach $30 \mathrm{Min}$. rühren eine Farbveränderung nach braun beobachtet werden. Nun wurde für weitere $2 \mathrm{~h}$ bei RT gerührt. Nach Filtrieren durch Celite und entfernen des Lösungsmittels des Filtrats wurde eine brauner Feststoff erhalten. Nach mehrfacher Umkristallisation in Toluol konnten $0.21 \mathrm{~g}, 0.30 \mathrm{mmol}, 60 \%$ eines hellbraunen mikrokristallinen Pulvers erhalten werden. IR (KBr) [ $\left.\mathbf{c m}^{-1}\right]$ : 434 (w), 465 (w), 504 (w), 555 (w), 639 (w), 693 (w), $728(\mathrm{w}), 759(\mathrm{w}), 797$ (m), $858(\mathrm{w}), 951$ (w), $985(\mathrm{~m}), 1020(\mathrm{w}), 1057$ (w), 1156 (s), 1247 (vs), 1314 (s), 1361 (w), 1384 (w), 1438 (w), 1463 (w), 1534 (w), 1623 (w), 2343 (w), 2361 (w), 2867 (w), 2926 (w), 2962 (w), 3059 (w); MS [EI, 70 eV] e/z (\%) = 699.2 (100) [MoO(xdt)(nacnac)] EA: Berechnet für $\mathrm{C}_{37} \mathrm{H}_{49} \mathrm{MoN}_{2} \mathrm{OS}_{2}(\%)$ : C: 63.68, H: 7.08; N: 4.01, S: 9.19; Gefunden (\%): C: 63.55, H: 7.06; N: 4.31; S: 9.46 
10.2.43 Synthese von [WO(xdt)(nacnac)] (25)

$0.45 \mathrm{~g}, 0.65 \mathrm{mmol}$ des blauen [ $\mathrm{WOCl}_{2}$ (nacnac)] wurde in THF gelöst und dieses dann mit einer THF-Lösung aus dem Natriumsalz des xdt-Ligandens bei $-80^{\circ} \mathrm{C}$ versetzt. Nach direkter Zugabe konnte keine Farbänderung festgestellt werden. Die Lösung wurde nun langsam auf RT erwärmt und für $3 \mathrm{~d}$ bei RT gerührt. Nach Filtration und Entfernung des Lösungsmittels aus dem Filtrat konnten $0.19 \mathrm{~g}, 0.24 \mathrm{mmol}, 37 \%$ eines hellbraunen Feststoffs isoliert werden. IR (KBr) [cm ${ }^{-1}$ ]: 474 (w), 507 (w), $555(\mathrm{w}), 640$ (w), 669 (w), 704 (w), 746 (w), 801 (vs), 865 (w), 1022 (s), 1099 (s), 1155 (m), 1262 (s), 1309 (w), 1414 (w), 2362 (w), 2964 (w), 3447 (w); MS [EI, 70 eV] e/z (\%) = 785.3 (100) [WO(xdt)(nacnac)]; EA: Berechnet für $\mathrm{C}_{37} \mathrm{H}_{49} \mathrm{~N}_{2} \mathrm{OS}_{2} \mathrm{~W}(\%)$ : C: 56.56, H: 6.29; N: 3.57, S: 8.16; Gefunden (\%): C: 56.99, H: 6.56; N: 3.89; $\mathrm{S}: 8.36$ 


\section{Kapitel 11}

Anhang 
11.1 Auftragungen der elektrochemischen Untersuchungen

(Wolframverbindungen in rot, Molybdänverbindungen in schwarz)

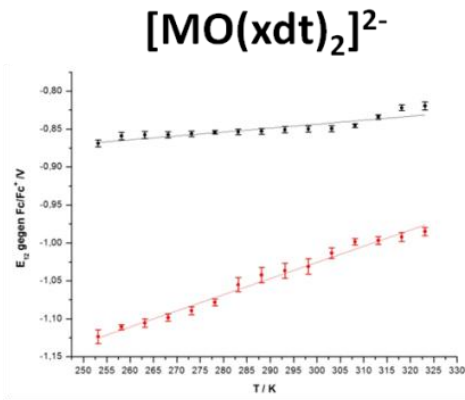

$\left[\mathrm{MO}(\mathrm{tdt})_{2}\right]^{2-}$

$\left[\mathrm{MO}(\mathrm{vdt})_{2}\right]^{2-}$

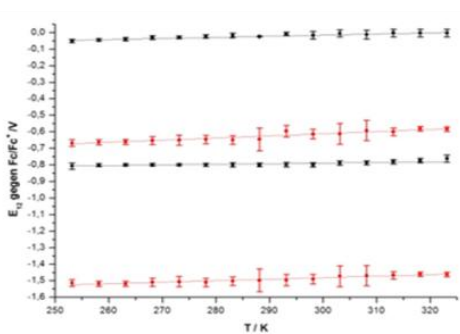

$\left[\mathrm{MO}(\mathrm{qdt})_{2}\right]^{2-}$

$\left[\mathrm{MO}(\mathrm{ntdt})_{2}\right]^{2-}$
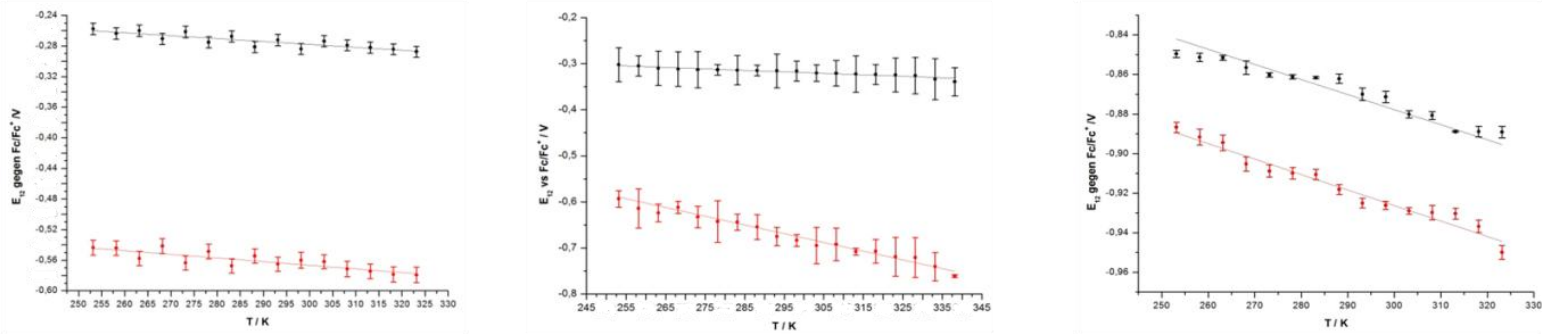

$\left[\mathrm{MO}(\mathrm{tldt})_{2}\right]^{2-}$

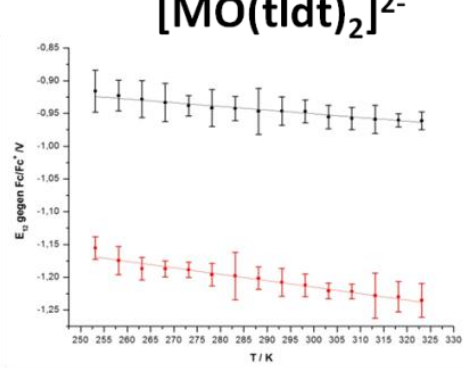

$\left[\mathrm{MO}(\mathrm{cdt})_{2}\right]^{2-}$

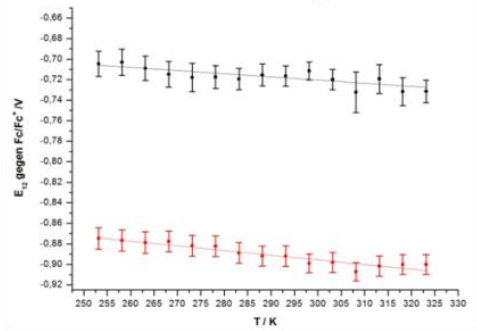

$\left[\mathrm{MO}(\mathrm{tcdt})_{2}\right]^{2-}$

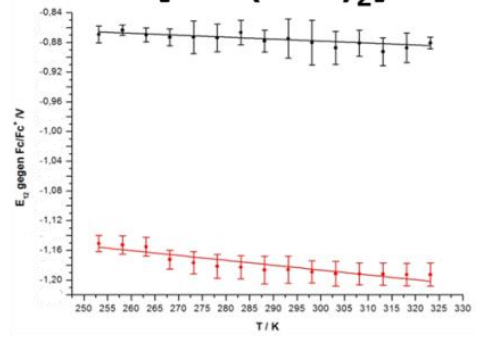

$\left[\mathrm{MO}(\mathrm{cydt})_{2}\right]^{2-}$

$\left[\mathrm{M}\left(\mathrm{OEt}_{2}\right)_{2}(\mathrm{tdt})_{2}\right]^{2-}$

$\left[\mathrm{MO}_{2}(\mathrm{CN})_{4}\right]^{4-}$
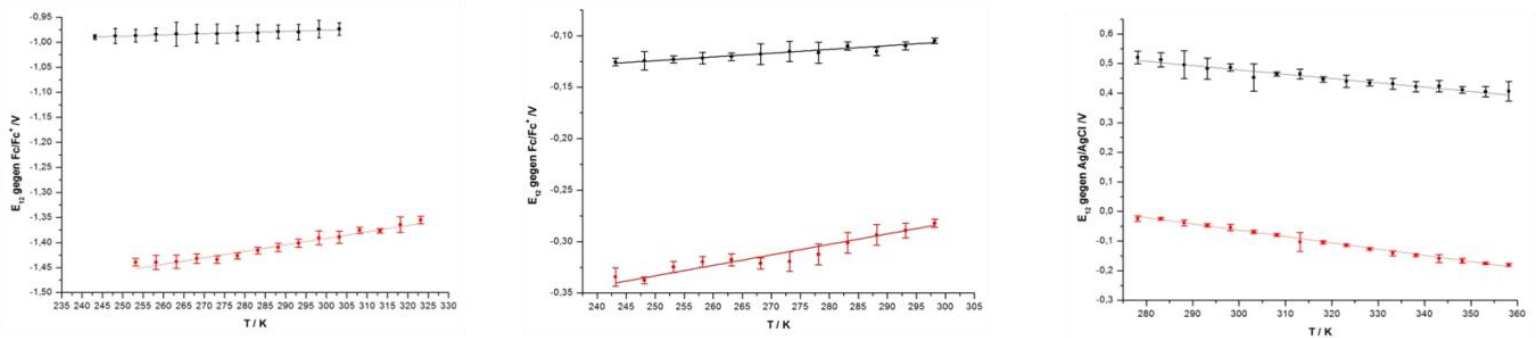

$\left[\mathrm{M}(\mathrm{CN})_{8}\right]^{4-}$

$\left[\mathrm{MCl}_{4}(\mathrm{MeCN})_{2}\right]$

$\left[\mathrm{MCp}_{2} \mathrm{Cl}_{2}\right]$ in $\mathrm{MeCN}$
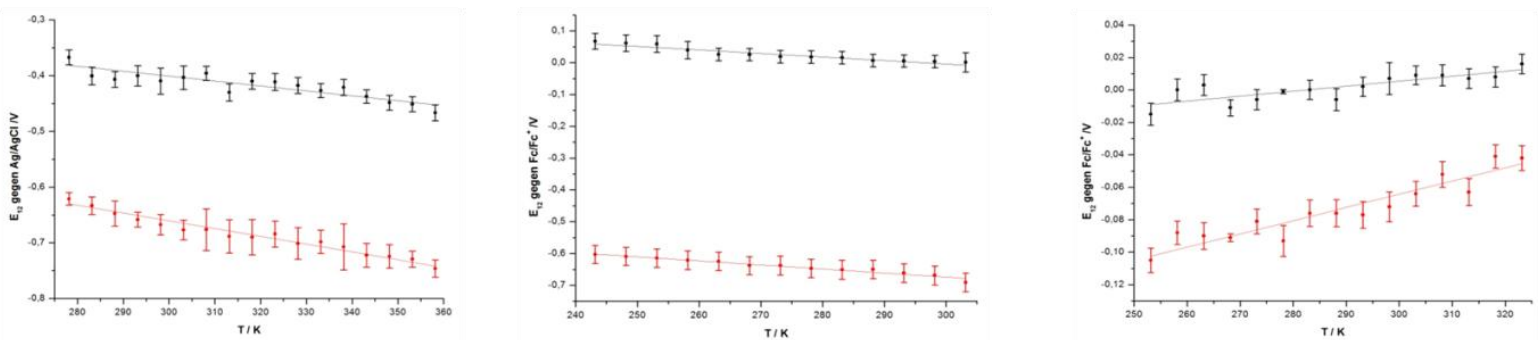

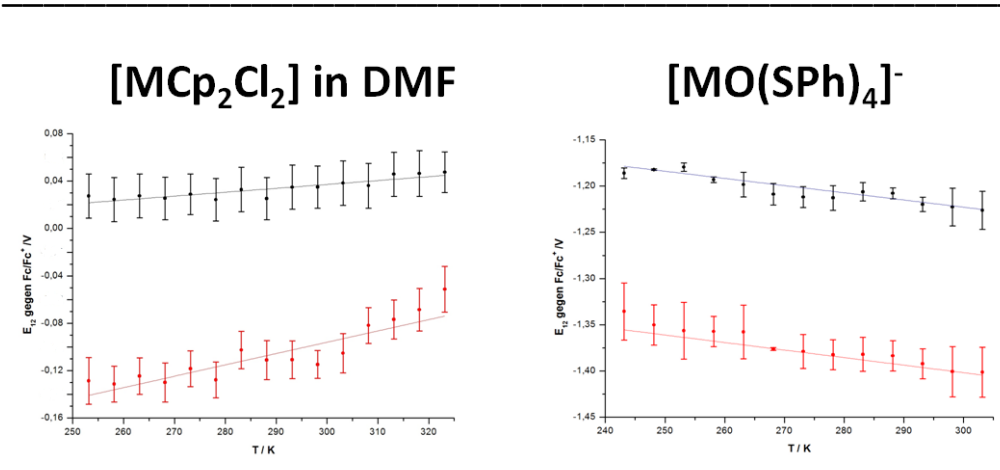

Einige CVs und DPVs bei jeweils der niedrigsten (grün), bei $25^{\circ} \mathrm{C}$ (blau) und bei der höchsten Temperatur (rot)
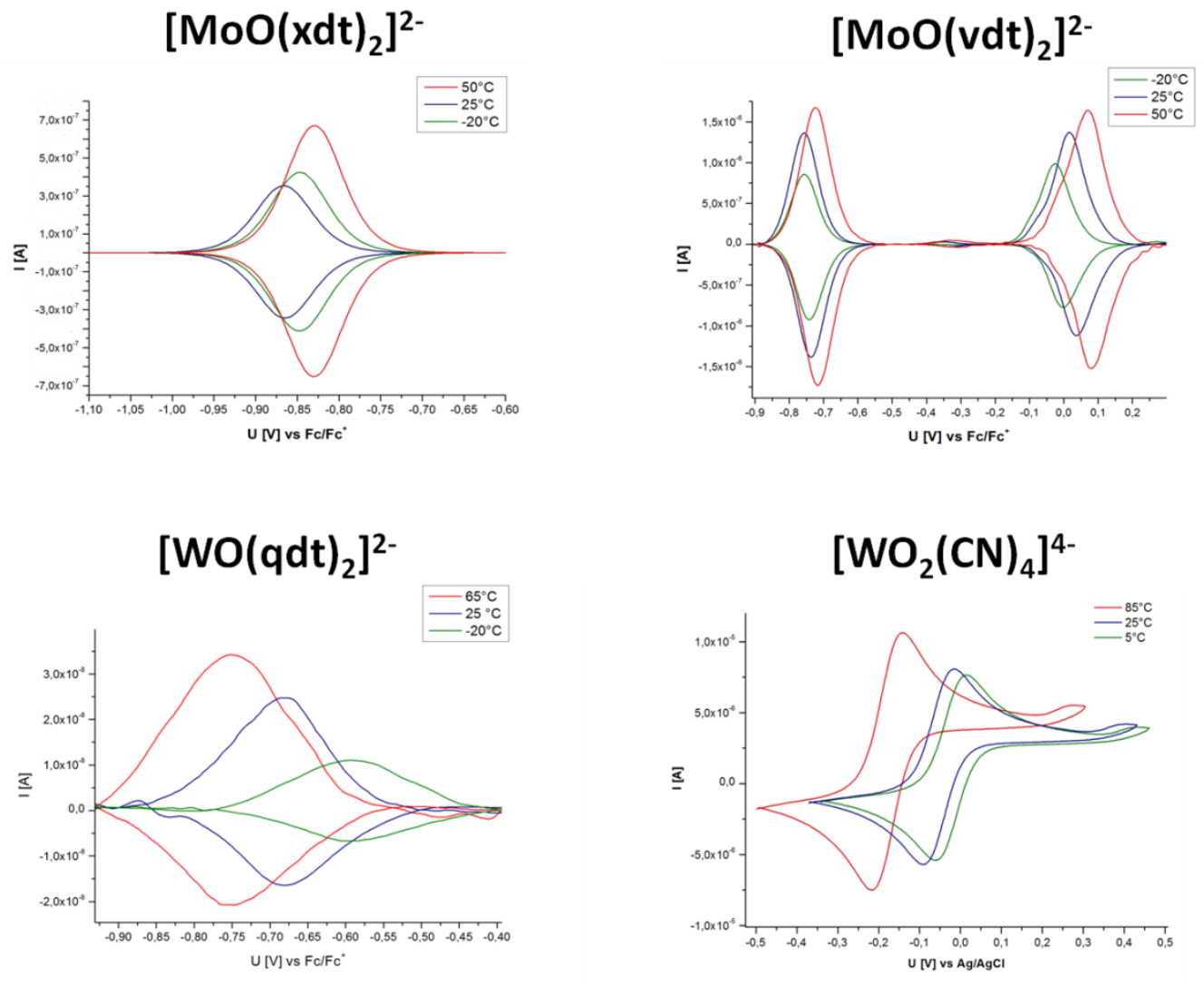

$\left[\mathrm{WCp}_{2} \mathrm{Cl}_{2}\right]$ in $\mathrm{MeCN}$
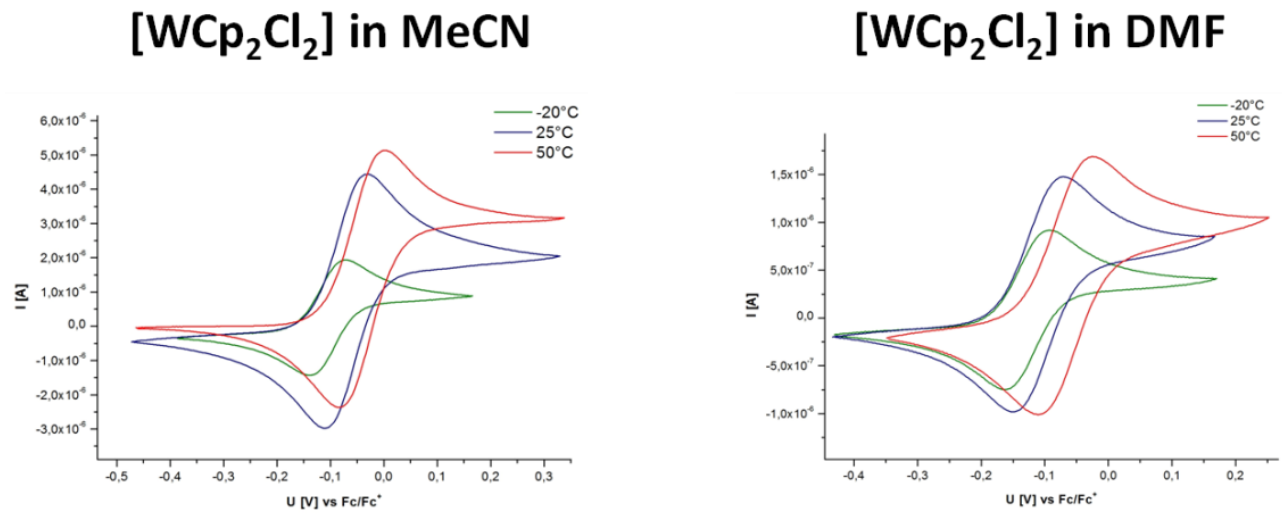
11.2 Auftragungen der temperaturabhängigen katalytischen Reaktionen
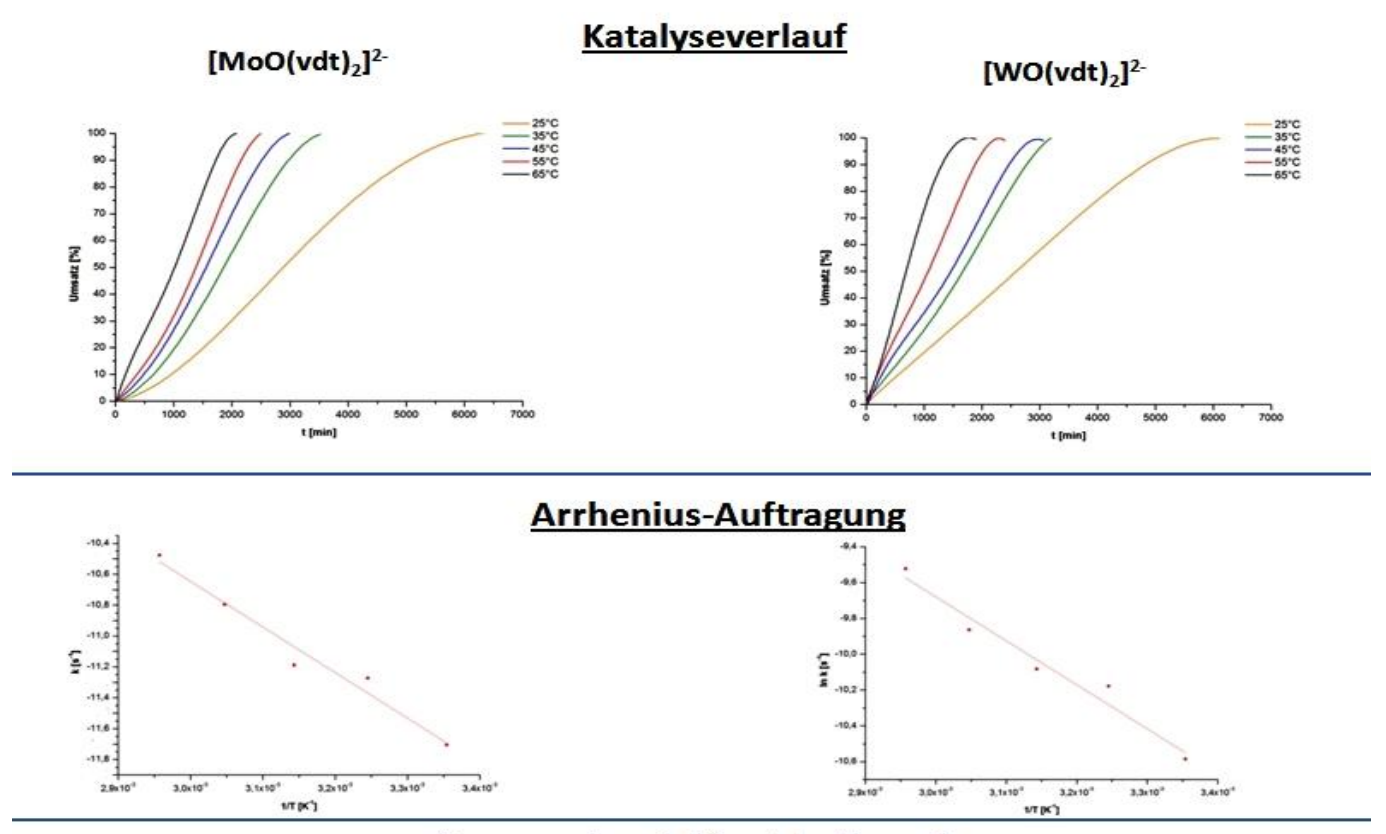

Temperaturabhängigkeit von $\mathbf{k}$
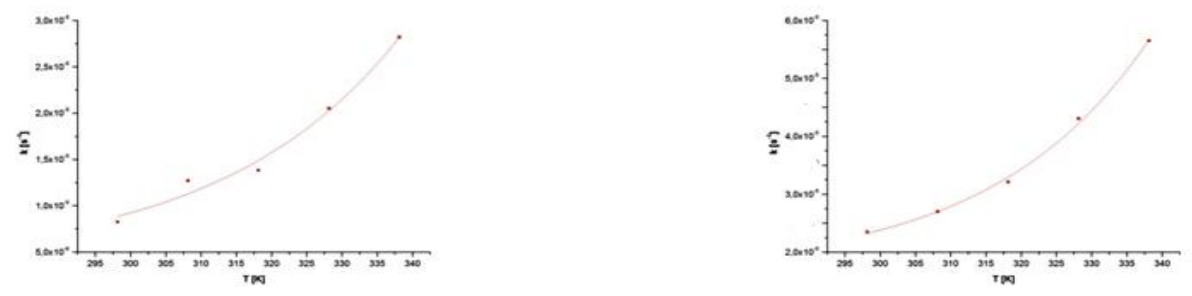

$\left[\mathrm{MoO}(\mathrm{qdt})_{2}\right]^{2-}$

$\underline{\text { Katalyseverlauf }}$

$\left[\mathrm{WO}(\mathrm{qdt})_{2}\right]^{2-}$
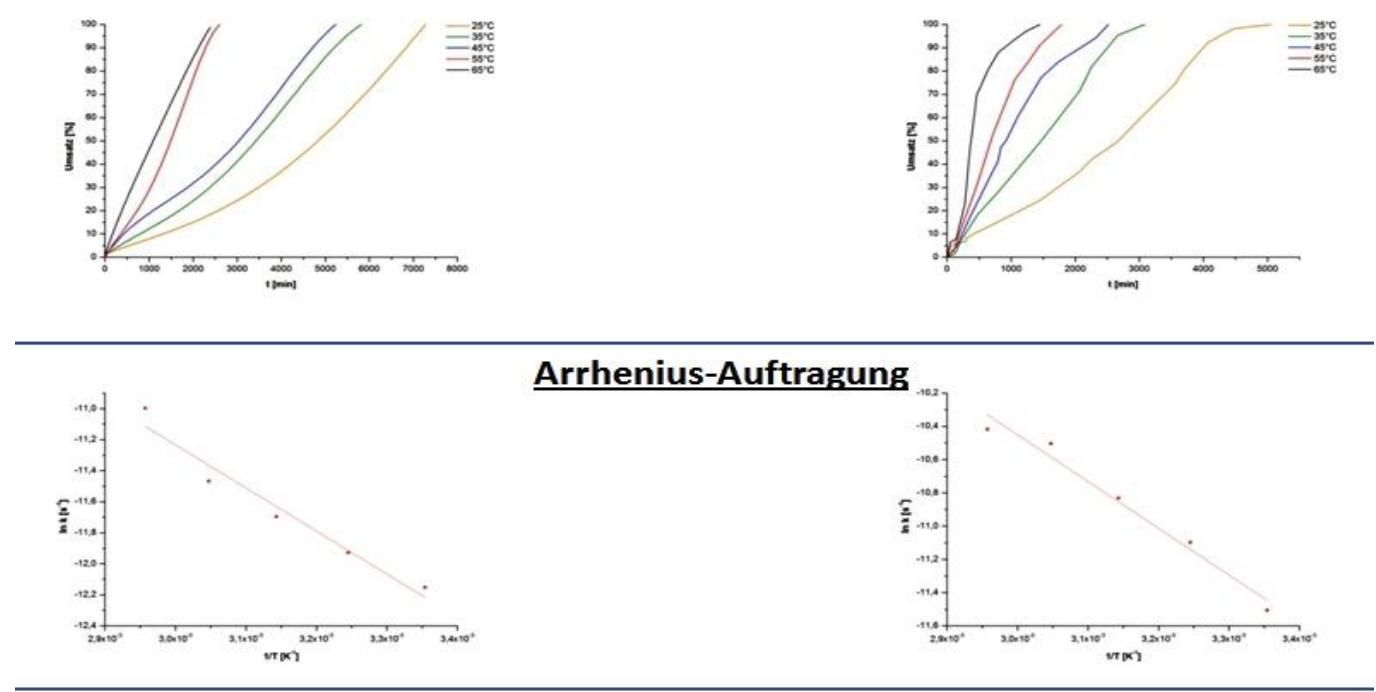

Temperaturabhängigkeitvon $\mathbf{k}$
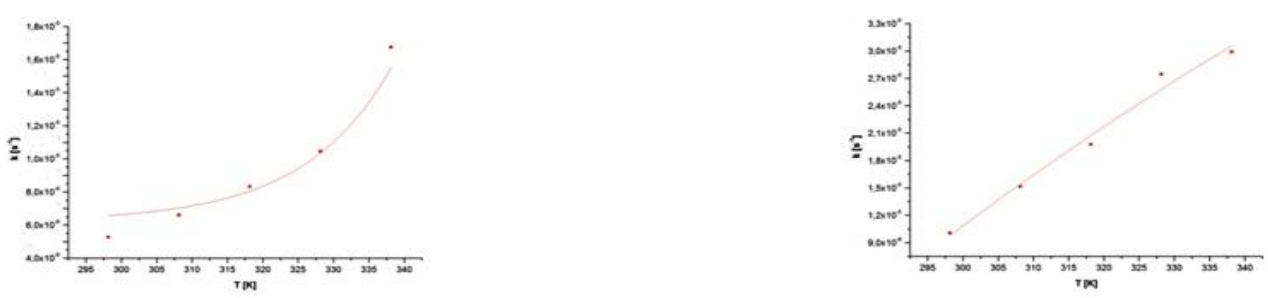
11.3 Geometrieparameter aus DFT-Rechnungen

\begin{tabular}{|c|c|c|c|c|c|c|}
\hline \multirow[b]{2}{*}{ Oxstufe } & \multicolumn{3}{|c|}{$\left[\mathrm{MoO}(\mathrm{xdt})_{2}\right]^{2-}$} & \multicolumn{3}{|c|}{$\left[\mathrm{WO}(\mathrm{xdt})_{2}\right]^{2-}$} \\
\hline & $(+I V)$ & $(+\mathrm{V})$ & $(+\mathrm{VI})$ & $(+I V)$ & $(+V)$ & $(+\mathrm{VI})$ \\
\hline $\mathrm{M}-\mathrm{S}_{4}$ & 0.752 & 0.784 & 0.809 & 0.725 & 0.755 & 0.766 \\
\hline $\mathbf{M}(\mathbf{1})-\mathbf{O}(\mathbf{1 0})$ & 1.71109 & 1.70652 & 1.69845 & 1.72227 & 1.71455 & 1.70667 \\
\hline $\mathbf{M}(1)-S(2)$ & 2.45972 & 2.42835 & 2.39557 & 2.44222 & 2.42834 & 2.38920 \\
\hline$M(1)-S(3)$ & 2.45969 & 2.42834 & 2.39557 & 2.44222 & 2.42834 & 2.38920 \\
\hline$M(1)-S(4)$ & 2.45969 & 2.44180 & 2.42259 & 2.44222 & 2.42485 & 2.41400 \\
\hline$M(1)-S(5)$ & 2.45972 & 2.44180 & 2.42258 & 2.44222 & 2.42485 & 2.41400 \\
\hline $\mathbf{M}(1)-\bar{S}$ & 2.45971 & 2.43507 & 2.40908 & 2.44222 & 2.42660 & 2.40160 \\
\hline$S(2)-C(8)$ & 1.77909 & 1.77882 & 1.76160 & 1.78566 & 1.78136 & 1.77064 \\
\hline$S(3)-C(9)$ & 1.77910 & 1.77882 & 1.76160 & 1.78566 & 1.78136 & 1.77064 \\
\hline$S(4)-C(7)$ & 1.77910 & 1.76945 & 1.74478 & 1.78566 & 1.77509 & 1.75585 \\
\hline$S(5)-C(6)$ & 1.77909 & 1.76945 & 1.74478 & 1.78566 & 1.77509 & 1.75585 \\
\hline$C(6)-C(7)$ & 1.41479 & 1.40834 & 1.41855 & 1.41156 & 1.40593 & 1.41352 \\
\hline $\mathrm{C}(8)-\mathrm{C}(9)$ & 1.41480 & 1.41142 & 1.42413 & 1.41156 & 1.41012 & 1.41983 \\
\hline FW (M1)-S(1-2)-C(8-9) & $165.72^{\circ}$ & $146.55^{\circ}$ & $140.09^{\circ}$ & $167.03^{\circ}$ & $147.88^{\circ}$ & $137.66^{\circ}$ \\
\hline FW (M1)-S(1-2)-C(8-9) von $M=O$ & $14.28^{\circ}$ & $33.46^{\circ}$ & $39.91^{\circ}$ & $12.97^{\circ}$ & $32.12^{\circ}$ & $42.34^{\circ}$ \\
\hline FW (M1)-S(3-4)-C(6-7) zu M=O & $165.72^{\circ}$ & $173.16^{\circ}$ & $162.12^{\circ}$ & $167.03^{\circ}$ & $179.37^{\circ}$ & $158.62^{\circ}$ \\
\hline$(3-4)-C(6-7$ & $14.28^{\circ}$ & $6.84^{\circ}$ & $17.88^{\circ}$ & $12.97^{\circ}$ & $0.63^{\circ}$ & $21.38^{\circ}$ \\
\hline & \multicolumn{3}{|c|}{$\left[\mathrm{MoO}(\mathrm{tdt})_{2}\right]^{2-}$} & \multicolumn{3}{|c|}{$\left[\mathrm{WO}(\mathrm{tdt})_{2}\right]^{2-}$} \\
\hline Oxstufe & $(+\mathbf{I V})$ & & $(+\mathrm{VI})$ & $(+\mathbf{I V})$ & & $(+\mathrm{VI})$ \\
\hline M-S & 0.753 & 0.785 & 0.810 & 0.726 & 0.756 & 0.767 \\
\hline $\mathbf{M}(\mathbf{1})-\mathbf{O}(\mathbf{1 0})$ & 1.71107 & 1.70642 & 1.69817 & 1.72237 & 1.7 & 1.70624 \\
\hline$M(1)-S(2)$ & 2.45860 & 2.44048 & 2.39481 & 2.44134 & 2.43394 & 2.41070 \\
\hline$M(1)-S(3)$ & 2.45952 & 2.44213 & 2.39500 & 2.44219 & 2.43516 & 2.41594 \\
\hline M(1) & 2.45952 & 2.42904 & 2.42457 & 2.44219 & 878 & 917 \\
\hline $\mathbf{M}(\mathbf{1})$ & 2.45860 & 2.42745 & 2.41871 & 2.44134 & 2.42756 & 890 \\
\hline $\mathbf{M}($ & 2.45906 & 2.43478 & 2.40827 & 2.4 & 2.4 & 2.40118 \\
\hline$S(2)-C(8)$ & 1.77944 & 1.76954 & 1.76086 & 1.78598 & 1.77511 & 1.75519 \\
\hline$S(3)-C(9)$ & 1.77929 & 1.76958 & 1.76321 & 1.78580 & 1.77508 & 696 \\
\hline$S(4)$ & 1.77929 & 1.77 & 1.7 & 1.7 & 117 & 1.77193 \\
\hline$S(5)$ & 1.77944 & 1.77 & 1.74389 & 1.78598 & 125 & 1.77013 \\
\hline $\mathrm{C}(6$ & 1.41829 & & & & & 104 \\
\hline$C(8)-$ & 1.41828 & 1.41005 & 1.42545 & 1.41495 & 1. & 1.41557 \\
\hline$S(1-2)-C(8-$ & $165.69^{\circ}$ & $146.64^{\circ}$ & $140.10^{\circ}$ & $166.96^{\circ}$ & $148.32^{\circ}$ & $138.01^{\circ}$ \\
\hline FW (N & $14.31^{\circ}$ & 33 & $39.90^{\circ \circ}$ & $04^{\circ}$ & $31.68^{\circ}$ & $41.99^{\circ}$ \\
\hline FW (M1)-S(3-4)-C(6-7) zu M=O & $165.69^{\circ}$ & $173.22^{\circ}$ & $161.63^{\circ}$ & $166.96^{\circ}$ & $179.44^{\circ}$ & $157.85^{\circ}$ \\
\hline \multirow[t]{2}{*}{ FW (M1)-S(3-4)-C(6-7) von $\mathrm{M}=\mathrm{O}$} & $14.31^{\circ}$ & $6.78^{\circ}$ & $18.37^{\circ}$ & $13.04^{\circ}$ & $0.56^{\circ}$ & $22.15^{\circ}$ \\
\hline & \multicolumn{3}{|c|}{$\left[\mathrm{MoO}(\mathrm{vdt})_{2}\right]^{2-}$} & \multicolumn{3}{|c|}{$\left[\mathrm{WO}(\mathrm{vdt})_{2}\right]^{2-}$} \\
\hline Oxstufe & $(+\mathbf{I V})$ & $(+\mathrm{V})$ & $(+\mathrm{VI})$ & $(+\mathbf{I V})$ & $(+\mathrm{V})$ & $(+\mathrm{VI})$ \\
\hline $\mathbf{M}-\mathbf{S}_{4}$ & 0.750 & & & & 0.755 & 0.770 \\
\hline $\mathbf{M}(\mathbf{1})-\mathbf{O}(\mathbf{1 0})$ & 1.71090 & 1.7 & 896 & 1.72223 & 1.71479 & 1.70759 \\
\hline$M(1)-S(2)$ & 2.45902 & 2.44121 & 2.42638 & 2.44131 & 2.42697 & 2.39113 \\
\hline$M(1)-S(3)$ & 2.45902 & 2.44121 & 2.42637 & 2.44131 & 2.42697 & 2.39113 \\
\hline$M(1)-S(4)$ & 2.45902 & 2.42758 & 2.40006 & 2.44131 & 2.43394 & 2.41595 \\
\hline$M(1)-S(5)$ & 2.45902 & 2.42758 & 2.40006 & 2.44131 & 2.43394 & 2.41595 \\
\hline $\mathbf{M}(1)-\bar{S}$ & 2.45902 & 2.43440 & 2.41322 & 2.44131 & 2.43046 & 2.40354 \\
\hline$S(2)-C(8)$ & 1.78125 & 1.76961 & 1.74301 & 1.78788 & 1.78202 & 1.76695 \\
\hline$S(3)-C(9)$ & 1.78125 & 1.76961 & 1.74301 & 1.78788 & 1.78202 & 1.76695 \\
\hline$S(4)-C(7)$ & 1.78125 & 1.77879 & 1.75781 & 1.78788 & 1.77597 & 1.75386 \\
\hline$S(5)-C(6)$ & 1.78125 & 1.77879 & 1.75781 & 1.78788 & 1.77597 & 1.75386 \\
\hline$C(6)-C(7)$ & 1.40869 & 1.40659 & 1.42514 & 1.40546 & 1.39993 & 1.41050 \\
\hline $\mathrm{C}(8)-\mathrm{C}(9)$ & 1.40869 & 1.40308 & 1.41791 & 1.40546 & 1.40471 & 1.41982 \\
\hline FW (M1)-S(1-2)-C(8-9) zu M=O & $165.73^{\circ}$ & $146.02^{\circ}$ & $140.59^{\circ}$ & $166.99^{\circ}$ & $147.04^{\circ}$ & $137.36^{\circ}$ \\
\hline FW (M1)-S(1-2)-C(8-9) von $M=O$ & $14.27^{\circ}$ & $33.98^{\circ}$ & $39.41^{\circ}$ & $13.01^{\circ}$ & $32.96^{\circ}$ & $42.65^{\circ}$ \\
\hline FW (M1)-S(3-4)-C(6-7) zu M=O & $165.73^{\circ}$ & $173.19^{\circ}$ & $164.76^{\circ}$ & $166.99^{\circ}$ & $179.25^{\circ}$ & $149.86^{\circ}$ \\
\hline FW (M1)-S(3-4)-C(6-7) von $M=0$ & $14.27^{\circ}$ & $6.81^{\circ}$ & $15.24^{\circ}$ & $13.01^{\circ}$ & $0.75^{\circ}$ & $30.14^{\circ}$ \\
\hline
\end{tabular}




\begin{tabular}{|c|c|c|c|c|c|c|}
\hline \multirow[b]{2}{*}{ Oxstufe } & \multicolumn{3}{|c|}{$\left[\mathrm{MoO}(\mathrm{ndt})_{2}\right]^{2-}$} & \multicolumn{3}{|c|}{$\left[\mathrm{WO}(\text { ndt })_{2}\right]^{2-}$} \\
\hline & $(+\mathrm{IV})$ & $(+V)$ & $(+\mathrm{VI})$ & $(+\mathrm{IV})$ & $(+V)$ & $(+\mathrm{VI})$ \\
\hline $\mathbf{M}-\mathbf{S}_{4}$ & 0.756 & 0.788 & 0.809 & 0.727 & 0.757 & 0.767 \\
\hline $\mathbf{M}(1)-\mathbf{O}(10)$ & 1.70413 & 1.70040 & 1.69297 & 1.71587 & 1.70889 & 1.70136 \\
\hline $\mathrm{M}(\mathbf{1})-\mathrm{S}(\mathbf{2})$ & 2.45482 & 2.44209 & 2.42221 & 2.43829 & 2.43595 & 2.41417 \\
\hline $\mathbf{M}(\mathbf{1})-\mathbf{S}(\mathbf{3})$ & 2.45482 & 2.44209 & 2.42221 & 2.43829 & 2.43595 & 2.41417 \\
\hline$M(1)-S(4)$ & 2.45482 & 2.42901 & 2.39666 & 2.43829 & 2.42911 & 2.39229 \\
\hline$M(1)-S(5)$ & 2.45482 & 2.42901 & 2.39666 & 2.43829 & 2.42911 & 2.39229 \\
\hline $\mathbf{M}(1)-\bar{S}$ & 2.45482 & 2.43555 & 2.40944 & 2.43829 & 2.43253 & 2.40323 \\
\hline $\mathbf{S}(2)-\mathbf{C}(8)$ & 1.76312 & 1.75941 & 1.74205 & 1.76972 & 1.76418 & 1.75298 \\
\hline $\mathbf{S}(\mathbf{3})-\mathbf{C}(\mathbf{9})$ & 1.76312 & 1.75941 & 1.74205 & 1.76972 & 1.76418 & 1.75298 \\
\hline$S(4)-C(7)$ & 1.76312 & 1.76883 & 1.76050 & 1.76972 & 1.77070 & 1.76870 \\
\hline$S(5)-C(6)$ & 1.76312 & 1.76883 & 1.76050 & 1.76972 & 1.77070 & 1.76870 \\
\hline$C(6)-C(7)$ & 1.42482 & 1.41881 & 1.42544 & 1.42149 & 1.41482 & 1.42186 \\
\hline $\mathrm{C}(\mathbf{8})-\mathrm{C}(9)$ & 1.42482 & 1.41669 & 1.42415 & 1.42149 & 1.41471 & 1.42019 \\
\hline FW (M1)-S(1-2)-C(8-9) zu M=O & $166.06^{\circ}$ & $148.00^{\circ}$ & $141.33^{\circ}$ & $167.22^{\circ}$ & $179.72^{\circ}$ & $139.47^{\circ}$ \\
\hline FW (M1)-S(1-2)-C(8-9) von $M=O$ & $13.94^{\circ}$ & $32.00^{\circ}$ & $38.67^{\circ}$ & $12.78^{\circ}$ & $30.23^{\circ}$ & $40.53^{\circ}$ \\
\hline FW (M1)-S(3-4)-C(6-7) zu M=O & $166.06^{\circ}$ & $172.59^{\circ}$ & $158.40^{\circ}$ & $167.21^{\circ}$ & $149.77^{\circ}$ & $154.45^{\circ}$ \\
\hline \multirow[t]{2}{*}{ FW (M1)-S(3-4)-C(6-7) von $M=0$} & $13.94^{\circ}$ & $7.42^{\circ}$ & $21.60^{\circ}$ & $12.79^{\circ}$ & $0.28^{\circ}$ & $25.55^{\circ}$ \\
\hline & \multicolumn{3}{|c|}{$\left[\mathrm{MoO}(\mathrm{qdt})_{2}\right]^{2-}$} & \multicolumn{3}{|c|}{$\left[\mathrm{WO}(\mathrm{qdt})_{2}\right]^{2}$} \\
\hline Oxstufe & $(+\mathbf{I V})$ & $(+\mathbf{V})$ & $(+\mathrm{VI})$ & $(+\mathbf{I V})$ & $(+\mathrm{V})$ & $(+\mathrm{VI})$ \\
\hline $\mathbf{M}-\mathbf{S}_{4}$ & 0.765 & 0.784 & 0.782 & 0.736 & 0.758 & 0.743 \\
\hline $\mathbf{M}(1)-\mathbf{O}(10)$ & 1.70492 & 1.70187 & 1.69490 & 1.71669 & 1.70996 & 1.70300 \\
\hline $\mathbf{M}(\mathbf{1})-\mathbf{S}(\mathbf{2})$ & 2.45971 & 2.43495 & 2.39754 & 2.44457 & 2.43512 & 2.41074 \\
\hline $\mathbf{M}(\mathbf{1})-\mathbf{S}(\mathbf{3})$ & 2.45971 & 2.43495 & 2.39754 & 2.44457 & 2.43510 & 2.41074 \\
\hline $\mathbf{M}(\mathbf{1})-\mathbf{S}(4)$ & 2.45971 & 2.44174 & 2.41678 & 2.44457 & 2.43507 & 2.39366 \\
\hline $\mathbf{M}(\mathbf{1})-\mathbf{S}(\mathbf{5})$ & 2.45971 & 2.44174 & 2.41678 & 2.44457 & 2.43496 & 2.39366 \\
\hline $\mathbf{M}(\mathbf{1})-\bar{S}$ & 2.45971 & 2.43835 & 2.40716 & 2.44457 & 2.43506 & 2.40220 \\
\hline $\mathbf{S}(2)-\mathbf{C}(8)$ & 1.75873 & 1.77264 & 1.77290 & 1.76379 & 1.77231 & 1.76870 \\
\hline $\mathbf{S}(3)-\mathbf{C}(\mathbf{9})$ & 1.75873 & 1.77264 & 1.77290 & 1.76379 & 1.77237 & 1.76870 \\
\hline$S(4)-C(7)$ & 1.75873 & 1.76643 & 1.75734 & 1.76379 & 1.77228 & 1.78128 \\
\hline $\mathbf{S}(\mathbf{5})-\mathbf{C}(\mathbf{6})$ & 1.75873 & 1.76643 & 1.75734 & 1.76379 & 1.77196 & 1.78128 \\
\hline$C(6)-C(7)$ & 1.47296 & 1.45736 & 1.45309 & 1.46927 & 1.45800 & 1.45095 \\
\hline $\mathrm{C}(\mathbf{8})-\mathrm{C}(9)$ & 1.47296 & 1.46056 & 1.45355 & 1.46927 & 1.45819 & 1.45066 \\
\hline FW (M1)-S(1-2)-C(8-9) zu M=O & $164.25^{\circ}$ & $149.36^{\circ}$ & $141.02^{\circ}$ & $165.07^{\circ}$ & $159.49^{\circ}$ & $140.36^{\circ}$ \\
\hline FW (M1)-S(1-2)-C(8-9) von $M=0$ & $15.75^{\circ}$ & $30.64^{\circ}$ & $38.98^{\circ}$ & $14.93^{\circ}$ & $10.51^{\circ}$ & $39.64^{\circ}$ \\
\hline FW (M1)-S(3-4)-C(6-7) zu M=O & $164.25^{\circ}$ & $175.35^{\circ}$ & $155.52^{\circ}$ & $165.07^{\circ}$ & $161.14^{\circ}$ & $152.72^{\circ}$ \\
\hline \multirow[t]{2}{*}{ FW (M1)-S(3-4)-C(6-7) von M=O } & $15.75^{\circ}$ & $4.65^{\circ}$ & $24.48^{\circ}$ & $14.93^{\circ}$ & $8.86^{\circ}$ & $27.28^{\circ}$ \\
\hline & \multicolumn{3}{|c|}{$\left[\mathrm{MoO}(\text { ntdt })_{2}\right]^{2-}$} & \multicolumn{3}{|c|}{$\left[\text { WO }(\text { ntdt })_{2}\right]^{2-}$} \\
\hline Oxstufe & $(+\mathrm{IV})$ & $(+\mathrm{V})$ & $(+\mathrm{VI})$ & $(+\mathbf{I V})$ & $(+\mathrm{V})$ & $(+\mathrm{VI})$ \\
\hline $\mathbf{M}-\mathbf{S}_{4}$ & 0.757 & 0.795 & 0.838 & 0.731 & 0.764 & 0.788 \\
\hline $\mathbf{M}(\mathbf{1})-\mathbf{O}(\mathbf{1 0})$ & 1.71043 & 1.70668 & 1.69883 & 1.72183 & 1.71495 & 1.70741 \\
\hline $\mathbf{M}(\mathbf{1})-\mathbf{S}(2)$ & 2.47169 & 2.44742 & 2.41949 & 2.45270 & 2.44146 & 2.40925 \\
\hline $\mathbf{M}(\mathbf{1})-\mathbf{S}(\mathbf{3})$ & 2.45317 & 2.43910 & 2.43596 & 2.43608 & 2.43218 & 2.42380 \\
\hline $\mathbf{M}(\mathbf{1})-\mathbf{S}(\mathbf{4})$ & 2.45085 & 2.42428 & 2.40096 & 2.43257 & 2.42275 & 2.39031 \\
\hline$M(1)-S(5)$ & 2.47199 & 2.43474 & 2.40704 & 2.45400 & 2.43359 & 2.39589 \\
\hline $\mathbf{M}(\mathbf{1})-\bar{S}$ & 2.46193 & 2.43639 & 2.41586 & 2.44384 & 2.43250 & 2.40481 \\
\hline $\mathbf{S}(2)-\mathbf{C}(\mathbf{8})$ & 1.74523 & 1.74101 & 1.71301 & 1.75228 & 1.74865 & 1.73822 \\
\hline $\mathbf{S}(3)-\mathbf{C}(\mathbf{9})$ & 1.79569 & 1.77503 & 1.74416 & 1.80367 & 1.78302 & 1.76637 \\
\hline$S(4)-C(7)$ & 1.79565 & 1.78395 & 1.75739 & 1.80387 & 1.78836 & 1.75715 \\
\hline $\mathbf{S}(5)-\mathbf{C}(6)$ & 1.74507 & 1.75108 & 1.72669 & 1.75254 & 1.75594 & 1.72621 \\
\hline$C(6)-C(7)$ & 1.36572 & 1.36323 & 1.38629 & 1.36290 & 1.36017 & 1.37050 \\
\hline $\mathrm{C}(\mathbf{8})-\mathrm{C}(\mathbf{9})$ & 1.36525 & 1.36134 & 1.38067 & 1.36241 & 1.35691 & 1.38086 \\
\hline FW (M1)-S(1-2)-C(8-9) zu M=O & $165.73^{\circ}$ & $148.36^{\circ}$ & $142.66^{\circ}$ & $166.18^{\circ}$ & $149.51^{\circ}$ & $138.01^{\circ}$ \\
\hline FW (M1)-S(1-2)-C(8-9) von $M=O$ & $14.27^{\circ}$ & $31.64^{\circ}$ & $37.34^{\circ}$ & $13.82^{\circ}$ & $30.49^{\circ}$ & $41.99^{\circ}$ \\
\hline FW (M1)-S(3-4)-C(6-7) zu M=O & $168.63^{\circ}$ & $173.89^{\circ}$ & $167.36^{\circ}$ & $170.26^{\circ}$ & $177.92^{\circ}$ & $164.42^{\circ}$ \\
\hline FW (M1)-S(3-4)-C(6-7) von $M=O$ & $11.37^{\circ}$ & $6.11^{\circ}$ & $12.65^{\circ}$ & $9.74^{\circ}$ & $2.08^{\circ}$ & $15.58^{\circ}$ \\
\hline
\end{tabular}




\begin{tabular}{|c|c|c|c|c|c|c|}
\hline \multirow[b]{2}{*}{ Oxstufe } & \multicolumn{3}{|c|}{$\left[\mathrm{MoO}(\mathrm{tldt}) \mathrm{C}^{2-}\right.$} & \multicolumn{3}{|c|}{$\left[W O(t \mid d t)_{2}\right]^{2-}$} \\
\hline & $(+I V)$ & $(+\mathrm{V})$ & $(+\mathrm{VI})$ & $(+\mathrm{IV})$ & $(+\mathrm{V})$ & $(+\mathrm{VI})$ \\
\hline $\mathbf{M}-\mathbf{S}_{4}$ & 0.763 & 0.799 & 0.837 & 0.735 & 0.769 & 0.787 \\
\hline $\mathbf{M}(\mathbf{1})-\mathbf{O}(\mathbf{1 0})$ & 1.71207 & 1.70739 & 1.69976 & 1.72313 & 1.71563 & 1.70847 \\
\hline$M(1)-S(2)$ & 2.46684 & 2.44428 & 2.42561 & 2.44907 & 2.43793 & 2.41463 \\
\hline$M(1)-S(3)$ & 2.45369 & 2.43383 & 2.42494 & 2.43691 & 2.42784 & 2.41393 \\
\hline$M(1)-S(4)$ & 2.45369 & 2.42183 & 2.39603 & 2.43691 & 2.42020 & 2.38496 \\
\hline$M(1)-S(5)$ & 2.46684 & 2.43270 & 2.40419 & 2.44907 & 2.43153 & 2.39298 \\
\hline $\mathbf{M}(1)-\bar{S}$ & 2.46027 & 2.43316 & 2.41269 & 2.44299 & 2.42938 & 2.40163 \\
\hline$S(2)-C(8)$ & 1.76040 & 1.75103 & 1.72145 & 1.76715 & 1.75918 & 1.73538 \\
\hline $\mathbf{S}(\mathbf{3})-\mathbf{C}(\mathbf{9})$ & 1.79348 & 1.77213 & 1.74102 & 1.80072 & 1.78028 & 1.75355 \\
\hline S(4)-C(7) & 1.79348 & 1.78053 & 1.75382 & 1.80072 & 1.78567 & 1.76335 \\
\hline$S(5)-C(6)$ & 1.76040 & 1.75929 & 1.73425 & 1.76715 & 1.76476 & 1.74559 \\
\hline$C(6)-C(7)$ & 1.36679 & 1.36955 & 1.39639 & 1.36390 & 1.36583 & 1.39052 \\
\hline $\mathrm{C}(\mathbf{8})-\mathrm{C}(\mathbf{9})$ & 1.36679 & 1.36592 & 1.38822 & 1.36390 & 1.36112 & 1.37657 \\
\hline FW (M1)-S(1-2)-C(8-9) zu M=O & $167.51^{\circ}$ & $147.18^{\circ}$ & $141.99^{\circ}$ & $168.77^{\circ}$ & $148.06^{\circ}$ & $136.91^{\circ}$ \\
\hline FW (M1)-S(1-2)-C(8-9) von $M=O$ & $12.49^{\circ}$ & $32.82^{\circ}$ & $38.01^{\circ}$ & $11.23^{\circ}$ & $31.94^{\circ}$ & $43.09^{\circ}$ \\
\hline FW (M1)-S(3-4)-C(6-7) zu M=O & $167.51^{\circ}$ & $175.31^{\circ}$ & $168.81^{\circ}$ & $168.77^{\circ}$ & $179.31^{\circ}$ & $165.76^{\circ}$ \\
\hline FW (M1)-S(3-4)-C(6-7) von $M=0$ & $12.49^{\circ}$ & $4.69^{\circ}$ & $11.19^{\circ}$ & $11.23^{\circ}$ & $0.69^{\circ}$ & $14.24^{\circ}$ \\
\hline & \multicolumn{3}{|c|}{$\left[\mathrm{MoO}(\mathrm{tedt})_{2}\right]^{2-}$} & \multicolumn{3}{|c|}{$\left[\mathrm{WO}(\mathrm{tcdt})_{2}\right]^{2-}$} \\
\hline Oxstufe & $(+\mathrm{IV})$ & $(+V)$ & $(+\mathrm{VI})$ & $(+\mathbf{I V})$ & $(+\mathrm{V})$ & $(+\mathrm{VI})$ \\
\hline $\mathbf{M}-\mathbf{S}_{4}$ & 0.763 & 0.800 & 0.843 & 0.734 & 0.771 & 0.788 \\
\hline $\mathbf{M}(\mathbf{1})-\mathbf{O}(\mathbf{1 0})$ & 1.71173 & 1.70720 & 1.69951 & 1.72269 & 1.71536 & 1.70827 \\
\hline $\mathbf{M}(\mathbf{1})-\mathbf{S}(2)$ & 2.45828 & 2.42844 & 2.40096 & 2.44056 & 2.43315 & 2.41037 \\
\hline $\mathbf{M}(\mathbf{1})-\mathbf{S}(\mathbf{3})$ & 2.44714 & 2.41945 & 2.39512 & 2.43049 & 2.42429 & 2.41328 \\
\hline $\mathbf{M}(\mathbf{1})-\mathbf{S}(4)$ & 2.44714 & 2.42985 & 2.42438 & 2.43049 & 2.41795 & 2.38457 \\
\hline $\mathbf{M}(\mathbf{1})-\mathbf{S}(\mathbf{5})$ & 2.45828 & 2.43897 & 2.42076 & 2.44056 & 2.42726 & 2.38966 \\
\hline $\mathbf{M}(\mathbf{1})-\bar{S}$ & 2.45271 & 2.42918 & 2.41031 & 2.43553 & 2.42566 & 2.39947 \\
\hline$S(2)-C(8)$ & 1.76580 & 1.76259 & 1.73690 & 1.77385 & 1.76243 & 1.73743 \\
\hline $\mathbf{S}(3)-\mathbf{C}(9)$ & 1.79824 & 1.78294 & 1.75526 & 1.80636 & 1.78326 & 1.75651 \\
\hline$S(4)-C(7)$ & 1.79824 & 1.77541 & 1.74365 & 1.80636 & 1.78822 & 1.76411 \\
\hline$S(5)-C(6)$ & 1.76580 & 1.75417 & 1.72295 & 1.77385 & 1.76824 & 1.74869 \\
\hline $\mathrm{C}(6)-\mathrm{C}(7)$ & 1.36410 & 1.36452 & 1.38795 & 1.36102 & 1.36509 & 1.39033 \\
\hline $\mathbf{C}(8)-\mathbf{C}(9)$ & 1.36410 & 1.36879 & 1.39543 & 1.36102 & 1.36013 & 1.37612 \\
\hline FW (M1)-S(1-2)-C(8-9) zu M=O & $166.31^{\circ}$ & $145.87^{\circ}$ & $141.85^{\circ}$ & $167.70^{\circ}$ & $146.99^{\circ}$ & $136.14^{\circ}$ \\
\hline FW (M1)-S(1-2)-C(8-9) von $M=O$ & $13.69^{\circ}$ & $34.13^{\circ}$ & $38.15^{\circ}$ & $12.30^{\circ}$ & $33.01^{\circ}$ & $43.86^{\circ}$ \\
\hline FW (M1)-S(3-4)-C(6-7) zu M=O & $166.31^{\circ}$ & $176.71^{\circ}$ & $168.89^{\circ}$ & $167.70^{\circ}$ & $179.37^{\circ}$ & $166.29^{\circ}$ \\
\hline FW (M1)-S(3-4)-C(6-7) von $M=0$ & $13.69^{\circ}$ & $3.29^{\circ}$ & $11.11^{\circ}$ & $12.30^{\circ}$ & $0.63^{\circ}$ & $13.71^{\circ}$ \\
\hline & \multicolumn{3}{|c|}{$\left[\mathrm{MoO}(\mathrm{cydt})_{2}\right]^{2-}$} & \multicolumn{3}{|c|}{$\left[\mathrm{WO}(\mathrm{cydt})_{2}\right]^{2-}$} \\
\hline Oxstufe & $(+I V)$ & $(+\mathrm{V})$ & $(+\mathrm{VI})$ & $(+\mathbf{I V})$ & $(+\mathrm{V})$ & $(+\mathrm{VI})$ \\
\hline $\mathbf{M}-\mathbf{S}_{4}$ & 0.750 & 0.794 & 0.836 & 0.722 & 0.761 & 0.785 \\
\hline $\mathbf{M}(1)-\mathbf{O}(\mathbf{1 0})$ & 1.71523 & 1.70952 & 1.70061 & 1.72625 & 1.71757 & 1.70909 \\
\hline$M(1)-S(2)$ & 2.46529 & 2.44311 & 2.42575 & 2.44676 & 2.43540 & 2.41608 \\
\hline $\mathbf{M}(\mathbf{1})-\mathbf{S}(\mathbf{3})$ & 2.46482 & 2.44185 & 2.42194 & 2.44625 & 2.43471 & 2.41298 \\
\hline $\mathbf{M}(\mathbf{1})-\mathbf{S}(4)$ & 2.46482 & 2.42559 & 2.39323 & 2.44625 & 2.42489 & 2.38431 \\
\hline $\mathbf{M}(\mathbf{1})-\mathbf{S}(\mathbf{5})$ & 2.46529 & 2.42611 & 2.39327 & 2.44676 & 2.42553 & 2.38455 \\
\hline $\mathbf{M}(\mathbf{1})-\bar{S}$ & 2.46506 & 2.43417 & 2.40855 & 2.44651 & 2.43013 & 2.39948 \\
\hline$S(2)-C(8)$ & 1.78642 & 1.76743 & 1.73402 & 1.79353 & 1.77586 & 1.74806 \\
\hline $\mathbf{S}(3)-C(9)$ & 1.78663 & 1.76680 & 1.73392 & 1.79384 & 1.77542 & 1.74755 \\
\hline$S(4)-C(7)$ & 1.78663 & 1.77762 & 1.75134 & 1.79384 & 1.78260 & 1.76256 \\
\hline$S(5)-C(6)$ & 1.78642 & 1.77637 & 1.75030 & 1.79353 & 1.78107 & 1.76091 \\
\hline$C(6)-C(7)$ & 1.35189 & 1.35804 & 1.38405 & 1.34925 & 1.35542 & 1.37853 \\
\hline $\mathrm{C}(\mathbf{8})-\mathrm{C}(\mathbf{9})$ & 1.35189 & 1.35605 & 1.37904 & 1.34925 & 1.35105 & 1.36818 \\
\hline FW (M1)-S(1-2)-C(8-9) z & $166.48^{\circ}$ & $147.82^{\circ}$ & $141.69^{\circ}$ & $167.56^{\circ}$ & $148.14^{\circ}$ & $137.07^{\circ}$ \\
\hline FW (M1)-S(1-2)-C(8-9) von $M=O$ & $13.53^{\circ}$ & 32.18 & $38.32^{\circ}$ & $12.44^{\circ}$ & $31.87^{\circ}$ & $42.93^{\circ}$ \\
\hline FW (M1)-S(3-4)-C(6-7) zu M=O & $166.48^{\circ}$ & $173.20^{\circ}$ & $165.6^{\circ}$ & $167.56^{\circ}$ & $178.95^{\circ}$ & $163.27^{\circ}$ \\
\hline FW (M1)-S(3-4)-C(6-7) von $M=O$ & $13.53^{\circ}$ & $6.81^{\circ}$ & $14.4^{\circ}$ & $12.44^{\circ}$ & $1.06^{\circ}$ & $16.74^{\circ}$ \\
\hline
\end{tabular}




\begin{tabular}{|c|c|c|c|c|c|c|}
\hline \multirow[b]{2}{*}{ Oxstufe } & \multicolumn{3}{|c|}{$\left[\mathrm{MoO}\left(\mathrm{S}_{2} \mathrm{C}_{2} \mathbf{M e}_{2}\right)_{2}\right]^{2-}$} & \multicolumn{3}{|c|}{$\left[\mathrm{WO}\left(\mathbf{S}_{2} \mathrm{C}_{2} \mathbf{M e}_{2}\right)_{2}\right]^{2-}$} \\
\hline & $(+\mathbf{I V})$ & $(+\mathrm{V})$ & $(+\mathrm{VI})$ & $(+\mathrm{IV})$ & $(+\mathrm{V})$ & $(+\mathrm{VI})$ \\
\hline $\mathbf{M}-\mathbf{S}_{4}$ & 0.756 & 0.798 & 0.843 & 0.731 & 0.766 & 0.790 \\
\hline $\mathbf{M}(\mathbf{1})-\mathbf{O}(\mathbf{1 0})$ & 1.71651 & 1.71029 & 1.70132 & 1.72744 & 1.71824 & 1.70960 \\
\hline $\mathbf{M}(\mathbf{1})-\mathbf{S}(2)$ & 2.45935 & 2.43691 & 2.42441 & 2.44095 & 2.42975 & 2.41064 \\
\hline $\mathbf{M}$ & 2.45935 & 2.43691 & 2.42441 & 2.44095 & 2.42975 & 2.41064 \\
\hline $\mathbf{M}($ & 45935 & 2.42183 & 2.39230 & 2.44095 & 2.4 & 2.38188 \\
\hline $\mathbf{M}(1$ & 15935 & 2.42183 & 2.39230 & 2.44095 & 2.42 & 2.38188 \\
\hline $\mathbf{M}(\mathbf{1})$ & 45935 & 2.42937 & 2.40836 & 2.44095 & 2.42551 & 2.39626 \\
\hline $\mathbf{S}(2)-\mathbf{C}(8)$ & 1.79412 & 1.77355 & 1.73050 & 1.80225 & & 1.75050 \\
\hline & 1.79412 & 1.7 & 1.7 & 1.80225 & 1.7 & 1.75050 \\
\hline$S(4$ & 1.79412 & 1.78303 & 1.74834 & 1.80225 & 1.78792 & 1.76345 \\
\hline$S(5)-C(6)$ & 1.79412 & 1.78303 & 1.74834 & 1.80225 & 1.78792 & 1.76345 \\
\hline$C(6)-C(7)$ & 1.35268 & & 1.38 & 1.34980 & & 1.38113 \\
\hline C & 1.35268 & 1.3 & 1.3 & 1.34980 & 1.3 & 1.37130 \\
\hline FW (M1)-S(1-2 & $166.35^{\circ}$ & $147.42^{\circ}$ & $142.90^{\circ}$ & $167.54^{\circ}$ & $147.99^{\circ}$ & $137.60^{\circ}$ \\
\hline FW (M1)-S(1-2)-C(8-9) von $M=O$ & $13.65^{\circ}$ & $32.58^{\circ}$ & $37.10^{\circ}$ & $12.46^{\circ}$ & $32.01^{\circ}$ & $42.40^{\circ}$ \\
\hline FW (M1)-S(3-4)-C(6-7) & $166.35^{\circ}$ & $173.29^{\circ}$ & $167.00^{\circ}$ & 167.54 & $178.81^{\circ 0}$ & $163.34^{\circ}$ \\
\hline FW (M1)-S(3-4)-C(6-7) & $13.65^{\circ}$ & 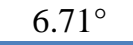 & $13.00^{\circ}$ & $12.46^{\circ}$ & $1.19^{\circ}$ & $16.66^{\circ}$ \\
\hline
\end{tabular}

\subsection{Energieunterschiede HOMO und LUMO}

Werte in [Hartree]

\begin{tabular}{|c|c|c|c|c|}
\hline & {$\left[\mathrm{MoO}(\mathrm{xdt})_{2}\right]^{2-}(\mathrm{IV})$} & {$\left[\mathrm{MoO}(\mathrm{xdt})_{2}\right]^{-}(\mathrm{V})$} & & {$\left[\mathrm{MoO}(\mathrm{xdt})_{2}\right](\mathrm{VI})$} \\
\hline HOMO-1 (99) & 0.0575 & -0.0952 & HOMO (99) & -0.2147 \\
\hline HOMO (100) & 0.0691 & -0.0727 & LUMO (100) & -0.1541 \\
\hline LUMO (101) & 0.2108 & 0.0570 & LUMO+1 (101) & -0.0720 \\
\hline \multirow[t]{2}{*}{$\Delta(101-100)$} & 0.1418 & 0.1297 & $\Delta(100-99)$ & 0.0606 \\
\hline & {$\left[\mathrm{WO}(\mathbf{x d t})_{2}\right]^{2-}(\mathrm{IV})$} & {$\left[\mathrm{WO}(\mathbf{x d t})_{2}\right]^{-}(\mathrm{V})$} & & {$\left[\mathrm{WO}(\mathrm{xdt})_{2}\right](\mathrm{VI})$} \\
\hline HOMO-1 (99) & 0.0558 & -0.0987 & HOMO (99) & -0.2177 \\
\hline HOMO (100) & 0.0844 & -0.0724 & LUMO (100) & -0.1458 \\
\hline LUMO (101) & 0.2149 & 0.0773 & LUMO+1 (101) & -0.0584 \\
\hline \multirow[t]{2}{*}{$\Delta(101-100)$} & 0.1305 & 0.1497 & $\Delta(100-99)$ & 0.0719 \\
\hline & {$\left[\mathrm{MoO}(\mathrm{tdt})_{2}\right]^{2-}(\mathrm{IV})$} & {$\left[\mathrm{MoO}(\mathbf{t d t})_{2}\right]^{-}(\mathrm{V})$} & & {$\left[\mathrm{MoO}(\mathrm{tdt})_{2}\right](\mathrm{VI})$} \\
\hline HOMO-1 (91) & 0.0576 & -0.0973 & HOMO (91) & -0.2201 \\
\hline HOMO (92) & 0.0700 & -0.0745 & LUMO (92) & -0.1583 \\
\hline LUMO (93) & 0.2133 & 0.0558 & LUMO+1 (93) & -0.0784 \\
\hline \multirow[t]{2}{*}{$\Delta(93-92)$} & 0.1433 & 0.1303 & $\Delta(93-92)$ & 0.0618 \\
\hline & {$\left[\mathrm{WO}(\mathrm{tdt})_{2}\right]^{2-}(\mathrm{IV})$} & {$\left[\mathrm{WO}(\mathrm{tdt})_{2}\right]^{-}(\mathrm{V})$} & & {$\left[\mathrm{WO}(\mathrm{tdt})_{2}\right](\mathrm{VI})$} \\
\hline HOMO-1 (91) & 0.0559 & -0.1009 & HOMO (91) & -0.2232 \\
\hline HOMO (92) & 0.0853 & -0.0743 & LUMO (92) & -0.1497 \\
\hline LUMO (93) & 0.2169 & 0.0760 & LUMO+1 (93) & -0.0626 \\
\hline \multirow[t]{2}{*}{$\Delta(93-92)$} & 0.1316 & 0.1503 & $\Delta(93-92)$ & 0.0735 \\
\hline & {$\left[\mathrm{MoO}(\mathrm{vdt})_{2}\right]^{2-}(\mathrm{IV})$} & {$\left[\mathrm{MoO}(\mathrm{vdt})_{2}\right]^{-}(\mathrm{V})$} & & {$\left[\mathrm{MoO}(\mathrm{vdt})_{2}\right](\mathrm{VI})$} \\
\hline HOMO-1 (115) & 0.0592 & -0.0848 & HOMO (115) & -0.1944 \\
\hline HOMO (116) & 0.0662 & -0.0658 & LUMO (116) & -0.1431 \\
\hline LUMO (117) & 0.2133 & 0.0600 & LUMO+1 (117) & -0.0592 \\
\hline \multirow[t]{2}{*}{$\Delta(117-116)$} & 0.1471 & 0.1258 & $\Delta(117-116)$ & 0.0513 \\
\hline & {$\left[\mathrm{WO}(\mathrm{vdt})_{2}\right]^{2-}(\mathrm{IV})$} & {$\left[\mathrm{WO}(\mathrm{vdt})_{2}\right]^{-}(\mathrm{V})$} & & {$\left[\mathrm{WO}(\mathrm{vdt})_{2}\right](\mathrm{VI})$} \\
\hline HOMO-1 (115) & 0.0576 & -0.0877 & HOMO (115) & -0.1971 \\
\hline HOMO (116) & 0.0814 & -0.0657 & LUMO (116) & -0.1364 \\
\hline LUMO (117) & 0.2168 & 0.0798 & LUMO+1 (117) & -0.0473 \\
\hline \multirow[t]{2}{*}{$\Delta(117-116)$} & 0.1354 & 0.1455 & $\Delta(117-116)$ & 0.0607 \\
\hline & {$\left[\mathrm{MoO}(\mathrm{ndt})_{2}\right]^{2-}(\mathrm{IV})$} & {$\left[\mathrm{MoO}(\mathrm{ndt})_{2}\right]^{-}(\mathrm{V})$} & & {$\left[\mathrm{MoO}(\mathrm{ndt})_{2}\right](\mathrm{VI})$} \\
\hline HOMO-1 (107) & -0.0050 & -0.1586 & HOMO (107) & -0.2468 \\
\hline HOMO (108) & 0.0109 & -0.1350 & LUMO (108) & -0.2227 \\
\hline LUMO (109) & 0.1111 & -0.0073 & LUMO+1 (109) & -0.1155 \\
\hline$\Delta(109-108)$ & 0.1002 & 0.1277 & $\Delta(109-108)$ & 0.0241 \\
\hline
\end{tabular}




\begin{tabular}{|c|c|c|c|c|}
\hline & {$\left[\mathrm{WO}(\text { ndt })_{2}\right]^{2-}(\mathrm{IV})$} & {$\left[\mathrm{WO}(\text { ndt })_{2}\right]^{-}(\mathrm{V})$} & & {$\left[\mathrm{WO}(\mathrm{ndt})_{2}\right](\mathrm{VI})$} \\
\hline HOMO-1 (107) & -0.0065 & 0.1590 & HOMO (107) & -0.2750 \\
\hline HOMO (108) & 0.0265 & -0.1315 & LUMO (108) & -0.2005 \\
\hline LUMO (109) & 0.1105 & 0.0004 & LUMO+1 (109) & -0.1207 \\
\hline \multirow[t]{2}{*}{$\Delta(109-108)$} & 0.0840 & 0.1311 & $\Delta(109-108)$ & 0.0745 \\
\hline & {$\left[\mathrm{MoO}(\mathrm{qdt})_{2}\right]^{2-}(\mathrm{IV})$} & {$\left[\mathrm{MoO}(\mathrm{qdt})_{2}\right]^{-}(\mathrm{V})$} & & {$\left[\mathrm{MoO}(\mathrm{qdt})_{2}\right](\mathrm{VI})$} \\
\hline HOMO-1 (109) & 0.0121 & -0.1255 & HOMO (109) & -0.2415 \\
\hline HOMO (110) & 0.0421 & -0.1115 & LUMO (110) & -0.1814 \\
\hline LUMO (111) & 0.1360 & 0.0233 & LUMO+1 (111) & -0.0970 \\
\hline \multirow[t]{2}{*}{$\Delta(111-110)$} & 0.0939 & 0.1348 & $\Delta(111-110)$ & 0.0601 \\
\hline & {$\left[\mathrm{WO}(\mathrm{qdt})_{2}\right]^{2-}(\mathrm{IV})$} & {$\left[\mathrm{WO}(\mathrm{qdt})_{2}\right]^{-}(\mathrm{V})$} & & {$\left[\mathrm{WO}(\mathrm{qdt})_{2}\right](\mathrm{VI})$} \\
\hline HOMO-1 (109) & 0.0111 & -0.1233 & HOMO (109) & -0.2443 \\
\hline HOMO (110) & 0.0586 & -0.1141 & LUMO (110) & -0.1718 \\
\hline LUMO (111) & 0.1353 & 0.0230 & LUMO+1 (111) & -0.0897 \\
\hline \multirow{2}{*}{$\Delta(111-110)$} & 0.0767 & 0.1371 & $\Delta(111-110)$ & 0.0725 \\
\hline & {$\left[\mathrm{MoO}(\mathrm{ntdt})_{2}\right]^{2-}(\mathrm{IV})$} & {$\left[\mathrm{MoO}(\text { ntdt })_{2}\right]^{-}(\mathrm{V})$} & & {$\left[\mathrm{MoO}(\text { ntdt })_{2}\right](\mathrm{VI})$} \\
\hline HOMO-1 (123) & 0.0506 & -0.0945 & HOMO (123) & -0.2078 \\
\hline HOMO (124) & 0.0538 & -0.0720 & LUMO (124) & -0.1515 \\
\hline LUMO (125) & 0.1287 & 0.0378 & LUMO+1 (125) & -0.0735 \\
\hline \multirow[t]{2}{*}{$\Delta(125-124)$} & 0.0749 & 0.1098 & $\Delta(125-124)$ & 0.0563 \\
\hline & {$\left[\mathrm{WO}(\mathrm{ntdt})_{2}\right]^{2-}(\mathrm{IV})$} & {$\left[\text { WO }(\text { ntdt })_{2}\right]^{-}(\mathbf{V})$} & & {$\left[\mathrm{WO}(\text { ntdt })_{2}\right](\mathrm{VI})$} \\
\hline HOMO-1 (123) & 0.0493 & -0.0976 & HOMO (123) & -0.2104 \\
\hline HOMO (124) & 0.0692 & -0.0716 & LUMO (124) & -0.1442 \\
\hline LUMO (125) & 0.1280 & 0.0376 & LUMO+1 (125) & -0.0646 \\
\hline \multirow[t]{2}{*}{$\Delta(125-124)$} & 0.0588 & 0.1092 & $\Delta(125-124)$ & 0.0662 \\
\hline & {$\left[\mathrm{MoO}(\mathrm{tldt})_{2}\right]^{2-}(\mathrm{IV})$} & {$\left[\mathrm{MoO}(\mathrm{tldt})_{2}\right]^{-}(\mathrm{V})$} & & {$\left[\mathrm{MoO}(\mathrm{tldt})_{2}\right](\mathrm{VI})$} \\
\hline HOMO-1 (111) & 0.0639 & -0.0701 & HOMO (111) & -0.2012 \\
\hline HOMO (112) & 0.0650 & -0.0622 & LUMO (112) & -0.1462 \\
\hline LUMO (113) & 0.1761 & 0.0649 & LUMO+1 (113) & -0.0671 \\
\hline \multirow[t]{2}{*}{$\Delta(113-112)$} & 0.1111 & 0.1271 & $\Delta(113-112)$ & 0.0550 \\
\hline & {$\left[\mathrm{WO}(\mathrm{tldt})_{2}\right]^{2-}(\mathrm{IV})$} & {$\left[\mathrm{WO}(\mathrm{tldt})_{2}\right]^{-}(\mathrm{V})$} & & {$\left[\mathrm{WO}(\mathrm{tldt})_{2}\right](\mathrm{VI})$} \\
\hline HOMO-1 (111) & 0.0623 & -0.0902 & HOMO (111) & -0.2041 \\
\hline HOMO (112) & 0.0804 & -0.0684 & LUMO (112) & -0.1391 \\
\hline LUMO (113) & 0.1759 & 0.0644 & LUMO+1 (113) & -0.0581 \\
\hline \multirow[t]{2}{*}{$\Delta(113-112)$} & 0.0955 & 0.1328 & $\Delta(113-112)$ & 0.0650 \\
\hline & {$\left[\mathrm{MoO}(\mathrm{tcdt})_{2}\right]^{2-}(\mathrm{IV})$} & {$\left[\mathrm{MoO}(\mathrm{tcdt})_{2}\right]^{-}(\mathrm{V})$} & & {$\left[\mathrm{MoO}(\text { tcdt })_{2}\right](\mathrm{VI})$} \\
\hline HOMO-1 (119) & 0.0527 & -0.0960 & HOMO (119) & -0.2099 \\
\hline HOMO (120) & 0.0541 & -0.0746 & LUMO (120) & -0.1552 \\
\hline LUMO (121) & 0.1613 & 0.0454 & LUMO+1 (121) & -0.0771 \\
\hline \multirow[t]{2}{*}{$\Delta(121-120)$} & 0.1072 & 0.1200 & $\Delta(121-120)$ & 0.0547 \\
\hline & $\left.\left[\mathrm{WO}(\mathrm{tcdt})_{2}\right]^{2-}(\mathrm{IV})\right)$ & $\left.[\text { WO(tcdt })_{2}\right]^{-}(\mathrm{V})$ & & {$\left[\mathrm{WO}(\text { tcdt })_{2}\right](\mathrm{VI})$} \\
\hline HOMO-1 (119) & 0.0509 & -0.1003 & HOMO (119) & -0.2123 \\
\hline HOMO (120) & 0.0686 & -0.0789 & LUMO (120) & -0.1482 \\
\hline LUMO (121) & 0.1611 & 0.0511 & LUMO+1 (121) & -0.0690 \\
\hline \multirow[t]{2}{*}{$\Delta(121-120)$} & 0.0925 & 0.1300 & $\Delta(121-120)$ & 0.0641 \\
\hline & $\begin{array}{c}{\left[\mathrm{MoO}(\mathrm{cydt})_{2}\right]^{2-}} \\
(\text { IV })\end{array}$ & $\begin{array}{c}{\left[\mathrm{MoO}(\mathrm{cydt})_{2}\right]^{-}} \\
(\mathrm{V})\end{array}$ & & $\begin{array}{c}{\left[\mathrm{MoO}(\mathrm{cydt})_{2}\right]} \\
\text { (VI) }\end{array}$ \\
\hline HOMO-1 (87) & 0.0883 & -0.0776 & HOMO (87) & -0.2109 \\
\hline HOMO (88) & 0.0909 & -0.0532 & LUMO (88) & -0.1465 \\
\hline LUMO (89) & 0.2361 & 0.0736 & LUMO+1 (89) & -0.0669 \\
\hline \multirow[t]{2}{*}{$\Delta(89-88)$} & 0.1452 & 0.1268 & $\Delta(89-88)$ & 0.0644 \\
\hline & $\begin{array}{c}{\left[\mathrm{WO}(\text { cydt })_{2}\right]^{2-}} \\
\text { (IV) }\end{array}$ & $\begin{array}{c}{\left[\mathrm{WO}(\mathrm{cydt})_{2}\right]^{-}} \\
(\mathrm{V})\end{array}$ & & $\begin{array}{c}{\left[\mathrm{WO}(\text { cydt })_{2}\right]} \\
\text { (VI) }\end{array}$ \\
\hline HOMO-1 (87) & 0.0865 & -0.0810 & HOMO (87) & -0.1818 \\
\hline HOMO (88) & 0.1062 & -0.0527 & LUMO (88) & -0.1599 \\
\hline LUMO (89) & 0.2483 & 0.0928 & LUMO+1 (89) & -0.0294 \\
\hline$\Delta(89-88)$ & 0.1421 & 0.1455 & $\Delta(89-88)$ & 0.0209 \\
\hline
\end{tabular}




\begin{tabular}{|c|c|c|c|c|}
\hline & {$\left[\mathrm{MoO}(\mathrm{mpt})_{2}\right]^{2-}(\mathrm{IV})$} & {$\left[\mathrm{MoO}(\mathbf{m p t})_{2}\right]^{-}(\mathbf{V})$} & & {$\left[\mathrm{MoO}(\mathrm{mpt})_{2}\right](\mathrm{VI})$} \\
\hline HOMO-1 (157) & 0.0460 & -0.0960 & HOMO (157) & -0.1790 \\
\hline HOMO (158) & 0.0480 & -0.0810 & LUMO (158) & -0.1550 \\
\hline LUMO (159) & 0.1290 & 0.0470 & LUMO+1 (159) & -0.0700 \\
\hline \multirow[t]{2}{*}{$\Delta(159-158)$} & 0.0820 & 0.1280 & $\Delta(159-158)$ & 0.0240 \\
\hline & {$\left[\mathrm{WO}(\mathrm{mpt})_{2}\right]^{2-}(\mathrm{IV})$} & {$\left[\mathrm{WO}(\mathrm{mpt})_{2}\right]^{-}(\mathrm{V})$} & & {$\left[\mathrm{WO}(\mathrm{mpt})_{2}\right](\mathrm{VI})$} \\
\hline HOMO-1 (157) & 0.0470 & -0.0950 & HOMO (157) & -0.1790 \\
\hline HOMO (158) & 0.0660 & -0.0790 & LUMO (158) & -0.1520 \\
\hline LUMO (159) & 0.1280 & 0.0590 & LUMO+1 (159) & -0.0670 \\
\hline$\Delta(159-158)$ & 0.0620 & 0.1380 & $\Delta(159-158)$ & 0.0268 \\
\hline
\end{tabular}




\begin{tabular}{|c|c|c|c|}
\hline Verbindungen & $1 a$ & $1 b$ & $2 a$ \\
\hline Empirische Formel & $\mathrm{C}_{62} \mathrm{H}_{108} \mathrm{Mo}_{2} \mathrm{~N}_{4} \mathrm{~S}_{8} \mathrm{O}_{2}$ & $\mathrm{C}_{42} \mathrm{H}_{60} \mathrm{~N}_{2} \mathrm{OS}_{4} \mathrm{~W}$ & $\mathrm{C}_{30} \mathrm{H}_{52} \mathrm{~N}_{2} \mathrm{OS}_{4} \mathrm{Mo}$ \\
\hline$M\left[\mathrm{~g} \mathrm{~mol}^{-1}\right]$ & 1389.97 & 921.01 & 680.92 \\
\hline $\mathbf{T}[\mathbf{K}]$ & $133(2)$ & $133(2)$ & $133(2)$ \\
\hline Kristallgröße [mm] & $0.23 \times 0.16 \times 0.11$ & $0.09 \times 0.07 \times 0.03$ & $0.45 \times 0.40 \times 0.33$ \\
\hline Kristallsystem & Monoklin & Triklin & Monoklin \\
\hline Raumgruppe & $\mathrm{P} 21 / \mathrm{c}$ & $P-1$ & $\mathrm{P} 2_{1}$ \\
\hline$a[\AA]$ & $9.2798(19)$ & $9.6497(19)$ & $9.2238(18)$ \\
\hline$b[\AA]$ & $14.016(3)$ & $15.672(3)$ & $13.832(3)$ \\
\hline$c[\AA]$ & $15.812(5)$ & $16.158(3)$ & $13.314(3)$ \\
\hline$\alpha\left[^{\circ}\right]$ & 90.00 & $117.92(3)$ & 90.00 \\
\hline$\beta\left[^{\circ}\right]$ & $120.81(2)$ & $94.62(3)$ & $97.83(3)$ \\
\hline$\gamma\left[{ }^{\circ}\right]$ & 90.00 & $96.55(3)$ & 90.00 \\
\hline$V\left[\AA^{3}\right]$ & $1766.35(21)$ & $2119.8(10)$ & $1682.9(6)$ \\
\hline$Z$ & 2 & 2 & 2 \\
\hline pber. $\left[\mathrm{g} \mathrm{cm}^{-3}\right]$ & 1.306 & 1.443 & 1.344 \\
\hline$\mu\left(\mathbf{M o}-K_{\alpha}\right)\left[\mathbf{m m}^{-1}\right]$ & 0.977 & 2.955 & 0.663 \\
\hline$F(000)$ & 860 & 944 & 720 \\
\hline$\theta$ Bereich $\left[{ }^{\circ}\right]$ & $2.64-26.94$ & $1.44-27.00$ & $1.54-26.95$ \\
\hline$h k l$ Bereich [h;k;l] & $-11 / 10 ; \pm 17 ; \pm 20$ & $\pm 12 ;-20 / 16 ;-18 / 20$ & $-11 / 10 ; \pm 17 ; \pm 16$ \\
\hline Gemessene Reflexe & 15101 & 15962 & 15217 \\
\hline Unabhängige Reflexe $\left[\mathbf{R}_{\text {int }}\right]$ & $3735[0.0430]$ & $9174[0.0941]$ & $6915[0.0649]$ \\
\hline Goodness-of-fit & 1.134 & 1.002 & 1.057 \\
\hline$R 1 . w R 2(I>2 \sigma(I))$ & 0.01370 .0305 & 0.0661 .0 .1158 & 0.0546 .0 .1431 \\
\hline R1. wR2 (alle Daten) & 0.01380 .0306 & 0.1140 .0 .1315 & 0.0551 .0 .1435 \\
\hline Restelektronendichte $\left[\mathbf{e}^{\circ} \AA^{-3}\right]$ & $0.767 / 0.736$ & $1.188 /-1.734$ & $1.494 /-1.352$ \\
\hline
\end{tabular}

$R 1=\Sigma|| F_{\mathrm{o}}|-| F_{\mathrm{c}}|| / \Sigma\left|F_{\mathrm{o}}\right| . w R 2=\left[\sum w\left(F_{\mathrm{o}}^{2}-F_{\mathrm{c}}^{2}\right)^{2} / \Sigma w\left(F_{\mathrm{o}}^{2}\right)^{2}\right]^{0.5}$. 


\begin{tabular}{|c|c|c|c|}
\hline Verbindungen & $2 c$ & $3 a$ & $3 b$ \\
\hline Empirische Formel & $\mathrm{C}_{32} \mathrm{H}_{52} \mathrm{~N}_{4} \mathrm{~S}_{4} \mathrm{~W}$ & $\mathrm{C}_{20} \mathrm{H}_{32} \mathrm{Mo} \mathrm{Na}_{2} \mathrm{O}_{9} \mathrm{~S}_{4}$ & $\mathrm{~W}_{2} \mathrm{~S}_{8} \mathrm{O}_{10} \mathrm{~N}_{4} \mathrm{C}_{81} \mathrm{H}_{92}$ \\
\hline$M\left[\mathrm{~g} \mathrm{~mol}^{-1}\right]$ & 804.87 & 686.62 & 1905.87 \\
\hline $\mathbf{T}[\mathbf{K}]$ & $133(2)$ & $133(2)$ & $133(2)$ \\
\hline Kristallgröße [mm] & $0.53 \times 0.27 \times 0.51$ & $0.49 \times 0.47 \times 0.34$ & $0.32 \times 0.21 \times 0.38$ \\
\hline Kristallsystem & Monoklin & Triklin & Triklin \\
\hline Raumgruppe & $\mathrm{C} 2 / \mathrm{c}$ & $P-1$ & $\mathrm{P} 1(1)$ \\
\hline$a[\AA]$ & $32.7425(65)$ & $8.3805(17)$ & $9.0131(18)$ \\
\hline$b[\AA]$ & $16.2364(32)$ & $9.2807(19)$ & $13.8037(28)$ \\
\hline$c[\AA]$ & $13.7608(28)$ & $20.013(4)$ & $18.7923(38)$ \\
\hline$\alpha\left[^{\circ}\right]$ & 90.00 & $101.83(3)$ & $95.88(3)$ \\
\hline$\beta\left[^{\circ}\right]$ & 91.69 & $97.32(3)$ & $96.19(3)$ \\
\hline$\gamma\left[{ }^{\circ}\right]$ & 90.00 & $100.05(3)$ & $94.79(3)$ \\
\hline$V\left[\AA^{3}\right]$ & 7312 & $1478.4(5)$ & 2301.6(13) \\
\hline$Z$ & 8 & 2 & 4 \\
\hline pber. $\left[\mathrm{g} \mathrm{cm}^{-3}\right]$ & 0.0978876 & 1.542 & 1.328 \\
\hline$\mu\left(\mathrm{Mo}-\mathrm{K}_{\alpha}\right)\left[\mathrm{mm}^{-1}\right]$ & 1.462 & 0.798 & 0.932 \\
\hline$F(000)$ & 3280 & 704 & 4321 \\
\hline$\theta$ Bereich $\left[{ }^{\circ}\right]$ & $2.02-25.78$ & $2.29-25.83$ & $2.21-25.83$ \\
\hline hkl Bereich [h;k;l] & $\pm 39 ; \pm 19 ; \pm 16$ & $\pm 10 ; \pm 11 ;-23 / 24$ & $\pm 28 ;-13 / 15 ; \pm 16$ \\
\hline Gemessene Reflexe & 27621 & 11820 & 33568 \\
\hline Unabhängige Reflexe $\left[\mathbf{R}_{\text {int }}\right]$ & 6897 & $5633[0.0322]$ & $5763[0.0350]$ \\
\hline Goodness-of-fit & 1.075 & 1.079 & 1.148 \\
\hline R1. wR2 $(I>2 \sigma(I))$ & 0.03400 .0733 & 0.0258 .0 .0706 & 0.0990 .0 .1078 \\
\hline R1. wR2 (alle Daten) & 0.06460 .0975 & 0.0281 .0 .0717 & 0.01252 .0 .1132 \\
\hline Restelektronendichte $\left[\mathrm{e} \AA^{-3}\right]$ & $4.027 / 1.927$ & $0.650 /-0.720$ & $1.049 /-0.756$ \\
\hline
\end{tabular}

$R 1=\Sigma|| F_{\mathrm{o}}|-| F_{\mathrm{c}}|| / \Sigma\left|F_{\mathrm{o}}\right| . w R 2=\left[\Sigma w\left(F_{\mathrm{o}}^{2}-F_{\mathrm{c}}^{2}\right)^{2} / \Sigma w\left(F_{\mathrm{o}}^{2}\right)^{2}\right]^{0.5}$. 


\begin{tabular}{|c|c|c|c|}
\hline Verbindungen & $5 a$ & $5 b$ & $5 c$ \\
\hline Empirische Formel & $\mathrm{C}_{42} \mathrm{H}_{52} \mathrm{MoN}_{6} \mathrm{OS}_{4}$ & $\mathrm{C}_{50} \mathrm{H}_{83} \mathrm{~N}_{7} \mathrm{OS}_{4} \mathrm{~W}$ & $\mathrm{C}_{8} \mathrm{H}_{10} \mathrm{~N}_{2} \mathrm{~S}_{2}$ \\
\hline$M\left[\mathrm{~g} \mathrm{~mol}^{-1}\right]$ & 881.08 & 1110.32 & 198.30 \\
\hline $\mathbf{T}[\mathbf{K}]$ & $133(2)$ & $133(2)$ & $133(2)$ \\
\hline Kristallgröße [mm] & $0.48 \times 0.44 \times 0.20$ & $0.50 \times 0.28 \times 0.26$ & $0.63 \times 0.06 \times 0.05$ \\
\hline Kristallsystem & Monoklin & Monoklin & Monoklin \\
\hline Raumgruppe & $\mathrm{P} 21 / \mathrm{c}$ & $\mathrm{P} 21 / \mathrm{c}$ & $\mathrm{P} 2_{1} / \mathrm{n}$ \\
\hline$a[\AA]$ & $18.978(4)$ & $19.723(4)$ & $3.8389(8)$ \\
\hline$b[\AA]$ & $12.596(3)$ & $17.245(3)$ & $9.2332(18)$ \\
\hline$c[\AA]$ & $17.724(4)$ & $16.654(3)$ & $18.627(4)$ \\
\hline$\alpha\left[{ }^{\circ}\right]$ & 90.00 & 90.00 & 90.00 \\
\hline$\beta\left[{ }^{\circ}\right]$ & $101.49(3)$ & $107.65(3)$ & $92.11(3)$ \\
\hline$\gamma\left[{ }^{\circ}\right]$ & 90.00 & 90.00 & 90.00 \\
\hline$V\left[\AA^{3}\right]$ & $4152.1(14)$ & $5397.7(18)$ & $659.8(2)$ \\
\hline$Z$ & 4 & 4 & 3 \\
\hline pber. $\left[\mathrm{g} \mathrm{cm}^{-3}\right]$ & 1.409 & 1.366 & 1.497 \\
\hline$\mu\left(\mathbf{M o}-K_{\alpha}\right)\left[\mathrm{mm}^{-1}\right]$ & 0.558 & 2.336 & 0.546 \\
\hline$F(000)$ & 1840 & 2312 & 312 \\
\hline$\theta$ Bereich $\left[{ }^{\circ}\right]$ & $1.95-27.02$ & $1.60-27.05$ & $2.19-27.01$ \\
\hline hkl Bereich [h;k;l] & $\pm 24 .-16 / 15 . \pm 22$ & $\pm 25 .-22 / 19 . \pm 21$ & $\pm 4 ; \pm 11 ; \pm 23$ \\
\hline Gemessene Reflexe & 36578 & 46115 & 5655 \\
\hline Unabhängige Reflexe $\left[R_{\text {int }}\right]$ & $8804[0.0364]$ & $11554[0.1061]$ & $1428[0.1602]$ \\
\hline Goodness-of-fit & 1.120 & 1.088 & 0.833 \\
\hline R1. wR2 $(I>2 \sigma(I))$ & 0.0283 .0 .0720 & 0.0653 .0 .0879 & 0.0518 .0 .1309 \\
\hline R1. wR2 (alle Daten) & 0.0349 .0 .0774 & 0.1057 .0 .0972 & 0.0618 .0 .1398 \\
\hline Restelektronendichte $\left[\mathrm{e} \AA^{-3}\right]$ & $0.364 /-0.445$ & $1.482 /-2.197$ & $0.401 /-0.351$ \\
\hline
\end{tabular}

$R 1=\Sigma|| F_{\mathrm{o}}|-| F_{\mathrm{c}}|| / \Sigma\left|F_{\mathrm{o}}\right| \cdot w R 2=\left[\Sigma w\left(F_{\mathrm{o}}^{2}-F_{\mathrm{c}}^{2}\right)^{2} / \Sigma w\left(F_{\mathrm{o}}^{2}\right)^{2}\right]^{0.5}$. 


\begin{tabular}{|c|c|c|c|}
\hline Verbindungen & $6 a$ & $10 a$ & $17 b$ \\
\hline Empirische Formel & $\mathrm{C}_{56} \mathrm{H}_{88} \mathrm{MoN}_{2} \mathrm{OS}_{4}$ & $\mathrm{C}_{38} \mathrm{H}_{56} \mathrm{Mo} \mathrm{N}_{2} \mathrm{OS}_{4}$ & $\mathrm{C}_{24} \mathrm{H}_{48} \mathrm{Mo} \mathrm{N}_{2} \mathrm{OS}_{6}$ \\
\hline$M\left[\mathrm{~g} \mathrm{~mol}^{-1}\right]$ & 1029.46 & 781.03 & 668.94 \\
\hline $\mathbf{T}[\mathbf{K}]$ & $133(2)$ & $133(2)$ & $133(2)$ \\
\hline Kristallgröße [mm] & $0.47 \times 0.19 \times 0.19$ & $0.31 \times 0.25 \times 0.24$ & $0.24 \times 0.13 \times 0.05$ \\
\hline Kristallsystem & Orthorhombisch & Monoklin & Monoklin \\
\hline Raumgruppe & P212121 & $\mathrm{P} 21 / \mathrm{c}$ & $\mathrm{P} 21 / \mathrm{n}$ \\
\hline$a[\AA]$ & $14.281(3)$ & $10.513(2)$ & $15.956(3)$ \\
\hline$b[\AA]$ & $14.491(3)$ & $22.232(4)$ & $12.071(2)$ \\
\hline$c[\AA]$ & $27.124(5)$ & $17.823(4)$ & $17.111(3)$ \\
\hline$\alpha\left[{ }^{\circ}\right]$ & 90.00 & 90.00 & 90.00 \\
\hline$\beta\left[^{\circ}\right]$ & 90.00 & $103.65(3)$ & $109.01(3)$ \\
\hline$\gamma\left[{ }^{\circ}\right]$ & 90.00 & 90.00 & 90.00 \\
\hline$V\left[\AA^{3}\right]$ & $5613(2)$ & 4048.1(14) & $3115.9(11)$ \\
\hline$Z$ & 4 & 4 & 4 \\
\hline pber. $\left[\mathrm{g} \mathrm{cm}^{-3}\right]$ & 1.218 & 1.282 & 1.426 \\
\hline$\mu\left(\mathbf{M o}-K_{\alpha}\right)\left[\mathrm{mm}^{-1}\right]$ & 0.420 & 0.560 & 0.843 \\
\hline$F(000)$ & 2208 & 1648 & 1408 \\
\hline$\theta$ Bereich $\left[{ }^{\circ}\right]$ & $1.50-25.86$ & $1.49-25.92$ & $1.52-27.07$ \\
\hline hkl Bereich [h;k;l] & $-16 / 17 ; \pm 17 ; \pm 33$ & $\pm 12 ; \pm 27 ; \pm 21$ & $-20 / 19 ;-15 / 15 ;-21 / 21$ \\
\hline Gemessene Reflexe & 49904 & 31651 & 27529 \\
\hline Unabhängige Reflexe $\left[R_{\text {int }}\right]$ & $10862[0.0378]$ & $7817[0.0741]$ & 6805 [0.1219] \\
\hline Goodness-of-fit & 1.010 & 1.030 & 0.961 \\
\hline R1. wR2 $(I>2 \sigma(I))$ & 0.0305 .0 .0690 & 0.0402 .0 .1042 & 0.0403 .0 .0846 \\
\hline R1. wR2 (alle Daten) & 0.0362 .0 .0706 & 0.0552 .0 .1099 & 0.0587 .0 .0898 \\
\hline Restelektronendichte $\left[\mathrm{e} \AA^{-3}\right.$ ] & $0.414 /-0.277$ & $0.598 /-0.756$ & $0.707 /-0.701$ \\
\hline
\end{tabular}

$R 1=\Sigma|| F_{\mathrm{o}}|-| F_{\mathrm{c}}|| / \Sigma\left|F_{\mathrm{o}}\right| \cdot w R 2=\left[\Sigma w\left(F_{\mathrm{o}}^{2}-F_{\mathrm{c}}^{2}\right)^{2} / \Sigma w\left(F_{\mathrm{o}}^{2}\right)^{2}\right]^{0.5}$. 


\begin{tabular}{|c|c|c|c|}
\hline Verbindungen & $17 c$ & 20 & 22 \\
\hline Empirische Formel & $\mathrm{C}_{8} \mathrm{H}_{20} \mathrm{NS}_{4} \mathrm{~K}$ & $\mathrm{C}_{56} \mathrm{H}_{96} \mathrm{~N}_{4} \mathrm{O}_{4} \mathrm{~S}_{12} \mathrm{Mo}_{4}$ & $\mathrm{C}_{36} \mathrm{H}_{49} \mathrm{Cl}_{2} \mathrm{Mo} \mathrm{N} \mathrm{N}_{2} \mathrm{O}$ \\
\hline$M\left[\mathrm{~g} \mathrm{~mol}^{-1}\right]$ & 297.59 & 1657.85 & 692.61 \\
\hline $\mathbf{T}[\mathbf{K}]$ & $133(2)$ & $133(2)$ & $133(2)$ \\
\hline Kristallgröße [mm] & $0.37 \times 0.34 \times 0.34$ & $0.36 \times 0.19 \times 0.29$ & $0.50 \times 0.37 \times 0.21$ \\
\hline Kristallsystem & Monoklin & Orthorhombisch & Orthorhombisch \\
\hline Raumgruppe & $\mathrm{P} 2{ }_{1} / \mathrm{n}$ & Pbca & $\mathrm{Pbca}$ \\
\hline$a[\AA]$ & $7.0770(14)$ & $12.4565(3)$ & $19.382(4)$ \\
\hline$b[\AA]$ & $17.280(4)$ & $17.3548(5)$ & $18.065(4)$ \\
\hline$c[\AA]$ & $11.906(2)$ & $32.7301(7)$ & $20.419(4)$ \\
\hline$\alpha\left[^{\circ}\right]$ & 90.00 & 90.00 & 90.00 \\
\hline$\beta\left[{ }^{\circ}\right]$ & $106.05(3)$ & 90.00 & 90.00 \\
\hline$\gamma\left[{ }^{\circ}\right]$ & 90.00 & 90.00 & 90.00 \\
\hline$V\left[\AA^{3}\right]$ & $1399.3(5)$ & $7075.6(3)$ & $7149(2)$ \\
\hline$Z$ & 4 & 8 & 8 \\
\hline pber. $\left[\mathrm{g} \mathrm{cm}^{-3}\right]$ & 1.413 & 1.556 & 1.287 \\
\hline$\mu\left(\mathbf{M o}-K_{\alpha}\right)\left[\mathbf{m m}^{-1}\right]$ & 0.944 & 1.091 & 0.545 \\
\hline$F(000)$ & 632 & 3408 & 2904 \\
\hline$\theta$ Bereich $\left[{ }^{\circ}\right]$ & $2.13-27.00$ & $1.24-25.63$ & $1.84-25.86$ \\
\hline$h k l$ Bereich [h;k;l] & $-8 / 9 ; \pm 22 / \pm 15$ & $-15 / 12 ; \pm 21 . \pm 39$ & $-20 / 23 ;-21 / 22 ;-25 / 24$ \\
\hline Gemessene Reflexe & 12554 & 55893 & 57436 \\
\hline Unabhängige Reflexe $\left[R_{\text {int }}\right]$ & $3012[0.0420]$ & $6653[0.1110]$ & $6584[0.0322]$ \\
\hline Goodness-of-fit & 1.212 & 1.077 & 1.037 \\
\hline$R 1 . w R 2(I>2 \sigma(I))$ & $0.0625 ; 0.2103$ & 0.0361 .0 .0792 & 0.0273 .0 .0657 \\
\hline R1. wR2 (alle Daten) & $0.0625 / 0.2137$ & 0.0449 .0 .0827 & 0.0346 .0 .0682 \\
\hline Restelektronendichte $\left[\mathrm{e} \AA^{-3}\right.$ ] & $0.761 /-2.308$ & 0.860. -0.757 & $0.565 /-0.326$ \\
\hline
\end{tabular}

$R 1=\Sigma|| F_{\mathrm{o}}|-| F_{\mathrm{c}}|| / \Sigma\left|F_{\mathrm{o}}\right| \cdot w R 2=\left[\Sigma w\left(F_{\mathrm{o}}^{2}-F_{\mathrm{c}}^{2}\right)^{2} / \Sigma w\left(F_{\mathrm{o}}^{2}\right)^{2}\right]^{0.5}$. 


\subsection{Literaturverzeichnis}

[1] A. Kletzin, M. W. W. Adams, Microbiol. Rev. 1996, 18, 5-63.

[2] R. Hille, Trend. Biochem. Sci. 2002, 27, 360-367.

[3] a)C. Scott, T. W. Lyons, A. Bekker, Y. Shen, S. W. Poulton, X. Chu, A. D. Anbar, Nature 2008, 452, 456-460; b)K. K. Bertine, K. K. Turekian, Cosmochim. Acta 1973, 37, 1415-1434; c)S. R. Taylor, S. M. McLennan, Rev. Geophys. 1995, 33, 241-265.

[4] C. Schulzke, Dalton Trans. 2005, 713-720.

[5] J. H. Enemark, J. J. A. Cooney, J. J. Wang, R. H. Holm, Chem. Rev. 2004, 104, 11751200 .

[6] J. L. Johnson, K. V. Rajagopalan, Proc. Nat. Acad. Sci. USA 1982, 79, 6856-6860.

[7] S. P. Kramer, J. L. Johnson, A. A. Ribeiro, D. S. Millington, K. V. Rajagopalan, J. Biol. Chem. 1987, 262, 16357-16363.

[8] O. Einsle, F. A. Tezcan, S. L. A. Andrade, B. Schmid, M. Yoshida, J. B. Howard, D. C. Rees, Science 2002, 297, 1696-1700.

[9] R. Hille, Chem. Rev. 1996, 96, 2757-2816.

[10] B. G. Seiffert, G. M. Ullmann, A. Messerschmidt, B. Schink, P. M. H. Kroneck, O. Einsle, Proc. Nat. Acad. Sci. USA 2007, 104, 3073-3077.

[11] E. Karakas, H. L. Wilson, T. N. Graf, S. Xiang, S. Jaramillo-Busquets, K. V. Rajagopalan, C. Kisker, J. Biol. Chem. 2005, 280, 33506-33515.

[12] R. M. Garrett, K. V. Rajagopalan, J. Biol. Chem. 1996, 271, 7387-7391.

[13] C. C. Page, C. C. Moser, X. Chen, P. L. Dutton, Nature 1999, 402, 47-52.

[14] Y. Kubo, N. Ogura, H. Nakagawa, J. Biol. Chem. 1999, 263, 1968-1971.

[15] a)C. A. Kipke, M. A. Cusanovich, G. Tollin, R. A. Sunde, J. H. Enemark, Biochemistry 1988, 27, 2918-2926; b)E. P. Sullivan Jr., J. T. Hazzard, G. Tollin, J. H. Enemark, Biochemistry 1993, 32, 12465-12470.

[16] a)J. M. Berg, R. H. Holm, J. Am. Chem. Soc. 1985, 107, 917-925; b)J. M. Berg, R. H. Holm, J. Am. Chem. Soc. 1985, 107, 925-932.

[17] M. L. Mader, M. D. Carducci, J. H. Enemark, Inorg. Chem. 2000, 3, 525-531.

[18] B. S. Lim, M. W. Willer, M. Miao, R. H. Holm, J. Am. Chem. Soc. 2001, 123, 83438349 .

[19] a)M. J. Romão, M. Archer, I. Moura, J. J. G. Moura, J. LeGall, R. Engh, M. Schneider, P. Hof, R. Huber, Science 1995, 270, 1170-1176; b)R. Huber, P. Hof, R. O. Duarte, J. J. G. Moura, I. Moura, M. Y. Liu, J. LeGall, R. Hille, M. J. Romão, J. Proc. Nat. Ac. Sci USA 1996, 93, 8846-8851. 
[20] a)C. J. Doonan, D. J. Nielsen, P. D. Smith, J. W. White, G. N. George, C. G. Young, J. Am. Chem. Soc. 2006, 128, 305-316; b)L. J. Laughlin, A. E. Aston, N. G. Graham, R. T. T. Edward, C. G. Young, Inorg. Chem. 2007, 46, 939-948.

[21] H. Schindelin, C. Kisker, J. Hilton, K. V. Rajagopalan, D. C. Rees, Science 1996, 272, 1615-1621.

[22] J. C. Boyington, V. N. Gladyshev, S. V. Khangulov, T. C. Stadtman, P. D. Sun, Science 1997, 275, 1305-1308.

[23] P. Ellis, T. Conrads, R. Hille, P. Kuhn, Structure 2001, 9, 125-132.

[24] J. M. Dias, M. E. Than, A. Humm, R. Huber, G. P. BBourenkov, H. D. Bartunik, S. Bursakov, J. Calvete, J. Caldeira, C. Carneiro, J. J. G. Moura, I. Moura, M. J. Ramão, Structure 1999, 7, 65-79.

[25] M. J. Maher, J. Santini, I. J. Pickering, R. C. Price, J. M. Macy, G. N. George, Inorg. Chem. 2004, 43, 402-404.

[26] A. Bruce, J. L. Corbin, P. L. Dahlstrom, R. Hyde, M. Minelli, E. I. Stiefel, J. T. Spence, J. Zubieta, Inorg. Chem. 1982, 21, 917-926.

[27] I. W. Boyd, I. G. Dance, A. E. Landers, A. G. Wedd, Inorg. Chem. 1979, 18, 19751885 .

[28] S. Boyde, S. R. Ellis, C. D. Garner, W. Cleeg, Chem. Commun. 1986, 1541-1543.

[29] a)E. S. Davies, R. L. Beddoes, A. Collison, A. Dinsmore, A. Docrat, J. A. Joule, C. R. Willson, C. D. Garner, Dalton Trans. 1997, 3985-3996; b)H. Oku, N. Ueyama, M. Kondo, A. Nakamura, Inorg. Chem. 1994, 33, 209-216; c)M. A. Ansari, J. Chandrasekaran, S. Sarkar, Inorg. Chim. Acta. 1987, 133, 133-136; d)D. Coucouvanis, A. Hadjikyriacou, A. Toupadakis, S.-M. Koo, O. Ileperuma, M. Draganjac, A. Salifoglou, Inorg. Chem. 1991, 30, 754-767; e)H. Oku, N. Ueyama, H. Nakagawa, Inorg. Chem. 1997, 36, 1504-1516; f)G. Matsubayashi, T. Nojo, T. Tanaka, Inorg. Chem. 1988, 54, 133-135; g)J. A. McCleverty, J. Locke, B. Ratcliff, E. J. Wharton, Inorg. Chim. Acta. 1969, 3, 283-286; h)S. K. Das, P. K. Chaudhury, D. Biswas, S. Sarkar, J. Am. Chem. Soc. 1994, 116, 9061-9070; i)J. P. Donahue, C. R. Goldsmith, U. Nadiminti, R. H. Holm, J. Am. Chem. Soc. 1998, 120, 12869-12881.

[30] H. Sugimoto, M. Harihara, M. Shiro, K. Sugimoto, K. Tanaka, H. Miyake, H. Tsukube, Inorg. Chem. 2005, 44, 6386-6392.

[31] a)B. S. Lim, R. H. Holm, J. Am. Chem. Soc. 2001, 123, 1920-1930; b)J. J. Whang, C. Tessier, R. H. Holm, Inorg. Chem. 2006, 45, 2979-2988.

[32] S. Sarkar, S. K. Das, Proc. Ind. Ac. Sci. - Chem. Sci. 1992, 104, 533-534. 
[33] H. Sugimoto, K. Sugimoto, Inorg. Chem. Commun. 2008, 11, 77-80.

[34] S. Groysman, R. H. Holm, Inorg. Chem. 2007, 46, 4090-4102.

[35] J. A. McCleverty, Prog. Inorg. Chem. 1968, 10, 49-221.

[36] N. Svenstrup, J. Becher, Synthesis 1995, 3, 215-235.

[37] E. I. Stiefel, Dithiolene Chemistry, Vol. 52, John Wiley \& Sons New Jersey, 2004.

[38] a)R. Eisenberg, J. A. Ibers, Inorg. Chem. 1965, 605-608; b)G. N. Schrauzer, M. V., J. Am. Chem. Soc. 1962, 84, 3221; c)D. Sartain, M. R. Truter, J. Am. Chem. Soc. 1967, 1264-1272.

[39] a)R. Eisenberg, J. A. Ibers, J. Am. Chem. Soc. 1965, 87, 3776-3778; b)R. Eisenberg, J. A. Ibers, Inorg. Chem. 1966, 5, 411-416; c)R. Eisenberg, H. B. Gray, Inorg. Chem. 1967, 6, 1844-1849; d)A. E. Smith, G. N. Schrauzer, V. P. Mayweg, W. J. Heinrich, J. Am. Chem. Soc. 1965, 87, 5798-5799.

[40] S. Alvarez, R. Vicente, R. Hoffmann, J. Am. Chem. Soc. 1985, 107, 6253-6277.

[41] R. Gupta, M. Gupta, S. Paul, R. Gupta, A. Loupy, Org. Lett. 2008, 5, 153-157.

[42] F. Drawert, K. Reuer, H. Born, Chem. Ber. 1960, 93, 3056-3065.

[43] M. S. Newman, M. D. Earbman, H. Hipsher, Organic Synthesis Coll. Vol.III 1955, 188-192.

[44] E. Klingsberg, Synthesis 1972, 29-30.

[45] P. R. Asthon, U. Girreser, D. Giuffida, F. H. Kohnke, J. P. Mathias, F. M. Raymo, A. M. Z. Slawin, J. F. Stoddart, D. J. Williams, J. Am. Chem. Soc. 1993, 115, 5422-5249.

[46] a)D. M. Giolando, K. Kirschbaum, Synthesis 1992, 5, 451-452; b)G. D. Figuly, C. K. Loop, J. C. Martin, J. Am. Chem. Soc. 1989, 111, 654-658.

[47] D. C. Morrison, A. Furst, J. Org. Chem. 1965, 21, 470-471.

[48] B. Ma, J.-H. Lu, H. F. S. III, N. L. Allinger, J. Phys. Chem. 1996, 100, 8763-8769.

[49] M. J. Bennett, M. Cowie, J. L. Martin, J. Takats, J. Am. Chem. Soc. 1973, 95, 75047505 .

[50] M. E. Helton, N. E. Gruhn, R. L. McNaughton, M. L. Kirk, Inorg. Chem. 2000, 39, 2273-2278.

[51] N. N. Greenwood, A. Earnshaw, Vol. 2. , VCH, Würzburg, 1990, p. 1291.

[52] K. Starke, Georg-August-Universität (Göttingen), 2007.

[53] a)H. Sugimoto, K. Sugimoto, Inorg. Chem. Commun. 2008, 11, 77-80; b)D. H. Evans, P. J. Jimencz, M. J. Kelly, J. Electroanal. Chem. 1984, 163, 145-149.

[54] H. Sugimoto, M. Harihara, M. Shiro, K. Sugimoto, K. Tanaka, H. Miyake, H. Tsukube, Inorg. Chem. 2005, 44, 6386-6392. 
[55] H. Sugimoto, K. Suyama, K. Sugimoto, H. Miyake, I. Takahashi, S. Hirota, S. Itoh, Inorg. Chem. 2008, 47, 10150-10157.

[56] J. J. Wang, S. Groysman, S. C. Lee, R. H. Holm, J. Am. Chem. Soc. 2007, 129, 75127513.

[57] A. Döring, C. Schulzke, Q. Zhang, Inorganica Chimica Acta, In Press, Corrected Proof.

[58] M.-M. Rohmer, M. Bénard, C. R. Chim. 2005, 8, 1093-1099.

[59] A. Döring, C. Schulzke, Dalton Trans. 2010, 39, 5623-5629.

[60] R. P. Van Duyne, C. R. Reilley, Anal. Chem. 1972, 44, 142-158.

[61] M. S. Shumann, I. Shain, Anal. Chem. 1969, 41, 1818.

[62] C. Amatore, J. Pinson, J. M. Savéant, J. Electroanal. Chem. 1982, 137, 143-146.

[63] a)E. D. Eastman, J. Am. Chem. Soc. 1928, 50, 292-297; b)E. D. Eastman, J. Am. Chem. Soc. 1926, 48, 1482-1493; c)A. J. de Bethune, T. S. Licht, N. Swedeman, J. Electrochem. Soc. 1959, 106, 616-625.

[64] A. J. Bard, L. R. Faulkner, Electrochemical Methods - Fundamentals and Applications, Vol. 2nd edn, New York, Chinchester, Weinheim, Brisbane, Singapore, Toronto, 2001.

[65] R. Pyykkö, Chem. Rev. 1988, 88, 563-594.

[66] S. A. Lerke, D. H. Evans, J. Am. Chem. Soc. 1995, 117, 11768-11772.

[67] R. R. Schrock, R. M. Kolodziej, A. H. Liu, W. M. Davis, M. G. Vale, J. Am. Chem. Soc. 1990, 112, 4338-4345.

[68] H. K. Joshi, J. H. Enemark, J. Am. Chem. Soc. 2004, 126, 11784-11785.

[69] J. W. Lauher, R. Hoffmann, J. Am. Chem. Soc. 1976, 98, 1729-1742.

[70] J. J. A. Cooney, M. A. Cranswick, N. E. Gruhn, H. K. Joshi, J. H. Enemark, Inorg. Chem. 2004, 43, 8110-8118.

[71] U. Ryde, C. Schulzke, K. Starke, J. Biol. Inorg. Chem. 2009, 14, 1053-1064.

[72] B. S. Lim, D. V. Formitchev, R. H. Holm, Inorg. Chem. 2001, 40, 4257-4262.

[73] M. J. Frisch, G. W. Trucks, H. B. Schlegel, G. E. Scuseria, M. A. Robb, J. R. Cheeseman, J. A. Montgomery, T. Vreven, K. N. Kudin, J. C. Burant, J. M. Millam, S. S. Iyengar, J. Tomasi, V. Barone, B. Mennucci, M. Cossi, G. Scalmani, N. Rega, G. A. Petersson, H. Nakatsuji, M. Hada, M. Ehara, K. Toyota, R. Fukuda, J. Hasegawa, M. Ishida, T. Nakajima, Y. Honda, O. Kitao, H. Nakai, M. Klene, X. Li, J. E. Knox, H. P. Hratchian, J. B. Cross, V. Bakken, C. Adamo, J. Jaramillo, R. Gomperts, R. E. Stratmann, O. Yazyev, A. J. Austin, R. Cammi, C. Pomelli, J. W. Ochterski, P. Y. 
Ayala, K. Morokuma, G. A. Voth, P. Salvador, J. J. Dannenberg, V. G. Zakrzewski, S. Dapprich, A. D. Daniels, M. C. Strain, O. Farkas, D. K. Malick, A. D. Rabuck, K. Raghavachari, J. B. Foresman, J. V. Ortiz, Q. Cui, A. G. Baboul, S. Clifford, J. Cioslowski, B. B. Stefanov, G. Liu, A. Liashenko, P. Piskorz, I. Komaromi, R. L. Martin, D. J. Fox, T. Keith, A. Laham, C. Y. Peng, A. Nanayakkara, M. Challacombe, P. M. W. Gill, B. Johnson, W. Chen, M. W. Wong, C. Gonzalez, J. A. Pople, Gaussian 03, Revision C.02, 2003.

[74] L. Onsager, J. Am. Chem. Soc. 1936, 58, 1486-1493.

[75] A. Klamt, G. Schuurmann, J. Chem. Soc. - Perkin Trans. 1993, 2, 799-803.

[76] S. Miertus, E. Scrocco, J. Tomasi, J. Chem. Phys. 1981, 55, 117-119.

[77] W.-M. Hoe, A. J. Cohen, N. H. Handy, Chem. Phys. Letters 2001, 341, 319-328.

[78] a)A. D. Becke, Phys. Rev. A 1988, 38; b)J. P. Perdew, Electronic Structure of Solids`91, Akademie Verlag, Berlin, 1991; c)A. D. Becke, J. Chem. Phys. 1993, 98, 5648-5650.

[79] a)C. Adamo, V. Barone, J. Chem. Phys. 1998, 108, 664-676; b)B. J. Lynch, Y. Zhao, D. G. Truhlar, J. Phys. Chem. A 2003, 107, 1384-1388.

[80] C. Adamo, V. Barone, J. Chem. Phys. 1999, 110, 6158-6163.

[81] J. P. Perdew, K. Burke, M. Ernzerhof, Phys. Rev. Lett. 1996, 77, 3865-3868.

[82] E. E. Dahlke, D. G. Truhlar, J. Phys. Chem. B 2005, 109, 15677-15683.

[83] R. H. Holm, Coord. Chem. Rev. 1990, 100, 183-221.

[84] B. E. Schultz, R. H. Holm, Inorg. Chem. 1993, 32, 4244-4248.

[85] B. E. Schultz, R. Hille, R. H. Holm, J. Am. Chem. Soc. 1995, 117, 827-828.

[86] J. Topich, J. T. Lyons, Inorg. Chem. 1984, 23, 3202-3206.

[87] M. S. Reynolds, J. M. Berg, R. H. Holm, Inorg. Chem. 1984, 23, 3057-3062.

[88] a)R. H. Holm, J. M. Berg, Acc. Chem. Res. 1986, 19, 363-370; b)B. E. Schultz, S. F. Gheller, M. C. Muetterties, M. J. Scott, R. H. Holm, J. Am. Chem. Soc. 1993, 115, 2714-2722.

[89] A. Majumdar, K. Pal, S. Sarkar, J. Am. Chem. Soc. 2006, 128, 4196-4197.

[90] H. H. Szmant, O. Cox, J. Org. Chem. 1966, 31, 1595-1598.

[91] J. Yadav, S. K. Das, S. Sarkar, J. Am. Chem. Soc. 1997, 119, 4315-4316.

[92] N. Ueyama, H. Oku, A. Nakamura, J. Am. Chem. Soc. 1992, 114, 7310-7311.

[93] K. Tatsumi, R. Hoffmann, Inorg. Chem. 1980, 19, 2656-2658.

[94] a)R. H. Holm, Chem. Rev. 1987, 87, 1401-1449; b)H. Taube, ACS Symp. Ser. 1982, 198, 151-179. 
[95] a)H. Oku, N. Ueyama, A. Nakamura, Chemistry Letters 1996, 25, 1131-1132; b)J. Jiang, R. H. Holm, Inorg. Chem. 2005, 44, 1068-1072.

[96] A. Thapper, R. Deeth, E. Nordlander, Inorg. Chem. 2002, 41, 6695-6702.

[97] a)C. E. Webster, M. B. Hall, J. Am. Chem. Soc. 2001, 123, 5820-5821; b)J. P. McNamara, I. H. Hillier, T. S. Bhachu, C. D. Garner, Dalton Trans. 2005, 3572-3579.

[98] H. Lineweaver, D. Burk, J. Am. Chem. Soc. 1934, 56, 658-667.

[99] a)C. S. Hanes, Biochemical Journal 26 1932, 1406-1421; b)J. T.-F. Wong, W. Thompson, Biographical Memories of Fellows of the Royal Society 1994, 39, 149155.

[100] a)L. Bourget-Merle, M. F. Lappert, J. R. Severn, Chem. Rev. 2002, 102, 3031-3065; b)M. Rahim, N. J. Taylor, S. Xin, S. Collins, Organometallics 1998, 17, 1315-1323; c)W. E. Piers, D. J. H. Emslie, Coord. Chem. Rev. 2002, 233-234, 131-151; d)H. W. Roesky, S. Singh, V. Jancik, V. Chandrasekhar, Acc. Chem. Res. 2004, 37, 969-981; e)D. J. Mindiola, Acc. Chem. Res. 2006, 39, 813-821; f)C. J. Cramer, W. B. Tolman, Acc. Chem. Res. 2007, 40, 601-608; g)P. L. Holland, Acc. Chem. Res. 2008, 41, 905914.

[101] R. H. Holm, G. W. Everett Jr., A. Chakravorty, Prog. Inorg. Chem. 1966, 7, 83-214.

[102] a)P. L. Holland, W. B. Tolman, J. Am. Chem. Soc. 1999, 121, 7270-7271; b)D. W. Randall, S. D. George, P. L. Holland, B. Hedman, K. O. Hodgson, W. B. Tolman, E. I. Solomon, J. Am. Chem. Soc. 2000, 122, 11632-11648.

[103] J. P. Smit, W. Purcell, A. Roodt, J. Leipoldt, Polyhedron 1993, 12, 2271-2277.

[104] A. Roodt, S. S. Basson, J. Leipoldt, Polyhedron 1994, 13, 599-607.

[105] a)J. van de Poel, H. M. Neumann, Inorg. Synth. 1968, 11, 53-54; b)J. Leipoldt, L. D. C. Bok, P. J. Cilliers, Z. Anorg. Allgem. Chem. 1974, 409, 343-346.

[106] a)H. Baadsgaard, W. D. Treadwell, Helvetica Chimica. Acta. 1955, 201, 1669-1679; b)E. A. Heintz, Inorg. Synth. 1963, 7, 142-146.

[107] F. Stoffelbach, D. Saurenz, R. Poli, Eur. J. Inorg. Chem. 2001, 10, 2699-2703.

[108] C. Persson, C. Andersson, Inorg. Chemica. Acta. 1993, 3, 235-238.

[109] G. R. Willey, T. J. Woodman, M. G. B. Drew, J. Organomet. Chem. 1996, 510, $213-$ 217.

[110] V. Kolesnichenko, D. C. Swenson, L. Messerie, Inorg. Chem. 1998, 37, 3257-3262.

[111] I. W. Boyd, I. G. Dance, K. S. Murray, A. G. Wedd, Aust. J. Chem. 1978, 31, 278-284.

[112] J. W. McDonald, F. G. D., L. D. Rosenhein, W. E. Newon, Inorg. Chim. Acta. 1983, 72, 205-209. 
[113] V. C. Gibson, T. P. Kee, A. Shaw, Polyhedron 1988, 7, 579-580.

[114] A. J. Nielson, R. A. Andersen, Inorg. Synth. 1985, 23, 195-199.

[115] C. A. Rice, C. G. Benson, C. A. McAuliffe, Inorg. Chim. Acta. 1982, 59, 33-39.

[116] M. Draganjac, E. Simhon, L. T. Chan, M. Kanatzidis, N. C. Baenziger, D. Coucouvanis, Inorg. Chem. 1982, 21, 3321-3324.

[117] D. P. Tate, W. R. Knipple, J. M. Augl, Inorg. Chem. 1962, 2, 433-434.

[118] G. N. Schrauzer, V. P. Mayweg, J. Am. Chem. Soc. 1965, 87, 1483-1486.

[119] R. Adams, W. Reifschneider, A. Ferretti, Org. Synth. 1962, 42, 22-25.

[120] C. M. Fendrick, L. D. Schertz, E. A. Mintz, T. J. Marks, Inorg. Synth. 1992, 29, 193198.

[121] V. T. Kamble, V. S. Jamode, N. S. Joshi, A. V. Biradar, R. Y. Deshmukh, Tetrahedron Lett. 2006, 47, 5573-5575.

[122] B. A. Jazdzewski, P. L. Holland, M. Pink, C. G. Young, D. J. E. Spencer, W. B. Tolmann, Inorg. Chem. 2001, 40, 6097-6107.

[123] G. M. Sheldrick, SHELXS-97, Program for Crystal Structure Solution 1997, Universität Göttingen.

[124] G. M. Sheldrick, SHELXL-97, Program for Crystal Structure Refinement 1997, Universität Göttingen.

[125] G. M. Sheldrick, Acta Crystallogr. Sect. A 2008, 64, 112-122.

[126] C. Lee, W. Yang, R. G. Parr, Phys. Rev. B 1988, 37, 785-789.

[127] P. J. Hay, W. R. Wadt, J. Chem. Phys. 1985, 82, 270-273.

[128] N. D. Lowe, C. D. Garner, Dalton Trans. 1993, 2197-2207.

[129] D. Wöhrle, M. Eskes, K. Shigehara, A. Yamada, Synthesis 1993, 194-196.

[130] A. K. Bhattacharya, A. G. Hortmann, J. Org. Chem. 2008, 1, 95-97.

[131] a)D. E. Ward, M. Sales, C. C. Man, J. Shen, P. K. Sasmal, C. Guo, J. Org. Chem. 2002, 67, 1618-1629; b)G. N. Rule, M. R. Detty, J. E. Kaeding, J. A. Sinicropi, J. Org. Chem. 1995, 60, 1665-1673.

[132] H. G. O. Becker, W. Berger, G. Domschke, E. Fanghänel, J. Faust, M. Fischer, F. Gentz, K. Gewald, R. Gluch, R. Mayer, K. Müller, D. Pavel, H. Schmidt, K. Schollberg, E. Seiler, G. Zeppenfeld, in Organikum, Vol. 21., Wiley-VCH, Weinheim, New York, Chichester, Brisbane, Singapore, Toronto, 2001. 


\section{Danksagung}

Meiner Doktormutter Prof. Dr. Carola Schulzke danke ich für die Möglichkeit, diese Arbeit in ihrem Arbeitskreis durchführen zu dürfen und die vollkommende Freiheit bei diesem Forschungsthema und die jederzeitige Diskussionsbereitschaft in Göttingen sowie in Dublin.

Außerdem möchte ich mich bei Prof. Dr. Oliver Wenger für die die kurzfristige Übernahme des Zweitreferendariats bedanken und dass ich ihn in mein „Thesis Committee aufnehmen durfte.

Prof. Franc Meyer danke ich für die Hilfestellungen während meiner Doktorarbeitszeit.

Ich möchte mich außerdem bei allen Mitgliedern der Prüfungskommission Prof. Dr. CAROLA Schulzke, Prof. Dr. Oliver Wenger, Prof. Dr. Andreas Pack, Jun.-Prof. Dr. Christian Ducho, Prof. Dr. Franc Meyer und Jun.-Prof. Guido Clever recht herzlich für die Teilnahme an meiner Disputation und das Interesse an meiner Arbeit bedanken.

Ich möchte mich ausdrücklich bei meinen Mitstreitern im Arbeitskreis bedanken: Carlos Enrique Abad, Prinson P. Samuel, Gunther Speichert, Muxin Han, Vanessa Brake, Dr. Monika Heinz, Dr. Kerstin Starke, Dr. Quingwai Zhang und Dr. Xiaoli Ma ohne eure Anwesenheit wäre der Laboralltag sehr eintönig gewesen. Besonders hier herausheben möchte ich Carlos und Prinson. Mit Euch habe ich die längste und schönste Zeit dieser Promotion verbracht, sowie auch viele Stunden außerhalb des Labors.

Gunther Speichert danke ich recht herzlich für die Vorarbeiten die er Dublin vor meinen „Messtagen“ getätigt hat. Außerdem für die schöne Zeit mit ihm in Göttingen und die Durchhaltekraft bei meinen langen Messungen in Dublin.

Ich möchte außerdem allen Mitarbeitern Dr. Anukul Jana, Dr. Sarish Sankaranarayana Pillai, Dr. Bijan Nekoueishahraki, Dr. Gaŝper Tavcar, Dr. Jianfeng Li, Dr. Rajendra S. Ghadwal, Dr. Shabana Khan, Dr. Azhakar Ramachandran, Dr. Arun Vasudevan Nair, Dr. Sakya S. Sen und Dr. Zhensheng Zhang des Arbeitskreises von Prof. Roesky danken, mit denen ich aufgrund der Benutzung des gleichen Labors ebenfalls viel Zeit verbracht habe. 
Besonders möchte ich mich bei meinen Freunden Sakya, Prinson und Sarish bedanken. Mit euch hatte ich auch außerhalb des Labors viel Spaß. Dank Euch ist mir die indische Kultur ein wenig näher gebracht worden.

Außerdem möchte ich auch den anderen Personen danken, die mit mir im Praktikumstrakt ihre Forschung bestritten haben. Dr. Animesh Das, Thomas Bayer, Stefan Fischer, Torben Böhnisch, die immer für einen kleinen Plausch auf dem Gang zu haben waren.

Für die gemeinsame Zeit im Chemiepraktikum für Biologen möchte ich mich außerdem bei allen Assistenten bedanken, sowie den neuen Leitern am Ende des Praktikums Prof. Oliver Wenger und Jun.-Prof. Guido Clever.

Dr. Noltemeyer danke ich für die gute Zusammenarbeit im Biologenpraktikum und die Hilfe bei manchen Kristallographischen Problemen.

Mathias Hesse danke ich für die jederzeitige schneller Besorgung und Entsorgung der benötigten Chemikalien, auch außerhalb der Öffnungszeiten seiner Chemikalienausgabe, sowie nette Gespräche über Gott und die Welt.

Den Mitarbeitern der NMR-Abteilung Dr. Michael John, Wolfgang Zolke und Ralf Schöne danke ich recht herzlich für die Messungen meiner Proben und die Hilfe bei anstehenden Fragen.

Den Mitarbeitern der Massenspektrometrie danke ich recht herzlich, da besonders Thomas Schuchardt und Jörg Schöne durch die vielen Messungen den entscheiden Hinweis bei vielen Verbindungen gegeben haben.

Dem Analytischen Labor und seinen Mitarbeitern Angelika Wraage, Susanne Petrich und Diana Kumpart danke ich für die Messungen der Elementaranalysen

Den Mitarbeitern der Elektrowerkstätten sowie Mechanikwerkstätten bin ich zu Dank verpflichtet. Ohne die Bereitstellung der funktionsfähigen Geräte wäre diese ganz Arbeit nicht möglich gewesen. Sowie den Mitarbeitern der Glasbläserei unter Leitung von Hans-Joachim Schlette.

Frau Dr. A. Claudia Stückl danke ich für die Messung der ESR-Proben und die vielen Versuche bis zu den erhaltenen Spektren. Dr. Serhiy Demeshko danke ich für die Messung der SQUID-Proben. Dr. Sebastian Dechert danke ich für die vielen Hilfestellungen bei Problemen mit dem Diffraktometer. 
Dr. Rainer Oswald danke ich die Beantwortung meiner Fragen in den Anfängen meiner DFTRechnungen.

Ich möchte mich bei allen Abteilungspraktikanten (Anna Noll, Muxin Han, Lin Wu, Jing Zhou, Yue Qiu, Stefanie Wohnig, Alexander Walter, Diana Petersen, Philip Lottmann, Daniela Diedrich, Edna Ofosua-Nyarko und Julia Kaup) bedanken, die mich mal mehr, mal weniger erfolgreich bei meinen Arbeiten unterstützt haben.

Ich danke für die moralische Unterstützung meiner Familie und für Aufmunterung in manchmal schweren Zeiten. Abschließend möchte ich mich noch bei meiner Verlobten Kerstin bedanken ohne Dich diese ganze Arbeit nicht möglich gewesen wäre und entschuldige mich für die vielen Entbehrungen die durch die Uniarbeit in den letzten drei Jahren entstanden sind. 


\section{Liste der wissenschaftlichen Beiträge}

\section{Publikationen}

[1] Anukul Jana, Herbert W. Roesky, Carola Schulzke, Alexander Döring „Reactions of Tin(II) Hydride Species with Unsaturated Molecules“ Angew. Chem. Int. Ed. 48, 2009, 1106-1109

[2] Anukul Jana, Herbert W. Roesky, Carola Schulzke, Alexander Döring, Tobias Beck, Aritra Pal, Regine Herbst-Irmer "Facile Access of Stable Divalent Tin Compounds with Terminal Methyl, Amide, Fluoride and Iodide Substituents" Inorg. Chem. 48, 2009, 193-197

[3] Jianfeng Li, Sebastian Merkel, Julian Henn, Kathrin Meindl, Alexander Döring, Herbert W. Roesky, Rajendra S. Ghadwal, Dietmar Stalke „Lewis-Base-Stabilized Dichlorosilylene: A Two-Electron $\sigma$-Donor Ligand“ Inorg. Chem. 49, 2010, 775-777

[4] Anukul Jana, Sarish Sankaranarayana Pillai, Herbert W. Roesky, Carola Schulzke, Alexander Döring, Michael John "Facile Access of Well-Defined Stable Divalent Lead Compounds with Small Organic Substituents" Organometallics 28, 2009, 2563-2567

[5] Zhensheng Zhang, Herbert W. Roesky., Thomas Schulz, Dietmar Stalke, Alexander Döring „A Chlorine-Centered Cluster of Composition [ $\left(\mathrm{Me}_{3} \mathrm{Si}\right)_{2} \mathrm{NC}(\mathrm{NCy})_{2}$ $\left.\mathrm{SmCl}_{2}\right]_{5}(\mathrm{thf})_{2}$ and a Comparison with Heavier Ytterbium Congener $\left[\mathrm{Me}_{3} \mathrm{Si}\right)_{2}$ $\left.\mathrm{NC}(\mathrm{NCy})_{2} \mathrm{YbCl}_{2}\right]_{2}(\mathrm{LiCl})_{2}(\mathrm{thf})_{4}$ " Eur. J. Inorg. Chem. 2009, 4864-4869

[6] Jianfeng Li, Carola Schulzke, Sebastian Merkel, Herbert W. Roesky, Prinson P. Samuel, Alexander Döring, Dietmar Stalke "Synthesis and Characterization of N-neterocyclic Carbene Complexes of Titanium (IV) and Titanium (III)" Z. Anorg. Allg. Chem. 636, 2010, 511-514

[7] Anukul Jana, Herbert W. Roesky, Carola Schulzke, Prinson P. Samuel, Alexander Döring "Synthesis and Reaction of Monomeric Germanium(II) and Lead(II) Dimethylamide and the Synthesis of Germanium(II) Hydrazide by Clevage of one N-H bond of Hydrazine" Inorg. Chem. 49 2010, 5554-5559

[8] Alexander Döring, Carola Schulzke "Tungsten's redox potential is more temperature sensitive than that of molybdenum" Dalton Trans. 39, 2010, 5623-5629 (overpage)

[9] Alexander Döring, Carola Schulzke, Qingwei Zhang Synthesis, characterization and structure analysis of isostructural dinuclear molybdenum and tungsten oxo-bis- $\mu$ sulfido-benzenedithiolene complexes" Inorg. Chim. Acta. 2010, 4140-4144

[10] Kajsa G. V. Havelius, Stefan Reschke, Sebastian Horn, Alexander Döring, Dimitri Niks, Russ Hille, Carola Schulzke, Silke Leimkühler, Michael Haumann "The structure of the molybdenum site in YedY, a sulfite oxidase homologue from Escherichia coli" 2010, Inorg. Chem. akzeptiert 
[11] Alexander Döring, Prinson P. Samuel, Carola Schulzke, "Influence of hetero atoms in the ring in the dithiolene complexes of molybdenum and tungsten" in Bearbeitung

[12] Alexander Döring, Claudia A. Stückl, Carola Schulzke „EPR-studies of dithiolene complexes of molybdenum and tungsten compounds". in Bearbeitung

[13] Alexander Döring, Carola Schulzke "Temperature dependent catalytic reactions of molybdenum and tungsten dithiolens" in Bearbeitung

\section{Vorträge}

Alexander Döring, Carola Schulzke Vergleichende elektrochemische Untersuchungen an Molybdän und Wolframverbindungen 1. Göttinger Chemieforum (Juli 2007)

Alexander Döring, Carola Schulzke Vergleichende Temperaturabhängige elektrochemische Untersuchungen an Paaren von Molybdän- und Wolframverbindungen, 11. Norddeutsches Doktoranden Kolloquium der Anorganischen Chemie 2008, Burg Warberg, Braunschweig (September 2008)

Alexander Döring, Carola Schulzke Vergleichende Untersuchungen an Molybdän- und Wolframverbindungen, 6. Koordinationschemikertreffen 2010, Mainz (Februar 2010)

\section{Posterpräsentationen}

Alexander Döring, Carola Schulzke Vergleichende elektrochemische Untersuchungen von Molybdän und Wolframverbindungen, 10. Norddeutsches Doktoranden Kolloquium 2007, Bremen (September 2007)

Alexander Döring, Carola Schulzke Vergleichende Untersuchungen an Molybdän- und Wolframverbindungen, 3. Göttingen Chemieforum (Juli 2009)

Alexander Döring, Carola Schulzke Katalytische Aktivitäts Untersuchungen von Molybdänund Wolframverbindungen, 12. Norddeutsches Doktoranden Kolloquium 2009, Rastede, Oldenburg (Oktober 2009) 


\section{Lebenslauf}

Name:

Geburtsdatum:

Geburtsort:

Staatsangehörigkeit:

Werdegang:

$1991-1993$
$1993-1997$

Juni 1997

$1997-2000$

Juni 2000

$2000-2001$

Juni 2001

Oktober 2001

Juli $2006-$

Mai 2007

Mai 2007-

$2001-2005$

April 2007-

September 2010

Beschäftigungen:
Besuch der Ernst-Leinius Schule (Grundschule) in Kassel

Besuch der Förderstufe Harleshausen in Kassel

Besuch der Heinrich-Schütz-Schule (Gesamtschule-Sekundarstufe I) in Kassel

Realschulabschluss

Besuch der Elisabeth-Knipping-Schule. Schwerpunkt Chemietechnik. Berufliches Gymnasium in Kassel. mit den Leistungskursen Chemie und Biologie

Allgemeine Hochschulreife

Besuch des 14. Schuljahres der ElisabethKnipping-Schule mit der Ausbildung zum Chemisch-Technischen-Assistenten

Staatlich anerkannter Chemisch-TechnischerAssistent

Beginn des Studiengangs Diplomchemie an der Georg-August Universität in Göttingen

Diplomarbeit bei JP Dr. Carola Schulzke mit dem Thema: Elektrochemische Untersuchungen von Molybdän- und Wolframkomplexen an der Georg-August Universität in Göttingen

Beginn der Promotion bei JP Dr. Carola Schulzke mit dem Thema: Verbindunggen von Molybdän und Wolfram in den Oxidationsstufen IV - VI als Modelle für Molybdän- und Wolfram-Cofaktoren

Angestellter im Analytischen Labor Dr. Alfons Wüsteneck in Felsberg-Altenburg

Wissenschaftlicher Mitarbeiter im Institut für Anorganische Chemie 